\title{
Tribological and Electrochemical Corrosion Behaviours of Titanium Nitride and Chromium Nitride Based PVD Coating Systems
}

\author{
by \\ Feng Cai \\ M.A.Sc., Carleton University, 2005
}

A thesis submitted to the Faculty of Graduate and Postdoctoral Affairs in partial fulfillment of the requirements for the degree of

Doctor of Philosophy

Ottawa-Carleton Institute for Mechanical and Aerospace Engineering

Department of Mechanical and Aerospace Engineering

\author{
Carleton University \\ Ottawa, Ontario \\ Canada
}

April 2011

C 2011, Feng Cai 


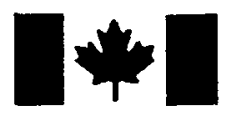

Library and Archives

Canada

Published Heritage

Branch

395 Wellington Street Ottawa ON K1A ON4 Canada
Bibliothèque et

Archives Canada

Direction du

Patrimoine de l'édition

395 , rue Wellington

Ottawa ON K1A ON4

Canada

Your file Votre référence

ISBN: 978-0-494-81545-8

Our file Notre référence

ISBN: $978-0-494-81545-8$

NOTICE:

The author has granted a nonexclusive license allowing Library and Archives Canada to reproduce, publish, archive, preserve, conserve, communicate to the public by telecommunication or on the Internet, loan, distribute and sell theses worldwide, for commercial or noncommercial purposes, in microform, paper, electronic and/or any other formats.

The author retains copyright ownership and moral rights in this thesis. Neither the thesis nor substantial extracts from it may be printed or otherwise reproduced without the author's permission.
AVIS:

L'auteur a accordé une licence non exclusive permettant à la Bibliothèque et Archives Canada de reproduire, publier, archiver, sauvegarder, conserver, transmettre au public par télécommunication ou par l'Internet, prêter, distribuer et vendre des thèses partout dans le monde, à des fins commerciales ou autres, sur support microforme, papier, électronique et/ou autres formats.

L'auteur conserve la propriété du droit d'auteur et des droits moraux qui protège cette thèse. $\mathrm{Ni}$ la thèse ni des extraits substantiels de celle-ci ne doivent être imprimés ou autrement reproduits sans son autorisation.
In compliance with the Canadian Privacy Act some supporting forms may have been removed from this thesis.

While these forms may be included in the document page count, their removal does not represent any loss of content from the thesis.
Conformément à la loi canadienne sur la protection de la vie privée, quelques formulaires secondaires ont été enlevés de cette thèse.

Bien que ces formulaires aient inclus dans la pagination, il n'y aura aucun contenu manquant. 
The undersigned recommend to

the Faculty of Graduate and Postdoctoral Affairs

acceptance of the thesis

Tribological and Electrochemical Corrosion Behaviours of Titanium Nitride and Chromium Nitride Based PVD Coating Systems

\author{
Submitted by Feng Cai \\ M.A.Sc. \\ In partial fulfillment of the requirements for the degree of \\ Doctor of Philosophy
}

Dr. Xiao Huang, Thesis Supervisor

Dr. Qi Yang, Thesis Co-supervisor

Dr. John Rodgers, External Examiner

Dr. Metin I. Yara, Chair, Department of Mechanical and Aerospace Engineering

Carleton University

2011 


\title{
To parents
}

Guizhen Tan and Lianhai Cai

\author{
with love
}




\section{Abstract}

This thesis investigates the effects of deposition process and microstructure on tribological properties and corrosion behaviour of various coating systems. The coating systems studied were electroplated and physical vapor deposited (PVD) using electron beam (EB), cathodic arc (CA) and plasma enhanced magnetron sputtering (PEMS). Several coating compositions were also studied, including nickel, nickel with carbon nano-tubes, TiN, $\mathrm{CrN}$ and $\mathrm{CrSiCN}$. Post-deposition treatments with polymethyl methacrylate (PMMA) were applied to the TiN coating systems to modify and study the corrosion behaviours with respect to the effect of defects.

All coating samples were evaluated for their composition, microstructure and surface morphology. The coating mechanical properties of hardness and Young's modulus were also studied to investigate their correlation to wear and erosion. Potentiodynamic polarization and electrochemical impedance spectroscopy (EIS) tests were conducted to investigate the electrochemical corrosion behaviours of the PVD coating systems. Equivalent electrical circuit (EEC) models were then applied to analyze the relationship between electrochemical impedance and the key diffusion governing factors, i.e., diffusion coefficient and diffusion layer thickness. The correlations between these electrochemical factors and the coating microstructure were analyzed.

In this study, it was observed that in addition to hardness $(\mathrm{H})$ and Young's modulus (E), the $\mathrm{H}^{3} / \mathrm{E}^{2}$ ratio is an important parameter relating to the wear and erosion rates. In wear 
behaviour, the $\mathrm{H}^{3} / \mathrm{E}^{2}$ ratio is inversely proportional to the specific wear rate. In erosion behaviour, at low impingement angles, higher $\mathrm{H}^{3} / \mathrm{E}^{2}$ ratios relate to lower erosion rates; whereas at high impingement angles, higher $\mathrm{H}^{3} / \mathrm{E}^{2}$ ratios relate to higher erosion rates.

This study also found that in a coating system the effective diffusion coefficient is proportional to the coating porosity; and the diffusion layer thickness on substrate would be increased by the presence of a sufficient coating thickness. Thus coating defects and coating thickness are the two essential factors governing the electrochemical corrosion in a coating/substrate system. 


\section{Acknowledgements}

I express my sincere gratitude to my supervisor Dr. Xiao Huang, who continuously encouraged me to take more academic challenges. It was her leadership that initialized the key idea which led to the major finding in this work. I want to thank her for her

patience and support during the period of this academic pursuit. I believe that these experiences will benefit my life in future.

I would also express great thanks to my co-supervisor Dr. Qi Yang. His important and valuable advice in experimental research methodologies provided a solid guidance for this research endeavour. I believe that his help will continually benefit me in my career.

I thank the Institute for Aerospace Research of the National Research Council of Canada, where I conducted experimental studies for this thesis.

I also thank all the people who provided help, gave advice and showed encouragement to me during this journey.

I feel grateful to be able to complete the writing of this thesis at the beautiful seaside village of Port Jefferson in Long Island, New York.

Finally, I am deeply moved by the unbending love of my parents, their constant encouragement and support in difficult times. In the very depths of my heart, I owe the completion of this work to their greatest love. Thank You!

Feng Cai

Carleton University, Ottawa, Canada 


\section{Table of Contents}

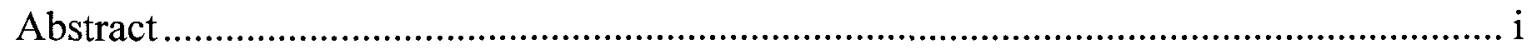

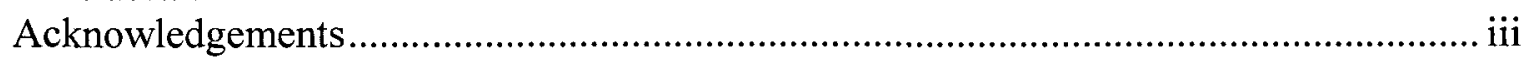

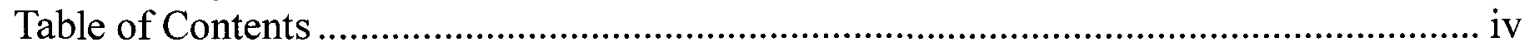

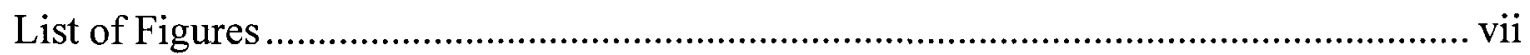

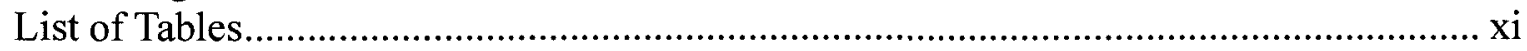

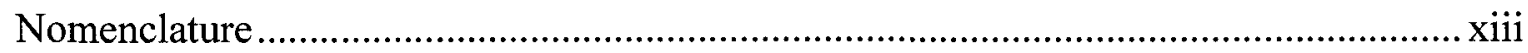

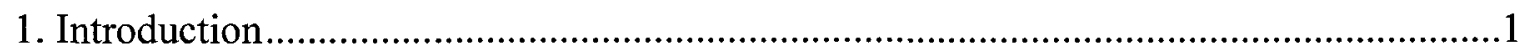

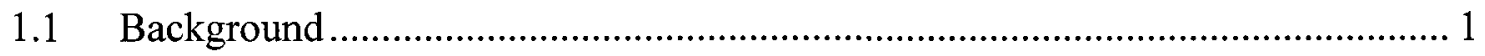

1.2 Development of Surface Engineering in Protective Applications ...................... 2

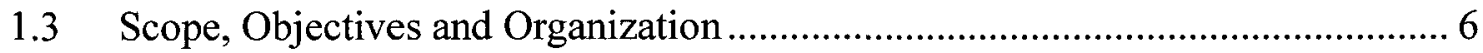

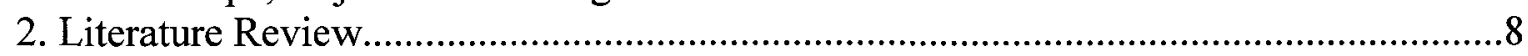

2.1 Metal Component Surface Degradation....................................................... 8

2.2 Degradation Mechanisms..................................................................... 9

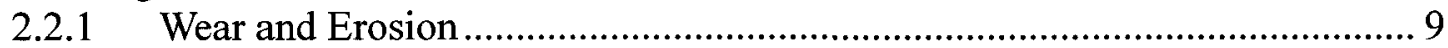

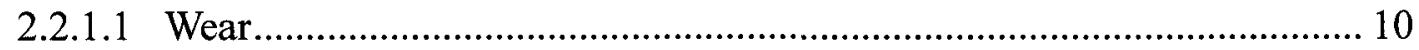

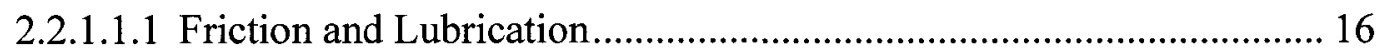

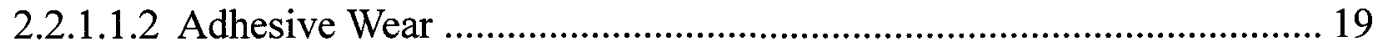

2.2.1.1.3 Abrasive Wear ................................................................................. 20

2.2.1.1.4 Models for Predicting Abrasive Wear ............................................ 22

2.2.1.1.5 Abrasive Wear Test...................................................................... 24

2.2.1.2 Erosive Damage .......................................................................... 26

2.2.1.2.1 Types of Erosion and Mechanism ............................................... 26

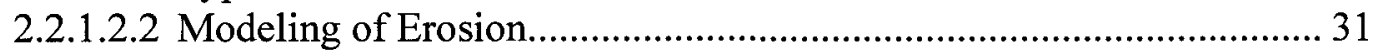

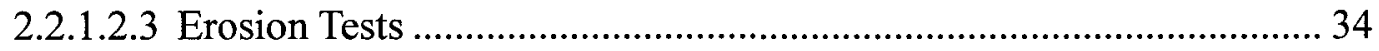

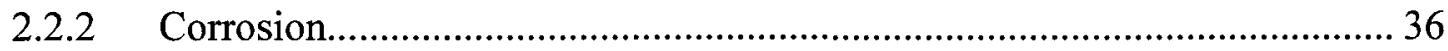

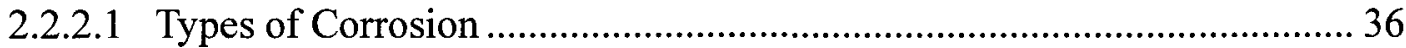

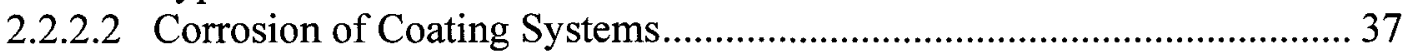

2.2.2.3 Electrochemical Corrosions ....................................................... 40

2.2.2.4 Experimental Approaches to Studying Corrosion Properties................... 46

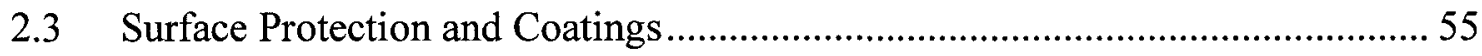

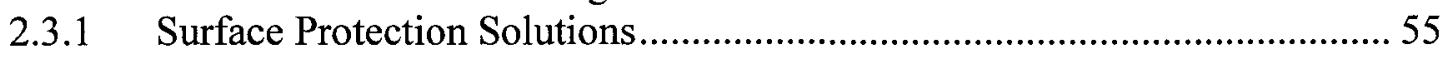

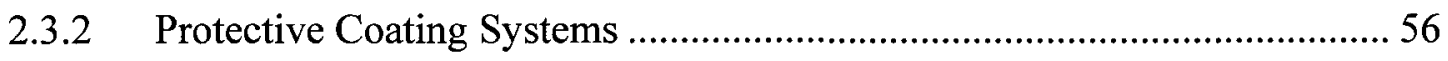

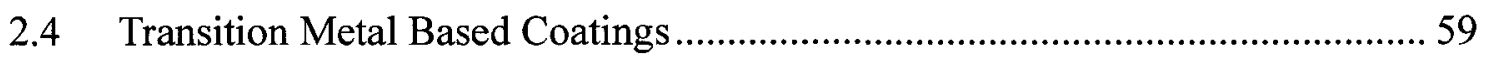

2.4.1 Transition Metals and Their Compounds............................................... 59

2.4.2 Development in Transition Metal Based Coatings ................................. 61

2.4.2.1 Coating Composition Development ................................................. 61

2.4.2.2 Layered Coating Structure and New Microstructure Development ......... 64 
2.4.3 Transition Metals and Compounds: Processes and Properties...................... 73

2.4.3.1 Coating Deposition Processes .................................................................. 73

2.4.3.1.1 Aqueous Deposition: Electroplating .................................................. 74

2.4.3.1.2 Physical Vapor Depositions................................................................. 76

2.4.3.2 Coating Microstructure and Properties................................................... 82

2.4.3.2.1 Effect of Coating Microstructure …………....................................... 82

2.4.3.2.2 Coating Mechanical Properties ............................................................ 84

2.4.3.2.3 Wear Behaviours of Transition Metal Nitrides and Carbides.............. 89

2.4.3.2.4 Erosion Behaviours of Transition Metal Nitrides and Carbides ......... 98

2.4.3.2.5 Corrosion Behaviours of Transition Metal Nitrides............................ 105

2.4.3.2.6 Coating Defects and Their Impacts ................................................... 114

2.4.3.2.7 Correlations between Coating Properties........................................... 121

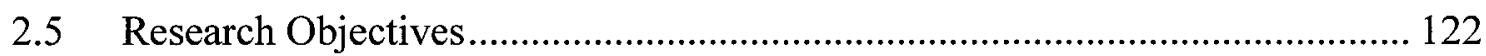

3. Materials and Experimental Procedures ....................................................................125

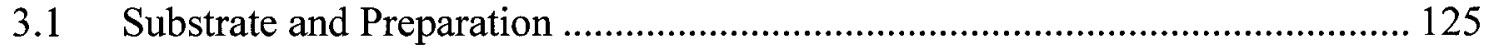

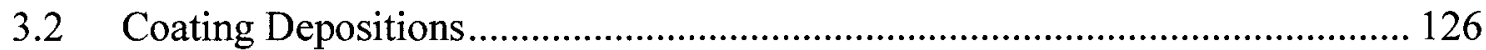

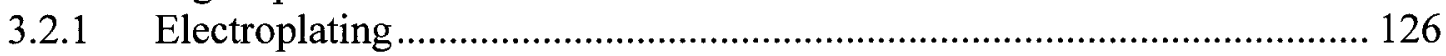

3.2.2 Electrolyte Preparation......................................................................... 129

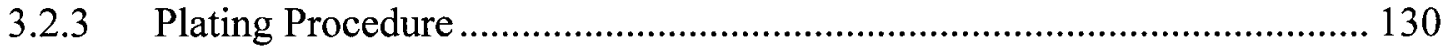

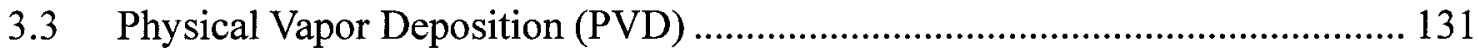

Electronic Beam (EB) Evaporation Deposition..................................................... 131

Cathodic Arc (CA) Deposition............................................................................... 131

Plasma Enhanced Magnetron Sputtering (PEMS) Deposition ................................. 132

3.4 Post Deposition Treatment ............................................................................... 133

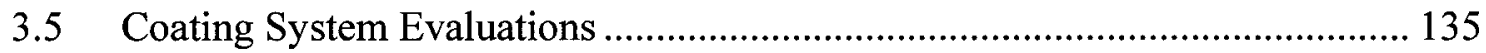

3.5.1 Coating Thickness Measurement .............................................................. 135

3.5.2 Scanning Electron Microscope (SEM) Analyses .......................................... 137

3.5.3 X-Ray Diffraction (XRD) Analysis ....................................................... 138

3.5.4 Mechanical Properties......................................................................... 138

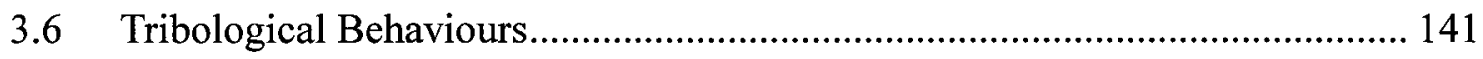

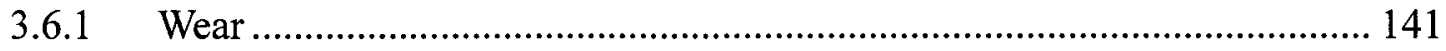

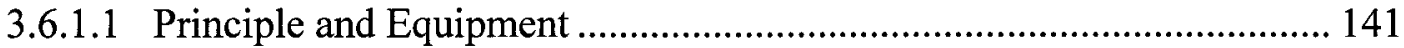

3.6.1.2 Data Processing …………................................................................ 144

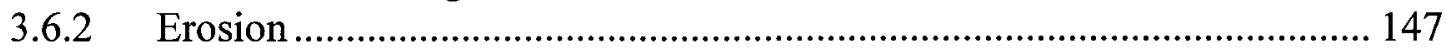

3.6.2.1 Principle and Equipment .................................................................. 147

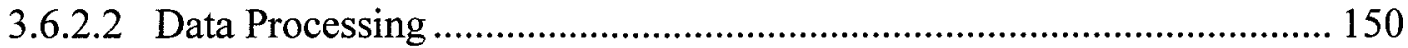

3.7 Corrosion Behaviours of Coating-substrate Systems ....................................... 151

3.7.1 Experimental Setup.............................................................................. 152

3.7.2 Operational Procedures.......................................................................... 154

3.7.3.1 Potentiodynamic Polarization Test .......................................................... 154

3.7.3.2 Electrochemical Impedance Spectroscopy (EIS) Test.............................. 154

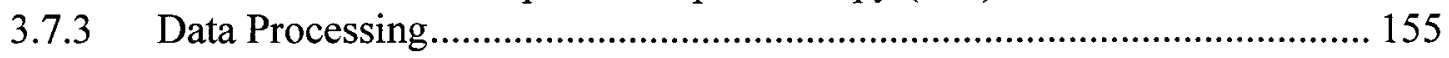

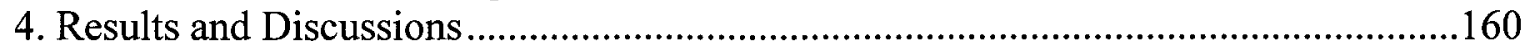


4.1 Coating Characteristics and Properties ......................................................... 160

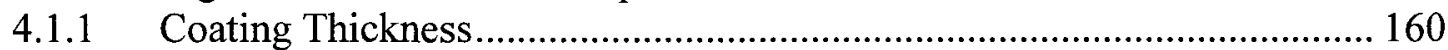

4.1.2 Scanning Electron Microscope (SEM) Analysis........................................ 163

4.1.3 X-Ray Diffraction (XRD) Analysis ......................................................... 183

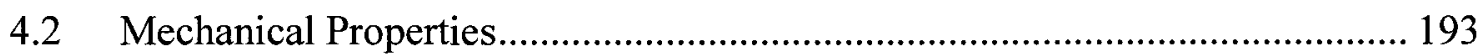

4.3 Coating System Tribological Behaviours .................................................. 199

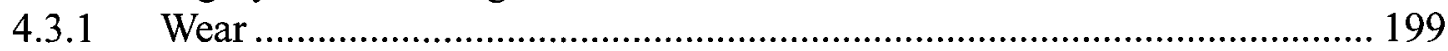

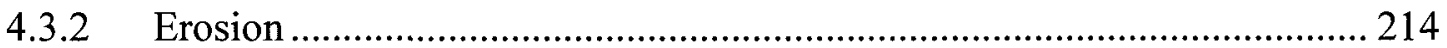

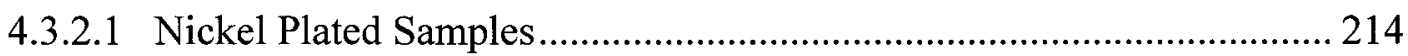

4.3.2.2 Physical Vapor Deposition (PVD) Coatings......................................... 217

4.3.3 Correlations between $\mathrm{H}^{3} / \mathrm{E}^{2}$ Ratio and Tribological Properties of PVD

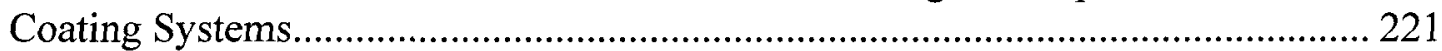

4.4 Coating System Corrosion Behaviours..................................................... 222

4.4.1 Potentiodynamic Polarization Tests ........................................................ 223

4.4.2 Electrochemical Impedance Spectroscopy (EIS) .................................... 232

4.4.3 Scanning Electron Microscope (SEM) Analyses after Corrosion Tests .... 260

4.4.4 Electrical Equivalent Circuit (EEC) Analysis.......................................... 278

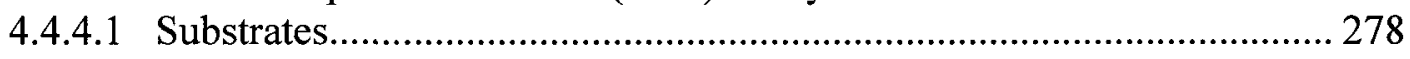

4.4.4.2 Titanium Nitride (TiN) Coating Systems .............................................. 288

4.4.4.3 Chromium Nitride Based Coating Systems.............................................. 300

4.4.4.4 Diffusion and Coating System Corrosion Resistance ............................. 306

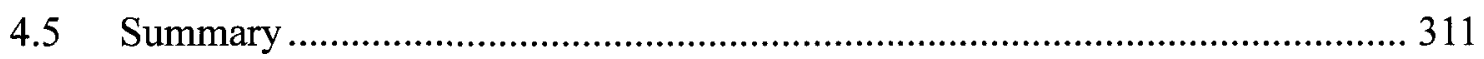

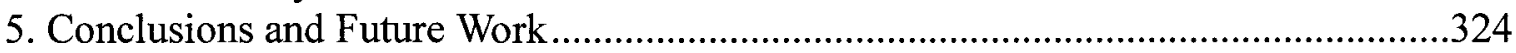

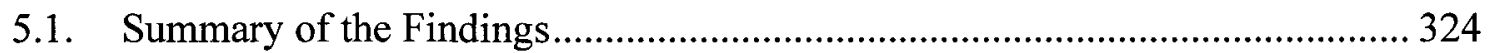

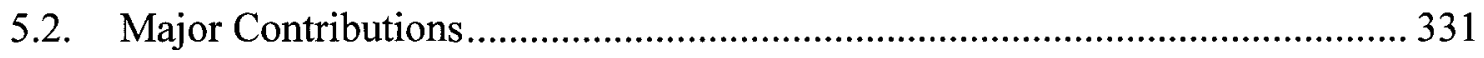

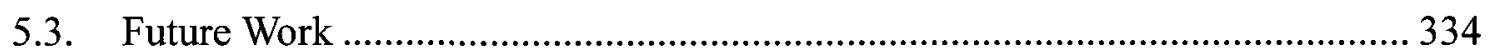

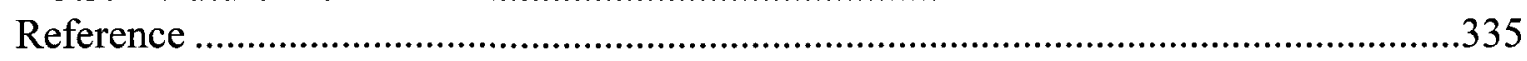

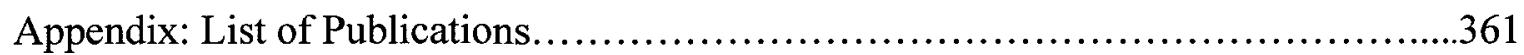




\section{List of Figures}

Figure 1-1 The development of superalloys and heat resistant coating systems. .............. 4

Figure 2-1 Erosive and corrosive damages on components............................................ 9

Figure 2-2 Wear and wear rate behaviour as a function of time, number of over-rolling or sliding distance

Figure 2-3 The change of the coefficient of friction and a roughness as a function of time, number of over rolling or sliding distance of a contact under constant operating conditions.

Figure 2-4 Wear tracks on PVD CrN coatings. ............................................................ 13

Figure 2-5 SEM images of processes of abrasive wears................................................. 21

Figure 2-6 Schematic illustration of various types of abrasive wear tests........................ 25

Figure 2-7 Mechanisms of erosions for ductile and brittle materials. .............................. 27

Figure 2-8 Erosion resistances vs. impingement angle................................................... 29

Figure 2-9 Erosion rate as a function of particle kinetic energy .................................... 30

Figure 2-10 Schematics of erosion test systems. .............................................................. 35

Figure 2-11 Schematic of corrosion mechanisms of coating systems with defects......... 38

Figure 2-12 Schematic for pitting corrosion mechanism in a coating/substrate system and damage to the coating. ............................................................................................ 40

Figure 2-13 Schematic diagram of three-electrode electrochemical cell........................ 43

Figure 2-14 A typical hypothetical polarization curve. .................................................... 44

Figure 2-15 Schematic of linear polarization curve and extraction of polarization resistance.

Figure 2-16 Potentiodynamic polarization curves for TiN, $\mathrm{TiBN}, \mathrm{CrN}$ and $\mathrm{Nb}_{2} \mathrm{~N}$ coating

systems and $\mathrm{HSS}$ substrate in $0.8 \mathrm{M} \mathrm{NaCl}$ solution...............................................49

Figure 2-17 Schematic polarization curve showing Tafel extrapolation. .........................51

Figure 2-18 EIS curves for Ti- and $\mathrm{Cr}$-based nitride coatings in $3.5 \% \mathrm{NaCl}$ solution. ... 53

Figure 2-19 Nanohardness vs. third element for various metal nitrides. .......................... 62

Figure 2-20 Cross section images of multilayer and superlattice coating systems.......... 65

Figure 2-21 Cross-sectional TEM micrograph multilayered coating with superlattice structure

Figure 2-22 Mechanical properties of a multilayered coating as a function of the number

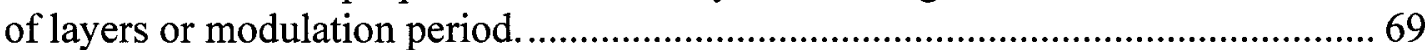

Figure 2-23 Microstructures of nano-composite coatings. .............................................. 71

Figure 2-24 Comparisons of mechanical properties for nano-composite coatings........... 72

Figure 2-25 Schematic of a typical electroplating setup.................................................. 74

Figure 2-26 Schematic of a cathodic arc deposition process.......................................... 78

Figure 2-27 Schematic diagram of the sputtering process.............................................. 79

Figure 2-28 Schematics magnetron sputtering configurations. ..........................................81 81

Figure 2-29 SEM images of fracture surfaces. ............................................................... 83

Figure 2-30 Hardness of a material as a function of the grain size................................. 85

Figure 2-31 Coating hardness and average crystallite size vs. substrate bias votage. ..... 86 
Figure 2-32 Effect of substrate bias on the microstructure (inset) and erosion behaviours for TiN and TiAlN coatings tested under $60 \mathrm{~m} / \mathrm{s}$ and $30^{\circ}$ and $90^{\circ}$ impingement angles.

Figure 2-33 Coating mechanical properties of as function of substrate temperature. .... 87

Figure 2-34 Weight losses with the number of cycles for uncoated and TiN coated

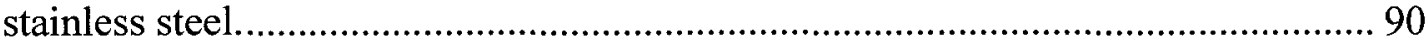

Figure 2-35 Wear behaviours of hard components............................................. 91

Figure 2-36 Abrasion wear volume as a function of wear time and microhardness....... 92

Figure 2-37 Maximum flank wear as a function of time. ......................................... 93

Figure 2-38 Microhardness and friction coefficient of coating vs. Si contents............. 95

Figure 2-39 Coefficient of friction and wear depths for the $\mathrm{CrN}$ and $\mathrm{CrN}-\mathrm{Wx}$ coatings

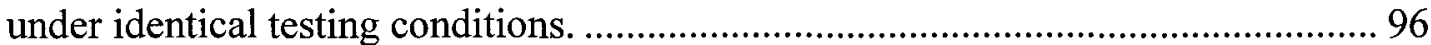

Figure 2-40 Effect of Mo on tribological behaviours of TiMoN coatings.....................97

Figure 2-41 Influence of Si content on coating tribological behaviours....................... 98

Figure 2-42 Erosion behaviours for 17-4PH steel, electroless nickel coating, and TiN.. 99

Figure 2-43 Erosion resistance of coatings WC and TiN and uncoated Ti-6Al-4V steel.

Figure 2-44 Erosion rate variation of uncoated and coated INCO 718 with impingement angle.

Figure 2-45 Erosion rates of coatings with temperature. 102

Figure 2-46 Erosion rates of TiN and TiAlN coating systems. 102

Figure 2-47 Influences of impingement angle and particle velocity on erosion rates for

TiN and TiAlN-\#3 coating systems.

Figure 2-48 Erosion resistance of various coatings with 50 micron $\mathrm{Al}_{2} \mathrm{O}_{3}$ erodent. .... 104

Figure 2-49 Log erosion-corrosion rate, erosive rate and flow-induced corrosion rate vs. impact velocity for the TiN coating and an uncoated $\alpha$-Ti alloy.

Figure 2-50 Potentiodynamic curves of coatings 1040 steel substrates in $1 \mathrm{~N}$ sulfuric

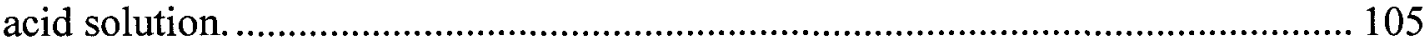

Figure 2-51 Potentiodynamic polarization curves of coating and substrate steels. ...... 107

Figure 2-52 Potential polarization curves of the TiAlN and CrAlN coating systems. .. 108

Figure 2-53 A schematic illustration of oxygen diffusion mechanism for (a) columnar

(e.g. TiN), and (b) equiaxed (e.g. CrN) crystallite structures............................. 110

Figure 2-54 Polarization curves of coating systems with various thicknesses............. 111

Figure 2-55 Potentiodynamic polarization curves of TiN coatings produced by various deposition processes.

Figure 2-56 Effect of coating thickness on critical passivity current density and area ratio of pinhole defect.

Figure 2-57 Defect formations in coating systems. ......................................... 116

Figure 2-58 Images of irregularty and droplet. .................................................... 117

Figure 2-59 Typical SEM photographs of chromium nitride films deposited at the substrate bias voltage.

Figure 2-60 Variation in number of projections classified by diameters with substrate bias voltage.

Figure 2-61 Pinhole density vs. substrate bias voltage.

Figure 3-1 Nickel electroplating apparatus. 


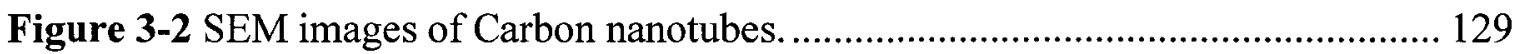

Figure 3-3 Setup of ball crater method of measuring thickness of coating. ….............. 136

Figure 3-4 Geometry of ball crater method of measuring coating thickness................. 137

Figure 3-5 Schematic of the nanoindentation load-displacement curve. ....................... 140

Figure 3-6 TEER POD 2 pin-on-disc wear tester. ………............................................ 142

Figure 3-7 Diagram of a pin-on-disc wear tester work principle................................... 143

Figure 3-8 Veeco Dektak ${ }^{\circledR} 150$ surface profiler....................................................... 145

Figure 3-9 A typical wear profile on a TiN coating sample measured using Veeco

Dektak $(150$ surface profiler. ........................................................................... 145

Figure 3-10 HME Airbrasive unit and working principle............................................. 148

Figure 3-11 SEM micrograph of $\mathrm{Al}_{2} \mathrm{O}_{3}$ particles used in erosion testing.................... 149

Figure 3-12 Sample and nozzle holder inside test chamber........................................... 150

Figure 3-13 Electrochemical experimental setup...................................................... 151

Figure 3-14 Electrochemical cell setup...................................................................... 152

Figure 3-15 A typical potentiodynamic polarization curve obtained from a test on a

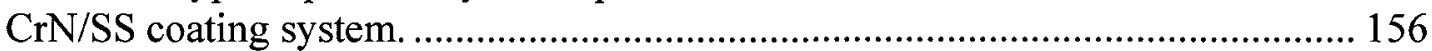

Figure 3-16 Nyquist plots measured from an EIS test on a TiN/MS coating system.... 157

Figure 3-17 Bode plots obtained from an EIS tests on a CrN/SS coating. ................... 158

Figure 4-1 Cross sectional images of $\mathrm{Ni}$ and $\mathrm{Ni}-\mathrm{CNT}$ plating samples under various deposition conditions.

Figure 4-2 Thickness of $\mathrm{Ni}$ and Ni-CNT plated samples as a function of pulse frequency.

Figure 4-3 SEM images of conventional Ni plated samples deposited at different

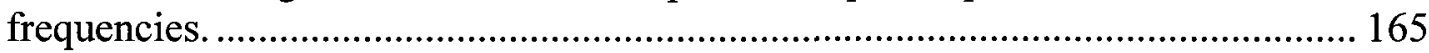

Figure 4-4 SEM images of Ni-CNT plated samples with CNTs dispersed. ................... 167

Figure 4-5 SEM images of the as-deposited EB TiN coating........................................ 172

Figure 4-6 SEM images of the as-deposited CA TiN coating. ..................................... 174

Figure 4-7 SEM images of PMMA-treated EB TiN coating. ……................................ 176

Figure 4-8 SEM images of PMMA-treated CA TiN coating........................................... 177

Figure 4-9 SEM images demonstrating morphologies. ................................................ 180

Figure 4-10 SEM images revealing defects on the coatings......................................... 182

Figure 4-11 XRD spectra of the Ni plated samples. ................................................. 185

Figure 4-12 XRD spectra of Ni-CNT plated samples.................................................. 187

Figure 4-13 XRD spectra of PVD TiN coating systems.............................................. 190

Figure 4-14 XRD spectra of CrN based PVD coating systems. ................................... 192

Figure 4-15 Average grain size vs. Si content in $\mathrm{CrN}$ based coating systems............... 193

Figure 4-16 Hardness of Ni plated and Ni-CNT plated samples.................................. 194

Figure 4-17 Young's modulus of Ni plated and Ni-CNT plated samples at various pulse

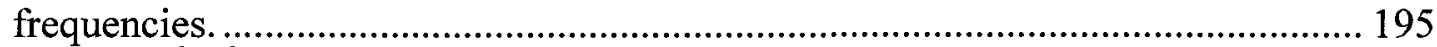

Figure 4-18 $\mathrm{H}^{3} / \mathrm{E}^{2}$ ratios for Ni plated and Ni-CNT plated samples............................. 196

Figure 4-19 Hardness and Young's modulus vs. Si content in CrN based coating

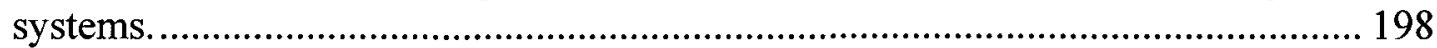

Figure 4-20 Coating oefficient of friction vs. sliding distance.................................... 200

Figure 4-21 Coefficient of friction and $\mathrm{H}^{3} / \mathrm{E}^{2}$ ratio vs. Si content in $\mathrm{CrN}$ based coating systems. 202 
Figure 4-22 Wear track cross-sectional profiles for test coating samples.

Figure 4-23 Wear behaviours vs. Si content in CrN based coating systems.

Figure 4-24 SEM images of wear tracks and EDS spectrum on worn debris for the test

coating systems (a) $\mathrm{CrN}$, (b) $\mathrm{CrSiCN}$ (1) and (c) $\mathrm{CrSiCN}(2)$, and (d) $\mathrm{CrSiCN}$ (3).. 210

Figure 4-25 Wear tracks and EDS spectrum on wear debris of the CA TiN coating. ... 212

Figure 4-26 SEM images of wear scars on WC-Co balls. ...................................... 213

Figure 4-27 Erosion rates for Ni plated and Ni-CNT plated samples......................... 216

Figure 4-28 SEM image of erosion tested Ni plated sample .................................... 216

Figure 4-29 Erosion rates for test PVD coating systems. ...................................... 218

Figure 4-30 SEM images of surface characteristics of $\mathrm{CrN}$ based coating systems after erosion tests at different impingement angles.

Figure 4-31 Potentiodynamic polarization curves of uncoated mild steel and 17-4PH

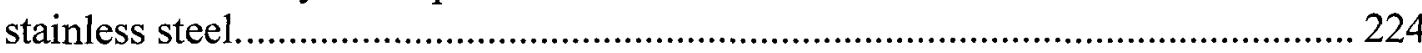

Figure 4-32 Potentiodynamic polarisation curves of TiN coating systems. ................. 226

Figure 4-33 Potentiodynamic polarization curves of TiN coating systems with various deposition conditions.

228

Figure 4-34 Potentiodynamic polarization curves of $\mathrm{CrN}$ based coating systems. ...... 230

Figure 4-35 EIS spectra of mild steel and 17-4PH steel substrates. ........................... 236

Figure 4-36 EIS spectra of EB TiN/SS coating systems........................................ 241

Figure 4-37 EIS spectra of CA TiN/SS coating systems. ....................................... 245

Figure 4-38 EIS spectra of CA TiN/MS coating systems....................................... 249

Figure 4-39 EIS spectra of $\mathrm{CrN}$ based coating systems....................................... 259

Figure 4-40 SEM analysis images for EB TiN/SS coating systems after immersions. . 264

Figure 4-41 SEM analysis images for CA TiN/SS coating system after immersions. .. 267

Figure 4-42 Optical and SEM images for CA TiN/MS coating system after immersion.

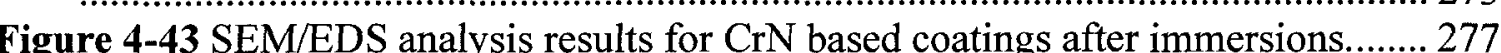

Figure 4-44 Schematic of CPE Model. ................................................................. 279

Figure 4-45 EEC fitting curves on MS substrate. ................................................. 280

Figure 4-46 Schematic of EEC models for diffusion controlled corrosions................ 285

Figure 4-47 EEC fitting curves on 17-4PH steel substrate...................................... 287

Figure 4-48 EEC fitting curves on EB TiN/SS coating systems................................ 289

Figure 4-49 EEC fitting curves on CA TiN/SS coating systems. .............................. 290

Figure 4-50 Schematic of EEC model for localized corrosion model. ........................ 296

Figure 4-51 EEC fitting curves on CA TiN/MS coating systems.............................. 297

Figure 4-52 EEC fitting curves for $\mathrm{CrN}$ based coating systems. ............................. 302

Figure 4-53 Mechanical properties vs. Si content in CrN based coating systems........ 313

Figure 4-54 Wear behaviour vs. Si content in $\mathrm{CrN}$ based coating systems.................. 314

Figure 4-55 Erosion behaviours of test coating systems..................................... 316

Figure 4-56 Polarization behaviours of test PVD coating systems............................. 318

Figure 4-57 EIS (Nyquist) plots of test PVD coating systems. ............................. 321 


\section{List of Tables}

Table 1-1 Mechanical component failure mechanisms..................................................... 2

Table 1-2 Summaries of metal degradation caused economic losses ............................... 2

Table 2-1 Distinction between mild and severe wear ....................................................... 14

Table 2-2 Summary of electrochemical measurements in corrosion studies ................... 54

Table 2-3 Summary of the development in protective coating systems …..................... 57

Table 2-4 Properties of binary transition metal nitrides and carbides................................. 61

Table 2-5 Summary of ternary and quaternary $\mathrm{Ti}$ and $\mathrm{Cr}$ based coating compositions... 63

Table 2-6 Summary of improvement due to nitrides and carbides ................................. 94

Table 2-7 Potentiodynamic polarization data for 1040 steel, TiN and $\mathrm{CrN}$ coatings on 1040 steels substrates at various temperatures determined from the polarization curves in $1 \mathrm{~N}$ sulfuric acid solutions................................................................... 106

Table 2-8 Potentiodynamic polarization data of the untreated and coated specimens recorded in $0.5 \mathrm{M} \mathrm{NaCl}$ aqueous solution after 1 hour exposure .......................... 107

Table 2-9 Potentiodynamic polarization data of $\mathrm{CrN}$ and CrAlN coatings deposited on mild steel substrates in $3.5 \% \mathrm{NaCl}$ solution at room temperature.

Table 2-10 Density of nodular "inhomogeneities", corrosion pits, corrosion current density, corrosion potential and calculated porosity of some $\mathrm{TiN}$ and $(\mathrm{Ti}, \mathrm{Al}) \mathrm{N}$ coatings, together with the coating thicknesses and $\mathrm{Al} / \mathrm{Ti}$ evaporation ratios........ 113

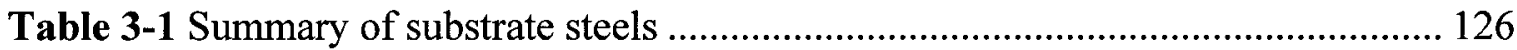

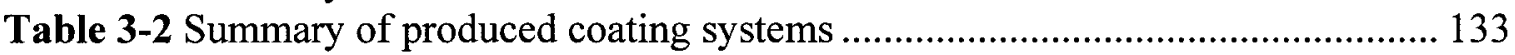

Table 3-3 Basic circuit elements used in EEC models.............................................. 159

Table 4-1 Summary of thicknesses $(\mu \mathrm{m})$ of Ni and Ni-CNT plated samples deposited using various pulse frequencies

Table 4-2 Summary of thicknesses of PVD coating systems......................................... 162

Table 4-3 Chemical composition (at.\%) for the CrN based coatings............................... 183

Table 4-4 Summary of defect densities of PVD coating systmes ................................... 183

Table 4-5 Average grain size of the Ni and Ni-CNT plated samples .......................... 188

Table 4-6 Average grain size of $\mathrm{CrN}$ based coating systems........................................ 192

Table 4-7 Summary of Vickers hardness and elastic modulus of Ni plated samples .... 196

Table 4-8 Summary of Vickers hardness and elastic modulus of PVD coating systems 197

Table 4-9 Wear test coatings and test conditions ...................................................... 199

Table 4-10 Summaries of wear testing data for the tested coating systems.................... 204

Table 4-11 Wear test data for WC-Co ball sliding against test coatings ....................... 212

Table 4-12 Poltetiodynamic polarization parameters for substrate steels...................... 224

Table 4-13 Potentiodynamic polarization test data for TiN coating systems ................ 229

Table 4-14 Poltetiodynamic polarization test data for $\mathrm{CrN}$ based coating systems...... 232

Table 4-15 EEC fitted data for mild steel (MS) substrate (CPE) ................................... 280

Table 4-16 EEC fitted data for 17-4PH steel substrate (OctWar) …………………......... 287 
Table 4-17 EEC fitted data for EB TiN/SS coating systems (OctWar)

Table 4-18 EEC fitted data for CA TiN/SS coating systems (OtcWar)

Table 4-19 EEC fitted data for CA TiN/MS coating systems (localized corrosion)...... 298

Table 4-20 EEC fitted data for CA PMMA TiN/MS coating systems (OtcWar) .......... 298

Table 4-21 EEC fitted data for CrN coating system (Otc-Cot-Hyper) ........................... 303

Table 4-22 EEC fitted data for CrSiCN(1) coating system (Otc-Cot-Hyper) ................. 303

Table 4-23 EEC fitted data for $\mathrm{CrSiCN}(2)$ coating system (Otc-Cot-Hyper) ................. 303

Table 4-24 EEC fitted data for $\mathrm{CrSiCN}(3)$ coating system (Otc-Cot-Hyper) .................. 303

Table 4-25 Summary of mechanical and wear properties of PVD coating systems ...... 312

Table 4-26 Summaries of coating thickness, porosities, polarization resistance $\left(R_{p}\right)$ and diffusion impedance $(Z)$ for the test PVD coating systems .................................... 322 


\section{Nomenclature}

A

$\mathrm{AC}$

$A_{\text {coatmg-wear }}$

AEGD

$A\left(h_{c}\right)$

ARE

$A_{r}$

$a$

$B$

$\mathrm{BCC}$

BSE

b

C

$\mathrm{CA}$

$C_{d l}$

CDC

CNT

COF

$C_{O}$

$C_{R}$

CVD

CPE

$c$

D

DC

$D_{e}$ real area of contact, electrode active surface area

alternating current

worn track cross-sectional area

arc enhanced glow discharge

projected contact area of the indenter tip

Plasma-assisted reactive evaporation

projected indentation area

Tafel equation constant

characteristic diffusion parameter

body centered cubic

back-scattered electron

Tafel equation constant

ion concentration

cathodic arc

double layer capacitance

circuit description code

carbon nano-tube

coefficient of friction

bulk concentration of diffusing oxidant

bulk concentration of diffusing reductant

chemical vapor deposition

constant phase element

constant, constrictivity

average grain size, diffusion coefficient

direct current

effective diffusion coefficient 
Do diffusion coefficient of oxidant

$D_{R} \quad$ diffusion coefficient of reductant

d pre-existing crack length

$d_{\text {scar }} \quad$ diameter of the scar on the abrasive ball

E modulus of elasticity or elastic modulus (Young's modulus), electrode potential

EB electron beam

EDS energy dispersive X-ray spectroscopy

EEC equivalent electrical circuit

$\mathrm{E}^{\circ} \quad$ standard electrode potential

EFM electrochemical frequency modulation

EIS

electrochemical impedance spectroscopy

$E_{\text {Indenter }} \quad$ Young's modulus for indenter

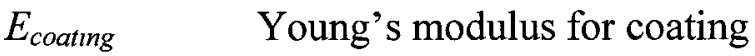

$E_{\text {corr }} \quad$ corrosion potential and

$E_{e q} \quad$ equilibrium potential

EMF (emf) electromotive force

$E_{m} \quad$ mass removed by a unit mass of erodent

$E_{o 2} \quad$ oxygen evolution potential

$\mathrm{E}_{\mathrm{OCP}} \quad$ open-circuit potential

$\mathrm{E}_{\mathrm{o}, \mathrm{H}} \quad$ open-circuit potential for hydrogen

$\mathrm{E}_{0, \mathrm{M}} \quad$ open-circuit potential for metal

$E_{p} \quad$ pitting potential

$E_{p a} \quad$ passive potential

$E_{p p} \quad$ primary passive potential

$E_{v} \quad$ volume of material removed by a unit mass of erodent

$\Delta E \quad$ difference in overpotential

$\Delta E_{\text {corr }} \quad$ difference in corrosion potentials between coated and bared substrates

F tangential force, friction force, Faraday constant

FCC face-centered cubic

$F_{\text {friction }} \quad$ measured friction force 


\begin{tabular}{|c|c|}
\hline FWHM & full width at half maximum \\
\hline g & gram \\
\hline $\mathrm{H}$ & Hardness \\
\hline HAZ & heat-affected zone \\
\hline HRTEM & high resolution transmission electron microscopy \\
\hline HSS & high speed steel \\
\hline$h$ & depth of wear \\
\hline$h_{c}$ & current contact depth \\
\hline$h_{\max }$ & maximum contact depth \\
\hline$I$ & electrode current \\
\hline$I_{\text {corr }}$ & corrosion current \\
\hline IP & ion plating \\
\hline$\Delta I$ & difference in electrode current \\
\hline$i$ & current density \\
\hline$i_{a}$ & anodic current density \\
\hline$i_{c}$ & cathodic current density \\
\hline$i_{\text {corr }}$ & corrosion current density \\
\hline$i_{L}$ & limiting current density \\
\hline$i_{\max }$ & maximum current density \\
\hline$i_{o}$ & exchange current density \\
\hline$i_{0, \mathrm{H}}$ & exchange current density for hydrogen \\
\hline$i_{\mathrm{O}, \mathrm{M}}$ & exchange current density for metal \\
\hline$i_{p}$ & passive current density \\
\hline$i_{s}$ & secondary current density \\
\hline$\Delta i$ & difference in current density \\
\hline$J$ & flux of diffusing specie \\
\hline$K$ & wear coefficient \\
\hline$K_{I c}$ & fracture toughness \\
\hline$k$ & wear coefficient, specific wear rate, XRD constant \\
\hline$k_{4}$ & erosion constants \\
\hline
\end{tabular}




\begin{tabular}{|c|c|}
\hline$k_{A}$ & Archard wear coefficient \\
\hline$k_{t}$ & erosion constant \\
\hline$k_{K}$ & Khurshchov wear coefficient \\
\hline$L$ & sliding distance, liter \\
\hline LP & linear polarization \\
\hline$M$ & molecular mass $(\mathrm{g} / \mathrm{mol})$, mole, metal \\
\hline $\mathrm{MBE}$ & molecular beam epitaxy \\
\hline$M_{e}$ & mass of erodent \\
\hline MMA & methyl methacrylate \\
\hline$M_{m}$ & mass of removed material \\
\hline MS & mild steel, magnetron sputtering \\
\hline$m$ & exponent \\
\hline $\mathrm{N}$ & load, normal force \\
\hline NDL & Nernst diffusion layer \\
\hline NHT & Nano Hardness Tester \\
\hline$n$ & exponent, number of electrons \\
\hline$n-Q_{c}$ & empirical exponent of CPE for coating \\
\hline$n-Q_{s}$ & empirical exponent of $\mathrm{CPE}$ for substrate \\
\hline $\mathrm{OCP}$ & open-circuit potential \\
\hline OEM & optical emission monitor \\
\hline OFLD & Open Finite-Length Diffusion \\
\hline$P$ & pressure, normal contact force, porosity, packing factor \\
\hline PBII & plasma-based ion implantation \\
\hline PEMS & plasma enhanced magnetron sputtering \\
\hline PH-SS & precipitation-hardening stainless steel \\
\hline$P_{\max }$ & maximum Hertizan contact pressure, maximum load \\
\hline PMMA & polymethyl methacrylate \\
\hline PTA & plasma transfer arc \\
\hline PVD & physical vapor deposition \\
\hline$P_{y}$ & contact pressure \\
\hline$R$ & universal gas constant, particle radius \\
\hline
\end{tabular}




\begin{tabular}{|c|c|}
\hline$R_{b}$ & radius of abrasive ball \\
\hline$R_{e}$ & resistance of electrolyte \\
\hline$R_{p}$ & polarization resistance \\
\hline$R_{\text {pore }}$ & resistance to the ionic current through the coating pores \\
\hline$R_{p s}$ & polarization resistance of substrate \\
\hline$R_{s}$ & charge-transfer resistance of the substrate, \\
\hline$R^{*}$ & constant determined by $\mu, k_{2}, k_{3}$, velocity, and substrate elasticity \\
\hline$r$ & contacting sphere radius \\
\hline $\mathrm{rms}$ & root mean square \\
\hline $\begin{array}{l}r_{s} \text { and } r_{c} \\
\text { rpm }\end{array}$ & $\begin{array}{l}\text { measured radii of small and large circle in ball-cratering method } \\
\text { revolutions per minute }\end{array}$ \\
\hline$r_{\text {wear }}$ & radius of wear track \\
\hline S & wear resisting term \\
\hline SCE & saturated calomel (mercury-mercury (II) chloride) electrode \\
\hline$S_{c, m}$ & mechanical severity of contact \\
\hline SE & secondary electron \\
\hline SEM & scanning electron microscope \\
\hline SHE & standard hydrogen electrode \\
\hline SS & stainless steel \\
\hline SWNT & single-walled nanotube \\
\hline$T$ & temperature \\
\hline$t$ & time \\
\hline TBC & thermal barrier coating \\
\hline TE & Tafel extrapolation \\
\hline TEC & thermal expansion coefficient \\
\hline TEM & transmission electron microscopy \\
\hline TMS (3MS) & trimethylsilane \\
\hline UV & ultraviolet \\
\hline$V$ & potential \\
\hline$V_{\text {ero }}$ & volume loss due to erosion \\
\hline$V_{m}$ & volume of removed material \\
\hline
\end{tabular}




\begin{tabular}{|c|c|}
\hline$V_{\text {wear }}$ & worn volume \\
\hline$V_{\text {coating-wear }}$ & worn volume of coating \\
\hline$V_{\text {ball-wear }}$ & worn volume of spherical cap \\
\hline$v$ & velocity \\
\hline$W$ & Warburg diffusion (element), Tungsten \\
\hline WE & working electrode \\
\hline$W_{\text {wear }}$ & specific wear rate \\
\hline XRD & X-Ray diffraction \\
\hline$x$ & distance from electrode surface \\
\hline$Y_{0}$ & admittance \\
\hline$Y_{0^{-}} Q_{c}$ & (modulus of) electrochemical admittance of $\mathrm{CPE}$ for coating \\
\hline$Y_{0}-Q_{s}$ & (modulus of) admittance of CPE for substrate \\
\hline$Y_{0}-O$ & (modulus of) OFLD admittance \\
\hline$Y_{0^{-}} W$ & (modulus of) Warburg admittance \\
\hline$Z$ & impedance \\
\hline$z$ & number of electrons \\
\hline$Z_{O}$ & OFLD impedance \\
\hline$Z_{W}$ & Warburg impedance \\
\hline$\alpha$ & symmetry factor \\
\hline$\beta_{a}$ & anodic polarization constant (Tafel slope of anodic corrosion reaction) \\
\hline$\beta_{c}$ & cathodic polarization constant (Tafel slope of cathodic corrosion reaction) \\
\hline$\delta$ & coating thickness, diffusion length, Nernst diffusion layer (NDL) thickness \\
\hline$\varepsilon$ & residual strain \\
\hline$\varepsilon_{t}$ & porosity available for transport \\
\hline$\eta$ & difference in potential \\
\hline$\eta_{a}$ & anodic overpotential \\
\hline$\eta_{c}$ & cathode overpotential \\
\hline$\theta$ & impact / impingement angle, Bragg angle \\
\hline$\lambda$ & wavelength of the $\mathrm{x}$-ray, modulation period \\
\hline$\mu$ & coefficient of friction \\
\hline
\end{tabular}




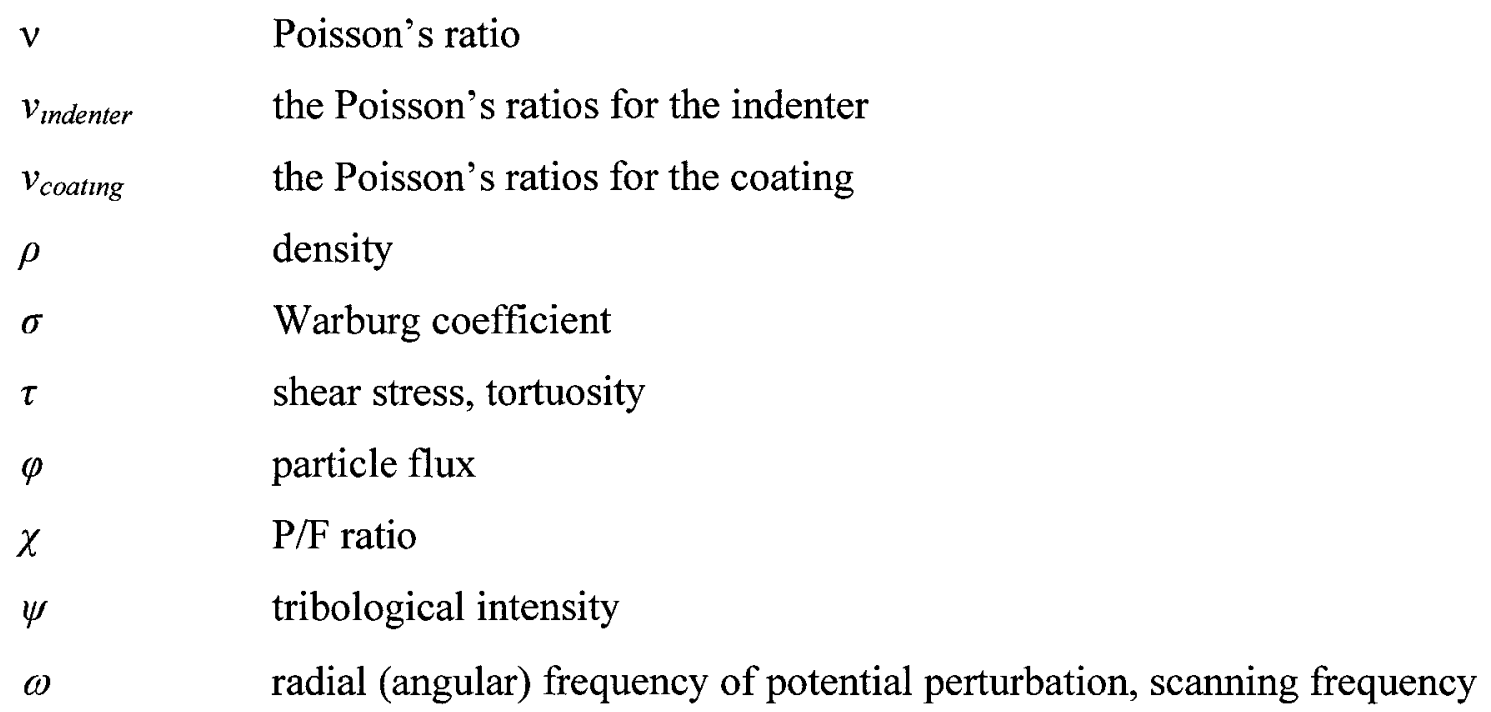




\section{Introduction}

\subsection{Background}

Mechanical component degradation has drawn more attentions in various industrial applications. In harsh working environments, mechanical and chemical attacks subject components to damage, and therefore components degrade over time. This can result in limited load-carrying capacities, reduced system efficiencies, shorter lifetimes, equipment or machinery shutdowns, and even fatal accidents sometimes [1]. The most common mechanical and chemical attacks are wear, erosion and corrosion, respectively.

Statistical studies conducted on the attack modes (Table 1-1) indicate that wear and corrosion are important factors that lead to high failure rates in engineering components, and corrosion plays a more significant role than wear; therefore corrosion causes more economic losses than wear or erosion (Table 1-2).

It has been estimated that about 25 to $40 \%$ of corrosion caused losses $[2,3]$ and $18.6 \%$ of wear caused losses [4] can be avoided by incorporating effective protection technologies, including improved component design, judicious selection of materials and coatings. There is a significant market demand to support preventing component degradation. 
Table 1-1 Mechanical component failure mechanisms (summarized from [5])

\begin{tabular}{|c|c|c|}
\hline & \multicolumn{2}{|c|}{ Percentage of failures } \\
\hline Attack manner & Non-aircraft components & Aircraft components \\
\hline Corrosion & 29 & 16 \\
\hline Wear/abrasion/erosion & 3 & 6 \\
\hline
\end{tabular}

Table 1-2 Summaries of metal degradation caused economic losses

\begin{tabular}{|l|c|c|c|}
\hline \multicolumn{1}{|c|}{ Country and year } & Attack & Economic loss & Reference \\
\hline Germany, 1975 & wear & $1 \%$ of GNP* & {$[6]$} \\
\hline US, 1978 & wear & $0.9 \%$ of GDP** & {$[4]$} \\
\hline US, per year & erosion & billions of dollars & {$[7]$} \\
\hline Germany, 1975 & corrosion & $5 \%$ of GNP* & {$[8]$} \\
\hline US, 1978 & corrosion & $3.5 \%$ of GDP** & {$[4]$} \\
\hline US, 1978 & corrosion & $3.1 \%$ of GDP** & {$[2]$} \\
\hline Australia, per year & corrosion & 3 to $4 \%$ of GNP & {$[9]$} \\
\hline Great Britain, per year & corrosion & 3 to $4 \%$ of GNP & {$[9]$} \\
\hline Japan, per year & corrosion & 3 to $4 \%$ of GNP & {$[9]$} \\
\hline
\end{tabular}

* Germany GNP in 1975 was 10 billion DM

** US GDP in 1978 was 2,276 billion US dollars

\subsection{Development of Surface Engineering in Protective}

\section{Applications}

In harsh working environments, the most common degradation initializes on the surfaces of mechanical components [10]. As a result, component degradation remedies typically resort to surface engineering [11] to improve the resistance of components to the attacks from wear, erosion and corrosion $[12,13]$. 
Surface engineering is a multidisciplinary subject of tailoring properties of component surfaces to achieve desired function and serviceability [4]. Protective surface modification is to apply proper treatments to the surface or near-surface regions, attempting to impart the surface with distinct functions from that of the original raw material [14]. These treatments can be in the form of metallurgical, mechanical, chemical, or overlay coatings [4]. Surface-engineered metallic components possess improved characteristics, including corrosion resistance, oxidation or sulfidation resistance, wear resistance, reduced frictional energy losses, enhanced fatigue resistance, and toughness.

The development of the automobile industry is a major driving force for rapid progress in surface modifications. The techniques used in these modifications include electrolytic plating to improve corrosion resistance and to lower the coefficient of friction (COF) of the cylinder bore and piston; and physical vapor deposition (PVD) or chemical vapor deposition (CVD) coatings to enhance wear and seizure resistance, and to lower friction [15].

In the aeronautical and gas turbine industries, component surface enhancement techniques have also undergone development. The development of jet enging-propelled aircraft imposed more demands for materials used in gas turbines. These new challenges included higher operating temperatures, lighter weight structures, and improved environmental resistance (resistance to sand, mist, salt, and impurity from low-grade fuels). Protective coatings are necessary to improve turbine component performance and 
extend operating temperature and durability [16]. Figure 1-1 shows that advanced thermal barrier coatings (TBC), combined with cooling systems and single crystal alloys, are becoming the only way to allow components to operate at high working temperatures [16]. Thermal barrier coatings offer protections for components working at temperatures up to $1320^{\circ} \mathrm{C}$ [17] A number of deposition technologies can be used to apply diffusion or overlay coatıngs, including CVD, PVD and thermal spraying technologies. Among them, electron beam physical vapor deposition (EB-PVD) can produce coatings with a high homogeneous thickness and a smooth surface [16].

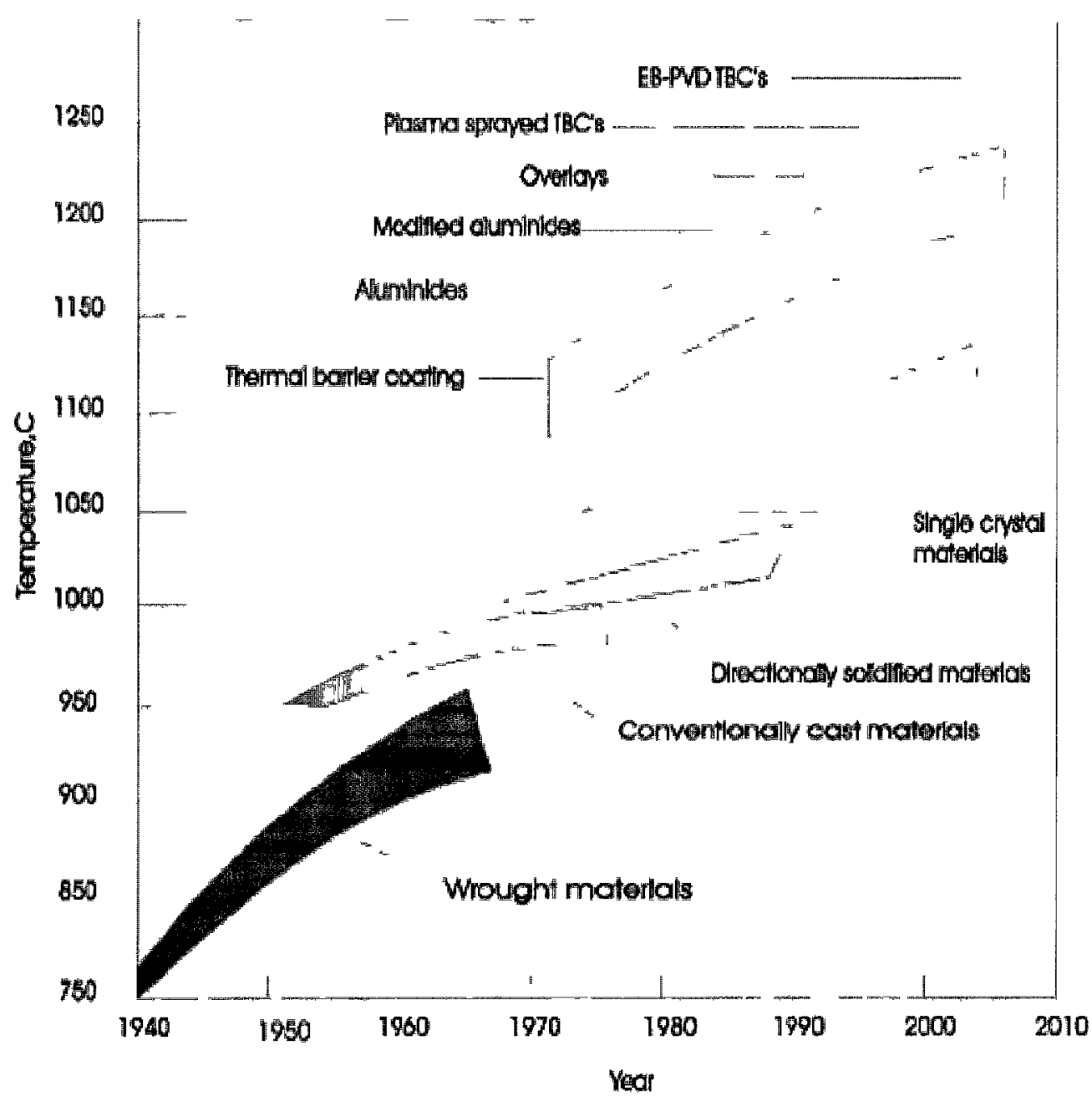

Figure 1-1 The development of superalloys and heat resistant coating systems [16]. 
Besides elevated temperature, gas turbine components, particularly vanes and blades, are vulnerable to erosion and corrosion [18 - 22]. Impacts of solid particles and liquid droplets on leading and trailing edges of turbine blades and vanes cause fatal consequences because they change the shape and dimensions [23 - 26].

For erosion protection, coatings were applied to engine components. The earliest solution was a varnish coating [16] that was intended to reduce erosion damages and corrosion attacks. In the past decades, PVD and CVD coated transition metal compound hard coatings became the dominant solutions to combat erosion [27 - 29] and corrosion [30]. However, because of the inherent defects (micro-cracks, pores, pinholes, grain boundaries) that resulted from most PVD deposition processes [31 - 34], corrosive media was able to reach the substrate material [35 - 38], attacking the coating-substrate interface $[36,39-41]$. These attacks eventually lead to failure of the protective coatings.

The primary objective of this thesis is to develop coating systems and associated deposition processes for improved triobological and corrosion resistances. A critical issue in this study to achieve this objective is to better understand the mechanisms of mechanical and chemical attacks and their correlations with the nature of coating systems. 


\subsection{Scope, Objectives and Organization}

As a part of a collaborative research project between Carleton University and Institute for Aerospace Research of National Research Council (NRC) of Canada, supported by Liburdi Turbine Services Inc., the main goal of this study is to gain a comprehensive understanding of the behaviours of transition metal coatings under erosion and corrosion environments which gas turbine compressors are subjected to; and to characterize coating microstructures and associated deposition processes. Within this scope, the main objectives of this thesis are:

1. To select a series of coating systems with potential capability to provide erosion, wear and corrosion protections for gas turbine compressor applications. Widely used transition metal nitride coating systems such as titanium nitride (TiN) and chromium nitride $(\mathrm{CrN})$ based coating systems $(\mathrm{CrSiCN})$ are selected in this study. These basic coating systems are produced using different deposition processes and with modified compositions to study the effects of deposition process and composition on microstructure and mechanical properties as well as their tribological and corrosion behaviours.

2. To conduct experimental studies of the selected coating systems under the conditions similar to that in the gas turbine compressor working environments.

3. To modify coating compositions and develop novel post-deposition treatments to improve corrosion resistance.

4. To examine the effect of nano-structure on the tribological resistance.

5. To analyze the effects of coating deposition processes on coating microstructures and performances in working environments. 
6. To investigate the effects of defects in coatings on their anti-corrosion performances.

This thesis includes the following chapters:

- Chapter 2 presents a literature review of the issues of surface degradation characteristics and mechanisms. The current trend in the development of solutions to surface degradations and the state-of-the-art of hard and super-hard coatings is studied.

- Chapter 3 details coating and coating characterization procedures. In particular, electroplating and PVD deposition techniques used for experimental studies are introduced. Characterizations of the coating systems were carried out using methods such as SEM, XRD, EDS, wear (pin-on-disk), erosion (solid particle impingement), and corrosion tests (potentiodynamic polarization and electrochemical impedance spectroscopy (EIS) measurements). Data obtained on corrosion tests was processed and analyzed using equivalent electrical circuit (EEC) technique.

- Chapter 4 systematically presents the experimental results on microstructures and mechanical properties of the coating systems studied; discusses the factors affecting coating system behaviours with respect to wear, erosion and corrosion and the correlation with their properties.

- Chapter 5 provides conclusions, indicates contributions based on the results and findings obtained, and recommends future work. 


\section{Literature Review}

\subsection{Metal Component Surface Degradation}

A general definition of material degradation is the loss of some desired performance in an engineering application. Metal degradation can be classified as due to physical, chemical or biological effects [8]. Physical degradation is associated with the effects of force and heat, which results in wear and thermal corrosion. Chemical degradation is related to destructive reactions, which cause the corrosions. Biological degradation is caused by interactions between life forms and engineering materials. This study emphasizes both physical and chemical degradation mechanisms.

The consequences of surface damage can be summarized into three categories, i.e., constant material volume and weight, material loss, and material gain. Even without weight or volume changes, damage can still arise due to structural changes, plastic deformation, and surface cracks. When material loss occurs it usually takes the form of erosion or wear. Material gain involves pickup of loose particles, embedding of erosive particle fragments into the component surface, or corrosion products. Any of these damages would deteriorate the desired functions of the components. As are shown in Figure 2-1 (a) and (b) sand and dust erosion caused severe damages to an aircraft turbine engine compressor. Detailed typical erosive and corrosive damages on a gas turbine compressor blade are shown in Figure 2-1 (c). 


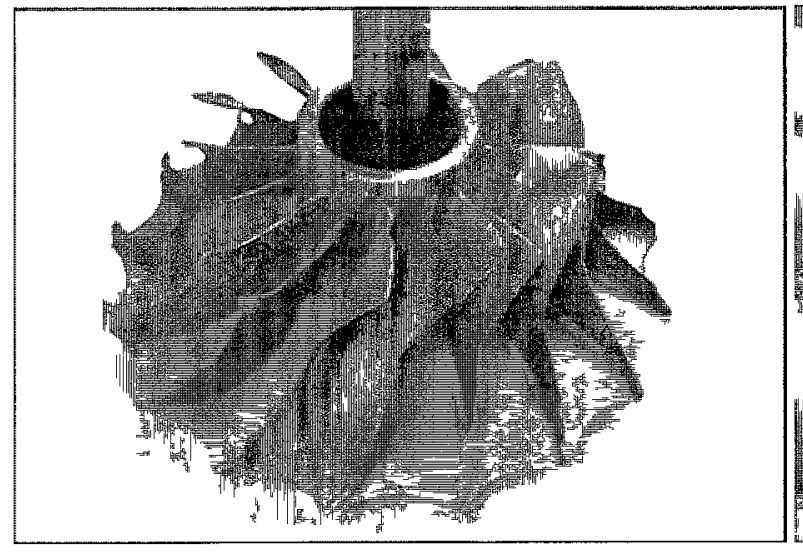

(a) Eroded centrifugal compressor [42]

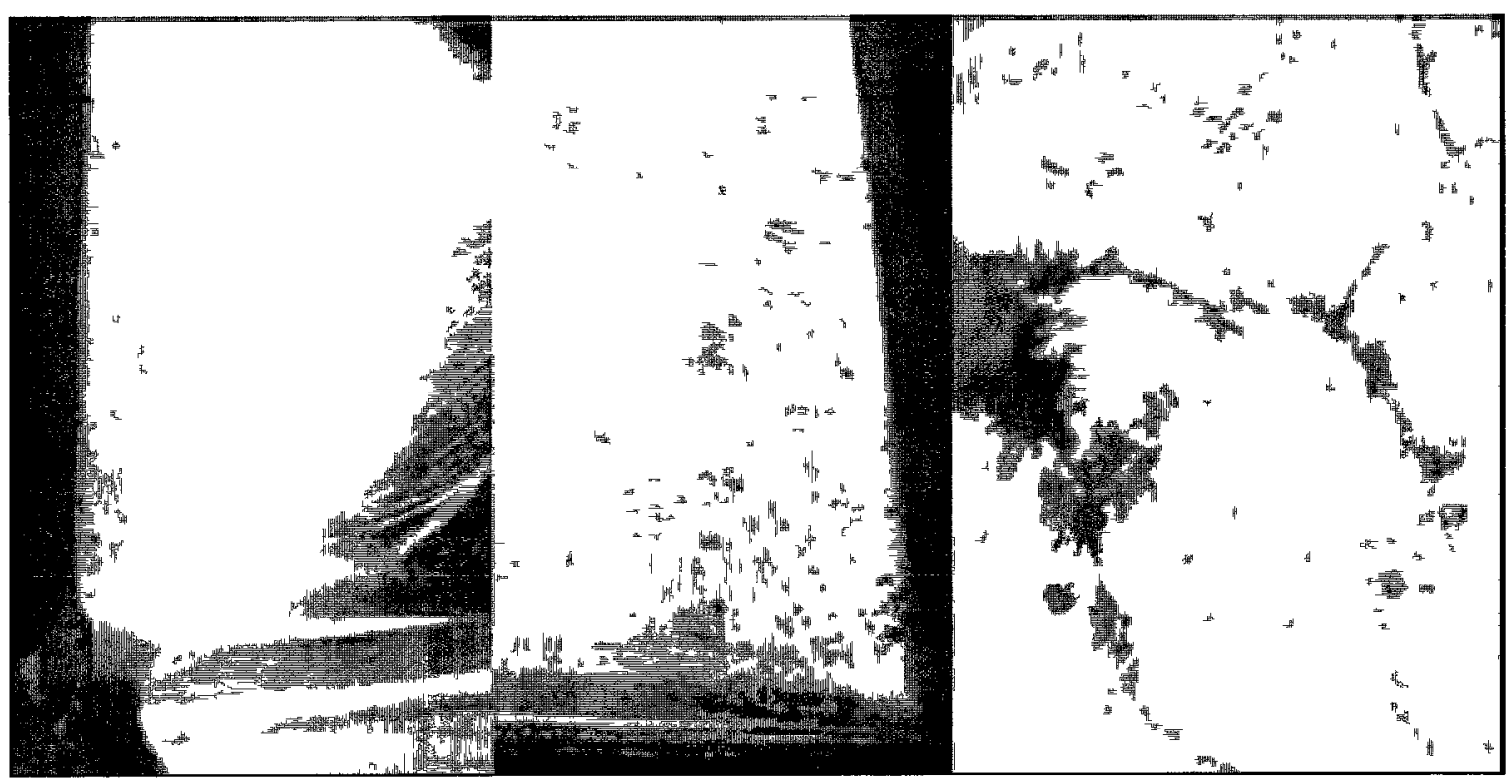

(c) Erosive and corrosive damages of the compressor blade [16]

Figure 2-1 Erosive and corrosive damages on components.

\subsection{Degradation Mechanisms}

\subsubsection{Wear and Erosion}

Physical attacks can be classified as dynamic impact and non-impact. Dynamic impact attacks are the attacks due to repetitive exposure to contacts from solid particulates or liquid droplets, which is termed erosion. Non-impact attacks, taking the form of sliding or 
scratching, are termed wear and cause the surface to roughen and material to be removed. The critical difference between erosion and wear is repeat impingements in erosion and abrasive sliding in wear. The force governing an erosion process is the deceleration of impinging particles, whereas the governing force in a wear process remains approximately constant [10].

Under normal conditions, the physical damages experience three stages: run-in, steady, and wear-out stages. Run-in period is an early stage, where the damage rate is high. In the following steady state process, most of the working lifetime of a component is consumed, but at a relative low rate of damage. In wear-out stage, a high rate of damage leads to fast failure [43] which should be avoided at all costs. Under increasingly harsh conditions, e.g., high temperatures, high strain rates, high stress and high sliding velocities, the steady stage is shortened, thus the run-in stage tends to merge with the wear-out stage, therefore drastically reducing the working lifetime for the component $[43,44]$.

\subsubsection{Wear}

Wear causes progressive loss of material from a solid surface, due to a relative motion between that surface and a contacting substance $[10,45,46]$.

The wear process usually experiences three typical stages [47]. In the initial period (type I), the wear rate is low. In the following period (type II), the wear rate is constant. In the tertiary period (type III), the wear rate increases drastically with time. These types of 
wear can be found in their corresponding regimes in a wear-time curve (Figure 2-2). In this curve, the types of wear correspond to three wear regimes: running-in or break-in or wear-in, the steady state, and accelerated wear/wear out $[47,48]$.
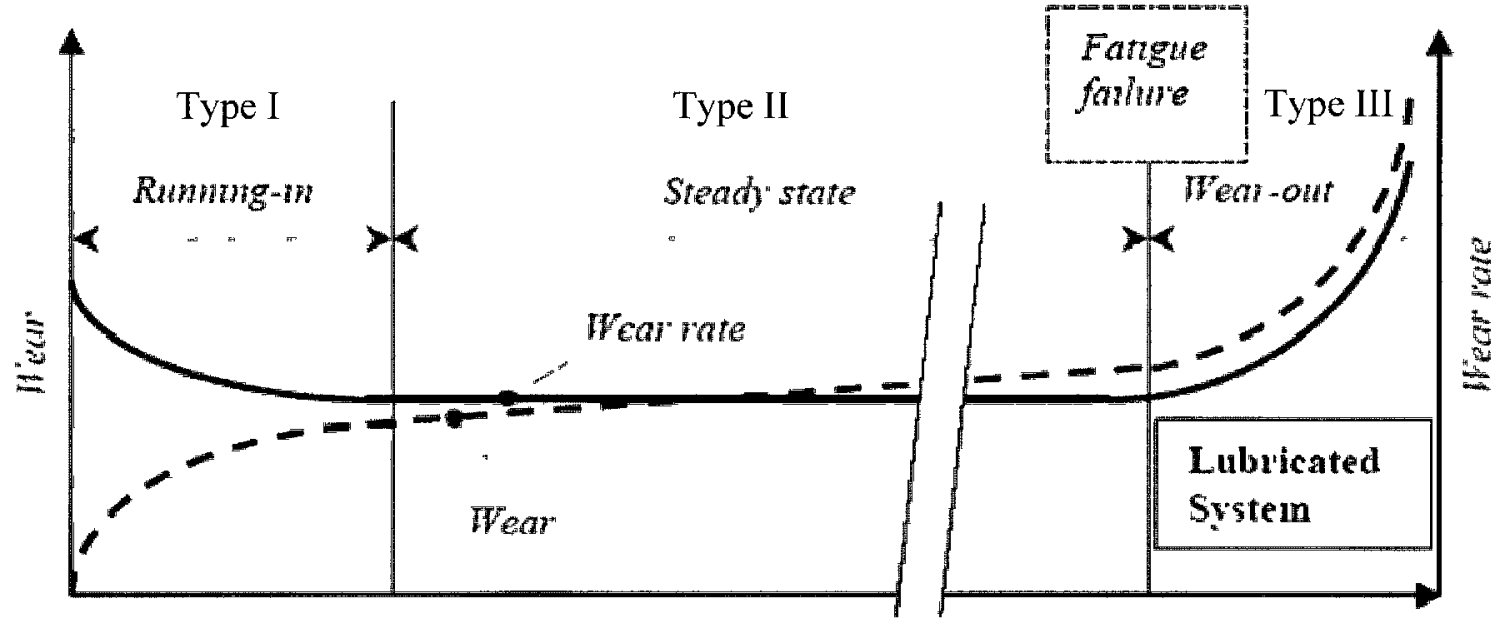

Tme, number of oren ollugs or siding distance

Figure 2-2 Wear and wear rate behaviour as a function of time, number of over-rolling or sliding distance [48].

Type I wear in the running-in regime causes changes in geometry and mechanical properties on the rolling/sliding surface [49]. This regime usually results in asperities, increased average contact area, decreased frictional work (Figure 2-3) and average roughness, lower surface temperature, and decreased wear rate [50]. Two mechanisms, i.e., plastic deformation and mild wear dominate this regime $[49,51]$. Plastic deformation is an important factor in changing the surface topography [52], for which many models were developed. The model of asperity change [48] due to plastic deformation in the rolling contact running-in period reached a good agreement with experiments. A deterministic elastic plastic contact model [53] was proposed for the surface topography of a rolling contact during the running-in phase. In the running-in regime, parameters such as load, sliding velocity, initial surface roughness, lubricant, and temperature have 
controlling effects [54]. A sufficient load benefits surface roughness reduction after running-in; therefore a proper running-in period is desirable for prolonging the lifetime of a system.

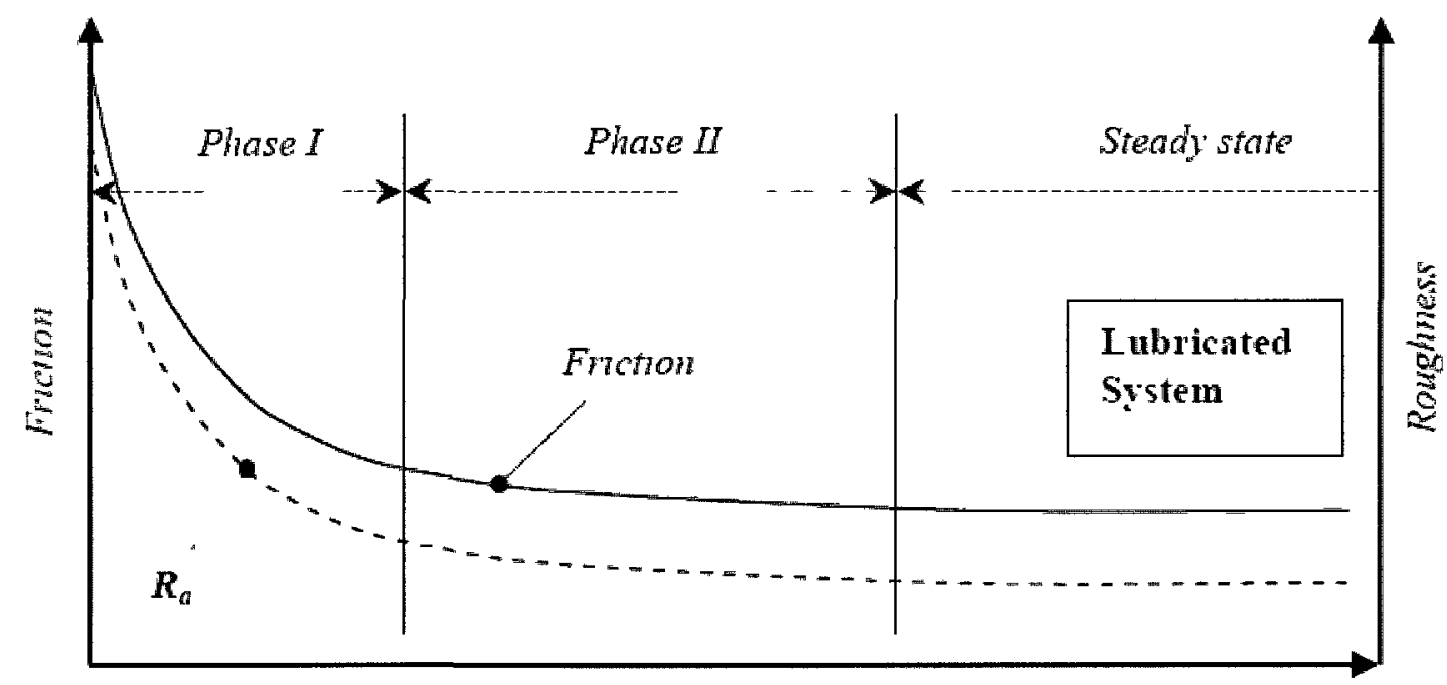

Time, number of overrollngs or sluding distance

Figure 2-3 The change of the coefficient of friction and a roughness as a function of time, number of over rolling or sliding distance of a contact under constant operating conditions [48].

In the steady state Type II wear, the wear rate demonstrates a linear wear vs. time relationship (Figure 2-2). In this phase, the average coefficient of friction, specific wear rate, and other specific parameters are constant [55]. The micro-hardness increases due to selective work hardening; and the coefficient of friction slightly decreases. This phase determines the working lifetime of the components, therefore it is desired that it be as long as possible [56]. As in the wear out region, dynamic loading causes fatigue, and results in material losses, resulting in rapid increases in the wear rate (Figure 2-2).

The extent of the damage and the mechanism of wear are two major concerns in the classification of wear. On the basis of the extent of damage, wear can be classified as 
mild or severe [57]. A comparison of mild wear and severe wear is summarized in Table 2-1. Generally, a mild wear results in polished surfaces (Figure 2-4 a), accompanied by a low wear rate, whereas a severe wear causes roughened surfaces (Figure 2-4 b) with a high wear rate.

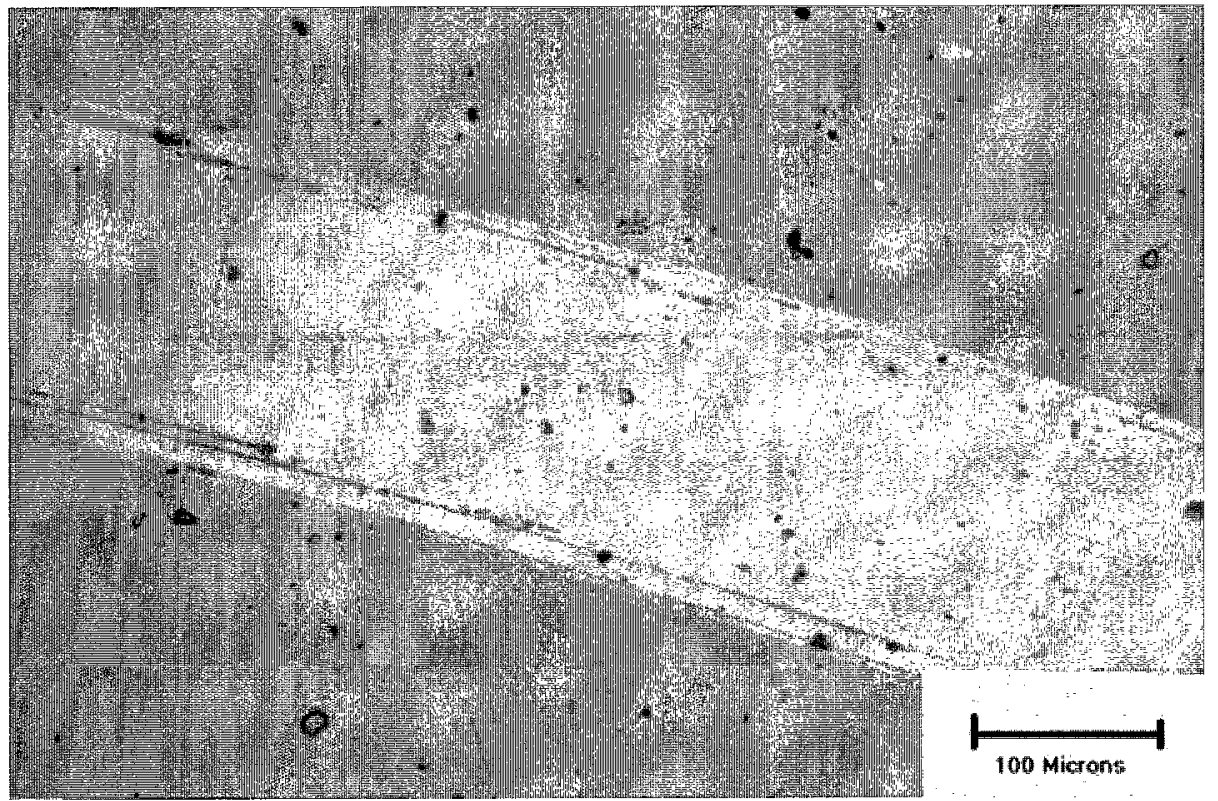

(a) Mild wear

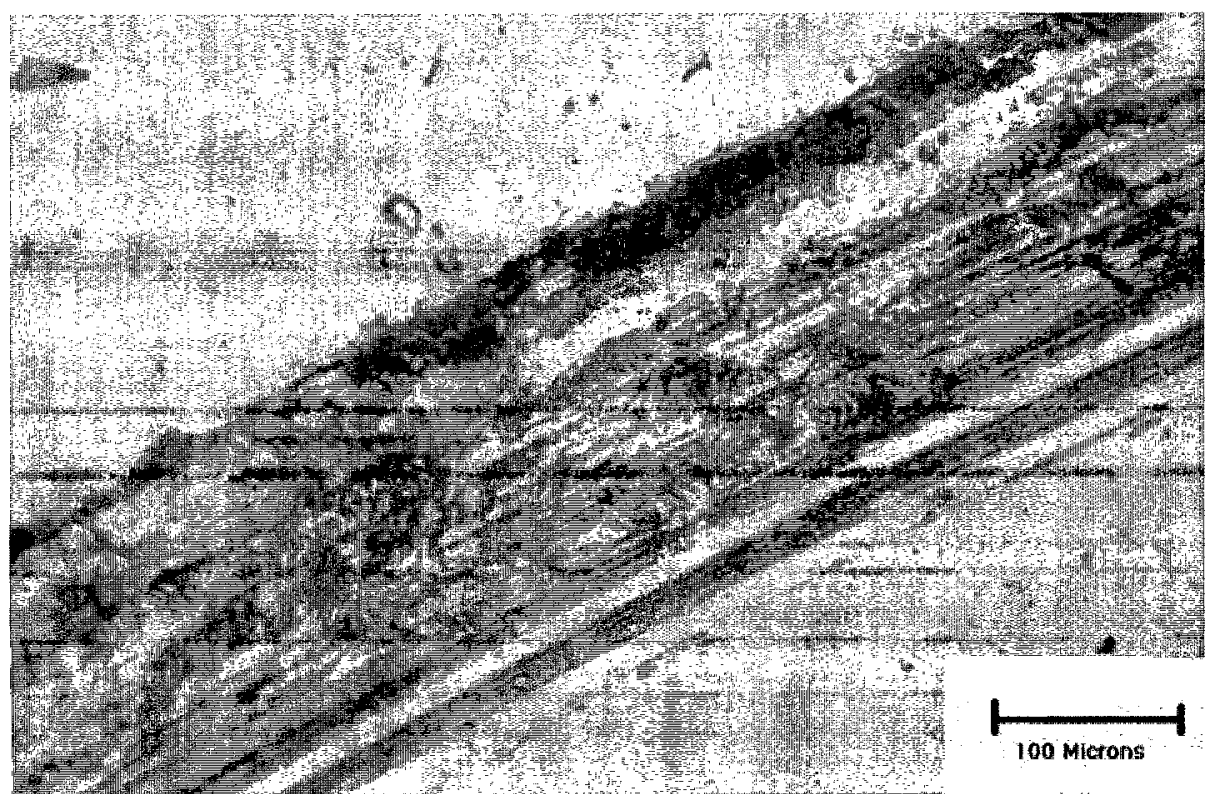

(b) Severe wear

Figure 2-4 Wear tracks on PVD CrN coatings [58]. 
In mild wear, tribochemistry and local plastic deformation are dominant [48]. Tribochemistry is a branch of chemistry about chemical and physical changes related to mechanical energy [59] and is concerned with the chemical reactions at the interface between the lubricant and the contact surfaces under boundary lubrication conditions [60]. In severe wear, material is lost by adhesive wear and material transfer, corrosive wear, cutting, plastic deformation, surface fracture and fatigue, tearing, and melting [1].

The criteria used to distinguish mild from severe wear are the coefficient of friction and the specific wear rate. The coefficient of friction, expressed as $\mu=F / N$, increases as a wear process transfers from sliding to deformation [61], indicating the transition from mild to severe wear, and from steady state to accelerated wear. The rapid increase in wear rate during transition from steady state to accelerated wear also indicates the transition from mild into severe wear [51].

Table 2-1 Distinction between mild and severe wear [51]

\begin{tabular}{|l|l|}
\hline \multicolumn{1}{|c|}{ Mild Wear } & \multicolumn{1}{c|}{ Severe Wear } \\
\hline Result in smooth surfaces & Result in rough, deeply torn surfaces \\
\hline Debris small than $100 \mathrm{~nm}$ & Large metallic wear debris up to $0.01 \mathrm{~mm}$ \\
\hline High electrical contact resistance & True metallic junctions formed \\
\hline
\end{tabular}

The transition from mild to severe wear would result in an acceleration of degradation. Predicting and prohibiting this transition are critical for governing the working lifetime of a component. The mechanical severity of contact $\left(S_{c, m}\right)$ has been proposed as one of the physical parameters for evaluating the transition from mild to severe wear, and is described as [57]: 


$$
S_{c, m}=\frac{(1+10 \mu) P_{\max } \sqrt{d}}{K_{I c}}
$$

where,

$\mu$ : coefficient of friction,

$P_{\max }:$ maximum Hertizan contact pressure,

$K_{I c}: \quad$ fracture toughness, and

$d$ : pre-existing crack length.

$S_{c, m}$ : mechanical severity of contact, is used to identify the transition from mild to severe wear, with a threshold value of 6 [57].

The general wear mechanism is the energy transfer accompanied by material removal or displacement [62]. Five basic specific wear mechanisms were summarized as adhesive wear, abrasive wear, surface fatigue [63], tribochemical reactions [62], and corrosive wear [64]. In adhesive wear, two smooth bodies slide against each other; fragments are pulled off one surface, adhering to the other [65] and is found in severe wear [63]. Abrasive wear takes place as a hard rough surface or a soft surface containing hard particles slides over a soft surface, and ploughs grooves. Fatigue wear takes place with repeated sliding or rolling over a surface, causing deformations or cracks [48]. In rubbing, chemical reactions occur at the interface between the contact materials [60] that accelerate material damage. In a corrosive environment, a reaction layer is generated. As the layer is removed by mechanical action, corrosive wear occurs $[48,64]$.

Wear processes are associated with two important tribological factors, friction and lubrication, which are described in the following sections, then the two important modes of wear, adhesive and abrasive, are reviewed in detail. 


\subsection{Friction and Lubrication}

\section{Friction}

Friction is defined as the resistance to relative lateral (tangential) motions between solid surfaces, fluid layers, or other material elements in contact.

Friction can be classified into several modes: dry friction, lubricated/fluid friction and internal friction. Dry friction consists of relative lateral motion between two unlubricated solid objects in contact. This friction can be further divided into static and kinetic. Static friction occurs between two solid objects at rest, opposes the relative sliding of the objects, and increases with the tangential force that attempts to move an object. When the tangential force overcomes the maximum static friction force (referred to as limiting friction force), the objects begin to slide relative to each other. At the moment that sliding occurs, static friction is no longer applicable and kinetic friction governs the dynamic sliding motion.

Lubricated friction [66] or fluid friction $[67,68]$ resists relative lateral motion of solid surfaces separated by a layer of fluid. This friction also describes the friction phenomenon between the fluid moving layers $[69,70]$.

A friction force is derived from electromagnetic force between charged particles such as electrons, protons, atoms, and molecules on two contacting surfaces. This force is difficult to calculate, so it is obtained in an empirical way. 
The adhesion theory of friction [71] discusses the cause of friction. When two surfaces are brought into contact under an applied load, junctions are formed where asperities contact. When contacting surfaces move relative to each other, the friction between the two surfaces converts kinetic energy into thermal energy. A kinetic friction is caused by chemical bonding between the surfaces [72] at asperities in micro- and nano-scale [73]. The total area of real surface contact at asperities is a function of load and penetration hardness of the softer material, and is approximated by the equation:

$$
A=N / H
$$

where,

$A$ : real area of contact

$N$ : load

$H$ : penetration hardness of the softer material

During sliding between two surfaces in contact, junctions are sheared. Therefore, the friction force $(F)$ is related to the shear stress $\tau$ of the softer material and the real contact area $A$ :

$$
F=\tau A
$$

Since $F=\mu N$, then $\mu=\tau / H$. The value of the ratio $\tau / H$, which equals the coefficient of friction, remains constant for most metals. With such a relationship, for clean, nonlubricated surfaces, the real effective contact area is increased as the surfaces wear, and increased surface energy generates more friction, therefore the coefficient of friction rises as the surfaces wear. 


\section{Lubrication}

Lubrication is a process or technique used to reduce the wear of surfaces, in contact and moving relative to each other, by interposing a third substance (in a form of film), termed a lubricant, between the surfaces to carry the load (pressure generated) between the surfaces. The lubricant can be a solid, a solid-liquid dispersion, a liquid, a liquid-liquid dispersion (greases) or a gas.

Three distinct situations can be observed with respect to the mode of lubrication, i.e., fluid film lubrication $[74,75]$, elastohydrodynamic lubrication $[75,76]$, and boundary film lubrication [75].

In a fluid film lubrication regime, through viscous forces the load is fully supported by the lubricant within the gap between the objects moving relative to each other, therefore solid contact is avoided. This mode of lubrication can be further divided into hydrostatic and hydrodynamic lubrications [75]. In a hydrostatic lubrication, an external pressure is applied to the lubricant, to maintain the fluid lubricant film; whereas in a hydrodynamic lubrication, the motion of the contacting surfaces is used to pump the lubricant around the moving object to maintain the lubricating film.

In elastohydrodynamic lubrication, an elastic deformation on the contacting surface is induced by viscous resistance of the lubricant which increases the load contacting area. In boundary film lubrication, chemically reactive components of the lubricant react with the contact surface at the elevated temperature and pressure conditions, forming a highly 
resistant film on the moving surfaces, which is referred as to a boundary film. This film is sufficient to support the load, and reduce asperity direct contact due to the normal force.

Adequate lubrication reduces wear between solid objects by preventing asperity contact; therefore it minimizes wear damage to the solid surfaces.

\subsection{Adhesive Wear}

Adhesive wear is a consequence of material transfer caused by direct contact and plastic deformation under a high normal load between the asperities in a rubbing pair. The asperities on the contacting surfaces penetrate the opposing surface, and generate a plastic zone surrounding the penetrating asperity. Adhesive wear is the most common form of wear and is commonly encountered on an unlubricated surface or in the case of lubricant failures. This type of wear is also referred to as cold welding wear, scoring, galling, seizing, or scuffing in engineering applications.

The mechanism of an adhesive wear is attributed to the contact that may result in a surface plastic deformation, where soft surface films or oxide layers are broken up and removed. Clean surfaces provide stronger adhesion [77] at the asperity contact, and increase the fragments pulled off due to repeated cold weld and separation [63]. In this type of wear, physical-chemical adhesive interaction between the surfaces plays a role in the initial asperity bonding process but the energy absorbed in plastic deformation and movement is the main cause for material transfer and wear. Oxidation films and other 
contaminants between the contact surfaces generally suppress adhesion wear but may contribute to abrasive wear [78].

The general rate of adhesive wear (i.e. volume removed per unit time), regardless the form of damage, is described by the Archard adhesive wear equation as [79]:

$$
w=k \frac{N L}{H}
$$

where,

$w$ : wear rate

$N$ : applied load,

$L$ : sliding distance,

$H$ : hardness of the softest material,

$k$ : wear coefficient.

\subsection{Abrasive Wear}

Abrasive wear behaviour is related to a variety of material characteristics [10]. Hardness is a primary property for abrasive wear resistance. The hardness and load together determine the contact surface area, which is proportional to the wear rate [80]. A high modulus results in high contact stresses that probably weaken this resistance. High yield strength would increase wear damages due to less fracture. Strain hardening could complicate the situation. A high melting temperature would increase the resistance to wear due to less softening.

Regarding crystal structure, wear rate for cubic crystals is about twice that for hexagonal crystals due to the differences in slip system and plasticity. With respect to microstructure, materials with high strain hardening exponent have better wear resistance. A high 
toughness increases the wear resistance, mainly for brittle materials. In general, alloys demonstrate higher wear resistances than pure metals. Carbides based materials or carbide reinforced metals usually have improved wear resistances [10]

Mechanisms of abrasive wear usually take the forms of cutting, wedge formation, plowing, and brittle fracture (cracking). In cutting wear, a chip forms in front of the cutting asperity. The material is removed from the surface, and the wear track (groove) is generated (Figure 2-5 a). In a wedge forming wear, the material is deformed, and accumulates in front of the sliding asperity (Figure 2-5 b). In plowing wear, the material is shifted to the sides of the wear groove, but is not removed from the surface (Figure 2-5 c). In cracking (brittle fracture) wear, the material cracks in the subsurface regions surrounding the wear groove (Figure 2-5 d).
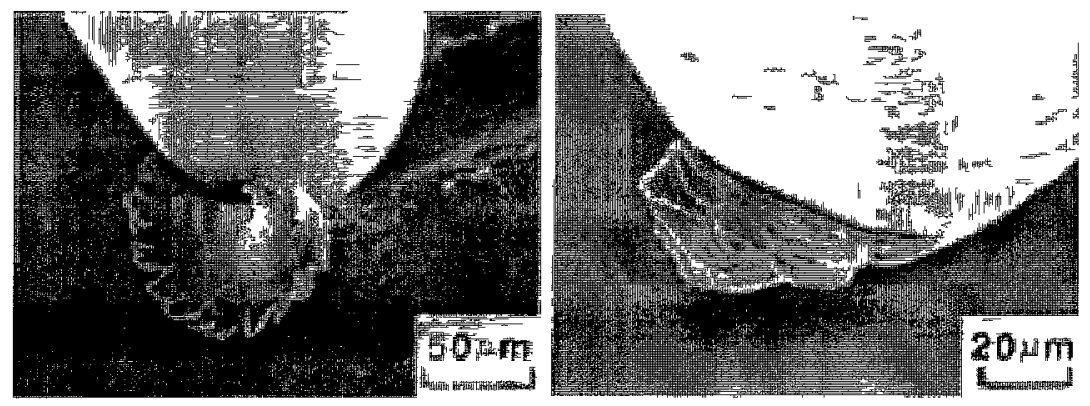

(a) Cutting [81]

(b) Wedge forming [81]
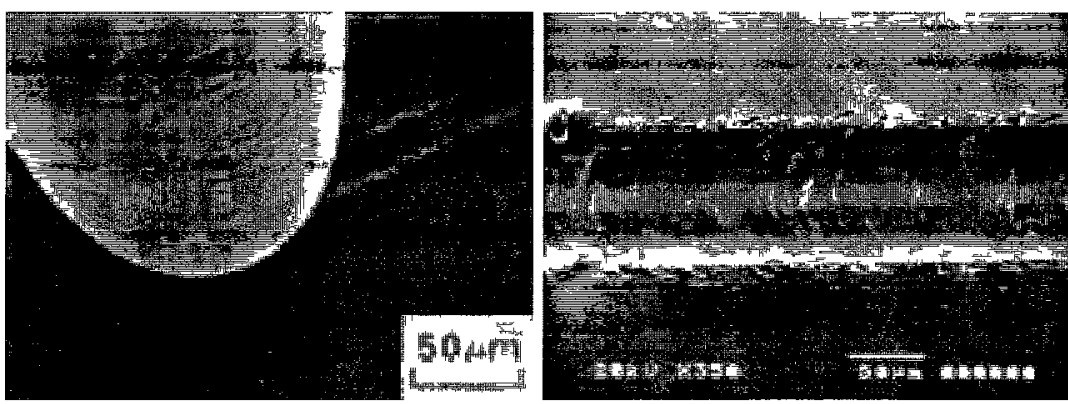

(c) Plowing [81]

(d) Cracking (brittle fracture) [82]

Figure 2-5 SEM images of processes of abrasive wears. 


\subsection{Models for Predicting Abrasive Wear}

An early analysis of wear was conducted by Holm [83] who established the relationship for the wear removed volume $\left(V_{\text {wear }}\right)$ in the sliding distance $L$ and the true contact area, which was followed by the work of Archard [65] who developed the Archard wear equation [84]:

$$
V_{\text {wear }}=k_{A} \frac{N L}{H}
$$

where,

$V_{\text {wear }}$ : the wear removed volume,

$k_{A}$ : Archard wear coefficient $(<1)[65,85]$.

$L$ : the sliding distance,

$N$ : the normal load,

$H$ : the hardness of the worn surface.

Replacing the wear coefficient $\left(k_{A}\right)$ and hardness of the surface $(\mathrm{H})$ with the wear rate $\left(k_{K}\right)$, Eq. (2-5) is simplified as [86]:

$$
V_{\text {wear }}=k_{K} N L
$$

This Archard wear equation (Eq. 2-6) postulates a proportional relationship between the wear rate (worn volume per unit sliding distance), load, and material combination.

A number of modified Archard's wear equations have been widely used in numerical simulations, especially for the mild wear situation, and have given satisfactory results [87 -91]. One of the equations uses a semi-empirical approach and relies on experimental data to determine the depth of wear $h$, and is presented as [92]: 


$$
h=\frac{K}{H} L^{n} P^{m}
$$

where

$K$ : wear coefficient,

$H$ : the hardness of the worn surface,

$L$ : sliding distance,

$P$ : pressure,

$m$ and $n$ : exponents determined by materials and test conditions [56].

The empirical equation (Eq. 2-7) indicates that Archard's linear wear law [65] involves the mechanical properties of the interacting materials. It states that the volume of the removed material is proportional to the sliding distance $(L)$, the pressure $(P)$, wear coefficient $(K)$, and is inversely proportional to the hardness of the contact surface $(\mathrm{H})$ [85].

Replacing wear coefficient $(K)$ and hardness of the surface $(\mathrm{H})$ with the dimensional wear rate $\left(k_{K}\right)$, Eq. $(2-7)$ is simplified as:

$$
h=k L^{n} P^{m}
$$

Eq. (2-8) is widely used for wear resistance comparison among materials.

Another modified Archard's wear equation, Khurshchov empirical equation [10], was developed incorporating the elastic properties of the interacting materials:

$$
V_{\text {wear }}=\frac{k_{K}}{E^{1.3}}
$$

where,

$E$ : modulus of elasticity, $k_{K}$ : the Khurshchov wear coefficient, and is determined through experiments 
In order to describe all phases during a wear process, i.e. running-in, steady state, and accelerated wear, a dynamic wear model was proposed [47], which is also modified from Archard's wear equation, and is presented as:

$$
V_{\text {wear }}=c F / S
$$

In this model, the wear rate is proportional to a force term $(F)$, which is contributed by frictional force, and is inversely proportional to the wear resisting term $(S)$, which is related to material strength near the surface. The constant $(c)$ can be determined experimentally or by using analytical methods.

Surveying the literature, it has been found that there have been over thirty two parameters and nearly two hundreds of equations proposed, involving various material properties and working conditions [93], in attempt to find a model that can describe and predict complex wear processes. However, not a simple and universal model has been found to be able to predict wear on the basis of mechanical properties and contact information [61]. This is primarily due to the complexity of the wear process which is highly dependant upon specific operating conditions. Experimentation in conjunction with semi-empirical approaches on a case by case basis seems to provide a more effective prediction.

\subsection{Abrasive Wear Test}

The wear testing methods include Taber ${ }^{\circledR}$ Abrasion test, sand rubber wheel abrasion test, ball-cratering method [94] (also referred as Calo ${ }^{\circledR}$ test), and pin-on-disk test $[95,96]$ as shown in Figure 2-6. 


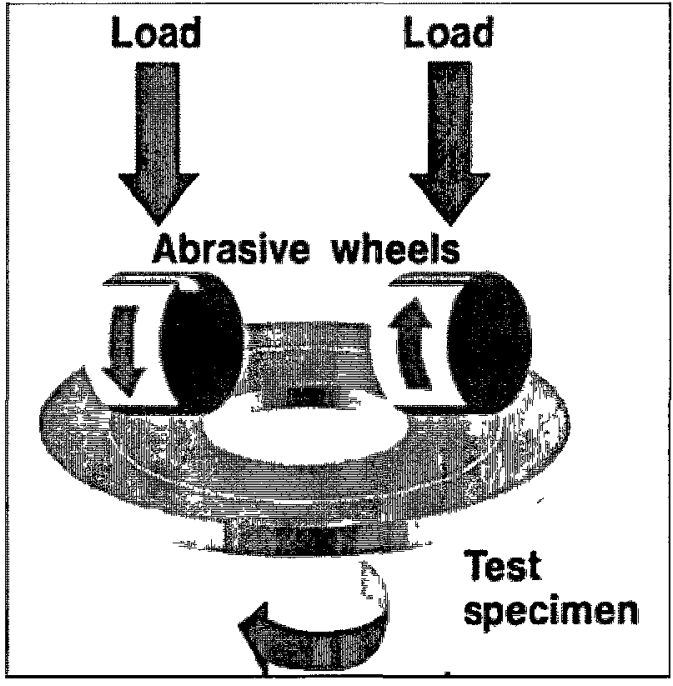

(a) Taber ${ }^{\circledR}$ Abrasion test [95]

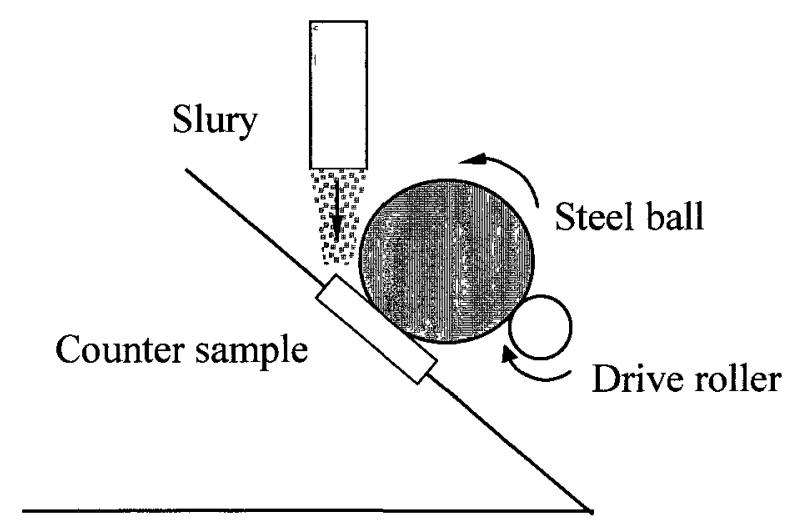

(c) Ball-cratering/Calo ${ }^{\circledR}$ test (redrawn from [97]) (d) Pin-on-disk test [96].

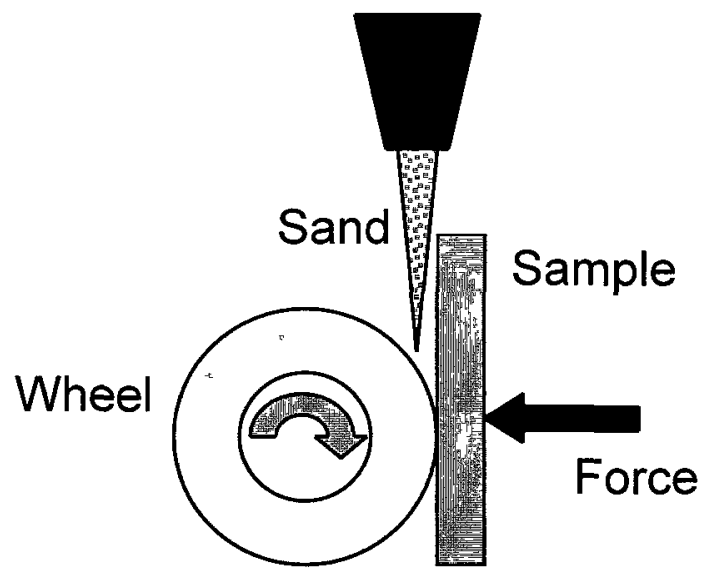

(b) Dry sand rubber wheel abrasion test

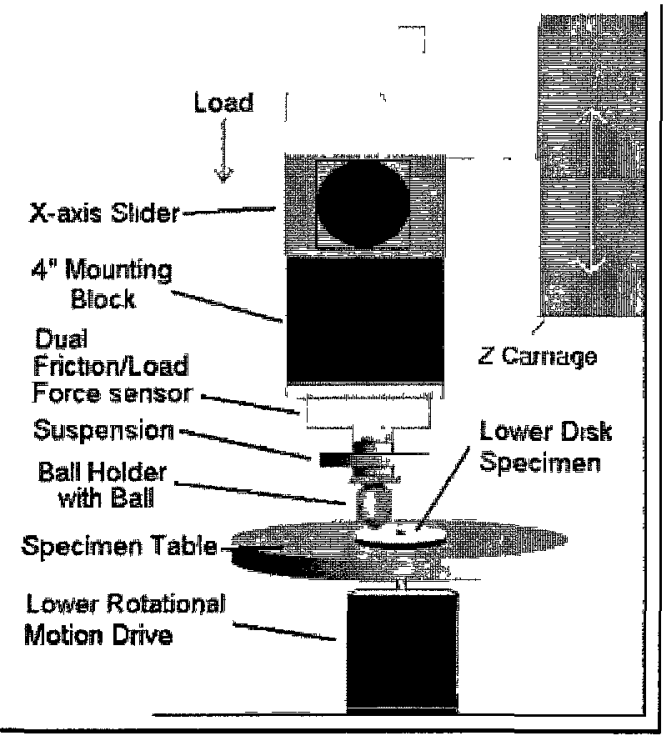

Figure 2-6 Schematic illustration of various types of abrasive wear tests.

The Taber ${ }^{\circledR}$ Abrasion test uses the rotary platform abrasion tester (Taber ${ }^{\circledR}$ Abraser) for evaluating resistance to rubbing abrasion. In this test, wear is caused by the rolling action of an abrading wheel against a spinning specimen [95]. This test has been widely used, and covers a wide spectrum of testing materials. The ball-crater and dimple-grinder 
methods have been further developed and used extensively to assess the abrasive wear resistance of thin hard coatings [94].

The pin-on-disk test is the most common abrasive wear test [98]. A fixed pin (usually a cylinder with a hemispheric end), under a certain load makes contact with a flat disk as it rotates at a certain velocity $[95,96]$. This test is designed for evaluating resistance to sliding wear, has been widely used, and is recognized as one of the standard wear evaluating methods [96].

\subsubsection{Erosive Damage}

Erosive damage is the destruction and loss of material as a consequence of repeated impacts from the abrasive action of a moving fluid or solid particles [10]. As a special case of wear, erosion involves material removal by cutting or surface fatigue [1], causing changes in volume / weight or shape of the attacked object [99 - 102]. The manifestation of particle erosion includes thinning of components, macroscopic scooping appearance, surface roughening, and formation of ripple patterns on metals [10].

\subsection{Types of Erosion and Mechanism}

The nature of the material and the erosion conditions govern the mechanism of an erosion process. The natures of materials include microstructure and various mechanical and physical properties $[103,104]$. For ductile materials, impacting particles cause severe and localized plastic strain that eventually cause fracture [105] (Figure 2-7 a). As for brittle 
materials, erosion is induced by the propagation and intersection of cracks [106] (Figure 2-7 b), and followed by fracture of the cracked micro pieces [103]. This form of erosion for brittle metals is attributed to Herzian stress (as indicated in Figure 2-7 b) [105, 107].

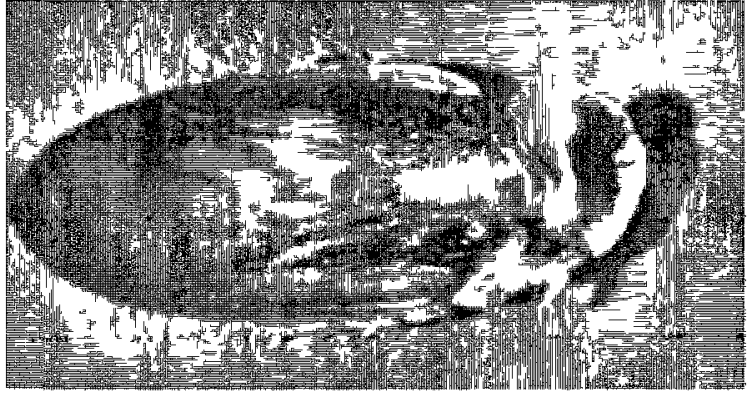

(6) Crater shape

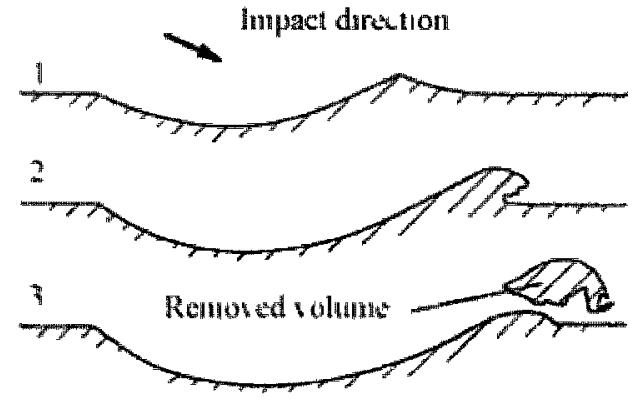

(h) Mutentul nemotal

(a) Ductile material erosion: cutting

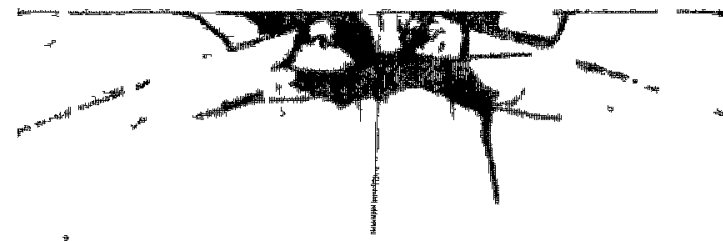

(a) Critur shape

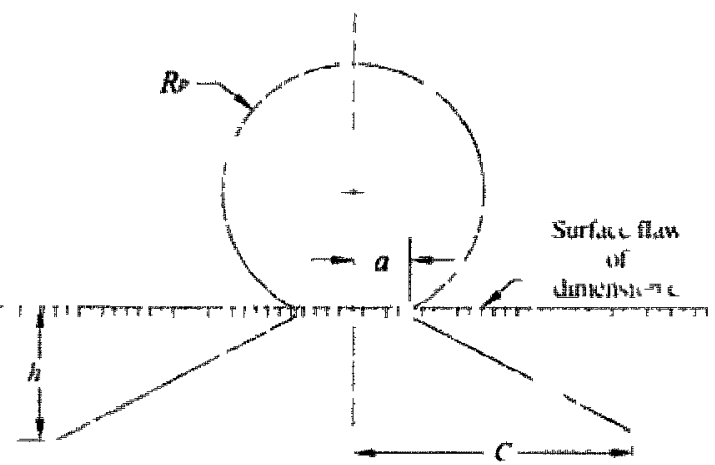

(b) Crack propdgatora

(b) Brittle material erosion: cracking

Figure 2-7 Mechanisms of erosions for ductile and brittle materials [108].

A contact surface involving many grains would reduce the rate of erosion $[100,109]$ due to the effect of grain boundary strengthening (Hall-Petch relationship), thus small grain sizes are preferred for erosion resistance. However, material heat treatment conditions should be also taken into account as well [110]. 
Erosion conditions, which are independent of the properties of the substrate, include impingement variables (impingement angle and erodent velocity), testing temperature, erodent feed rate, and erodent properties such as size, shape, density, hardness and friability. With respect to impingement angle, erosion can be further subdivided into abrasive and impingement modes. The abrasive erosion is a micromachining action of particles impacting the surface at low impingement angles, i.e., the relative motion of the abrasive particles is nearly parallel to the solid surface. This action involves low stresses, and does not fracture the surface. Impingement erosion is the impact of particles at large incident angles, which induces cracking and crack coalescence.

For hard and brittle materials, impingement induced cracking damage is proportional to the normal component of the impact momentum. At low impingement angles, the normal component of the impact momentum is low, and it is usually difficult to initiate cutting, therefore the erosion rate is low. High-angle erosions tend to be fatigue in nature. The erosion rate increases with impingement angle, approaching the maximum near a right angle for brittle materials, in which cracks can easily form and propagate [106].

Erosion rates for ductile materials are generally high due to their low strengths. The cutting mechanism dominates at low impingement angles due to high tangential impact force, while the work hardening effect dominates at high impingement angles. Materials consume impact energy mainly in the forms of plastic deformation and defect accumulation or micro cracking, depending on the hardness of materials. Erosion rates for ductile materials quickly increase with impingement angles in the low angle region, 
and reach the maximum values at low impingement angles (for example $30^{\circ}$ ); then decrease with a further increase of impingement angle, as the ductile materials can absorb most of impact energy at higher impingement angles $[106,111]$.

A comparison between the erosion rates with respect to material properties is shown in Figure 2-8. Understanding the impact angle related erosion mechanism is necessary for the selection of materials and their microstructures for different applications.



Figure 2-8 Erosion resistances vs. impingement angle [106].

Another important factor in erosion is particle impact velocity which significantly influences the erosion rate of brittle materials [112]. At low impact velocities, cracks take cone shapes, and radial cracking changes to lateral cracking with increase in further 
higher velocity, lateral cracks form. At high impact velocities, the damage pattern is essentially independent of the impacting particle geometry, impact angle, and surface hardness [113 - 115], and is predominantly governed by the kinetic energy of the impacting particles [116]. As shown in Figure 2-9, the erosion rate for brittle materials increases with impact velocities.

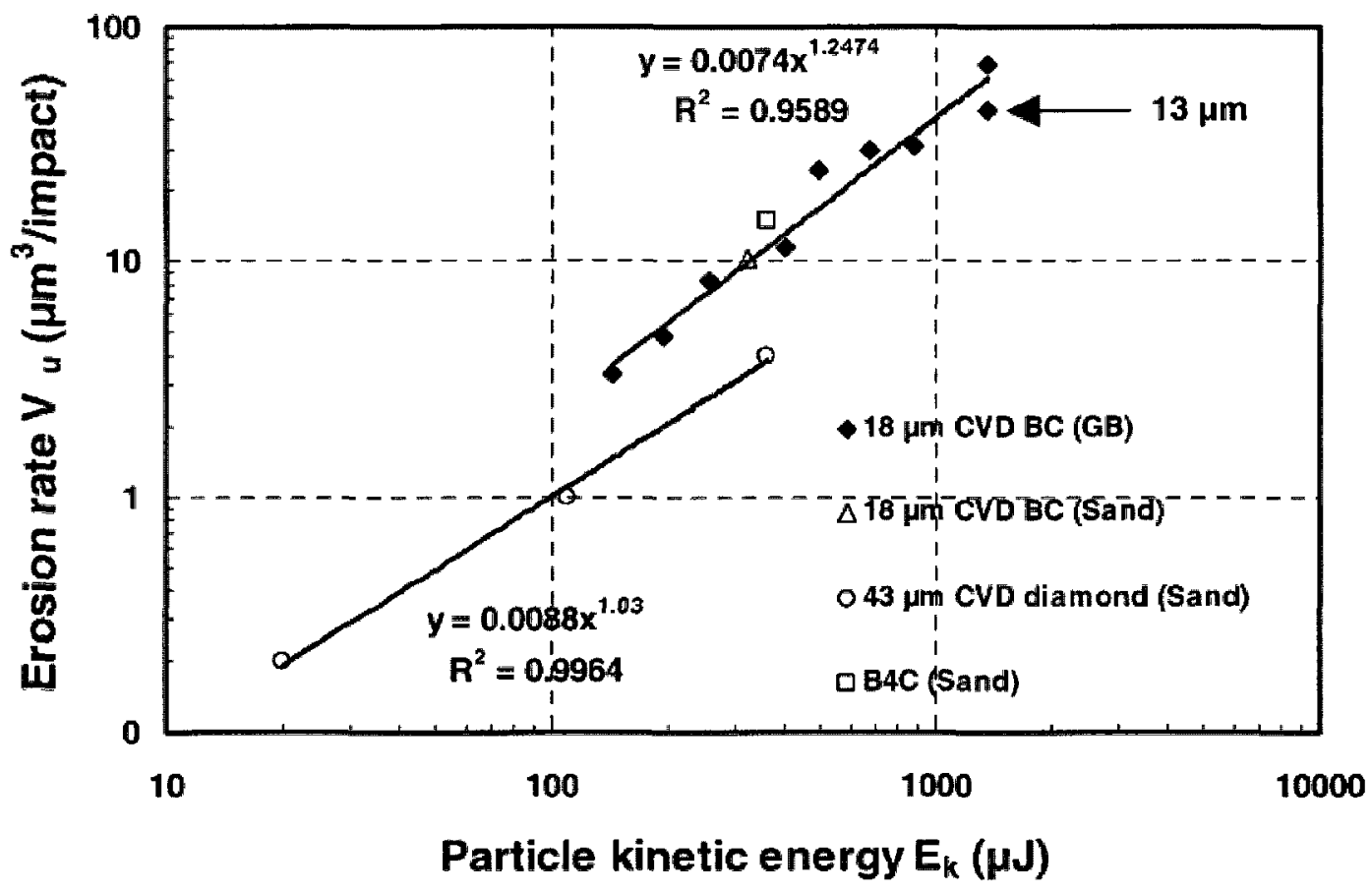

Figure 2-9 Erosion rate as a function of particle kinetic energy [117].

Temperature is another factor in an erosion process as surface properties of the metal significantly change due to oxide layer growth and annealing [118]. Under such conditions, erosion consists predominantly of three processes: erosion, erosion-oxidation and oxidation. Erosion-accelerated oxidation [119] is commonly seen in gas turbines where erosion removes the oxidation resistant layer rendering the substrate material more prone to oxidation. 


\subsection{Modeling of Erosion}

A common method of quantifying erosion is to measure the material loss normalized by the amount of erodent [94]. In a non-corrosive condition, an erosion process is mostly measured by erosion rate, $E_{m}$ or $E_{v}$, which is defined as the mass or volume of material removed by a unit mass of erodent, respectively, and can be simply described as:

$$
E_{m}=M_{m} / M_{e} \quad \text { or } \quad E_{v}=V_{m} / M_{e}
$$

where,

$M_{m}$ : mass of removed material,

$V_{m}$ : volume of removed material,

$M_{e}$ : mass of erodent.

This is the most common approach that has been employed in erosion evaluation. However, it can only describe relative erosion damage for a given test condition, and does not take erosion mechanism into account. Attempts have been made to involve other factors associated with erosion mechanisms under various test conditions. For example, kinetic factors were introduced to describe erosion rate in a dynamic mode [94] and described as [120]:

$$
E_{m}=(k / 2) \rho v^{2}
$$

where

$\rho$ : the density of the surface material, $k$ : specific wear rate or dimensional wear coefficient, and $v$ : abrasive particle velocity.

A tribological intensity $(\psi)$ (representing kinetic energy of the particle striking the unit surface per unit time) was introduced to characterize the relationships among particle velocity, impact angle, and erodent particle flow, and is written as:

$$
\psi=\varphi f(v, \theta)
$$


Eq. (2-13) indicates that the tribological intensity $(\psi)$ is linearly proportional to the particle flux $(\varphi)$ for a certain particle velocity vector determined by impact angle $(\theta)$ and particle velocity (v) [94]. This triobological factor, involving dynamic factors in the erosion process, can be used to modify other equations.

The volume loss due to erosion for ductile materials is described as [121]:

$$
V_{\text {ero }}=\frac{M_{e} v^{2}}{\psi \rho \chi}\left(\sin 2 \theta-\frac{6}{\chi} \sin ^{2} \theta\right)
$$

where,

$M_{e}$ : mass of erodent, $\rho$ : density of material,

$v$ : velocity of erodent,

$\theta$ : impact angle,

$\psi$ : the tribological intensity, the second erosion factor obtained from metal cutting experiments;

$\chi=P / F$,

where,

$P:$ normal contact force,

$F$ : friction force.

This equation assumes erosion abrasives bounce off the impacted surface with sufficient kinetic energy. In the case that an abrasive cuts into a ductile surface, and stops at a depth as its kinetic energy is exhausted, the volume loss can be expressed as [121]:

$$
V_{\text {ero }}=\frac{M_{e} v^{2}}{\psi \rho \chi}\left(\frac{\chi}{6} \cos ^{2} \theta\right)
$$

Eq. (2-14) and Eq. (2-15) involve the factors of material density, erodent velocity and impact angle. At a certain velocity, the materials with a lower density (or more porous) would be subjected to more loss. For ductile materials, the loss is proportional to the $2^{\text {nd }}$ 
power of erodent velocity or its kinetic energy. This indicates the predominant role of the impact velocity in an erosion process. A critical impact angle exists, usually lower than $30^{\circ}$, at which material loss reaches the maximum, and over which the loss begins to decrease. However, these two models lose their accuracies as impact angles approach $0^{\circ}$ and $90^{\circ}$, and are not valid at these two impact angles.

The volume loss due to erosion for brittle materials is described as [121]:

$$
V_{\text {ero }}=k_{4}\left(\frac{R^{6}}{R^{*}}\right)^{(06 \mu) /(\mu-2)} v^{(24 \mu) /(\mu-2)}
$$

where, $v$ : the velocity of erodent,

$R$ : the particle radius, $\mu$ : coefficient of friction, $k_{4}$ : the erosion constant that is determined by normal stress and velocity; $R^{*}$ : a constant determined by $\mu$, velocity, and elasticity of the substrate.

In this equation, for a given erosion condition, the item $k_{4}\left(\frac{R^{6}}{R^{*}}\right)^{(06 \mu) /(\mu-2)}$ is a constant; the erosion loss appears independent of the impact angle, and is mainly associated with the velocity of erodent and coefficient of friction. However, for a given material, the impact induced friction force is proportional to the normal component of the impact momentum (product of mass and velocity). Furthermore, for a given erodent with a certain impact velocity, the normal component of impact momentum increases with the impact angle. This indicates that higher impact angles would result in greater impact induced friction forces in addition to greater normal impact momentums, and therefore leads to higher erosion loss. This is in agreement with the foregoing discussion on the characteristics of brittle erosion. However, due to the fact that many parameters in this equation need to be 
determined by means of experiment, the accuracy of the prediction is dependent on the accuracies of experiments.

Other empirical equations have been developed with respect to specific erosion conditions $[7,103,107,117]$. However, those models, including the above mentioned, are only valid for certain materials under certain test conditions. As a special type of wear, erosion is a complex process involving numerous intrinsic and extrinsic factors. It is difficult to describe an erosion process using a purely mathematical model or a simplified

empirical equation. Instead, experimental investigation in combination with semiempirical equations is a more effective methodology in erosion evaluation.

\subsection{Erosion Tests}

Two widespread uses of erosion test are gas blasting and centrifugal accelerating, as shown in Figure 2-10. In a gas blast rig, erodent particles are accelerated through a nozzle by a stream of gas (dry nitrogen or argon) [122], shooting at the specimens [94]. In a centrifugal accelerating test, particles on a rapidly rotating disc are accelerated by the centrifugal force, flying to the specimens. In comparison with centrifugal accelerating test, a gas blasting test can provide a wider range of particle velocities, and does not need taking into consideration of the impact of rotating particles; a centrifugal accelerating test can closely control the erosion test parameters, and does not need a gas supply [123]. In this study, the erosion evaluations were conducted using blast test, under the same conditions of erodent properties, impingement velocity and angle, and in a comparison approach. Obtained data was processed using Eq. (2-11). 


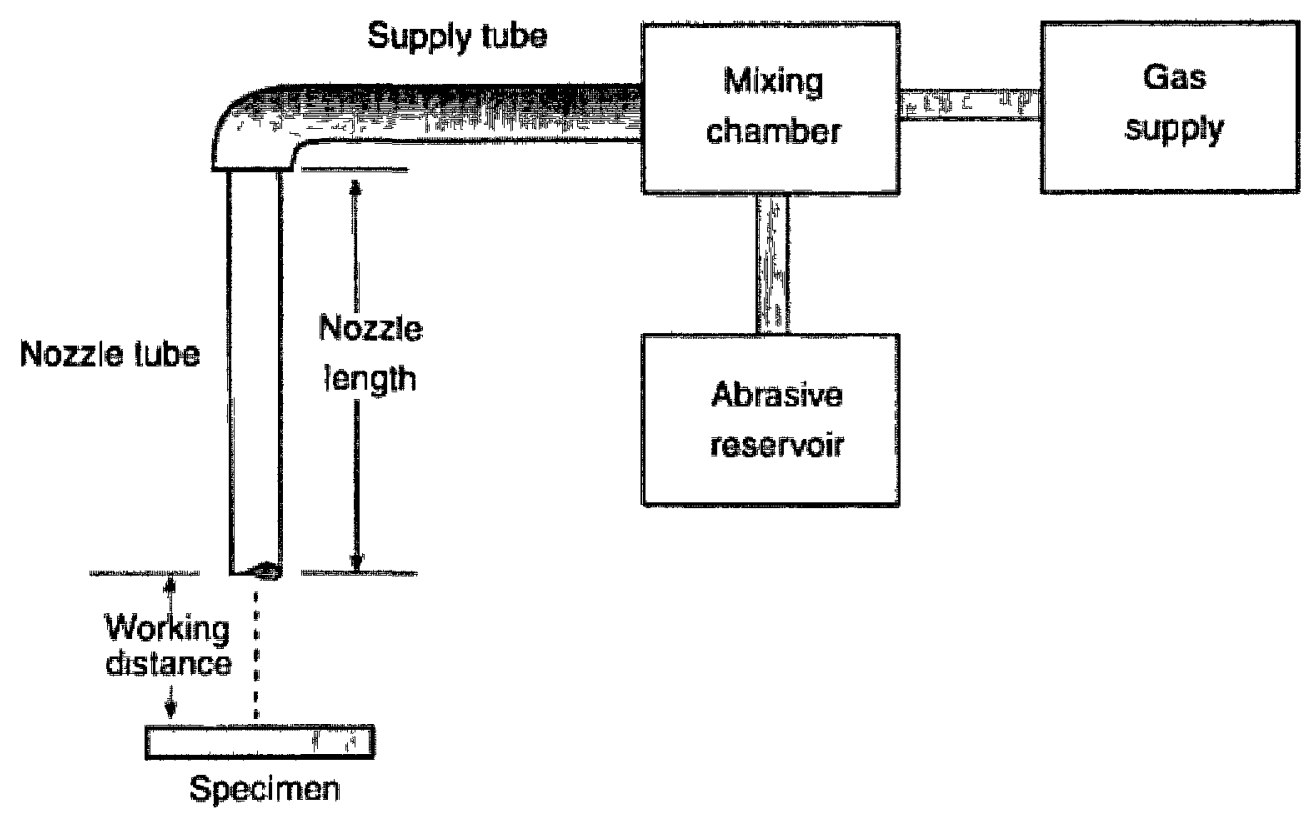

(a) Gas blast erosion test system [122]

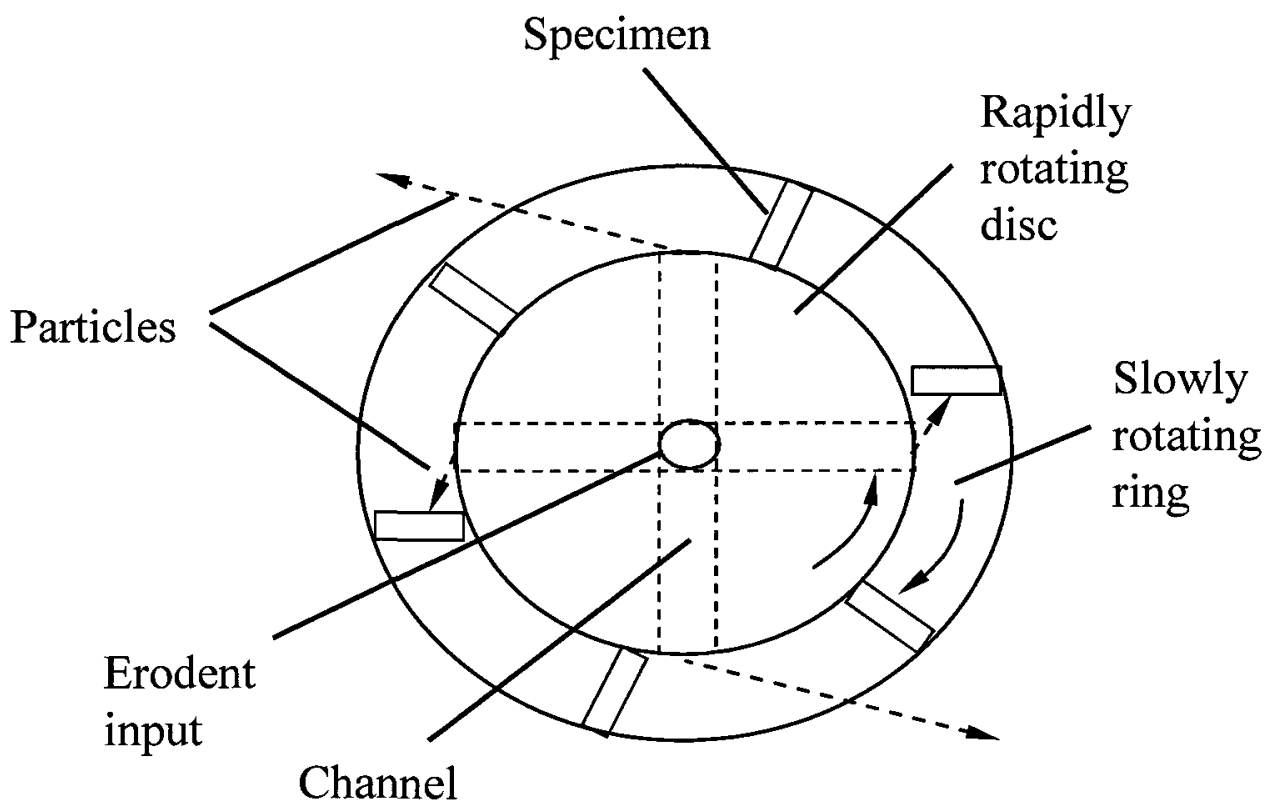

(b) Centrifugal erosion test system (redrawn from [124])

Figure 2-10 Schematics of erosion test systems. 


\subsubsection{Corrosion}

Corrosion is a phenomenon of destructive attack or deterioration of a metallic material due to chemical reaction with its environment $[125,126]$. The essence of corrosion is a chemical or electrochemical oxidation process, in which the metal transfers electrons to the environment, and results in a valence change from zero to positive values. The corrosion environment or media, can be in the form of liquid, gas or hybrid solid-liquid. These media are referred to as electrolytes in electrochemistry [127].

\subsubsection{Types of Corrosion}

Corrosion can be classified in many ways. With respect to corrosion media, corrosion of metallic materials can be divided into three groups: wet corrosion, molten matter corrosion, and dry corrosion $[125,128]$. Wet corrosion, a typical electrochemical process, is the most common type of corrosion occuring in water based electrolytes. Corrosion can also occur in other fluids, e.g., fused salts or molten metals. In a dry corrosive environment, the corrosion is an oxidation process.

Corrosion modes can be classified into general corrosion (uniform corrosion) and localized corrosion [127]. In general corrosion, reactions randomly take place across the whole surface of the material. General corrosion could take many forms [129]. Atmospheric corrosion is the most common type of corrosion on metal pieces. Galvanic corrosions occur between dissimilar metal/alloys or microstructural phases (e.g., pearlitic steels, copper alloys, and lead alloys). High temperature corrosion takes place on steels and forms a porous iron oxide phase. Fused salt corrosions occur due to molten salts. 
Localized corrosion concentrates at particular areas and also takes various forms [130]. Crevice corrosion is observed at interfaces on a metal/alloy surface like underneath a gasket or at lap joints under bolts or under rivet heads. As a special case of crevice corrosion, filiform corrosion occurs under a protective film. Pitting corrosion is an extremely localized corrosion [130], and is characteristized by having depth of penetration that is much greater than the diameter of the affected area.

Other types of corrosion are intergranular, selective leaching, corrosion-erosion, stress corrosion, and hydrogen embrittlement damage [131]. Corrosion mechanism and material microstructure both play key roles in a corrosion process. In this study a coating/metalsubstrate system is considered, where galvanic corrosion due to the presence of coating defects is the typical corrosion mode, and electrochemical reaction is the essential corrosion mechanism.

\subsubsection{Corrosion of Coating Systems}

In a coating/metal-substrate system, the metal substrate is not directly in contact with the corrosion environment. However corrosions may still occur due to defects in the coatings such as voids, pinholes, droplet, poor adhesion, differences of chemical composition between the coating matrix and droplet [32, 33, 132, 133], as summarized in Figure 2-11. These defects can lead to breakdown of the coating through coating delamination [37] or cracking [127]; and through these defects, corrosive media reach the substrate, and result in corrosive damages $[36,38,134,135]$. Therefore, these defects are the main causes of failure of a coating/metal system. 


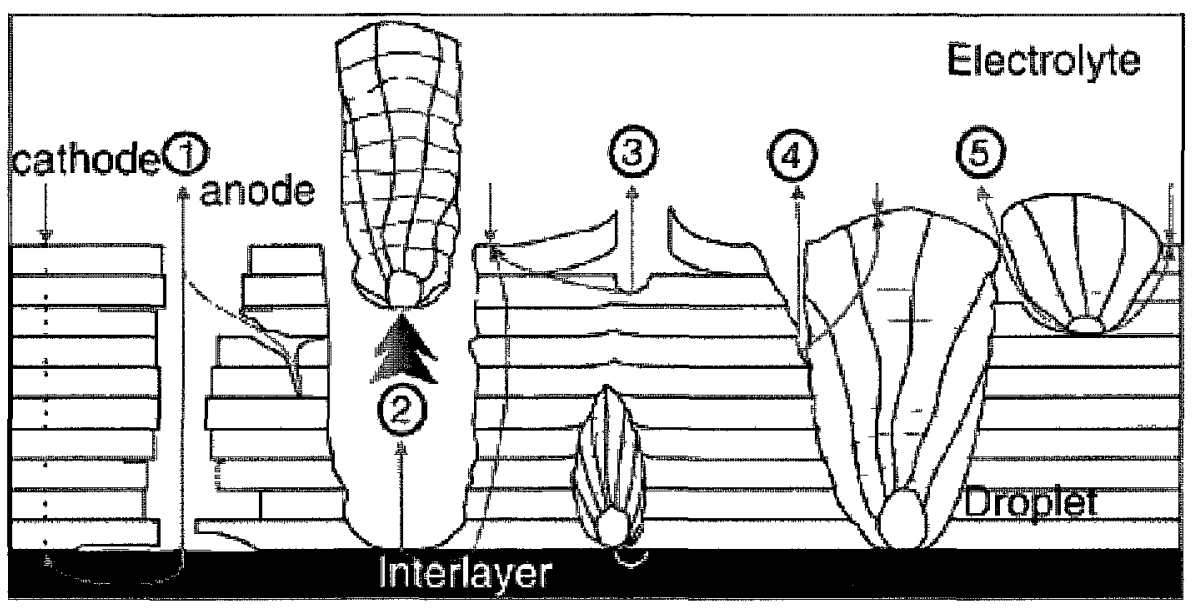

\section{Substrate}

Figure 2-11 Schematic of corrosion mechanisms of coating systems with defects [133].

In a coating/metal system, pitting is the result of localized galvanic corrosion at the interface $[33,39,40]$. In pitting corrosion, the two competing factors are pit plugging action and corrosion product dissolution. In a corrosion process, the corrosion product could accumulate at pit sites, plugging the local access for the electrolyte; the corrosion product could be further dissolved into the electrolyte. The plugging effect increases resistance to corrosion, whereas dissolution decreases this resistance.

Dissolution is caused by the hydrolysis of metal salts in the pits, and the process is responsible for the increase in acidity $[133,136]$. At this level, corrosion is diffusion governed, and the corrosion products limit oxygen access [137-139]. Once metal dissolution starts, localized attacks with a high local corrosion current density will occur [40], providing accesses for electrolyte to the substrate metal. Anodic and cathodic reactions occur at local sites, and continue toward the direction of gravity. A vertically 
oriented or downward defect retains the electrolyte, and assists the accumulation of corrosion products [127]. Therefore, a rough and heterogeneous surface is more susceptible to pitting corrosion [140 - 142].

Important factors affecting pitting corrosion include environment, coating morphology [143], compositional heterogeneity [127], and the local microscopic condition such as the microstructure and orientation of the pits [143].

Studies were conducted with regard to nucleation of pits and their distribution over metal surfaces. A model [144] was proposed to explain the formation of corrosion pits in a water based electrolyte with chlorine ions $\left(\mathrm{Cl}^{-}\right)$and oxygen molecules $\left(\mathrm{O}_{2}\right)$. As shown in Figure 2-12, $\mathrm{Cl}^{-}$ions penetrate via the irregularities or defects in the coating, moving towards the bottom of the pit where they react to form metal salts; oxygen molecules and the metal salts react with water molecules to casue oxidation [145]:

$$
\mathrm{O}_{2}+3 \mathrm{Cl}^{-}+\mathrm{M}+2 \mathrm{H}_{2} \mathrm{O} \rightarrow \mathrm{MCl}+2 \mathrm{OH}^{-}+2 \mathrm{HCl}
$$

Metal salts and hydroxyl ions $\left(\mathrm{OH}^{-}\right)$are produced in the process. With further reaction, metal chloride is hydrolyzed and free hydrochloric acid and metal hydroxide are generated. Free hydrochloric acid formed at the bottom of the pit results in an increase in the concentration of hydrogen ions, and consequently leads to high acidity near the bottom of the pit and high conductivity of the electrolyte [133]. As a result the electrochemical process through the pit electrolyte is accelerated. The metal hydroxide compound reacts with oxygen and water, forming corrosion products (oxides). As a consequence of the above reactions, the coating can be delaminated by the growth of 
corrosion product (Figure 2-12 b), which leaves an accumulation of corrosion products over the pit that can act as a barrier to the entry of the electrolyte, retarding further corrosion progress in certain instances [144].

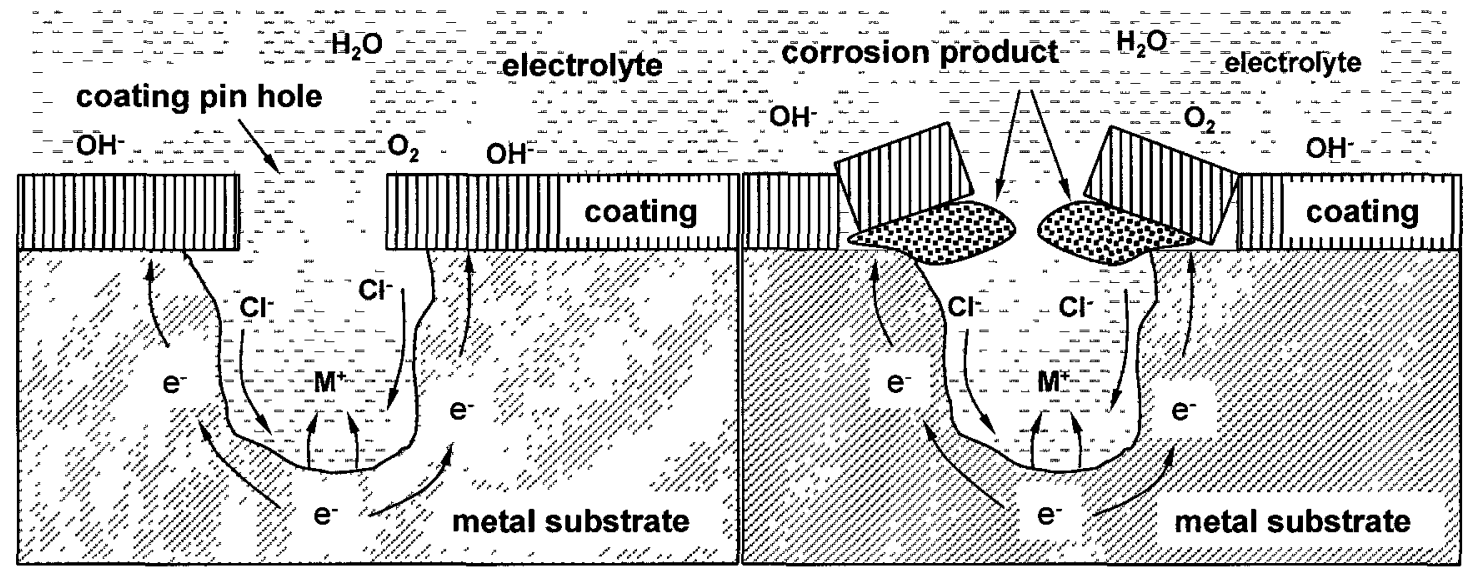

(a) Pitting corrosion mechanism

(b) Coating delamination as a result of pitting corrosion

Figure 2-12 Schematic for pitting corrosion mechanism in a coating/substrate system and damage to the coating.

\subsubsection{Electrochemical Corrosions}

Corrosion is an electrochemical reaction process, accompanying two simultaneously opposite reactions i.e., reduction and oxidation (referred to as redox reaction). In such a corrosion process, metal ions in the electrolyte are reduced and are deposited on the electrode surface (cathode); while the corroding metal atoms oxidize, become ions, and dissolve from the electrode (anode) surface into the electrolyte. Corrosion is governed by electrochemical kinetics [127]. The driving force is the difference in potentials of the electrodes, and the corrosion rate is related to the flow rate of electrons (electrode current) through a metal-electrolyte interface. The electrode current $(I)$ is the quantity monitored in investigating a corrosion process by means of electronic measurement. 
In an electrochemical process, electrode reactions induce deviations from equilibrium due to the electrical current passing through the electrochemical cell, and result in a deviation in the working electrode (WE) potential. This phenomenon is referred to as electrode polarization. In this process, the deviation from equilibrium causes a difference in electrical potential between the polarized and the equilibrium (un-polarized) electrodes. This potential difference is defined overpotential [145].

Two mechanisms, i.e., electrical charge transfer and mass transfer, govern an electrochemical polarization. In this study, the interest is on the charge-transfer governed polarization, referred to as activation polarization, in which the reaction controls the rate of electron flow through a metal surface that undergoes an oxidation [145].

In a polarization of an electrode from the open-circuit potential (OCP) under steady-state conditions, the potential-current relationship is quantitatively described by the ButlerVolmer equation $[146,147]$. This equation takes both cathodic and anodic reactions on the same electrode into account, and is given as:

$$
I=A \cdot i_{0} \cdot\left\{\exp \left[\frac{(1-\alpha) n \cdot F}{R T} \cdot\left(E-E_{e q}\right)\right]-\exp \left[-\frac{\alpha \cdot n \cdot F}{R T} \cdot\left(E-E_{e q}\right)\right]\right\}
$$

where:

I: electrode current,

$i_{o}$ : exchange current density,

$E$ : electrode potential,

$E_{e q}$ : equilibrium potential,

$A$ : electrode active surface area,

$T$ : absolute temperature,

$n$ : number of electrons involved in the electrode reaction

$F$ : Faraday constant $\left(9.65 \times 10^{-4} \mathrm{C} / \mathrm{mol}\right)$

$R$ : universal gas constant

$\alpha$ : symmetry factor, dimensionless 
As a relative parameter, electrode potential cannot be directly measured, and can only be evaluated by referring to an arbitrary zero point, which is the potential of a reference electrode. In electrochemistry, electromotive force (emf) is the potential difference between the two electrodes in an electrochemical cell without electronic current flow.

To measure electromotive force (emf), a standard hydrogen electrode (SHE) cell is introduced, as is shown in Figure 2-13, which consists of a working electrode, a counter electrode, and a reference electrode. Detailed working principles and apparatuses are introduced in the literatures $[127,146,147]$. A counter electrode is connected to the working electrode through the electrolyte to measure and control the potential drop across the surface of the working electrode and the electrolyte (referred to as interfacial potential). The counter electrode provides the current to the electrolyte, and changes polarity opposite to that of the working electrode.

To overcome the variation of the interfacial potential, a third electrode (reference electrode) is introduced between the working and counter electrodes (referred to as a three-electrode system). This system offers a controllable potential between the working and reference electrodes. In practice, two types of reference electrodes, i.e., silver-silver chloride electrode and saturated mercury-mercurous chloride (calomel electrode) electrode are used, with the latter one being the most widely used $[127,146]$. 


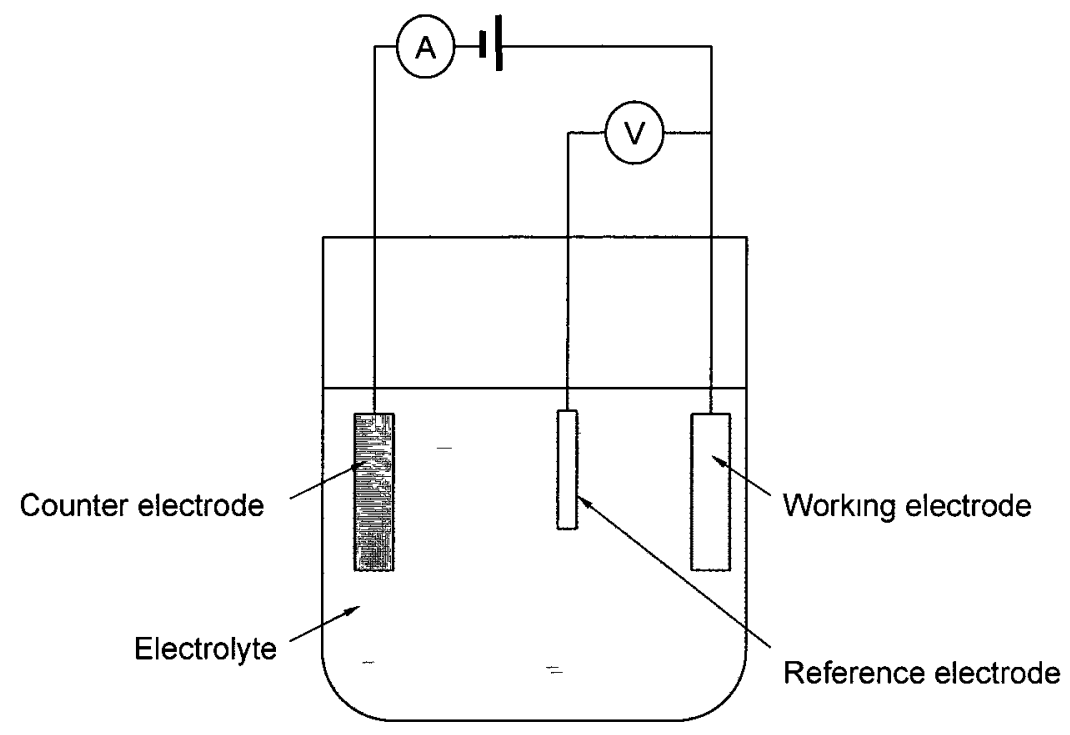

Figure 2-13 Schematic diagram of three-electrode electrochemical cell.

The potential-current relationship for the working electrode (WE) can be determined by the factor of either current or potential; and the corresponding approaches are the galvanostatic and the potentiostatic method, respectively. A galvanostatic approach, with a constant current applied between a working electrode and a counter electrode, measures the changes in potential of the working electrode relative to the reference electrode. Whereas in a potentiostatic approach, a fixed working electrode potential to reference electrode is maintained regardless of the variations in the current corresponding to the changes in the rates of corrosion. This method closely follows the potential behaviour of the metal. This potentiostatic method is favored in many applications, and was employed in this study.

As a determining factor for the rate of electrochemical corrosion, electrochemical kinetics is characterized by several primary polarization parameters, i.e., corrosion potential $\left(E_{c o r r}\right)$, and Tafel slopes $\left(\beta_{a}\right)$ and $\left(\beta_{c}\right)$. Evaluation of these parameters can determine the 
polarization resistance $\left(R_{p}\right)$, corrosion current density $\left(i_{c o r r}\right)$, and Faradaic corrosion rate $\left(C_{R}\right)$. The functional relationship among these electrochemical kinetic parameters can be obtained and described with assistance of a polarization curve shown in Figure 2-14.

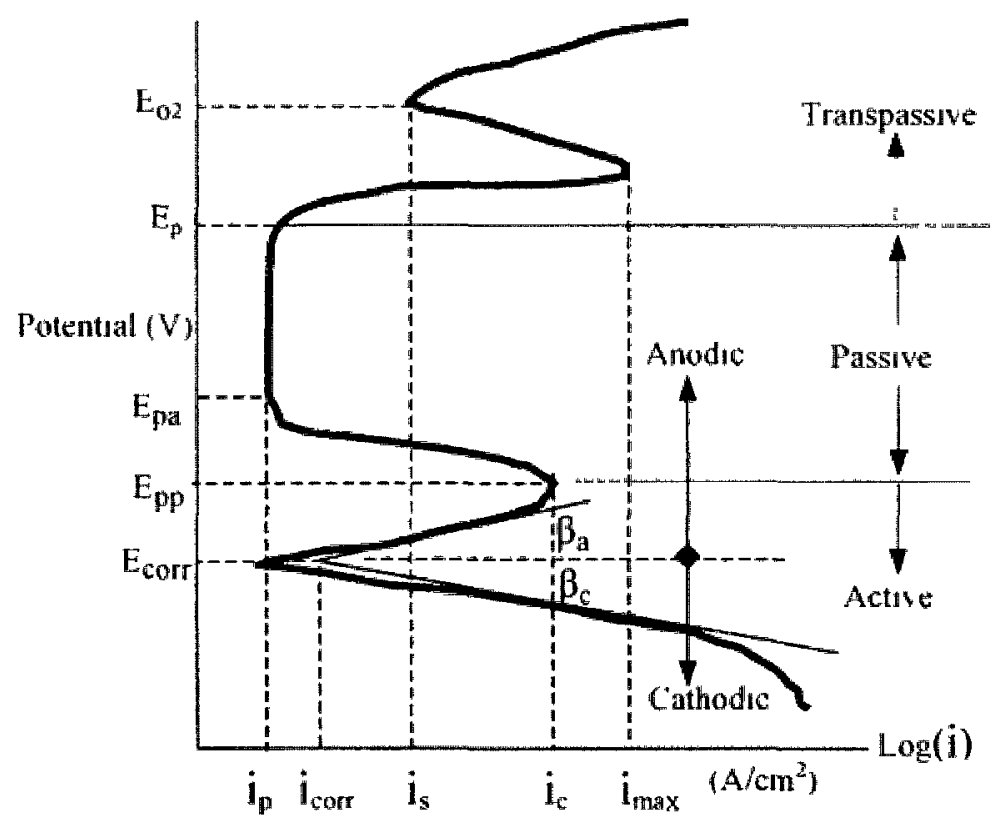

(where $i_{p}$ is the passive current density, $i_{c o r r}$ corrosion current density, $i_{s}$ secondary current density, $i_{c}$ critical current density, $i_{\max }$ maximum current density, $E_{p a}$ passive potential, $E_{\text {corr }}$ corrosion potential, $E_{O 2}$ oxygen evolution potential, $E_{p p}$ primary passive potential, and $E_{p}$ pitting potential)

Figure 2-14 A typical hypothetical polarization curve [127].

A polarization curve indicates two limiting cases for the Butler-Volmer equation [127]. In a low over potential region (polarization resistance region), the Butler-Volmer equation is simplified as [127]:

$$
i=i_{0} \frac{n F}{R T}\left(E-E_{e q}\right)
$$

whereas, in a high over potential region, the Butler-Volmer equation is simplified as [127]:

$$
\begin{array}{ll}
E-E_{e q}=a-b \log (i) & \text { for a cathodic reaction, or } \\
E-E_{e q}=a+b \log (i) & \text { for an anodic reaction, }
\end{array}
$$


This form of the Butler-Volmer equation is also referred as the Tafel equation, where $a$ and $b$ are Tafel equation constants (for a given reaction and temperature). In the Tafel equation, the anodic polarization due to an anodic over potential $\left(E-E_{e q}>0\right)$ corresponds to a metal surface oxidation in the form of losing electrons, consequently, the metal surface is positively charged.

On the other hand, cathodic polarization requires that electrons be supplied to the metal surface at a negative over potential, $E-E_{e q}<0$, thus implying $E<E_{e q}$. Under this condition, the Butler-Volmer equation is converted to the Stern-Geary relation $[148,149]:$

$$
i_{\text {corr }}=\frac{1}{2.303} \cdot \frac{\beta_{a} \beta_{b}}{\left(\beta_{a}+\beta_{b}\right)} \cdot \frac{1}{R_{p}}
$$

where

$\beta_{a}$ and $\beta_{c}$ : the Tafel slopes of the anodic and cathodic corrosion reactions, $R_{p}$ : polarization resistance corresponding to the resistance of the system to the corrosion process.

In an electrolytic cell system, electrode polarization is governed by two factors, i.e., electrical charge transfer and mass transfer [127]. The charge-transfer induced electrochemical polarization is referred to as activation polarization, where a redox stoichiometric reaction occurs. This reaction controls the rate of electron current flow from the electrode surface [145]. Therefore from this electric current the polarization behaviour can be investigated. 


\subsubsection{Experimental Approaches to Studying Corrosion Properties}

A metal corrosion process can be evaluated by direct current (DC) or alternating current (AC) electrochemical techniques. The direct current (DC) techniques include potentiostatic or potentiodynamic polarization, linear polarization, Tafel extrapolation, and cyclic polarization. The alternating current $(\mathrm{AC})$ techniques include electrochemical frequency modulation (EFM) and electrochemical impedance measurements (for example, electrochemical impedance spectroscopy (EIS)).

Polarization measurement is a powerful tool in the study of reaction mechanisms and corrosion kinetics [148]. Using this method the potential range of interest is close to the corrosion potential, and the applied currents are within the range of the corrosion current. The nature of the test surface is retained without significant changes, thus the reactions that occur during the polarization are the ones in the corrosion process [150].

Three polarization methods are widely used in the experimental investigation of corrosion, i.e. linear polarization (LP), Tafel extrapolation, and EIS [127]. The linear polarization (LP) technique covers both anodic and cathodic regions of the linear polarization curve (potential vs. current density) for determining the polarization resistance $\left(R_{p}\right)$. The Tafel extrapolation (TE) technique focuses on the linear regions of the anodic and cathodic curves for determining $\left(R_{p}\right)$. EIS, also named dielectric spectroscopy, is an alternating current (AC) polarization experimental technique characterizing electrochemical systems. This technique measures the impedance of a 
system over a range of frequencies corresponding to different interfaces in an electrolytic cell system. Using this method, a series of electrochemical parameters can be determined, including charge transfer resistance, double layer capacitance and ohmic resistance. More details with regard to the three test methods and procedures are further discussed in the following sections.

\section{Linear (Potentiostatic and Potentiodynamic) Polarization}

Potential control is commonly used in characterizing electrochemical behaviour of metallic materials. Potentiostatic and potentiodynamic are two potential control approaches used in developing polarization curves. The former is a stepwise technique and generates a steady-state current response. The latter one is a dynamic technique, in which a polarization curve can be obtained quickly at a low potential scan rate, yielding a transient current response [127].

The Potentiodynamic polarization technique is a DC electrochemical method measuring electrochemical current density that depends on the dynamic potential of a working electrode, from which corrosion rates are estimated. Important information that can be detected includes critical potential for passivity, the corrosion rate in the passive region, and the spontaneous passivity of a material in a specific solution. It also works with a

scan at a varying rate that provides thermodynamic and kinetic information simultaneously. 
Figure 2-15 shows a schematic of a linear polarization curve, which demonstrates the extraction of polarization resistance. The polarization resistance $\left(R_{p}\right)$ is estimated from the linear slope of the curve as:

$$
R_{p}=\frac{\Delta E}{\Delta i}=\frac{\eta}{\Delta i}
$$

where the difference in overpotentials is defined as $\Delta E=\eta=\eta_{a}-\eta_{c}$ and the corrosponding difference in corrsion current density is defined as $\Delta i=i_{o, 2}-i_{o, 1}[127]$.

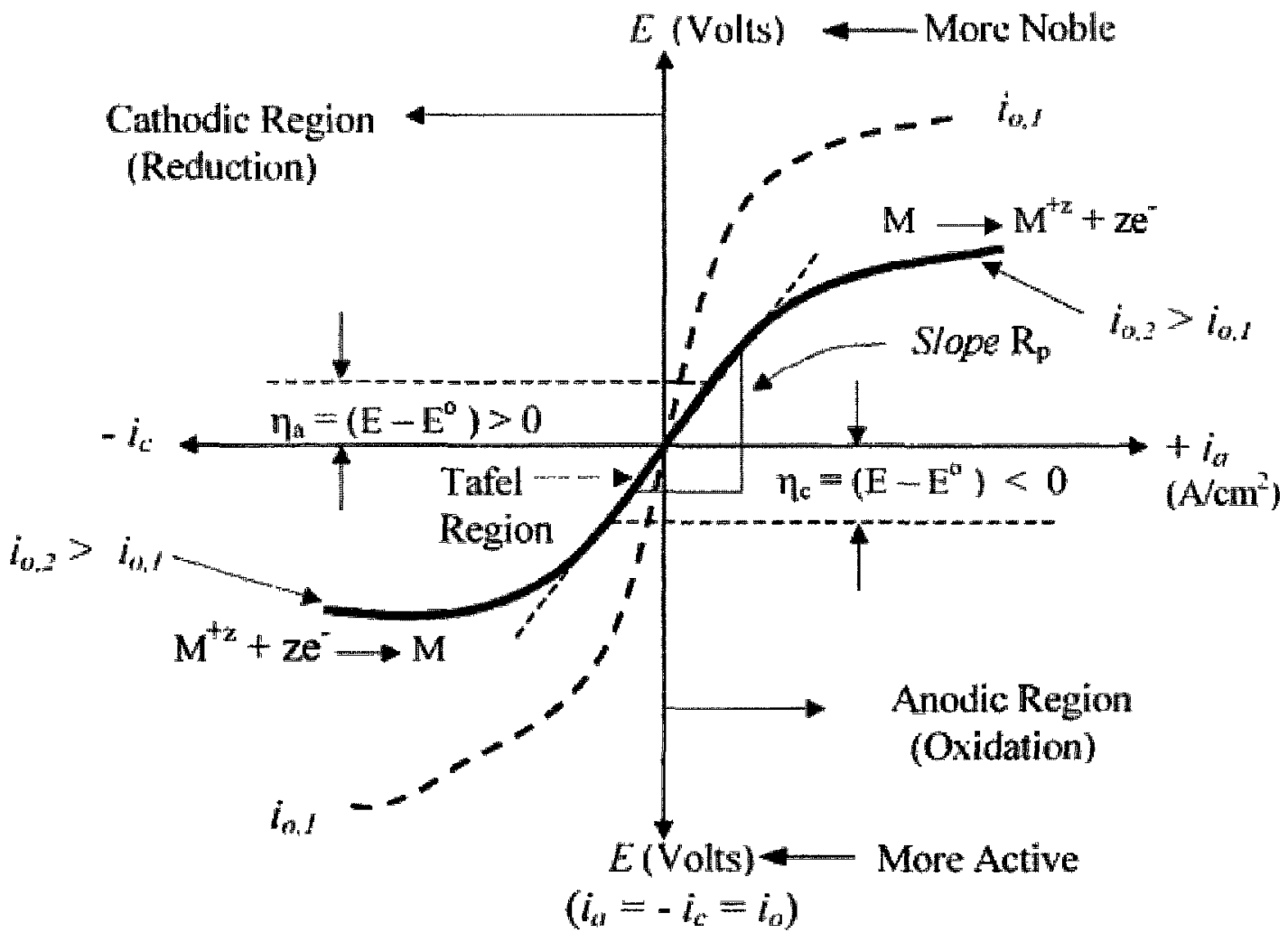

Figure 2-15 Schematic of linear polarization curve and extraction of polarization resistance [127]. 
Figure 2-16 shows an example of the polarization curve for TiN, TiBN, $\mathrm{CrN}$ and $\mathrm{Nb}_{2} \mathrm{~N}$ coating systems and HSS substrate, where the anti-corrosion characteristics of several coating systems and the high speed steel (HSS 1.3207) substrate can be compared by means of comparing their polarization characteristic regions and parameters such as corrosion potential $\left(E_{c o r r}\right)$, corrosion current density $\left(i_{c o r r}\right)$, active region, passivation potential, passive region, and pitting potential.

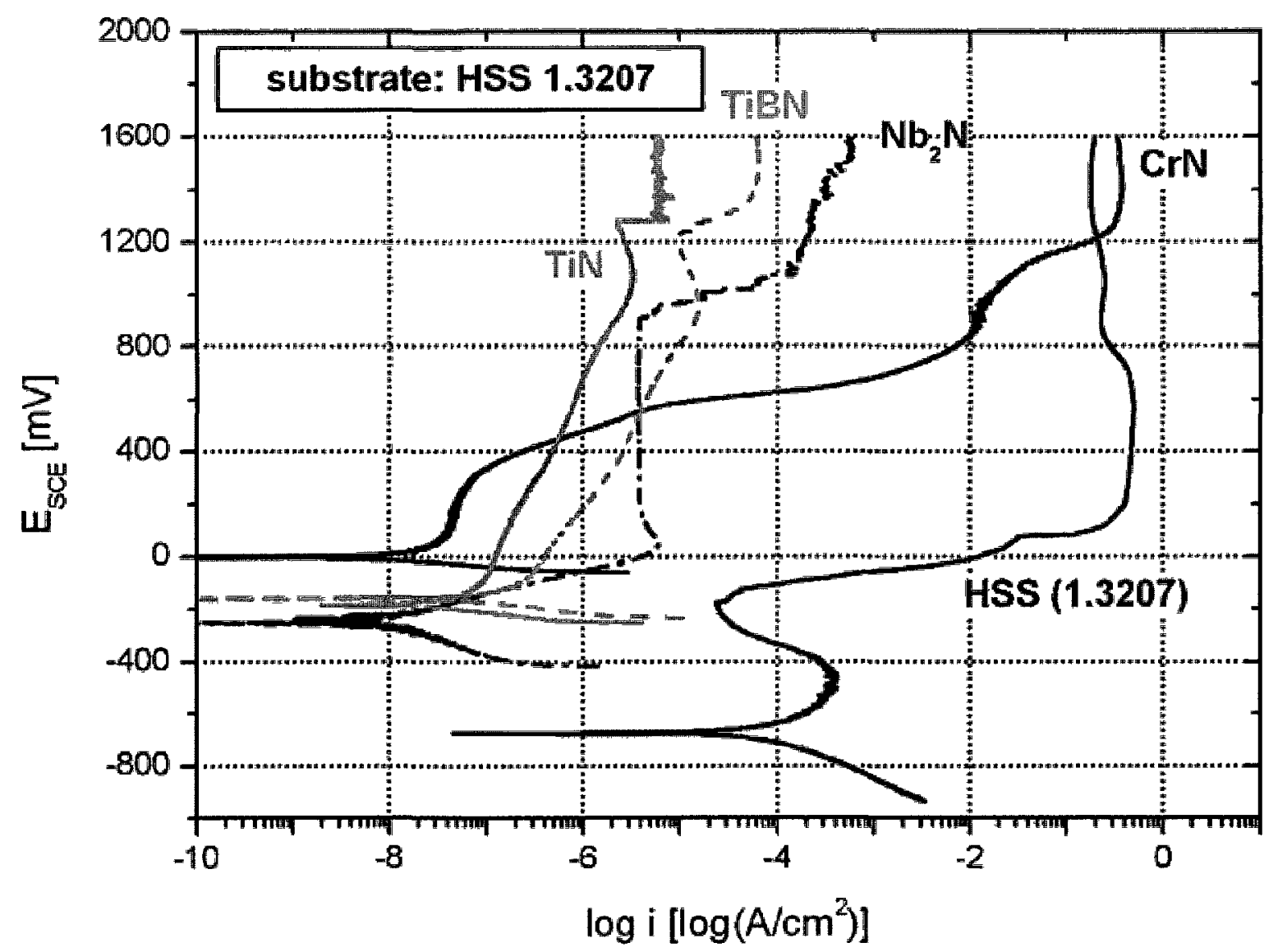

Figure 2-16 Potentiodynamic polarization curves for TiN, $\mathrm{TiBN}, \mathrm{CrN}$ and $\mathrm{Nb}_{2} \mathrm{~N}$ coating systems and HSS substrate in $0.8 \mathrm{M} \mathrm{NaCl}$ solution [151].

Polarization techniques can be used in kinetic corrosion studies and accurate measurement of low corrosion rates (less than $0.1 \mathrm{~mm} / \mathrm{y}$ ) $[146,152,153]$. Linear 
polarization measurements have been used when evaluating protection for corrosion control, comparison studies of corrosion rates of alloys, and evaluating coatings in corrosion protections [150]. However, there are uncertainties in this technique that include methods of plotting data, correct interpretation of critical regions in polarization curves, and abrupt changes in the slope of the overvoltage verses the logarithm of current density. These problems need detailed consideration when conducting experimental studies [148].

\section{Tafel Extrapolation Method}

In a polarization curve to extrapolate the Tafel anodic and cathodic linear regions, an intersection of curves can be used to obtain both $E_{c o r r}$ and $i_{c o r r}$. Figure 2-17 shows Tafel constants (in unit of V/decade) obtained from both the anodic and cathodic portions of the Tafel plot. As a basic curve, the Stern diagram (Figure 2-17) can be generated using the potentiodynamic polarization technique with a constant potential scan rate. This technique can measure extremely low corrosion rates, and is used for continuous monitoring of corrosion rates. A Tafel plot can measure the corrosion current which is related to the corrosion rate. However, this method is complex due to the effects of concentration polarization and resistance drop $[127,154]$. The concentration polarization stems from a high reaction rate at which the electro-active species cannot reach the electrode surface at a sufficient rate. In such a condition, the reaction rate is diffusion controlled. The resistance of the solution could cause a nonlinear Tafel behaviour at high currents. 


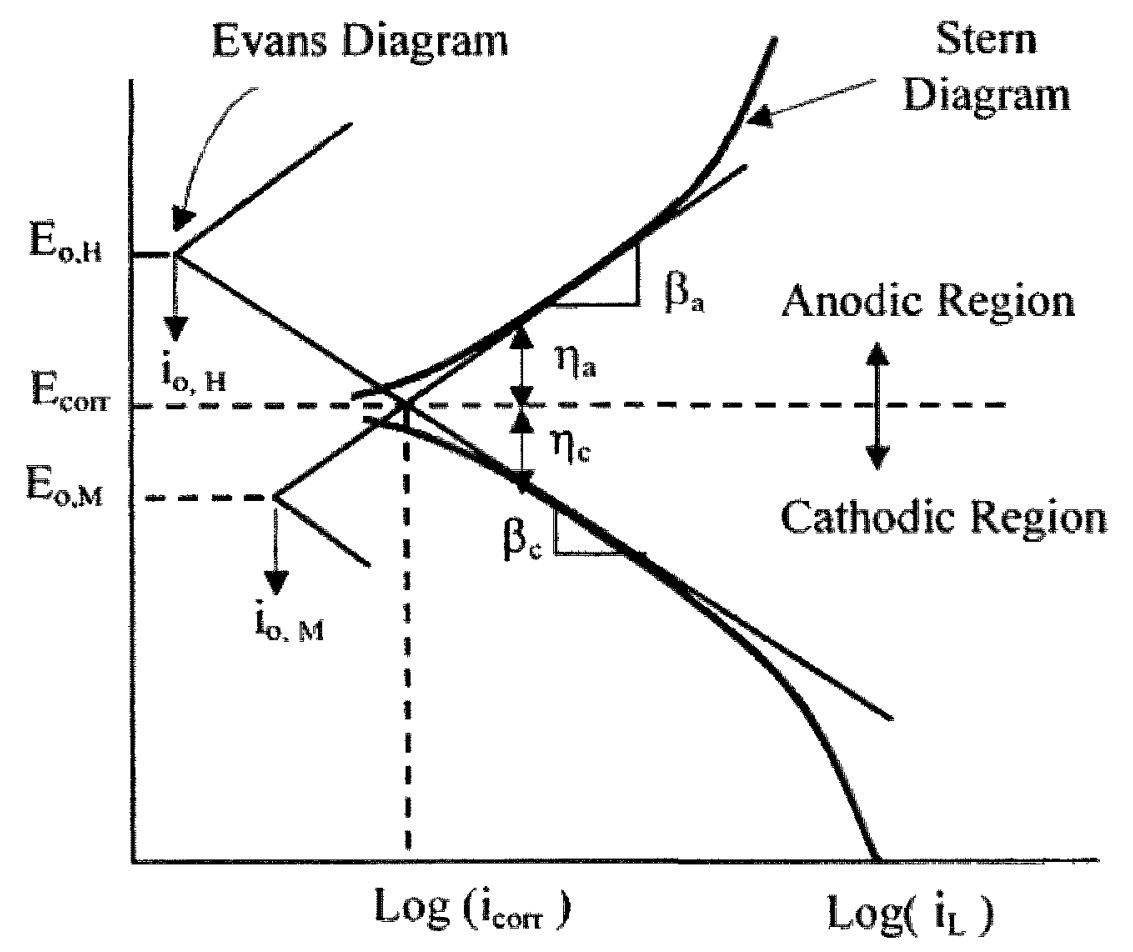

Figure 2-17 Schematic polarization curve showing Tafel extrapolation [127].

Coating porosity is an important parameter determining the corrosion of a coated metal system. Techniques have been proposed for evaluating the porosity of a coating $[35,155$, 156], including anodic current measurement, the coulometric method, and porosity calculations from $E_{c o r r}$ and $R_{p}$ measurement [157]. An empirical equation was developed for estimating the porosity of the coating as [158 -160]:

$$
P=\left(R_{p s} / R_{p}\right) \times 10^{-\left|\Delta E_{c o r r}\right| / \beta_{a}}
$$

where,

$P \cdot$ the total coating porosity,

$R_{p s}:$ the polarization resistance of the substrate,

$R_{p}$ : the polarization resistance of the coated steel,

$\Delta E_{\text {corr }}$ : the difference between the corrosion potentials of the coated steel and the substrate material,

$\beta_{a}$ : the anodic Tafel slope of the substrate. 


\section{Electrochemical Impedance Spectroscopy (EIS)}

EIS is a powerful experimental technique that has found widespread applications in the field of characterization of materials, coatings, batteries, fuel cells, and corrosion phenomena. Pioneering theoretical work including that of Epelboin, Smith et al. (AC polarography), Frumkin, and Armstrong et al has been enabled by EIS and has contributed to the understanding of detailed mechanisms of corrosion [161]. EIS provides information on reaction parameters such as corrosion rates, oxide integrity, surface porosity, coating integrity, mass transport, and electrode/interface characteristics [37] and is suitable for high-impedance interfaces, which is the case for an anti-corrosion coating system [162].

Figure 2-18 illustrates EIS characteristics for Ti- and Cr-based PVD coating systems in the forms of Nyquist plots (plots of the real component vs. the imaginary component of impedance) and Bode plots (plots of impedance magnitude and phase at different frequencies). This provides a more comprehensive approach to investigating an electrochemical corrosion behaviour for a coating system compared to a potentiodynamic polarization approach. 

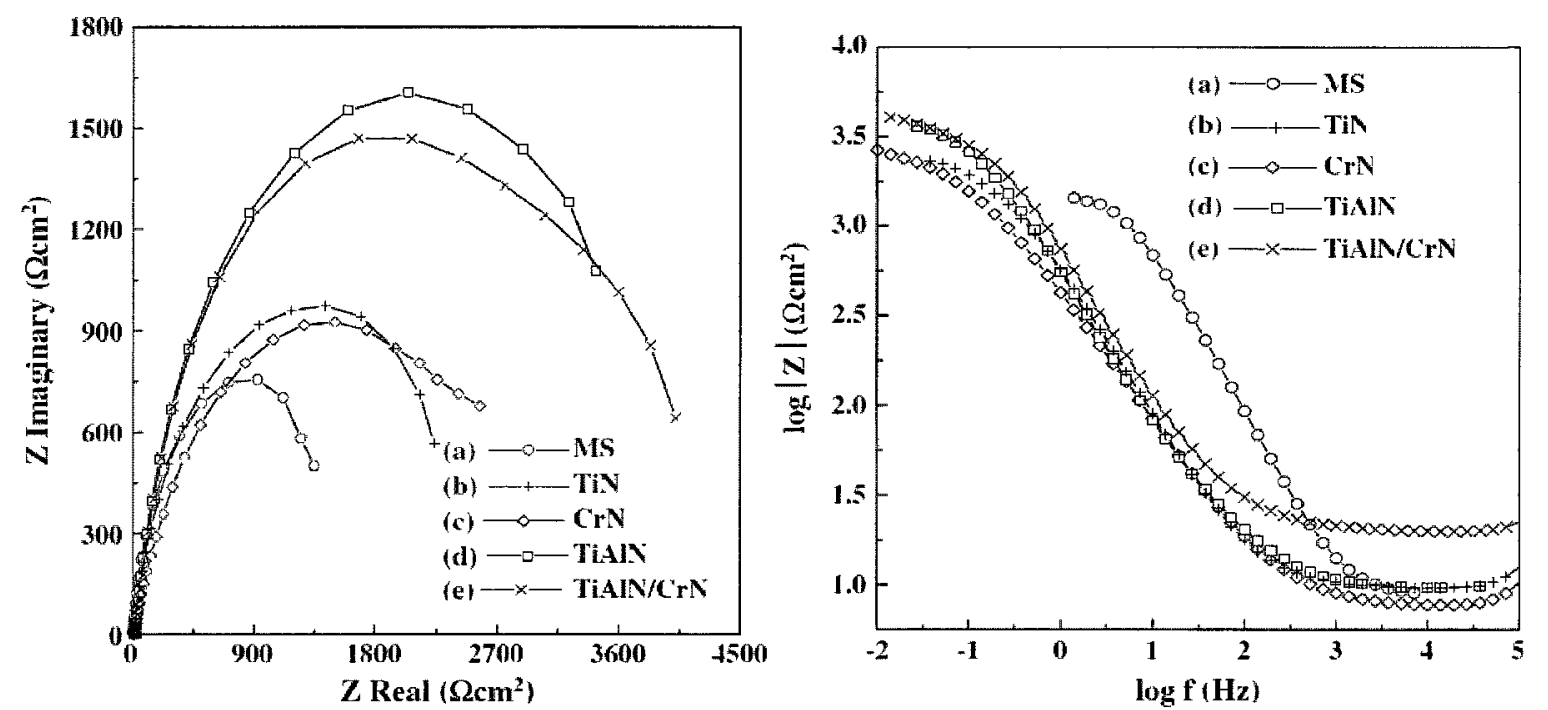

(a) Nyquist plots

(b) Bode plots $(\log |Z|$ vs. $\log f)$

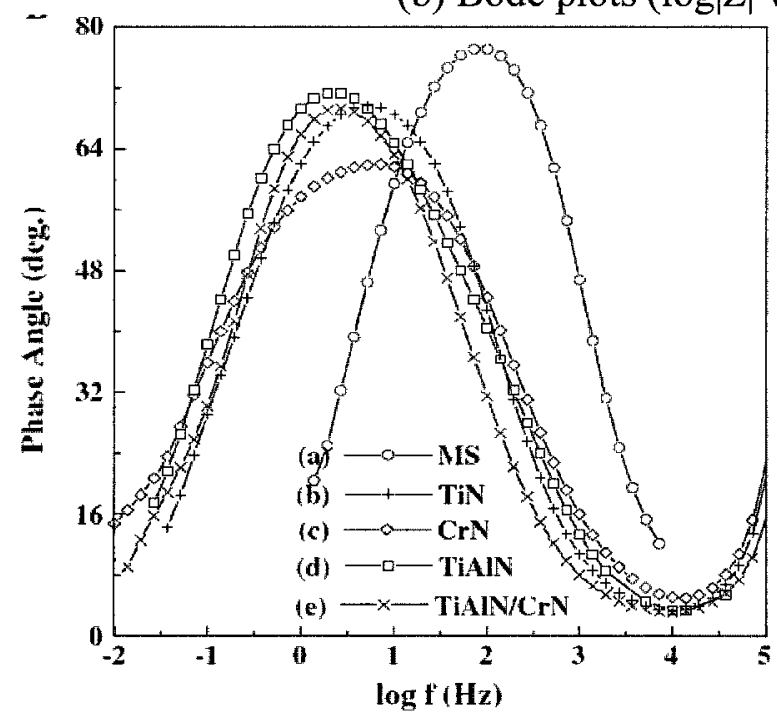

(c) Bode plots (phase angle vs. $\log f$ )

Figure 2-18 EIS curves for Ti- and Cr-based nitride coatings in 3.5\% $\mathrm{NaCl}$ solution [38].

Recently, equivalent electrical circuit (EEC) technique has been developed $[37,127,163$, 164 ]. Using this technique, an electrochemical system can be described with an equivalent electrical circuit, EIS experimental data is incorporated into equivalent electrical circuit models that corresponds to specific electrochemical corrosion systems, thus obtaining the values for the equivalent elements in the equivalent electrical circuit 
models. Therefore, it makes the EIS method more practical and powerful in the study of electrochemical corrosions.

The EIS technique is more powerful than traditional DC techniques in which it is very difficult or impossible to obtain mechanistic information [163, 164]. Being more sensitive, the EIS technique can also be used in situ and does not necessarily require artificial accelerating factors [146]. Table 2-2 summarizes the most frequently used electrochemical testing methods. This study uses both potentiodynamic polarization and EIS techniques to analyze the corrosion of coatings.

Table 2-2 Summary of electrochemical measurements in corrosion studies [165]

\begin{tabular}{|c|c|c|}
\hline Test & Conditions & Information \\
\hline Potentistatic & Constant votage & $\begin{array}{l}\text { Time dependence of corrosion } \\
\text { current at given potential }\end{array}$ \\
\hline Potentiodynamic & Variable votage & Corrosion mechanism \\
\hline Galvanostatic & Constant current & $\begin{array}{l}\text { Time dependence of corrosion } \\
\text { potential at given current }\end{array}$ \\
\hline Galvanodynamic & Varibale current & Corrosion mechanism \\
\hline Potential & $\mathrm{I}=0$ & Behaviour in galvanic corrosion \\
\hline $\begin{array}{l}\text { Polarization } \\
\text { resistance }\end{array}$ & $\begin{array}{l}\text { Variable votage, around } \\
\text { corrosion potential }\end{array}$ & Absolute corrosion rate \\
\hline Impedance & r.f. voltage & Changes in surface state \\
\hline
\end{tabular}




\subsection{Surface Protection and Coatings}

\subsubsection{Surface Protection Solutions}

Surface protection can be achieved using two general approaches, i.e., new materials development and surface modification $[1,166]$. In practical applications, the most commonly used solutions are surface modification and application of surface coatings [46].

Surface compositional and microstructural modifications were the earliest solutions to increase surface hardness and wear resistance for cutting tools. Compositional modification can be accomplished through thermochemical treatments involving carburizing, nitriding, carbonitriding, siliconizing, chromizing, boronizing (for carbon steels and high-speed steels), and ion implantation [11, 167, 168], while the surface microstructural manipulation uses laser hardening, electron beam hardening, and shot peening.

Several limitations exist to applying surface modifications. The dependence on the nature of the metal surface restricts modification techniques in compositions and microstructure. The drawbacks include limited composition choices, induced brittleness through the formation of intermetallic compounds, grain growth due to prolonged thermal treatment, and dimensional changes. To overcome these limitations, overlay protective coating solutions have been developed, which are independent of the nature of surface, and have 
many more applications. The following sections introduce various coating types and the associated applications.

\subsubsection{Protective Coating Systems}

The automobile industry started the development of protective coatings for wear and corrosion prevention in the late 1920 s and was the first to introduce electroplating techniques to improve component surface qualities $[15,169]$. The tool industry later pursued coating development to increase hardness and wear resistance for cutting tools, and drove the growth of hard protective coatings. In the mid $1960 \mathrm{~s}$, chemical vapor deposition (CVD) techniques were used for the deposition of hard and refractory compounds (e.g., $\mathrm{WC}, \mathrm{TiC}, \mathrm{TiN}, \mathrm{ZrN}$, and $\mathrm{HfN}$ ) to provide anti-wear properties for cutting tools $[168,170]$. The worldwide market for cutting tools is a multi-billion dollars business and nearly $70 \%$ of all cutting tools were coated as of 1996 [168].

In the aircraft industry, sand erosion, corrosion, and oxidation motivated coating development for protecting gas turbine engine components $[28,171-174]$. PVD was a major deposition technique in the development of hard coatings such as $\mathrm{CrN}$, TiN, $\mathrm{TiC}$, TiCN, and TiAlCN.

Table 2-3 summarizes the progress in wear, erosion and corrosion protection coatings. It shows a trend in the mainstream evolution of protective coatings, from plating to vapor deposition, from pure metals to ceramic compounds, from monophase to multiphase compounds, and from single to multiple functions. 
Table 2-3 Summary of the development in protective coating systems

\begin{tabular}{|c|c|c|c|}
\hline Year & Coating systems & Feature & Reference \\
\hline 1926 & $\mathrm{Ni}, \mathrm{Cr}$ electroplating & Anti-wear and corrosion & [169] \\
\hline 1938 & Ni bright plated & Anti-corrosion & [169] \\
\hline \multirow{2}{*}{$1960 \mathrm{~s}$} & Hard Cr plating & Anti-erosion & [28] \\
\hline & $\begin{array}{l}\text { Sputtered silicon carbide, plasma } \\
\text { sprayed tungsten carbide coatings }\end{array}$ & Anti-erosion & [28] \\
\hline 1965 & $\begin{array}{l}\text { Semibright nickel plus bright nickel } \\
\text { with microdiscontinuousc hromium }\end{array}$ & Anti-wear and corrosion & [169] \\
\hline 1968 & $\begin{array}{l}\text { Si and } \mathrm{Al} \text { based, modify elements: } \mathrm{V} \text {, } \\
\mathrm{Al}, \mathrm{Mo}, \mathrm{Cr} \text {, and } \mathrm{Ti}\end{array}$ & Anti-erosion & [175] \\
\hline \multirow{2}{*}{1969} & Impregnated flame sprayed carbides & $\begin{array}{l}\text { Increase life of gas turbine } \\
\text { components }\end{array}$ & [176] \\
\hline & $\begin{array}{l}\text { Combine high temperature strength } \\
\text { of dispersion strengthened alloys }\end{array}$ & gas turbine components & {$[177]$} \\
\hline 1970 & $\begin{array}{l}\text { Semibright nickel plus bright nickel } \\
\text { with trivalent chromium }\end{array}$ & Anti-wear, corrosion & [169] \\
\hline 1971 & $\begin{array}{l}\text { Fused silicided compositions and } \\
\text { flame sprays overlays }\end{array}$ & Gas turbine & [178] \\
\hline \multirow{2}{*}{1972} & $\begin{array}{l}\text { Stainless steel with high } \mathrm{Cr} \text { and } \mathrm{C} \\
\text { contents }\end{array}$ & Reduced abrasive wear & [99] \\
\hline & TIKOTE-C on Ti-6-4 & Surface crack propagation & [179] \\
\hline \multirow{2}{*}{1973} & $\begin{array}{l}\text { Reduce } \mathrm{Al} \text { and Ti contents in the } \\
\text { alloy }\end{array}$ & $\begin{array}{l}\text { Oxidation of nickel-base alloys in } \\
\text { gaseous and alkaline environment }\end{array}$ & {$[180]$} \\
\hline & $\begin{array}{l}\text { Combinations of refractory, reactive, } \\
\text { and rare earth metals in the alloys }\end{array}$ & $\begin{array}{l}\text { Hot corrosion resistance of } \\
\text { nickel-base superalloys }\end{array}$ & [181] \\
\hline 1974 & $\begin{array}{l}\text { Nickel plating (PWATM 36) for } \\
\text { titanium alloys (Ti-8Al-1Mo-1V, Ti- }\end{array}$ & Anti-erosion & [182] \\
\hline 1980 & $\begin{array}{l}\text { Multilayered nickel controlled by } \\
\text { STEP with microdiscontinuousc }\end{array}$ & Anti-corrosion & [169] \\
\hline \multirow{3}{*}{1991} & $\mathrm{Al}_{2} \mathrm{O}_{3}, \mathrm{TiN}, \mathrm{TiC}$ and $\mathrm{TiB}_{2}, \mathrm{SiC}, \mathrm{HfN}$ & WC tools & [183] \\
\hline & PVD $(\mathrm{Ti}, \mathrm{Al}) \mathrm{N}$ coatings & Anti-corrosion & {$[184]$} \\
\hline & Sputtered TiN & Anti-corrosion & {$[185]$} \\
\hline \multirow{3}{*}{1992} & $\begin{array}{l}\text { Oxides: } \alpha \mathrm{Al}_{2} \mathrm{O}_{3}, \mathrm{~V}_{2} \mathrm{O}_{3}, \mathrm{TiO}_{2} \text {, indium- } \\
\text { tin oxide }\end{array}$ & Cutting tools & [101] \\
\hline & $\begin{array}{l}\text { Inorganic coatings on the base of } \\
\text { salts of metals such as } \mathrm{Mg}, \mathrm{Ca}, \mathrm{Zn}\end{array}$ & Anti-corrosion & {$[186-189]$} \\
\hline & $\begin{array}{l}\text { Ternary coatings }(\mathrm{Ti}, \mathrm{Al}) \mathrm{N},(\mathrm{Cr}, \mathrm{Al}) \mathrm{N}, \\
(\mathrm{Ti}, \mathrm{Cr}) \mathrm{N},(\mathrm{Ti}, \mathrm{Y}) \mathrm{N},(\mathrm{Ti}, \mathrm{Pd}) \mathrm{N} .\end{array}$ & Anti-corrosion & [40] \\
\hline
\end{tabular}




\begin{tabular}{|c|c|c|c|}
\hline \multirow{3}{*}{1993} & $(\mathrm{Ti}, \mathrm{Al}) \mathrm{N}$ and $\mathrm{TiN}(\mathrm{Pt})(\mathrm{PVD})$ & $\begin{array}{l}\text { Protection of aero engine } \\
\text { compressor blades }\end{array}$ & {$[190]$} \\
\hline & $(\mathrm{Ti}, \mathrm{Al}) \mathrm{N}$ and $\mathrm{TiN}(\mathrm{Pt})(\mathrm{PVD})$ & Aero engine compressor blades & {$[190]$} \\
\hline & Ion-assisted deposition Ti-Cr-N & Anti-corrosion & {$[191]$} \\
\hline 1995 & WC(CVD), TiN(PVD) & Cutting tools & {$[103]$} \\
\hline \multirow{2}{*}{1996} & WC-(TixAly)N coatings & Anti-corrosion & [192] \\
\hline & $\mathrm{CrN},(\mathrm{Cr}, \mathrm{Al}) \mathrm{N})$ & Anti-corrosion & [193] \\
\hline 1997 & $\mathrm{CrN}$ & Anti-corrosion & [194] \\
\hline \multirow{2}{*}{1998} & nc-MeN/a-nitride & Anti- wear / erosion & {$[195-197]$} \\
\hline & nc-MeN/nc-nitride & Anti- wear / erosion & [195] \\
\hline \multirow{2}{*}{1999} & nc-MeN/metal & Anti- wear / erosion & {$[198-200]$} \\
\hline & $\begin{array}{l}\text { PVD TiN, CrN, TiAlN, TiAlCN; and } \\
\text { CVD DLC }\end{array}$ & $\begin{array}{l}\text { Anti- wear, seizure in auto } \\
\text { industry }\end{array}$ & {$[15]$} \\
\hline 2000 & $\mathrm{Cr}_{2} \mathrm{O}_{3}$ & Anti-corrosion & {$[201]$} \\
\hline \multirow{2}{*}{2003} & Ti-C-N/DLC & Gas turbine & {$[202]$} \\
\hline & (Ti, Al)N based multilayer coating & Gas turbine & {$[203]$} \\
\hline \multirow{3}{*}{2004} & TiN/NbB, TiN/VN, TiN/AIN (PVD) & Anti- wear & {$[204]$} \\
\hline & $\mathrm{TiCrN}$ & Anti- wear & {$[205]$} \\
\hline & Ion-assisted deposition $\mathrm{Ti}-\mathrm{Cr}-\mathrm{N}$ & Anti-corrosion & [191] \\
\hline \multirow{2}{*}{2005} & Quaternary $\mathrm{Cr}-\mathrm{Si}-\mathrm{C}-\mathrm{N}$ coatings & Anti-wear & [206] \\
\hline & $\begin{array}{l}\text { Nanocrystalline grain/amorphous } \\
\text { matrix systems }\end{array}$ & Anti-wear & [207] \\
\hline 2006 & $\begin{array}{l}\text { Cr-Al-Si-N nanocomposites } \\
\text { coatings }\end{array}$ & Anti-wear & {$[208]$} \\
\hline 2007 & $\begin{array}{l}\mathrm{Cr}-\mathrm{Al}-\mathrm{N}, \mathrm{Cr}-\mathrm{Si}-\mathrm{N} \text {, and } \mathrm{Cr}-\mathrm{Al}-\mathrm{Si}- \\
\mathrm{N} \text { hybrid coatings }\end{array}$ & Anti-wear & [209] \\
\hline 2008 & $\begin{array}{l}\mathrm{Ti}-\mathrm{Si}-\mathrm{C}-\mathrm{N} \text { based nanocomposite } \\
\text { coatings }\end{array}$ & Anti-wear / erosion & [210] \\
\hline 2009 & $\mathrm{Ti}-\mathrm{Al}-\mathrm{Si}-\mathrm{N}$ nanocomposite coatings & Anti-wear & [211] \\
\hline 2010 & $\begin{array}{l}\text { Ti-Al-Cr-Si-Y-N nano-crystalline } \\
\text { coatings }\end{array}$ & Anti-wear and oxidation & [212] \\
\hline
\end{tabular}




\subsection{Transition Metal Based Coatings}

Transition metal compounds, especially their carbides (e.g., $\mathrm{TiC}$ and $\mathrm{Cr}_{3} \mathrm{C}_{2}$ ) and nitrides (e.g., $\mathrm{CrN}$ and $\mathrm{TiN}$ ) have high hardness, high melting points, excellent electrical and thermal conductivities, high chemical and thermal stabilities, and good resistance to wear, erosion and corrosion attacks [213 - 218]; valuable properties which ensure their dominant role in coatings in a variety of industries [213, 219 - 221].

\subsubsection{Transition Metals and Their Compounds}

Transition element atoms contain incomplete d-orbital sub-shells, which are able to become cations with incomplete $d$ sub-shells. These elements correspond to the groups from 3 to 12 in the Periodic Table. In the forty transition metals, the groups IV, V, and VI, especially in the first row (for example $\mathrm{Ti}, \mathrm{V}$, and $\mathrm{Cr}$ ) demonstrate characteristics such as exceptional environmental resistance and hardness [222 - 225].

Mechanical properties of transition metal compounds, such as hardness and elasticity are associated with their electronic structures and bonding $[216,221,226,227]$. In the early transition metal elements, the $3 \mathrm{~d}, 4 \mathrm{~d}$, and $5 \mathrm{~d}$ sub-shells extend outward the periphery of the atom (or ion), and are strongly influenced by other atoms such as $\mathrm{C}, \mathrm{N}$ or $\mathrm{O}$ [221]. The involvements of these atoms via hybridization of the p- or d- orbital, offer directional bonds of metal-to-nonmetal and significantly increase the strength and elastic modulus of the compounds $[220,217]$. The quantity and location of these valence electrons are 
two critical factors governing the structure and characteristics of the respective compounds, especially hardness [221]. In general, the hardness increases with the quantity of valence electrons [216, 220, 221, 223].

The bonds in transition metal compounds can take the form of ionic (e.g., salt-like nitrides), covalent (e.g., covalent nitrides), or a combination of metallic, covalent, and ionic (e.g., interstitial nitrides) [7, 101, 228]. The bonding structures of hard coatings consist of a combination of localized metal-metal and metal-nonmetal interactions, resembling both covalent and metallic bonding features [228]. The properties of transition metal compounds are simultaneous determined by covalent, metallic, and ionic bonds [221].

Transition metal nitrides and carbides are important compounds for surface modificatioins because of their properties [219], such as high melting points (1500$\left.3400^{\circ} \mathrm{C}\right)$, high hardness $(20-30 \mathrm{GPa})$, good resistances to abrasive wear, erosion [27 - 29, 229]. Their high chemical stability ensures a high resistance to corrosion [30, 225]. For example, titanium nitride (TiN) is most commonly used in industrial applications because of its high hardness and elastic modulus [230]; chromium-based nitride ( $\mathrm{CrN})$ coatings provide high resistance to wear and corrosion $[33,209,231,232]$, lower coefficient of friction, and high toughness [233, 225]. 


\subsubsection{Development in Transition Metal Based Coatings}

\subsubsection{Coating Composition Development}

The properties (crystal structure, hardness, modulus of elasticity, thermal expansion coefficient (TEC), and melting point) of several binary transition metal nitrides and carbides are summarized in Table 2-4.

Table 2-4 Properties of binary transition metal nitrides and carbides

\begin{tabular}{|c|c|c|c|c|c|c|}
\hline Material & $\begin{array}{c}\text { Crystal } \\
\text { structure }\end{array}$ & $\begin{array}{c}\text { Hardness } \\
(\mathrm{GPa})\end{array}$ & $\begin{array}{c}\text { Elastic } \\
\text { modulus } \\
(\mathrm{GPa})\end{array}$ & $\begin{array}{c}\mathrm{TEC} \\
\left(10^{-6} /{ }^{\circ} \mathrm{C}\right)\end{array}$ & $\begin{array}{c}\text { Melting } \\
\text { point } \\
\left({ }^{\circ} \mathrm{C}\right)\end{array}$ & Reference \\
\hline $\mathrm{CrN}$ & cubic & 11.2 & 400 & 2.3 & 1773 & {$[216,234]$} \\
\hline $\mathrm{Cr}_{3} \mathrm{C}_{2}$ & orthorhombic & 12.75 & 400 & 10.3 & 1895 & {$[234]$} \\
\hline $\mathrm{HfN}$ & cubic & 15.69 & 380 & 6.9 & 3660 & {$[168,216,234]$} \\
\hline $\mathrm{HfC}$ & cubic & 26.48 & 460 & 6.6 & 3930 & {$[234,235]$} \\
\hline $\mathrm{NbN}$ & cubic & 13.73 & 480 & 10.1 & 2323 & {$[216,234,235]$} \\
\hline $\mathrm{NbC}$ & cubic & 23.54 & 580 & 6.6 & 3600 & {$[234,235]$} \\
\hline $\mathrm{TiN}$ & cubic & $18-21$ & 395 & 9.35 & 2950 & {$[168,216,234,235]$} \\
\hline $\mathrm{TiC}$ & cubic & $28-35$ & $410-510$ & 7.4 & 3070 & {$[168,234,235]$} \\
\hline $\mathrm{TaN}$ & hexagonal & 9.8 & $556-595$ & 3.6 & 3300 & {$[234,236]$} \\
\hline $\mathrm{TaC}$ & cubic & 14.71 & 450 & 7.4 & 3980 & {$[234,235,237]$} \\
\hline $\mathrm{VN}$ & cubic & 14.71 & 460 & 8.1 & 2450 & {$[216,234]$} \\
\hline $\mathrm{VC}$ & cubic & 28.44 & 430 & 6.2 & 2648 & {$[234,238]$} \\
\hline $\mathrm{WC}$ & hexagonal & 21.58 & 695 & 4.3 & 2770 & {$[234]$} \\
\hline $\mathrm{ZrC}$ & cubic & 25.5 & $350-440$ & 6.7 & 3420 & {$[239]$} \\
\hline $\mathrm{ZrN}$ & cubic & 15.69 & 500 & 7.1 & 2980 & {$[168,216,235]$} \\
\hline
\end{tabular}

The properties of a binary nitride compound (e.g. TiN, $\mathrm{ZrN}, \mathrm{NbN}$, and $\mathrm{CrN}$ ) such as hardness, elasticity, and chemical inertness, can be improved by adding a third element

(e.g., $\mathrm{Al}, \mathrm{B}, \mathrm{Cr}, \mathrm{Si}, \mathrm{Ge}$ ), to obtain a ternary compound [40, 198, 240 - 242]. Even a small amount of a third element plays a decisive role in the modification of chemical bonding, structure, and morphology of the deposited coatings [39, 40]. In ternary metallic nitride 
coatings, the hardness is enhanced by means of a solid solution of atoms in metal nitride lattice, or by forming a nanocomposite material (nc-MN) with an amorphous phase [243 246]. Figure 2-19 shows hardness changes for metal nitrides vs. the concentration of the third element. It indicates that the nanohardness increases with the concentration of the third element, reaching a maximum at the concentration of the third element (between 2 at.\% and 10 at. $\%$ ) before declining; and this was found in another research that the peak hardness for $\mathrm{CrN}$ based coatings was observed as the Si content reached 2.2 at.\% [247].
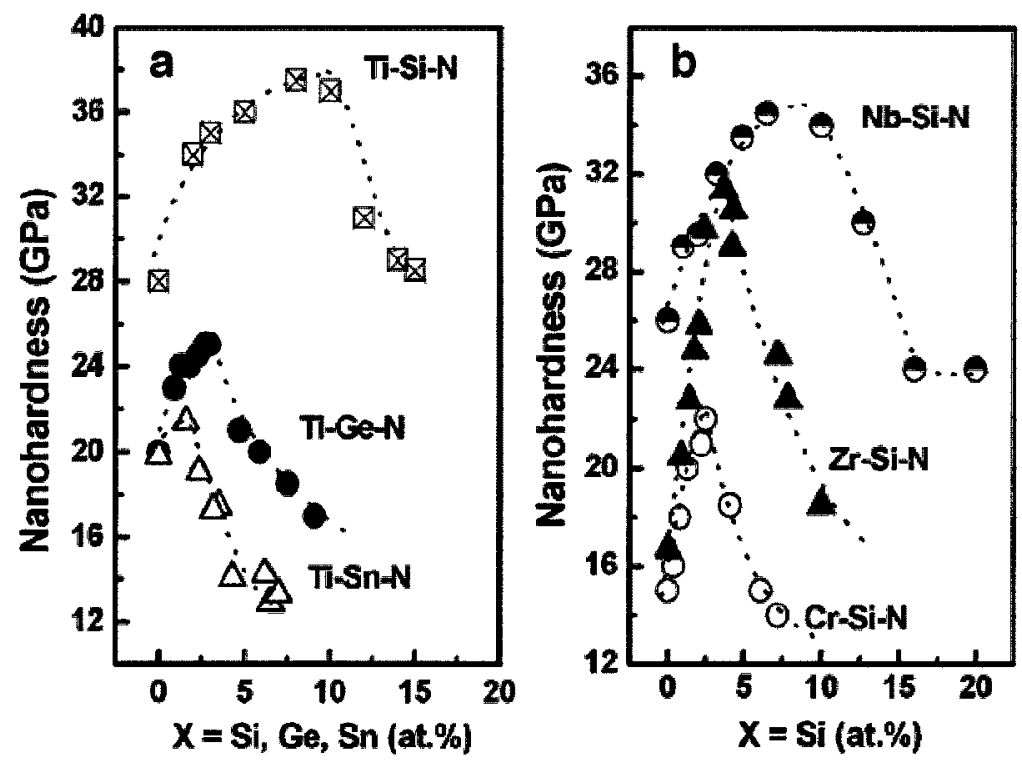

Figure 2-19 Nanohardness vs. third element for various metal nitrides [246].

In particular, the amorphous silicon nitride phase can be formed in the matrix as the result of adding $\mathrm{Si}$ and can improve the mechanical properties and oxidation resistance of the binary metal nitride coatings $[242,244,248]$. These were believed to be associated with the grain boundary hardening from a strong cohesive energy of the inter-phase boundaries [249] and solid solution effect from $\mathrm{Si}$ and amorphous silicon nitride, i.e., ncMeN/a-SiNx nanocomposite $[209,249,250]$. The amorphous phase can also act as an 
oxygen barrier to slow down the oxidation diffusion rate, resulting in enhanced oxidation resistance [251].

More recently, quaternary coating systems have been developed and investigated. A CrAlSiN system has demonstrated multi-functional coating characteristics with super hardness ( $\geq 40 \mathrm{GPa}$ ), excellent resistance to oxidation and wear [209]. The $\mathrm{CrSiCN}$ system has a fine composite microstructure consisting of nano-sized crystals $(\mathrm{Cr}, \mathrm{Si})(\mathrm{C}, \mathrm{N})$ that are well distributed in the amorphous phase of $\mathrm{Si}_{3} \mathrm{~N}_{4} / \mathrm{SiC}$ mixture. The hardness of $\mathrm{CrCN}$ coatings was significantly increased when the Si addition was increased to 9.2 at.\%. The friction coefficient of the $\mathrm{CrSiCN}$ coatings also decreased compared to $\mathrm{CrN}, \mathrm{Cr}(\mathrm{C}, \mathrm{N})$, and CrSiN coatings [206]. Compositions for ternary and quaternary Ti- and Cr-based coatings are summarized in Table 2-5.

Table 2-5 Summary of ternary and quaternary $\mathrm{Ti}$ and $\mathrm{Cr}$ based coating compositions

\begin{tabular}{|c|c|}
\hline Coating systems & Reference \\
\hline \multicolumn{2}{|l|}{ Ternary } \\
\hline$(\mathrm{Ti}, \mathrm{Al}) \mathrm{N},(\mathrm{Cr}, \mathrm{Al}) \mathrm{N},(\mathrm{Ti}, \mathrm{Cr}) \mathrm{N},(\mathrm{Ti}, \mathrm{Y}) \mathrm{N},(\mathrm{Ti}, \mathrm{Pd}) \mathrm{N}$ & [40] \\
\hline$(\mathrm{Ti}, \mathrm{Al}) \mathrm{N}, \mathrm{TiN}(\mathrm{Pt})$ & {$[190]$} \\
\hline$(\mathrm{Cr}, \mathrm{Al}) \mathrm{N}$ & [193] \\
\hline TiAlCN & [15] \\
\hline TiBN & [252] \\
\hline TiSiN & [252] \\
\hline Ti-Cr-N & {$[191,205]$} \\
\hline $\mathrm{Cr}(\mathrm{C}, \mathrm{N}), \mathrm{CrSiN}$ & {$[206]$} \\
\hline CrAlN, CrSiN & [209] \\
\hline \multicolumn{2}{|l|}{ Quaternary } \\
\hline $\mathrm{CrSiCN}$ & [206] \\
\hline CrAlSiN & [208] \\
\hline CrAiSiN & [209] \\
\hline TiSiCN & {$[210]$} \\
\hline TiAlSiN & [211] \\
\hline (TiAlV)N & [252] \\
\hline
\end{tabular}




\subsubsection{Layered Coating Structure and New Microstructure Development}

In addition to composition modification, the coating performance can be further improved through the development of multilayered or multiphase coatings. For example, to enhance the coating-substrate adhesion, improve hardness, and reduce stress, duplex or multilayered coatings were developed [170, 240, 253 - 256]. Figure 2-20 shows typical multilayered coatings. Early studies on multilayers of metals (each layer $<500 \mathrm{~nm}$ ) showed significant improvements in general strength $[257,258]$, which was attributed to inhibiting the Frank-Read source (dislocation formation) and crack propagation [259, 260], and crack deflection [261]. These effects were referred as to the toughening concept [226, 262 - 264].

The early modeling (by Koehler) [265] found that high shear strength coatings can be realized by means of alternating layers with high and low elastic modulus. Thin layers and the differences in elastic modulus between layers would inhibit dislocation formation and mobility [249]. The Hall-Petch relationship was reported between mechanical enhancements and laminated layer thicknesses in metal/ceramic and ceramic/ceramic laminate structures $[266,267]$. The overall hardness for a coating system can be higher than the individual components that make up the layers due to the formation of superlattices [268]. Superlattices contain multiple layers with a modulation period in the range of several lattice parameters (on the order of 1 to $10 \mathrm{~nm}$ ), as shown in Figure 2-21. 


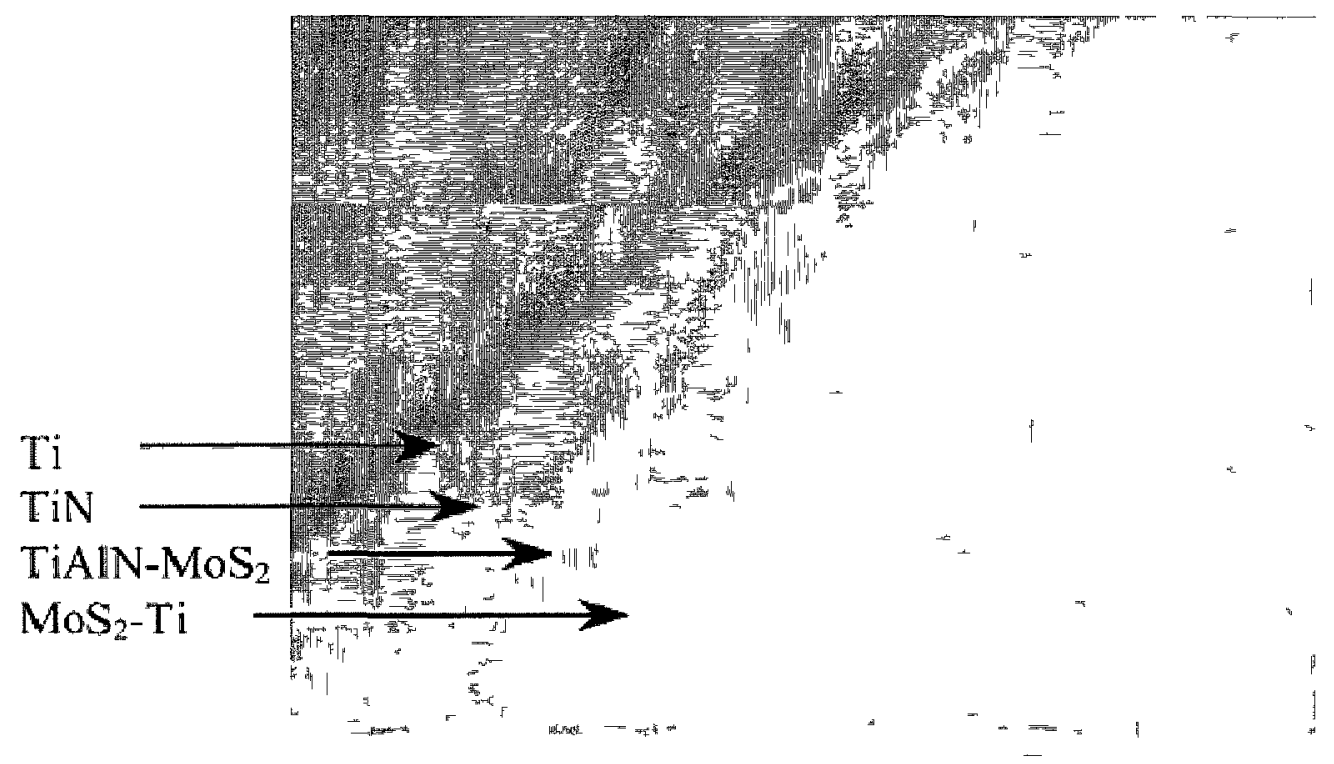

(a) SEM image of a $\mathrm{MoS}_{2}-\mathrm{Ti} / \mathrm{TiAlN}-\mathrm{MoS}_{2} / \mathrm{TiN} / \mathrm{Ti}$ multilayer coating [269]

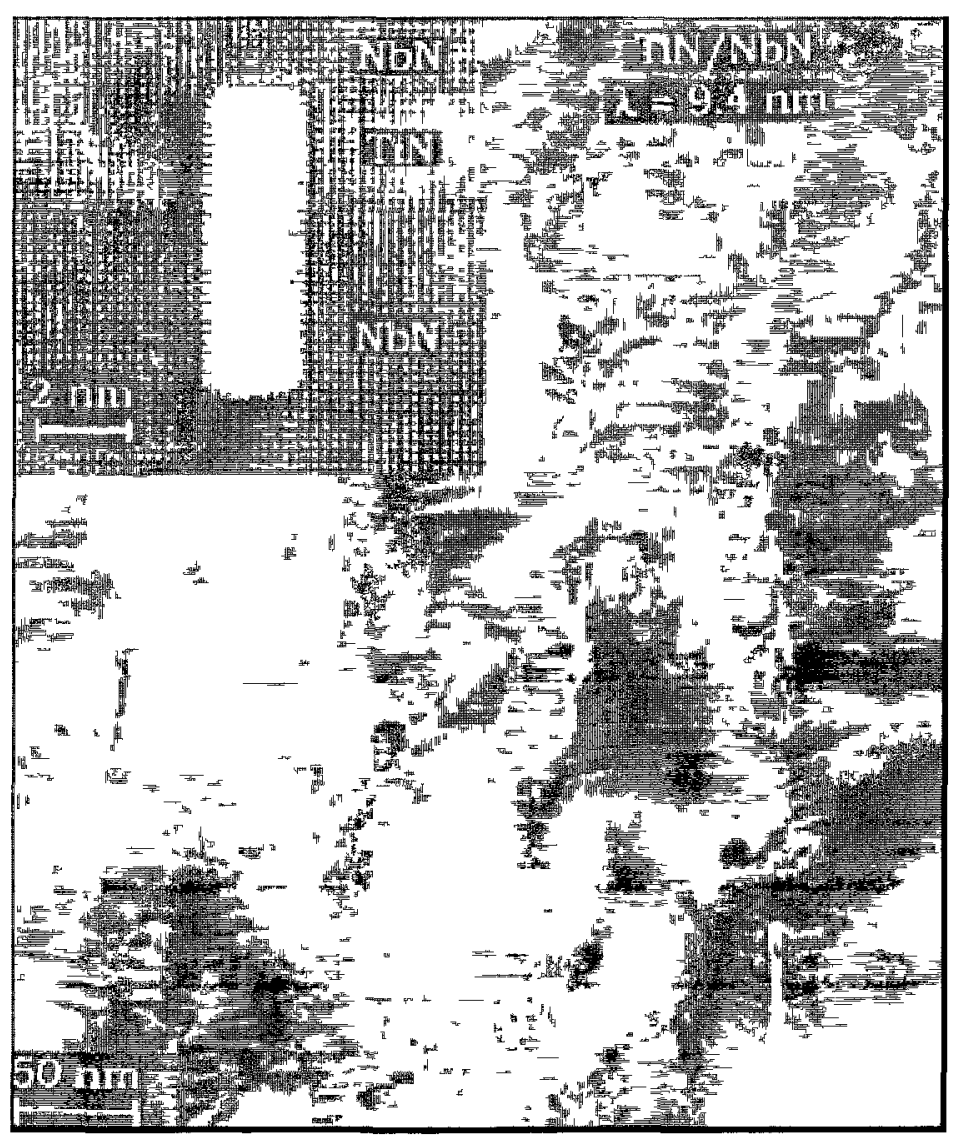

(b) TEM image of a TiN/NbN superlattice film. A HR lattice fringe image of a void situated at a low-angle column boundary is shown in upper-left [234]

Figure 2-20 Cross section images of multilayer and superlattice coating systems. 


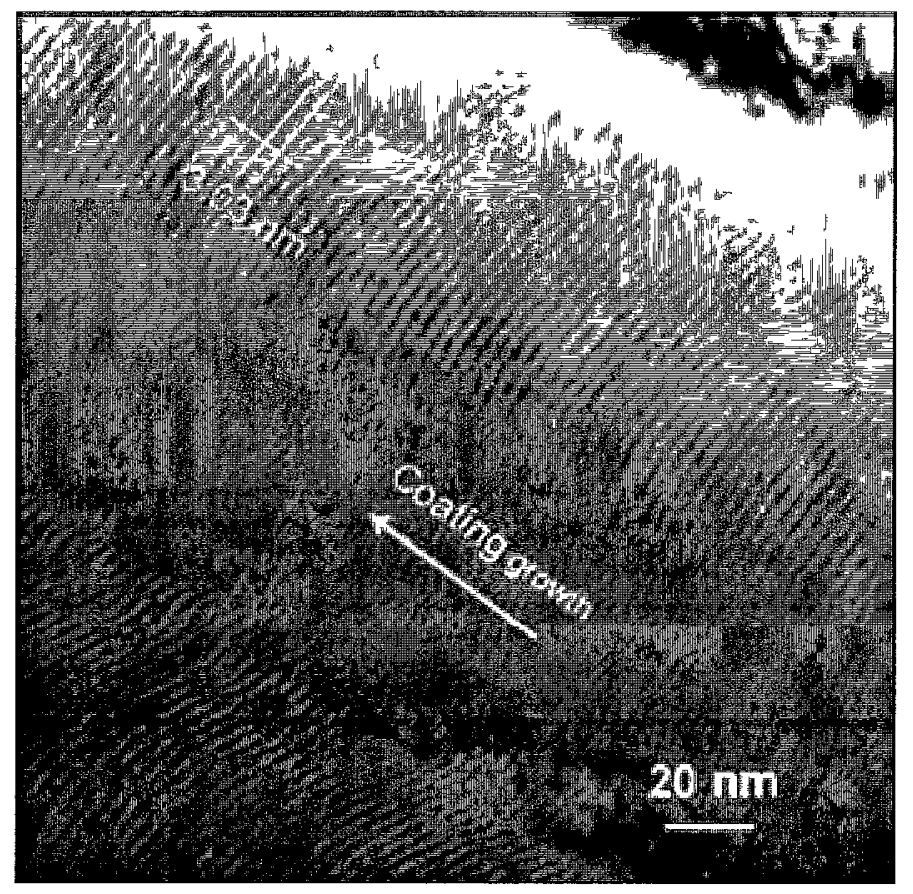

(a) nano-scale multilayered structure of the TiAlN/VN coating [270]



(b) $\mathrm{CrN} / \mathrm{ScN}$ coatıng (modulation period: $1.7 \mathrm{~nm}$, interface width: $0.18 \mathrm{~nm}$ ) [271]

Figure 2-21 Cross-sectional TEM micrograph multilayered coating with superlattice structure. 
Mechanical properties of multilayered coatings as a function the number of layers are shown in Figure 2-22. The microhardness of multilayered coatings $\mathrm{TiN} / \mathrm{ZrN}$ and $\mathrm{TiN} / \mathrm{NbN}$ increases with the number of layers; and for the multilayer coating $\mathrm{TiN} / \mathrm{CrN}$ the hardness increased, but reached a peak value at 80 layers (Figure 2-22 a). This behaviour of $\mathrm{TiN} / \mathrm{CrN}$ is associated with $(\mathrm{Ti}, \mathrm{Cr}) \mathrm{N}$ solution formation [272]. These observations suggest that the interphase boundaries and layer lattice mismatch are the main factors governing the increasing hardness of multilayered coatings [272]. As for the multilayer coating $\mathrm{CrN} / \mathrm{Si}_{3} \mathrm{~N}_{4}$ (Figure 2-22 b), the hardness is between those of monolithic $\mathrm{CrN}$ and $\mathrm{Si}_{3} \mathrm{~N}_{4}$ films, and increases with modulation period, remaining almost constant as the modulation period exceeds $10 \mathrm{~nm}$. The modulus of elasticity demonstrates a similar trend to that of hardness (Figure 2-22 c). For nitride superlattices (Figure 2-22 d), microhardness versus modulation period rapidly increased from $\lambda=0$, reaching the peak value at $\lambda=5-15 \mathrm{~nm}$, then decreases for increasing modulation period.

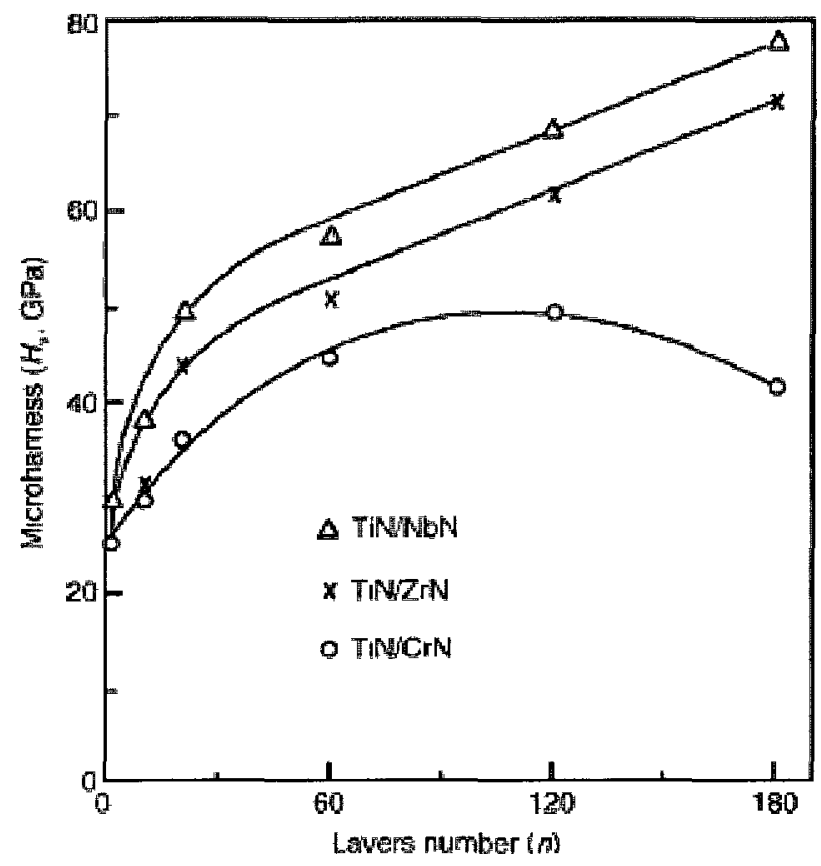

(a) Microhardness of multilayered coatings as a function of number of layers [272]

Figure 2-22 to be continued 


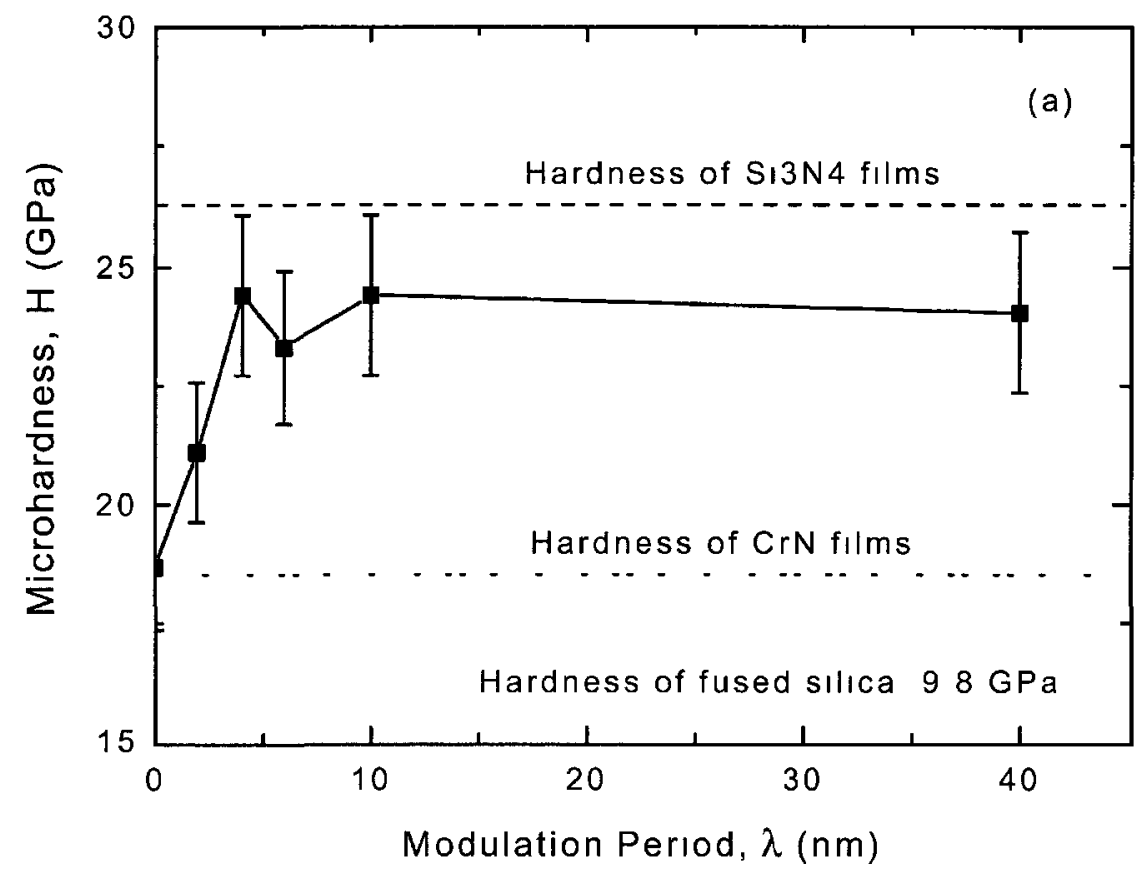

(b) Hardness of multilayered coatings as a function of modulation period [273]

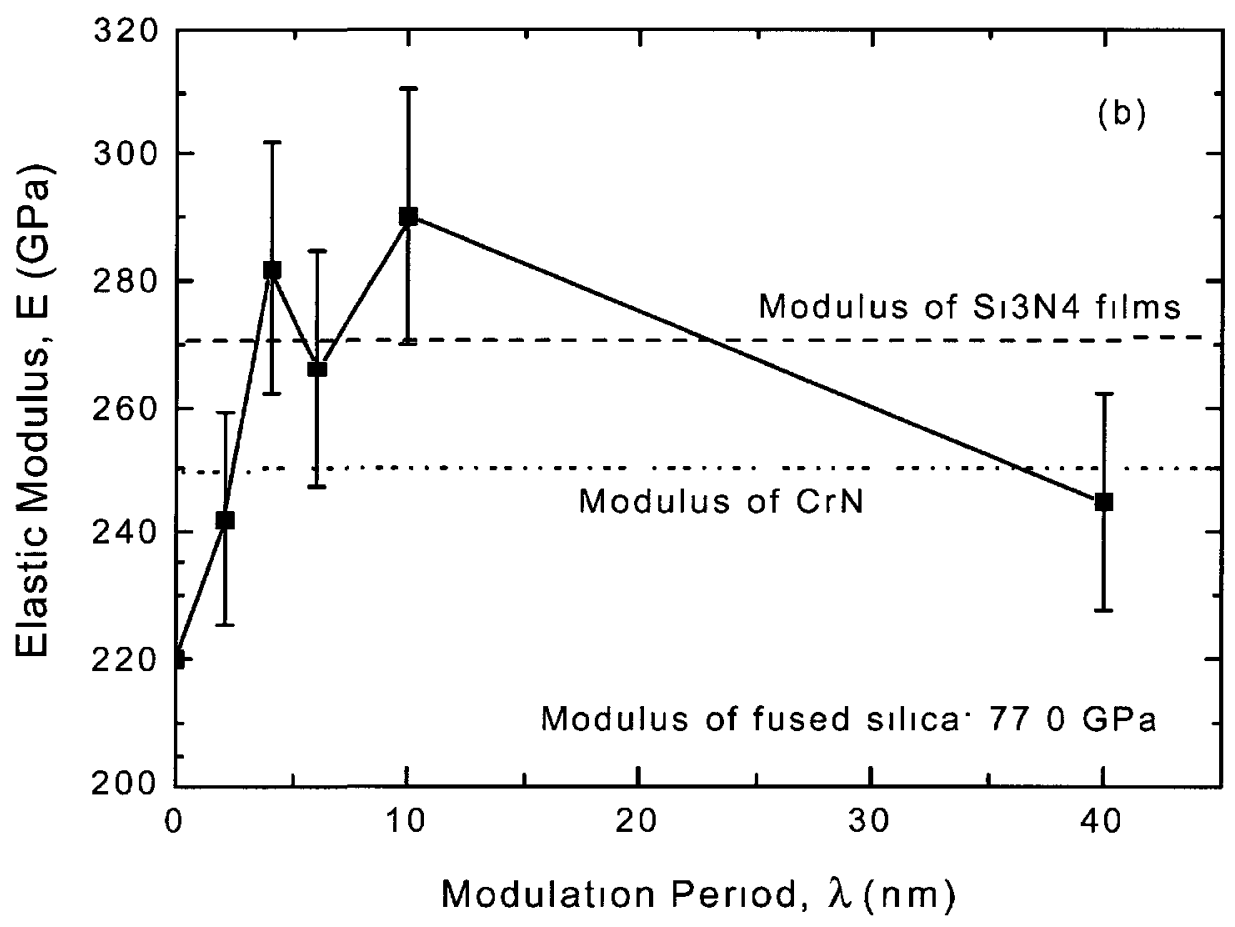

(c) Elastic modulus of multilayer coating as a function of modulation period [273]

Figure 2-22 to be continued 


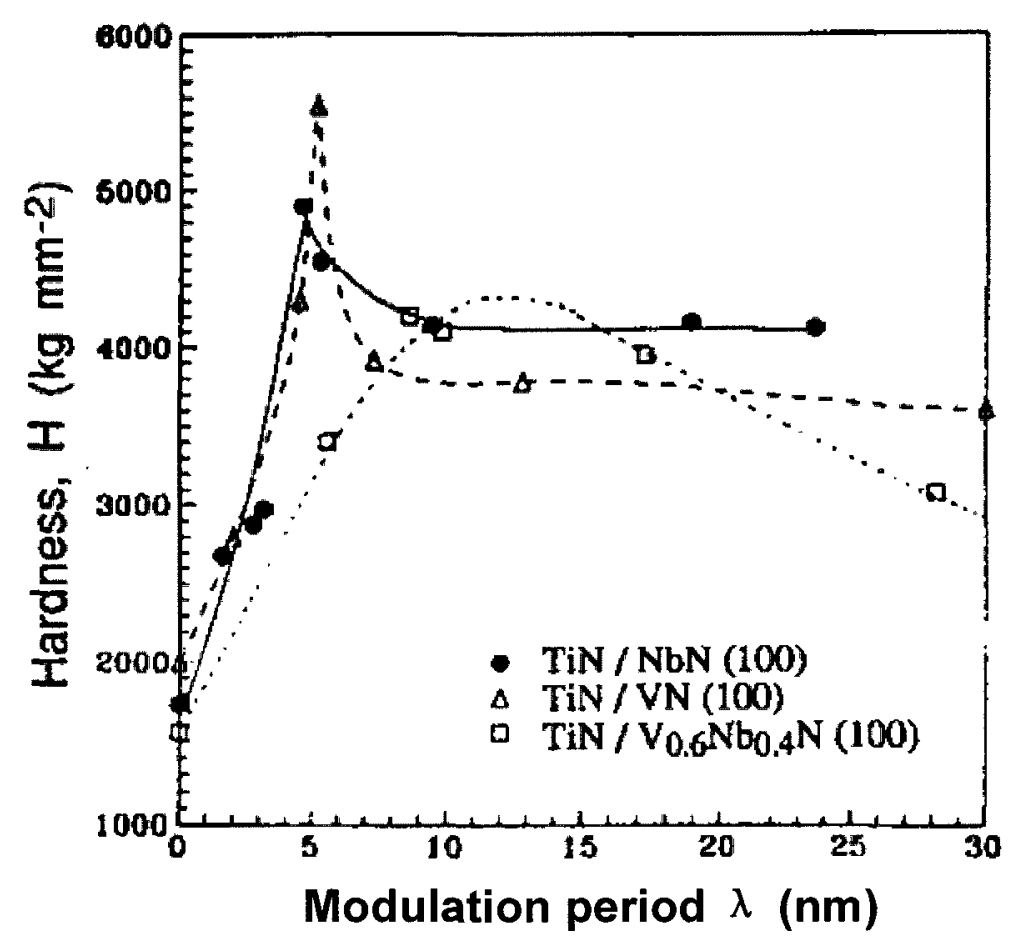

(d) Microhardness of multilayer coating as function of modulation period (modified from [234])

Figure 2-22 Mechanical properties of a multilayered coating as a function of the number of layers or modulation period.

Despite the advantages of multilayered coatings, it is difficult to apply them with uniform thicknesses on three-dimensional components and rough surfaces where the superlattice effect would be lost as the layers would not be in perfect periodicity [207]. In addition, extended exposure to elevated temperatures causes interdiffusion and subsequent intermixing of the superlattice structure $[274,275]$. As an alternative, nanocomposite coatings offer similar advantages to multilayered coatings, but their properties are not critically dependent on the layer thickness or substrate geometry [207].

With the development of nano-material science, nanotechnology has been incorporated into thin film/coating technology. Combining the concepts of composite materials and 
nano-scaled materials, nanocomposite coatings were developed $[170,276]$. A nanostructure (with grain size $<10 \mathrm{~nm}$ ) offers a strong interface that inhibits grain boundary sliding, high hardness, high toughness, good elastic recovery, chemical and thermal stabilities, low friction, and good tribological characteristics [202, 277 - 280].

A nanocomposite coating consists of at least two phases, which can be crystalline/amorphous or crystalline/crystalline $[170,202,276]$. Typical applied hard nanocomposite configurations include nc-MeN/a-nitride (where, nc-nanocrystalline, Metransition metals, a-amorphous) [195 - 197], nc-MeN/nc-nitride (nc-TiN/nc-BN)[195], and nc-MeN/metal (e.g., $\mathrm{Cu}, \mathrm{Ni}, \mathrm{Y}, \mathrm{Ag}$, and $\mathrm{Co}$ ) [198 - 200].

Figure 2-23 shows some developed systems for coating applications. Boundary regions play an important role in the atomic arrangement that relates to deformation [170]. The embedded nanocrystals in a thin amorphous matrix (Figure 2-23) offer better coherence at grain boundaries than do pure polycrystalline composites, and are more suitable for tribological applications compared to the single or polycrystalline multilayered coatings [249]. Figure 2-24 compares the mechanicial properties for those coatings, and shows that the nanocomposite and superlattice coatings demonstrate significant advantages in hardness and modulus of elasticity over monolithic or single-phase coatings. 


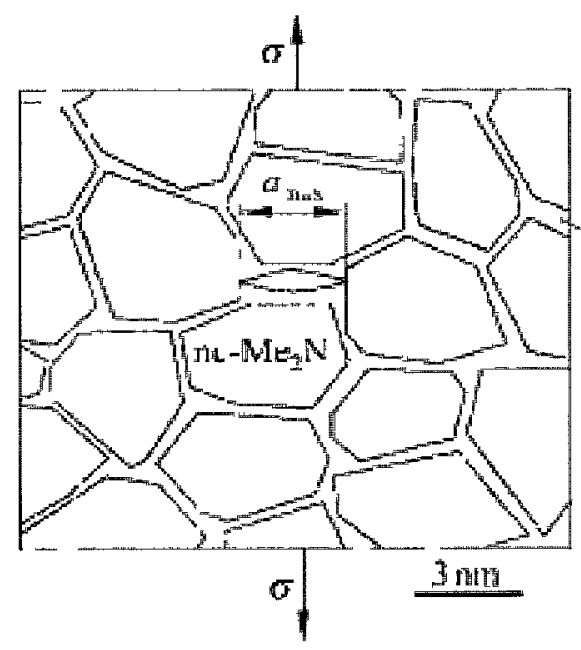

(a) Super-hard composite coating [207]


(c) YSZ/Au nanocomposite coating [207] (d)nc-CrN/a-Si $\mathrm{N}_{4}$ nanocomposite coating [247]

Figure 2-23 Microstructures of nano-composite coatings. 


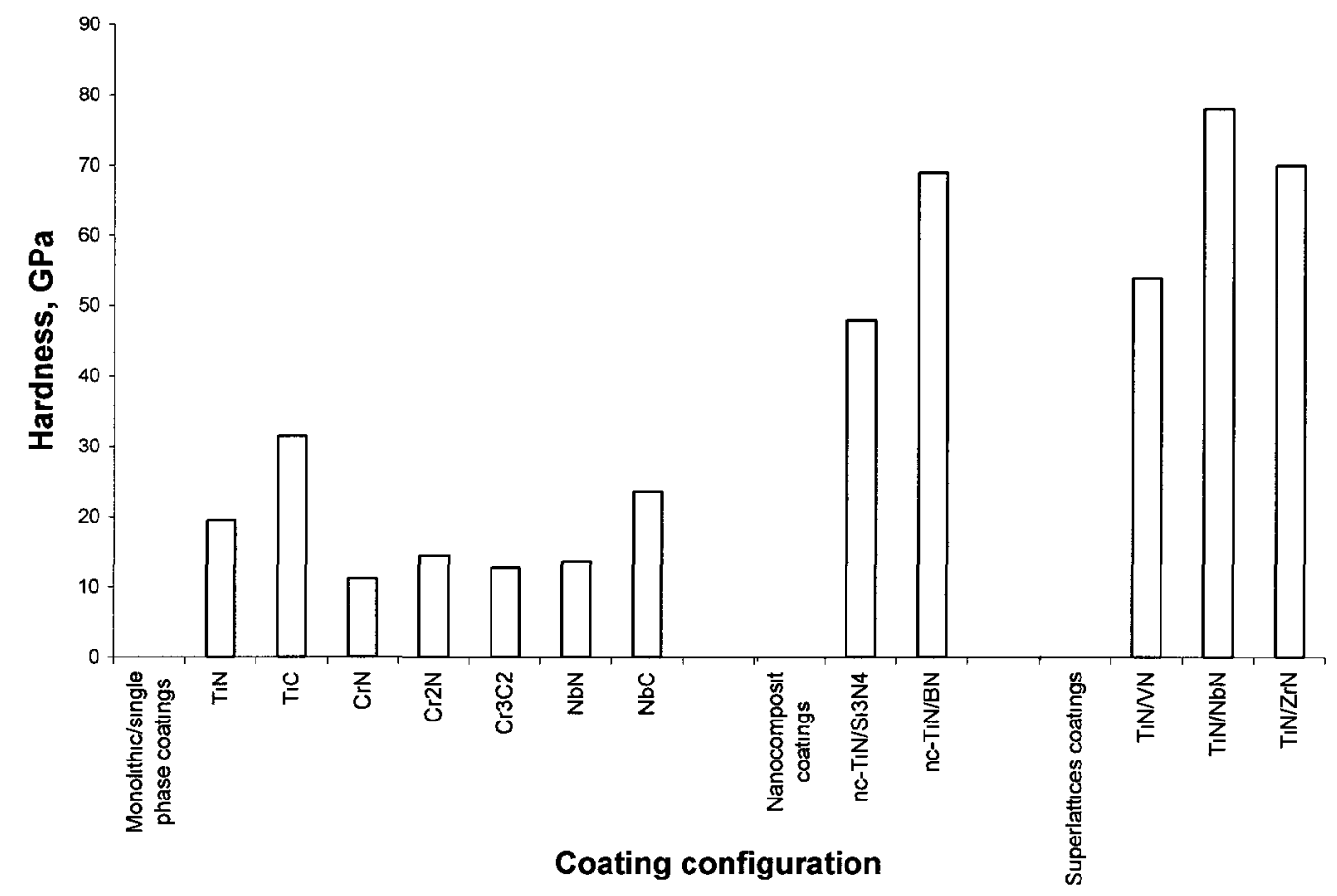

(a) Hardness

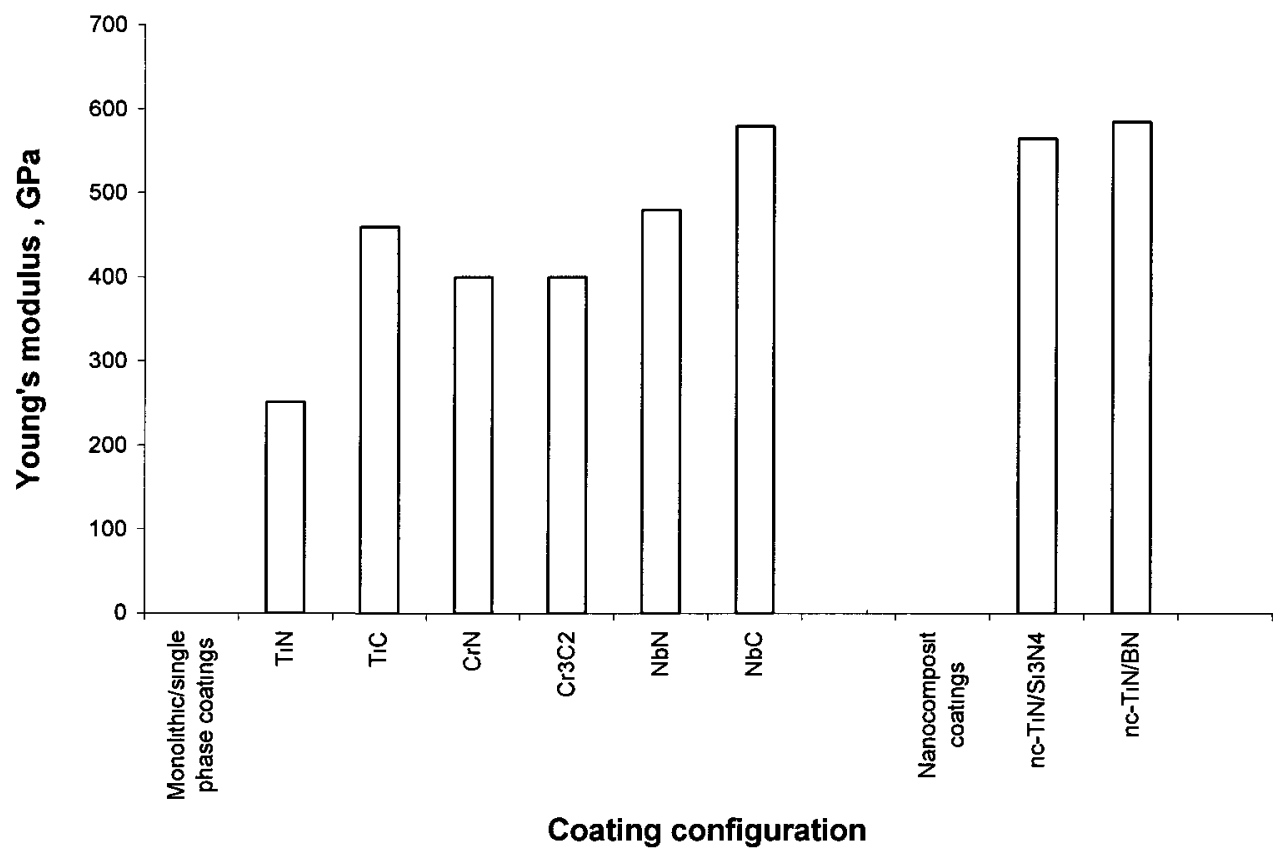

(b) Modului of elasticity

Figure 2-24 Comparisons of mechanical properties for nano-composite coatings (data sources: $[170,207,215,216,234,235,254,272,281,282])$. 
In nanocomposite coatings, a high-volume density of grain boundaries across grainmatrix interfaces limit initial crack sizes, promote crack deflections, and terminate crack propagation $[204,207,279,283]$. Smaller structures have fewer surface defects [284], which is another explanation for the reduced dislocation initiation in nanocomposite structures. Grain boundary sliding occurs instead of dislocation movement; and the result is a different microstructure deformation mechanism from that of large grain-sized materials $[202,277$ - 280]. Unlike the hard coatings where high plastic deformation can only be accommodated by hardness reduction, the nanocomposites allow high elastic strain with high hardness and toughness due to the effect of the combined hard and soft phases [170].

\subsubsection{Transition Metals and Compounds: Processes and Properties}

\subsubsection{Coating Deposition Processes}

Metal surface coating processes can be classified into three general forms, conversion, thin film, and overlay [103]. Conversion processes are chemical or electrochemical treatments such as carburizing, nitriding, carbonitriding, siliconizing, chromizing, boronizing, oxidizing, and phosphating. Thin film processes include aqueous-deposition (electro- or electroless- and other wet chemical depositions), vapor deposition such as physical vapor deposition (PVD) and chemical vapor deposition (CVD), and ion implantation. Overlay processes include plasma, laser, plasma transfer arc (PTA), thermal spray, and detonation gun. 


\subsection{Aqueous Deposition: Electroplating}

Electroplating is an electrochemical process classified as an aqueous deposition technique. In a metal salt electrolyte cell system, the metallic ions carrying a positive charge are attracted to the substrate that is negatively charged, which results in metallic ion being reduced to form a metallic coating on the substrate surface.

The main advantage of this technique is a metallurgically bonded coating with no heating required of the substrate. This reduces the heat-affected zone (HAZ), and minimizes the changes to the substrate properties [285]. Figure 2-25 shows a typical electroplating setup.

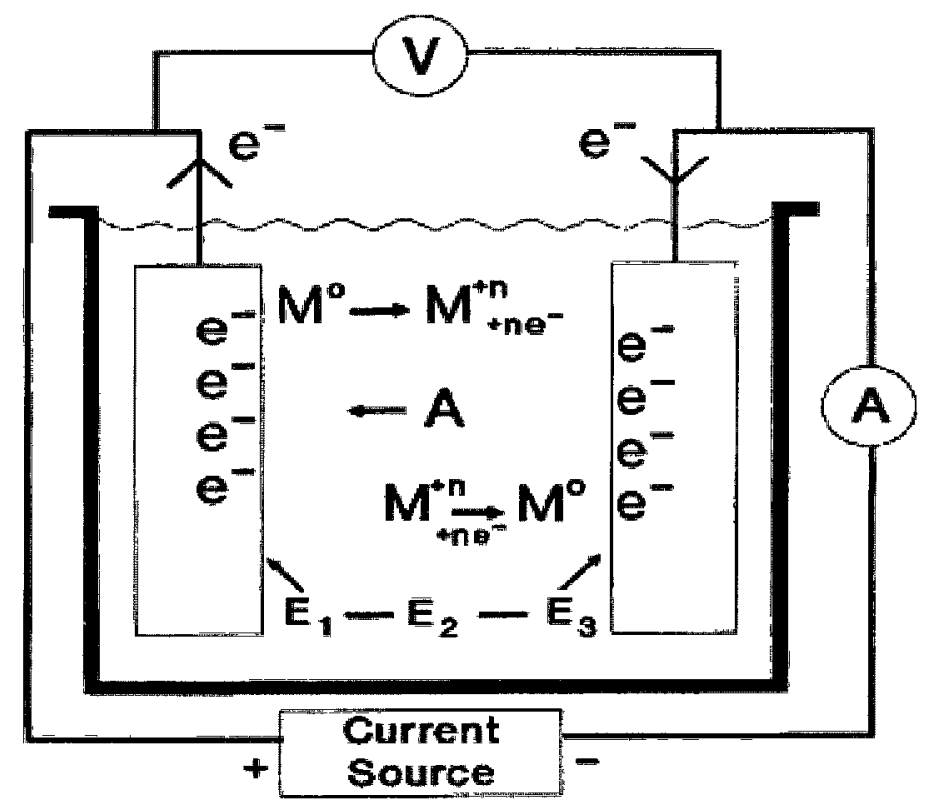

Figure 2-25 Schematic of a typical electroplating setup [286].

Process variables such as pulsed current and additives have been introduced into the conventional dc electrolytic plating and have brought benefits to depositions [287 - 291]. With a pulsed current, grain sizes and porosity can be reduced. Adhesion can also be 
improved with greater pulse peak voltages while allowing a moderate average local current density, so the burning effect is significantly alleviated and coating uniformity is improved. Additives such as carbon nanotubes and other nano particles in electroplating solutions change the brightness, grain size, and leveling of a coating; they can also modify the mechanical and physical properties and cause stress release and pitting reduction [95]. The grain refining and leveling are attributed to the effects of electrode kinetics and the structure of an electrical double layer at plating surfaces, which affect mass transport during the deposition process[95, 292, 293].

For example, the introduction of carbon nanotubes (CNT) into nickel electroplating generates a matrix of Ni-CNT coating. CNTs increase the number of nucleation sites for individual grain growth, therefore reducing the grain size [294]. CNTs also improve the mass transport rate for the ions in the electrolyte, thus enhancing current density and therefore increasing the growth rate [293]. As a result, the matrix of Ni-CNTs shows a significant improvement in mechanical properties over pure $\mathrm{Ni}$ electroplating [295, 296].

Other particulates such as nano $\mathrm{Al}_{2} \mathrm{O}_{3}$ particles [297-299], $\mathrm{ZrO}_{2}$ particles [300], $\mathrm{SiC}$ particles [294, 301, 302], and carbon nano-fibers [303] were also introduced into nickel electroplating. With the incorporation of nano $\mathrm{Al}_{2} \mathrm{O}_{3}$ particles, the grain size in the coating was reduced [299], the strength was increased [293], and microhardness was increased [298, 299]. The introduction of $\mathrm{ZrO}_{2}$ particles resulted in an increase in hardness [300]. With SiC particles, grain size was reduced, hardness was increased [294], the coefficient of friction was lowered [301], and the resistance to wear and corrosion 
was increased [294, 301, 302]. The incorporation of carbon nano-fibers modified the microstructure and mechanical propeties in the deposition composite [303].

\subsection{Physical Vapor Depositions}

The vapor deposition methods include physical vapor deposition (PVD) and chemical vapor deposition (CVD) $[239,288,304]$. PVD processes form coatings by means of material vapors and their successive condensation onto substrates. Vaporization techniques include evaporation, sputtering, laser ablating, ion plating, molecular beam epitaxy (MBE), and electron beam. CVD processes form coatings by depositing films from vapor-phased chemical reactions onto substrates.

Physical vapor deposition is an atomistic deposition process. In this process, solid materials are vaporized by physical means, and then are transported in the vapor phase to the substrate, and deposited as films [46, 305]. Compared to other deposition processes (electroplating, CVD, or plasma spraying) the PVD technique demonstrates several advantages such as versatile composition, unique microstructures, and the ability to modify crystal structure (e.g., amorphous deposits). Additionally, PVD techniques promise wide substrate temperature ranges, high deposition rates, high purities, excellent bonding to the substrate, and highly uniformed and dense structures [7, 306]. Evaporation and sputtering are two important vaporization mechanisms in PVD [307, 308]. 


\section{Thermal Evaporation Process}

In evaporation processes, materials are vaporized by means of heating mechanisms such as resistance, induction, electronic arc, electron beam, laser and plasma (ionization). The transition of solids or liquids into the vapor phase is an atomistic thermodynamic phenomenon [307]. The process requires a high vacuum environment (up to10 $0^{-5}-10^{-10}$ torr) to prevent collision between evaporated atoms with atmospheric gases.

Plasma-assisted reactive evaporation (ARE) is a process involving metal or alloy evaporation in a plasma reactive gas environment. The plasma assisted evaporation process results in a higher deposition rate compared to conventional evaporation process with simple heating.

\section{Cathodic Arc Process}

The cathodic arc deposition technique was developed to achieve higher ionization, current density, better coating adhesion, and a higher deposition rate [309]. Arc evaporation is induced by the striking of a high current, low voltage arc on a cathode surface (target), generating a small (i.e., a few micrometers) energetic emitting area (cathode spot), where a high velocity jet of vaporized cathode material is established. Figure 2-26 shows a cathodic arc PVD (CA-PVD) process. In the high density electron cloud many vaporized atoms are ionized. These ions are then accelerated to high energies by a controlled electric field within the vacuum chamber. For reactive depositions, the reactive gas atoms, introduced into the chamber during the process, are also ionized by 
the high energy electrons in the arc, and are accelerated before reacting with the target material. As a result of the reaction, the formed compound is bonded to the substrate.

A disadvantage of the CA-PVD technique is that it exhibits higher roughness compared to sputtering and electron-beam depositing techniques [32]. In a cathodic arc vaporization process, part of the material is ejected in the form of molten globules. These globules are deposited and embedded during the coating growth process and act as source of growth defect [310].

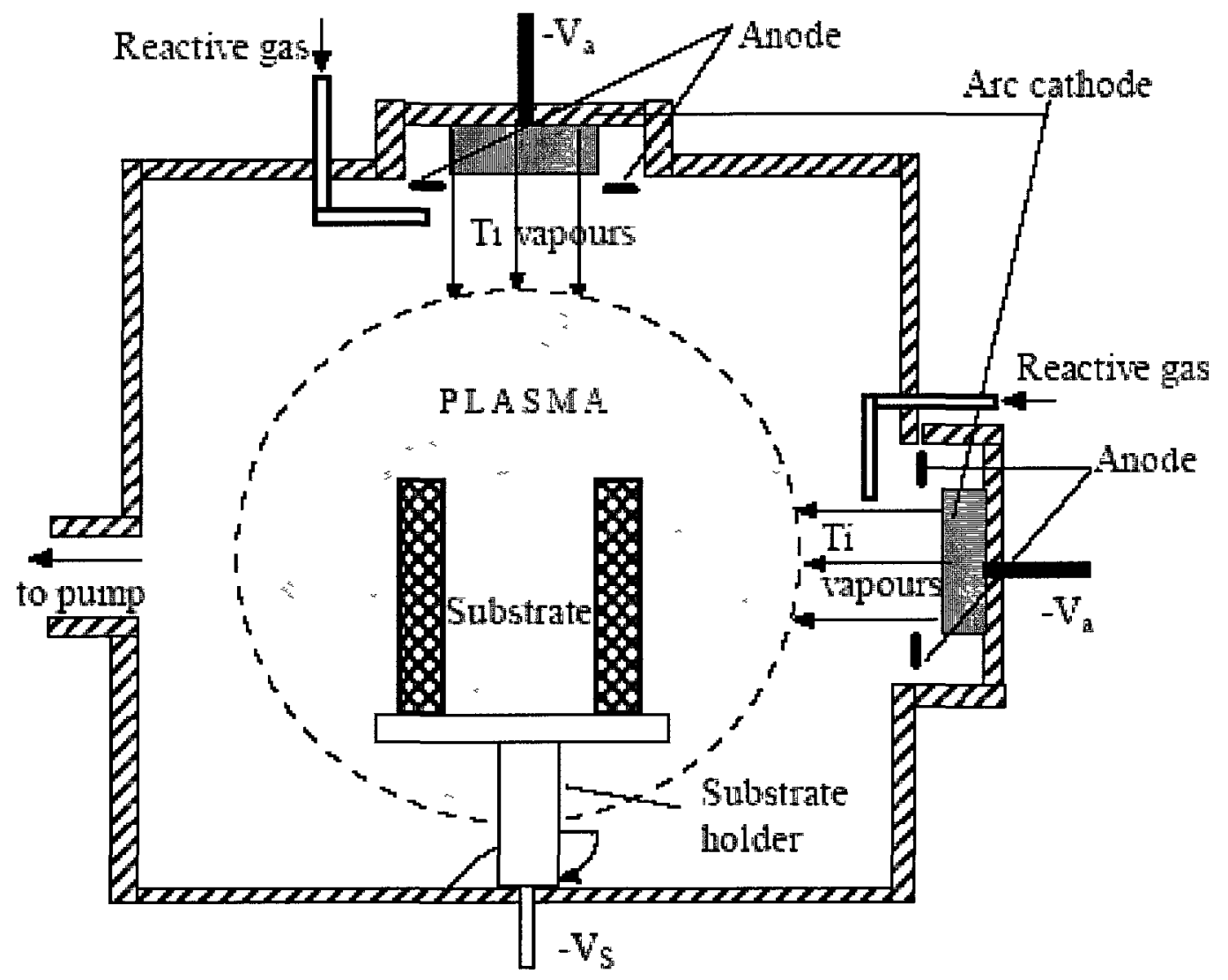

Figure 2-26 Schematic of a cathodic arc deposition process [311]. 


\section{Sputtering Process}

A sputtering deposition process makes use of the phenomena that momentum transfer from energetic species to a target material, and results in ejections of atoms or molecules from the surface by means of bombardment [225]. Figure 2-27 shows a typical sputtering deposition apparatus. In an evacuated chamber, the target (a source of coating material as cathode) is connected to a negative voltage supply. With an inert gas (typically argon) introduced into the chamber, a glow discharge is initiated between the cathode and anode (substrate holder). Due to the ion (e.g., $\mathrm{Ar}^{+}$) bombardment at the target surface, charged particles (e.g., secondary electrons or ions) and photons are emitted. In addition, under a constant electron bombardment, the substrate surface is deeply cleaned of surface oxides and other contaminants [312], which can improve the coating adhesion and density, and enhances the mobility of adsorbed species.

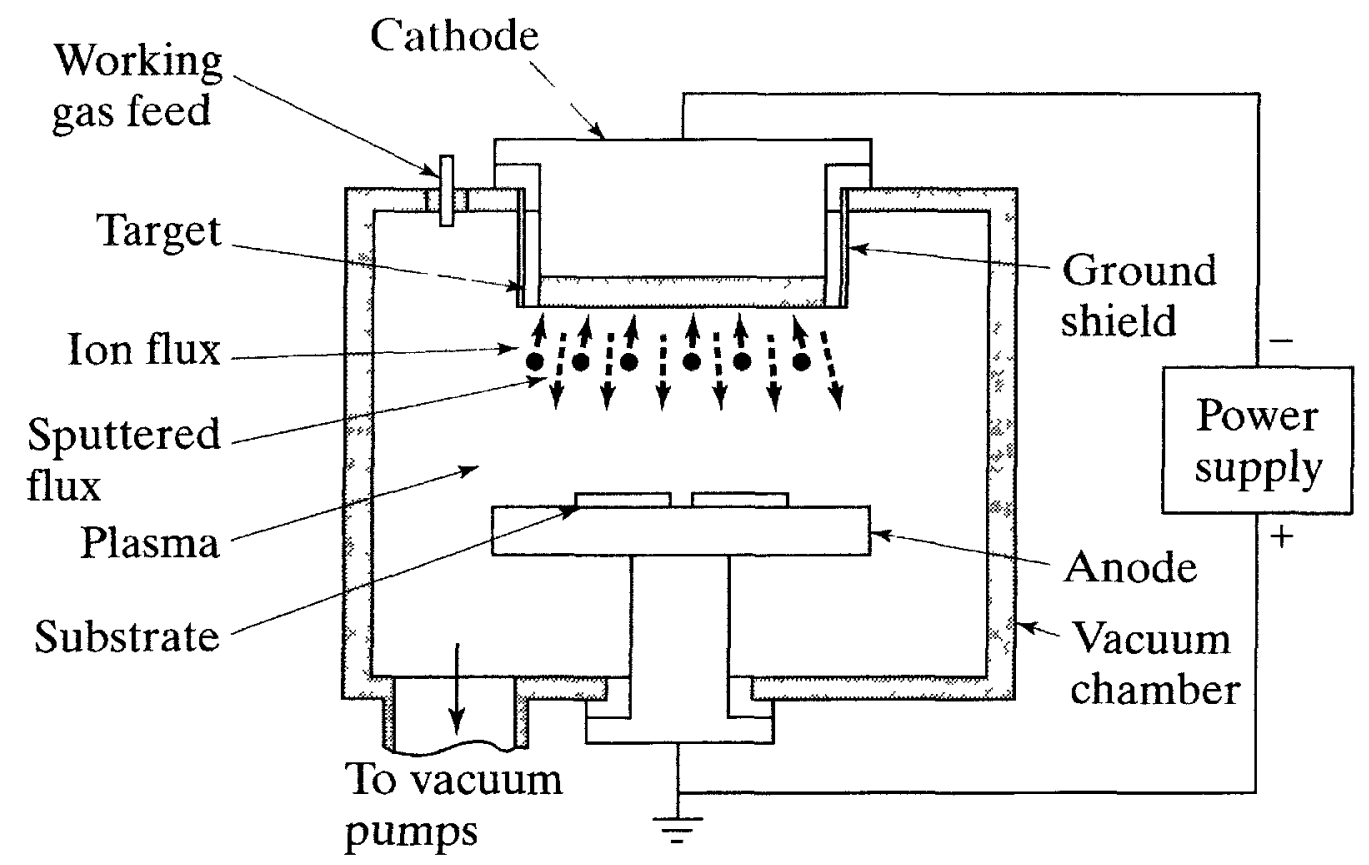

Figure 2-27 Schematic diagram of the sputtering process. The positively charged argon ions strike the negative cathode with sufficient energy to dislodge atoms, which then coat on the positively charged anode surfaces [313]. 
In a reactive sputtering, atoms or molecules from sputtered target and reactive gasses collide before they reach the substrate. This type of sputtering deposition is beneficial for depositing a compound as the phase in the coating structure (e.g., TiN form as a result of Ti reacts with $\mathrm{N}_{2}$ gas).

In magnetron sputtering, a magnetic-field generates spiraling electron paths to restrict the flow of electrons to the vacuum chamber wall and maximize the efficiency of gas ionization [314-316]. This process is further divided into balanced and unbalanced magnetron sputtering. In a balanced magnetron sputtering system, magnetic flux near the cathode surface goes to the magnetic core (Figure 2-28 a), therefore energetic electrons escape from the primary magnetic trap near the cathode surface, going toward the anode (substrate holder). To avoid the energetic particle bombardment on the growing film in balanced magnetron sputtering, an unbalanced magnetron configuration was introduced $[307,317,318]$. In an unbalanced magnetron configuration, stronger magnets are set on the outside of the existing field (Figure 2-28 b). This magnetic field traps secondary electrons escaping from the target surface to prevent collisions with the PVD chamber. When a negative bias is applied to the substrate, the incident ionized particle flux is increased, which is used to control the growth properties of the coating. Unbalanced magnetron sputtering creates coatings with increased density, lower stress, and lower droplet formations [319]; and has been commonly used to fabricate hard nitride/carbide coatings [307]. 


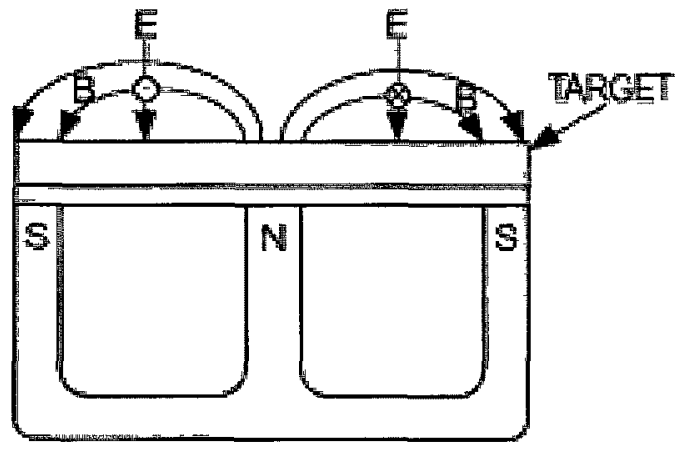

(a) Balanced configuration

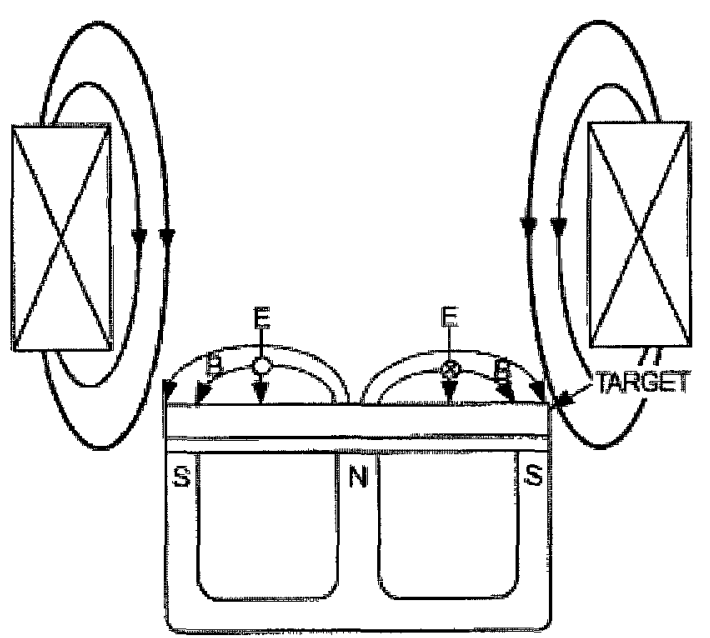

(b) Unbalanced configuration

Figure 2-28 Schematics magnetron sputtering configurations [308].

In general, magnetron sputtering allows for lower substrate temperatures [225], low working pressures (i.e., more compacted film structures) and better coating adhesion [315]. A wide range of ceramic, metallic or other compound films can be deposited using this technique. However, the power applied is limited so as to prevent the target material from melting [225], and the target utilization is low (only material on the track is being used) [320]. To enable higher power input, hence a higher deposition rate, high power impulse magnetron sputtering has been developed in which high voltage pulses (several $100 \mathrm{~V}$ to several $\mathrm{kV}$ ) are used to offer high plasma densities without overheating the target material [225].

Sputtering has been combined with other techniques such as ion beam and pulsed laser. Ion beam combined sputtering yields excellent adhesion and high purity due to low working pressures (about $0.1 \mathrm{mtorr}$ ) and low substrate temperature [307]. Magnetron 
sputter-assisted pulsed-laser deposition has been developed for depositing hard coatings such as TiN and Ti-C-N/DLC [202, 276, 305].

PVD techniques also have several drawbacks. Due to the effect of line-of-sight, it is difficult for PVD techniques to coat parts with complex shapes (no internal passages can be coated) and large surface areas (non-uniformity). PVD techniques need solid metal or oxide sources as target materials, which are time consuming to manufacture and costly [321]. High capital investment and operating costs are also a consideration when selecting PVD processes.

\subsubsection{Coating Microstructure and Properties}

In developing coatings for tribological applications, the major concerns are mechanical properties including hardness, elasticity, toughness and friction [216]. In corrosion applications, however, foci are placed on factors such as chemical stability, surface morphology, composition homogeneity, diffusion rate, and microstructure imperfections $[213-216,218]$.

\subsection{Effect of Coating Microstructure}

Figure 2-29 shows typical PVD coating fractures where features such as microstructure, thickness, interface, surface morphology, and defects can be observed. 


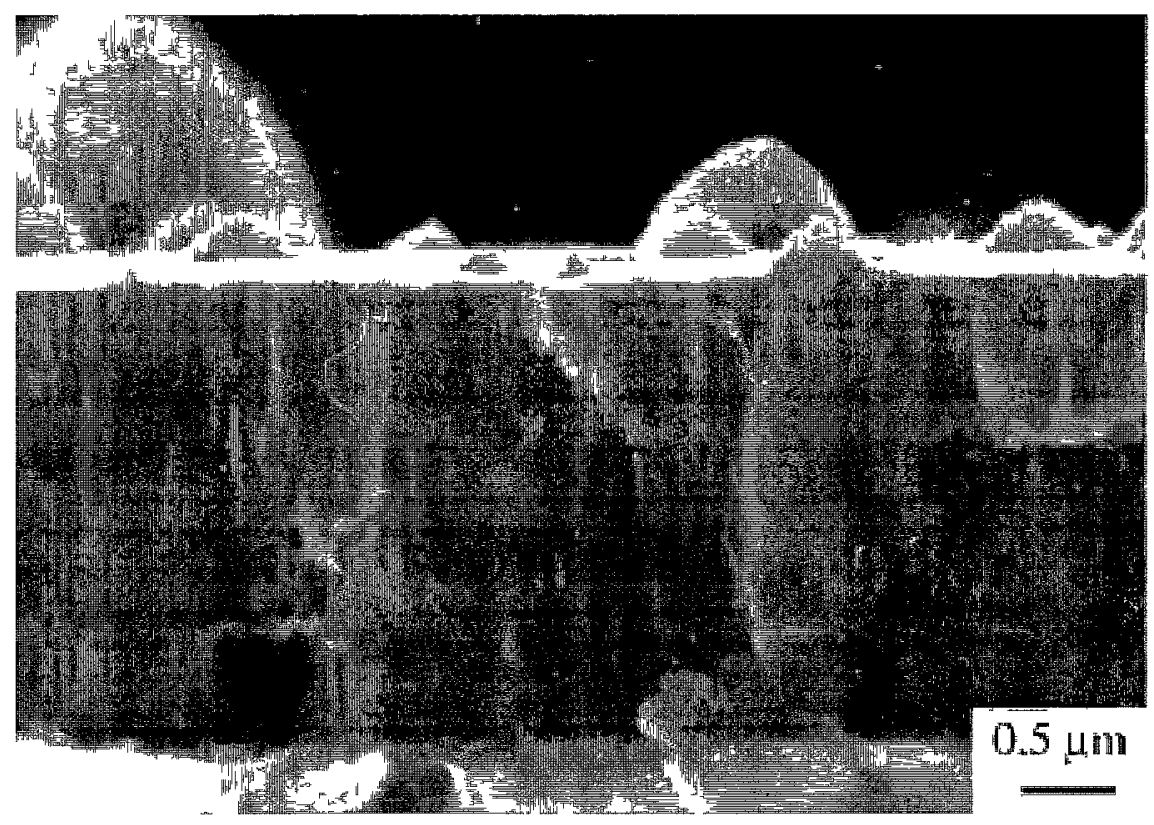

(a) $\operatorname{Ti}(\mathrm{C}, \mathrm{N})$ coating on HSS [322]

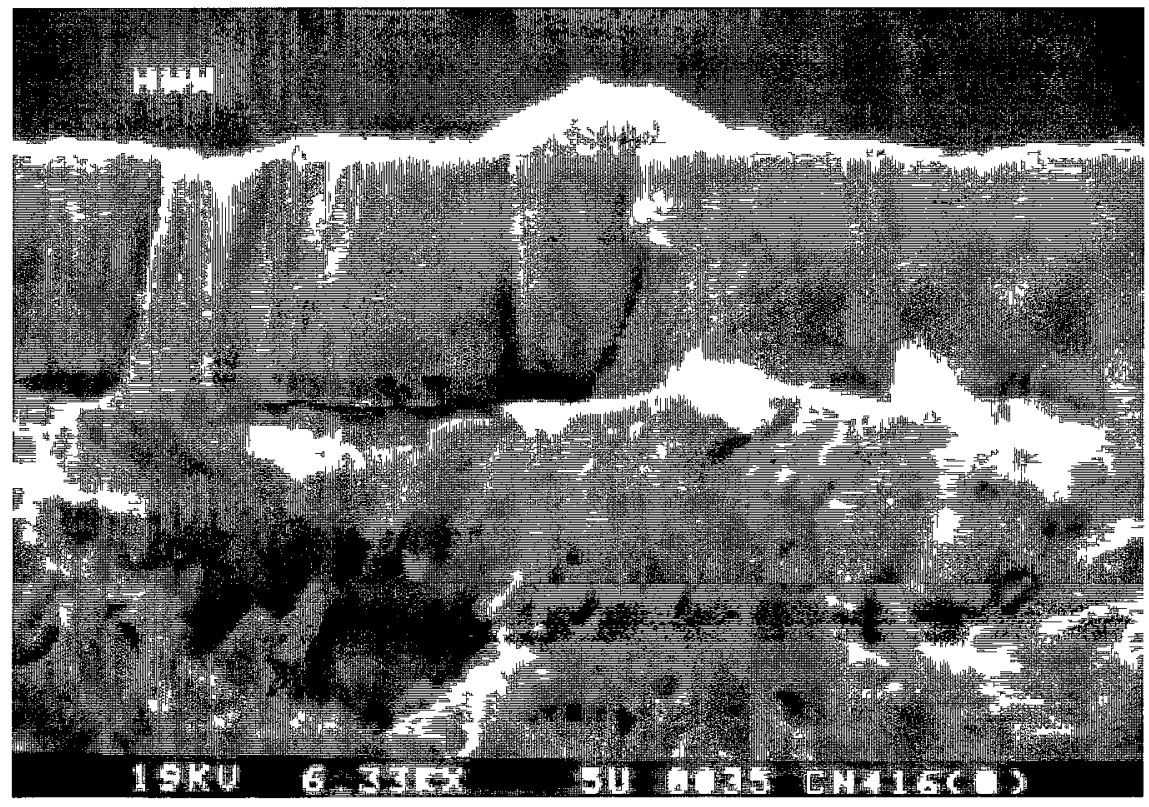

(b) $\mathrm{CrNr} / \mathrm{NbN}$ superlattice coating on MS [111]

Figure 2-29 SEM images of fracture surfaces.

In a coating/substrate system, the difference in thermal expansion between the coating and substrate generates stress. Incorporation of Ar into PVD sputtered films causes compressive stresses, whereas the incorporation of $\mathrm{N}$ interstitially in the lattice in 
tetrahedral positions results in lattice expansion in PVD films. Non-equilibrium growth conditions such as high biases, high rates or low temperatures could force nitrogen to occupy lattice positions, consequently increasing lattice strain. High density of grain boundaries and defects like dislocations generate internal stresses in coatings, which cause expansions in the lattice.

Plasma assisted physical vapor deposition techniques create low temperature deposition environments which reduces interdiffusions. A controlled low energy ion bombardment makes it possible to adjust the grain size and mechanical stress [323, 324], and to avoid columnar structures in the depositions [249].

\subsection{Coating Mechanical Properties}

Hardness and elasticity are two of the most important coating mechanical properties for many applications and are the ones considered in this work. However, the hardness for a coating is different from that of the bulk material of the same composition, which is due to the effect of the vapour deposition process in changing dislocation densities, grain size, strain hardening, and density $[98,111,249]$.

According to the Hall-Petch relationship, grain size reduction leads to increased strength (and hardness). However, with a grain size of about $10 \mathrm{~nm}$, hardness saturation occurs $[249,325]$, as demonstrated in Figure 2-30. For coatings with columnar microstructure and the presence of voids at the grain boundaries, a decrease in hardness results [216]. Among process parameters, bias voltage is a critical parameter governing the hardness of 
the coatings. As the bias voltage is increased to a sufficiently high value, ion bombardment on the coating during the growth results in a denser coating microstructure that can enhance the hardness [323, 324] (Figure 2-31 a, b) and erosion resistance (Figure 2-32) [326]. Growth temperature is another important factor being responsible for hardness. As shown in Figure 2-33 (a), for the ceramic coatings $\mathrm{TiN}, \mathrm{TiC}$, and $\mathrm{TiB}_{2}$, hardness increases with growth temperature; however, with higher temperatures, the hardness of the $\mathrm{TiC}$ and $\mathrm{TiB}_{2}$ coatings decrease. The hardness change with growth temperature could be associated with the changes in grain sizes or columnar grain boundary void densities at various growth temperatures [234]. As is shown in Figure 233 (b), the hardness and elastic modulus of TiN coatings increased as the substrate temperature increased from room temperature to $450^{\circ} \mathrm{C}$. With a further increase in substrate temperature, both values decreased.

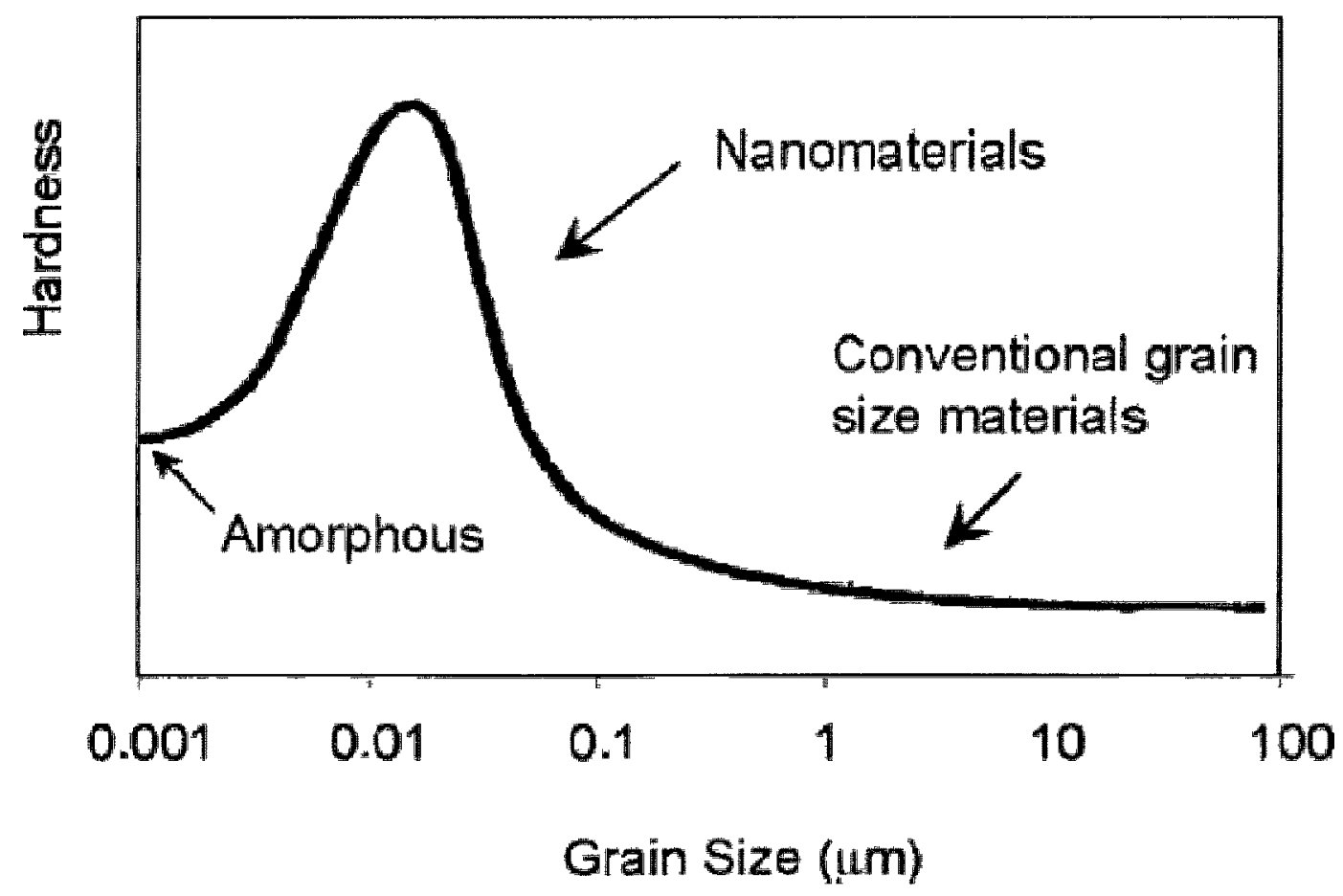

Figure 2-30 Hardness of a material as a function of the grain size [325]. 

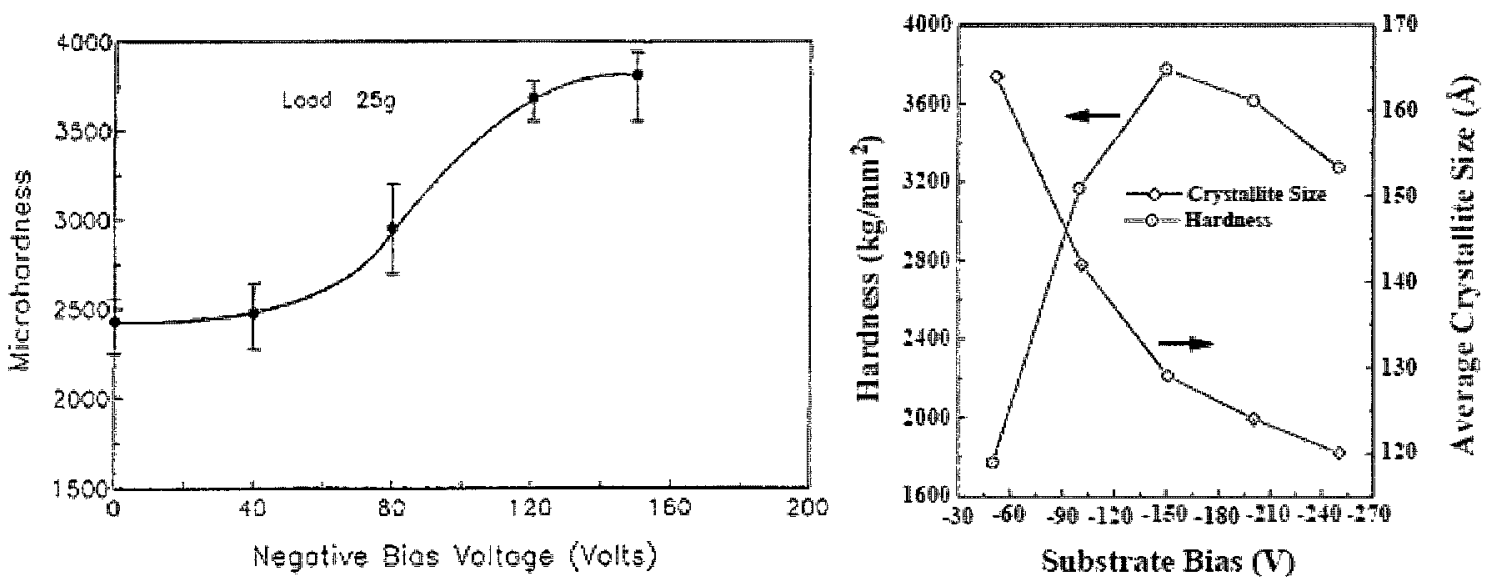

(a) $\mathrm{TiC}_{\mathrm{x}}$ coating [327]

(b) TiN/CrN multilayered coatings [328]

Figure 2-31 Coating hardness and average crystallite size vs. substrate bias votage.

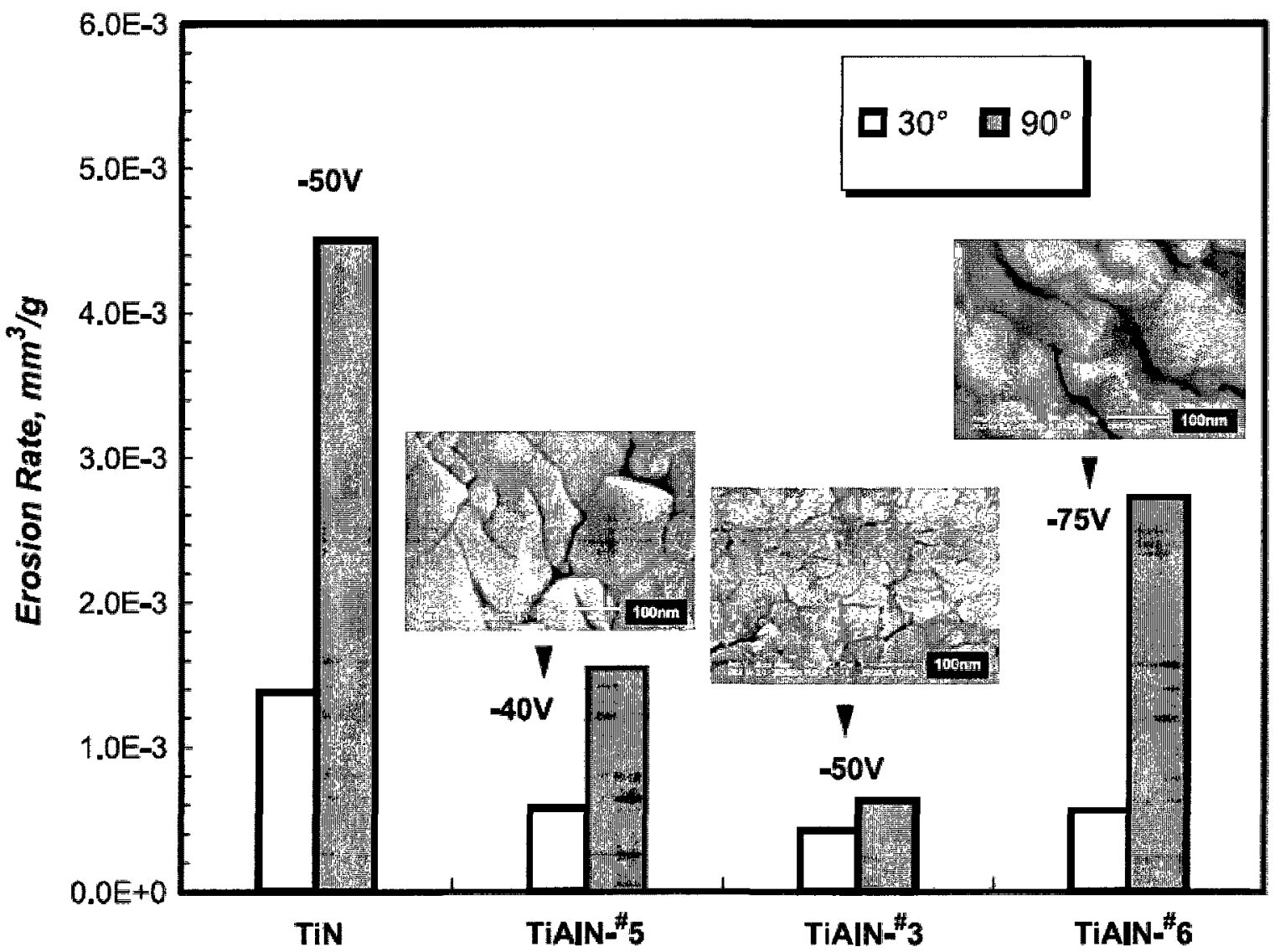

Figure 2-32 Effect of substrate bias on the microstructure (inset) and erosion behaviours for TiN and TiAlN coatings tested under $60 \mathrm{~m} / \mathrm{s}$ and $30^{\circ}$ and $90^{\circ}$ impingement angles [326]. 


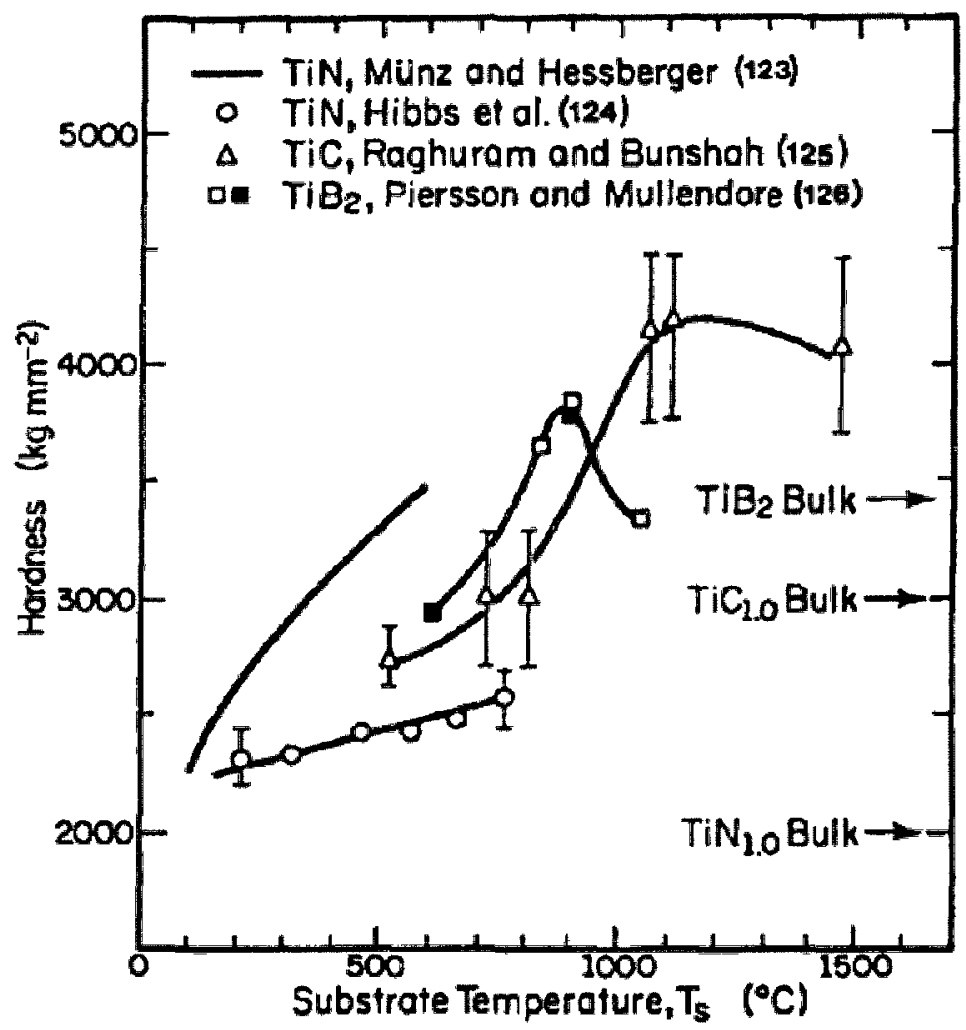

(a) Hardness as a function of growth temperature for some ceramic coatings [234]

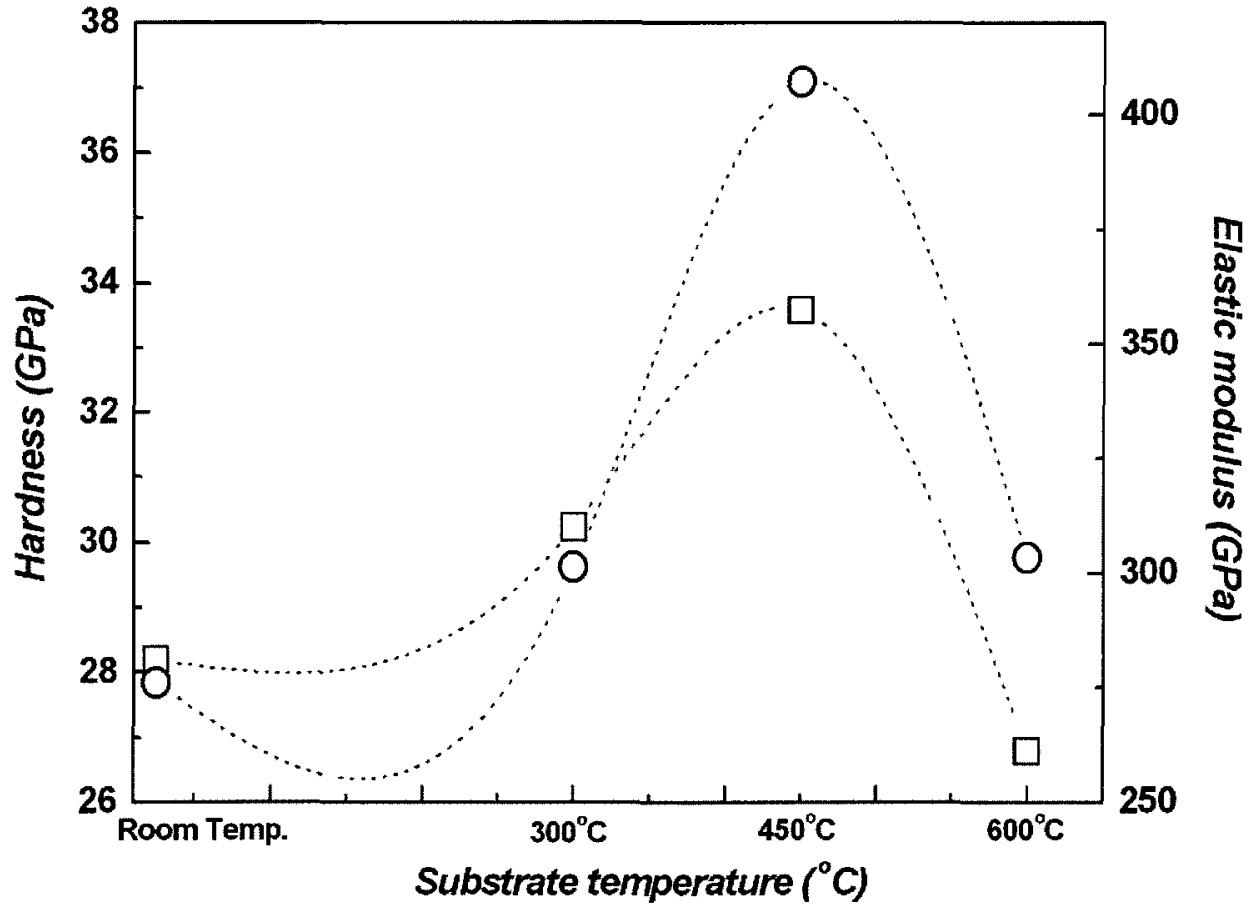

(b) Hardness ( $\square$ ) and modulus of elasticity (O) at a displacement $100 \mathrm{~nm}$ of TiN thin films as-grown at various substrate temperatures [329]

Figure 2-33 Coating mechanical properties of as function of substrate temperature. 
Coatings, in general, show very high elastic modulus and strength due to the effect of surface pinning of dislocations [98]. In addition to the influence of coating process parameters, the elastic properties of the coatings are also influenced by the coatingsubstrate interaction, and the stresses generated in the coating system [218]. The difference in thermal expansion between the coating and substrate generates stress. Incorporation of Ar into the deposited films causes compressive stresses, whereas the incorporation of $\mathrm{N}$ interstitially into the lattice results in lattice expansions in deposited films. High density of grain boundaries and defects like dislocations generate internal stresses in coatings.

Enhancements in both hardness and modulus of elasticity can be found in nanocrystalline/amorphous (nc/a) composites, where the nanocrystalline phase is smaller than $10 \mathrm{~nm}$, and the amorphous phase involving the nanocrystals is only a few atomic bond lengths [330], therefore sharp interfaces are provided between the nanocrystals and amorphous matrix. This is because the dislocations in the crystallites are prohibited from moving through the amorphous matrix. Randomly oriented nanocrystals embedded in a thin amorphous matrix yield a better coherence at the grain boundaries compared to pure polycrystalline. These properties make such materials more suitable for tribological coating applications than single or polycrystalline multilayered coatings [249].

Both hardness and modulus of elasticity of a coating are factors related to the wear behaviour of the coating [331]. These two factors together govern the contact pressure on a surface. The relationship can be described as [332]: 


$$
P_{Y}=0.78 r^{2}\left(H^{3} / E^{2}\right)
$$

where

$P_{y=}$ contact pressure,

$r=$ contacting sphere radius

According to Eq. (2-24), for a given contact pair, the ratio $\mathrm{H}^{3} / \mathrm{E}^{2}$ (plastic deformation resistance) is linearly proportional to the contact pressure. Therefore this combined parameter is an indicator that is used to evaluate the resistance of a coating to a plastic deformation [193] and in evaluating the wear behaviour of a coating.

\subsection{Wear Behaviours of Transition Metal Nitrides and Carbides}

Due to their advanced mechanical properties, transition metal nitride and carbide coatings have been applied in applications where wear is a factor. With the development of this group of coatings, theoretical and experimental studies on wear behaviours have been done. In an experimental study on a TiN coating, the wear rates (in the form of weight loss) for bare and TiN coated stainless steels under mild and severe Taber abrasive wear conditions were compared, as shown in Figure 2-34. Weight losses for TiN coatings were significantly lower than those for stainless steel substrates under both mild (Figure 2-34 a) and severe (Figure 2-34 b) wear conditions. The difference in weight loss between coated and uncoated samples increases with the number of cycles. Thicker coatings showed higher weight losses. This is due to microstructural effects and is attributed to lower densities and increased grain sizes with thicker coatings [333]. 


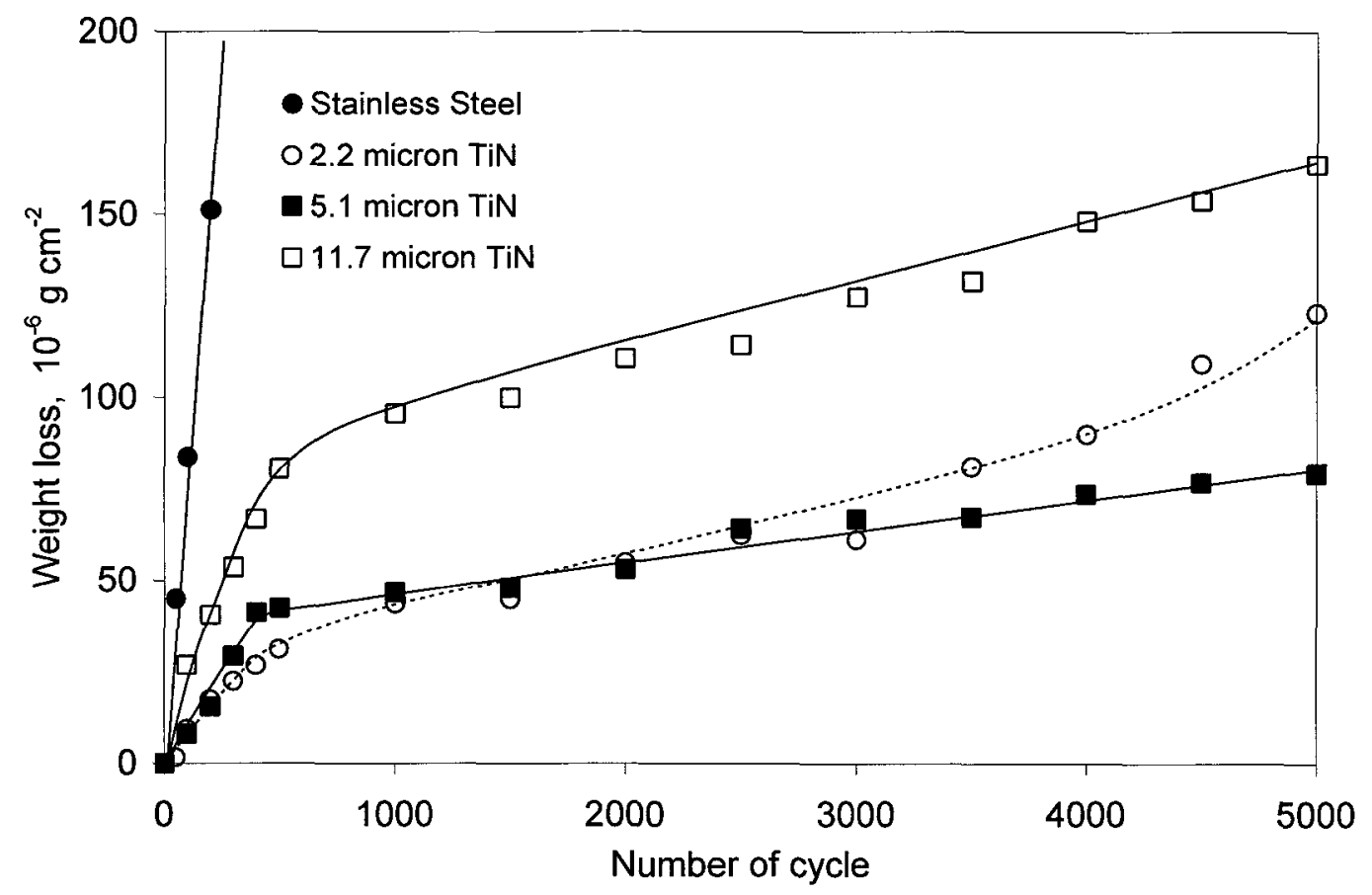

(a) Under mild abrasive wear conditions (CS-17 wheels under $1 \mathrm{~kg}$ load)

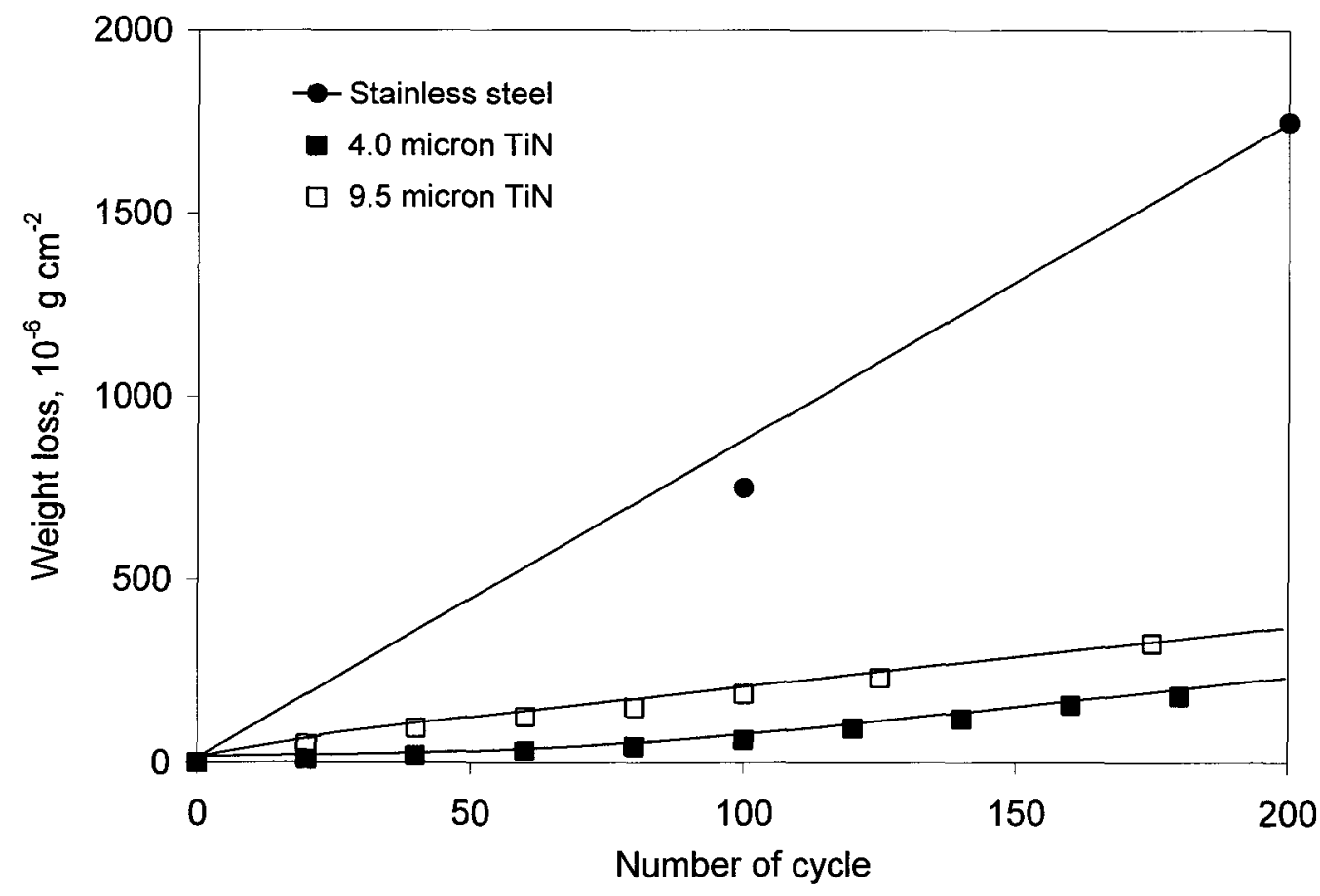

(b) Under severe abrasive wear conditions (H18 wheels under $1 \mathrm{~kg}$ load)

Figure 2-34 Weight losses with the number of cycles for uncoated and TiN coated stainless steel (Regenerated from [333]). 
Comparing nitrides and carbides, an overview of their resistance to flank wear and crater wear is shown in Figure 2-35 where flank wear is also referred as to as abrasive wear, and crater wear is caused by a chemical interaction between the rake face of a metal cutting insert and the hot metal chip. With regard to flank wear, most of the carbides showed a higher resistance than nitrides; this was attributed to the higher hardness of carbides (for example, TiC). With regard to crater wear, nitrides demonstrated higher resistances than carbides due to the high enthalpies of formation (e.g., TiN and HfN), which result in high resistances to chemical reactivity in tribological processes [203]. Additionally, higher melting points of $\mathrm{HfN}$ and $\mathrm{HfC}$ as compared to those for $\mathrm{TiC}$ and TiN are expected to be responsible for the improvement in the wear behaviours of HfN and HfC [203].

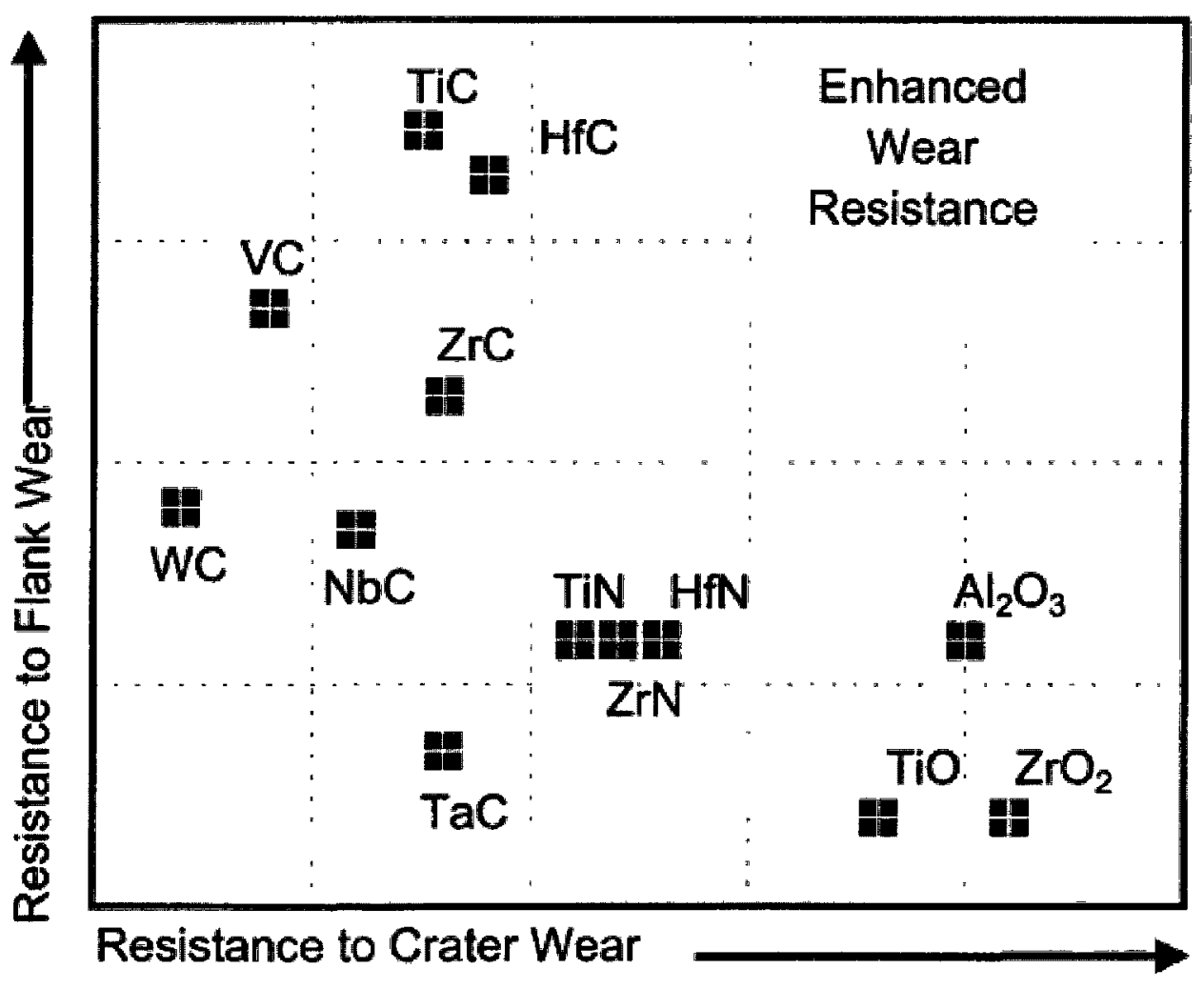

Figure 2-35 Wear behaviours of hard components [203]. 
The abrasive wear resistance of binary ( $\mathrm{TiN}$ and $\mathrm{TiB}_{2}$ ), ternary ( $\mathrm{TiBN}$ and $\mathrm{TiBC}$ ), and quaternary (TiBCN) coatings were also investigated (using a stylus-disc tribometer test). A comparison of the wear volume curves (Figure 2-36) indicates that the wear rate decreases with increased hardness of the coatings; it also reveals a trend that ternary and quaternary coatings demonstrate lower wear rates compared to binary coatings. This trend was also observed in another study on flank wear (in the form of cutting), as shown in Figure 2-37, ternary nitride coatings ( $\mathrm{TiCN}$ and TiAlN) demonstrate superior wear resistance to binary coatings (TiN). This is mainly attributed to the increased hardness of ternary and quaternary coatings, e.g., TiBC and (TiBC)N, compared to binary coatings. Additionally, the high resistance to thermal oxidation (e.g., TiAIN) is believed to be responsible for high resistance to wear.

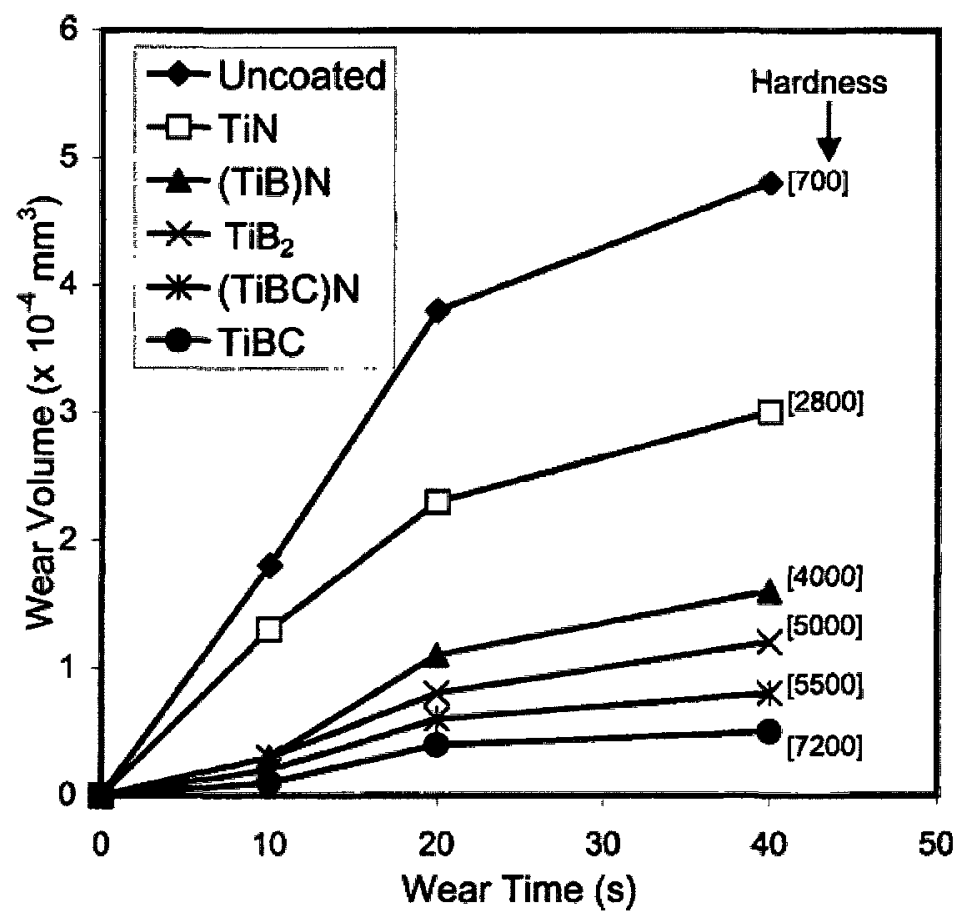

Figure 2-36 Abrasion wear volume as a function of wear time and microhardness $\left(\mathrm{HV}_{0.05}\right)$. Substrate: $\mathrm{S} 6-5-2$; counterpart: $100 \mathrm{Cr}$; interfacial medium: $\mathrm{Al}_{2} \mathrm{O}_{3}$; grit size: 1 $\mathrm{mm} ; \mathrm{F}=0.5 \mathrm{~N}$ [203]. 

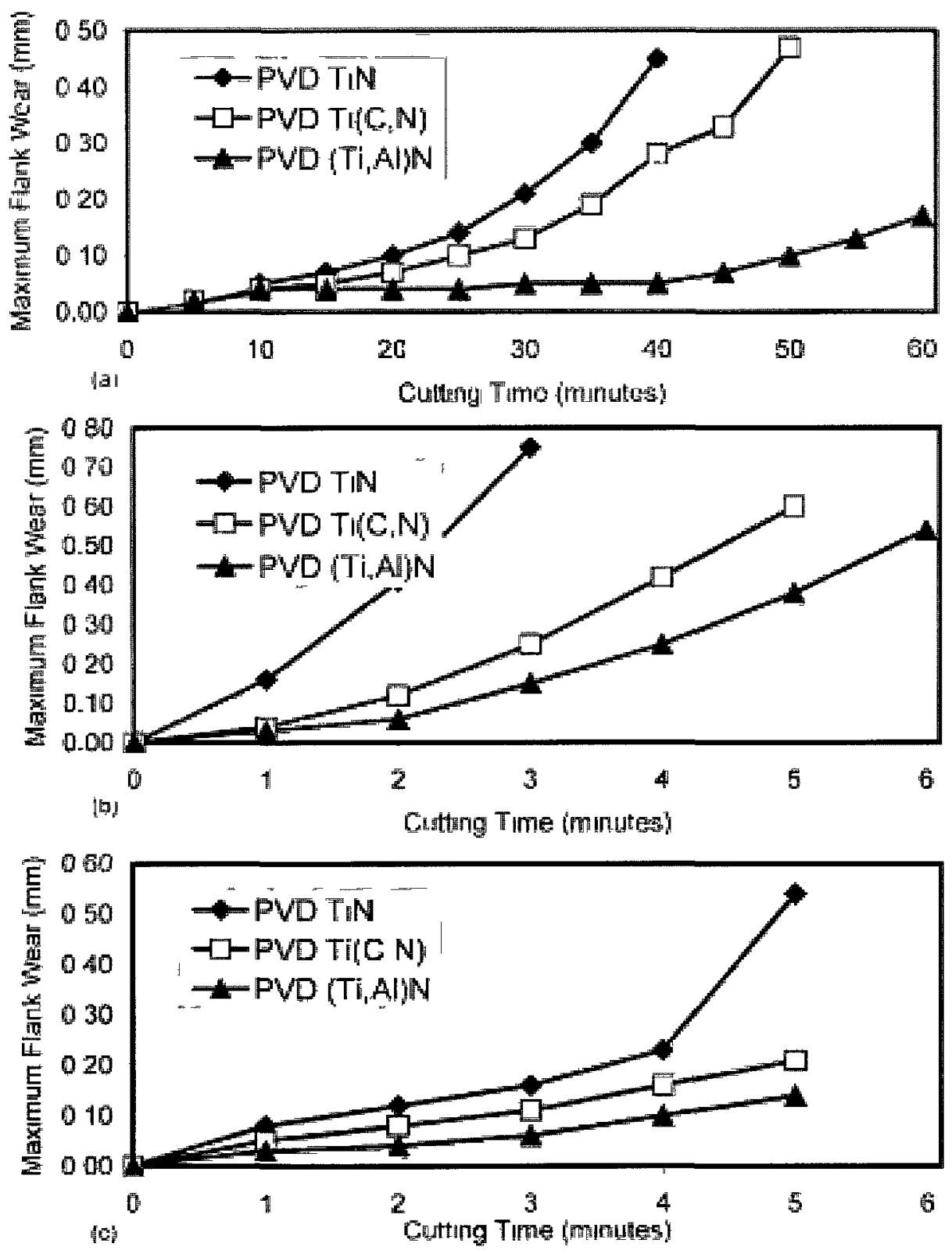

Figure 2-37 Maximum flank wear as a function of time for: (a) SAE 1045 steel (305 $\mathrm{m} / \mathrm{min})$; (b) ductile cast iron (244 $\mathrm{m} / \mathrm{min})$; and (c) Inconel $718(46 \mathrm{~m} / \mathrm{min})$ [203].

More results from experimental studies on nitride and carbide hard coatings in wear applications are summarized in Table 2-6, which shows significant improvements in wear resistance due to the application of nitrides and carbides. 
Table 2-6 Summary of improvement due to nitrides and carbides

\begin{tabular}{|c|c|c|c|c|c|}
\hline Tools substrate & $\begin{array}{c}\text { Coating } \\
\text { material/treatment }\end{array}$ & $\begin{array}{c}\text { Coating } \\
\text { thickness } \\
(\mu \mathrm{m})\end{array}$ & $\begin{array}{l}\text { Deposition } \\
\text { method }\end{array}$ & $\begin{array}{l}\text { Improvement } \\
\text { in wear life }\end{array}$ & Reference \\
\hline $\begin{array}{l}\text { HSS(M42) } \\
\text { cutting tool }\end{array}$ & $\mathrm{TiC}$ & $5-8$ & ARE & 3-8 times & [304] \\
\hline $\begin{array}{l}\text { Cemented carbide } \\
\text { cutting tool }\end{array}$ & TiN, TiC & 5 & IP, CVD & $\begin{array}{l}\text { IP comparable } \\
\text { to CVD }\end{array}$ & [304] \\
\hline HSS(M-10) drill & TiC, TiN & 2 & ARE & 20 time & [304] \\
\hline $\begin{array}{l}\text { Cemented carbide } \\
\text { cutting tool }\end{array}$ & TiN & 5 & MS & Several times & [304] \\
\hline $\begin{array}{l}\text { Cemented carbide } \\
\text { cutting tool }\end{array}$ & TiC, TiN & $8-10$ & CVD & Several times & [304] \\
\hline $\begin{array}{l}\text { Cemented carbide } \\
\text { cutting tool }\end{array}$ & $\begin{array}{l}\mathrm{HfN}, \mathrm{TiC} / \mathrm{Al}_{2} \mathrm{O}_{3}, \\
\mathrm{Al}_{2} \mathrm{O}_{3}, \mathrm{TiC} / \mathrm{TiN}\end{array}$ & - & CVD & $\begin{array}{l}\text { HfN most } \\
\text { superior }\end{array}$ & [304] \\
\hline HSS(M-10) drill & TiN & $1-2$ & MS & 50 times & [304] \\
\hline $\begin{array}{l}\text { Cemented carbide } \\
\text { cutting tool }\end{array}$ & $(\mathrm{Ti}, \mathrm{Al}) \mathrm{N}, \mathrm{TiN} / \mathrm{TiC}$ & - & CVD & $\begin{array}{l}3 \text { times better } \\
\text { than }(\mathrm{Ti}, \mathrm{Al}) \mathrm{N}\end{array}$ & [304] \\
\hline HSS(M-10) drill & $(\mathrm{Ti}, \mathrm{Al}) \mathrm{N}, \mathrm{TiN}$ & - & IP & $\begin{array}{l}3 \text { times better } \\
\text { than }(\mathrm{Ti}, \mathrm{Al}) \mathrm{N}\end{array}$ & {$[304]$} \\
\hline $\begin{array}{l}\text { Cemented carbide } \\
\text { cutting tool }\end{array}$ & $\mathrm{TiN}, \mathrm{HfN}, \mathrm{ZrN}$ & $8-18$ & CVD, IP & $\begin{array}{l}\text { High hardness } \\
\text { of IP, MS, ARE }\end{array}$ & [304] \\
\hline Coated steel & $\mathrm{Cr}_{7} \mathrm{C}_{3}$ & 10 & CVD & 20 times & [334] \\
\hline $\begin{array}{l}\text { cemented carbide } \\
\text { ball bearing }\end{array}$ & $\mathrm{TiC}$ & 2.5 & CVD & 5 times & [334] \\
\hline $\begin{array}{l}4118 \text { carburized } \\
\text { steel roller } \\
\text { bearing }\end{array}$ & TiN & 0.25 & PVD & 6 times & [334] \\
\hline $\begin{array}{l}4118 \text { carburized } \\
\text { steel roller } \\
\text { bearing }\end{array}$ & TiN & 1.0 & PVD & 4 time & [334] \\
\hline
\end{tabular}

Further studies on ternary and quaternary nitride coatings looked at important factors governing wear behaviours such as coefficient of friction and hardness. The microhardness of the $\mathrm{CrN}$ based coatings such as $\mathrm{CrN}, \mathrm{Cr}-\mathrm{Si}-\mathrm{N} \mathrm{Cr}-\mathrm{Al}-\mathrm{N}$ and $\mathrm{Cr}-\mathrm{Al}-\mathrm{Si}-$ $\mathrm{N}$ as a function of Si content, and average friction coefficients of these coatings against a steel ball are shown in Figure 2-38 (a) and (b) respectively. It can be seen that the harnesses of $\mathrm{Cr}-\mathrm{Si}-\mathrm{N}$ and $\mathrm{Cr}-\mathrm{Al}-\mathrm{Si}-\mathrm{N}$ increased with $\mathrm{Si}$, and reached a maximum at a $\mathrm{Si}$ content of around of 9 at.\%; meanwhile, the average coefficient of friction for the $\mathrm{CrN}$ based coatings decreased with the content of $\mathrm{Si}$. 


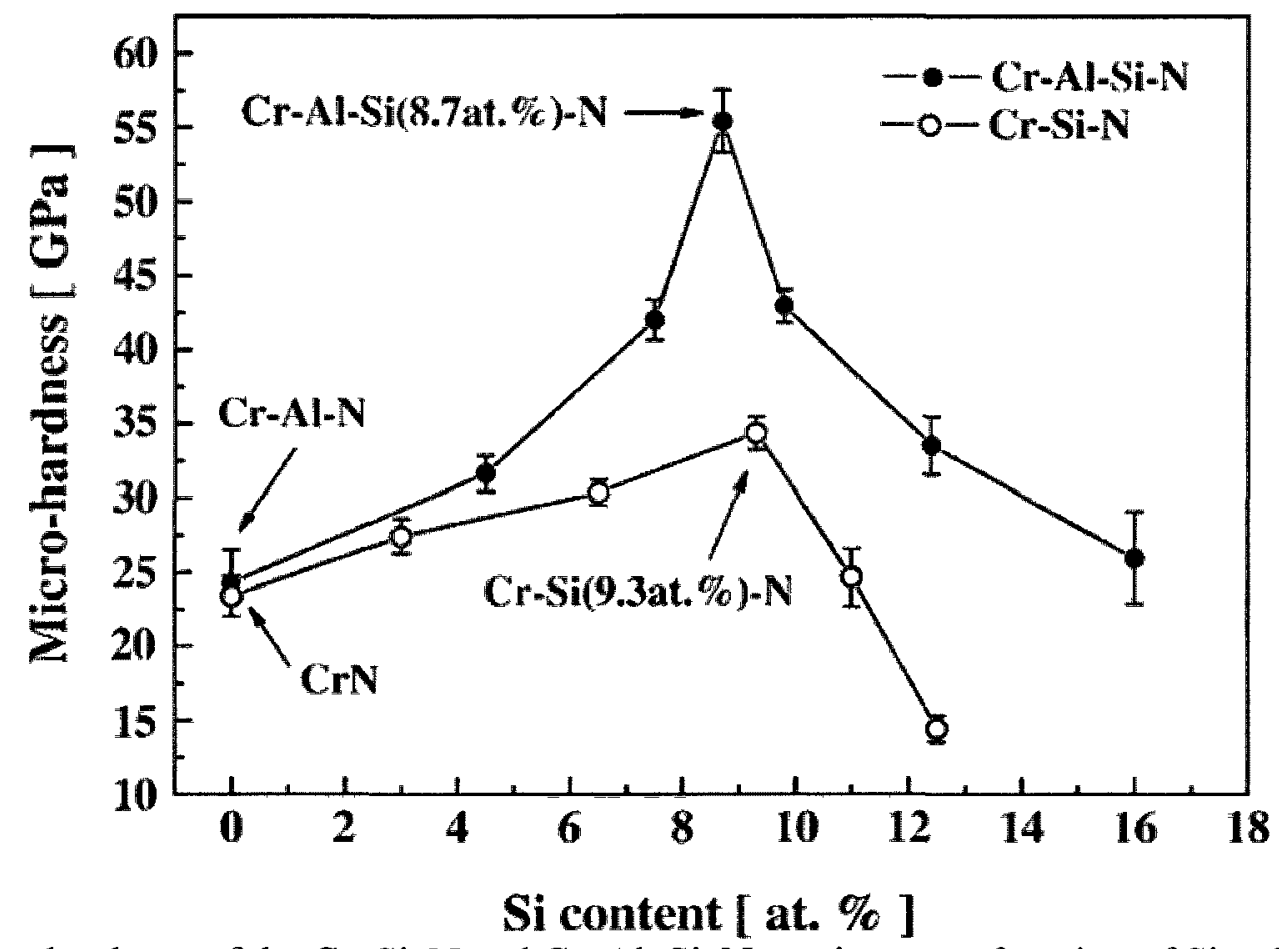

(a) Microhardness of the $\mathrm{Cr}-\mathrm{Si}-\mathrm{N}$ and $\mathrm{Cr}-\mathrm{Al}-\mathrm{Si}-\mathrm{N}$ coatings as a function of $\mathrm{Si}$ at.\%

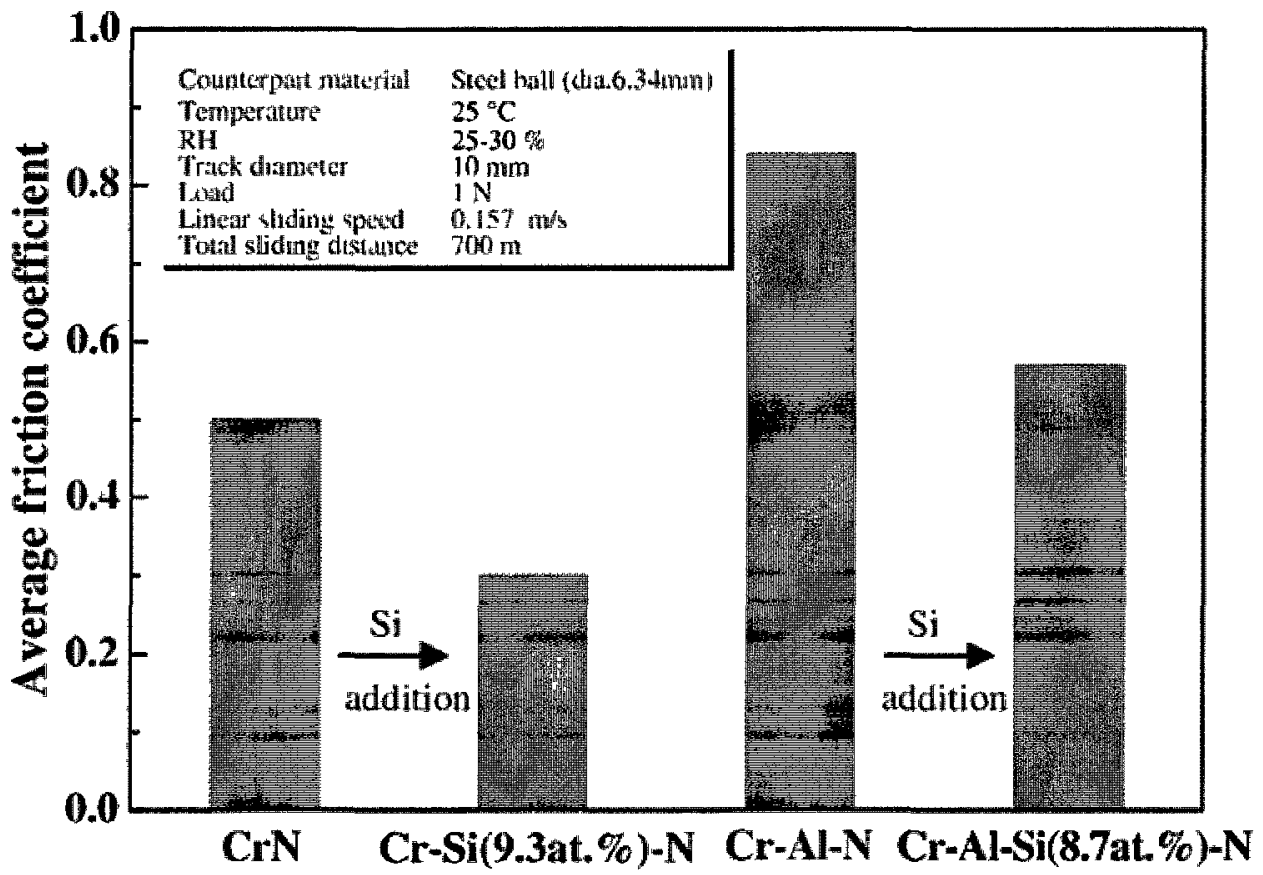

(b) Average friction coefficients of $\mathrm{CrN}, \mathrm{Cr}-\mathrm{Si}(9.3$ at.\%) $-\mathrm{N}, \mathrm{Cr}-\mathrm{Al}-\mathrm{N}$, and $\mathrm{Cr}-\mathrm{Al}-\mathrm{Si}$ (8.7 at. \%)-N coatings against a steel ball

Figure 2-38 Microhardness and friction coefficient of coating vs. Si contents [209]. 
In the study of $\mathrm{CrN}$ and $\mathrm{CrN}-\mathrm{Wx}$ series of coatings, the friction coefficients and wear depths for $\mathrm{CrN}$ and various $\mathrm{CrN}-\mathrm{Wx}$ coatings (against a 1045 steel cylinder) are summarized in Figure 2-39. The coefficient of friction and wear depth concurrently decrease with the content of $\mathrm{W}$, reaching a minimum value at a tungsten (W) content of 6 at. \%. This suggests a correlation between wear resistance, coefficient of friction and the addition of $\mathrm{W}$ in $\mathrm{CrN}$ coatings. It was found that the microstructure changed due to the addition of W. The coating microstructure was the most dense at the W content of 6 at.\%; and where the hardness reached the highest value [335].

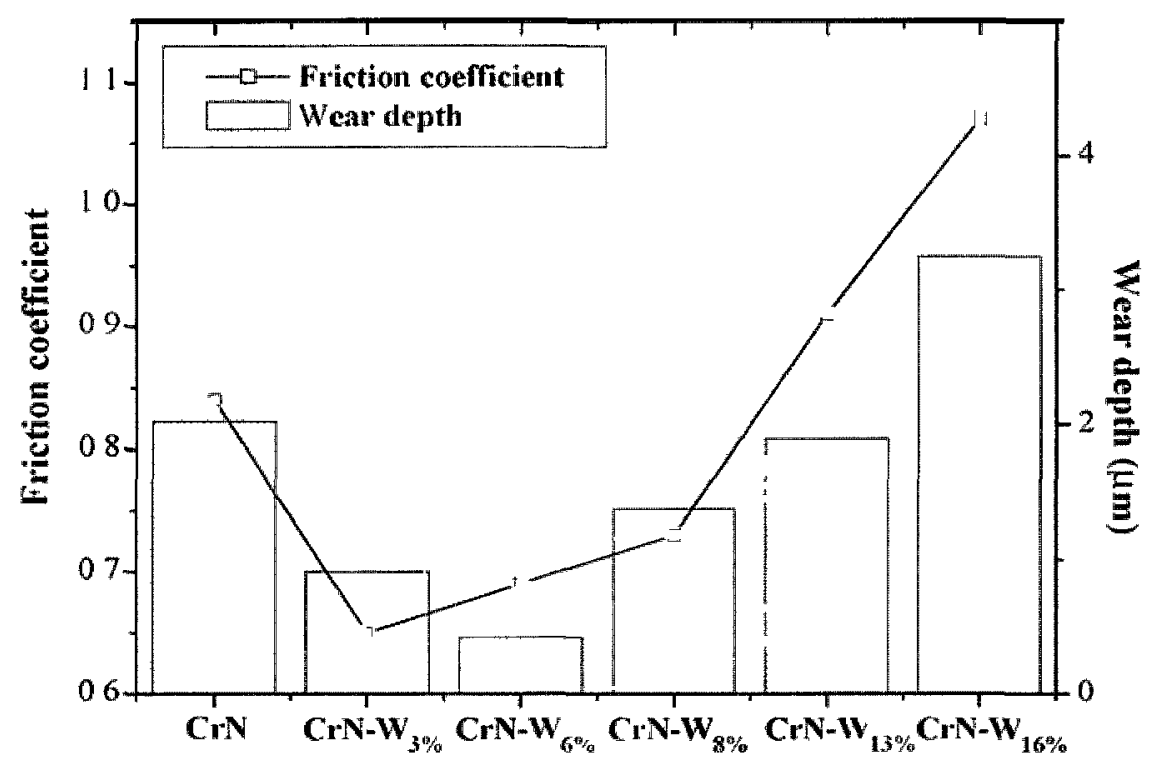

Figure 2-39 Coefficient of friction and wear depths for the $\mathrm{CrN}$ and $\mathrm{CrN}-\mathrm{Wx}$ coatings under identical testing conditions $(100 \mathrm{~N}$ normal load, $2 \mathrm{~mm}$ stroke, 6 min test duration, $50 \mathrm{~Hz}$ frequency, sliding against 1045 steel) [335].

In the study on TiMoN coatings, the average coefficient of friction and wear rate also change as a function of Mo content as shown in Figure 2-40 (a) and (b). Both properties decreased with the content of Mo; and reached the minimum values at the Mo content of 30 at. $\%$ and 50 at. $\%$, respectively. This is due to the tribo-oxidatively formed 
molybdenum trioxide $\left(\mathrm{MoO}_{3}\right)$ layer that with a low shear strength acted as a lubricant, resulting in reductions in coefficient of friction, and consequently lower wear rates [336].

Microhardness and coefficient of friction of various $\mathrm{CrSiCN}$ coating compositions vs. $\mathrm{Si}$ content are shown in Figure 2-41. Micro hardness of the coatings was observed to increase with Si content, and reached the peak value of $\sim 43 \mathrm{GPa}$ at a $\mathrm{Si}$ content of 9.2 at.\% (Figure 2-41 a). This is due to the evolution of the microstructure of $\operatorname{Cr}(\mathrm{CN})$ coatings with $\mathrm{Si}$ addition, which resulted in the formation of fine crystallite and nanocomposite structure, and enhanced hardness. Cohesive energy at interphase boundaries along with the percolation of the amorphous phase played a role in the hardness enhancement. Si dissolution into $\mathrm{Cr}(\mathrm{C}, \mathrm{N})$ crystal also resulted in solid solution hardening. Comparing the average coefficients of friction (Figure 2-41 b), it was found that the coefficient of friction of $\mathrm{CrN}$ coating decreased with Si content. This was due to the formation of an amorphous silicon nitride phase that acted as a solid lubricant [206].

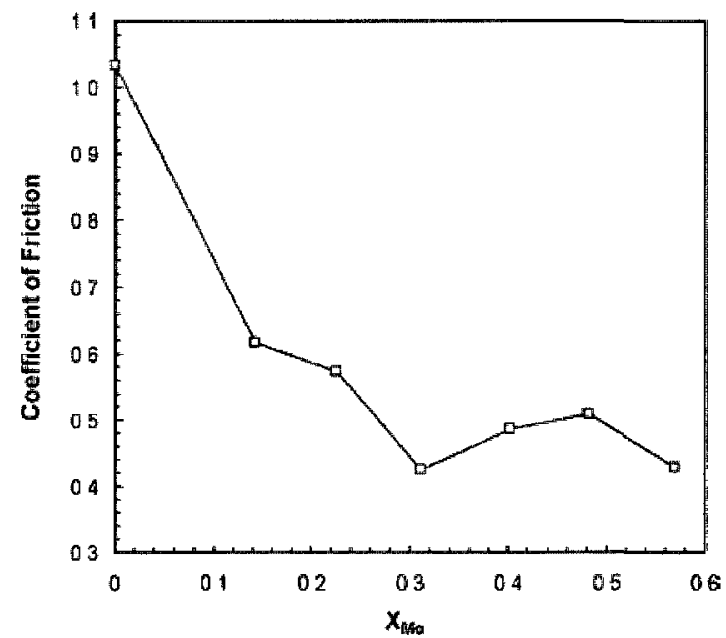

(a) Friction coefficients

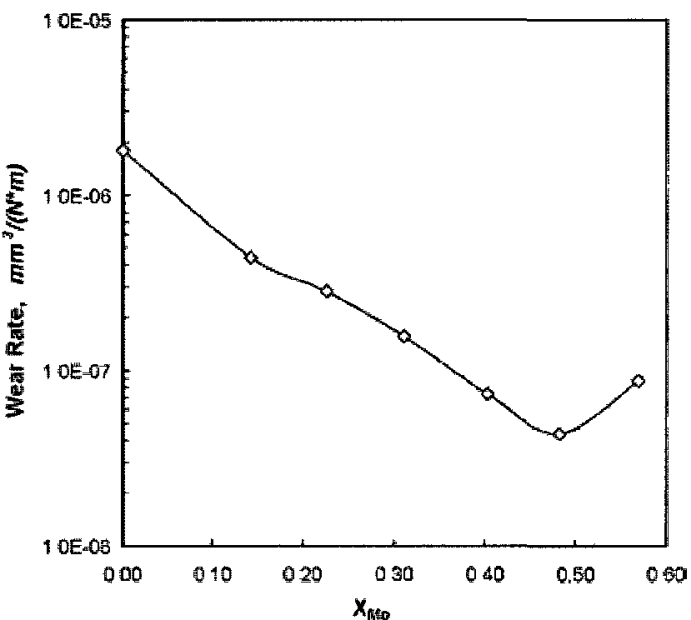

(b) Wear rates

Figure 2-40 Effect of Mo on tribological behaviours of TiMoN coatings (under pin-ondisc dry sliding with a WC-Co ball as the counterpart) [336]. 


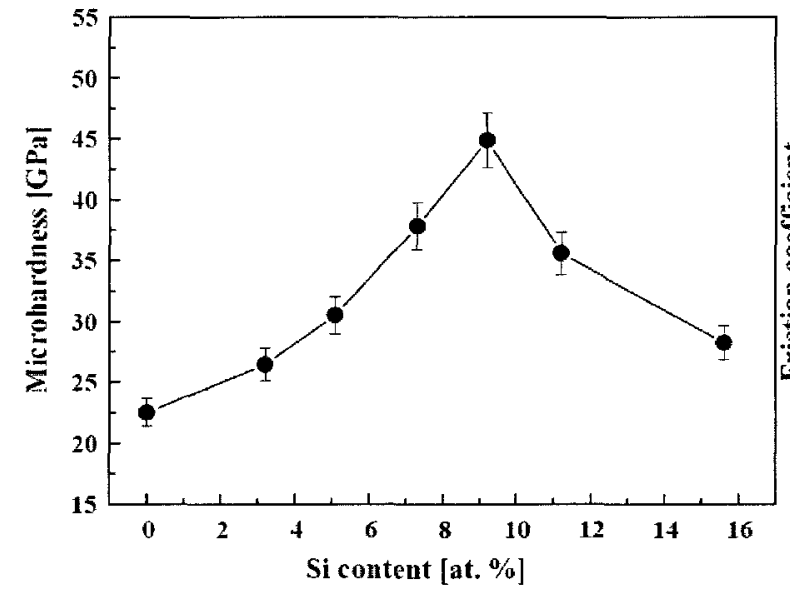

(a) Microhardness of $\mathrm{CrSiCN}$ coatings

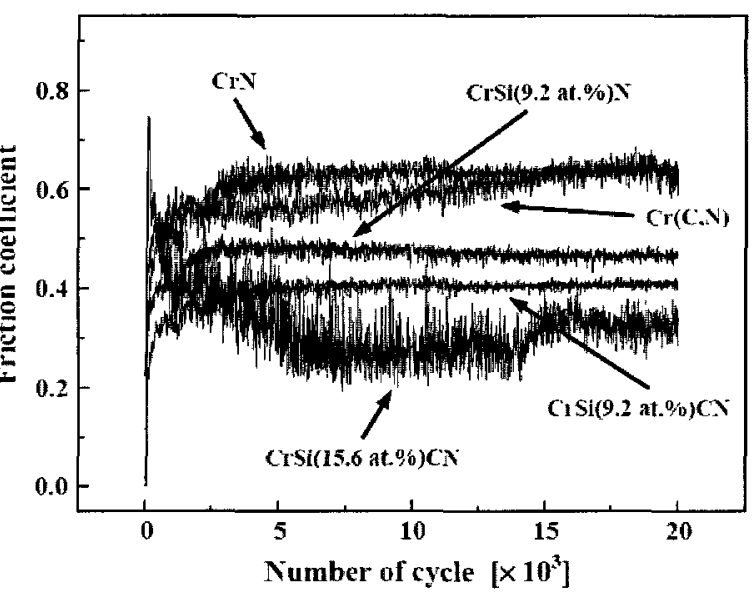

(b) Friction coefficients $\mathrm{CrN}$ coatings

Figure 2-41 Influence of Si content on coating tribological behaviours [206].

\subsection{Erosion Behaviours of Transition Metal Nitrides and Carbides}

As one of the most important anti-erosion coatings, TiN have been extensively studied.

The typical erosion behaviours of TiN coatings at various impingement angles are shown in Figure 2-42. TiN coatings, because they are harder, showed lower erosion rates compared to $17-4 \mathrm{PH}$ steel and electroless nickel coatings at all impingement angles, indicating their superior erosion performance. Unlike 17-4PH steel and electroless nickel coatings, erosion rates for the TiN coatings increased with impingement angle, and reached a maximum at high impingement angles $\left(60^{\circ}\right.$ to $\left.90^{\circ}\right)$, which is typical of brittle erosion. 


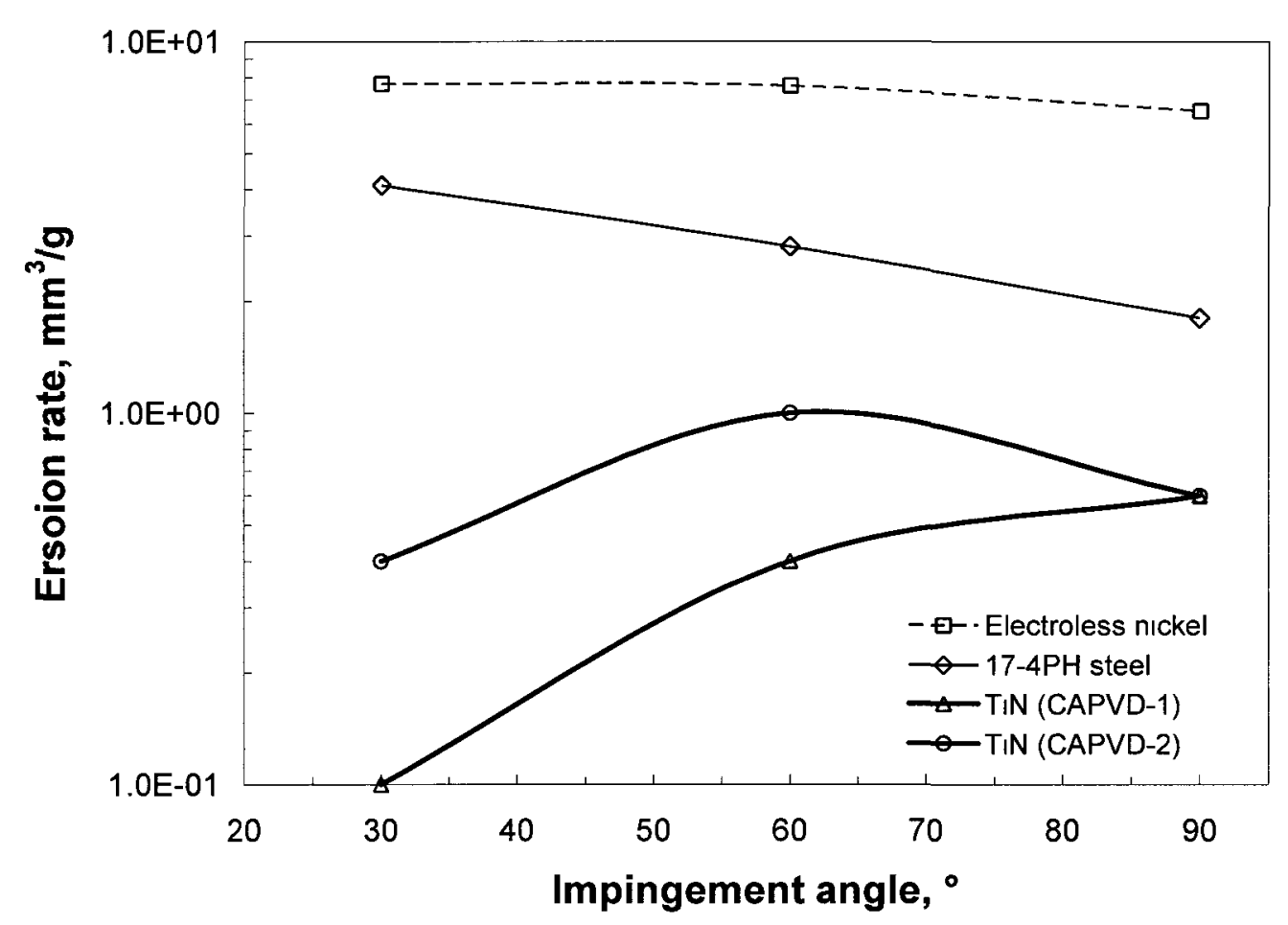

Figure 2-42 Erosion behaviours for 17-4PH steel, electroless nickel coating, and TiN (VAPVD-1) coating (plotted using data in [337])

Erosion resistances (in form of the time required to erode a unit length, i.e., s/mil, where mil $=25.4 \mu \mathrm{m}$ ) of tungsten carbide (WC) and titanium nitride (TiN) coatings and uncoated Ti-6Al-4V alloy at various impingement angles and particle velocities (at an impingement angle of $90^{\circ}$ ) are summarized in Figure 2-43. WC and TiN coatings showed similar erosion characteristics and their erosion resistances decreased with impingement angle, demonstrating brittle features, distinct from the titanium alloy Ti$6 \mathrm{Al}-4 \mathrm{~V}$. WC coating remained relatively erosion resistant at all impingement angles, which could be related to its fine grained microstructure compared to the TiN counterpart [338]. Both coatings show significant advantages (one order of magnitude) in erosion resistance over the Ti-6Al-4V alloy at a low impingement angle $\left(22.5^{\circ}\right)$. It can be observed that erosion resistance for both coatings at an impingement angle of $90^{\circ}$ 
decreased with increasing particle velocity. This was likely due to their brittle characteristics that weaken the resistance to erosion under high impact.

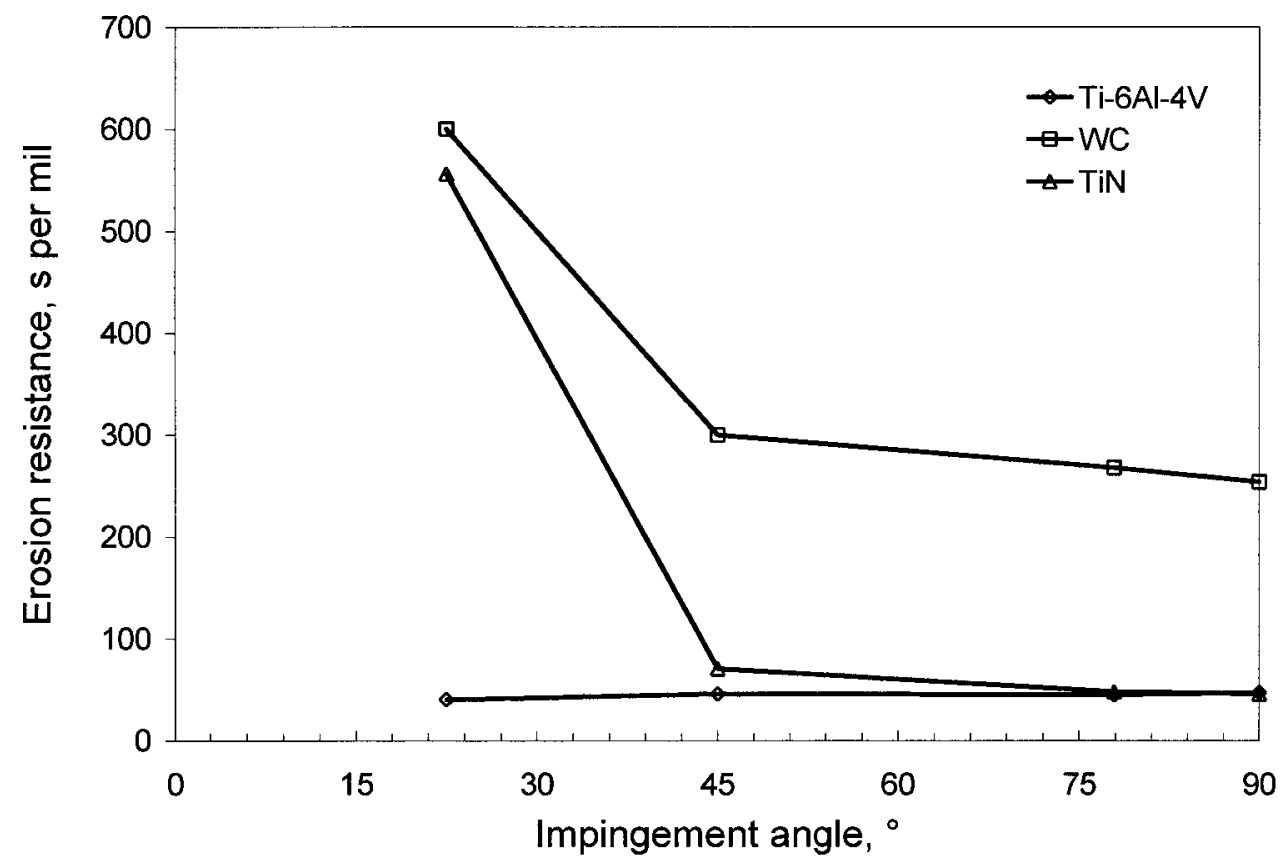

(a) At various impingement angles (at particle velocity of $93 \mathrm{~m} / \mathrm{s}$ )

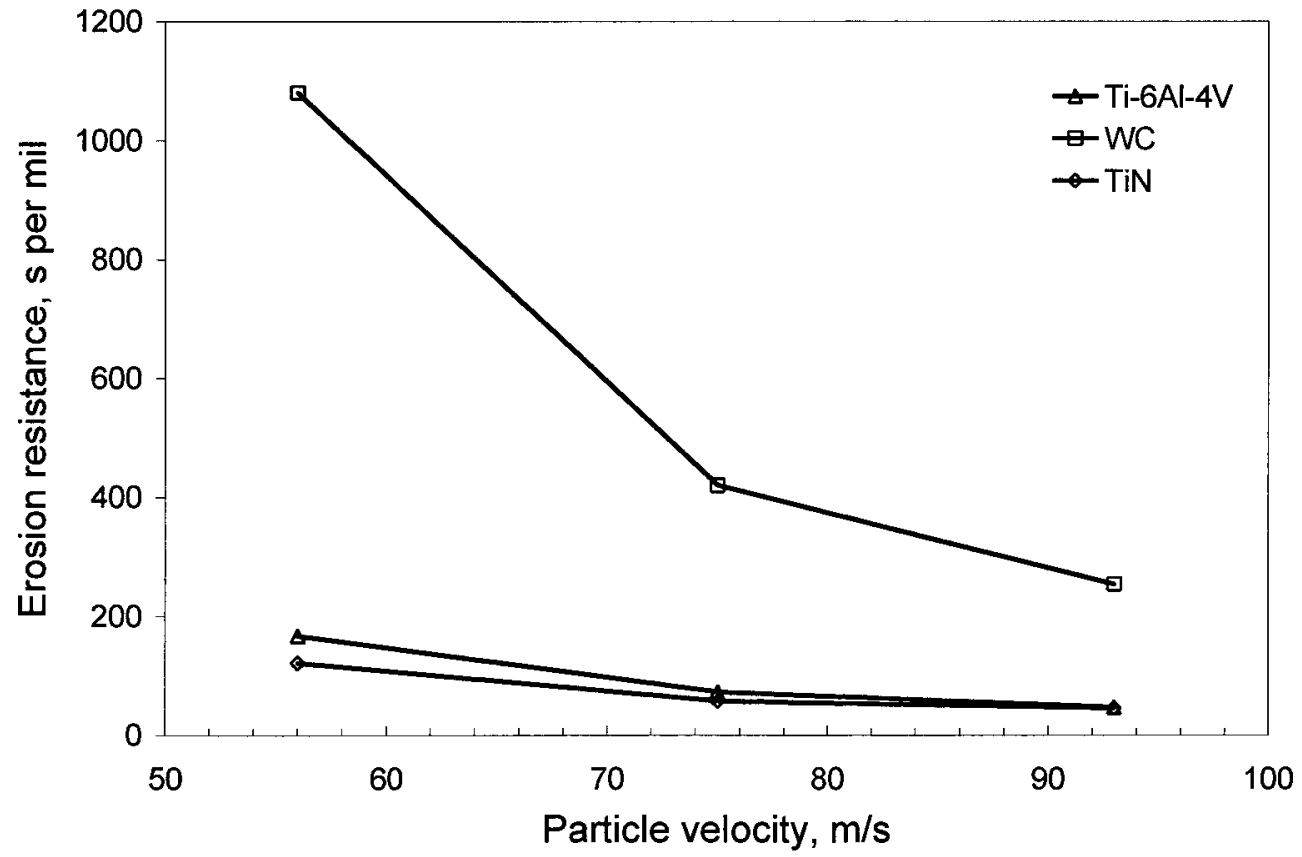

(b) At various particle velocities (at impingement angle of $90^{\circ}$ )

Figure 2-43 Erosion resistance of coatings WC and TiN and uncoated Ti-6Al-4V steel (plotted using data in [338]. 
At high temperatures, the erosion resistance of TiC, shown in Figure 2-44, demonstrated an improved erosion resistance over INCO 718 steel at low impingement angle. However, $\mathrm{TiC}$ reached a higher erosion rate at a high impingement angle $\left(90^{\circ}\right)$, indicating the brittle nature of the $\mathrm{TiC}$ coating even at elevated temperature.

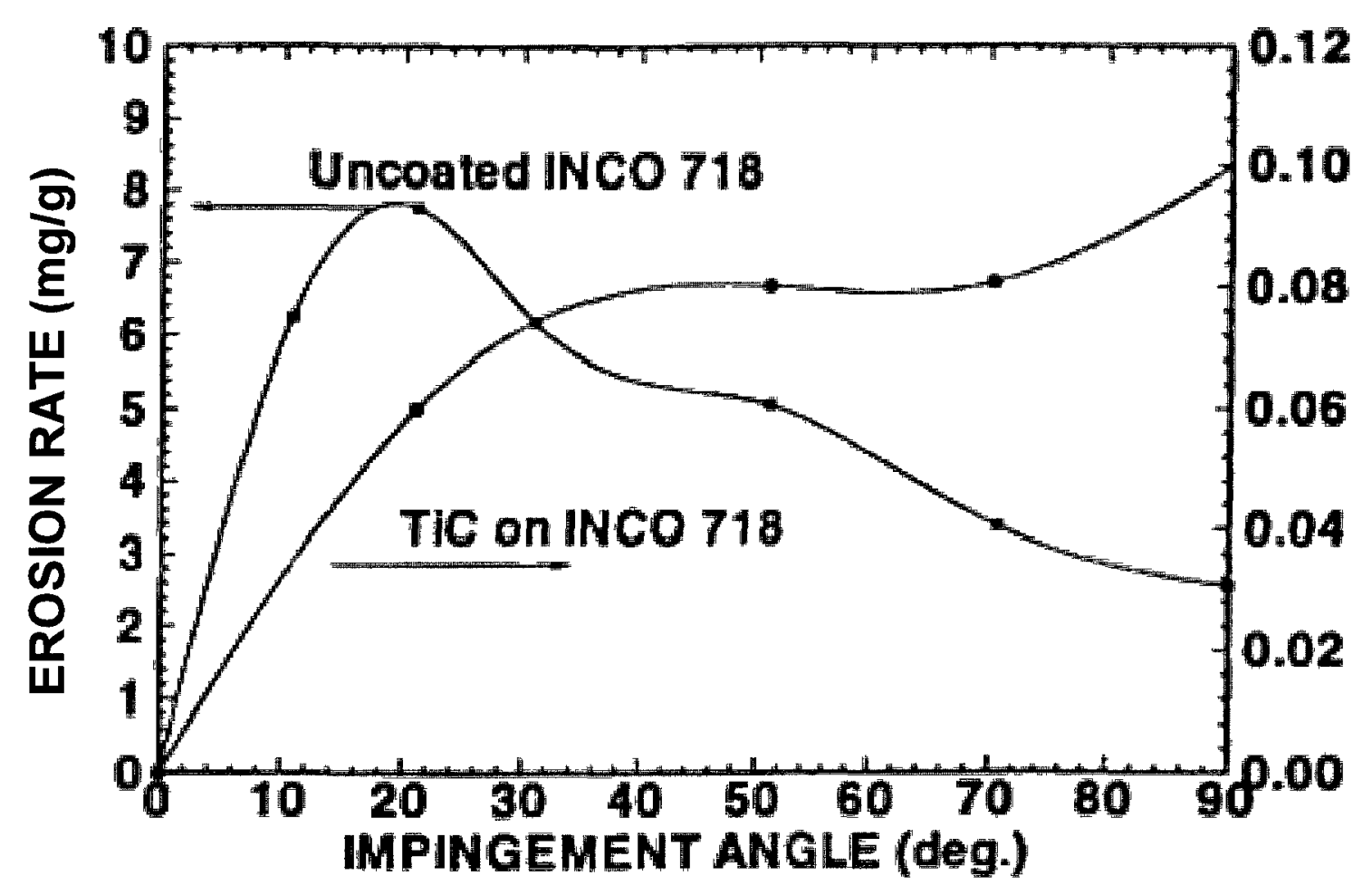

Figure 2-44 Erosion rate variation of uncoated and coated INCO 718 with impingement angle $\left(\mathrm{T}=538^{\circ} \mathrm{C}, \mathrm{V}=305 \mathrm{~m} / \mathrm{s}\right.$ of grit velocity, $20 \mathrm{~g}$ of grit) (modified from [339]).

Other coating compositions such as titanium nitrides, carbides and alumina demonstrated stable erosion resistances at elevated temperatures. As shown in Figure 2-45, the erosion rates for $\mathrm{TiN}$ and $\mathrm{TiC}$ coatings at a $90^{\circ}$ impingement angle (maximum erosion rates) are an order of magnitude lower than the INCO 718 steel. 


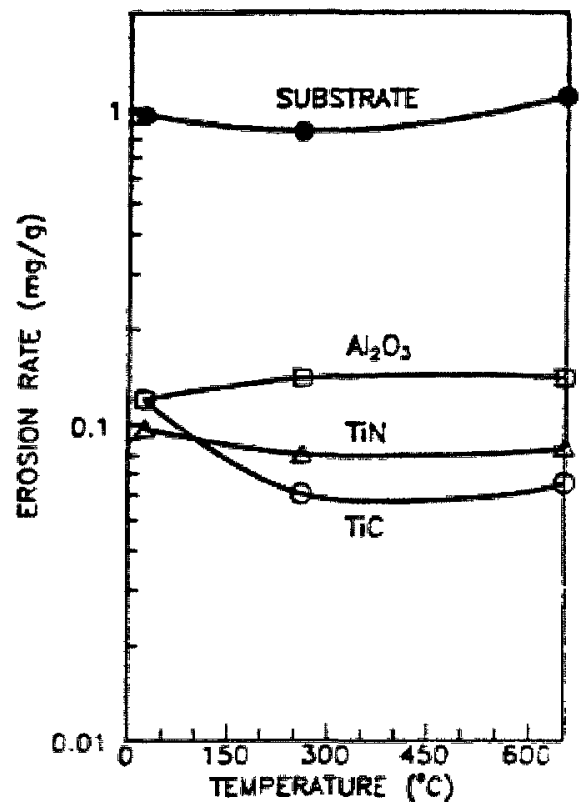

Figure 2-45 Erosion rates of coatings with temperature (at $90^{\circ}$ impingement angle, and $140 \mathrm{~m} / \mathrm{s}$ grit velocity) [339].

In a study of ternary coatings TiAlN, the erosion rates, displayed in Figure 2-46, were found to be in the order of TiAlN-\#4 $>$ TiAlN-\#1 $>$ TiAlN-\#2 $>$ TiAlN-\#3), where from TiAlN-\#1 to \#4, Al concentrations increased from 10 to 35 at.\%, which indicates that an optimal Al concentration for erosion resistance exists.

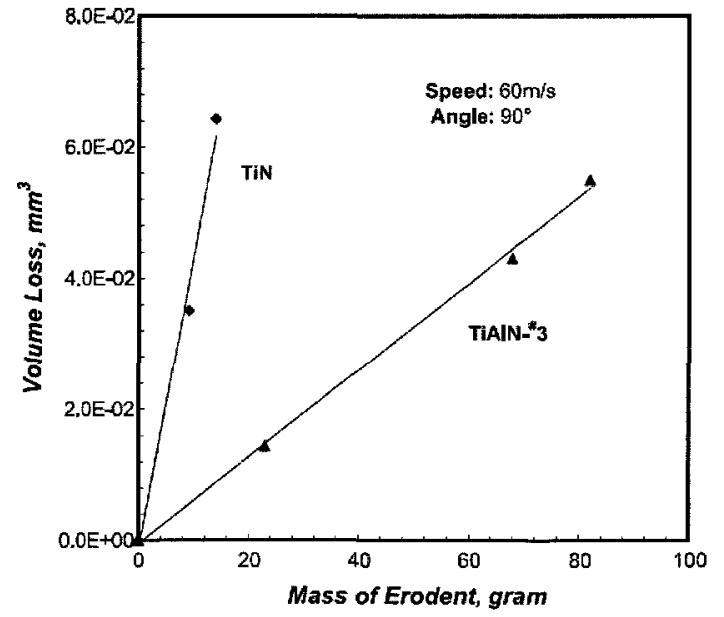

(a) Erosion volume loss vs. mass of erodent

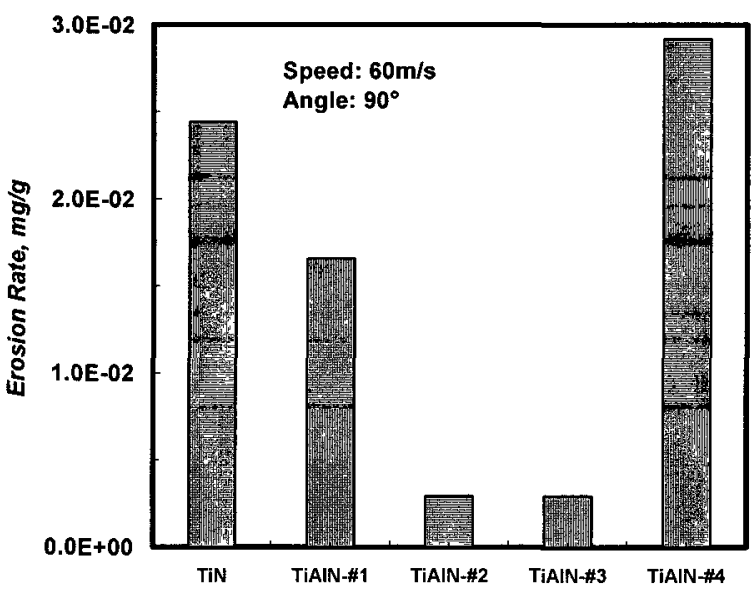

(b) Comparison with tested coatings

Figure 2-46 Erosion rates of TiN and TiAlN coating systems [326](Regenerated (b)). 
The effects of impingement angle and particle velocity on erosion rates of TiN and TiAlN-\#3 (with lowest erosion rate among the TiAlN coatings) coatings are further compared in Figure 2-47. The erosion rates for both coatings increase with impingement angle, indicating brittle erosion characteristics. Coating TiAlN-\#3 showed a consistently lower erosion rate than $\mathrm{TiN}$ at all impingement angles and even at a higher particle velocity than that applied to the TiN coating.

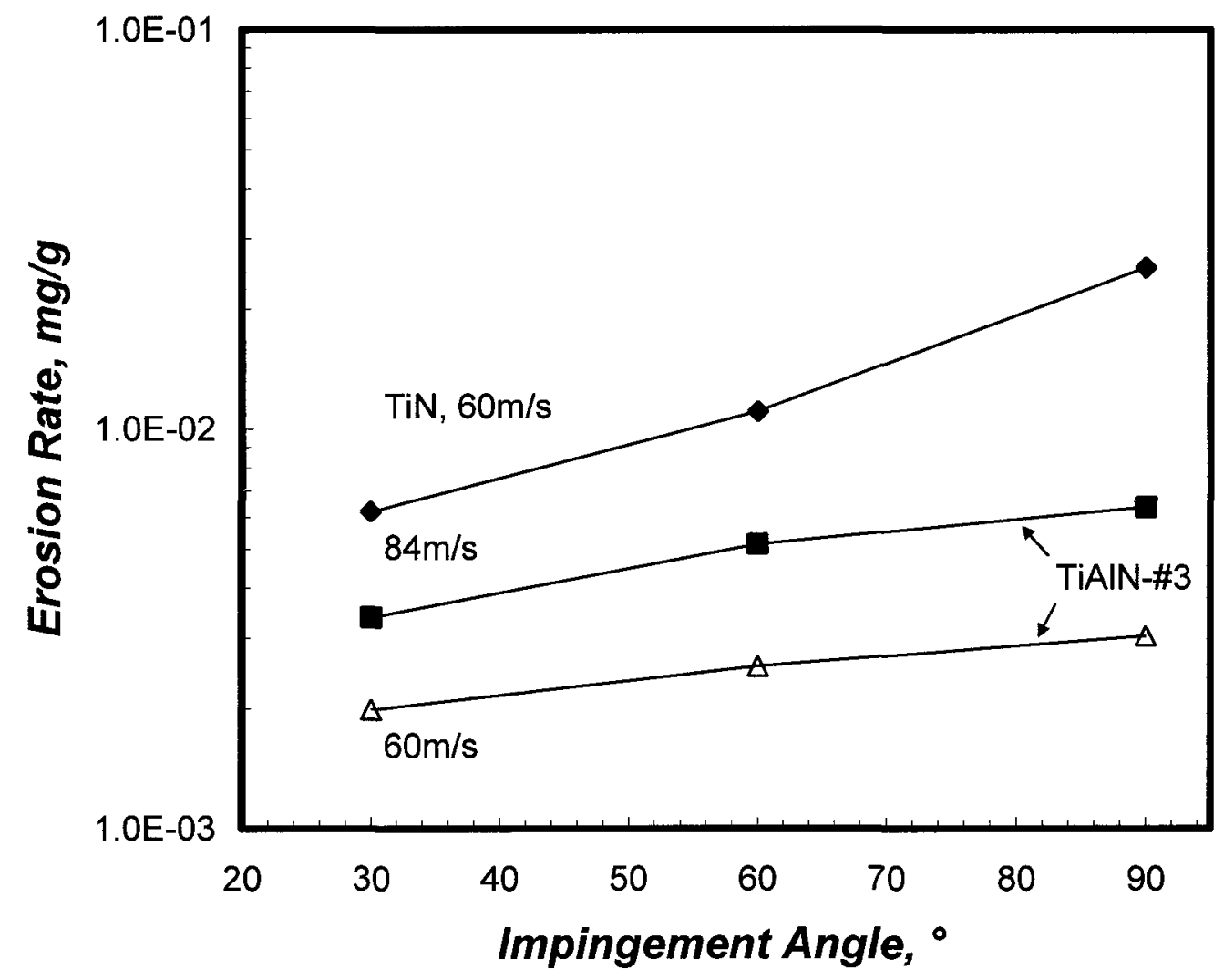

Figure 2-47 Influences of impingement angle and particle velocity on erosion rates for TiN and TiAlN-\#3 coating systems (regenerated from [326]).

Erosion behaviour of quaternary carbonitrides ( $\mathrm{TiSiCN}$ and $\mathrm{ZrSiCN}$ ) in the form of weight loss, are shown in Figure 2-48. The quaternary carbonitride coatings demonstrated a significant increase in erosion resistance relative to the bare substrates of stainless steel and titanium. 


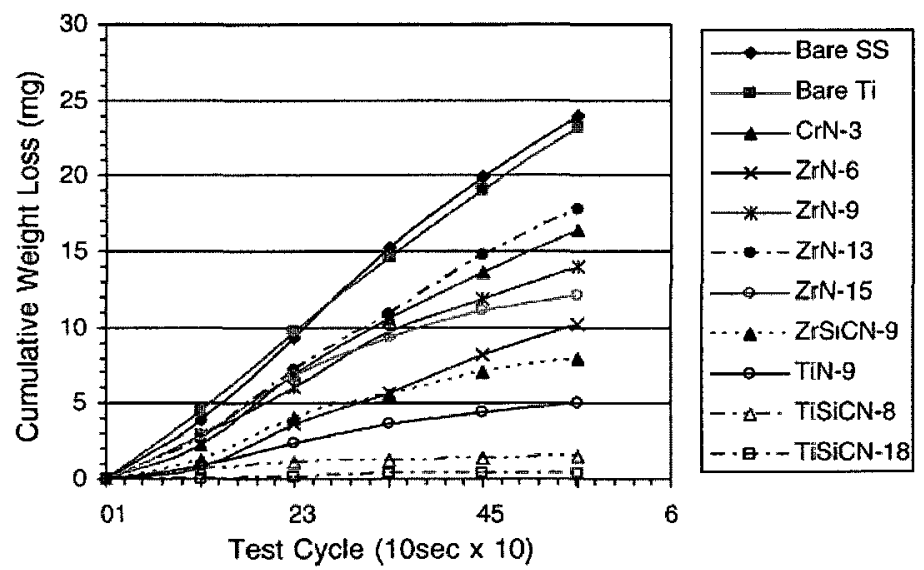

Figure 2-48 Erosion resistance of various coatings with 50 micron $\mathrm{Al}_{2} \mathrm{O}_{3}$ erodent [340].

In an experimental study on combined erosion and corrosion behaviours of TiN coatings on $\alpha$-Ti alloy substrate with saline slurry (sodium chloride, $0.91 \mathrm{wt} . \%$ normal solution), the erosion-corrosion rates, erosion rates and flow-induced corrosion rates vs. impact velocity are shown in Figure 2-49. It can be seen that the TiN coated samples demonstrate advantages in all three properties (erosion-corrosion rate, erosion rate and flow-induced corrosion rate) due to the primary protection of the TiN coating from erosive attack.

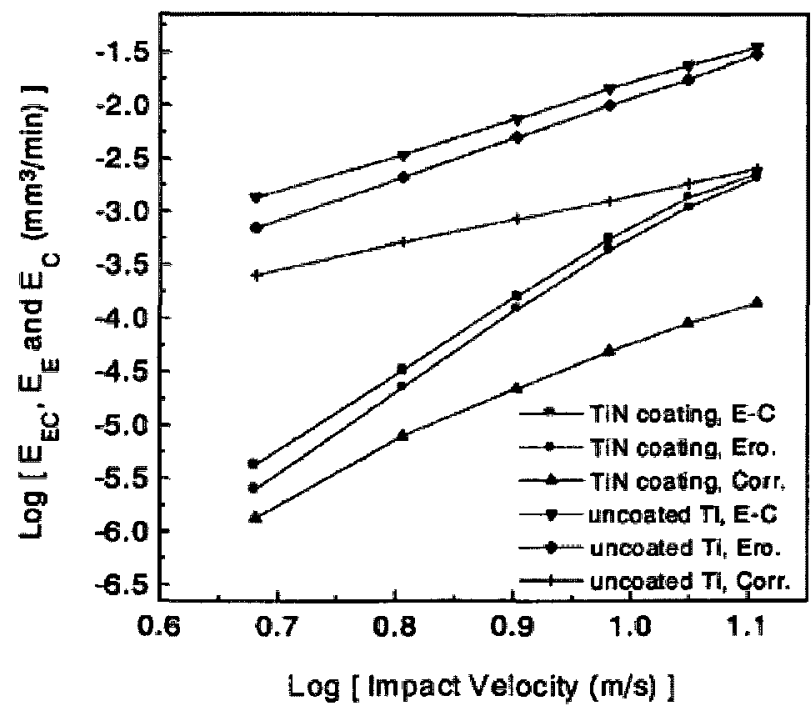

Figure 2-49 Log erosion-corrosion rate, erosive rate and flow-induced corrosion rate vs. impact velocity for the TiN coating and an uncoated $\alpha$-Ti alloy [341]. 


\subsection{Corrosion Behaviours of Transition Metal Nitrides}

Corrosion behaviours of TiN and $\mathrm{CrN}$ coatings in sulfuric acid at various temperatures are demonstrated in the form of a potentiodynamic polarization curve as shown in Figure 2-50, and the test results are summarized in Table 2-7. Corrosion current densities, critical and minimum passive current densities for the $\mathrm{TiN}$ and $\mathrm{CrN}$ coatings were two to three orders of magnitude lower than that for the 1040 steel substrate, indicating their advantages in corrosion resistances in sulfuric acid. After heat treatments at 600 and 650 ${ }^{\circ} \mathrm{C}$, the corrosion resistances of the TiN and $\mathrm{CrN}$ coatings were further improved (Figure 2-50 a, b)(Table 2-7). This was attributed to the diffusion of $\mathrm{Ti}$ and $\mathrm{Cr}$ into the steel substrate, generating corrosion resistant layers at the coating/substrate interface [342].

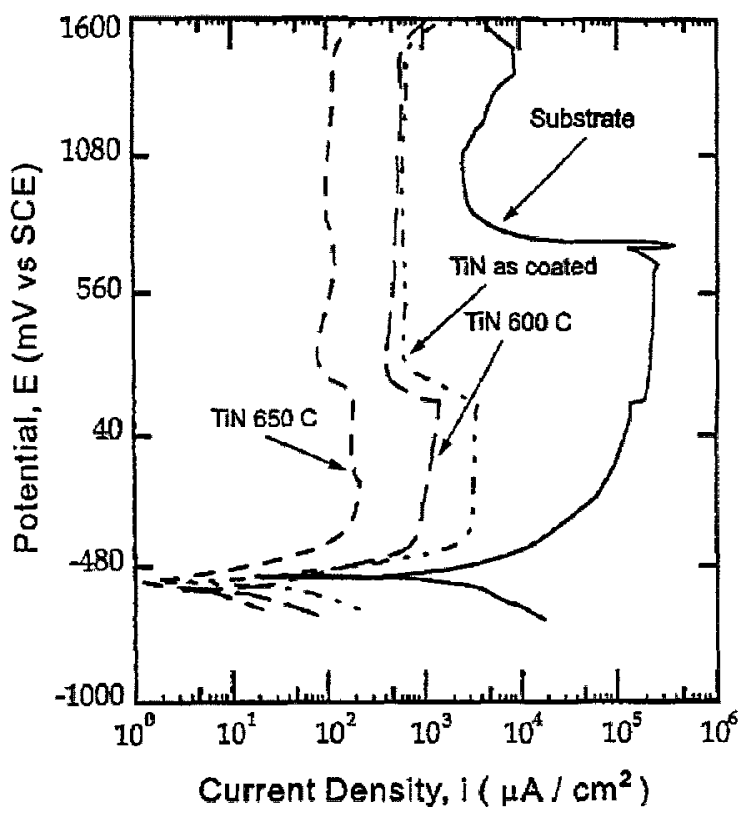

(a) TiN coatings

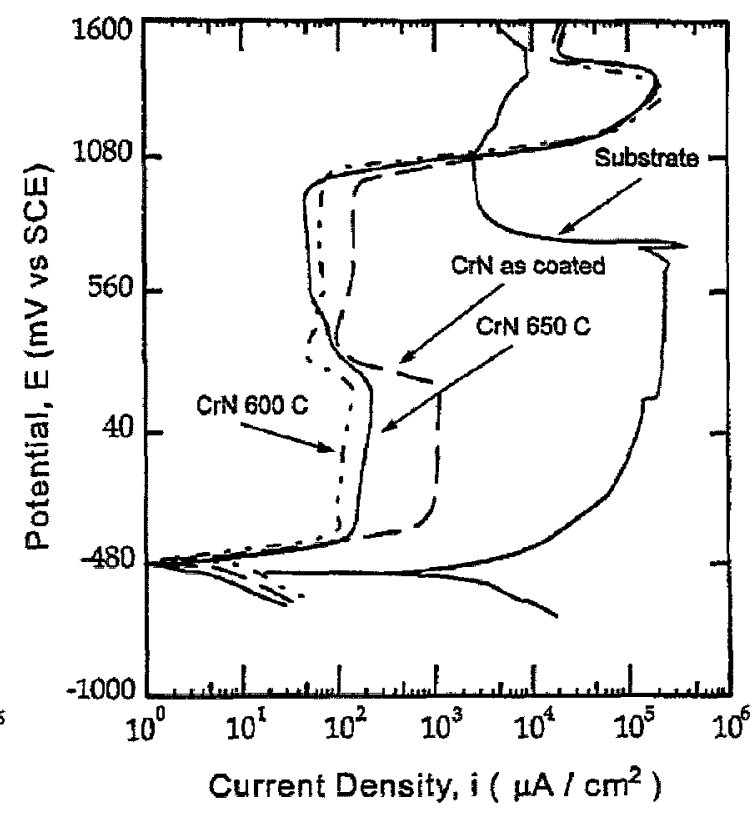

(b) CrN coatings

Figure 2-50 Potentiodynamic curves of coatings 1040 steel substrates in $1 \mathrm{~N}$ sulfuric acid solution [342]. 
Table 2-7 Potentiodynamic polarization data for 1040 steel, TiN and $\mathrm{CrN}$ coatings on 1040 steels substrates at various temperatures determined from the polarization curves in $1 \mathrm{~N}$ sulfuric acid solutions [342]

\begin{tabular}{|l|c|c|c|c|}
\hline \multicolumn{1}{|c|}{ Sample } & $\begin{array}{c}E_{\text {corr }} \\
(\mathrm{mV} \text { vs.SCE })\end{array}$ & $\begin{array}{c}i_{\text {corr }} \\
\left(\mu \mathrm{A} / \mathrm{cm}^{2}\right)\end{array}$ & $\begin{array}{c}i_{c r} \\
\left(\mu \mathrm{A} / \mathrm{cm}^{2}\right)\end{array}$ & $\begin{array}{c}i_{m i n} \\
\left(\mu \mathrm{A} / \mathrm{cm}^{2}\right)\end{array}$ \\
\hline Substrate 1040 steel & -554 & 3,387 & 266,000 & 3,000 \\
\hline TiN as-coated & -547 & 42 & 3,762 & 625 \\
\hline TiN heated $600^{\circ} \mathrm{C}$ & -571 & 35 & 1,480 & 400 \\
\hline TiN heated $650^{\circ} \mathrm{C}$ & -559 & 6 & 238 & 83 \\
\hline CrN as-coated & -499 & 9 & 1,256 & 102 \\
\hline $\mathrm{CrN}$ heated $600^{\circ} \mathrm{C}$ & -473 & 10 & 143 & 43 \\
\hline $\mathrm{CrN}$ heated $650^{\circ} \mathrm{C}$ & -489 & 7 & 244 & 46 \\
\hline
\end{tabular}

Experimental studies on binary and ternary titanium and chromium nitride coatings were conducted. The potentiodynamic polarization curves of the coatings TiN, TiAlN, and SB40-P40 steel substrate, and the coatings $\mathrm{CrN}, \mathrm{CrAlN}$ and mild steel substrates are shown in Figure 2-51 (a) and (b), respectively; and the test results summarized in Table 2-8 and Table 2-9. It can be observed from Figure 2-51 that the polarization curves of the coatings have lower corrosion current densities (three orders of magnitude lower) than the bare substrates, indicating their superior corrosion resistances. This observation was further confirmed by the increased values of polarization resistances $\left(R_{p}\right)$ (Table 2-8 and Table 2-9). Additionally, in the passivity region (Figure 2-51 a), passivity current densities of the coatings TiN and TiAlN are about three orders of magnitude lower than the substrate (passivity current density is a current density that remains stable as the electric potential increases within a certain potential zone). It is also noted that the ternary TiAlN and CrAlN coatings have higher polarization resistances than binary TiN and $\mathrm{CrN}$ coatings, respectively. This could be due to the denser microstructures of the ternary coatings TiAlN and CrAlN which restrict the corrosive medium from contacting the 
substrate $[343,344]$. The presence of $\mathrm{Al}$ in the ternary coatings formed an $\mathrm{Al}_{2} \mathrm{O}_{3}$ layer on the surface of the coating, acting as a protection from further attack [343].
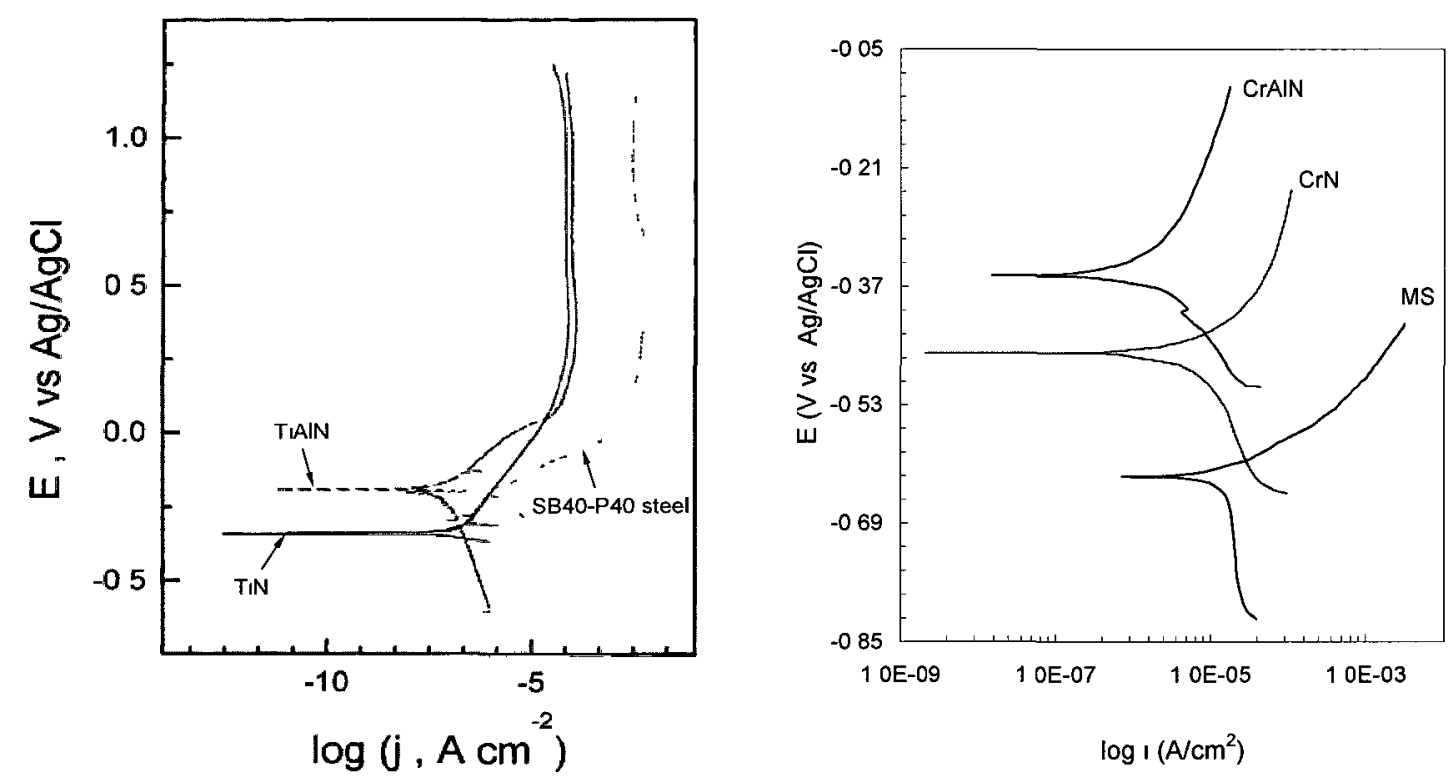

(a) In $0.5 \mathrm{M} \mathrm{NaCl}$ solution [345]

(b) in $3.5 \% \mathrm{NaCl}$ solution (regenerated from [343]

Figure 2-51 Potentiodynamic polarization curves of coating and substrate steels.

Table 2-8 Potentiodynamic polarization data of the untreated and coated specimens recorded in $0.5 \mathrm{M} \mathrm{NaCl}$ aqueous solution after 1 hour exposure [345]

\begin{tabular}{|l|c|c|c|}
\hline \multicolumn{1}{|c|}{ Sample } & $E_{\text {corr }}(\mathrm{V} \mathrm{vs} \mathrm{Ag/AgCl})$ & $i_{\text {corr }}\left(\mu \mathrm{A} / \mathrm{cm}^{2}\right)$ & $R_{p}\left(\mathrm{k} \Omega \mathrm{cm}^{2}\right)$ \\
\hline MB 40-P40 steel & -0.195 & 11.49 & 3.29 \\
\hline PVD TiN & -0.349 & 0.066 & 164 \\
\hline PVD TiAlN & -0.212 & 0.063 & 535 \\
\hline
\end{tabular}

Table 2-9 Potentiodynamic polarization data of $\mathrm{CrN}$ and CrAlN coatings deposited on mild steel substrates in 3.5\% NaCl solution at room temperature [343]

\begin{tabular}{|l|c|c|c|c|c|}
\hline \multicolumn{1}{|c|}{ Sample } & $E_{\text {corr }}(\mathrm{V}$ vs Ag/AgCl $)$ & $i_{\text {corr }}\left(\mu \mathrm{A} / \mathrm{cm}^{2}\right)$ & $\beta_{c}(\mathrm{~V} / \mathrm{dec})$ & $\beta_{a}(\mathrm{~V} / \mathrm{dec})$ & $R_{p}\left(\mathrm{k} \Omega \mathrm{cm}^{2}\right)$ \\
\hline Mild steel & -0.62 & 16.71 & 0.28 & 0.05 & 1.30 \\
\hline CrN & -0.46 & 6.95 & 0.17 & 0.09 & 5.90 \\
\hline CrAlN & -0.34 & 1.03 & 0.09 & 0.08 & 19.90 \\
\hline
\end{tabular}


Potentiodynamic polarization curves of ternary coatings TiAlN and CrAlN are shown in Figure 2-52. Both curves show obvious passive stages, which are due to the formation of passive oxide layers that seal pores in the coating [344]. In the passive regions the corrosion current densities remained at very low levels $\left(\sim 2 \mu \mathrm{A} / \mathrm{cm}^{2}\right)$. As the potentials increased to certain values (pitting potential), pitting occurred due to the dissolution of the oxide layers [344]. The higher pitting potential of the CrAlN coating (Figure 2-52) revealed its better corrosion resistance in comparison to the TiAlN coating.

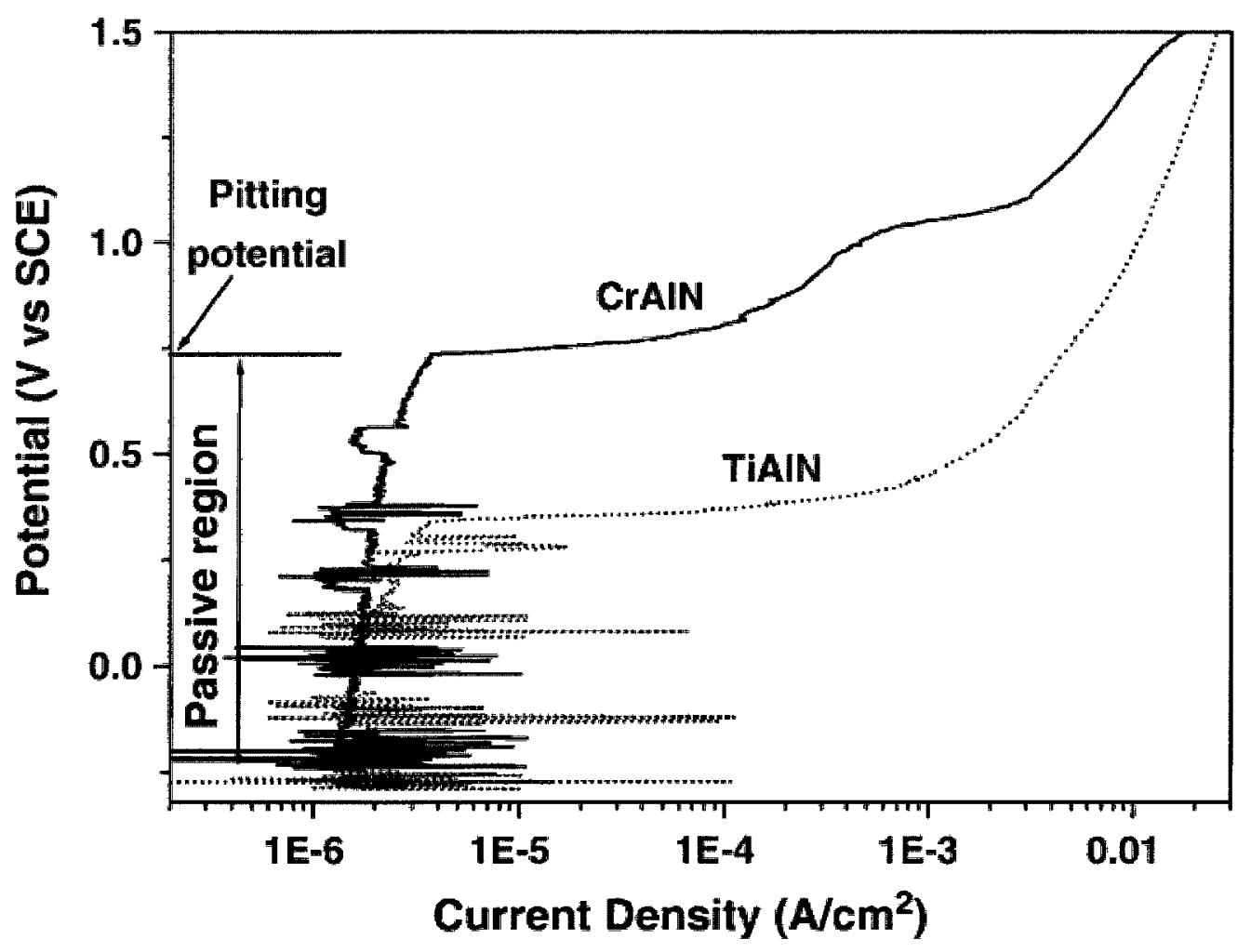

Figure 2-52 Potential polarization curves of the TiAlN and CrAlN coating systems [344].

Corrosion behaviours of nitride coatings TiN, CrN, TiAlN TiAlN/CrN (multilayered), and an uncoated mild steel substrate after one hour immersion in a corrosive medium were measured, and presented in the forms of EIS plots (Nyquist and Bode)(Figure 2- 
18). The Nyquist plots (Figure 2-18 a) and the $\log |Z|$ vs. $\log f$ plot (Figure 2-18 b) indicate that corrosion resistance increased in the order of: substrate, TiN, CrN, TiAlN and TiAlN/CrN. The phase angle vs. $\log f$ plot (Figure 2-18 c) shows that the minimum phase angles of all the coatings $\left(\sim 70^{\circ}\right)$ are less than that of the substrate $\left(\sim 78^{\circ}\right)$, indicating inhomogeneous surface features of the coatings.

EIS test results of the $\mathrm{TiN}$ and $\mathrm{CrN}$ coatings after multiple-day immersions indicated that $\mathrm{CrN}$ based coatings generally demonstrated higher polarization resistances than the TiN based coatings under the same conditions (substrate, corrosive media and immersion time).

This can be explained based on both composition and microstructure. Both TiN and $\mathrm{CrN}$ coatings can form thin passive oxide layers on the coating surface and at open pores, and therefore delay or eliminate localized corrosion. Chromium oxide layers are denser than titanium oxide layers, and therefore chromium nitride based coatings usually show higher resistances to corrosion and oxidation. Also, the passive oxide layers on $\mathrm{CrN}$ and $\mathrm{TiN}$ coating surfaces were assumed to possess $\mathrm{p}$ - and n-type semiconductor characteristics, respectively. The better corrosion resistance of $\mathrm{CrN}$ based coating is attributed to the $\mathrm{p}$ type semiconducting oxide layer, which significantly slows the reduction rate, and consequently lowers corrosion rate [344].

In a coating/substrate system, the corrosion behaviours are governed by the diffusion mechanism of the reactant species (e.g. oxygen) through the coating in an 
electrochemical process. The diffusivities of these species are influenced by the coating microstructure such as pores and grain boundaries. Titanium nitride coatings usually have straight columnar structures (Figure 2-53 a).

The concentration of reactive species gradually decreases across the electrolyte/coating interface. This is referred to as a semi-infinite-length diffusion mode; whereas in chromium nitride coatings, the zig-zag grain boundaries and fine equiaxed structures (Figure 2-53 b) restrict the diffusion of reactive species crossing the electrolyte-coating interface in a finite-length diffusion mode. The difference in coating microstructure is therefore another reason for the difference in corrosion resistances of these two nitride coatings [344].

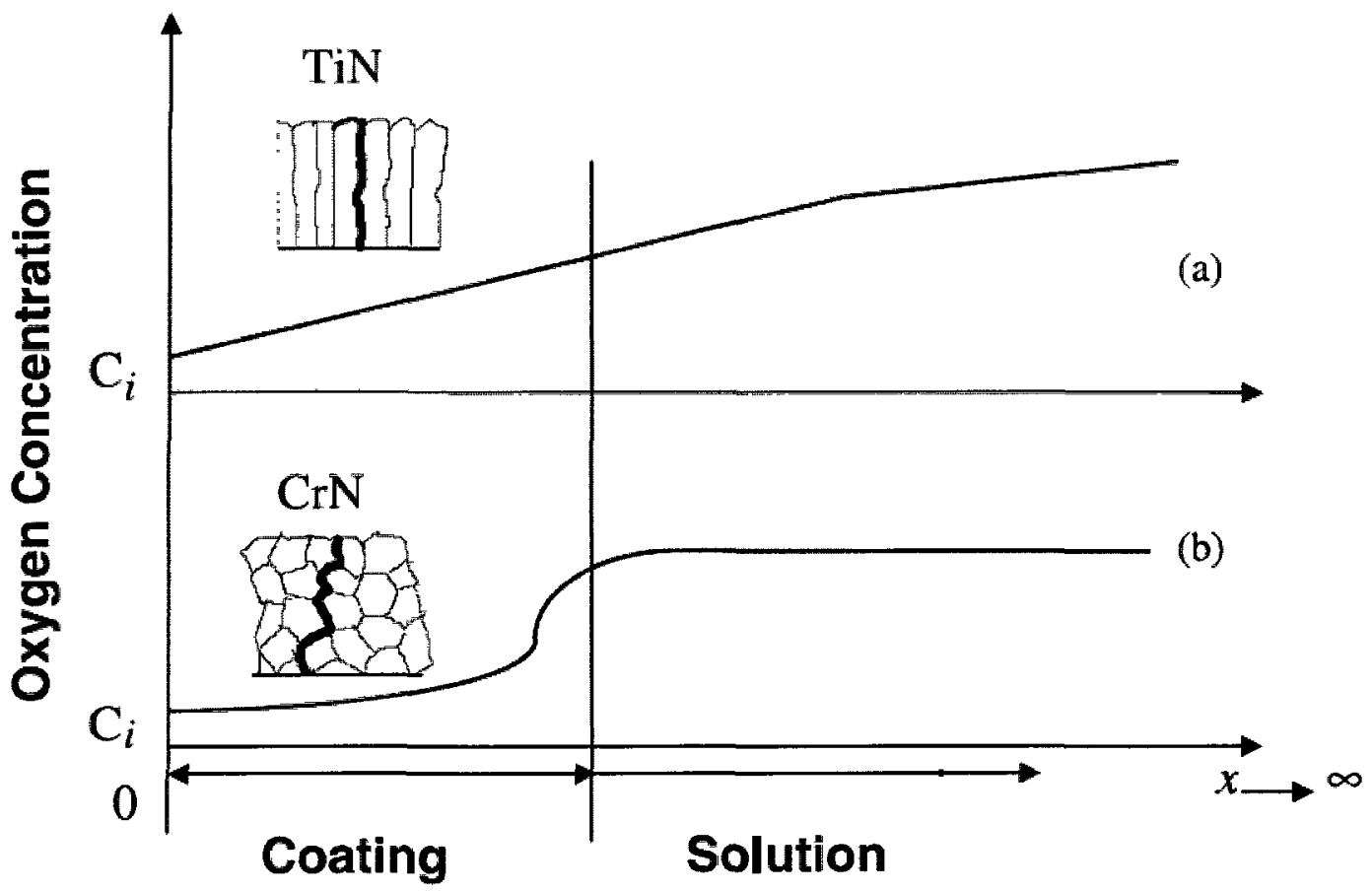

Figure 2-53 A schematic illustration of oxygen diffusion mechanism for (a) columnar (e.g. TiN), and (b) equiaxed (e.g. CrN) crystallite structures [346]. 
The coating thickness has been observed to affect the corrosion resistance as well. The polarization curves of various types of TiN coatings with various thicknesses are shown in Figure 2-54. It can be observed that the critical passivity current densities of the TiN coatings were much lower than those of the substrate. Increasing coating thickness resulted in further reduction in critical passivity current density for all TiN coatings. This indicates that a sufficient coating thickness in a coating system is desired for optimized corrosion resistance.

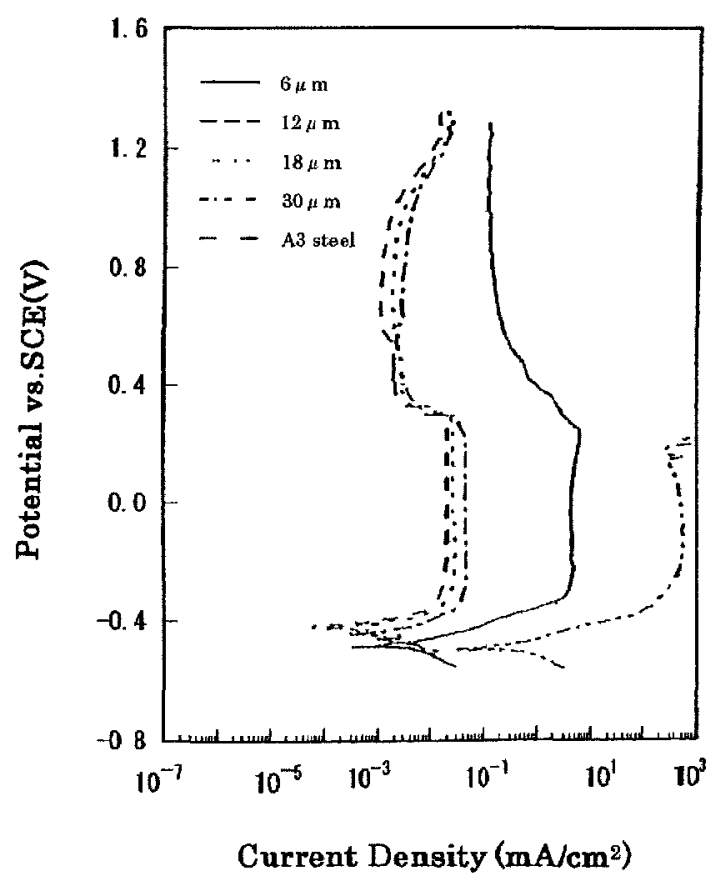

(a) TiN coatings in $1 \mathrm{~N} \mathrm{H}_{2} \mathrm{SO}_{4}$ solution [347]

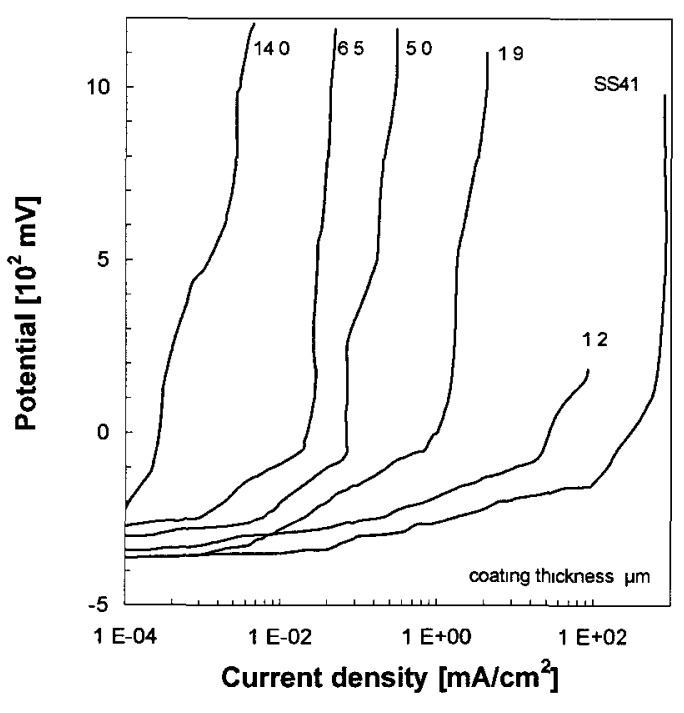

(b) TiN coatings in $\mathrm{HCl}$ (regenerated [36])

Figure 2-54 Polarization curves of coating systems with various thicknesses.

When coatings with different thicknesses are prepared using different techniques, the trend between the corrosion resistance and thickness may not be obvious. As shown in Figure 2-55, the potentiodynamic polarization curves of TiN coatings prepared using various deposition processes (plasma-based ion implantation (PBII), sputtering 
deposition, shielded vacuum arc deposition, and plasma spraying) revealed that the thinnest coating (PBII sample) exhibited the best corrosion resistance, i.e., the lowest corrosion current density and passivity current density. The lowest defect population was also found in the PBII TiN coating [348]. The result suggests that defect is a dominant factor governing corrosion behaviours of a coating system and that the defect density is strongly determined by the deposition process.

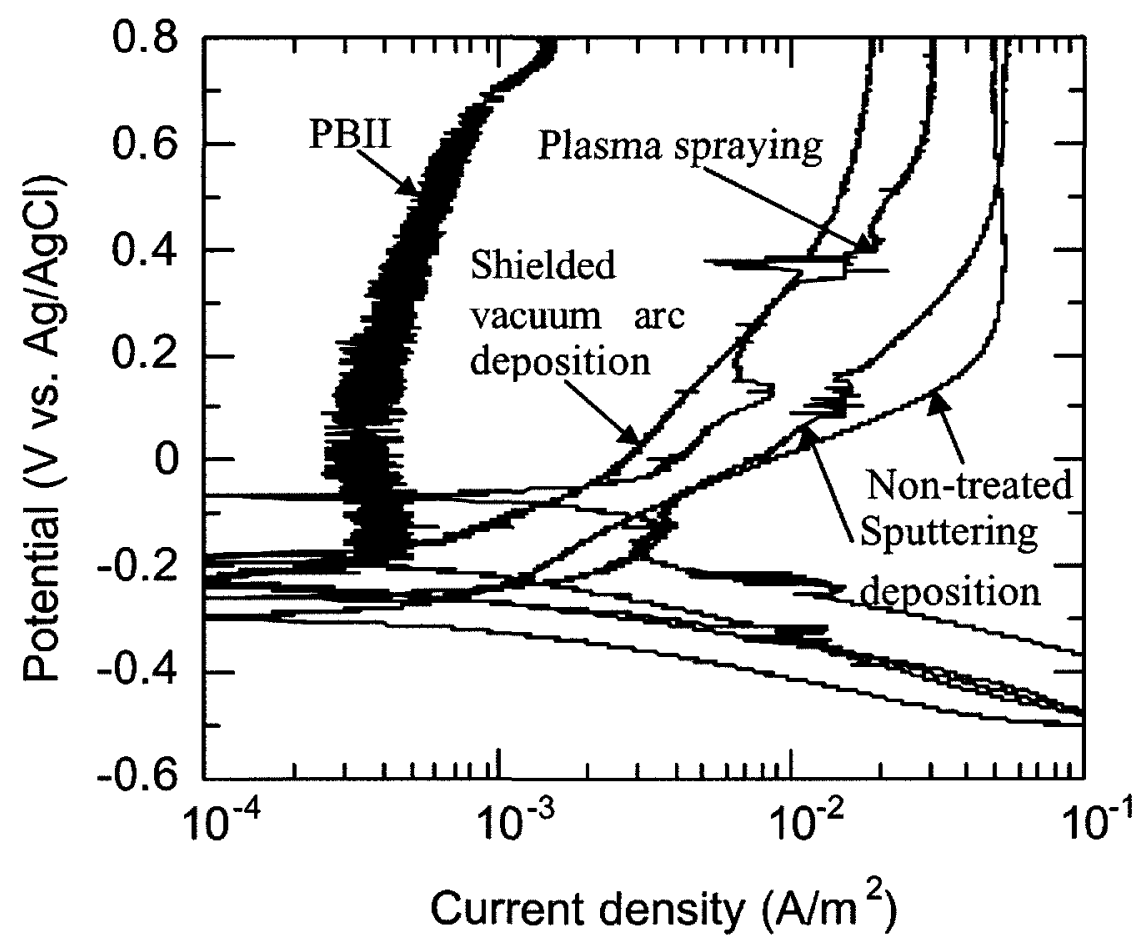

Coating thicknesses $(\mu \mathrm{m})$ : PBII: 0.03 , sputtering: 1 , vacuum arc: 1 , plasma spraying: 30 Figure 2-55 Potentiodynamic polarization curves of TiN coatings produced by various deposition processes [348].

As shown in Figure 2-56, all three TiN coatings (deposited using various techniques) demonstrated simultaneous changes in critical passivity current densities and area ratios of pinhole defects with an increase in coating thickness. This indicates a certain correlation of corrosion behaviour and defects in a coating system. Further quantitative 
studies on coating surface pit density and total volumetric porosity revealed a close dependence of corrosion current density and corrosion potential on coating defects as summarized in able 2-10. Therefore, coating defects are a priority issue in developing coating systems, particularly for corrosion applications.

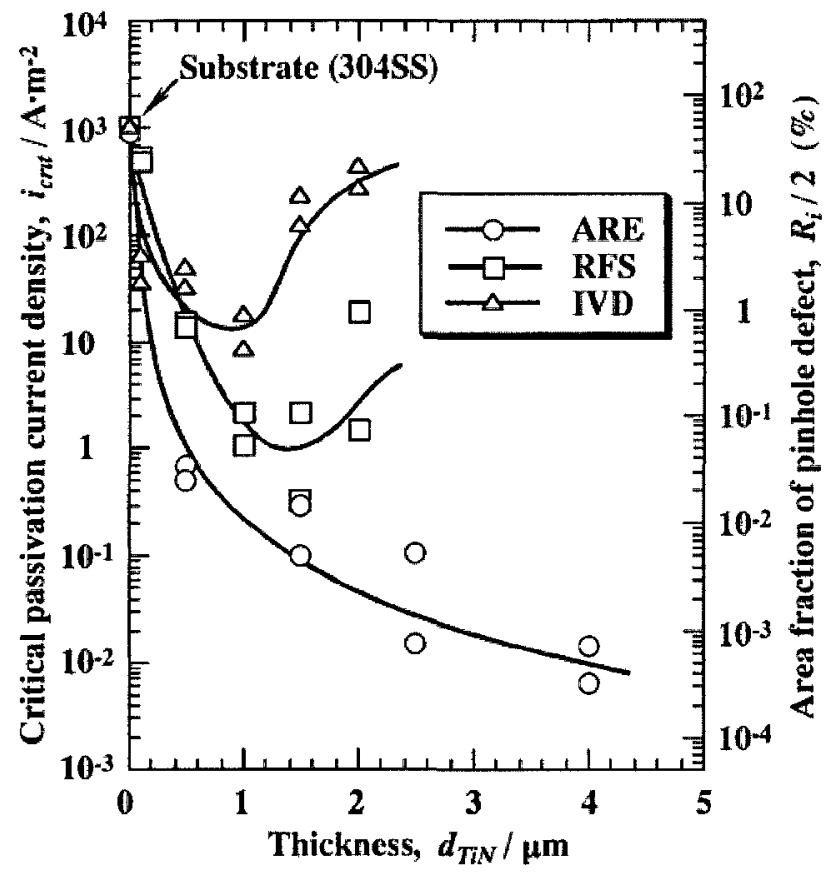

Figure 2-56 Effect of coating thickness on critical passivity current density and area ratio of pinhole defect [134].

Table 2-10 Density of nodular "inhomogeneities", corrosion pits, corrosion current density, corrosion potential and calculated porosity of some TiN and ( $\mathrm{Ti}, \mathrm{Al}) \mathrm{N}$ coatings, together with the coating thicknesses and $\mathrm{Al} / \mathrm{Ti}$ evaporation ratios [184]

\begin{tabular}{|c|c|c|c|c|c|c|c|}
\hline Coating & $\begin{array}{c}\text { Thickness } \\
(\mu \mathrm{m})\end{array}$ & $\begin{array}{c}\mathrm{Al} / \mathrm{Ti} \\
\text { ratio }\end{array}$ & $\begin{array}{c}\text { Defect } \\
\text { density } \\
\left(\mathrm{cm}^{-2}\right)\end{array}$ & $\begin{array}{c}\text { Pit } \\
\mathrm{density}_{\left(\mathrm{cm}^{-2}\right)}\end{array}$ & $\begin{array}{c}i_{\text {corr }} \\
\left(\mu \mathrm{A} / \mathrm{cm}^{2}\right)\end{array}$ & $\begin{array}{c}E_{\text {corr }} \\
(\mathrm{mV}(\mathrm{SCE}))\end{array}$ & Porosity \\
\hline$(\mathrm{Ti}, \mathrm{Al}) \mathrm{N}$ & 3.3 & 0.56 & 1,000 & 700 & 7.8 & -413 & $6.88 \times 10^{-4}$ \\
\hline$(\mathrm{Ti}, \mathrm{Al}) \mathrm{N}$ & 3.3 & 1.40 & 1,200 & 1100 & 6.6 & -421 & $7.14 \times 10^{-4}$ \\
\hline$(\mathrm{Ti}, \mathrm{Al}) \mathrm{N}$ & 3.4 & 2.40 & 6,700 & 2300 & 28 & -427 & $2.70 \times 10^{-3}$ \\
\hline $\mathrm{TiN}$ & 18 & - & 7,000 & 2 & $\mathbf{8}$ & -392 & $1.22 \times 10^{-4}$ \\
\hline $\mathrm{TiN}$ & 4.2 & - & 10,000 & 93 & 24 & -408 & $3.28 \times 10^{-4}$ \\
\hline $\mathrm{TiN}$ & 5.3 & - & 18,000 & 950 & 35 & -425 & $3.51 \times 10^{-3}$ \\
\hline
\end{tabular}




\subsection{Coating Defects and Their Impacts}

In a coating system, defects can be classified into three types: (1) open porosities where the pores are interconnected, (2) closed porosities where the pores are isolated, and (3) through-porosities (pores or pinholes) that extend through the deposit into the substrate $[98,349]$. Through-porosities are the main contributor to corrosion of the substrate as it is through these porosities that aqueous corrosive media access the substrates $[111,133,98$, $349,350]$.

Defects in coatings can be classified according to formation mechanism as lattice defects and growth defects $[322,349]$. Lattice defects are the vacancies resulting from missing atoms or clusters at lattice positions, and lattice dislocations. As a typical lattice defect, voids are closed internal pores that do not connect to a free surface [349]. The aggregation of vacancies result in microvoids along grain and column boundaries, going through the deposition (Figure 2-57 a) [234]. Abrupt interfaces form interfacial voids; rapid diffusion causes capillary voids at grain boundaries [349]; voids also form at triple points between grains and grain boundary during annealing [351] (Figure 2-57 b, c). Additionally, voids form due to the embedded droplets [133](Figure 2-57 d). 




(a) Cross-sectional TEM micrograph showing the evolution of microstructure in a TiN film sputter-deposited at $\mathrm{Ts}=300^{\circ} \mathrm{C}$. The negative substrate bias Vs was changed stepwise without interrupting the growth. The void regions along column boundaries (indicated by arrows) become dense when increasing Vs from 80 to $120 \mathrm{~V}$ [234]

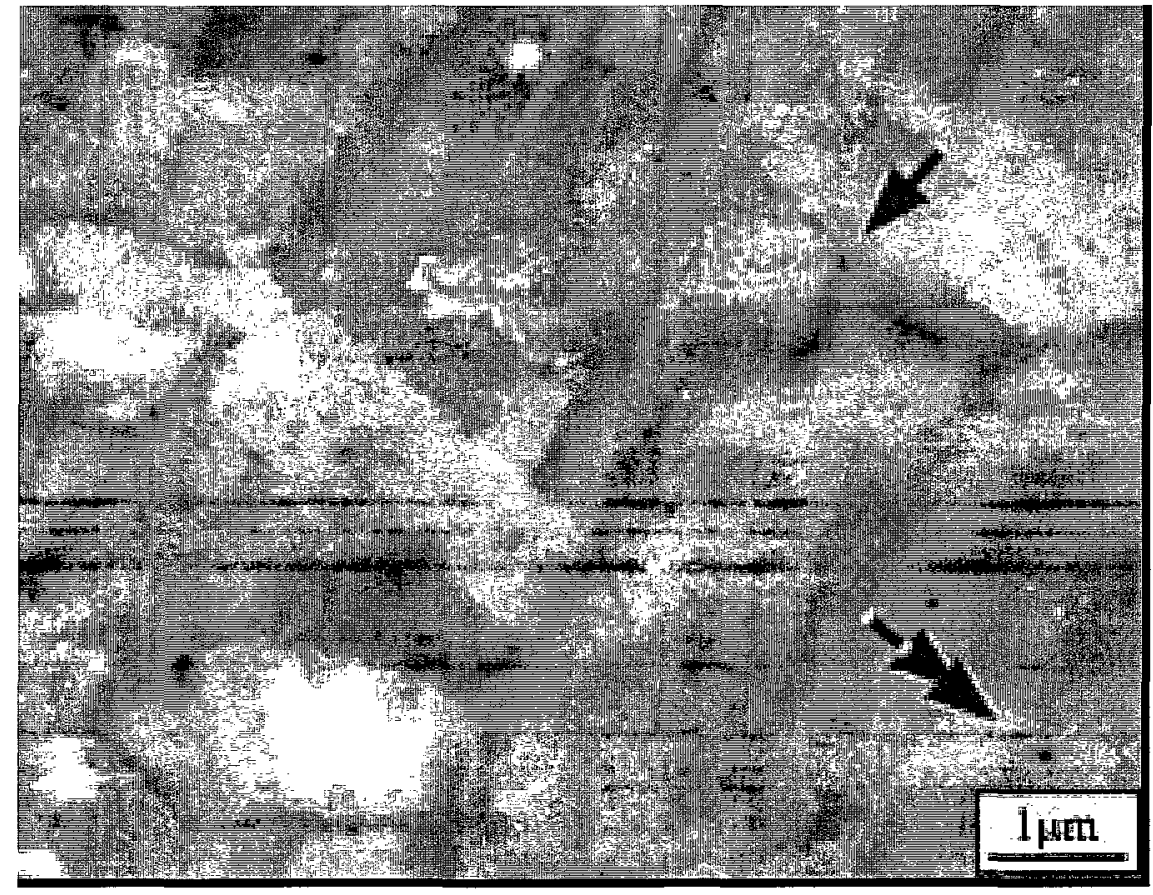

(b) SEM image after thermal cycling. A single arrow indicates void formation along the grain boundary. A double arrow indicates void formation in the grain interior [352]

Figure 2-57 to be continued 


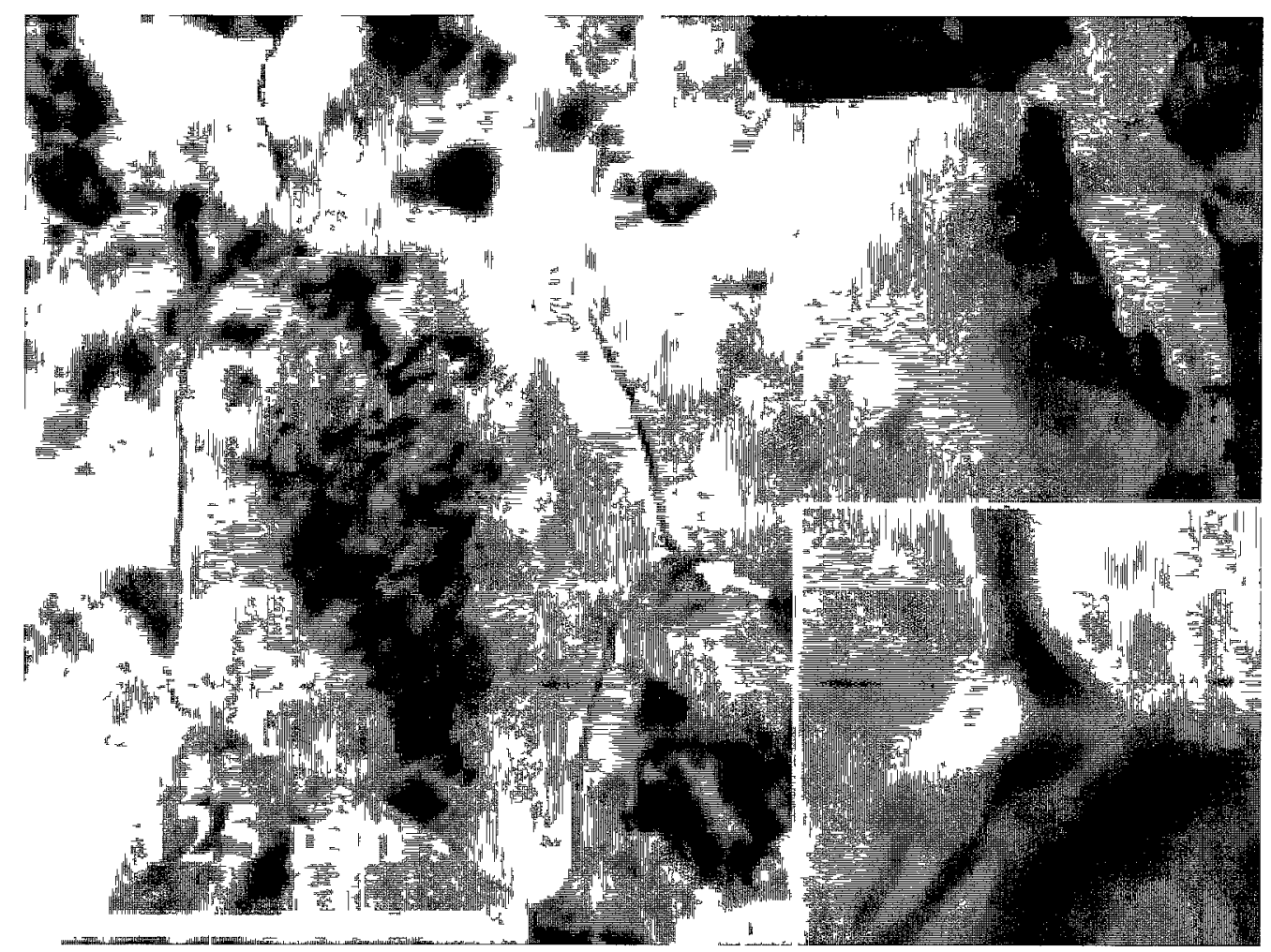

(c) A typical void in a coating after annealing at $900^{\circ} \mathrm{C}$ for 2 hours [351]

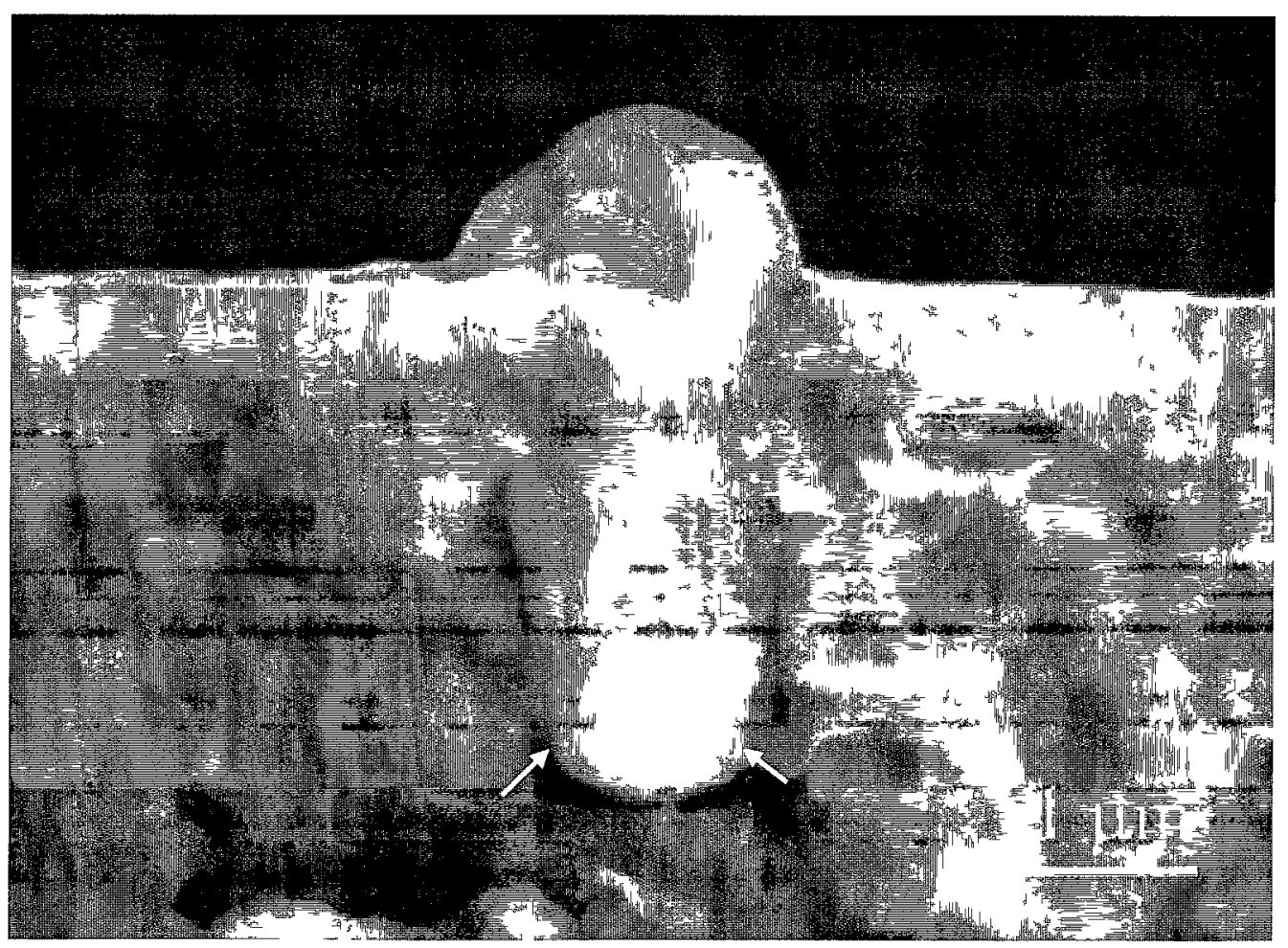

(d) A droplet in a coating and resulted in voids [353].

Figure 2-57 Defect formations in coating systems. 
Two general sources of cores are foreign particles and droplets. Foreign particles stem from contamination (e.g., dusts and debris) on the substrate surface. This type of particle can be minimized through cleaning the substrates, chambers, and targets (for PVD) by physical means. The other source of cores are droplets generated during the deposition process. Physically cleaning substrates and deposition chambers, and optimizing deposition parameters would minimize the source of droplets, therefore lower the rate of growth defects. In PVD processes, droplets can be generated due to cathode arcing [32,111], molten vapor condensation $[32,111,354,355]$, and preferential nucleation caused by surface irregularities or inhomogeneities [32] (Figure 2-58). These defect formations and growths are affected by deposition parameters, including bias voltage and duty cycle [32]. They can be minimized by increasing the energy of bombarding ions, eliminating the nucleation sites, increasing the atom mobility and surface diffusion, and increasing the duty cycle [32].

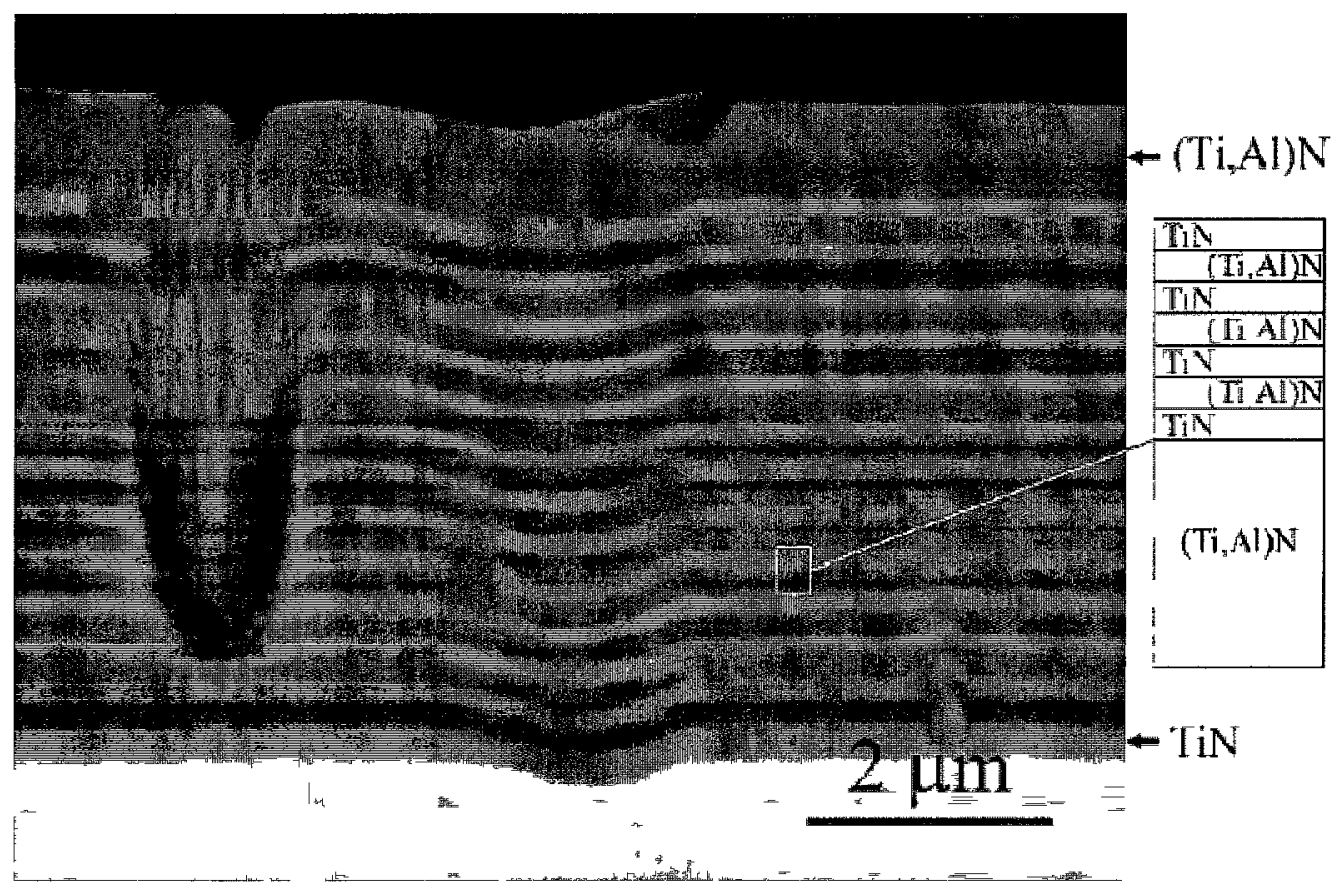

Figure 2-58 Images of irregularty and droplet [353]. 
Substrate bias voltage is an important parameter governing the crystal structure and morphology of deposited coatings. Surface morphologies of $\mathrm{CrN}$ coatings deposited at various bias voltages are shown in Figure 2-59. The surface roughness decreased with an increase in bias voltage, which was attributed to the change in number and size of projections on the surface of the coatings. These results were in agreement with a statistical study about the relationship between numbers of projections and substrate bias voltage. As can be seen in Figure 2-60, the number of projections and the diameter of projections decreased with increasing bias voltage.
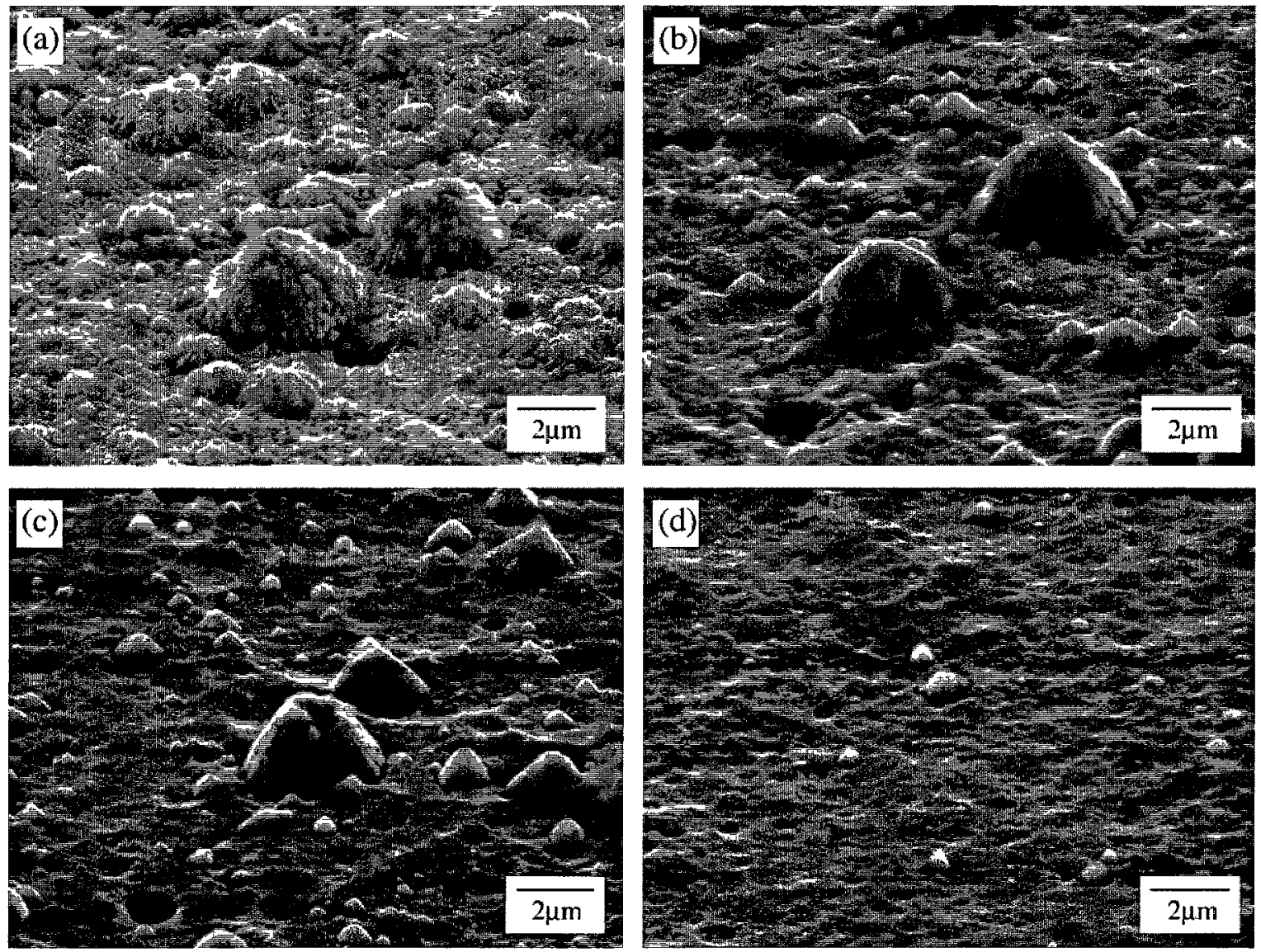

Figure 2-59 Typical SEM photographs of chromium nitride films deposited at the substrate bias voltage of (a) $0 \mathrm{~V}$, (b) $-20 \mathrm{~V}$, (c) $-50 \mathrm{~V}$, and (d) $-500 \mathrm{~V}$ [356]. 


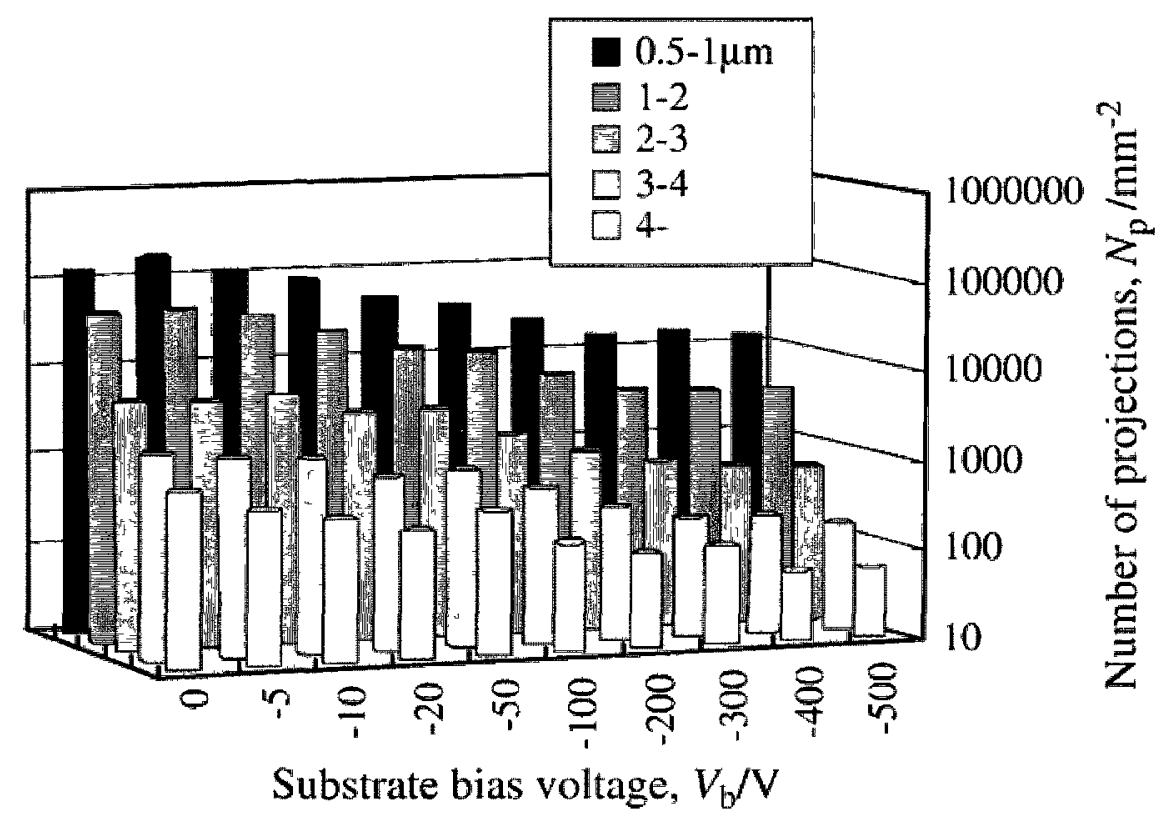

Figure 2-60 Variation in number of projections classified by diameters with substrate bias voltage [356].

Pinhole densities in TiN and $\mathrm{CrN}$ coatings at various bias voltages are shown in Figure 2-61. TiN coatings exhibited the maximum pinhole density at a bias voltage of $100 \mathrm{~V}$ (Figure 2-61 a). With increasing bias voltage, pinhole density decreased. $\mathrm{CrN}$ based coatings with multiple phases prepared at low bias have the highest pinhole density (Figure 2-61 b); whereas, a single phase coating deposited at high nitrogen pressure and high substrate bias has the lowest density. The change in pinhole density with bias voltage is related to its effect that controls the ion bombardment energy on on coating growth and internal stress of deposited TiN coatings [323, 324].

Porosity affects the properties of coatings in many ways. High surface area in a porous material makes it easy to contaminate, oxidate, and deform. The voids at the coatingsubstrate interface reduce the adhesion due to the decrease in effective contact area, acting as stress concentration defects that initiate a path for fracture propagation. Also, 
interfacial voids result in increased contact resistances between the coating and substrate, and decreased thermal conductance across the interface $[98,349]$.

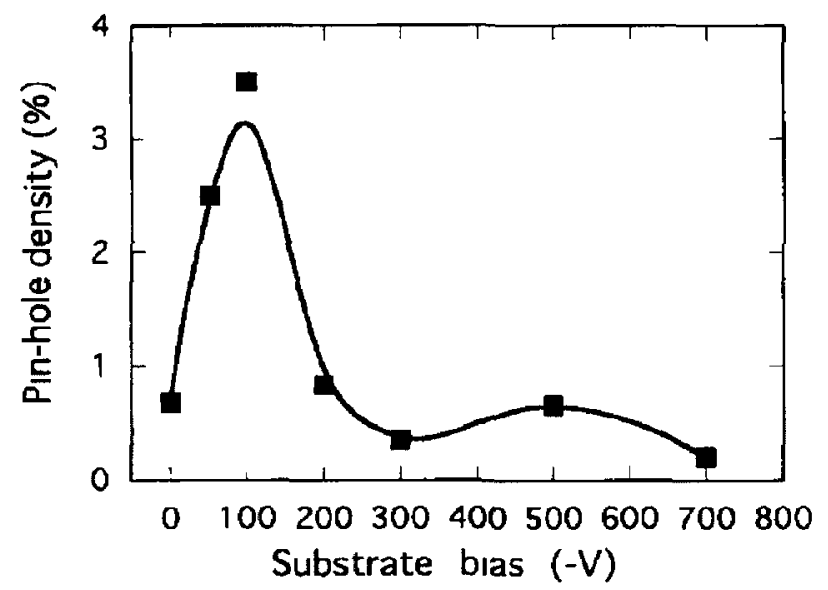

(a) TiN coatings on stainless steels

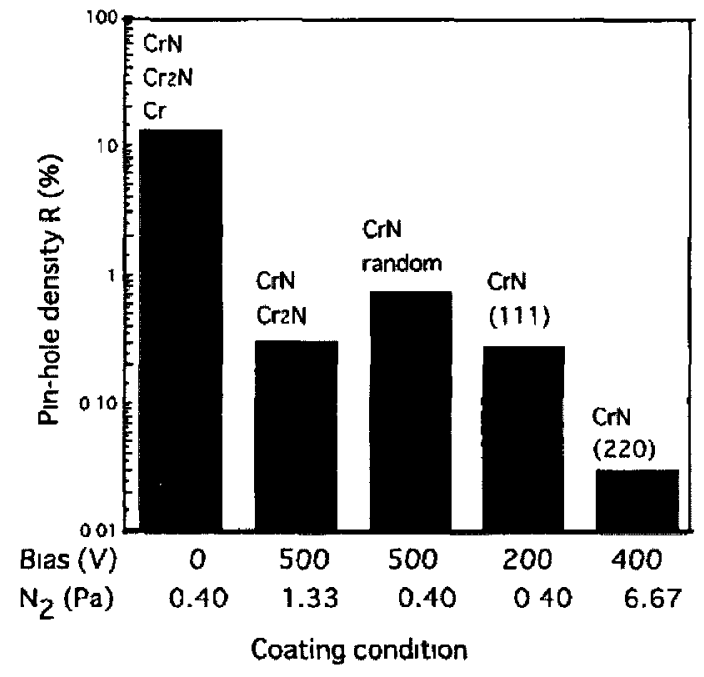

(b) $\mathrm{CrN}$ coatings on stainless steels

Figure 2-61 Pinhole density vs. substrate bias voltage [357].

Density is important in determining coating properties including mechanical deformation and corrosion resistance $[98,349]$. Porosity measurement is a good evaluation of defect densities, which can be evaluated by means of electrochemical methods [158 - 160]. Due to the fact that porosity is inversely proportional to the packing factor, the packing factor (P) can be employed as a quantitative measurement of the defect density (pores or pinholes) [101, 133].

Coating defects are inevitable in hard coating depositions. Post-deposition treatments are conducted in an attempt to interrupt the defect and block the access path for the corrosive media [111]. Some experimental studies have shown the effect of post-deposition treatment in closing the open pores $[358,359]$. 


\subsection{Correlations between Coating Properties}

To possess enhanced tribological and corrosion resistances, it is expected that a coating system should have high hardness, sufficient elastic modulus, and low defect density. PVD hard coatings such as TiN and $\mathrm{CrN}$ have good wear resistance, and are moderately corrosion resistant. However, due to their specific microstructures, columnar (for TiN) or equiaxed crystalline (for $\mathrm{CrN}$ ) [346], growth defects are difficult to avoid, which could lead to severe corrosion and ultimately loss of mechanical integrity. Experimental results revealed that low-density coatings do not necessarily show open porosity, whereas open porosity can be found for coatings with higher densities [215].

The superhard coating systems, such as multilayer, nanocomposite, and superlattices, possess excellent hardness and elasticity. Also, multiple interfaces in multilayer and superlattices act as barriers to corrosion attackes to substrates. However, any intrinsic defects in the coating structure such as voids (Figure 2-57), irregularties and droplets (Figure 2-58), could undermine this advantage. This result suggests that a complex coating system must be well designed in terms of both compositions and microstructure in order to have enhanced the mechanical and electrochemical properties.

In summary, it is a challenge for a coating system to provide good anti-corrosion properties, while retaining excellent mechanical behaviour [133], such as high hardness, high elesticity, and a low cofficient of friction. The difficulties stem from the nature of the coatings and the deposition processes. Understanding these properties and their correlations is fundmental for the development of new future coating systems. 


\subsection{Research Objectives}

From the literature review it is concluded that coating is an effective way to provide protection to metal components under tribological and corrosive attacks. For a given coating system, the responses to tribological and corrosive attacks are directly determined by composition, microstructure and mechanical properties. As such the research objectives for this thesis will focus on the effects of coating compositions and microstructures on these behaviours under tribological and corrosive attacks. More specifically, this thesis is focused on the following four main objectives:

\section{Effect of Coating Deposition Conditions on Coating Microstructure and Properties}

The effect of deposition process and parameters on coating microstructure and properties will be studied through a series electroplated nickel coatings. In electroplating, different pulse frequencies (and nano sized additives) are used to modify coating microstructures (grain size and thickness) and consequently the properties of the coating such as hardness and modulus of elasticity. To investigate the effect of nano additives, carbon nano-tubes, will be introduced in the electroplating process to impart changes to microstructure. The effect of changing microstructures on various properties will also be investigated. This study helps better understand the correlation between deposition conditions and coating properties; and is expected to support the further studies of the PVD hard coating systems in this work. 


\section{Effect of Coating Composition on Mechanical Properties and Tribological Behaviours}

The effect of coating composition on microstructure, mechanical properties, and behaviours of wear, erosion and corrosion will be studied with a series of chromium nitride based PVD coating systems with various $\mathrm{C}, \mathrm{Si}$ and $\mathrm{N}$ contents. The coating composition will be changed gradually from $\mathrm{CrN}$ to $\mathrm{CrSiCN}$, with increasing amounts of Si and C. With composition changes, microstructure, and mechanical properties and their wear, erosion and corrosion resistances are expected to change. The reasons for such changes will be explored.

\section{Effect of Coating Defect and Post-deposition Treatment on Corrosion Behaviours}

First the effect of defects in PVD coating systems on corrosion behaviour will be further studied. Subsequently, post-deposition treatment with a dielectric substance poly (methyl methacrylate) (PMMA) will be applied to the coating surfaces to fill the coating defects, and any changes to corrosion mechanism and rate will be measured. Polarization measurement and EIS techniques will be used in this study.

\section{Quantitative Analysis on Corrosion Mechanisms in Coating Systems}

To study the corrosion mechanism(s) of PVD coating systems with different compositions and microstructure, the EEC models are proposed and applied to corrosion analyses. Through interpreting and comparing EIS parameters from alternative EEC fitting results, further information on detailed diffusion related corrosion mechanisms will be obtained. 
Mathematical analyses on electrochemical impedance or admittance will be involved to determine the correlations between the changes in impedance or admittance and microstructure in coating systems. Experimental data will be compared with the mathematical analyses.

\section{Expected Outcomes}

The expected outcome of this study is an understanding of the effects of coating composition, microstructure, and post-deposition treatment on the properties of PVD coating systems and their tribological and corrosion resistances. This understanding is expected to make a contribution to the development of new wear, erosion, and corrosion resistant PVD coating systems. 


\section{Materials and Experimental Procedures}

This chapter introduces substrate material types and surface preparations, deposition facilities and procedures, and post-deposition treatments. Coating processes include electroplating and physical vapor deposition (PVD). Evaluations on coating microstructure and properties such as wear, erosion, and corrosion resistances in various environments are detailed in this study.

\subsection{Substrate and Preparation}

The substrates used included AISI 1040 carbon steel and 17-4 PH stainless steel. The sample dimensions and surface treatment conditions are summarized in Table 3-1. All the 17-4 PH stainless steel substrates were subjected to a heat treatment, which is equivalent to that applied to turbine compressor blades. The heat treatment process consists of a solid solution treatment followed by a precipitation treatment and a stress relief treatment. For the solid solution treatment, the substrates were heated for two hours at $1038 \pm 15^{\circ} \mathrm{C}$, then air cooled to room temperature. For the precipitation treatment, the substrates were heated for four hours at $649 \pm 8^{\circ} \mathrm{C}$, then air cooled to room temperature. The stress relief treatment consists of heating for two hours at $515 \pm 5^{\circ} \mathrm{C}$ then air cooling to room temperature. 
Table 3-1 Summary of substrate steels

\begin{tabular}{|l|c|c|c|l|}
\hline \multicolumn{1}{|c|}{ Material } & $\begin{array}{c}\text { Thickness } \\
(\mathrm{mm})\end{array}$ & $\begin{array}{c}\text { Dimension } \\
(\mathrm{mm} \times \mathrm{mm})\end{array}$ & $\begin{array}{c}\text { Deposition } \\
\text { technique }\end{array}$ & \multicolumn{1}{|c|}{ Test } \\
\hline AISI 1040 (MS) & 1.6 & $76 \times 25$ & Electroplating & Erosion \\
\hline AISI 1040 (MS) & 1.6 & $16($ diameter $)$ & PVD & Corrosion \\
\hline 17-4 PH (SS) & 1.6 & $16($ diameter) & PVD & Corrosion \\
\hline 17-4 PH (SS) & 3.2 & $50($ diameter) & PVD & Wear/erosion \\
\hline
\end{tabular}

All the substrates were ground using abrasive papers with grit sizes from $\# 400$ to $\# 1200$, and polished using a $1 \mu \mathrm{m}$ diamond suspension fluid, then rinsed with distilled water and ethanol, air dried and individually sealed in plastic bags.

\subsection{Coating Depositions}

In this section, two deposition techniques, i.e., electroplating and PVD, were used to deposit the coatings on the substrate.

\subsubsection{Electroplating}

Nickel plating was conducted with an electroplating apparatus in the Department of Mechanical \& Aerospace Engineering, Carleton University. The apparatus consists of a pulsed direct current (DC) power supply system, an electrolyte bath, and a hotplate magnetic stirrer (Corning PC-42, S50448HP) (Figure 3-1 a). The pulsed DC power supply system is composed of a DC power supply (GW Instek GPS - 3303), a function generator (GW Instek GFG - 8210), and a solid state relay (SSRDC100VDC12). The DC power supply provides a direct current for nickel plating. The desired current is set by 
adjusting the voltage output, and the pulse frequency is set by adjusting the function generator. In this study, the function generator was set to square wave output with a duty cycle of 50/100. The signal from the function generator controls the solid state relay that is connected in series in the circuit to produce the pulsed current. An oscilloscope displays the waveforms of the pulsed current.

In the electrolyte bath $(1000 \mathrm{ml})$ (Figure 3-1), a substrate and a basket with nickel balls (commercially pure nickel balls $6.35 \mathrm{~mm}$ in diameter) are submerged into the electrolyte. The substrate is connected to the DC power supply as the cathode, and the basket is connected to the DC power supply, through the solid state relay (controlled by the function generator), as the anode. To avoid contamination of the electrolyte by dissolution of the copper anode pole connection, an "anti-capillary" cathode was created. A polypropylene beaker with drilled holes was inserted in the basket and suspended such that about half of the nickel balls were immersed in the electrolyte. A section of Tygon tube, filled of nickel balls, is set atop the stack and the anode pole connection made to the nickel balls at the top of the tube so that the electrolyte will not contact the copper wire connection (Figure 3-1 b).

The electrolyte bath was set on a hot plate with magnetic stirrer (Figure 3-1), which maintains a desired temperature for the electrolyte cell, and provides agitation to the electrolyte. Agitation maintains ion concentration uniformity near the electrode surfaces, and stabilizes the open-circuit potential [127]. 


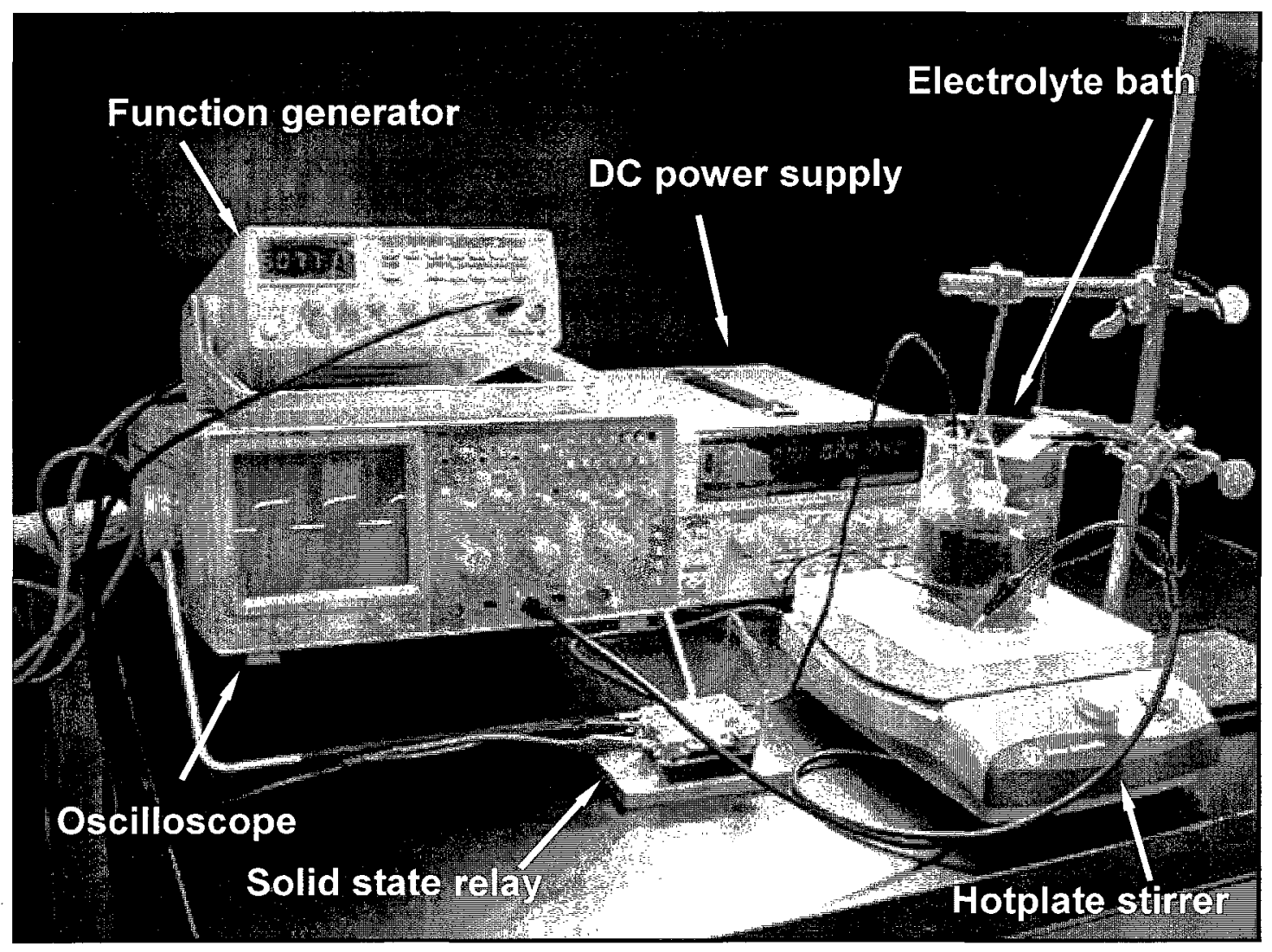

(a) Nickel electroplating setup

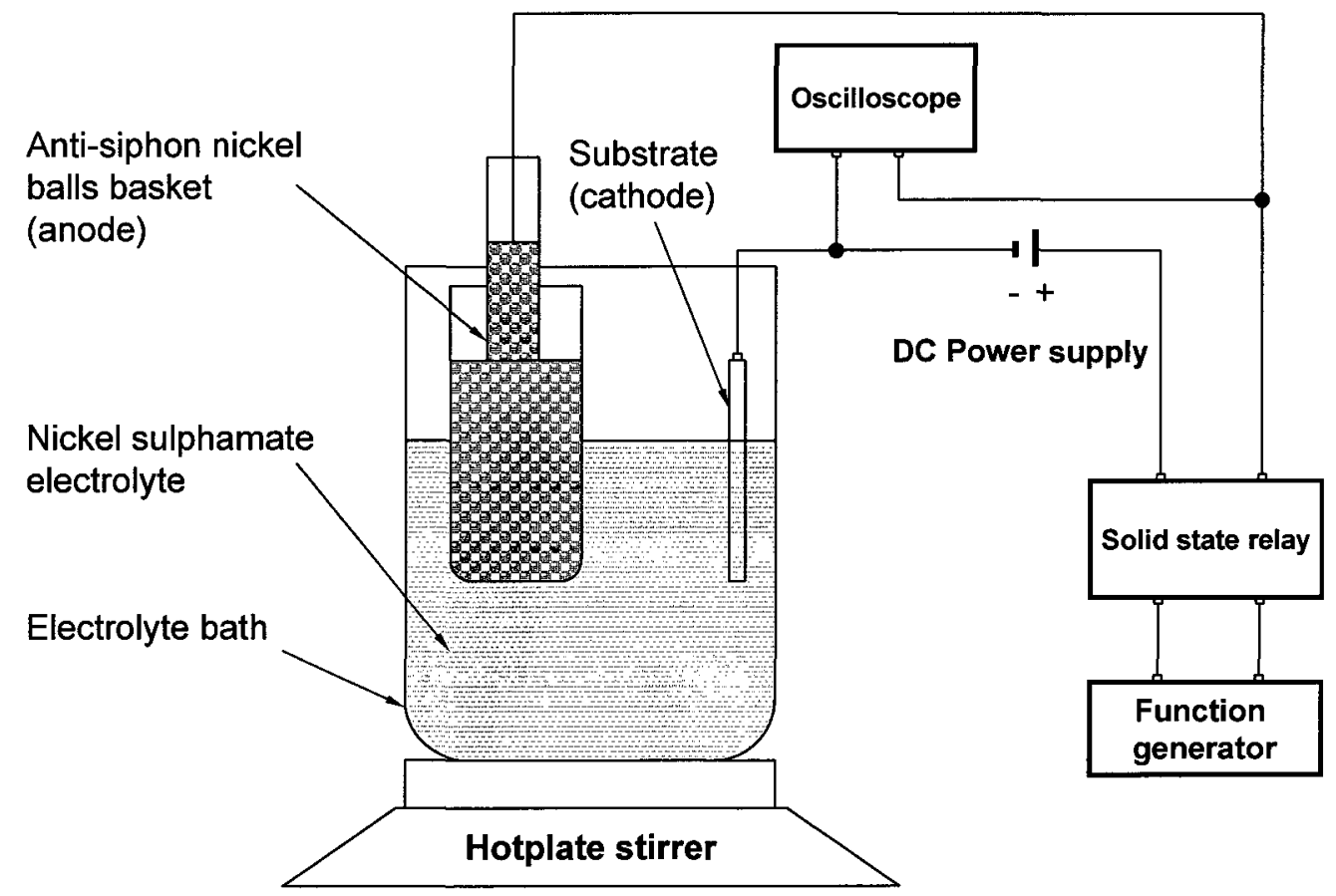

(b) Schematics of nickel electroplating setup and connection

Figure 3-1 Nickel electroplating apparatus. 


\subsubsection{Electrolyte Preparation}

In this study, the electrolyte was an aqueous solution made of $367 \mathrm{~g} / \mathrm{l}$ nickel sulphamate $\left(\mathrm{Ni}\left(\mathrm{NH}_{2} \mathrm{SO}_{3}\right)_{2}\right), 12 \mathrm{~g} / \mathrm{l}$ nickel chloride $\left(\mathrm{NiCl}_{2} \cdot 6 \mathrm{H}_{2} \mathrm{O}\right)$, and $36 \mathrm{~g} / \mathrm{l}$ boric acid $\left(\mathrm{H}_{3} \mathrm{BO}_{3}\right)$. A typical $\mathrm{pH}$ value of the solution was 1.5 . The solution was allowed to stabilize for 24 hours prior to the plating process.

The carbon nano-tube (CNT) dispersed nickel sulphamate electrolyte was prepared based on the conventional nickel sulphamate solution, as described above, with the addition of CNT. The CNT was CarboLex single-walled nanotube (SWNT) with a mean diameter of $1.4 \mathrm{~nm}[360]$. To help with the dispersion of CNT, a premixed solution containing CNTs $(2 \mathrm{~g} / \mathrm{l})$ and polyacrylic acid $\left(2 \times 10^{-5} \mathrm{M} / \mathrm{l}\right)$ were added to the nickel sulphamate electrolyte [361]. For further dispersion of CNTs, the electrolyte was subject to magnetic stirring (600 to $900 \mathrm{rpm}$ ) for at least 2 hours prior to each deposition processes. Sufficient soaking time helps in CNTs dispersion [295, 303, 362, 363].

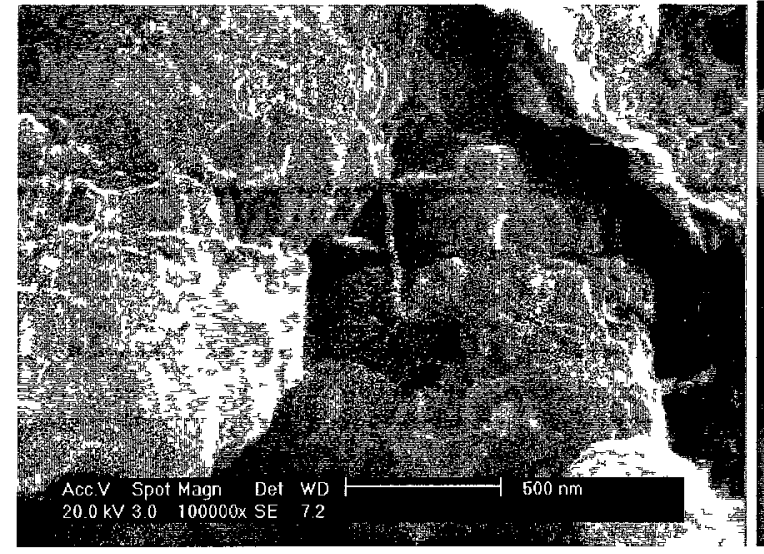

(a) Before dispersion

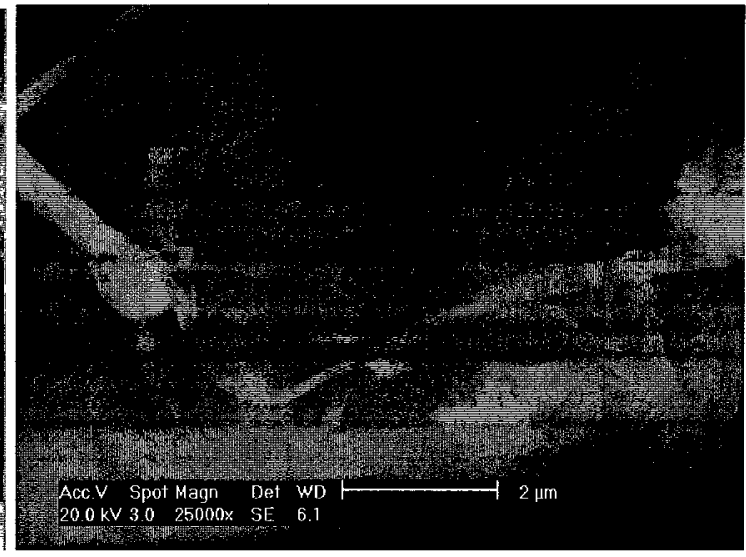

(b) After dispersion

Figure 3-2 SEM images of Carbon nanotubes. 
SEM images (Figure 3-2) show the CNTs before and after dispersion. Figure 3-2 (a) shows CNTs before dispersion in solution, where the sample was prepared by taping a thin layer of CNT onto conductive carbon tape. The images in Figure 3-2 (b) show the CNTs after dispersion in solution, where these samples were prepared by dropping diluted CNT dispersed solution on a polished stainless plate; after the water evaporated, the state of CNT dispersion was observed. It can be seen that the original CNTs conglomerate in the form of granules, while the dispersed CNTs show sufficient separation.

\subsubsection{Plating Procedure}

The average working current is determined by multiplying the average current density by the immersed total surface area of the substrate where the average current density is the product of the peak current density and the duty cycle [364]. In this study, the average current density was set at $20 \mathrm{~mA} / \mathrm{cm}^{2}$ which was in the typical range of current density for nickel plating $[365,366]$. By controlling sample size and immersion depth, the effective immersed substrate surface area was set to $12 \mathrm{~cm}^{2}$, so an average working current of $240 \mathrm{~mA}$ was employed.

During the plating process, the current stability was monitored. Normally, the working current decreases with plating progress, which is associated with the increase in hydrogen near electrodes and over potential on the electrode surface [127, 367]; this has been referred to as electrical double layer (or Helmholtz layer) which behaves as a charged capacitor [127]. An agitation helps prevent the current decrease [127]. It is critical to 
maintain the current at the initial value to avoid cracking of the plated surface due to micro-stresses generated during deposition with variable rates.

In this study, all deposition conditions were fixed, except the electrolyte and pulse frequency. The plating specifications are summarized in Table 3-2.

\subsection{Physical Vapor Deposition (PVD)}

In this study, physical vapor deposition (PVD) coatings were made using several deposition techniques with various deposition conditions. The coating systems included TiN and $\mathrm{CrN}$ based with various substrates. The coating specifications are summarized in Table 3-2.

\section{Electronic Beam (EB) Evaporation Deposition}

TiN coatings were deposited on 17-4PH steel substrates with diameters of 50 and $16 \mathrm{~mm}$, using an electron beam (EB) assisted PVD evaporation technique at Liburdi Turbine Services Inc. Due to the proprietary nature of process, the detailed operation parameters are not described here.

\section{Cathodic Arc (CA) Deposition}

TiN coatings were deposited on AISI 1040 mild steel (MS) and 17-4PH stainless steel (PH-SS) substrates using cathodic arc deposition technique (Metaplas MZR-304 
unfiltered cathodic arc (CA) coater installed at IAR, NRC). Prior to the deposition, the substrates were subjected to an ion cleaning using arc enhanced glow discharge (AEGD) with a titanium cathode.

Four metallic Ti cathodes and $\mathrm{N}_{2}$ gas were employed to form $\mathrm{TiN}$ through a reactive process. During the deposition process, the chamber pressure was controlled at $8 \times 10^{-3}$ mbar for a total of 480 ampere-hours. The substrates were negatively biased at $200 \mathrm{~V}$, and heated to $400^{\circ} \mathrm{C}$ with an electrical resistance heater.

\section{Plasma Enhanced Magnetron Sputtering (PEMS) Deposition}

The $\mathrm{CrN}$ based coatings containing $\mathrm{Si}$ and $\mathrm{C}$ additions (including $\mathrm{CrN}, \mathrm{CrSiCN}(1)$, $\mathrm{CrSiCN}(2)$ and $\mathrm{CrSiCN}(3)$ ) were deposited using a plasma enhanced magnetron sputtering (PEMS) coater at Southwest Research Institute, San Antonio, USA. The substrates were subject to an Ar sputter cleaning for 60 to 90 minutes to remove the residual oxide. A solid $\mathrm{Cr}$ target was employed as a source of $\mathrm{Cr}[368,369]$. In addition to a mixture of $\mathrm{Ar}$ and $\mathrm{N}_{2}$ gases, trimethylsilane $\left(\left(\mathrm{CH}_{3}\right)_{3} \mathrm{SiH}\right.$ or $\left.3 \mathrm{MS}\right)$ was introduced during the deposition process; where, the dissociation of trimethylsilane provides $\mathrm{Si}$ and $\mathrm{C}$ to form the series of $\mathrm{CrSi}_{\mathrm{x}} \mathrm{C}_{\mathrm{y}} \mathrm{N}_{z}$ coatings. The gas flow rates were regulated using an optical emission monitor (OEM), and the chamber pressure was maintained between 0.33 and $0.47 \mathrm{~Pa}$ ( 2.5 to $3.5 \times 10^{-3}$ Torr). The substrate surface temperature was maintained at about $400^{\circ} \mathrm{C}$ during the deposition process. 
Table 3-2 Summary of produced coating systems

\begin{tabular}{|l|c|c|c|}
\hline Coating system & Content & Substrate & Deposition process \\
\hline \multicolumn{4}{|c|}{ Electroplating } \\
\hline Ni-0 & Nickel & MS & Electroplating, no pulsed current \\
\hline Ni-5 & Nickel & MS & Electroplating, pulsed 5 Hz \\
\hline Ni-100 & Nickel & MS & Electroplating, pulsed $100 \mathrm{~Hz}$ \\
\hline Ni-1000 & Nickel & MS & Electroplating, pulsed 1000 Hz \\
\hline Ni-CNT-0 & Nickel + CNTs & MS & Electroplating, no pulsed current \\
\hline Ni-CNT-5 & Nickel + CNTs & MS & Electroplating, pulsed 5 Hz \\
\hline Ni-CNT-100 & Nickel + CNTs & MS & Electroplating, pulsed 100 Hz \\
\hline Ni-CNT-1000 & Nickel + CNTs & MS & Electroplating, pulsed 1000 Hz \\
\hline \multicolumn{5}{|c|}{ PVD } \\
\hline EB TiN & TiN & $17-4$ PH & Electron beam \\
\hline CA TiN/MS & TiN & MS & Cathodic arc \\
\hline CA TiN/SS & TiN & $17-4$ PH & Cathodic arc \\
\hline CrN & CrN & $17-4$ PH & PEMS \\
\hline CrSiCN(1) & CrSiCN & $17-4$ PH & PEMS \\
\hline CrSiCN(2) & CrSiCN & $17-4$ PH & PEMS \\
\hline CrSiCN(3) & CrSiCN & $17-4$ PH & PEMS \\
\hline
\end{tabular}

\subsection{Post Deposition Treatment}

For comparison purposes with regard to the factor of coating defect in corrosion behaviours, some of the PVD coated samples were subject to surface treatment with polymethyl methacrylate (PMMA) to seal or limit the coating defects; and were tested for corrosion behaviours. Polymethyl methacrylate (PMMA) is synthetic polymer of methyl methacrylate (MMA). PMMA exhibits excellent optical transparency and ultraviolet 
(UV) resistance, is highly inert to acids and alkalis, and has a low water absorption rate [370]. PMMA has been widely used in the fabrication of nanoporous structures, where PMMA fills the nano-scaled pores by polymerization of the MMA monomer. Benzoyl peroxide acts as a radical supplying agent [371 - 373].

In this study, the PMMA solution was prepared with 16.6 wt.\% poly(methyl methacrylate)(PMMA, $\left.\left(\mathrm{C}_{5} \mathrm{O}_{2} \mathrm{H}_{8}\right)_{\mathrm{n}}\right), 78.4$ wt.\% methyl methacrylate(MMA) and 5 wt.\% benzoyl peroxide. Because sufficient time is required for the solution to equilibrate, the PMMA solution was sealed in a glass container at room temperature, and stored in a dark place for 24 hours [361, 374].

The samples were cleaned with acetone in an ultrasonic bath for 20 minutes, and then air dried. The samples were then individually immersed in the PMMA solution, with the coating surface facing upward. The container was then sealed and stored in a dark place for 24 hours at room temperature. In the first few hours, ultrasonic agitation was used to help remove air from the pores or open voids in the coating and enhance solution penetration.

After 24 hours of immersion, the samples were removed from the solution and immediately cleaned with a dry soft tissue to remove any residual PMMA solution. The cleaned samples were then cured under ultraviolet light at $30^{\circ} \mathrm{C}$ for 8 hours to assist polymerization of the PMMA. The finished samples were individually sealed in labeled plastic bags. 


\subsection{Coating System Evaluations}

In this chapter, experimental procedures for characterizing the coatings are described. These include experimental evaluations of coating microstructure (phase and grain size), surface morphology, defects, chemical composition, mechanical properties (hardness and modulus of elasticity), and other properties which include functional responses to wear, erosion and corrosion that were evaluated using pin-on-disk sliding wear, sand erosion, polarization, and EIS tests.

\subsubsection{Coating Thickness Measurement}

Two methods were used to measure coating thickness, cross sectional images and the ball crater method. The former is a direct measurement and can be used for almost all types of coatings. And the latter one is an indirect measurement based on geometrical calculation and can only be applied to a coating system on which a through crater can be generated with an abrasive ball without inducing heavy plastic deformation to the coating.

Because of their ductility, the thicknesses of the Ni plating samples were measured using cross sectional image analysis with the assistance of Clemex Vision PE 4.0 software, whereas, the brittle, PVD coating thickness was measured using the ball crater method.

In a ball crater setup (Figure 3-3), the specimen is fixed on a tilt clamp. An abrasive ball (25.4 mm diameter WC-Co was used) is set between the specimen and a driving roller. 
Abrasive corundum $(0.5 \mu \mathrm{m})$ grinding paste was applied on the contact surfaces between the WC-Co ball and the coating. With the abrasive ball spinning, a crater on the coating sample is created. After the coating is ground through, the radii of the two circles generated on the coating surface, which correspond to the coating surface and the substrate surface (Figure 3-4), can be used to calculate the thickness of the coating by simple application of Pythagorus theorem (Eq. 3-1)

$$
\delta=H_{s}-H_{c}=\sqrt{R_{b}^{2}-r_{s}^{2}}-\sqrt{R_{b}^{2}-r_{c}^{2}}
$$

where,

$R_{b}$ : the radius of abrasive ball, $r_{s}$ and $r_{\mathrm{c}}$ : the measured radii of small and large circle.

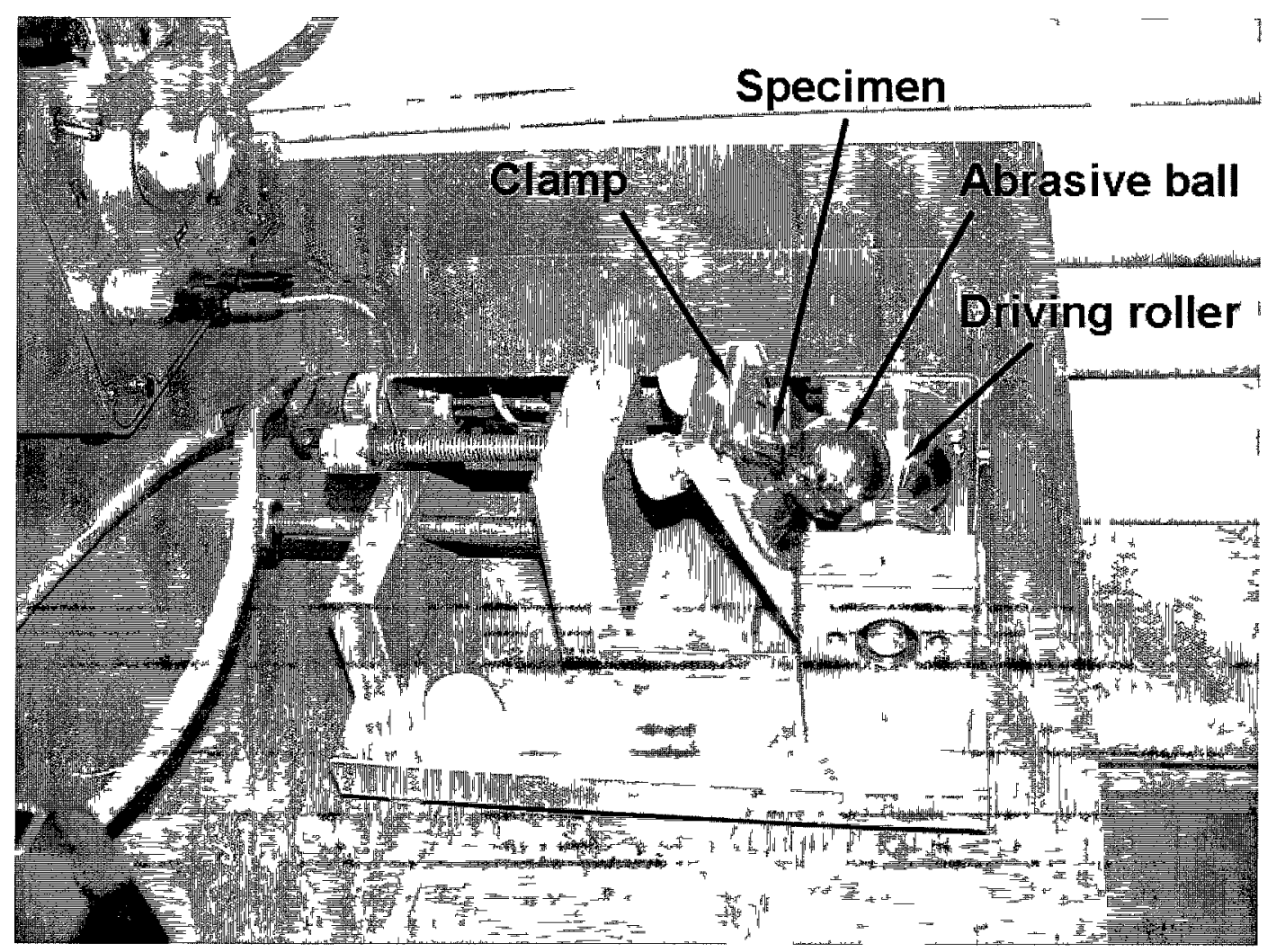

Figure 3-3 Setup of ball crater method of measuring thickness of coating (IAR-NRC Canada). 


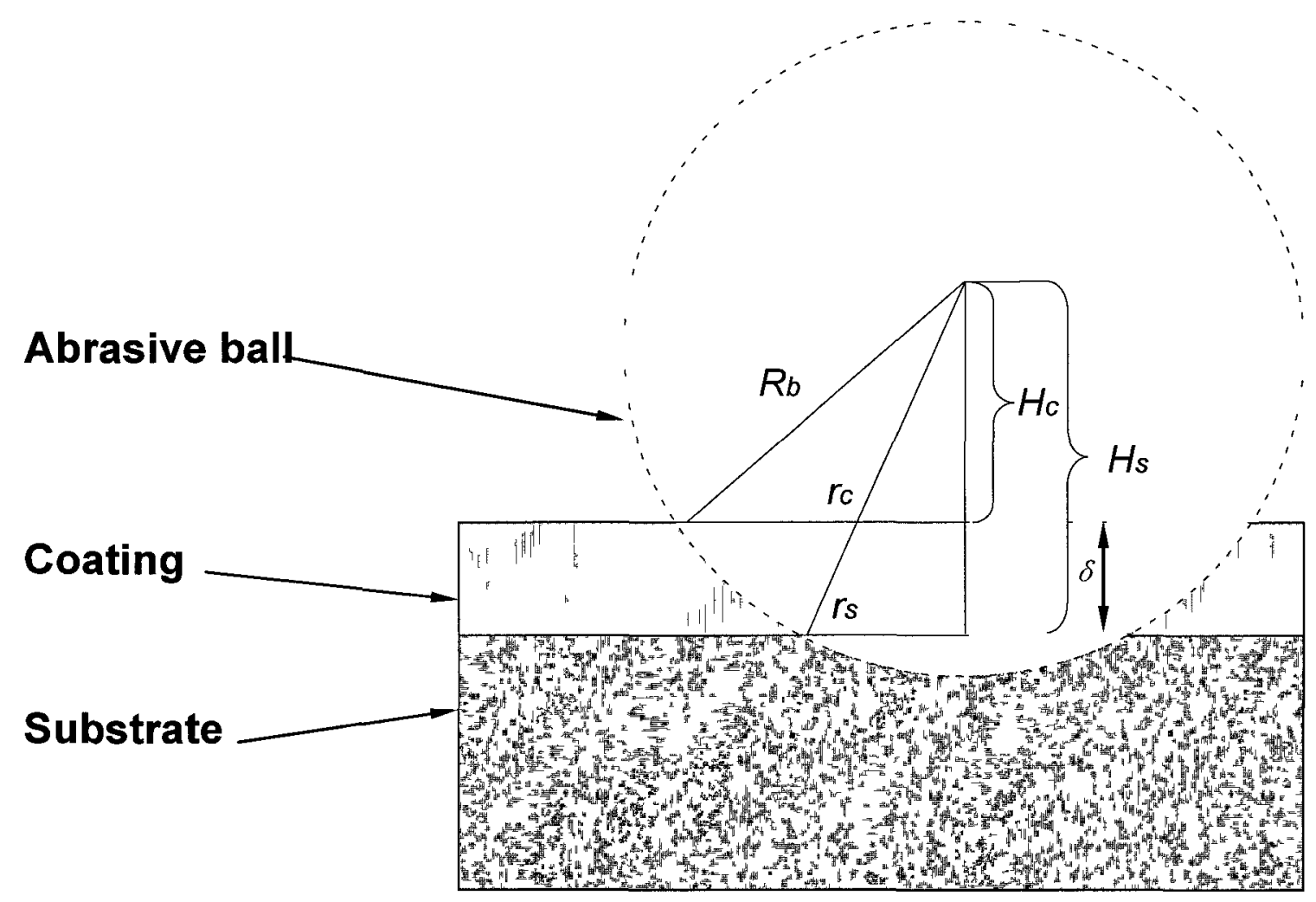

Figure 3-4 Geometry of ball crater method of measuring coating thickness.

\subsubsection{Scanning Electron Microscope (SEM) Analyses}

Scanning electron microscopy (SEM) (Philips XL30-S FEG) was used to determine surface topography, microstructure, and chemical composition of the coatings.

Surface topography was examined by SEM under a secondary electron (SE) mode. With very narrow electron beams multi-direction emitting, SEM (SE mode) produces micrographs with a deep field and a three-dimensional characteristic, which is beneficial to understanding surface structure. Back-scattered electron (BSE) images were also 
generated in which the regions containing different compositions exhibit different contrast.

In this study, the grain sizes of the coating systems were estimated from morphology images. Deposition defects including embedded nodule, void, and pinholes were observed, and were then statistically evaluated with the assistance of image analysis software (Clemex Vision PE 4.0). The images of coatings after mechanical and chemical attacks were used in the estimation of wear, erosion and corrosion.

\subsubsection{X-Ray Diffraction (XRD) Analysis}

In this study, an X-Ray diffractor (XRD) (Rigaku CN 2631 X-Ray diffractometer) was employed for the investigation of crystal structures of the coating systems. A Cu Ka XRay source with a wavelength $(\lambda)$ of $0.154 \mathrm{~nm}$ was utilized, and scan ranges $(2 \theta)$ were set from 20 to $100^{\circ}$. The rates of scanning were set according to the desired resolutions. The X-Ray peak identifications and lattice parameter calculations were conducted with a DataScan 3.1 software package (Materials Data, Inc).

\subsubsection{Mechanical Properties}

The mechanical properties of the samples, i.e., hardness $(\mathrm{H})$ and modulus of elasticity (E), were measured using a nano-indentation hardness tester, a CSM Nano Hardness Tester (NHT). 
For thin film materials, the correlation between load and projected residual macro- or micro-indentation area is not as certain as that for bulk materials [101,375], therefore nano-indentation techniques were used.

In a nano-indentation test, light loads are applied to an indenter with a nano scaled and a high precision shaped tip (usually a Berkovich tip with a three-side pyramid geometry), which allows the properties of the coatings to be measured without any influence from substrate. The curves of load vs. depth of penetration for both loading and unloading stages are recorded. From the curves, mechanical properties (hardness and Young's modulus) of the material can be inferred using the Oliver-Pharr method [376].

The hardness is given by the following equation, which relates the maximum load to the projected indentation area:

$$
H=\frac{P_{\max }}{A_{r}}
$$

where,

$P_{\max }$ : maximum load,

$A_{r}: \quad$ projected indentation area.

Note that in a nano-indentation test, to avoid the influence from the substrates, the depths of indentation must be controlled to be less than $1 / 10$ thickness of the coating $[98,377$, 378]. In this study, a load of 50 or $100 \mathrm{mN}$ were applied depending on the thickness of the coatings. Both loading and unloading rates were set to $100 \mathrm{mN} / \mathrm{min}$ for the load of $50 \mathrm{~g}$ or $200 \mathrm{mN} / \mathrm{min}$ for the load of $100 \mathrm{~g}$. For each sample, at least 12 measurements were randomly conducted over the test area, and the average values used. 
Elastic properties can be determined from the maximum penetration depth (when under loading) in comparison with the residual depth of the indentation (upon unloading) [98]. The elastic modulus (Young's modulus, E) of bulk material is the stress versus strain for the material under an elastic (reversible) deformation, which can be deduced from the slope of the unloading section in the load-displacement curve (Figure 3-5), using the Oliver and Pharr method [379]:

$$
E=\frac{1}{2} \sqrt{\left.\frac{\pi}{A\left(h_{c}\right)} \frac{d P}{d h}\right|_{h=h_{\max }}}
$$

where,

$A\left(h_{c}\right):$ projected contact area of the indenter tip, $h_{c}$ and $h_{\max }$ : current and maximum contact depth, respectively.

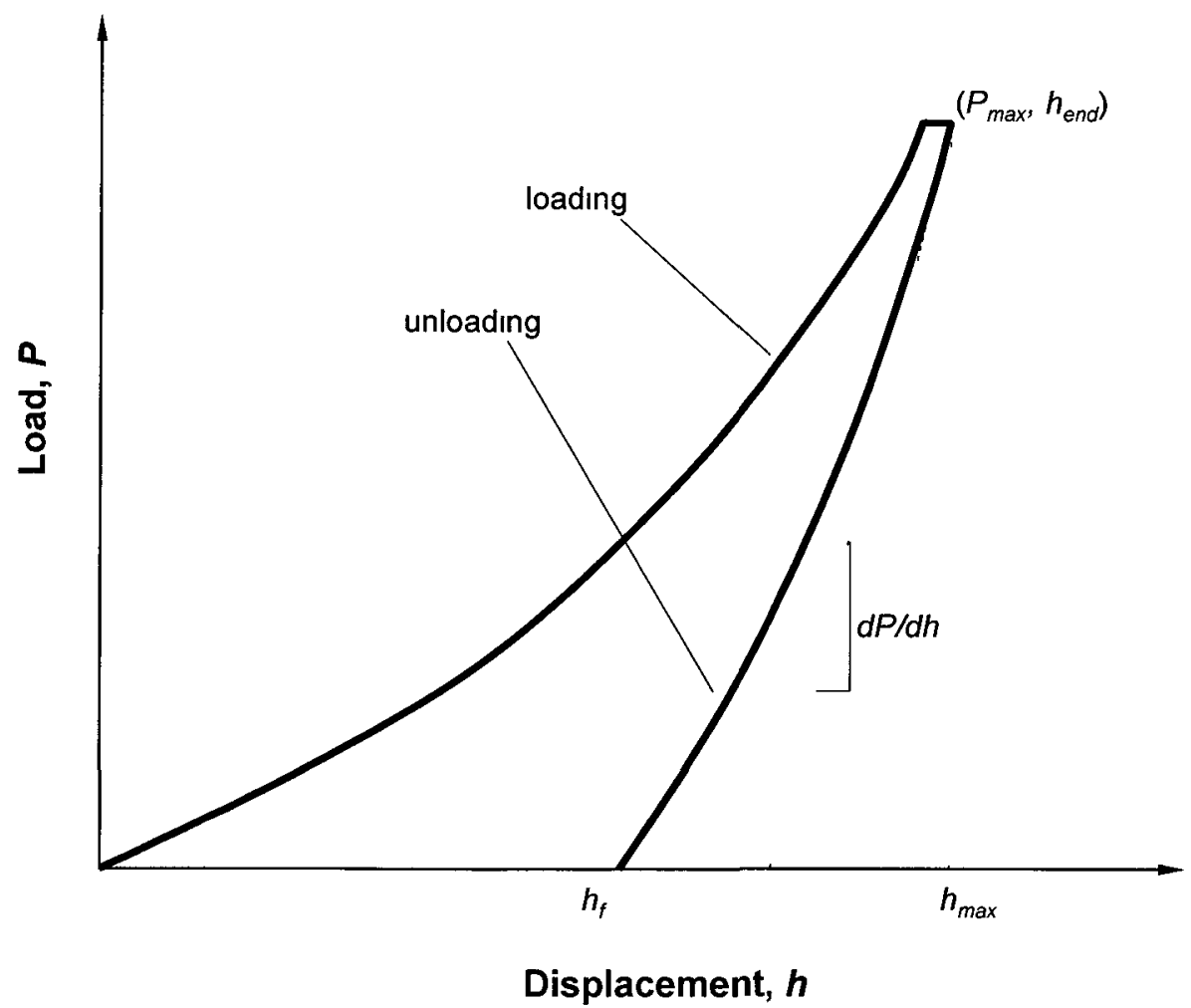

Figure 3-5 Schematic of the nanoindentation load-displacement curve (Oliver and Pharr model) (modified from [379]). 
For a specific nano-indentation tester and a specific sample, the coating's modulus $E_{\text {coatng }}$ can be calculated according to the Hertzian equation:

$$
\frac{1}{E}=\frac{\left(1-v_{\text {indenter }}^{2}\right)}{E_{\text {indenter }}}+\frac{\left(1-v_{\text {coating }}^{2}\right)}{E_{\text {coating }}}
$$

where,

$v_{\text {indenter }}$ and $v_{\text {coatng }}$ : the Poisson's ratios for the indenter and coating, respectively; $E_{\text {indenter }}$ and $E_{\text {coating: }}$ : the corresponding Young's modulus, in this study, assuming $v_{\text {tndenter }}=0.07$ and $E_{\text {indenter }}=1140 \mathrm{GPa}$ for the diamond indenter; and the Poisson's ratio $v$ for the coating materials was 0.3 .

\subsection{Tribological Behaviours}

\subsubsection{Wear}

\subsubsection{Principle and Equipment}

Wear of the coatings was evaluated by means of dry sliding using a TEER POD 2 pin-ondisc wear tester (TEER Coatings Ltd., UK)(Figure 3-6) at room temperature. The apparatus consists of a sample setup table, a driving system, a controlling and measuring system, and a data processing system.

The working principle of the TEER POD 2 pin-on-disc wear tester is demonstrated in a diagram (Figure 3-7). The specimen is horizontally clamped on the sample table. The wear pin is set against the specimen surface and offset from the rotating center. The offset distance is the radius of the wear track. The driving system rotates the sample table under 
the loaded wear pin. The control/measurement system controls the test facility, and measures test parameters. The data is stored, and processed by the TEER POD 2 software package.

In this study, the wear pin was a WC-Co $(6 \% \mathrm{Co})$ ball with a diameter of $5 \mathrm{~mm}$. The radius of the wear track was set to $4 \mathrm{~mm}$ (measured from the center of the pin to the centre of the specimen holder), and the rotational speed was set to $477 \mathrm{rpm}$, which corresponds to a linear speed of $200 \mathrm{~mm} / \mathrm{s}$.

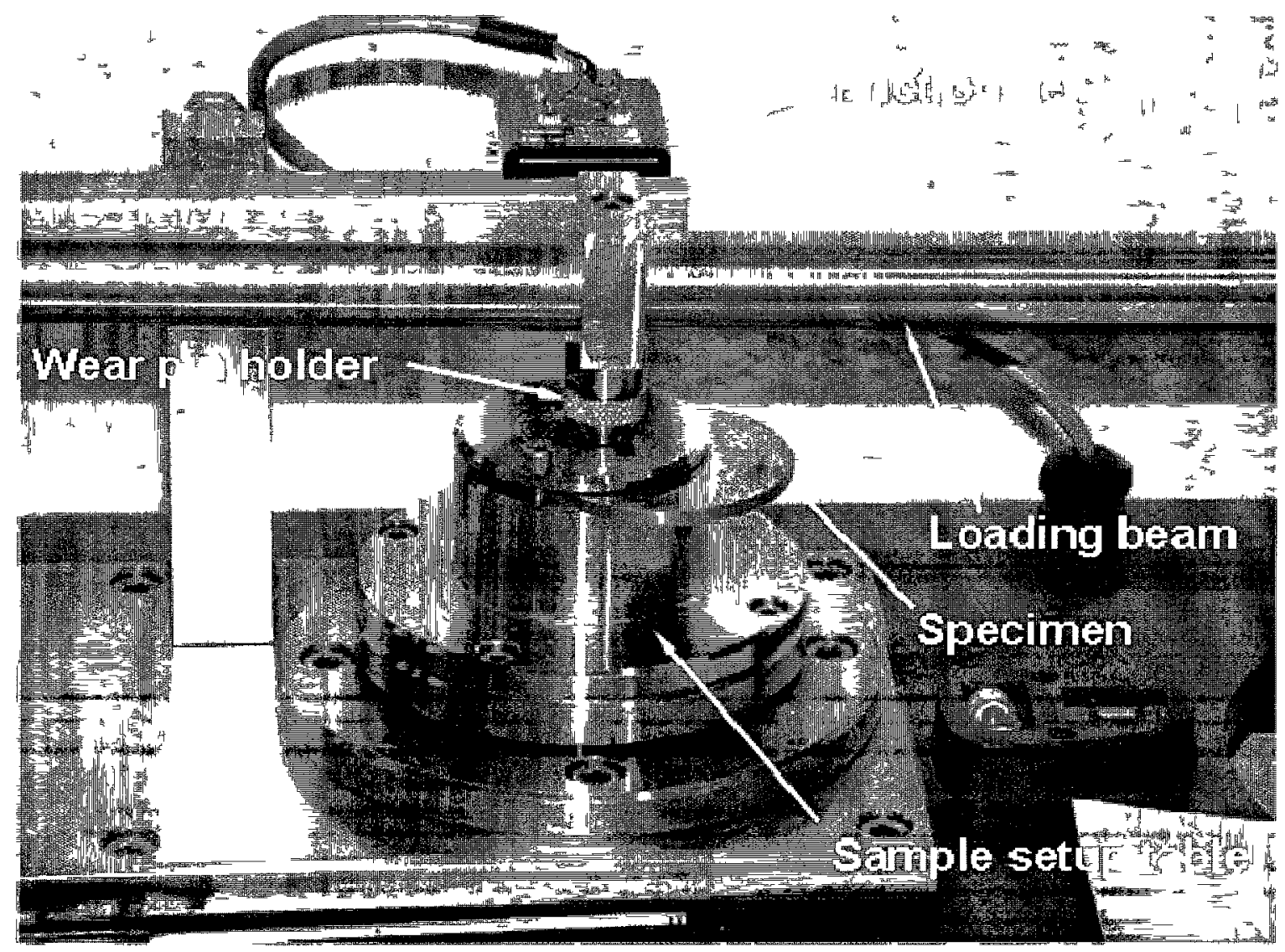

Figure 3-6 TEER POD 2 pin-on-disc wear tester (IAR-NRC Canada). 

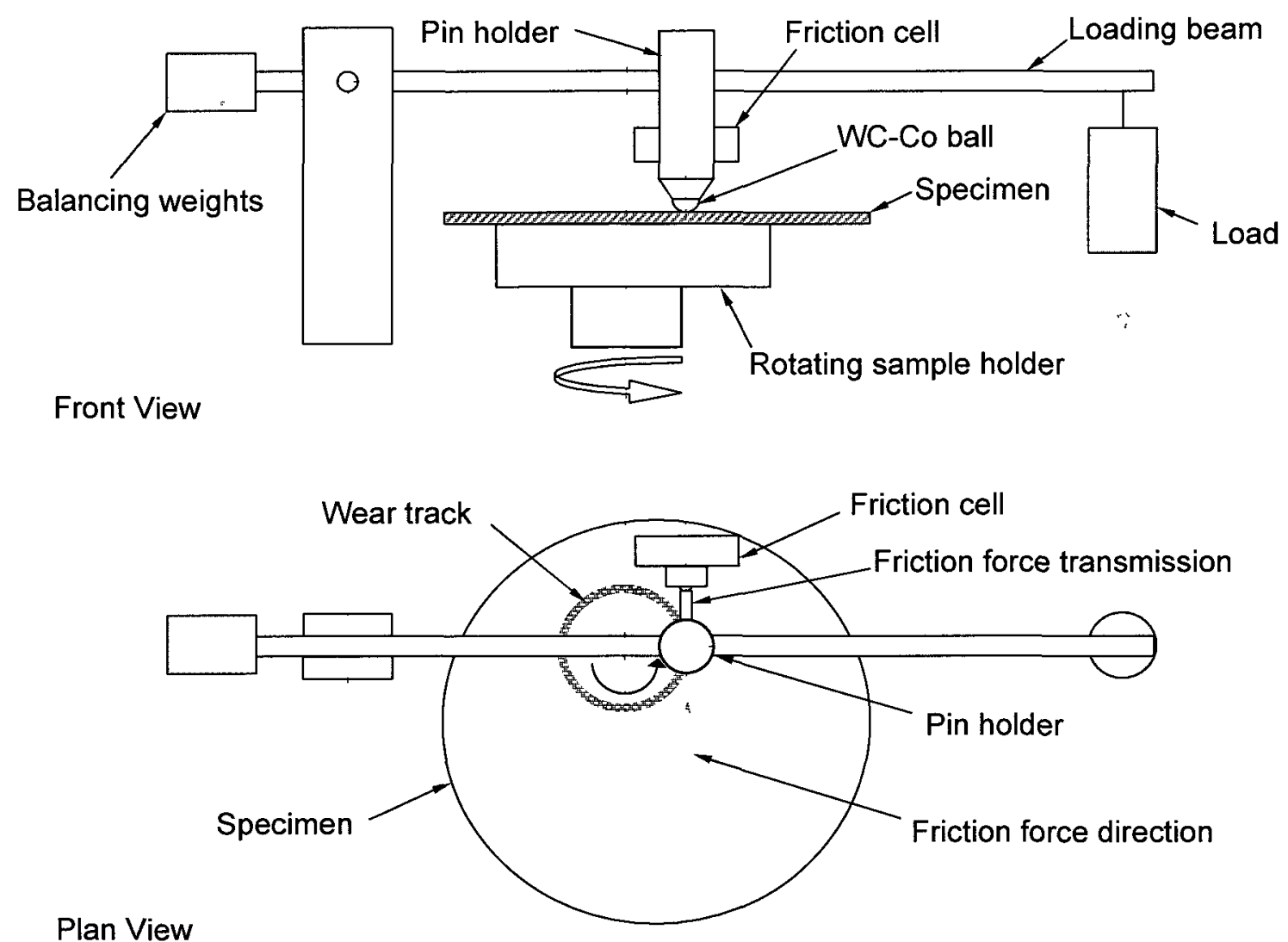

Figure 3-7 Diagram of a pin-on-disc wear tester work principle.

A properly selected load (dead weight) is applied to the end of the loading beam, and is transferred through the loading beam and WC-Co ball to the contact point between the ball and specimen. With the sample table spinning, the ball slides against the surface, generating a wear track circle. Frictional force, generated by the sliding motion between the ball and test surface, is measured by the friction cell. 


\subsubsection{Data Processing}

During the sliding test, frictional forces $\left(F_{\text {frnction }}\right)$ were measured by the friction cell, and the data was stored by the software TEER POD 2. The coefficient of friction (COF) $(\mu)$ between coatings and the WC-Co sliding ball can be calculated by dividing frictional forces $\left(F_{\text {frction }}\right)$ with the applied normal load $(\mathrm{N})$.

In the standard wear tests (ASTM Standard G99 [380]), the wear damage to the material is evaluated in terms of volume loss. To calculate the worn volume, the depth of the worn profile, and hence the cross sectional area is needed. In this study, the depths of the worn profiles were measured using a Veeco Dektak ${ }^{\circledR} 150$ Surface Profiler (Figure 3-8), and a typical wear profile is shown in Figure 3-9. The test data was processed using the software Veeco Dektak V. 9, with which the cross-sectional area $\left(A_{\text {coatng-wear }}\right)$ of the worn track can be obtained. Consequently, the worn volume $\left(V_{\text {coattng-wear }}\right)$ can be calculated using the average worn track cross-sectional area $\left(A_{\text {coattng-wear }}\right)$ and the radius of the wear track $\left(r_{\text {wear }}\right)$, as described by:

$$
V_{\text {coating-wear }}=2 \pi r_{\text {wear }} A_{\text {coating-wear }}
$$

As for the WC-Co sliding ball, using a geometrical relationship, the worn volume of spherical cap $\left(V_{\text {ball-wear }}\right)$ can be calculated as:

$$
V_{\text {ball-wear }}=\frac{2}{3} \pi R_{b}^{3}\left(1-\sqrt{1-\left(\frac{d_{\text {scar }}}{2 R_{b}}\right)^{2}}\right)-\frac{1}{3} \pi\left(\frac{d_{\text {scar }}}{2}\right)^{2} \sqrt{R_{b}^{2}-\left(\frac{d_{\text {scar }}}{2}\right)^{2}}
$$

where,

$R_{b}$ : the radius of WC-Co ball, $d_{s c a r}$ : the diameter of the scar on the abrasive (WC-Co) ball. 


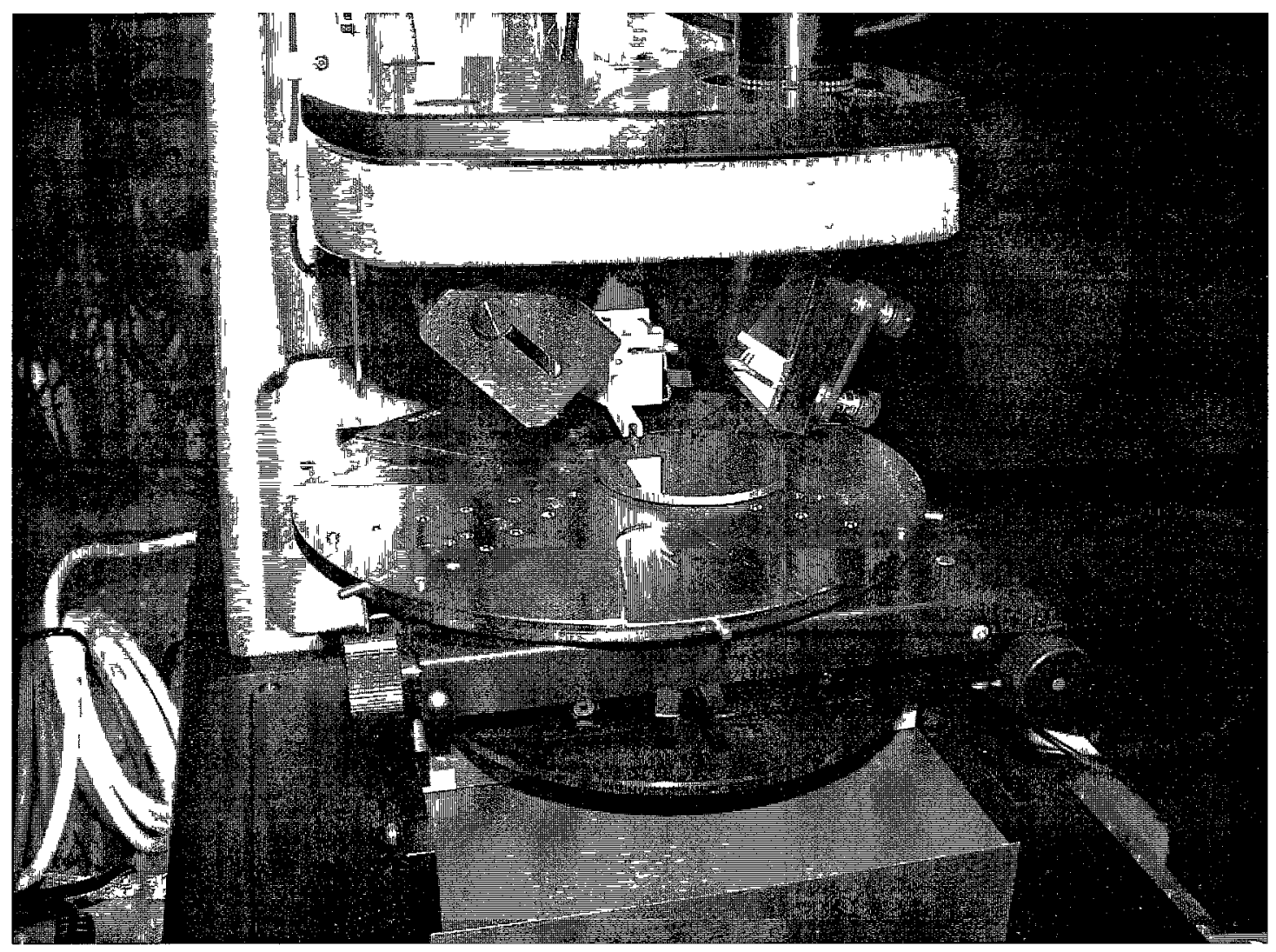

Figure 3-8 Veeco Dektak ${ }^{\circledR} 150$ surface profiler (IAR-NRC Canada).

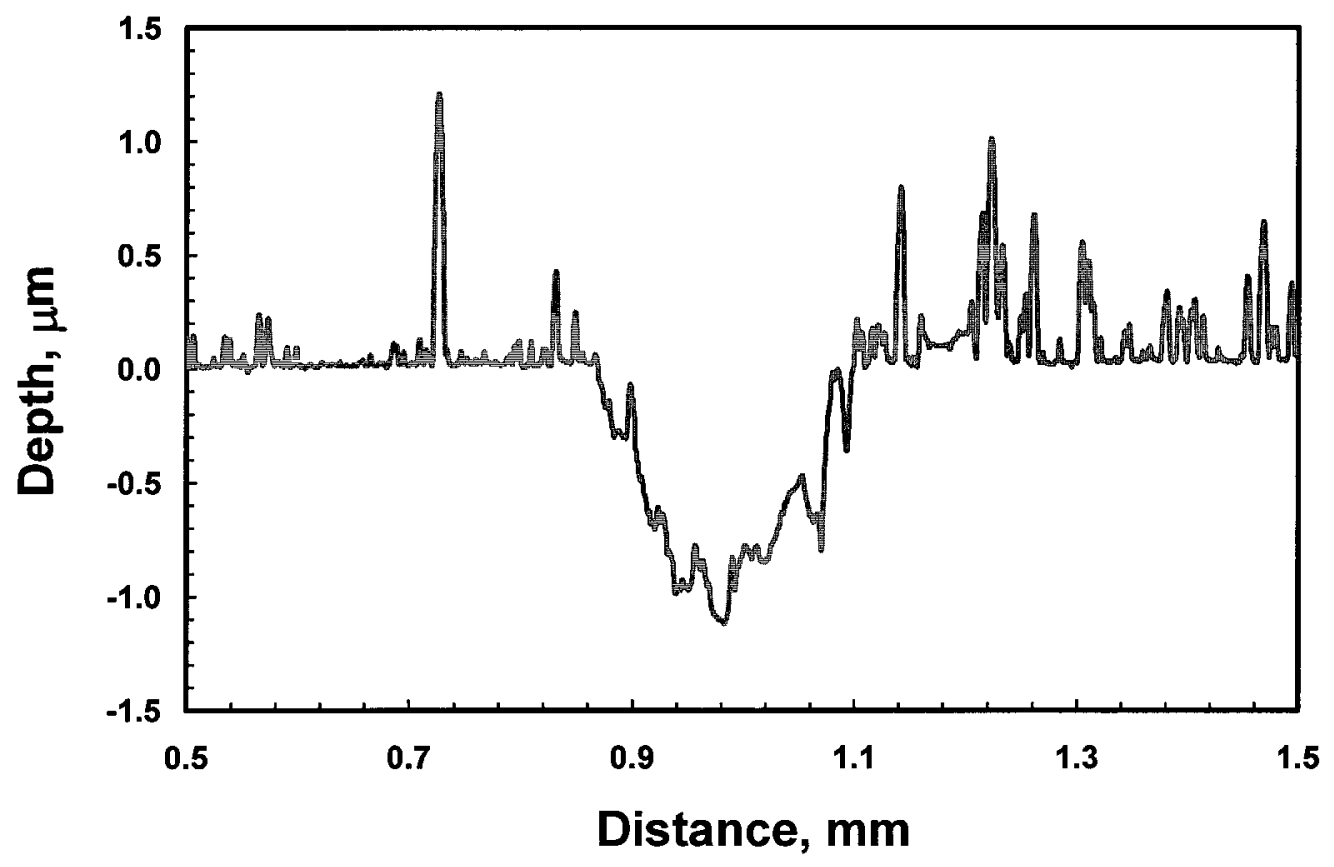

Figure 3-9 A typical wear profile on a TiN coating sample measured using Veeco Dektak ${ }^{\circledR} 150$ surface profiler. 
With wear volumes obtained, specific wear rates for both the coating and the ball can be obtained for a given normal load $(\mathrm{N})$ and the total sliding distance (L), using the following relationship:

$$
W_{\text {wear }}=\frac{V_{\text {wear }}}{N L}
$$

To take the hardness of the interacting materials into account in the wear behaviour, Archard linear wear law [65] was applied, which is given as [85]:

$$
V_{\text {wear }}=k_{A} \frac{N L}{H}
$$

Where,

$H$ : the hardness for one of the interacting materials, $k_{A}$ : Archard wear coefficient $(<1)[65,85]$.

Therefore, Archard wear coefficient $\left(k_{A}\right)$ is:

$$
k_{A}=\frac{V_{\text {wear }} H}{N L}
$$

It can be seen that $k_{A}$ describes a wear property associated with both specific wear rate and the hardness of the interacting materials.

At least 3 wear tests were randomly conducted on each sample, and at least 3 measurements were randomly conducted on each wear track; and the average values were obtained and used in analyses. 


\subsubsection{Erosion}

\subsubsection{Principle and Equipment}

In this study, the coated samples were evaluated for solid particle erosion behaviour in an inert environment at room temperature. Experiments were conducted according to the ASTM standard G76-02 [381], using a HME Airbrasive Unit (S.S. White Industrial, USA) test facility (Figure 3-10 a).

The test facility (Figure 3-10 c) consists of a test chamber, an erodent particle (sand) supply system, a compressed carrying gas supply system, and a dust collector, as are shown in Figure 3-10 (b). The gas supply system provides the carrier for the particulate flow. In this study, the carrying gas was compressed dry nitrogen gas $\left(\mathrm{N}_{2}\right)$, which also provides an inert environment surrounding the test samples avoiding oxidation to the freshly eroded surface that would affect the nature of the erosion process [382]. The sand supply system provides erodent, which in this study was angular AccuBRADE ${ }^{\circledR}-50$ Blend \#3 alumina $\left(\mathrm{Al}_{2} \mathrm{O}_{3}\right)$ powders (S.S. White Industrial, USA) with an average particle size of $50 \mu \mathrm{m}$ (Figure 3-11). The sand supply system is connected through a hose to the gas supply system. Sand in the holding tank is released with a magnetic vibration device, and blown by the carrying gas into a Teflon hose connected to the nozzle, thus generating a particulate stream. A powder regulation adjustment knob controls vibration voltage to the magnetic vibration device, thus controlling the erodent feed rate. A gas pressure adjustment knob controls the gas valve, and consequently the pressure of the carrying gas and the speed of the particulate stream. A scale (with an accuracy of $1 \mathrm{~g}$ ) is set under the 
sand container to monitor the weight change of the sand container, and calculates sand consumption during an erosion process.

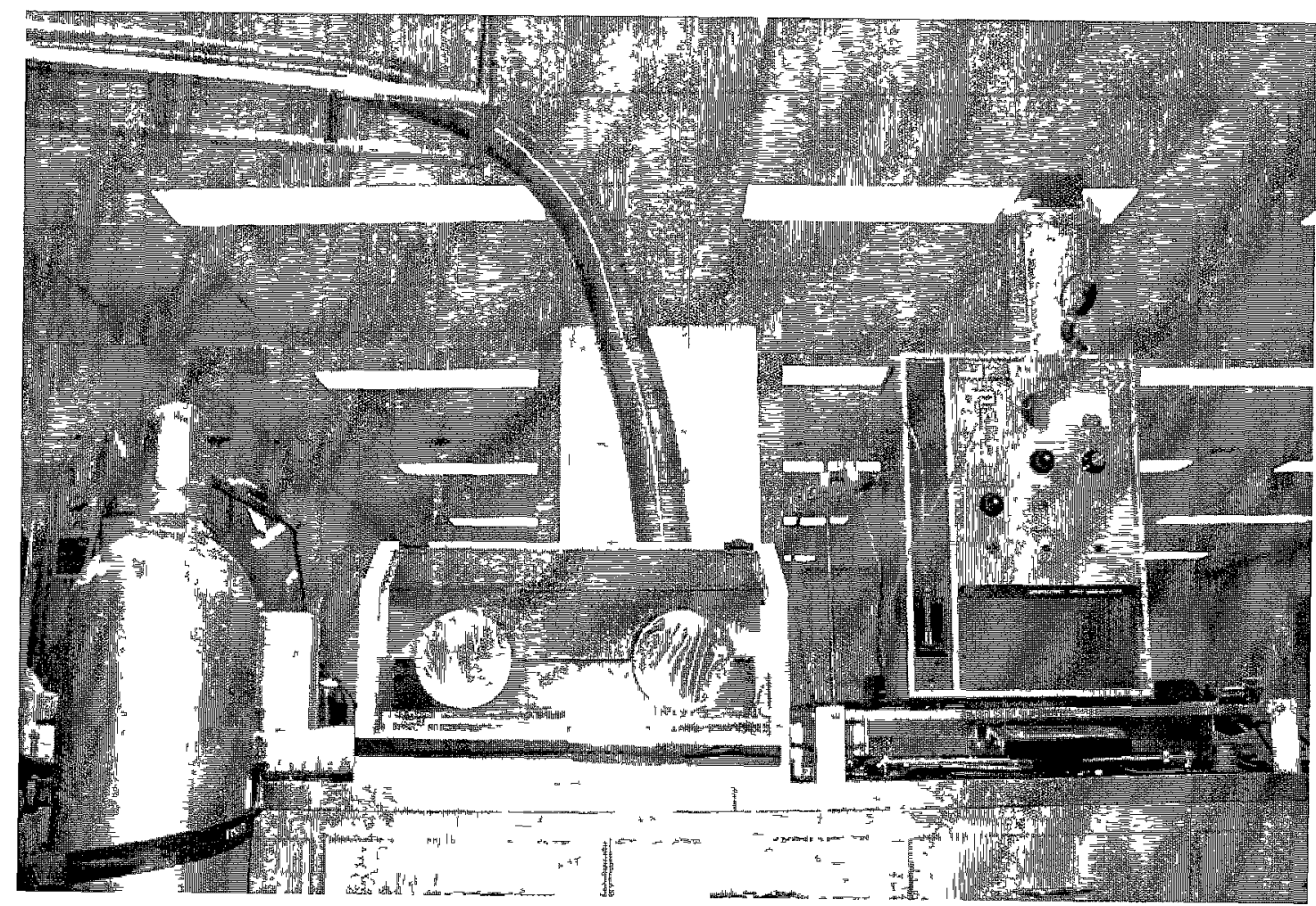

(a) HME Airbrasive unit (IAR-NRC Canada)

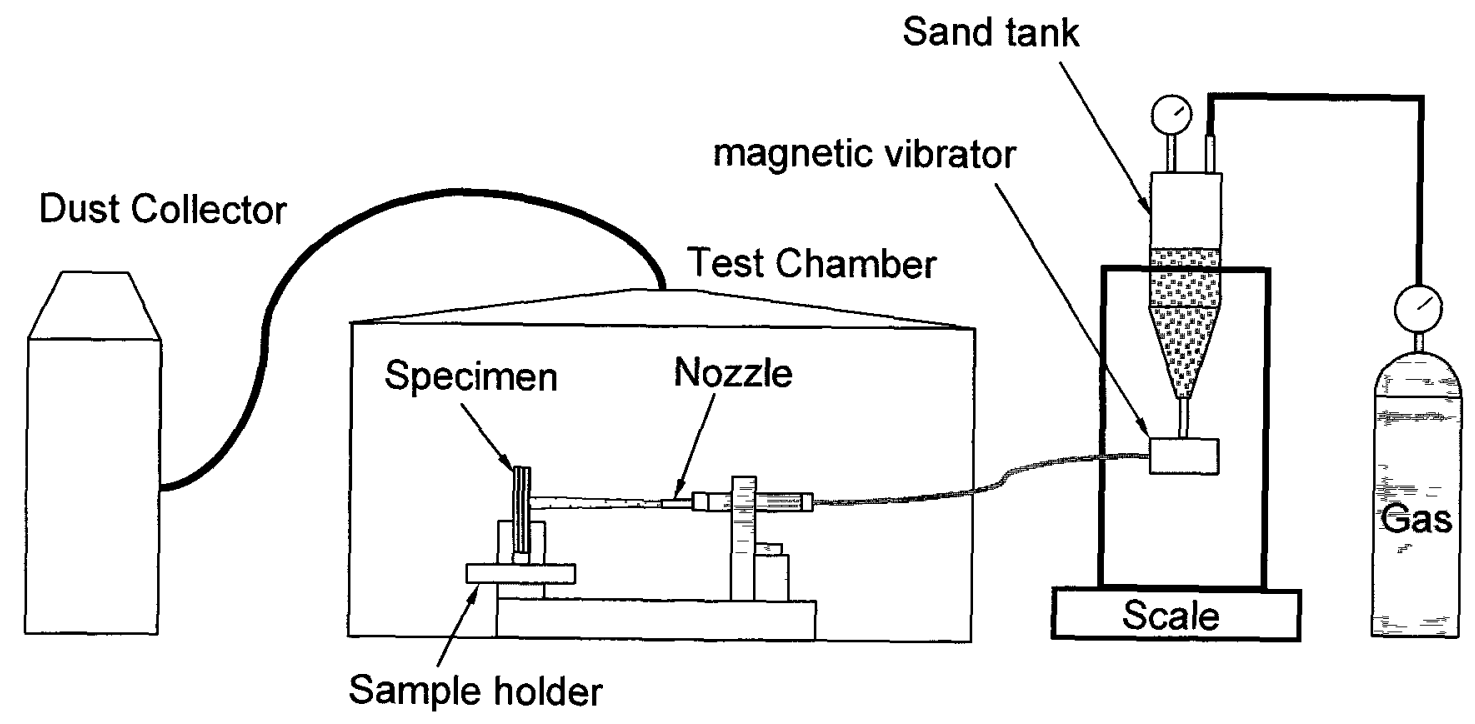

(b) Schematics of HME Airbrasive Unit working principle

Figure 3-10 HME Airbrasive unit and working principle. 




Figure 3-11 SEM micrograph of $\mathrm{Al}_{2} \mathrm{O}_{3}$ particles used in erosion testing

In the test chamber (Figure 3-12), the sample and a silicon carbide nozzle are fixed on a sample holder and a nozzle holder, respectively. The sample can be adjusted at various angles that correspond to impingement angles required in the erosion test; the nozzle can be adjusted to a height that matches the targeted area on the sample. The distance from the nozzle tip to the nominal face of the sample is fixed at $38 \mathrm{~mm}$. During the erosion test, debris is collected by a dust collector. The sample is weighed using a scale with an accuracy of $10^{-5} \mathrm{~g}$, before and after each erosion process cycle; and the weight loss is calculated. 


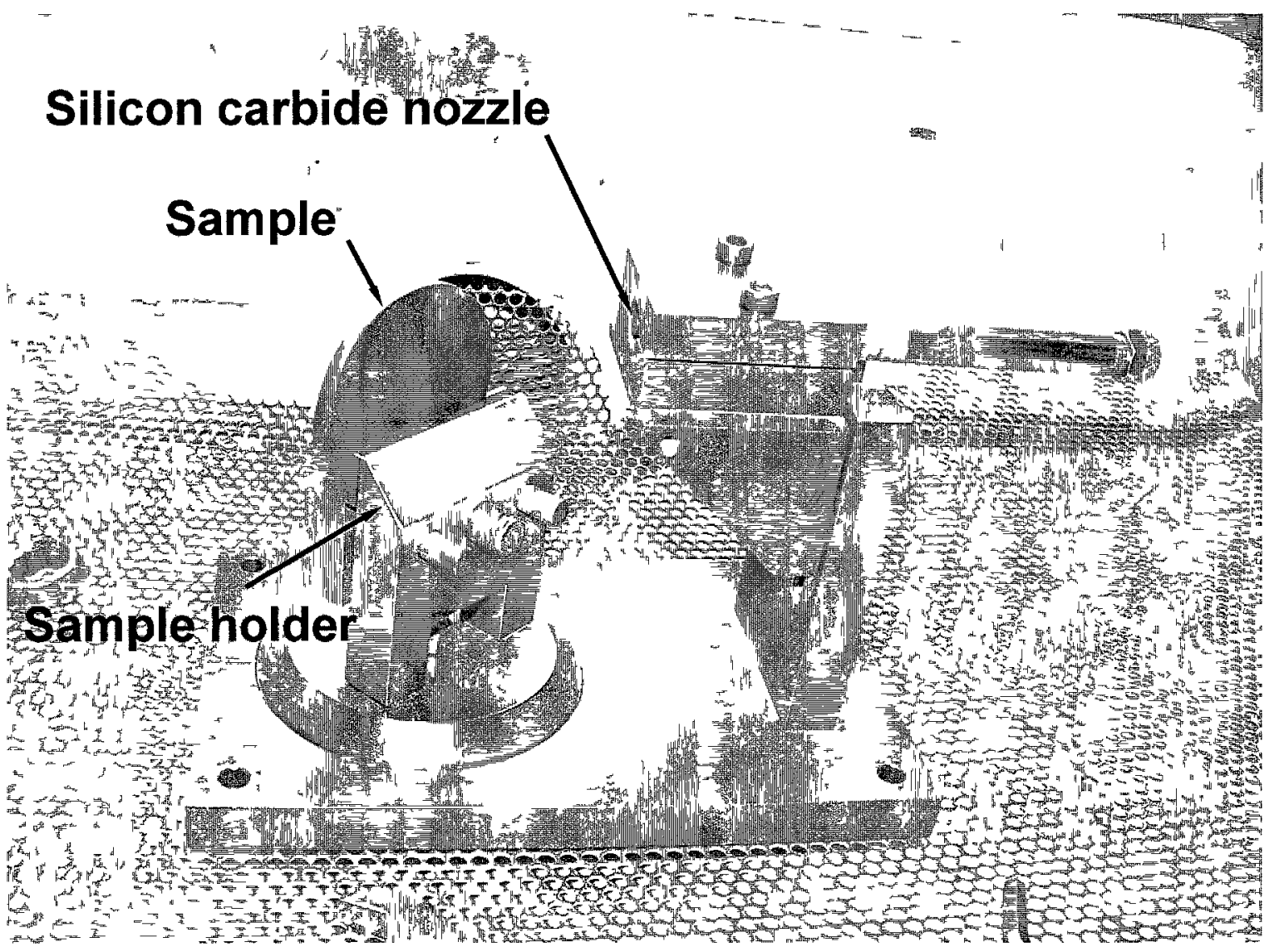

Figure 3-12 Sample and nozzle holder inside test chamber (IAR-NRC Canada).

\subsubsection{Data Processing}

The data obtained in erosion tests includes the mass of erodent used and the weight loss of coated samples. For a given density of the coating material, the corresponding volume changes can be calculated from the weight loss.

In this study, erosion is evaluated using cumulative weight loss. Using multiple measurements at each time interval, the errors inherent in the weight measurements can be minimized by averaging. Erosion rate is expressed as a rate of weight or volume of the coating material lost per unit of erodent and may take into account impingement angle. 


\subsection{Corrosion Behaviour of Coating-substrate Systems}

Corrosion was studied using potentiodynamic polarization and EIS, using a Gamry ${ }^{\mathrm{TM}}$ $\mathrm{PC} 14^{\mathrm{TM}}$ potentiostat system (Figure 3-13).

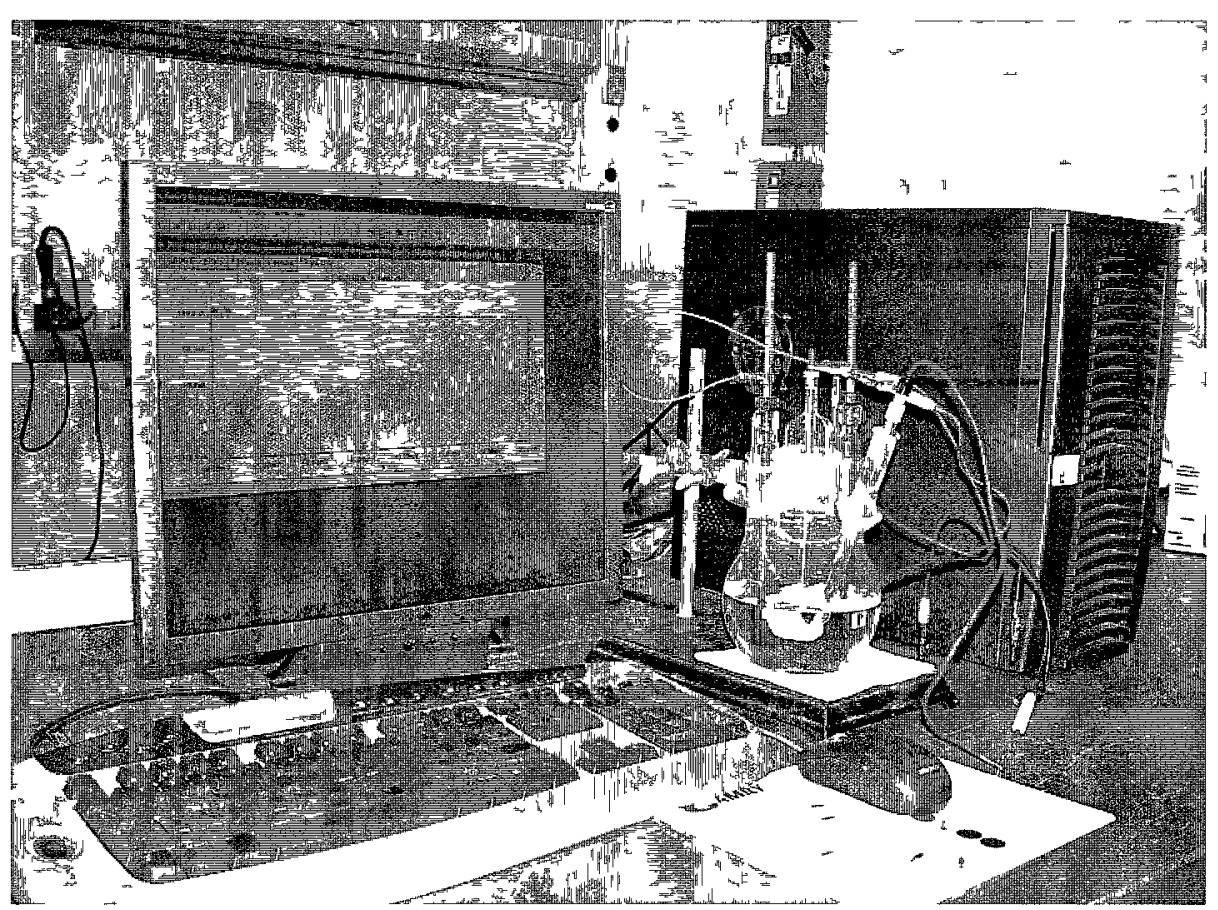

(a) Electrochemical experimental setup (IAR-NRC Canada)

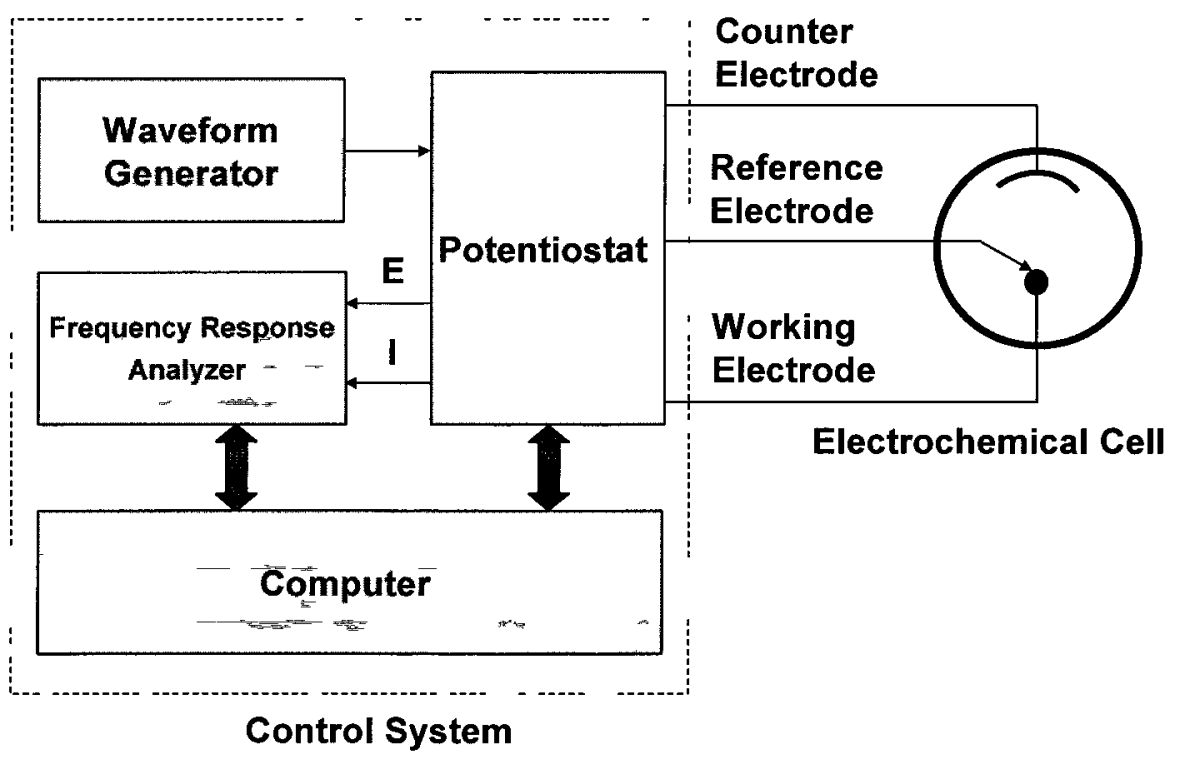

(b) Schematic diagram of the setup

Figure 3-13 Electrochemical experimental setup. 


\subsubsection{Experimental Setup}

The experimental system consists of an electrochemical cell and a control system that includes a data acquisition and analysis system. The electrochemical cell provides the environment for the measurement of electrochemical parameters, and consists of four primary components: (1) cell body, (2) electrolyte, (3) electrodes, and (4) devices monitoring and controlling the environment (Figure 3-14).



Figure 3-14 Electrochemical cell setup (IAR-NRC Canada). 
In this study, the cell body was a glass cell, which can be used in temperatures ranging from room temperature to $100^{\circ} \mathrm{C}$, and is inert to the common electrolyte ( $3.5 \mathrm{wt} . \%$ sodium chloride $(\mathrm{NaCl})$ aqueous solution in this study). Electrodes include a working electrode (test sample), auxiliary or counter electrodes (two carbon rods), and a reference electrode. The test sample (a disk with a diameter of $16 \mathrm{~mm}$ and thickness of $1.6 \mathrm{~mm}$ ) was mounted on a Teflon sample holder. The test surface of the sample is pressed against the O-ring and is exposed to the electrolyte through the aperture (with a diameter of $10 \mathrm{~mm})$.

To measure and control the potential across the interface between the specimen surface and electrolyte (interfacial potential) a counter electrode is introduced, which is electrically connected to the working electrode through the electrolyte. To overcome the variation in the interfacial potential, a reference electrode is introduced between the working and counter electrodes. This system is referred as to a three-electrode system, which provides a controllable potential between the working and reference electrodes [383]. In this study a saturated mercury-mercury (II) chloride (calomel) electrode (SCE) was used.

The control system consists of a computer, a potentiostat, a waveform generator, and frequency response analyzer. The potentiostat keeps the working electrode potential at a constant level with respect to the reference electrode. The waveform generator provides the electrical signal that gates the flow of current to the cell. Data was collected by the computer, and then out put to the frequency response analyzer for processing. 


\subsubsection{Operational Procedures}

Based on the American Society for Testing Materials ASTM G59 [384], testing methods ASTM G102 [385], and ASTM G5-94 [386], electrochemical experimental procedures were developed for this study. The procedures for potentiodynamic polarization and EIS tests are introduced in the following sections.

\subsubsection{Potentiodynamic Polarization Test}

The specimen was immersed in the $\mathrm{NaCl}$ solution for a few minutes until the solution reached equilibrium to establish the steady state open-circuit potential ( $\left.E_{O C P}\right)$ [38]. In the potentiodynamic polarization test, the polarization scan started at $-300 \mathrm{mV}$ vs. open circuit potential and ended at $1400 \mathrm{mV}$ vs. SCE with a scan rate of $5 \mathrm{mV} / \mathrm{s}$. The scan could be terminated if the current density reached $1 \mathrm{~mA} / \mathrm{cm}^{2}$. Experimental data were collected and then analyzed with Gamry Electrochemistry DC105 ${ }^{\mathrm{TM}}$ software.

\subsubsection{Electrochemical Impedance Spectroscopy (EIS) Test}

In this study, all EIS tests and measurements were carried out at an open circuit potential. A sinusoidal AC perturbation of $10 \mathrm{mV}$ (rms) amplitude coupled with the corrosion potential was applied to the electrode in the frequencies from $1 \times 10^{-2}$ to $3 \times 10^{5} \mathrm{~Hz}$ using five points/decade. As the corrosion potential reached a stable state (a variation of less than $5 \mathrm{mV}$ in $5 \mathrm{~min}$ ), the electrode potential was recorded. The electrode potentials were recorded at regular intervals throughout the test process. The test could last several days, 
depending on the specimen's corrosion performance. Note that, for an EIS test the sample must be immersed in the solution until the last data is recorded.

\subsubsection{Data Processing}

Electrochemical measurement data was concurrently recorded during the course of the experiments and was later processed using software (Gamry Electrochemistry EIS300 ${ }^{\mathrm{TM}}$ ).

From potentiodynamic polarization test data, a potentiodynamic polarization curve can be plotted, where $\mathrm{x}$ - and $\mathrm{y}$-axes correspond to the two primary parameters, current density (i) and potential $(E)$, respectively as shown in Figure 3-15. Characteristic regions can be identified on the curve, such as active, passive, and transpassive regions. Several parameters corresponding to those regions can be determined, including corrosion potential, current density, passivity potential, and pitting potential.

Combining Tafel extrapolation, important parameters such as corrosion current $\left(I_{c o r r}\right)$ can be obtained using Stern-Geary equation $[146,127]$ :

$$
I_{\text {corr }}=\frac{\beta_{a} \beta_{c}}{2.3039\left(\beta_{a}+\beta_{c}\right) R_{p}}
$$

Tafel slopes $\left(\beta_{a}\right)$ and $\left(\beta_{c}\right)$ corresponding to anode and cathode respectively, can be obtained from Tafel plot. Polarization resistance $\left(R_{p}\right)$ can be obtained from the following relationship:

$$
R_{p}=\left.(\Delta E / \Delta I)\right|_{\Delta E \rightarrow 0}
$$


With these parameters, coating porosity $(P)$ can be estimated with the empirical equation $[158-160]:$

$$
P=\left(R_{p s} / R_{p}\right) \times 10^{-\mid \Delta E_{\text {corr }} / \beta_{a}}
$$

where,

$R_{p s}: \quad$ the polarization resistance of the substrate,

$\Delta E_{c o r r}$ : the difference in corrosion potential between the coated and bare substrates.

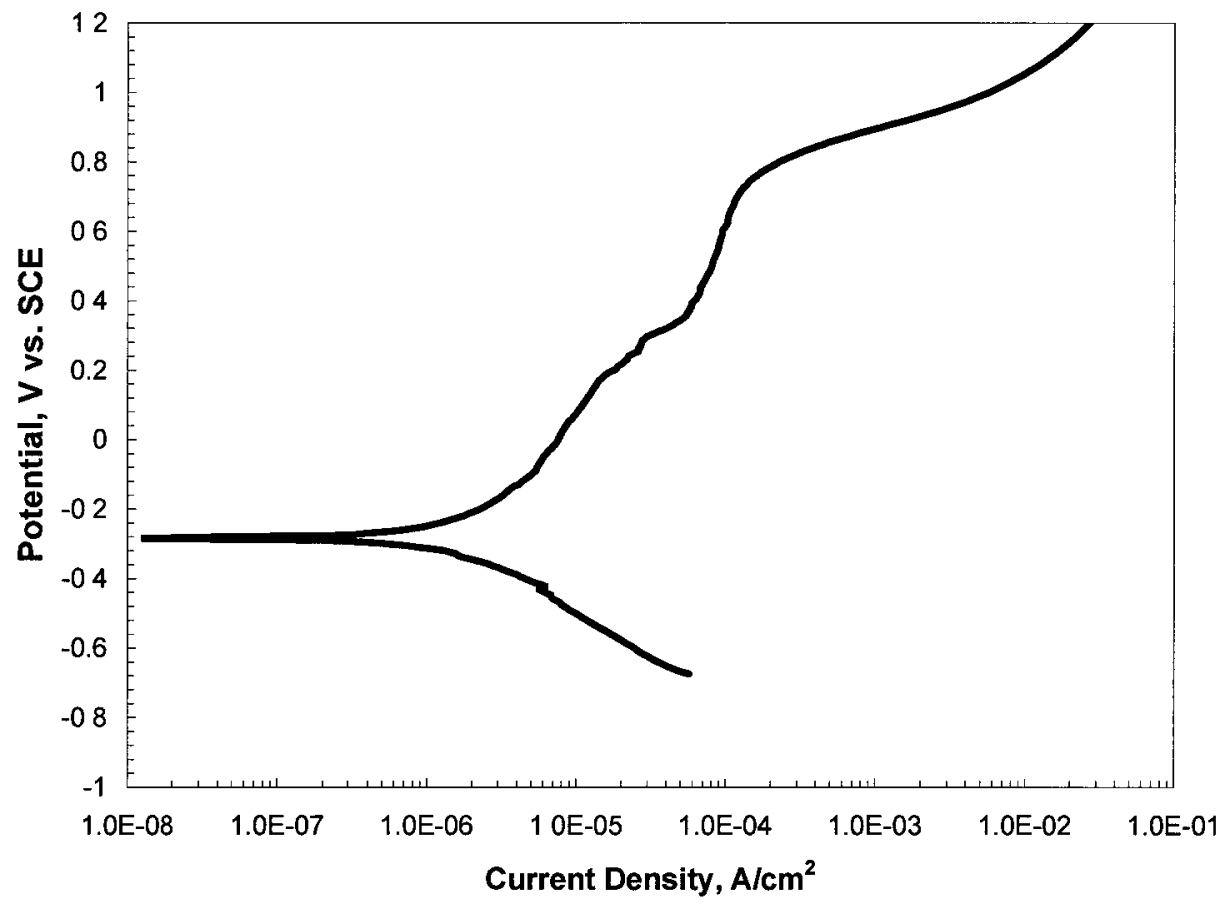

Figure 3-15 A typical potentiodynamic polarization curve obtained from a test on a $\mathrm{CrN} / \mathrm{SS}$ coating system.

For the EIS test, electrochemical impedance $Z(\omega t-\varphi)$, a complex number, is composed of a modulus $|Z|$ and a phase angle $(\varphi)$, both varying with frequency $(\omega)$. The real and imaginary components of the electrochemical impedance obtained at various frequencies can be plotted on $\mathrm{x}$ - and $\mathrm{y}$ - axes, respectively, generating a Nyquist plot as shown in Figure 3-16. The high and low frequency data are on the far left and right side of the 
plot, respectively. Each point on the curve represents data obtained at a certain scanning frequency $(\omega)$; and the angle between the vector and the x-axis represents the phase shift $(\varphi)$.

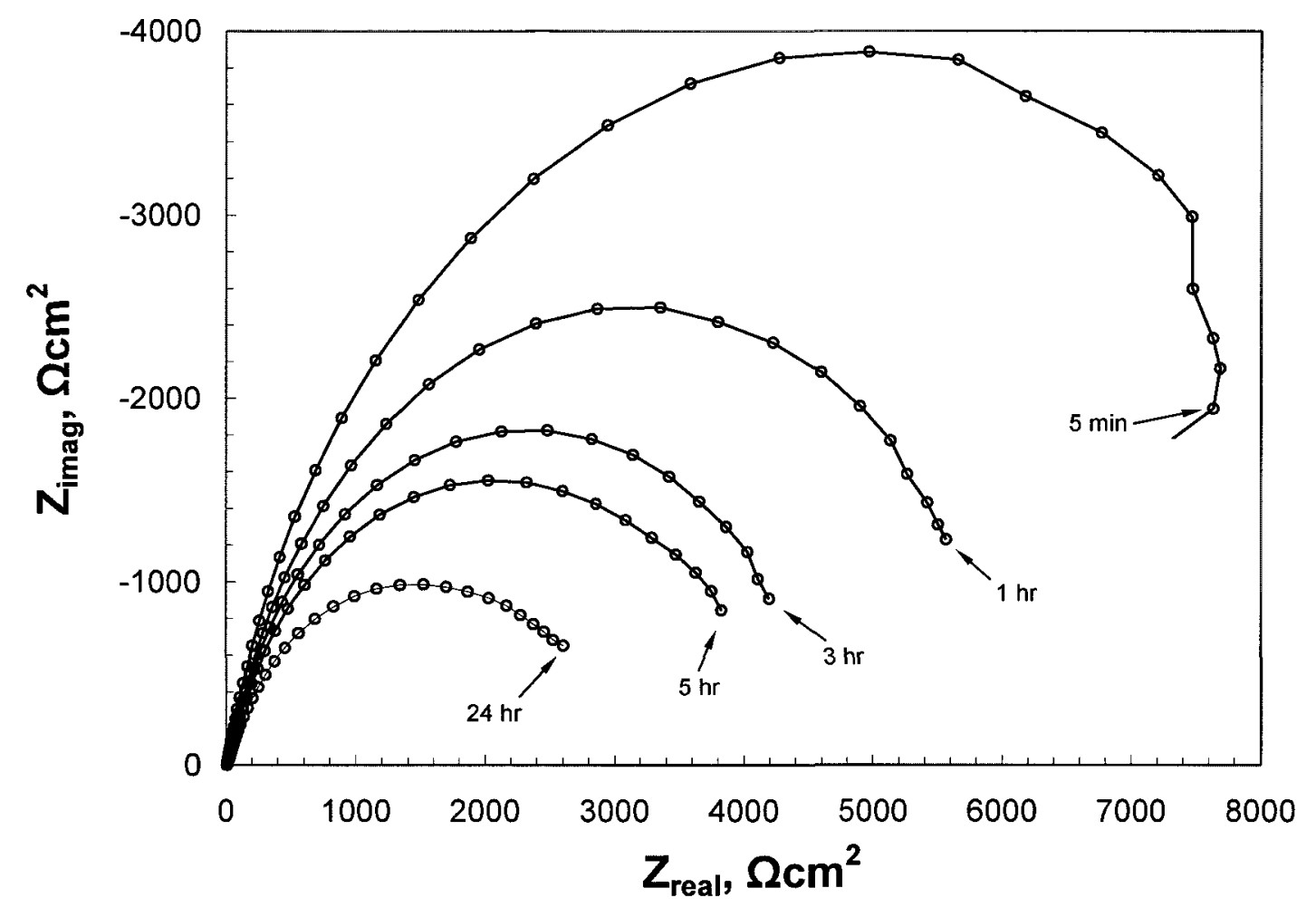

Figure 3-16 Nyquist plots measured from an EIS test on a TiN/MS coating system.

A Nyquist plot offers an overview of the data and a qualitative interpretation. A larger geometrical size of a Nyquist curve indicates a greater impedance value at the corroding interface compared to that at electrolyte-coating interface, revealing a higher resistance or a better corrosion behaviour for a coating system. However, a Nyquist plot does not indicate the frequency corresponding to the point. Bode plots include the forms of $\log |Z|$ vs. $\log f$ and phase angle vs. $\log f$, where $|\mathrm{Z}|$ is the modulus of impedance and $f$ is frequency. The impedance is plotted with $\log f$ on $\mathrm{x}$-axis and both the impedance modulus value and phase shift angle on y-axis (Figure 3-17). The Bode plots explicitly 
display data for all the frequencies along with the absolute impedance values, indicating the effect of angular frequency on the impedance and phase angle.

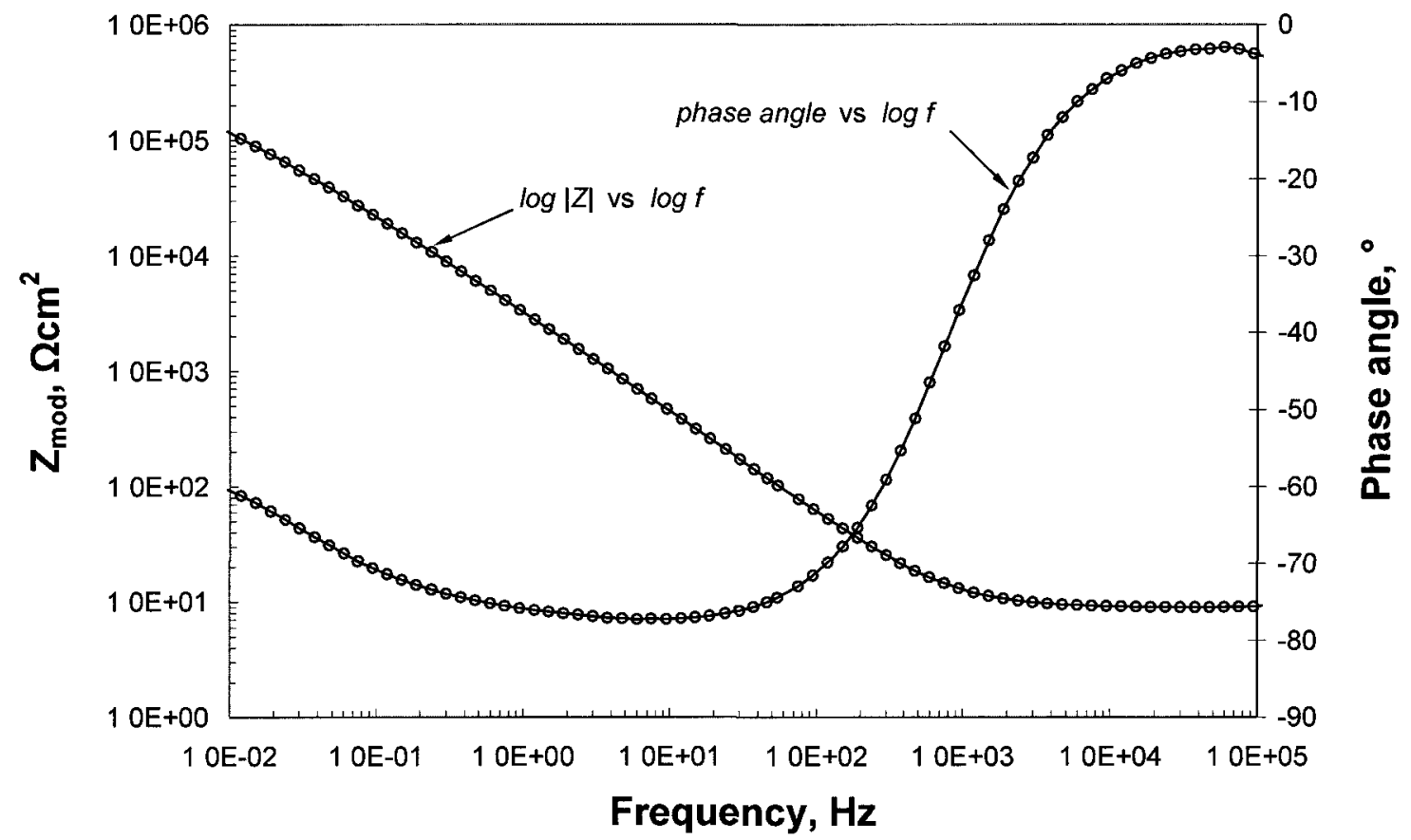

Figure 3-17 Bode plots obtained from an EIS tests on a CrN/SS coating.

To conduct quantitative interpretations and analyses, the test results were interpreted through curve fitting by equivalent electrical circuit (EEC) analyses using Gamry Echem Analyst program (V. 1.35). The electrochemical corrosion behaviours can be analyzed or explained through the interpretation of the variable values of equivalent electrical elements. Some basic EEC models have been developed, which contain the basic circuit elements presented in Table 3-3. The corresponding descriptions for the admittance and impedance are provided for each element. 
The relative variables used in these equations are $R, C, L, Y_{o}, B$, and $n$. Gamry Echem Analyst program (V. 1.35) incorporates these variables as parameters into a fitting operation.

Table 3-3 Basic circuit elements used in EEC models [387]

\begin{tabular}{|l|l|l|}
\hline \multicolumn{1}{|c|}{ Equivalent element } & \multicolumn{1}{c|}{ Admittance } & \multicolumn{1}{c|}{ Impedance } \\
\hline Resistor (R) & $1 / R$ & $R$ \\
\hline Capacitor (C) & $j \omega C$ & $1 / j \omega C$ \\
\hline Inductor (L) & $1 / j \omega L$ & $j \omega L$ \\
\hline Infinite Warburg (W) & $Y o \sqrt{ }(j \omega)$ & $1 / Y o \sqrt{ }(j \omega)$ \\
\hline Finite/porous bounded Warburg & $Y o \sqrt{ }(j \omega) \operatorname{coth}(B \sqrt{ }(j \omega))$ & $\tanh (B \sqrt{ }(j \omega)) / Y o \sqrt{ }(j \omega)$ \\
\hline Bounded Warburg (T) & $Y o \sqrt{ }(j \omega) \tanh (B \sqrt{ }(j \omega))$ & $\operatorname{coth}(B \sqrt{ }(j \omega)) / Y o \sqrt{ }(j \omega)$ \\
\hline Q(CPE) & $Y o(j \omega)^{n}$ & $1 / \operatorname{Yo}(j \omega)^{n}$ \\
\hline
\end{tabular}

In order to minimize errors in EEC fitting in this study, various initial values were applied to each fitting and run several times until a stable result was obtained with an acceptable error (less than 10\%) [37, 387, 388]. 


\section{Results and Discussions}

This section presents the results obtained in this research along with discussion based on the observation. Furthure analyses are conducted on electrochemical corrosion.

\subsection{Coating Characteristics and Properties}

\subsubsection{Coating Thickness}

\section{Nickel Plating}

Cross sectional images of Ni plated samples are shown in Figure 4-1 From these images, the thicknesses of the coatings were measured with the assistance of Clemex Vision PE 4.0 Image Analysis software, and the results are summarized in Table 4-1.

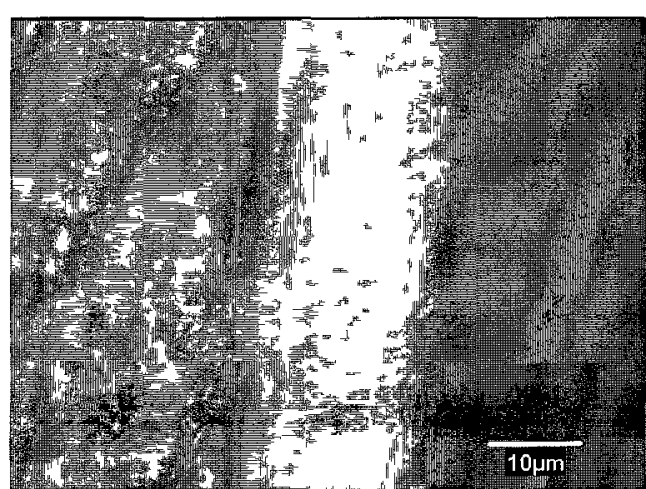

(a) $\mathrm{Ni}-0$

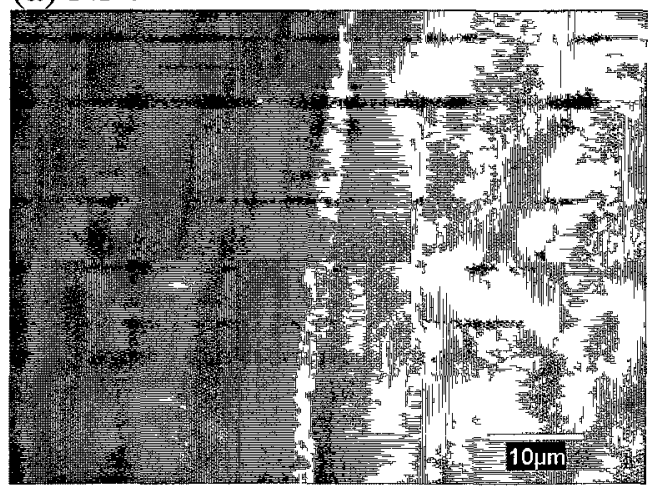

(c) $\mathrm{Ni}-100$

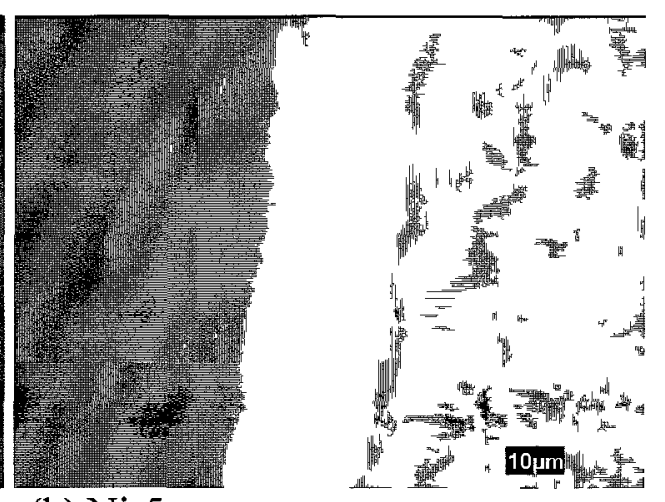

(b) Ni-5

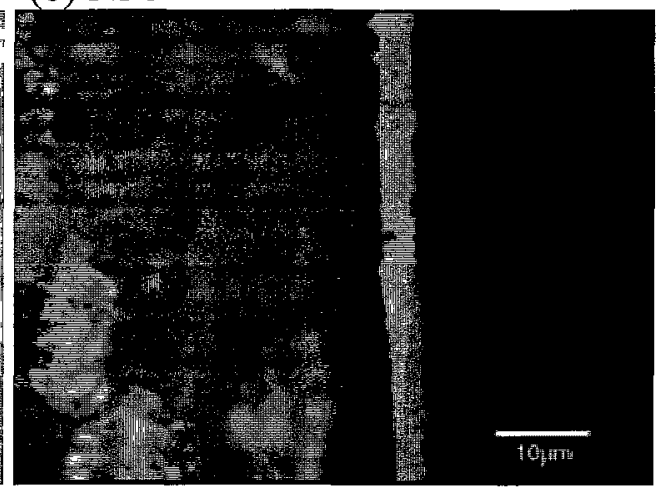

(d) Ni-1000

Figure 4-1 to be continued 

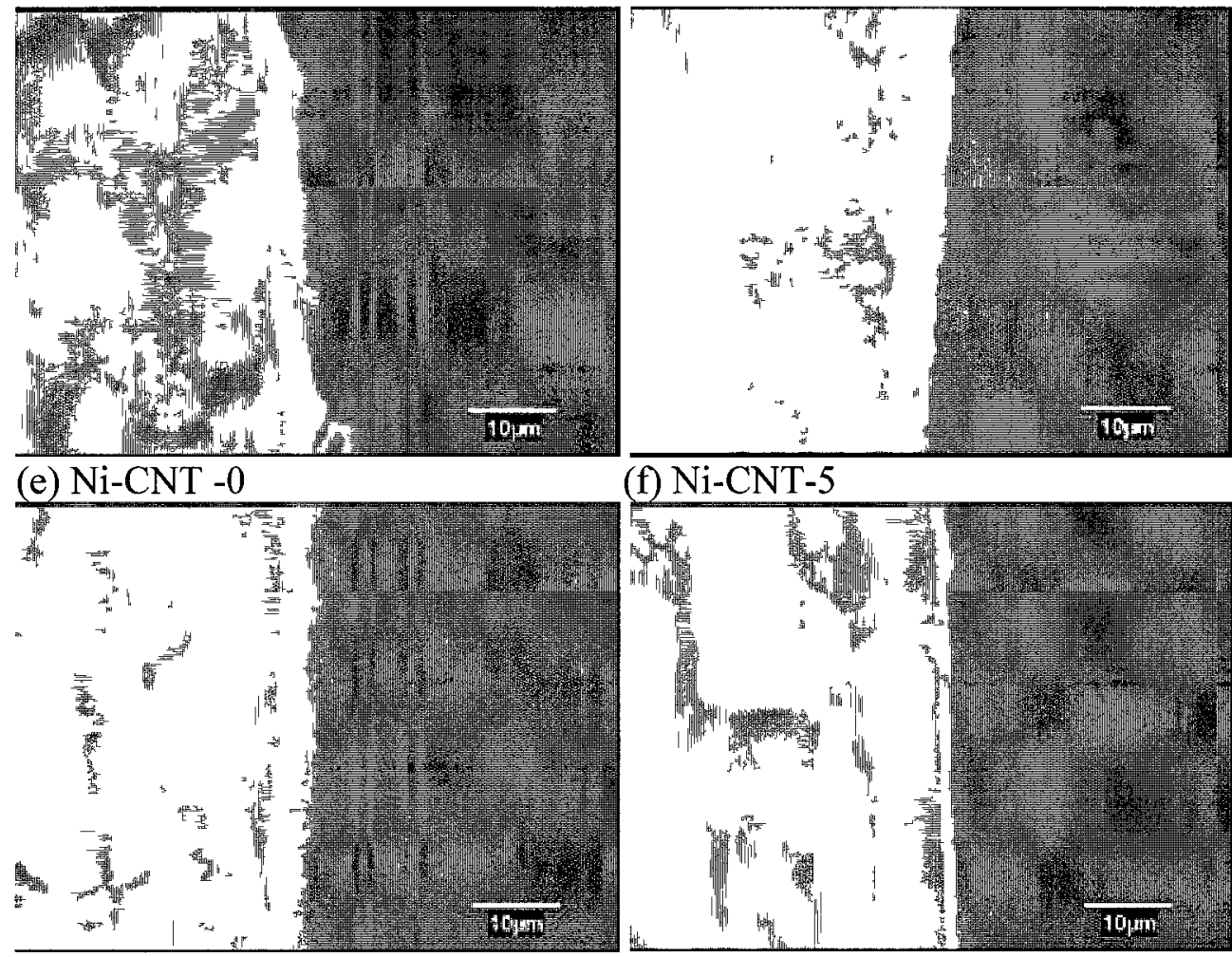

(f) Ni-CNT-5

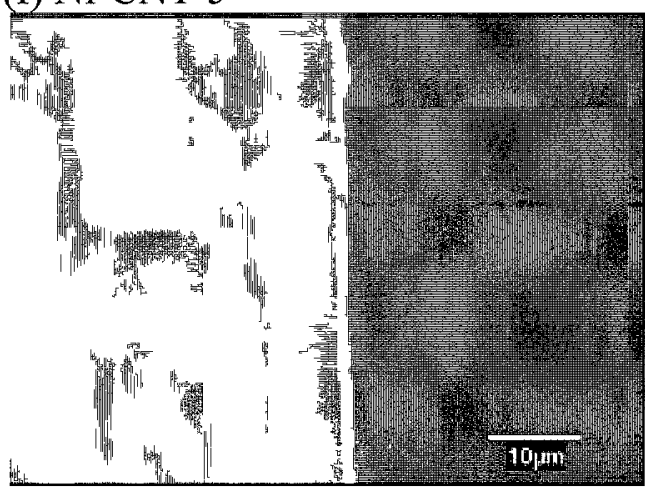

(g) Ni-CNT-100

(h) Ni-CNT-1000

Figure 4-1 Cross sectional images of $\mathrm{Ni}$ and Ni-CNT plating samples under various deposition conditions.

Table 4-1 Summary of thicknesses $(\mu \mathrm{m})$ of Ni and Ni-CNT plated samples deposited using various pulse frequencies

\begin{tabular}{|l|c|c|c|c|}
\hline \multicolumn{1}{|c|}{ Pulse Frequency } & $0 \mathrm{~Hz}$ & $5 \mathrm{~Hz}$ & $100 \mathrm{~Hz}$ & $1 \mathrm{kHz}$ \\
\hline Ni plating & 19.87 & 12.76 & 3.12 & 3.64 \\
\hline Ni-CNT plating & 5.43 & 5.17 & 4.83 & 1.6 \\
\hline
\end{tabular}

The general trend in thickness change with plating parameters is shown in Figure 4-2. An increase in the pulse frequency and the incorporation of CNTs, both result in a decrease in the thickness (or deposition rate) of the plating. In pure Ni plating (without CNTs), the deposition thickness is more sensitive to the pulse frequency than it is when CNTs are added. The plating thicknesses were reduced about $80 \%$ as the pulse frequency changed from 0 (Ni-0) to $1000 \mathrm{~Hz}(\mathrm{Ni}-1000)$. With the CNT embedded nickel plated samples (Ni-CNT), the plating thickness was significantly reduced at all pulse 
frequencies, indicating that the growth of the coating is affected more by the presence of the CNTs than by changes in the pulse frequency. This phenomenon is associated with the disturbance of the deposit nucleation $[95,293,294]$.

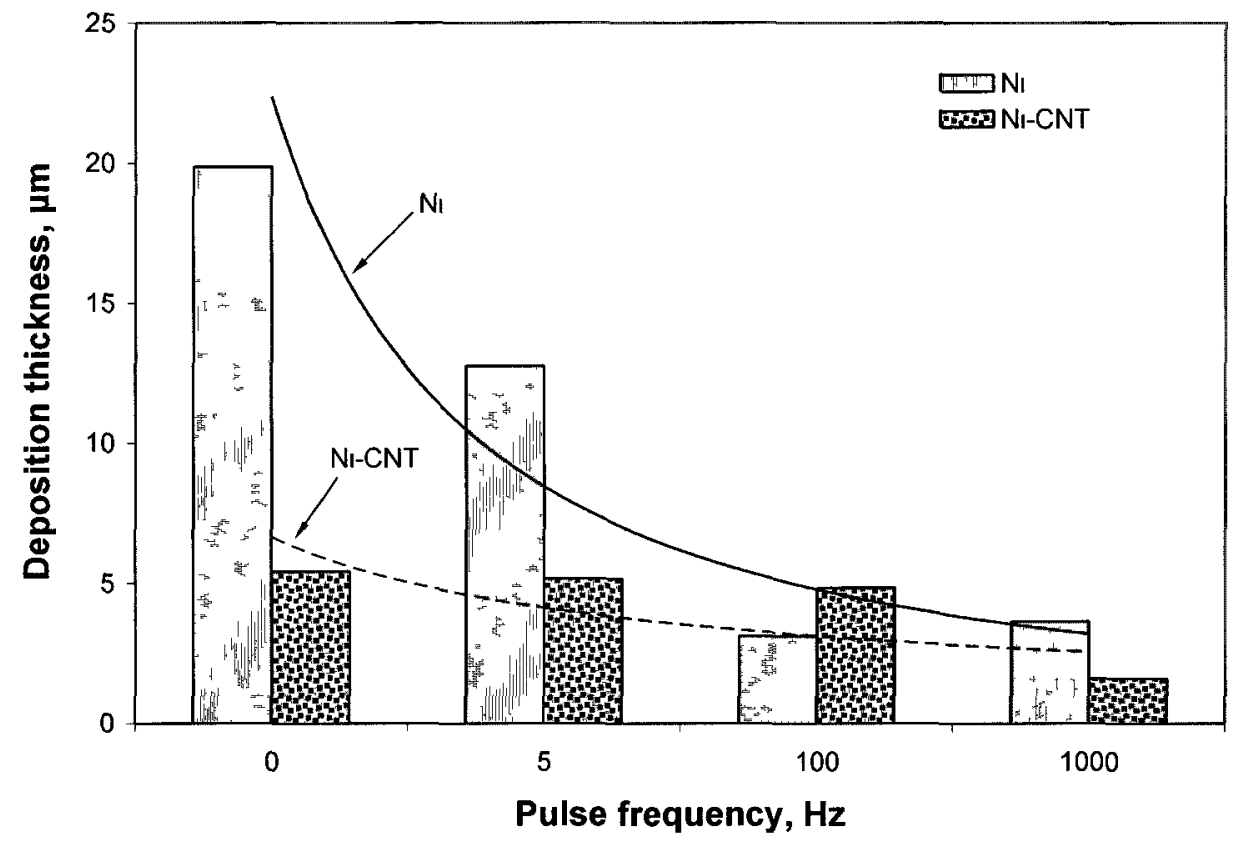

Figure 4-2 Thickness of Ni and Ni-CNT plated samples as a function of pulse frequency. PVD Coating Systems

The measurements of PVD coating thicknesses were conducted using an abrasive ball crater, and the results are summarized in Table 4-2

Table 4-2 Summary of thicknesses of PVD coating systems

\begin{tabular}{|l|c|}
\hline \multicolumn{1}{|c|}{ Coating system } & Thickness of coating $(\mu \mathrm{m})$ \\
\hline EB TiN/SS & 1.5 \\
\hline CA TiN/MS & 2.8 \\
\hline CA TiN/SS & 2.8 \\
\hline CrN & 20.2 \\
\hline CrSiCN(1) & 18.9 \\
\hline CrSiCN $(2)$ & 21.1 \\
\hline CrSiCN $(3)$ & 19.6 \\
\hline
\end{tabular}




\subsubsection{Scanning Electron Microscope (SEM) Analysis}

The coating surface morphologies were examined using scanning electron microscopy

(SEM); and their chemical compositions were measured using energy dispersive X-ray spectroscopy (EDS).

\section{Nickel Plating}

The conventional Ni plated samples exhibited surface morphologies that are dependant on the pulse frequency. As shown in Figure 4-3, the ones obtained with pulsed current had much finer surface features, and the surface smoothness increased with pulse frequency, which is in agreement with previous studies [389].

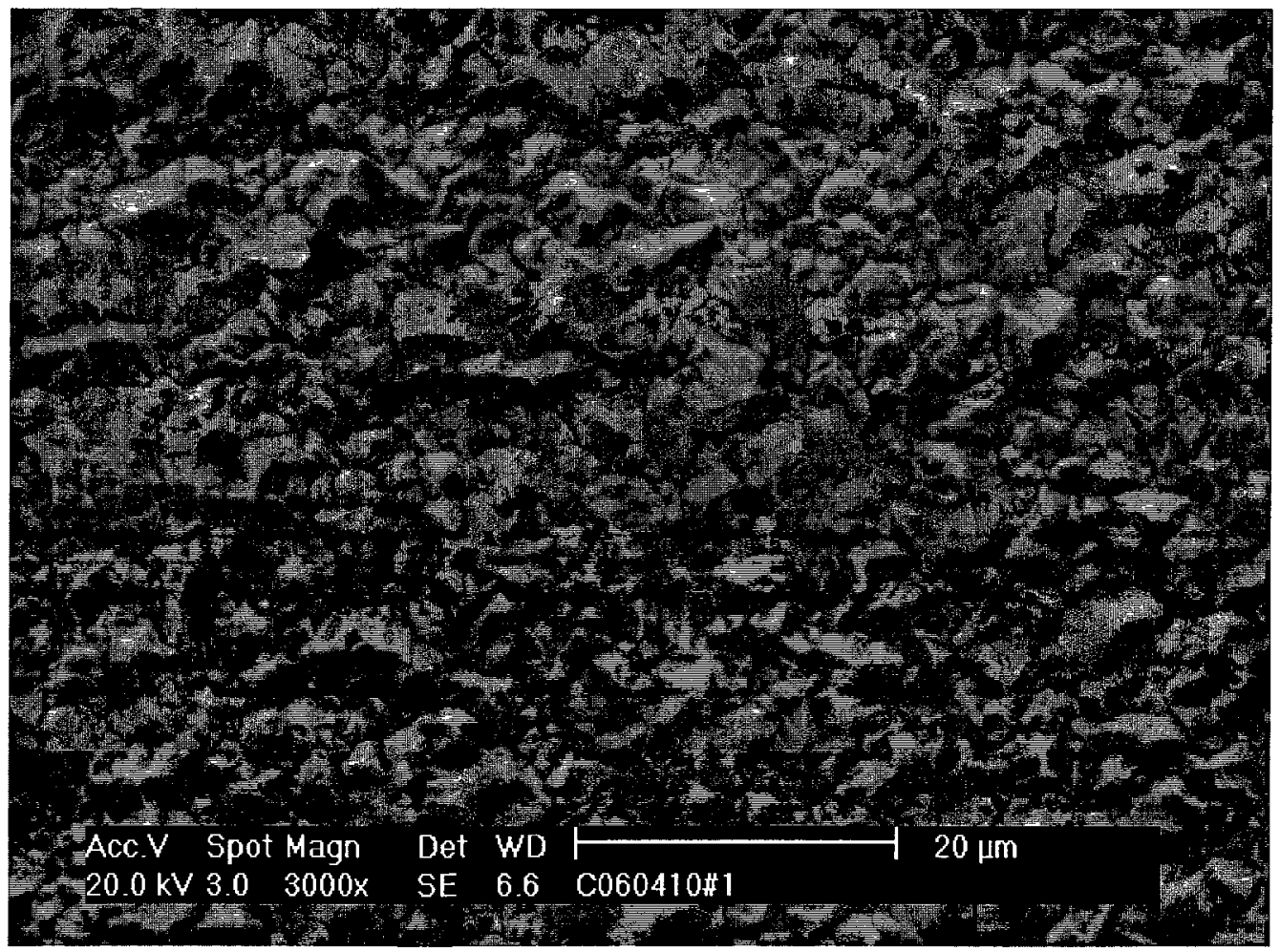

(a) $\mathrm{Ni}-0$

Figure 4-3 to be continued 


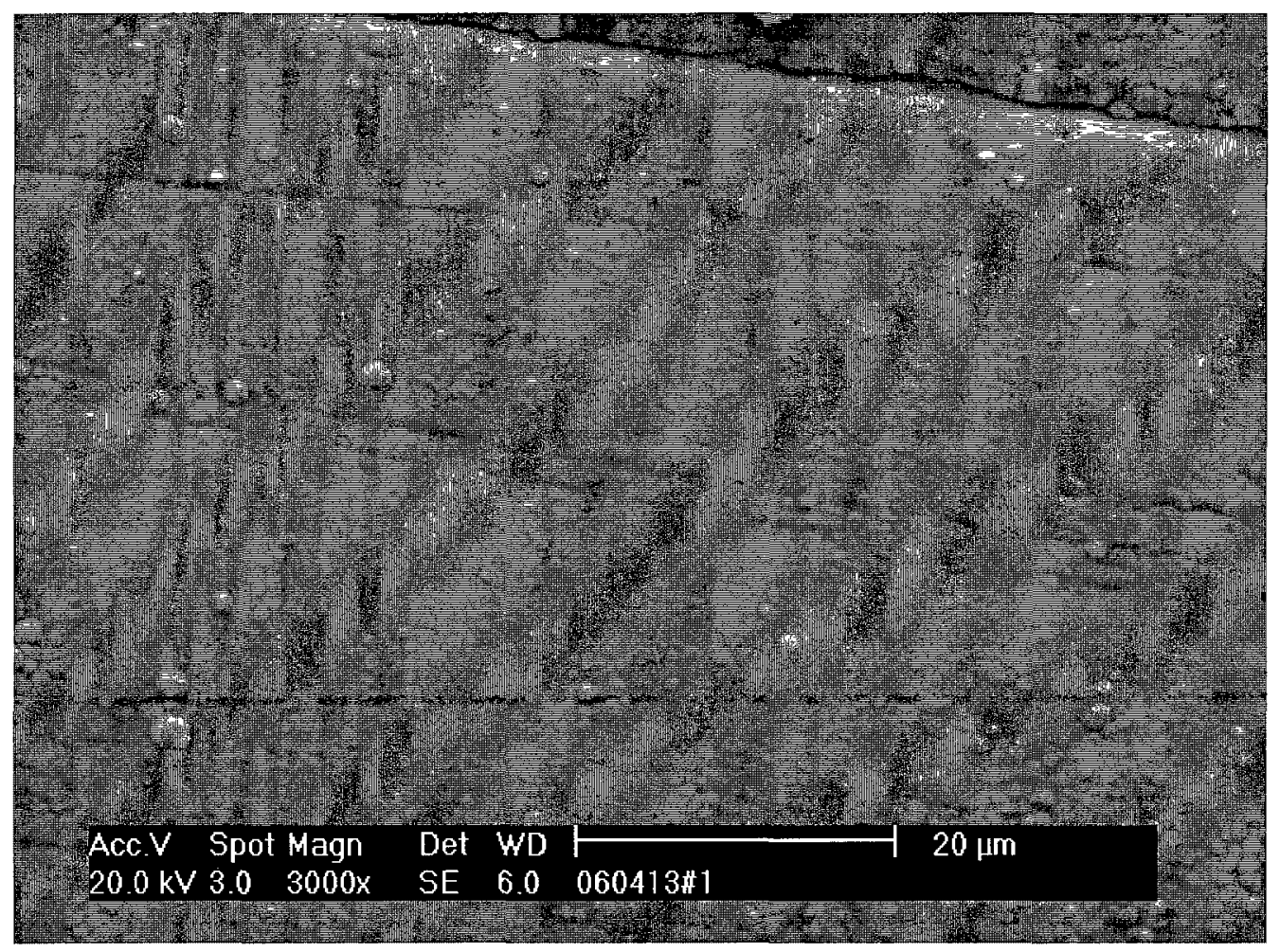

(b) Ni-5

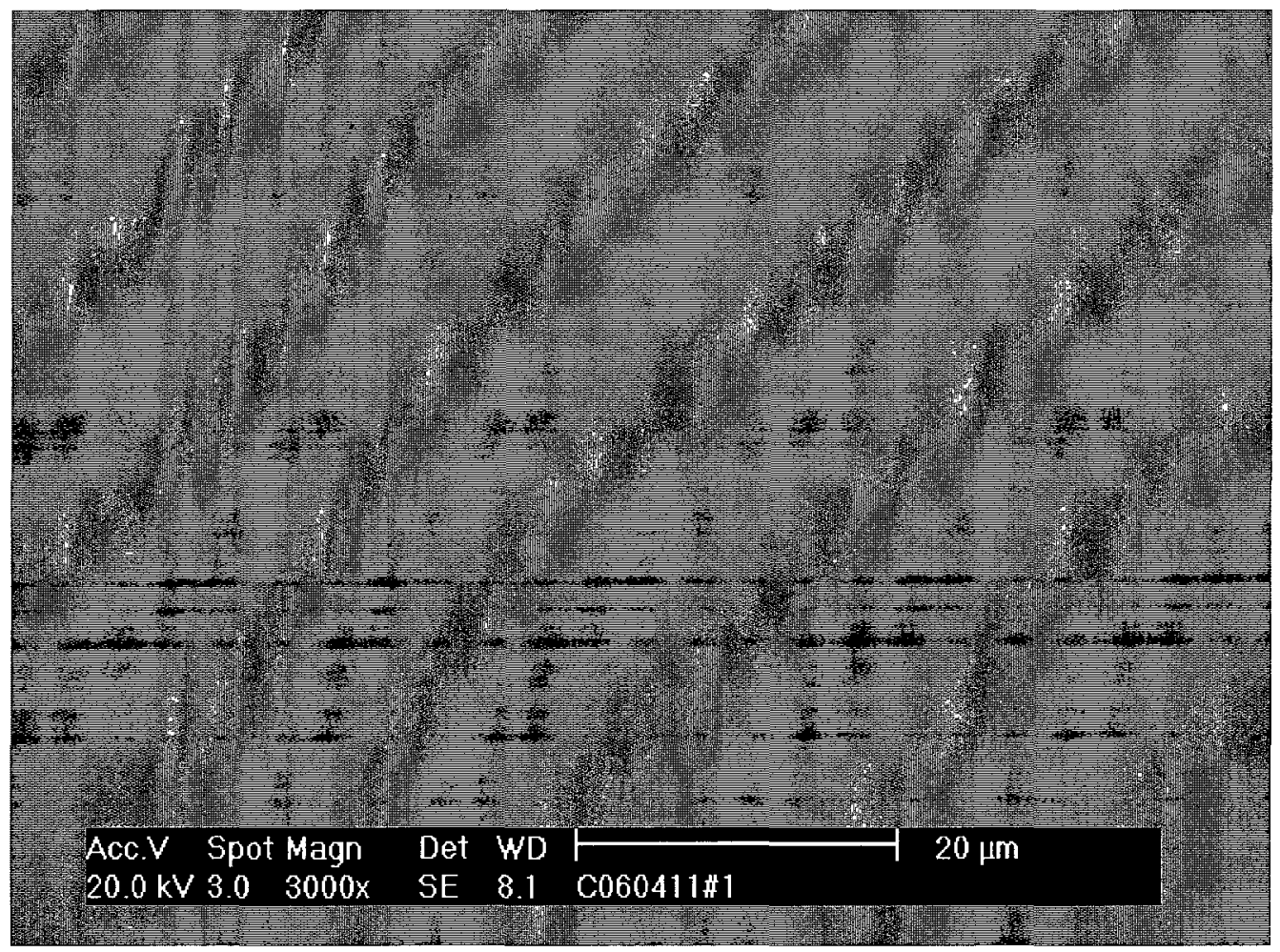

(c) $\mathrm{Ni}-100$

Figure 4-3 to be continued 




(d) $\mathrm{Ni}-1000$

Figure 4-3 SEM images of conventional Ni plated samples deposited at different frequencies.



(a) Ni-CNT-0

Figure 4-4 to be continued 


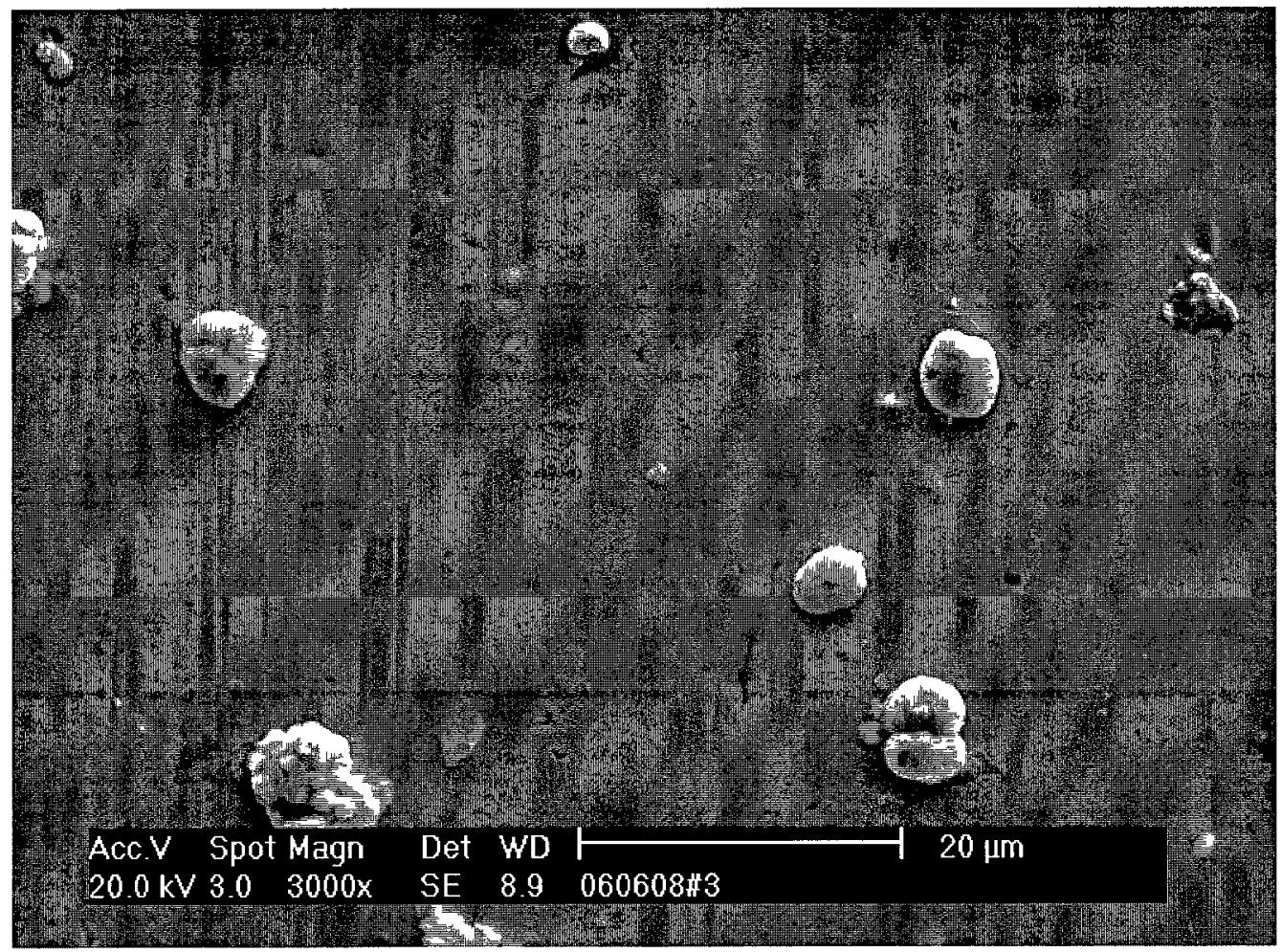

(b) Ni-CNT-5

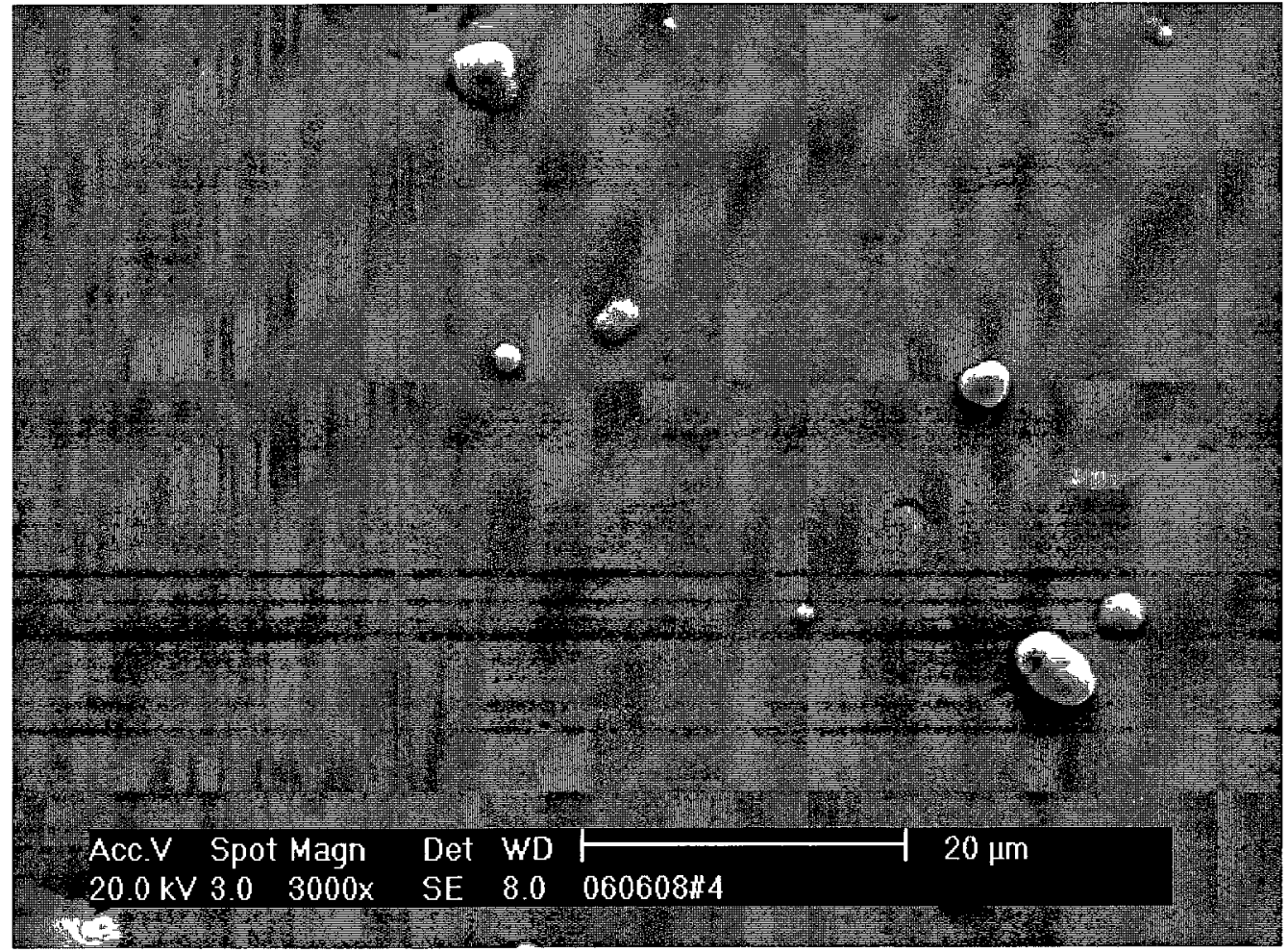

(c) Ni-CNT-100

Figure 4-4 to be continued 


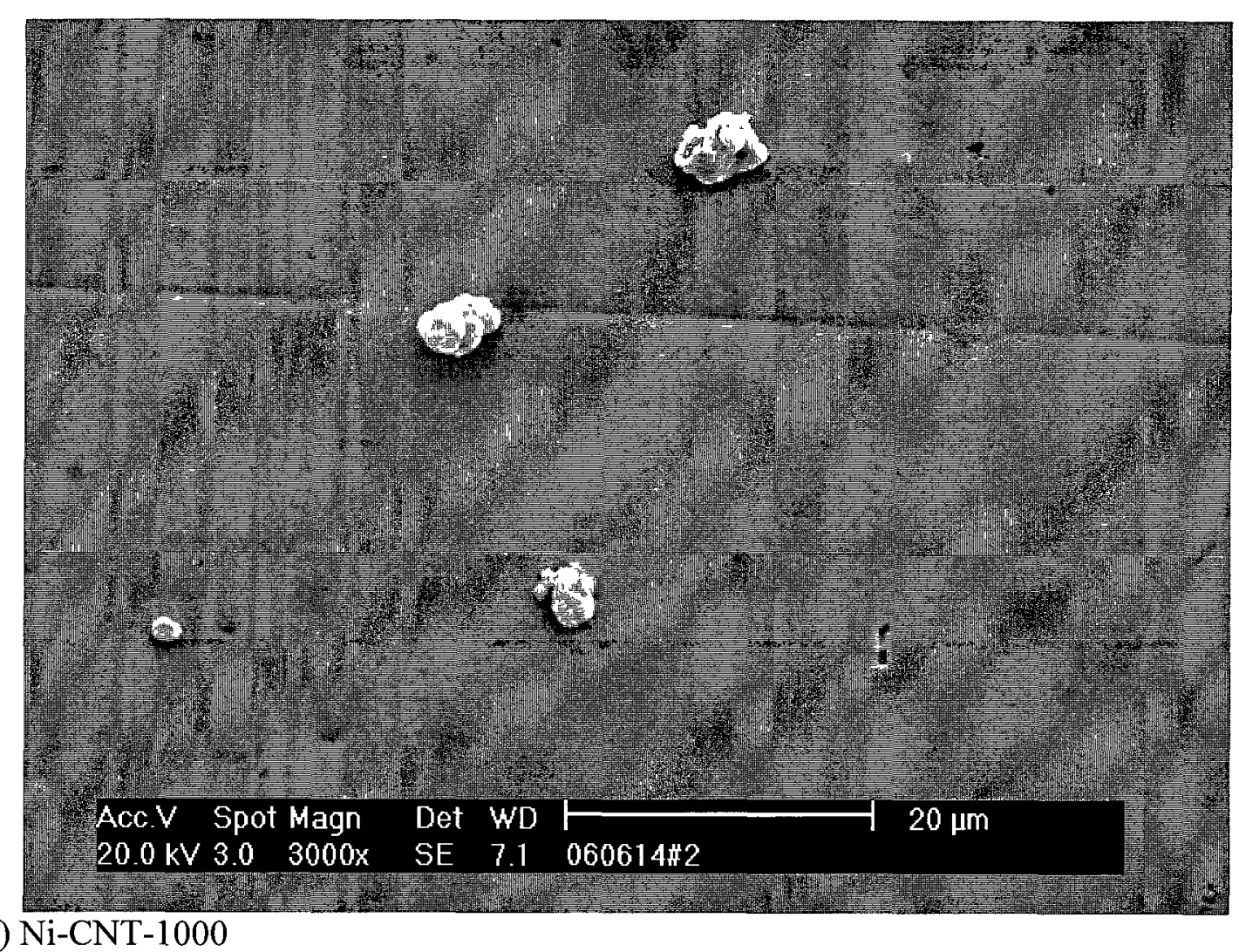

Figure 4-4 SEM images of Ni-CNT plated samples with CNTs dispersed.

The surface morphologies of the Ni-CNT plated samples at various pulse frequencies are shown in Figure 4-4. The incorporation of CNTs contributes to a grain refining of the deposition and a change in surface morphology as is evident by comparing the images in Figure 4-3 with the corresponding images in Figure 4-4. The surface with CNTs is composed of fine spherical grains, while pure Ni plating assumes a rough surface. With pulsed current, even finer surface morphology results. A noticeable difference from the conventional Ni plating is the clusters of CNTs on the surface, which stemmed from the not completely dispered CNTs in the electrolyte. 


\section{TiN Coating Systems}

SEM images for the morphologies of the TiN coatings deposited with EB and CA techniques and their PMMA-treated counterparts are shown in Figure 4-5 to Figure 4-8. The surfaces of the TiN coatings were smooth with occasional particulate defects (Figure 4-5 a, b)(Figure 4-6 a, b). The CA TiN (Figure 4-6) coating had a much higher defect density than its EB counterpart, as summarized in Table 4-4. The coating defects including nodules and nodule-detached craters are associated with the effect of ion bombardment in the cathodic arc process which typically generates a rougher surface than sputtering or EB techniques [32, 234].

A typical defect is an embedded nodule (Figure 4-5 b and Figure 4-6 c) with a cauliflower-like feature, showing apparent separations or gaps between the nodules and the coating (Figure 4-5 $\mathrm{b}$ and Figure 4-6 b). These nodules, having the same composition as the bulk coating, are growth defects formed mainly on the metal-rich droplets (appearing white under backscatter electron mode, Figure 4-6 c) ejected from the cathode arc spot during coating deposition. The droplets land on the substrate and act as preferred nucleation sites for the coating, thus causing the droplet region of the coating to grow at a faster rate than the bulk and produce a shadowing effect on the adjacent areas $[31,32]$. As the deposition continues to grow, gaps or weak bonds form between the nodules and the surrounding coating [32]. Due to either high internal stresses [390] or external forces, some of the nodular particles detach from the coating, leaving craters on the coating surface. The crater surfaces, as shown in Figure 4-5 (e) and Figure 4-6 (d), are covered by a thin layer of the TiN coating and are rough and porous. As shown in 
Figure 4-5 (d), nodules may also develop on a foreign particle (in this case, an $\mathrm{Al}_{2} \mathrm{O}_{3}$ particle, which appeared dark in back-scattered electron (BSE) mode).

Depending on when the nodules formed and detached during the coating process, the crater bottoms could either have a rough and porous coating appearance, which is typical (Figure 4-5 e and f), or exposed substrate surface in some instances. The nodules and craters left by the detached nodules can act as potential sites for localized corrosion as they provide channels for corrosive media to penetrate to the substrate surface.



(a) Coating surface at low magnification

Figure 4-5 to be continued 


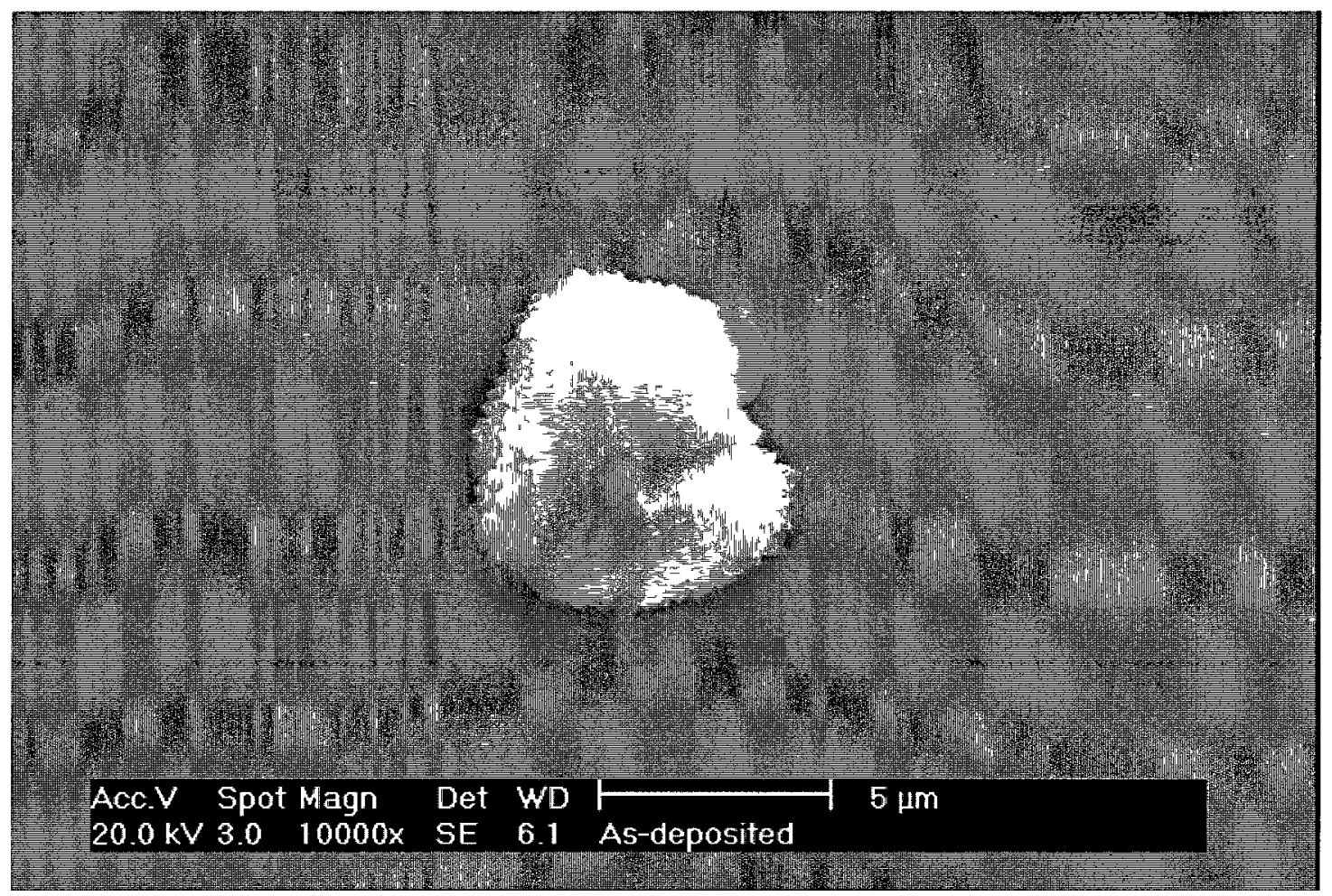

(b) The presence of a defect nodule on coating surface



(c) Cross sectional image showing a defect nodule in coating

Figure 4-5 to be continued 




(d) Cross sectional image showing a defect nodule in coating

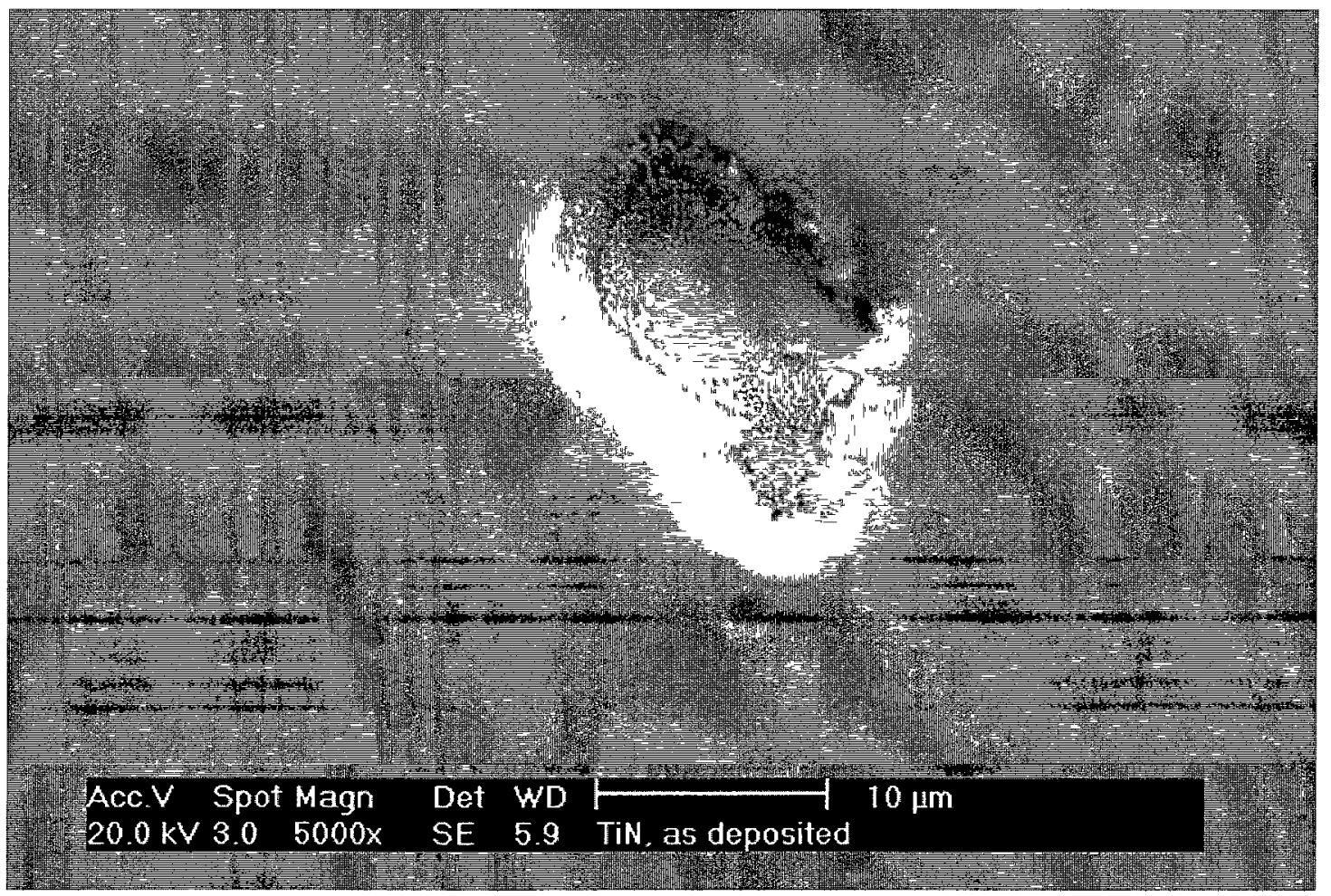

(e) A nodule-detached crater on coating surface

Figure 4-5 to be continued 


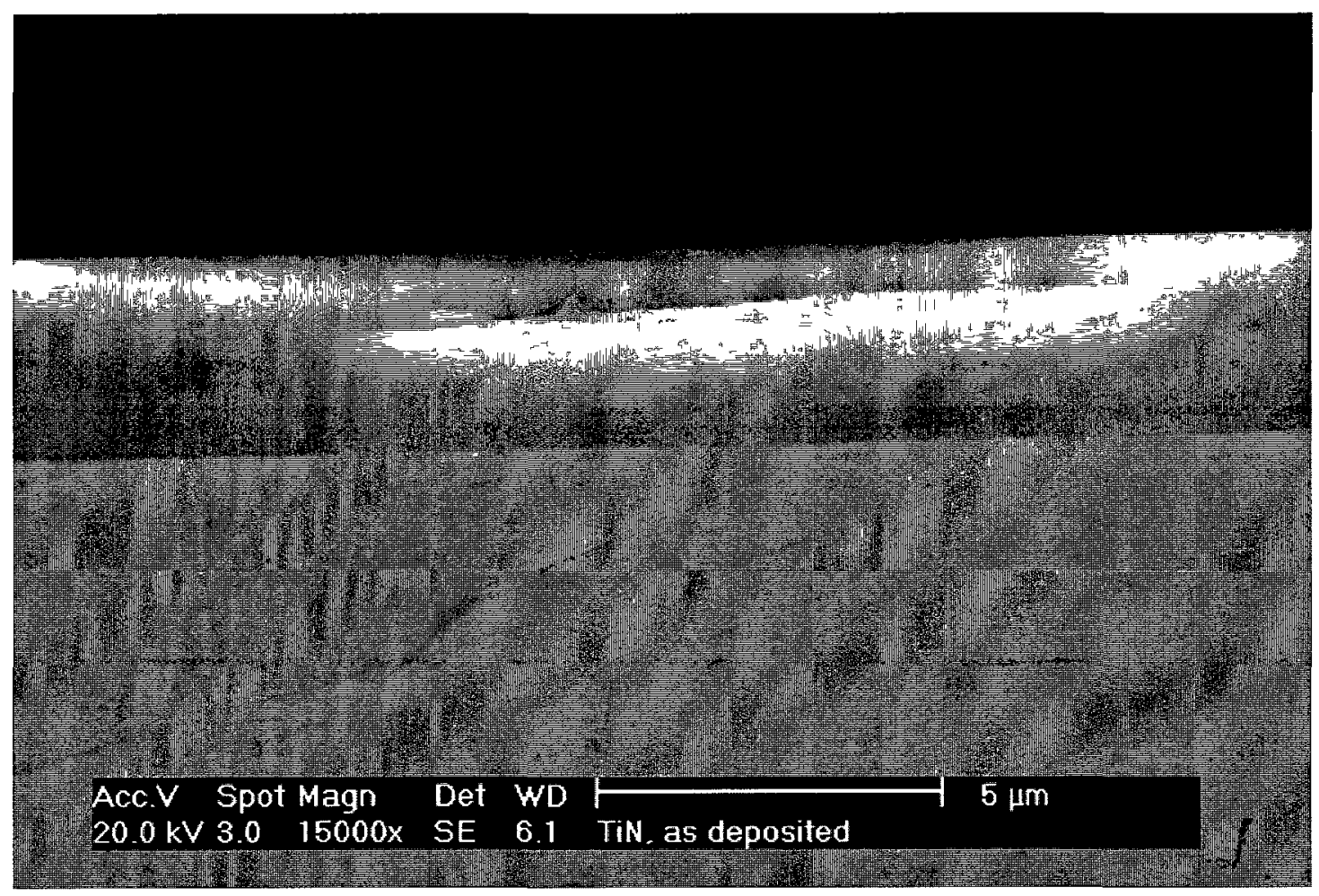

(f) Cross sectional image showing a nodule-detached crater in coating

Figure 4-5 SEM images of the as-deposited EB TiN coating.

A high substrate bias voltage benefits atom mobility and increases surface diffusion, therefore leading to a better coverage of surface imperfections [349, 391]. In this study, the CA TiN coatings were deposited under a high value of substrate bias voltage $-200 \mathrm{~V}$, and a high substrate temperature of $400^{\circ} \mathrm{C}$. As a result, the growth defects appeared well bonded to the coating with smaller gaps between them (Figure 4-6 b, d) compared to their EB counterparts (Figure 4-5 b, e). 




(a) Coating surface at low magnification

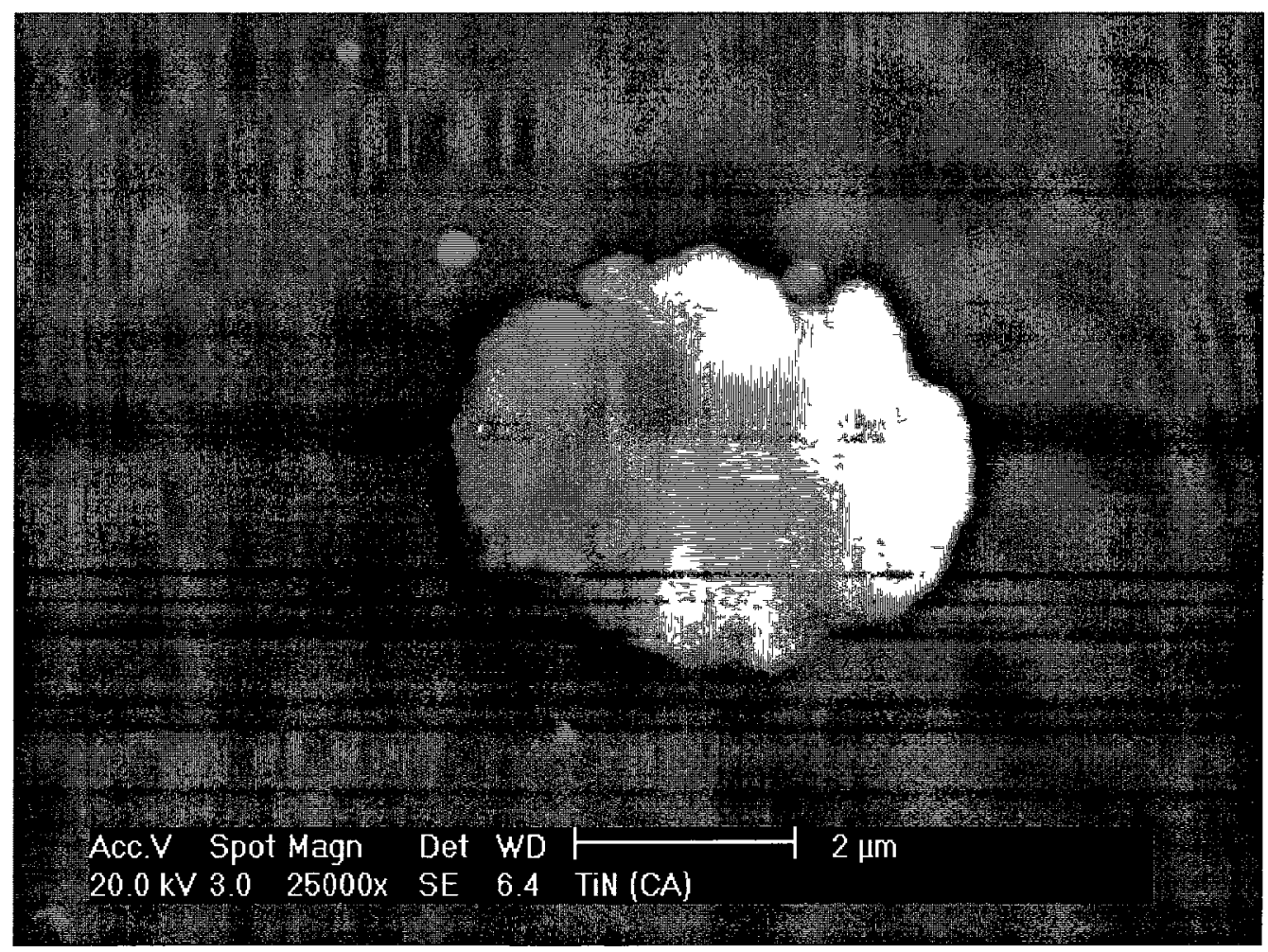

(b) The presence of a defect nodule on coating surface

Figure 4-6 to be continued 


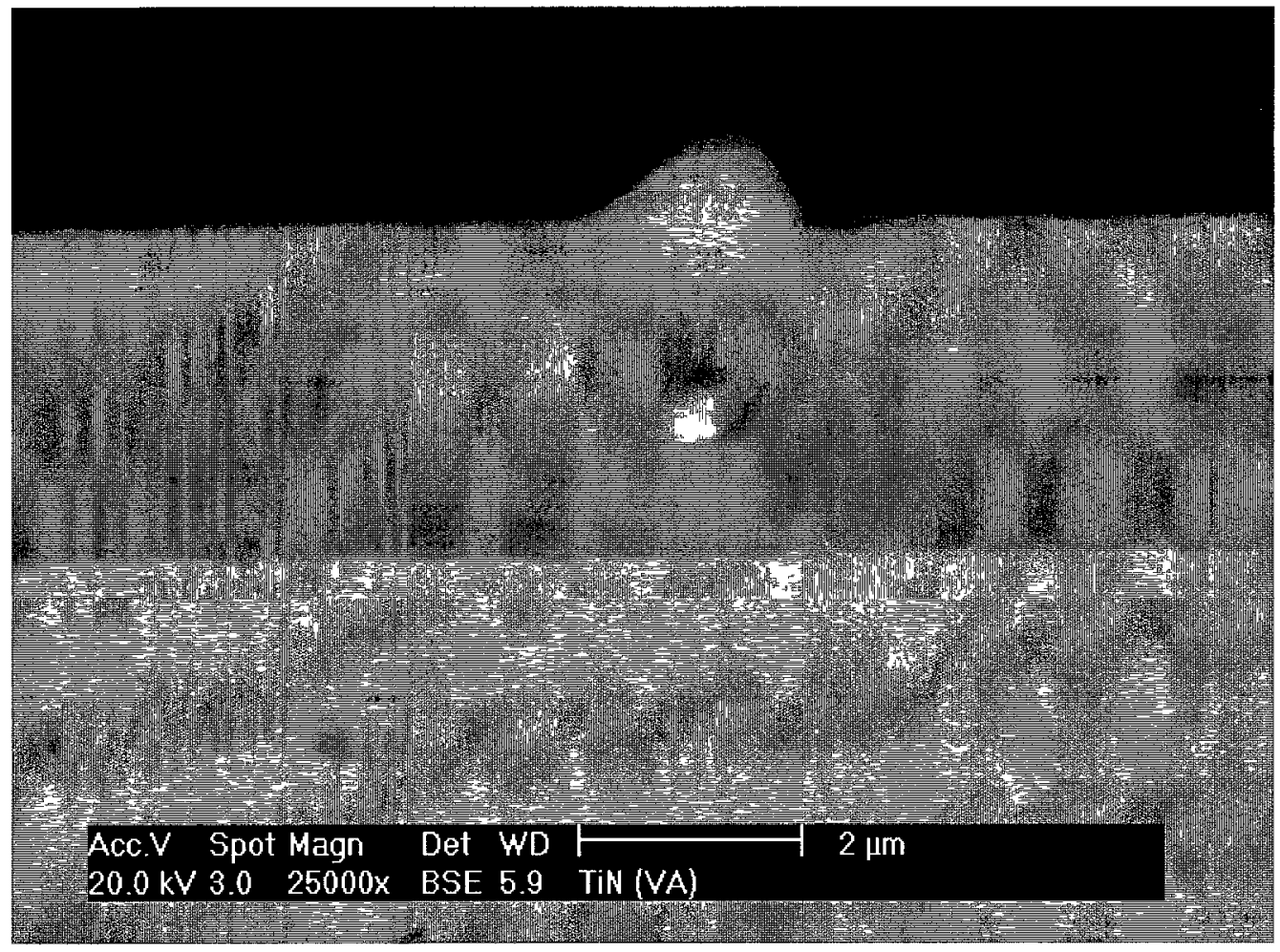

(c) Cross sectional image showing a defect nodule in coating

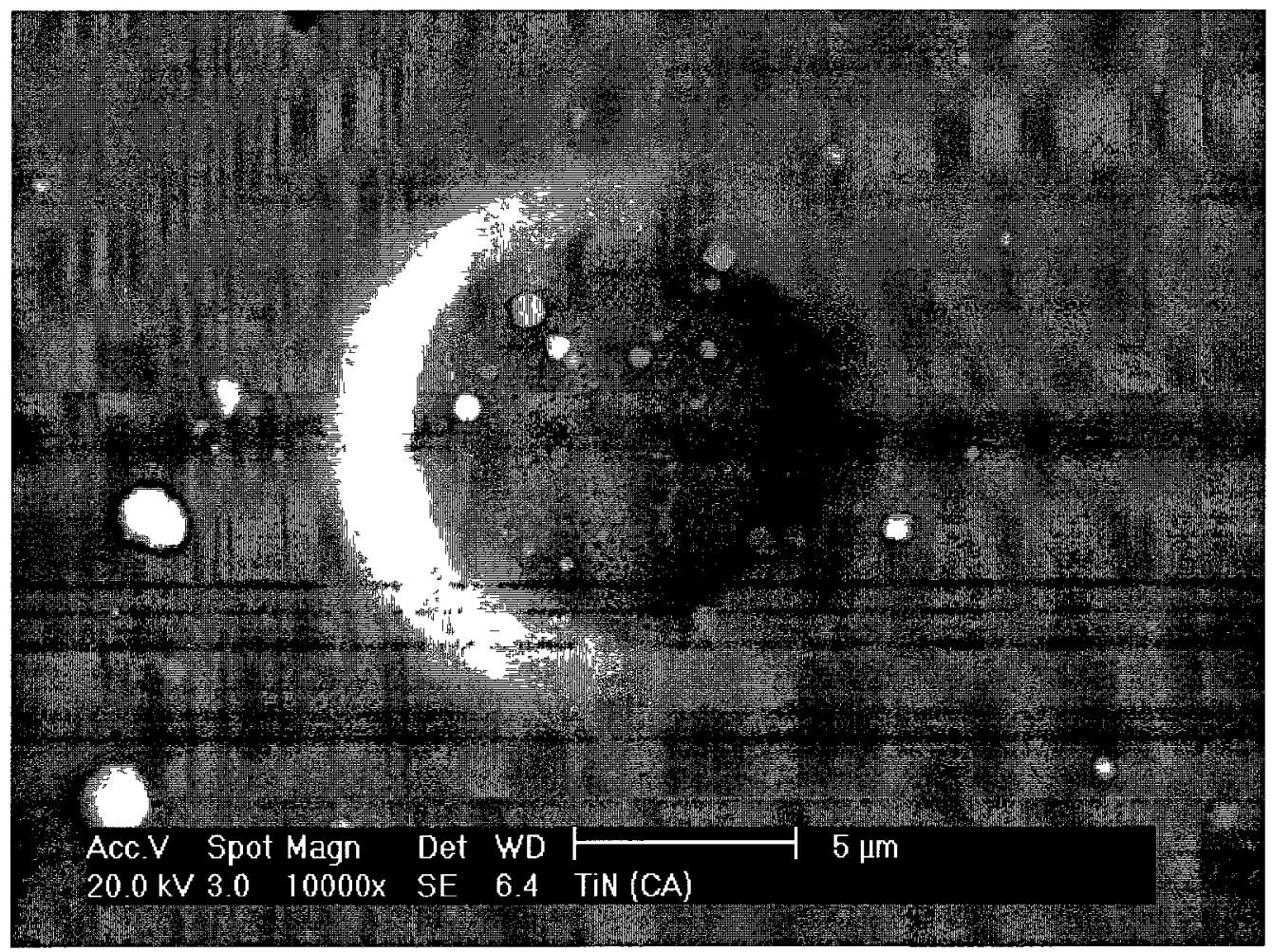

(d) A nodule-detached concave on coating surface

Figure 4-6 SEM images of the as-deposited CA TiN coating. 
In the PMMA-treated TiN coatings, SEM did not detect the presence of PMMA on the coating surface, except in the areas adjacent to nodules (Figure 4-7 a and Figure 4-8 a) and in the crater (Figure 4-7 b). The gap between the nodules and the surrounding coating appeared dark in BSE mode (Figure 4-7 a and Figure 4-8 a), and where strong combined carbon and oxygen peaks were observed in the EDS spectrum (Figure 4-7 c and Figure 4-8 b) (the main constituents of PMMA), indicating that PMMA filled the defect gap, and covered the crater (Figure 4-7 b). The EDS analyses of areas away from the defects detected no PMMA constituents. Only Ti, N and W peaks were found (Ti and $\mathrm{N}$ are the contents of the as-deposited coatings, and $\mathrm{W}$ could be an impurity in the target materials). These results suggest that the PMMA treatment successfully covered defect areas (nodule-detached crater surface and surrounding areas of nodules) without covering the rest of the coating surface.

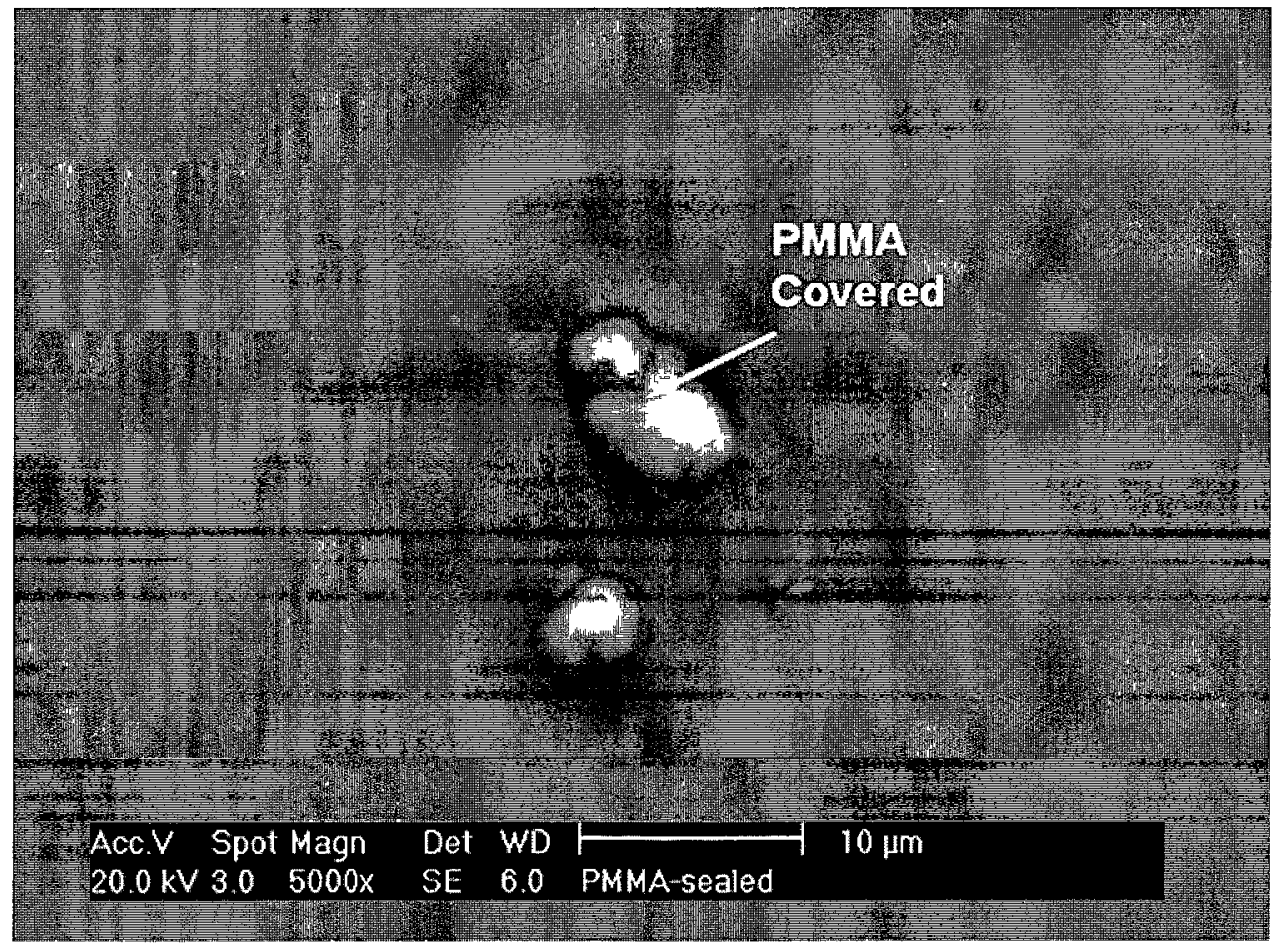

(a) Gap areas surrounding the nodules

Figure 4-7 to be continued 


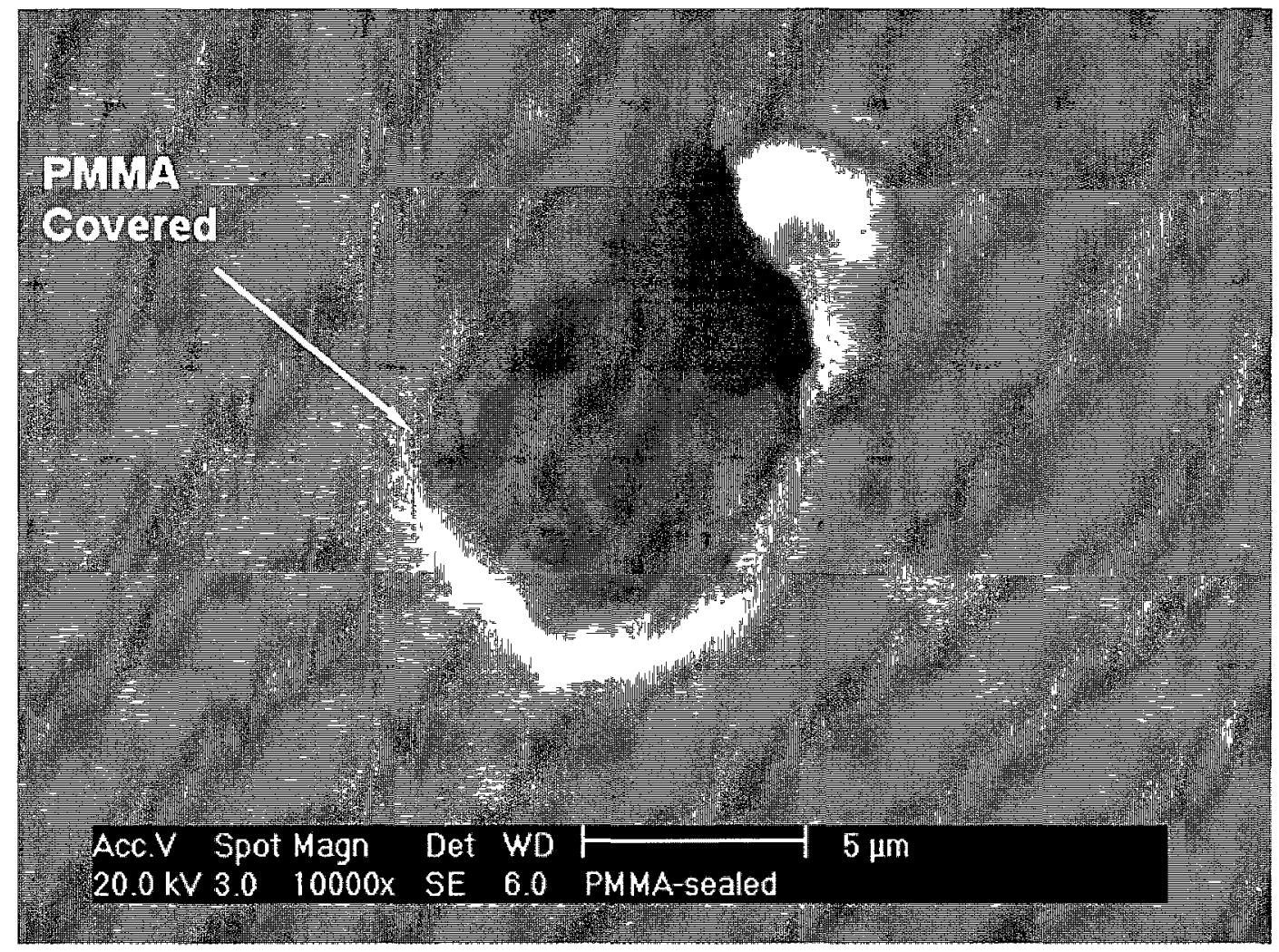

(b) Nodule-detached area are covered by PMMA

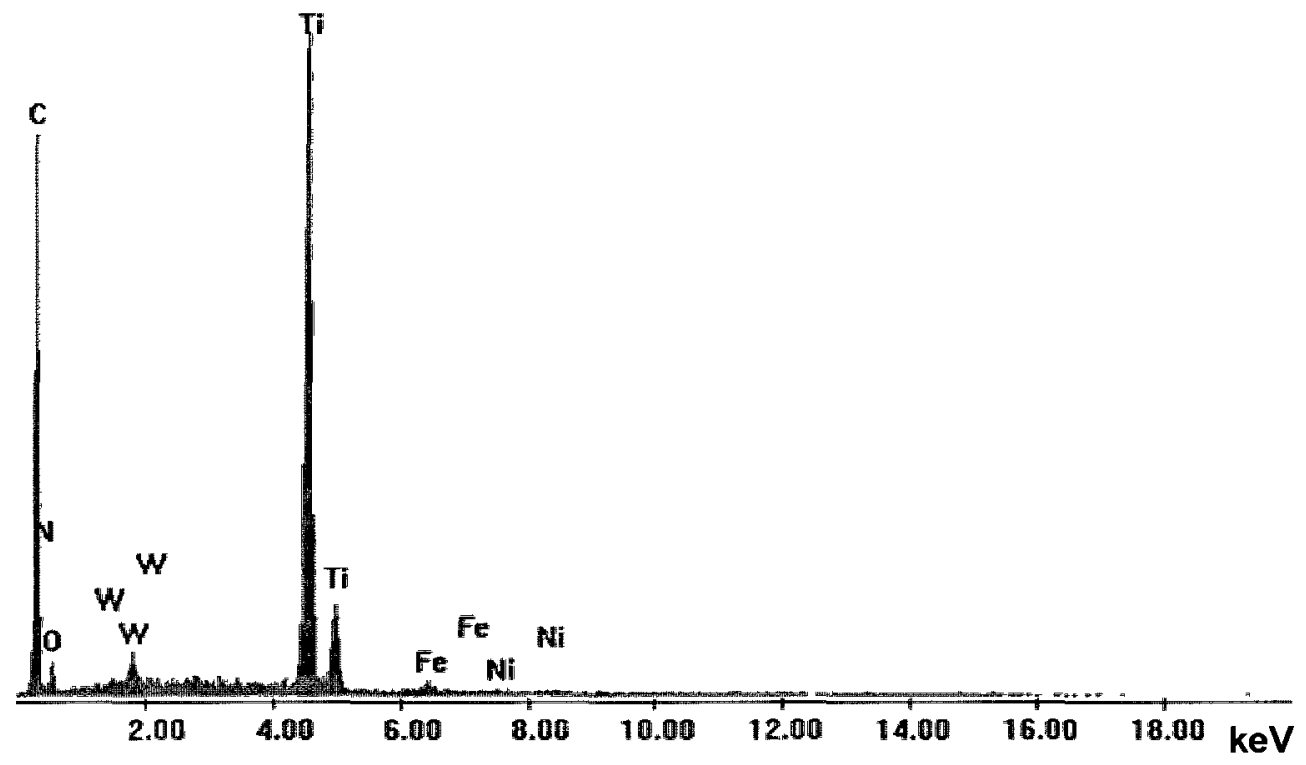

(c) EDS shows carbon peaks accompanied by oxygen at gap areas in (a) indicating that PMMA filled the gap areas surrounding nodules

Figure 4-7 SEM images of PMMA-treated EB TiN coating. 




(a) PMMA-covered gap areas surrounding nodules

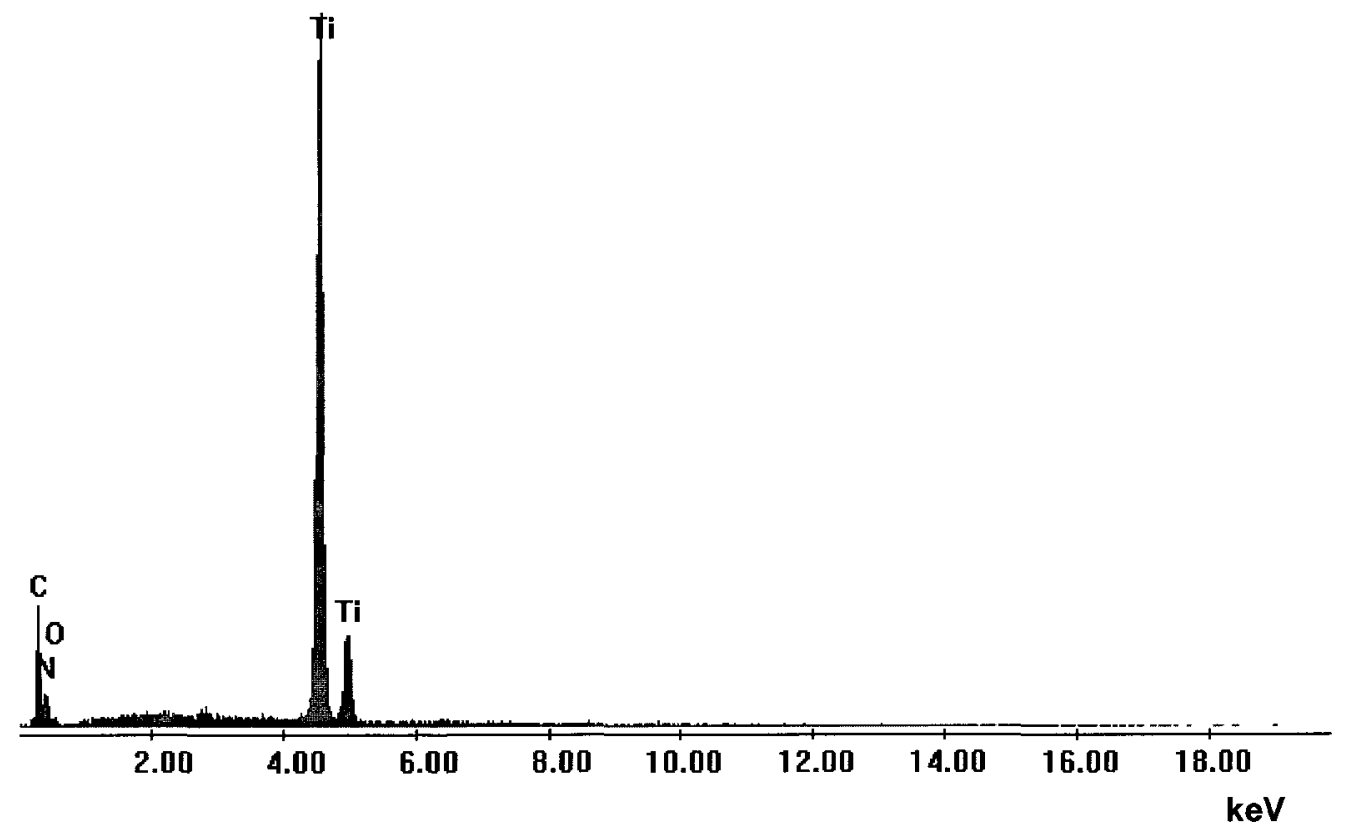

(b) EDS shows carbon peaks accompanied by oxygen at gap areas as shown in (a) indicating that PMMA filled the gap areas surrounding nodules

Figure 4-8 SEM images of PMMA-treated CA TiN coating. 


\section{CrN Based Coating Systems}

The surface morphologies of the four $\mathrm{CrN}$ based coating systems are shown in the SEM images of Figure 4-9. All coating samples displayed cellular structures in addition to the presence of surface defects (embedded nodules with various sizes ranging from 2 to 15 $\mu \mathrm{m}$ ) on the coating surfaces as shown in Figure 4-10. The defects on $\mathrm{CrN}$ (Figure 4-10 a), $\mathrm{CrSiCN}(1)$ (Figure 4-10 b), and $\mathrm{CrSiCN}(2)$ (Figure 4-10 c) appeared larger than those on the $\mathrm{CrSiCN}(3)$ (Figure 4-10 d). Other defects observed include voids or notches along grain boundaries formed during the coating growth. Due to the coarse grain structures in the coatings $\mathrm{CrSiCN}(2)$ and $\mathrm{CrSiCN}(3)$, more voids/notches were expected.

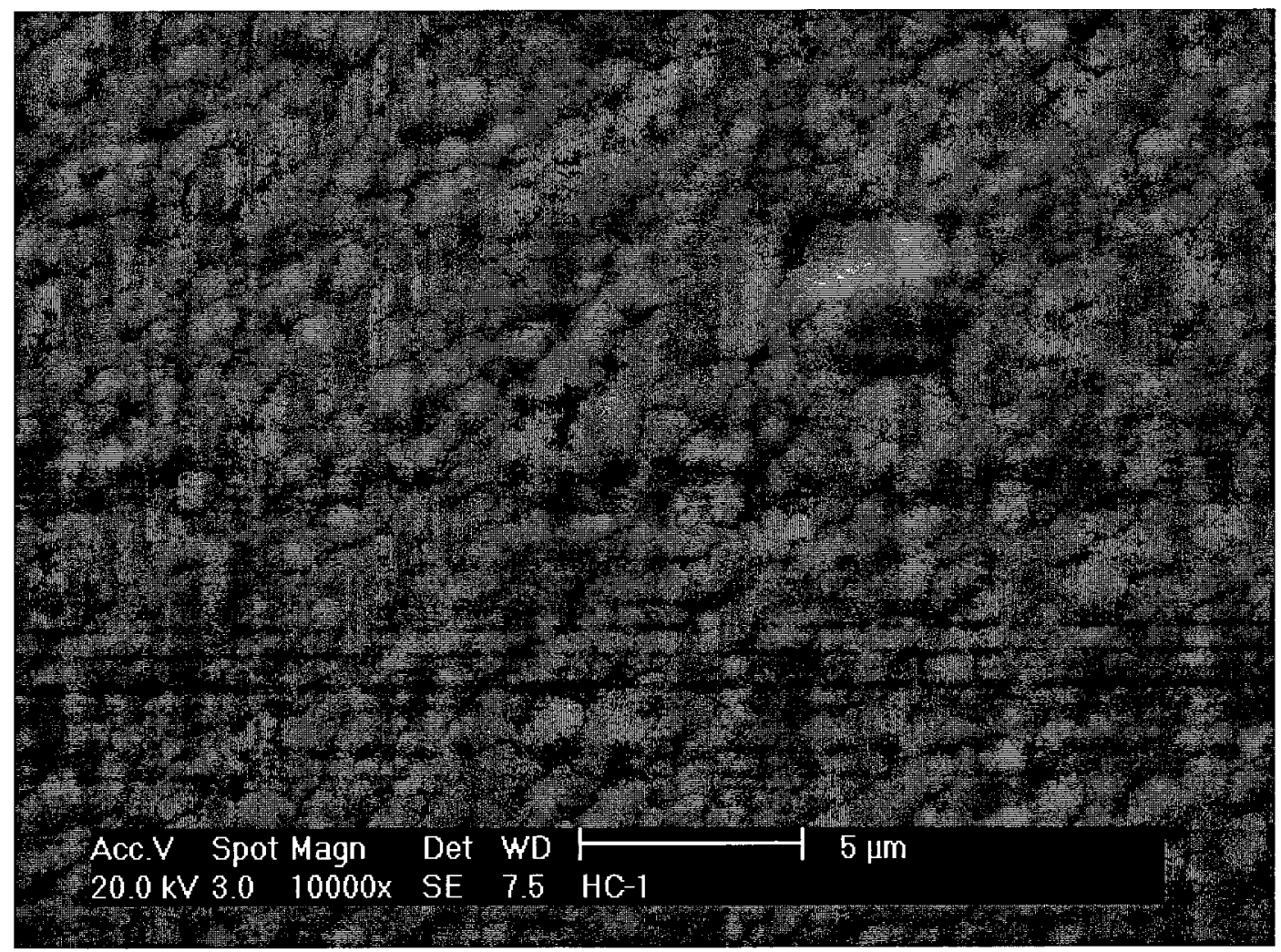

(a) $\mathrm{CrN}$ coating

Figure 4-9 to be continued 




(b) $\mathrm{CrSiCN}$ (1) coating

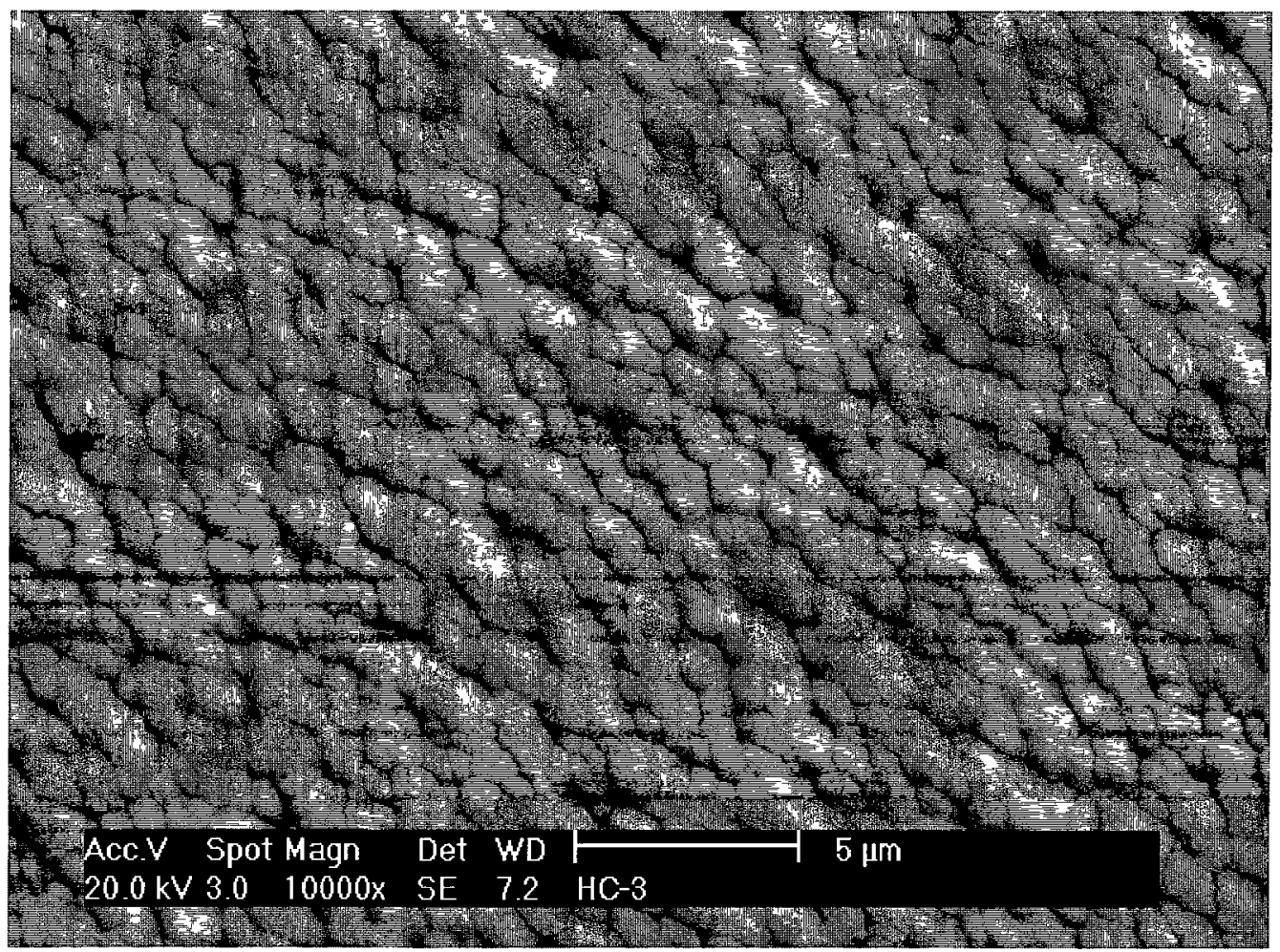

(c) $\mathrm{CrSiCN}(2)$ coating

Figure 4-9 to be continued 


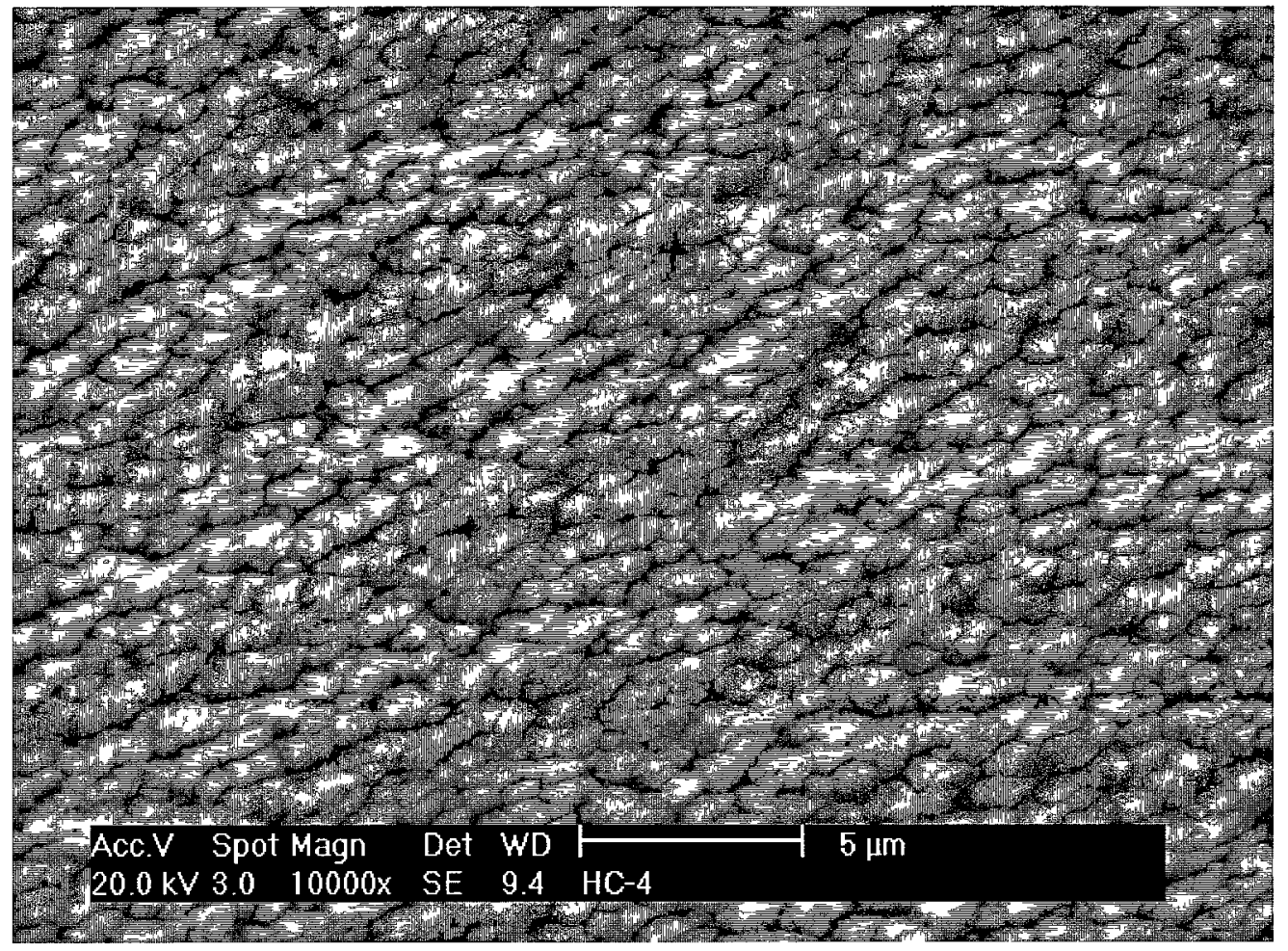

(d) $\mathrm{CrSiCN}(3)$ coating

Figure 4-9 SEM images demonstrating morphologies.

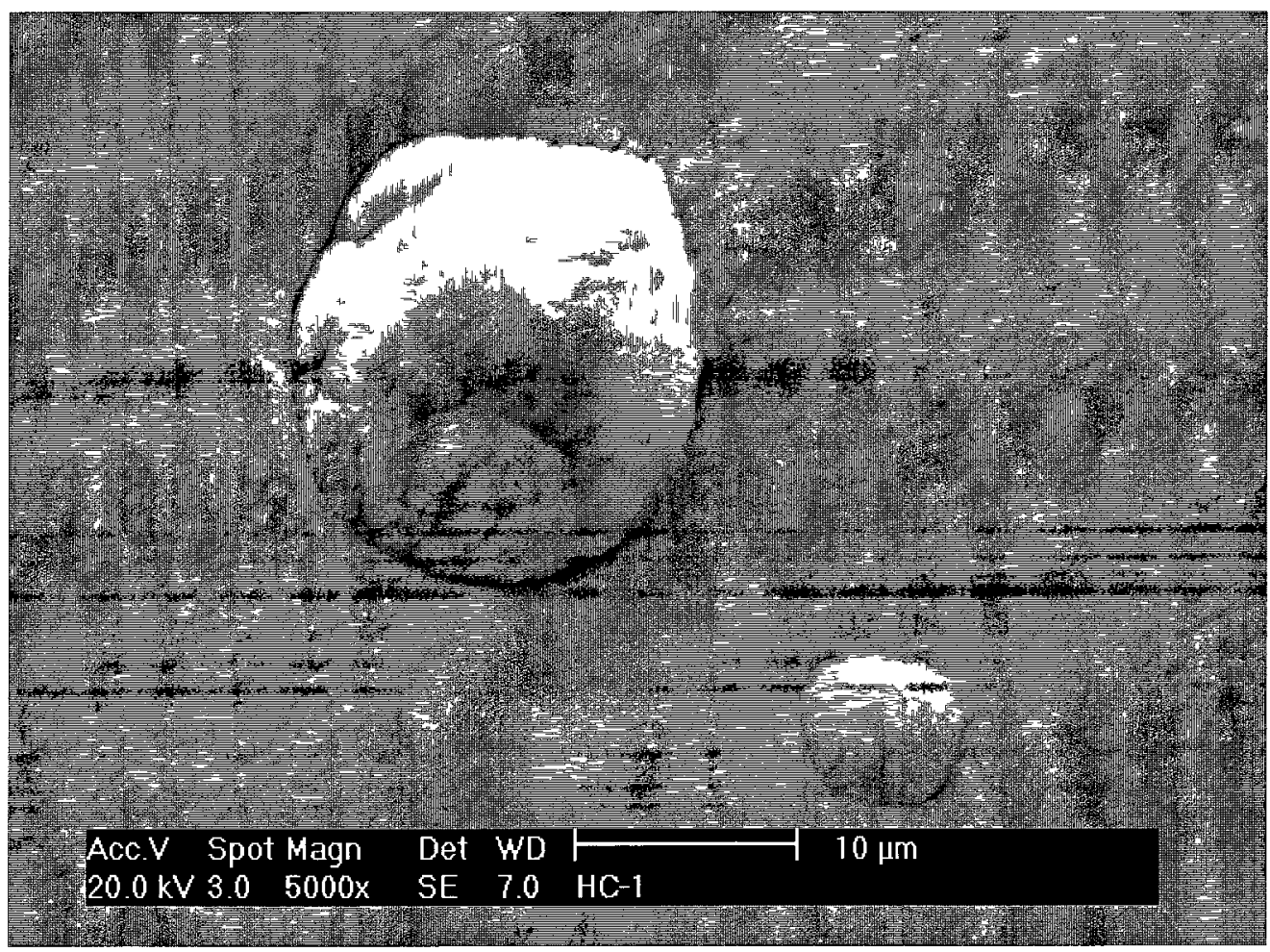

(a) $\mathrm{CrN}$ coating

Figure 4-10 to be continued 


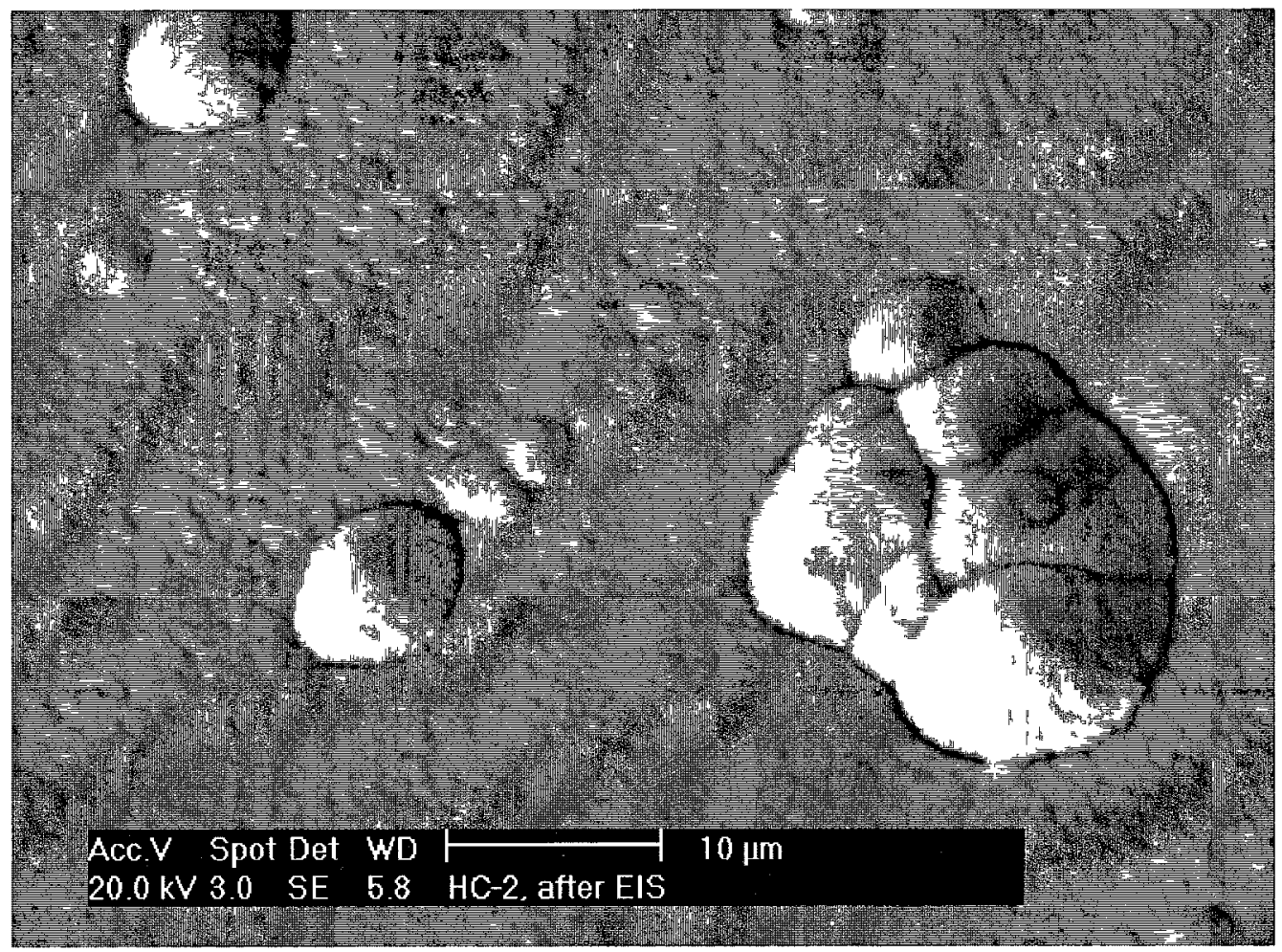

(b) $\mathrm{CrSiCN}(1)$ coating

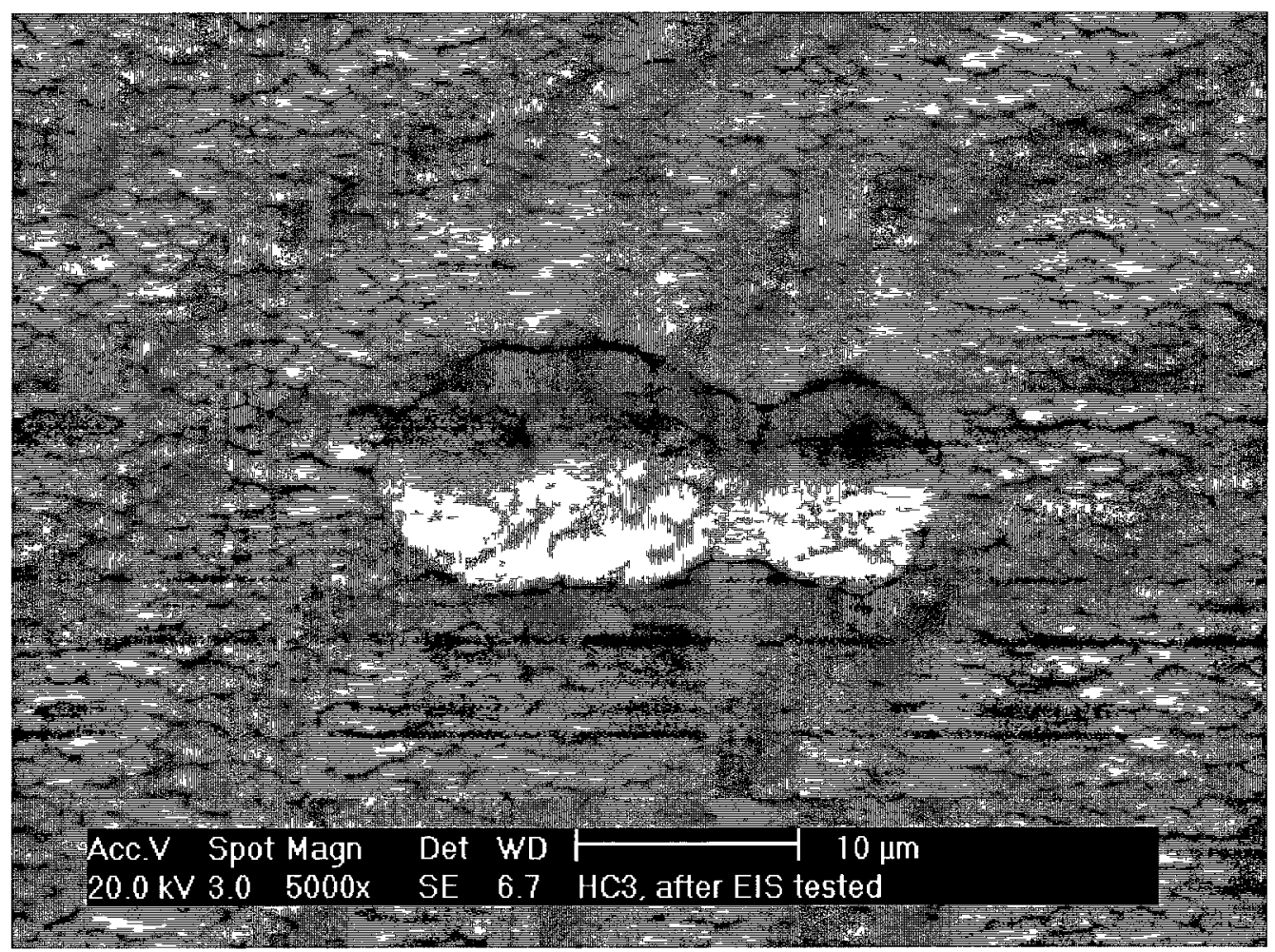

(c) $\mathrm{CrSiCN}(2)$ coating

Figure 4-10 to be continued 


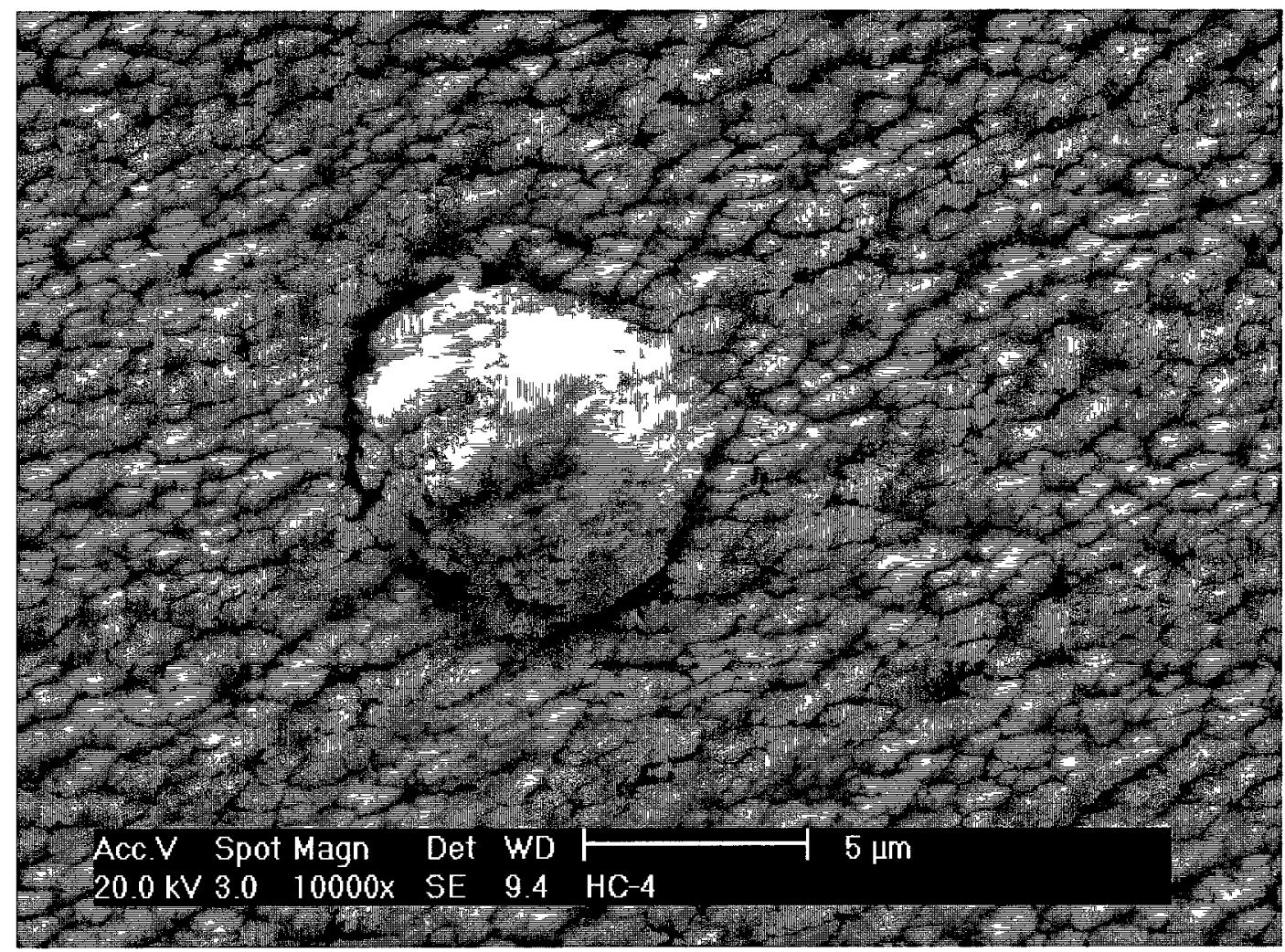

(d) $\mathrm{CrSiCN}(3)$ coating

Figure 4-10 SEM images revealing defects on the coatings.

Chemical compositions of the four $\mathrm{CrN}$ based coatings were examined at 4-5 different locations on each sample using EDS (using spot and area scanning), and the results are summarized in Table 4-3. As the solid solution limits are exceeded for $\mathrm{Si}$ and $\mathrm{C}$ in the metal nitride lattice, the formation of a second phase can occur [243 246]. The $\mathrm{N}$ level was approximately constant for all four coating compositions. Note that the content of $\mathrm{Si}$ in $\mathrm{CrSiCN}(3)$ was selected to be above the solid solution limit of $\mathrm{Si}$ in $\mathrm{Cr}-\mathrm{Si}-\mathrm{N}$ system (7.3 at.\% $\mathrm{Si}$ of the total $\mathrm{Si}+\mathrm{Cr})$ in an attempt to form the silicon nitride $\left(\mathrm{Si}_{3} \mathrm{~N}_{4}\right)$ phase [206]. 
Table 4-3 Chemical composition (at.\%) for the $\mathrm{CrN}$ based coatings

\begin{tabular}{|c|c|c|c|c|}
\hline Coating & $\mathrm{Cr}$ & $\mathrm{Si}$ & $\mathrm{C}$ & $\mathrm{N}$ \\
\hline $\mathrm{CrN}$ & 68.2 & - & - & 31.8 \\
\hline $\mathrm{CrSiCN}(1)$ & 54.5 & 1.3 & 14.0 & 30.2 \\
\hline $\mathrm{CrSiCN}(2)$ & 45.5 & 2.6 & 22.4 & 29.5 \\
\hline $\mathrm{CrSiCN}(3)$ & 43.0 & $3.4(7.33 \%$ for $\mathrm{Si}:(\mathrm{Cr}+\mathrm{Si}))$ & 24.9 & 28.7 \\
\hline
\end{tabular}

A summary of the defect density along with defect sizes in various coating systems are summarized in Table 4-4.

Table 4-4 Summary of defect densities of PVD coating systmes

\begin{tabular}{|l|c|c|}
\hline \multicolumn{1}{|c|}{ Coating system } & $\begin{array}{c}\text { Defect density } \\
(\text { count/mm }\end{array}$ & Defect size $(\mu \mathrm{m})$ \\
\hline EB TiN & $28-35$ & $1-10$ \\
\hline CA TiN/SS & $2200-2700$ & $1-10$ \\
\hline CA TiN/MS & $2200-2700$ & $1-10$ \\
\hline CrN & $185-225$ & $2-15$ \\
\hline CrSiCN(1) & $1115-1210$ & $2-15$ \\
\hline CrSiCN(2) & $75-95$ & $2-15$ \\
\hline CrSiCN(3) & $185-225$ & $2-15$ \\
\hline
\end{tabular}

\subsubsection{X-Ray Diffraction (XRD) Analysis}

The XRD patterns of the Ni plated and Ni-CNT plated samples at various pulse frequencies are illustrated in Figure 4-11 and Figure 4-12. Distinct Ni (111) and Ni (200) peaks appear at $2 \theta=44.5^{\circ}$ and $51.8^{\circ}$, respectively. 


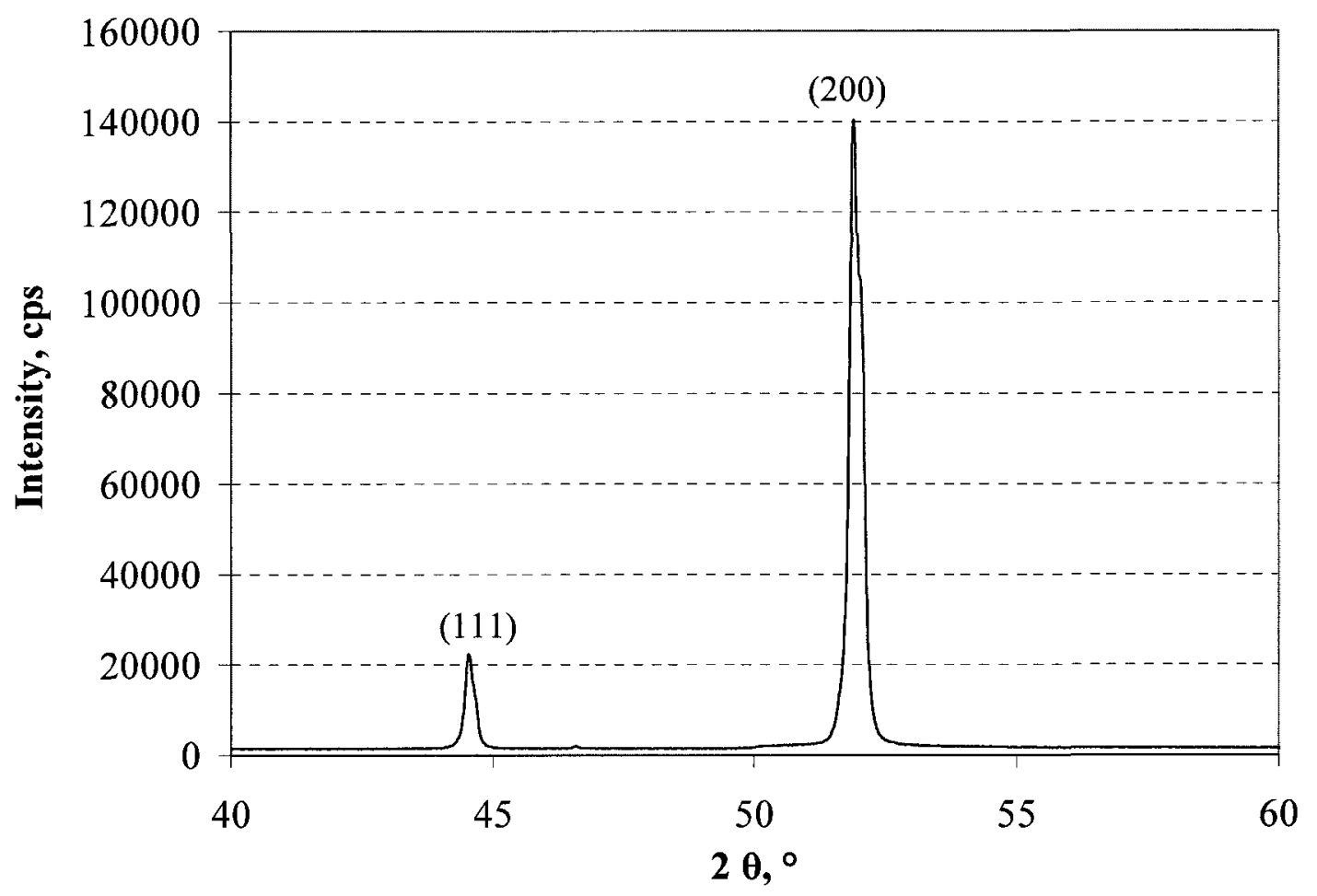

(a) $\mathrm{Ni}-\mathrm{O}$

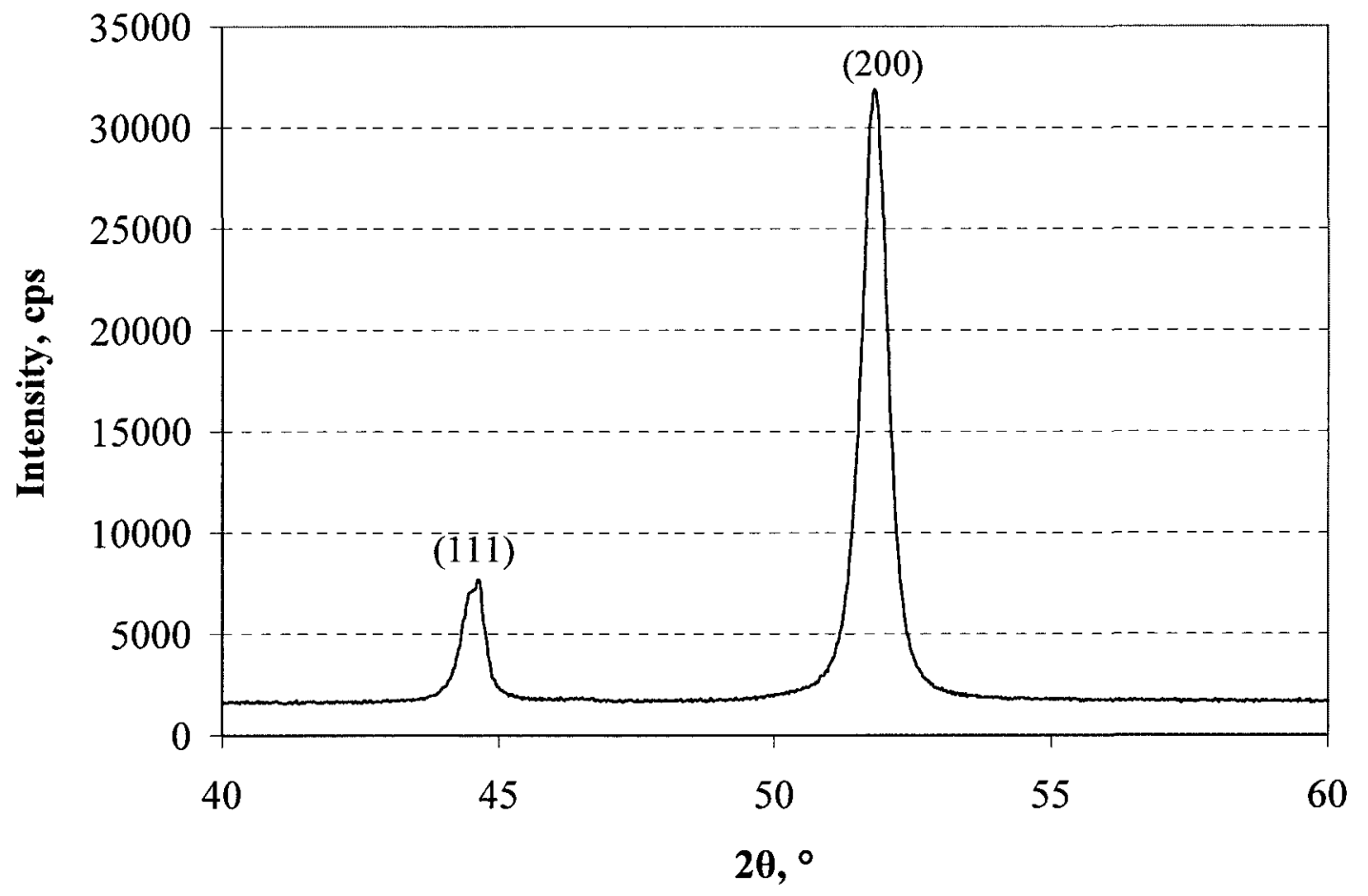

(b) Ni-5

Figure 4-11 to be continued 


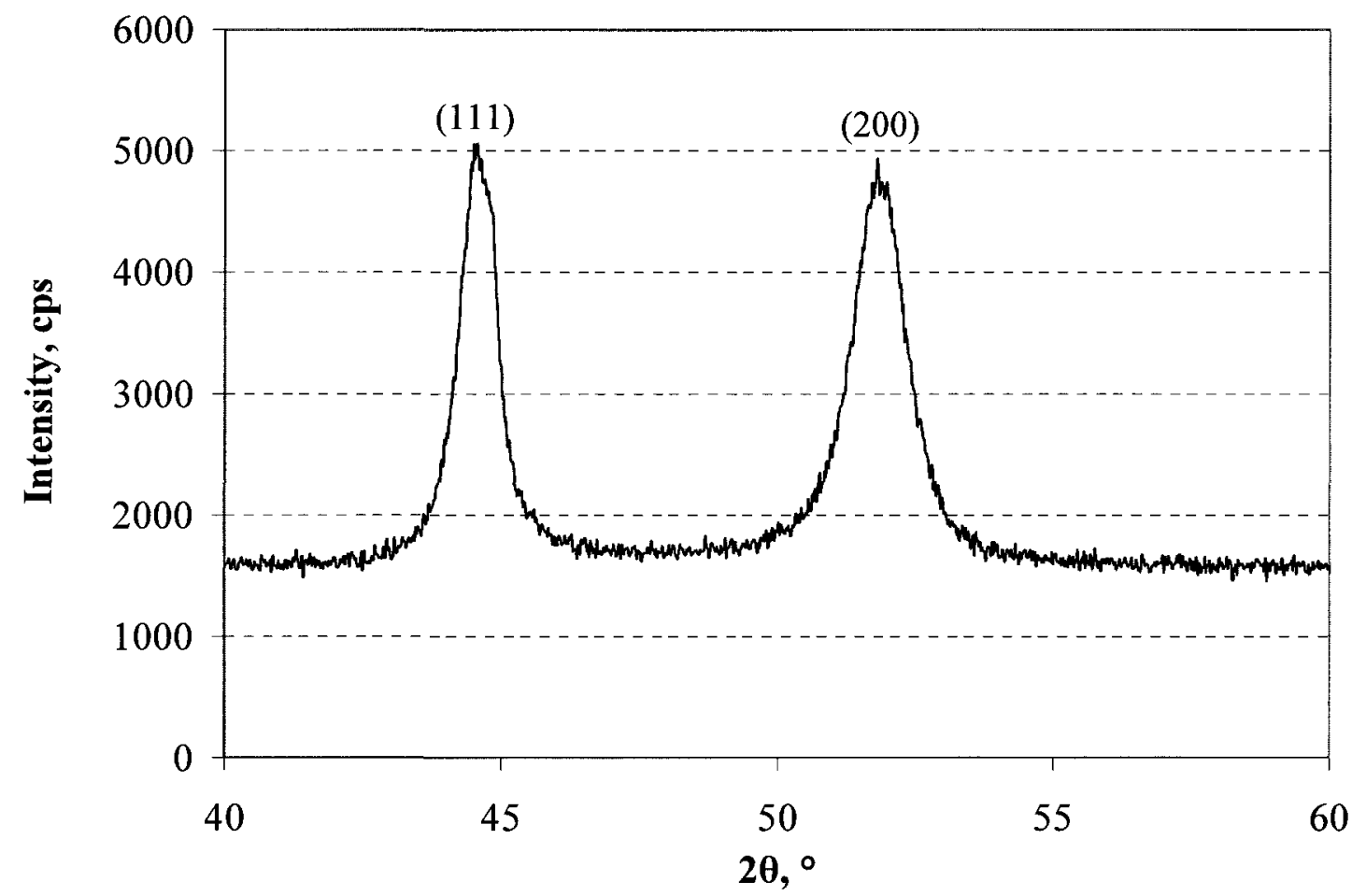

(c) $\mathrm{Ni}-100$

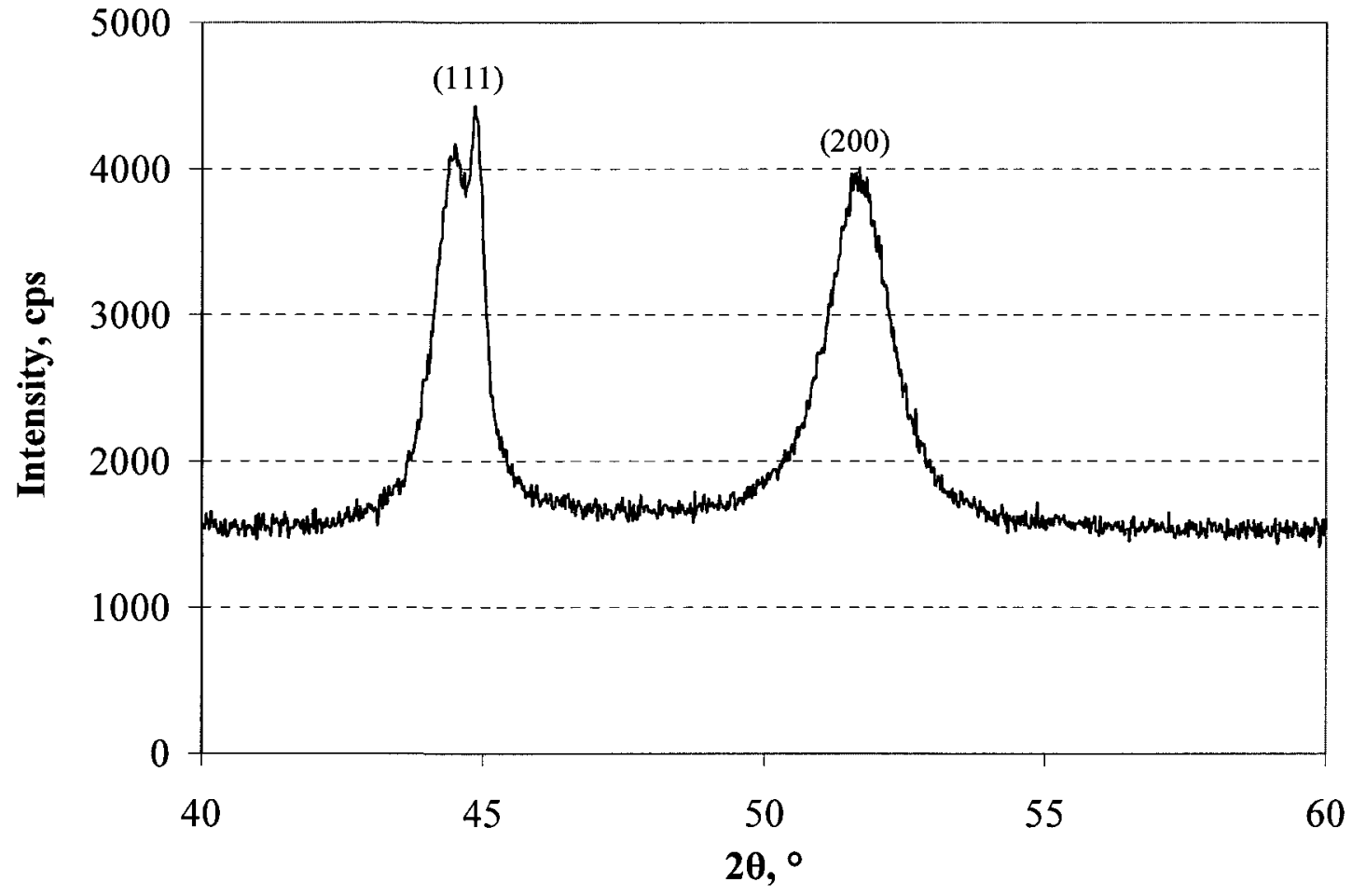

(d) $\mathrm{Ni}-1000$

Figure 4-11 XRD spectra of the Ni plated samples. 


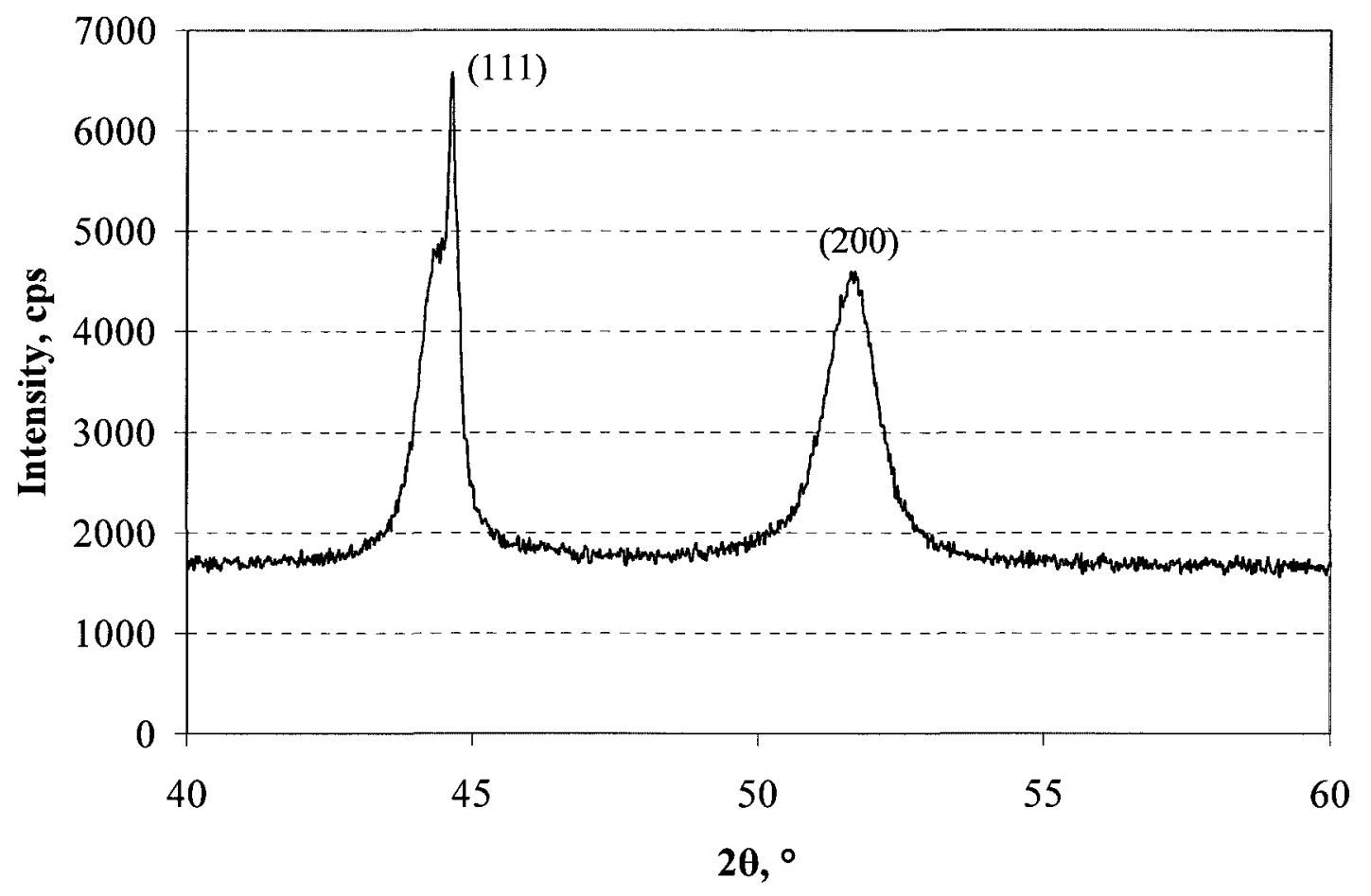

(a) Ni-CNT-0

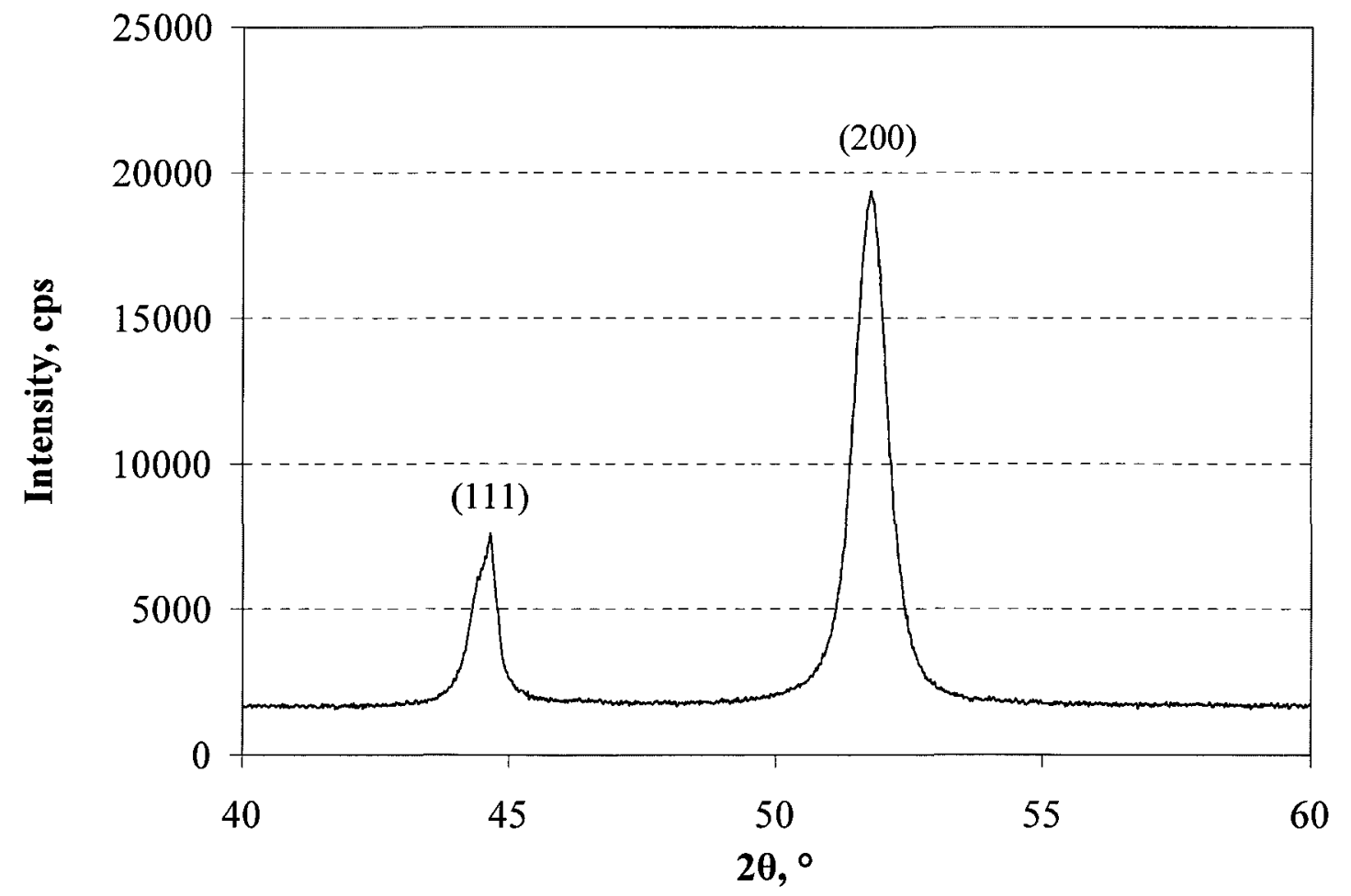

(b) Ni-CNT-5

Figure 4-12 to be continued 


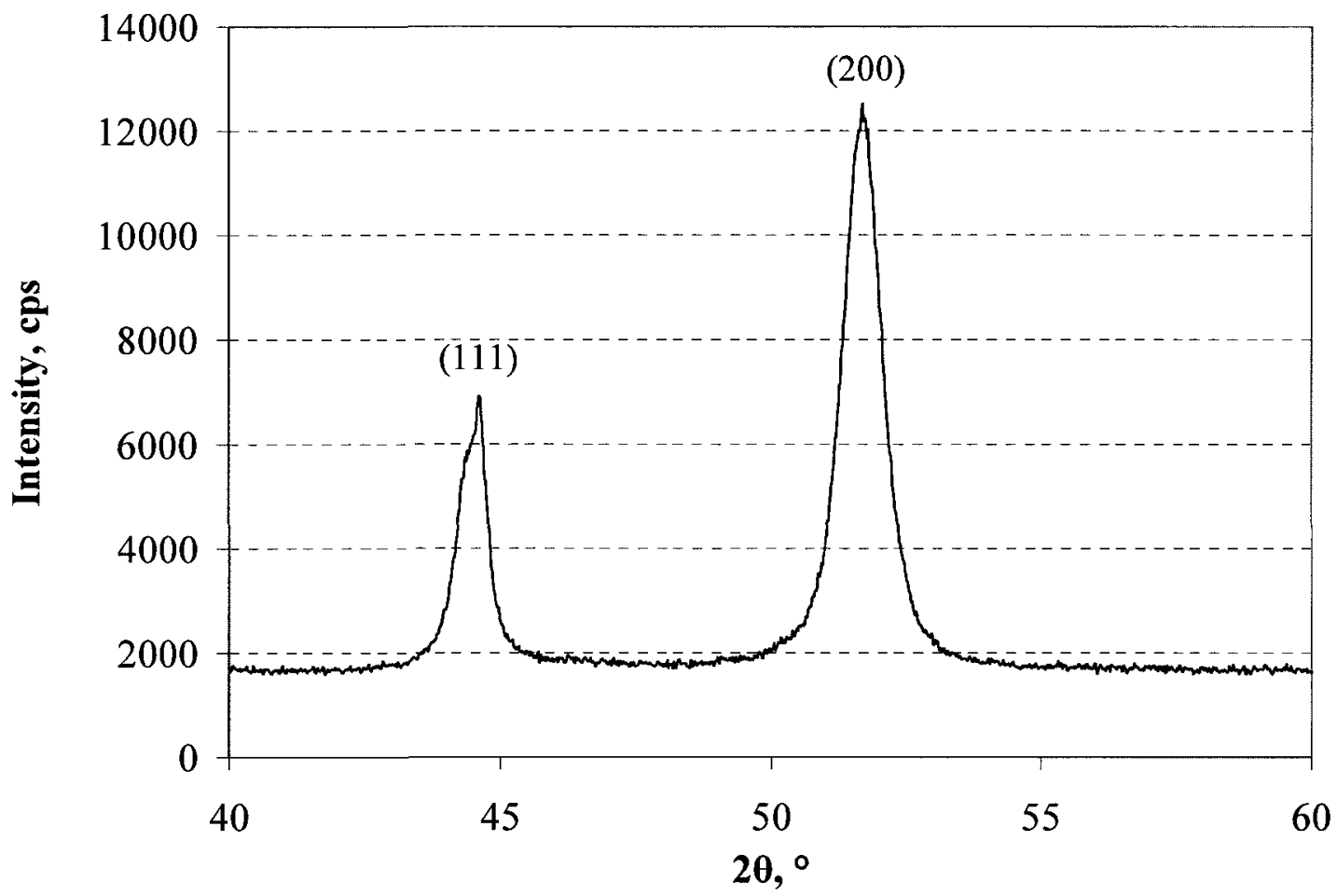

(c) Ni-CNT-100

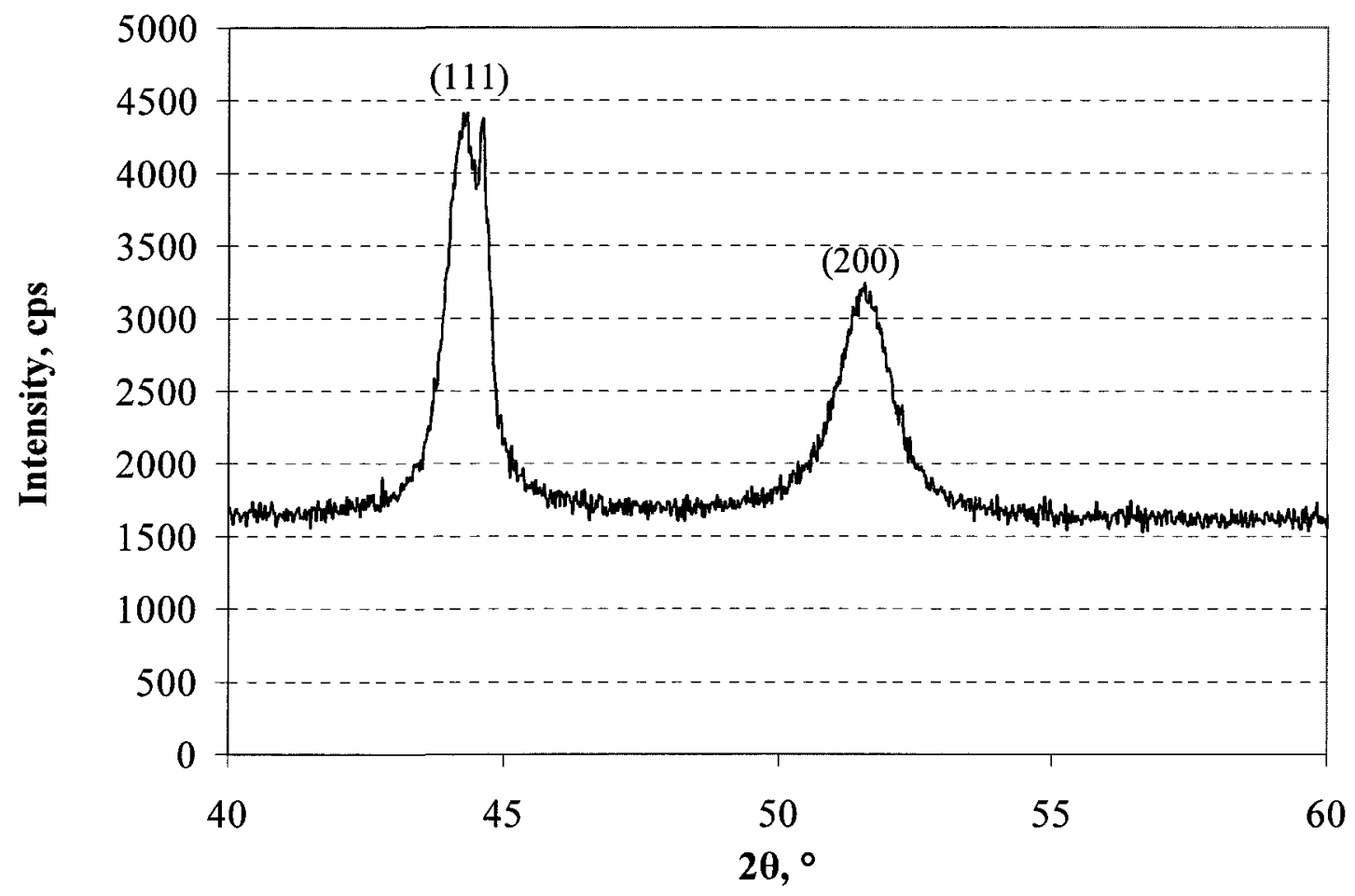

(d) Ni-CNT-1000

Figure 4-12 XRD spectra of Ni-CNT plated samples. 
Using FWHM method or Scherrer equation [392], the grain sizes for the plated samples were obtained, and are summarized in Table 4-5.

Table 4-5 Average grain size of the Ni and Ni-CNT plated samples

\begin{tabular}{|l|l|c|}
\hline \multicolumn{2}{|c|}{ Plating conditions } & Average grain size D (nm) \\
\hline \multirow{4}{*}{ Ni plated samples } & Ni-0 & 51.3 \\
\cline { 2 - 3 } & Ni-5 & 34.7 \\
\cline { 2 - 3 } & Ni-100 & 18.5 \\
\cline { 2 - 3 } & Ni-1000 & 9.4 \\
\hline \multirow{4}{*}{ Ni-CNT plated samples } & Ni-CNT-0 & 10 \\
\cline { 2 - 3 } & Ni-CNT-5 & 48 \\
\cline { 2 - 3 } & Ni-CNT-100 & 19 \\
\cline { 2 - 3 } & Ni-CNT-1000 & 15.1 \\
\hline
\end{tabular}

It is apparent that the pulse frequency affects the grain size of Ni plating. The grain size decreased from $51(\mathrm{Ni}-0)$ to $9 \mathrm{~nm}(\mathrm{Ni}-1000)$ as the pulse frequency increased from 0 to $1000 \mathrm{~Hz}$. These results are in agreement with other works [367, 389], and are consistent with the observation in Section 4.1.2. Without pulsed current, the addition of CNTs decreased the grain size from $51 \mathrm{~nm}(\mathrm{Ni}-0)$ to $10 \mathrm{~nm}(\mathrm{Ni}-\mathrm{CNT}-0)$, but no obvious trend can be observed on the effect of pulse frequency on grain size in the case of added CNTs.

In equiaxed polycrystalline nickel, the intensity ratio between peaks (111) and (200) is 100:48 (Powder diffraction file PDF\#04-0850, PDF-2 database sets 1-43, International Centre of Diffraction Data). Figure 4-11(a) reveals that the grain growth of Ni plating is along a preferred direction [200] (perpendicular to the (200) plane) during the deposition of the sample Ni- 0 . With the introduction of a pulsed current, the tendency for a preferred growth direction [200] is gradually reduced, but with a pulse frequency of $1000 \mathrm{~Hz}$ 
(sample Ni-1000), this preferred orientation still remained as evident in the (111) and (200) peak intensities (Figure 4-11 d). The change in crystal orientation with a pulsed current is attributed to a disruption in the grain growth as the current is switched. The introduction of CNTs in the electrolyte may offer a similar effect to the pulsed current, and be able to disrupt the grain growth along the [200] direction as shown in Figure 4-12 (a), where a lower Ni (200) peak can be observed in sample Ni-CNT-0 as compared to that in Figure 4-11 (a). Also note that in the XRD spectra (Figure 4-11 and Figure 412), additional peaks are observed near the $\mathrm{Ni}$ (111) peak for sample Ni-1000 and NiCNT-1000, which was identified as an X-ray diffraction from the mild steel substrate (body centered cubic - BCC).

XRD spectra for EB and CA TiN coating systems are illustrated in Figure 4-13. Strong TiN peaks from (111), (002), (220), and (222) planes exist in the EB TiN coating spectrum. In contrast, only two strong (111) and (222) TiN peaks can be found in the CA TiN coating spectrum. This difference stems from the different deposition conditions for EB and CA TiN coatings. To minimize the total energy under strain-energy-dominated growth, the (111) plane that has the lowest strain energy (due to anisotropy in modulus of elasticity) tends to align perpendicularly to the growing direction [393, 225]. In the CA deposition process, high bias voltage results in high atom mobility and increased surface diffusion, which could facilitate alignment of the growth along the [111] direction. The EB TiN coating also showed several more peaks reflecting from the substrate due to the limited thickness of the EB TiN coating $(1.5 \mu \mathrm{m})$. 

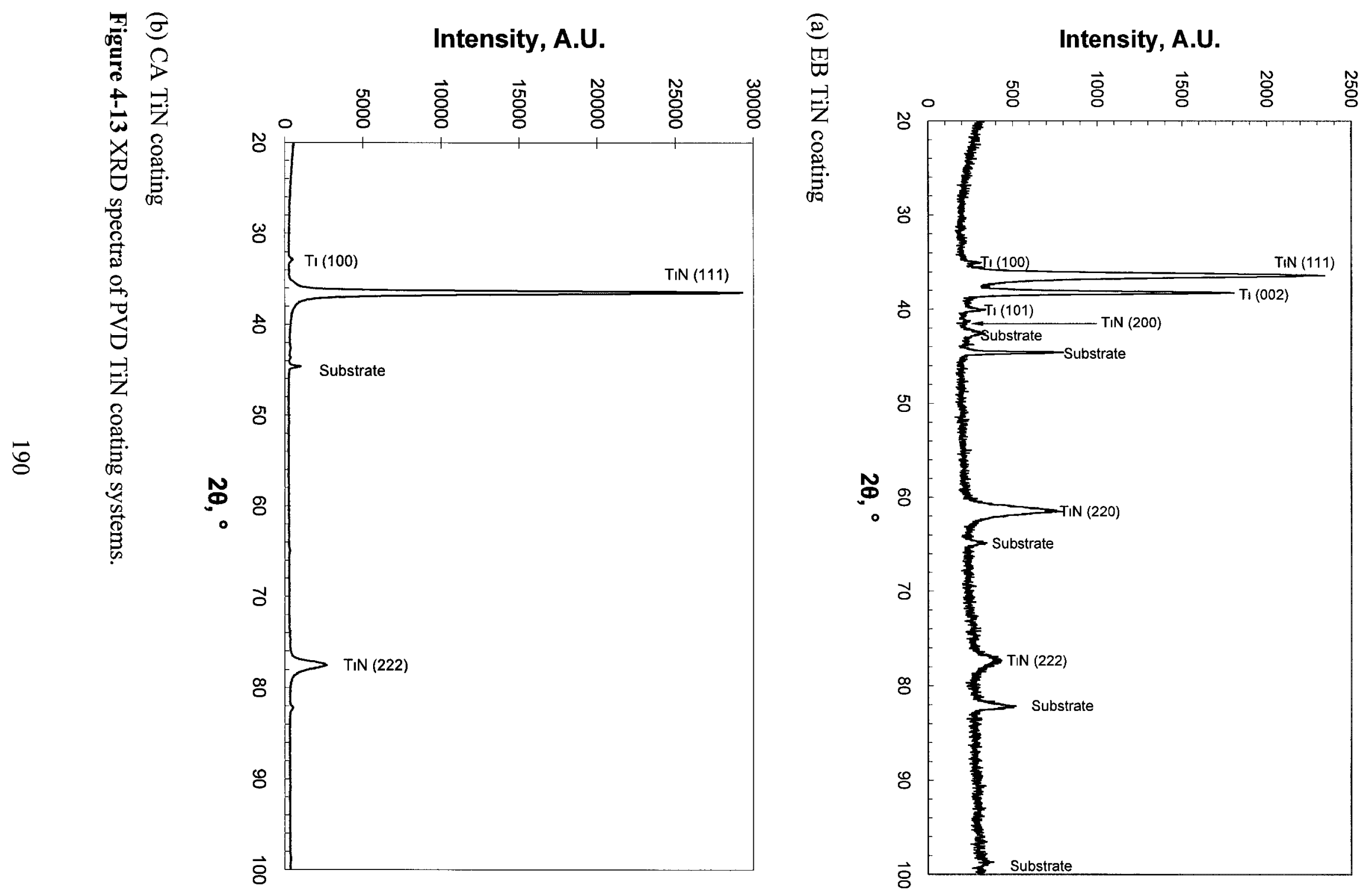
The XRD spectra for the four CrN based coating systems are given in Figure 4-14. The diffraction pattern of $\mathrm{CrN}$ coating shows a weak and broad peak at $2 \theta=43.8^{\circ}$ with a preferred orientation of (200). This broad and low intensity peak indicates the presence of a fine crystalline face-centered cubic (FCC) $\mathrm{B} 1-\mathrm{NaCl}$ crystal structure. The $\mathrm{CrSiCN}(1)$ coating with low contents of $\mathrm{Si}$ and $\mathrm{C}$ exhibits a $\mathrm{B} 1-\mathrm{NaCl}$ crystal structure with a (200) orientation at $2 \theta=44.4^{\circ}$. The $\mathrm{CrSiCN}(2)$ coating, with further increase in $\mathrm{Si}$ and $\mathrm{C}$, shows a B1-NaCl crystal structure with a (200) orientation at $2 \theta=44.1^{\circ}$. The $\mathrm{CrSiCN}(3)$ coatings, with higher contents of $\mathrm{Si}$ and $\mathrm{C}$, shows all the distinct diffraction peaks of $\mathrm{B} 1 \mathrm{NaCl}$ crystal structures indicating no preferred orientations; and the narrow and distinct (111) and (200) peaks revealed larger grain sizes compared to other coatings. Furthermore, in the enlarged region near the (111) peak for $\mathrm{CrSiCN}(3)$ coating, diffraction peaks from crystalline $\mathrm{Si}_{3} \mathrm{~N}_{4}$ were found as demonstrated by the insert in Figure 4-14. Published research indicated that the addition of $\mathrm{Si}$ to $\mathrm{CrN}$ coating was observed to induce the formation of amorphous $\mathrm{Si}_{3} \mathrm{~N}_{4}$ near the grain boundary [247, 340, 394]. However, crystalline $\mathrm{Si}_{3} \mathrm{~N}_{4}$ was observed in this study. The reason for the formation of the crystalline $\mathrm{Si}_{3} \mathrm{~N}_{4}$ phase in this high-carbon content coating (24.9 at.\%)(Table 4-3) or if the formation of the crystalline $\mathrm{Si}_{3} \mathrm{~N}_{4}$ phase is associated with the high-carbon content is not clear at present. Also, further investigations could be needed to reveal if amorphous $\mathrm{Si}_{3} \mathrm{~N}_{4}$ phase exists.

Using FWHM method or Scherrer equation [392], the average grain size for each coating was calculated, and is shown in Figure 4-15. The grain size decreased from $7.6 \mathrm{~nm}$ for $\mathrm{CrN}$ coating to $5 \mathrm{~nm}$ for $\mathrm{CrSiCN}(1)$ with slight addition of $\mathrm{Si}(1.3$ at.\%). With further 
increase in $\mathrm{Si}$, the grain size then increased to $20.4 \mathrm{~nm}$ for $\mathrm{CrSiCN}(2)$ and $44.5 \mathrm{~nm}$ for $\mathrm{CrSiCN}(3)$. This trend in grain size change is consistent with the surface morphology observation where $\mathrm{CrSiCN}(2)$ coating showed a coarse grain structure (Figure 4-9 c), and $\mathrm{CrSiCN}(3)$ coating exhibites a coarse facet structure (Figure 4-9 d).

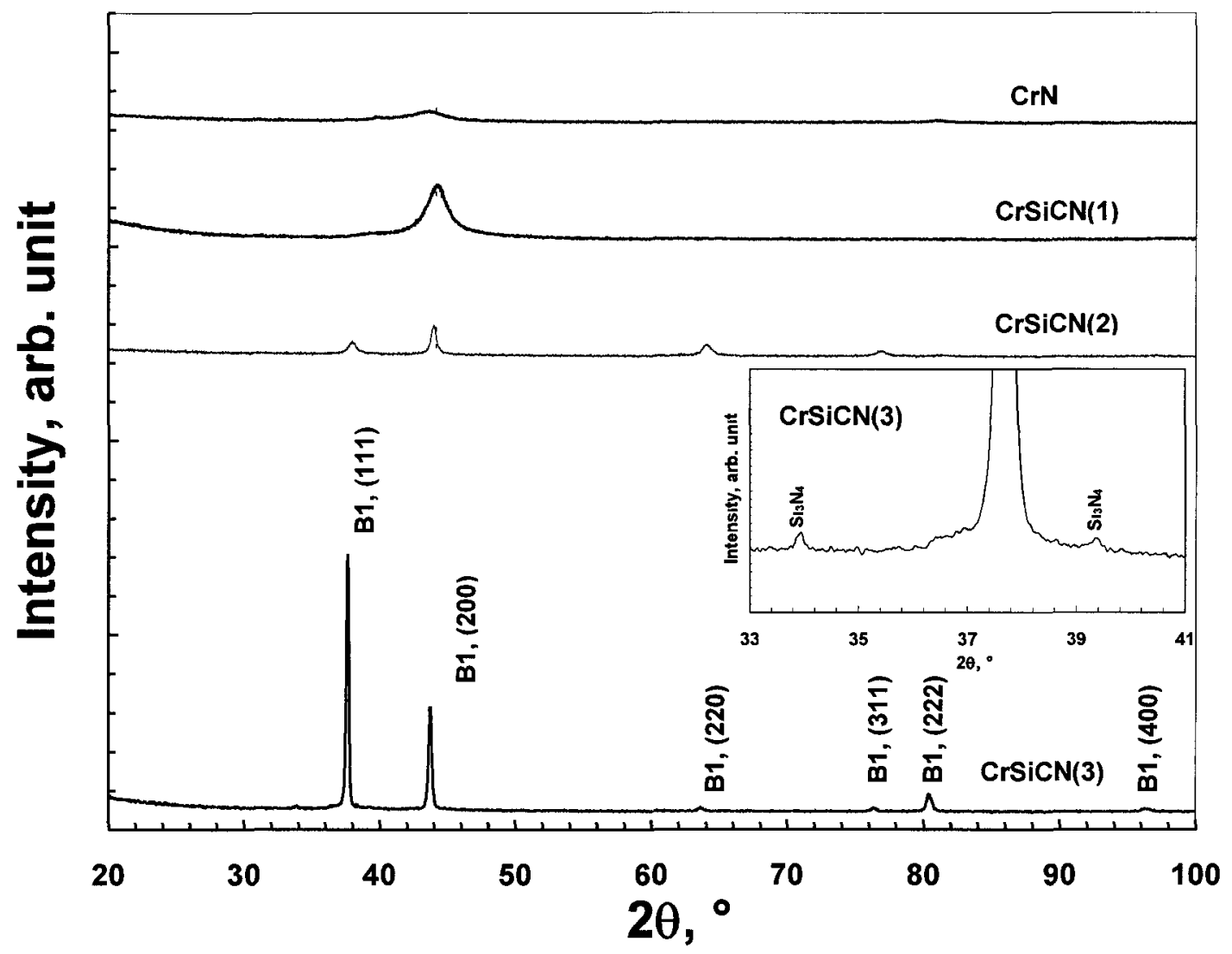

Figure 4-14 XRD spectra of CrN based PVD coating systems.

Table 4-6 Average grain size of $\mathrm{CrN}$ based coating systems

\begin{tabular}{|l|ll|}
\hline \multicolumn{1}{|c|}{ Coating } & \multicolumn{3}{|c|}{ Grain size (nm) } \\
\hline $\mathrm{CrN}$ & 7.6 & $(\mathrm{CrN}(200))$ \\
\hline $\mathrm{CrSiCN}(1)$ & 5 & $(\mathrm{CrN}(200))$ \\
\hline $\mathrm{CrSiCN}(2)$ & 14.27 & $(\mathrm{CrN}(111))$ \\
\hline \multirow{2}{*}{$\mathrm{CrSiCN}(3)$} & 43.67 & $(\mathrm{CrN}(111))$ \\
\cline { 2 - 3 } & 44.53 & $(\mathrm{CrN}(200))$ \\
\hline
\end{tabular}




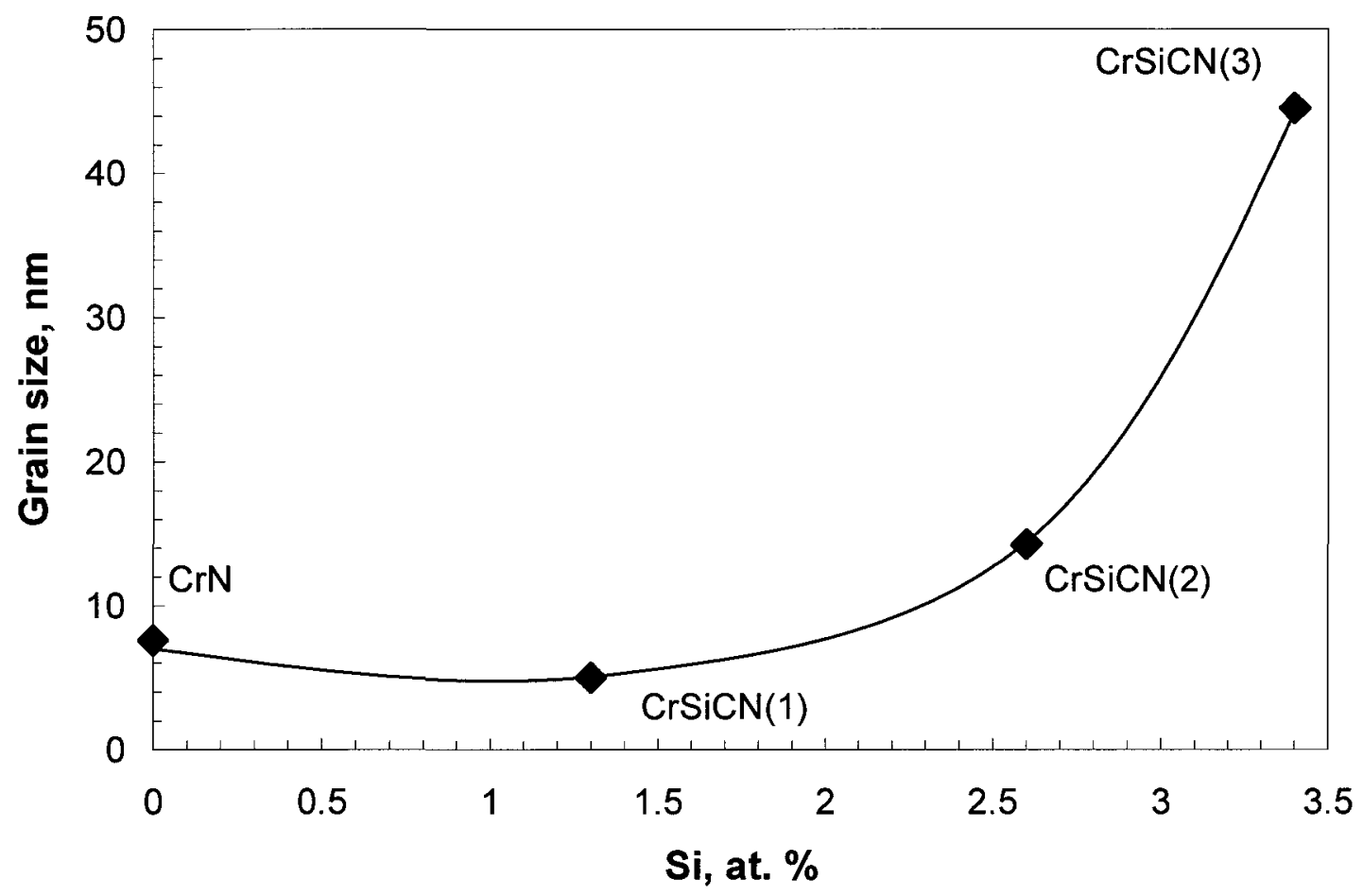

Figure 4-15 Average grain size vs. Si content in $\mathrm{CrN}$ based coating systems.

\subsection{Mechanical Properties}

Hardness values of the Ni plating and Ni-CNT plating are presented in Figure 4-16. The hardness of conventional $\mathrm{Ni}$ plating is sensitive to the pulse frequency, with hardness values increasing with the pulse frequency, from $3.72(\mathrm{Ni}-0)$ to $9.15 \mathrm{GPa}(\mathrm{Ni}-1000)$. This trend is consistent with the observed grain size reduction with increasing pulse frequency (Section 4.1.3). Fine grains result in a larger grain boundary area, which can impede dislocation motion, and therefore increase the strength (and hardness).

The hardness is also significantly improved by the incorporation of CNTs for the samples $\mathrm{Ni}-0$ and Ni-CNT-0; the average hardnesses were 3.72 and $8.2 \mathrm{GPa}$, respectively and the 




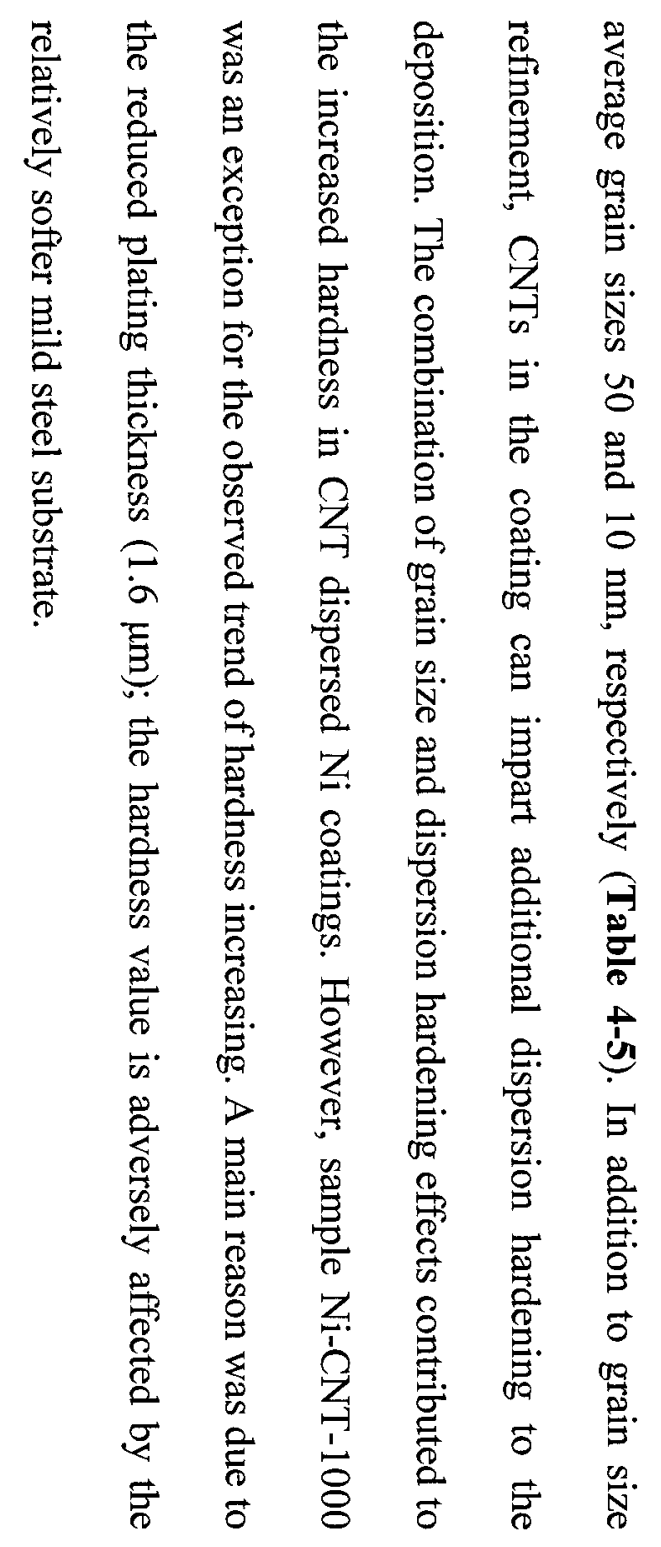


direct current, but the changes in Young's modulus (E) for the samples deposited at various conditions are much less significant than the changes in hardness.

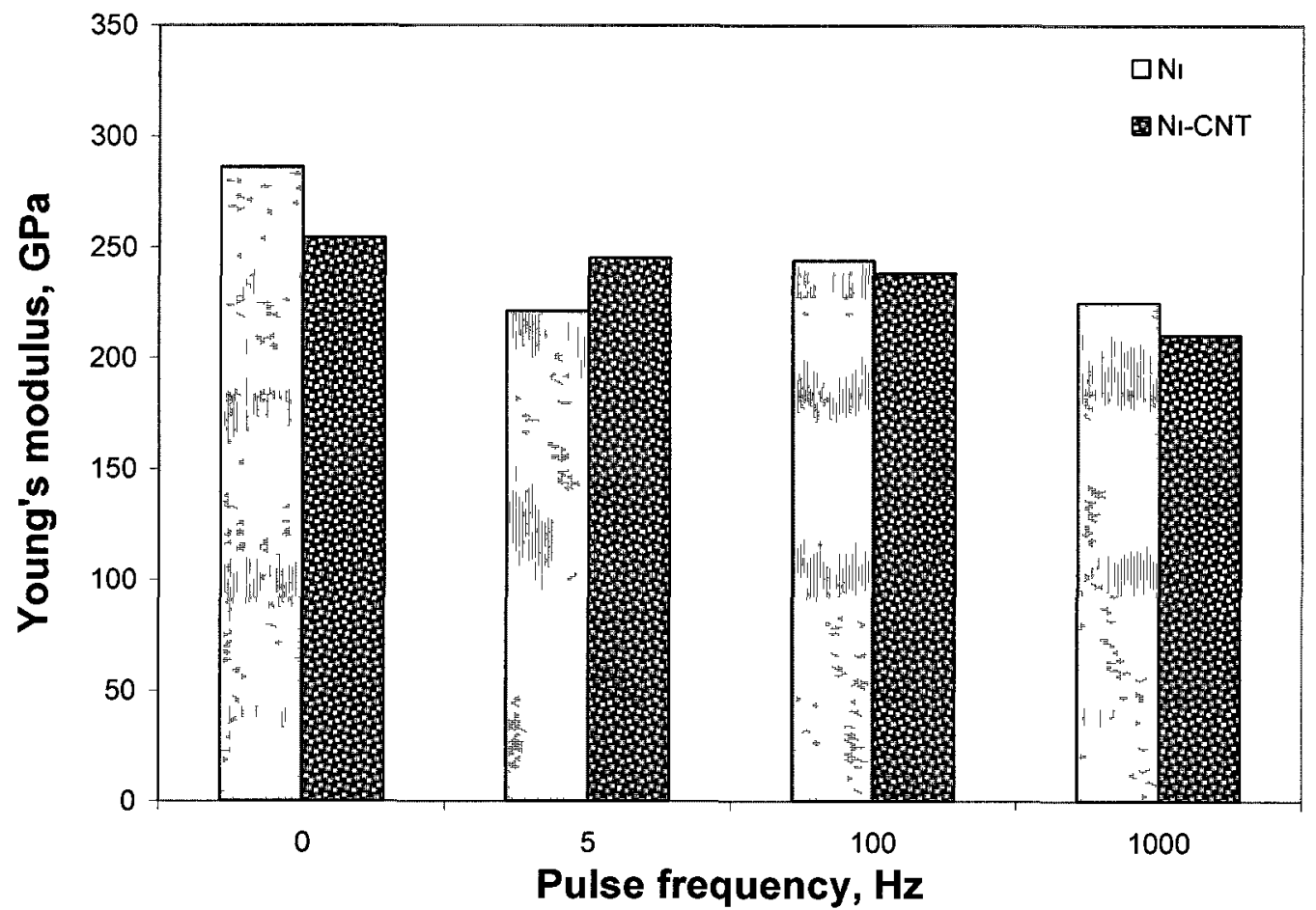

Figure 4-17 Young's modulus of Ni plated and Ni-CNT plated samples at various pulse frequencies.

The $\mathrm{H}^{3} / \mathrm{E}^{2}$ ratios, which have been used as an indicator for evaluation of the resistance to plastic deformation [193], are displayed in Figure 4-18 for the Ni plated samples. It can be seen that the trend is consistent with that of the hardness values. For the pure Ni plated samples, the $\mathrm{H}^{3} / \mathrm{E}^{2}$ ratios were found to increase with pulse frequency; but for the NiCNT plated samples, no clear trend was observed with frequency. 


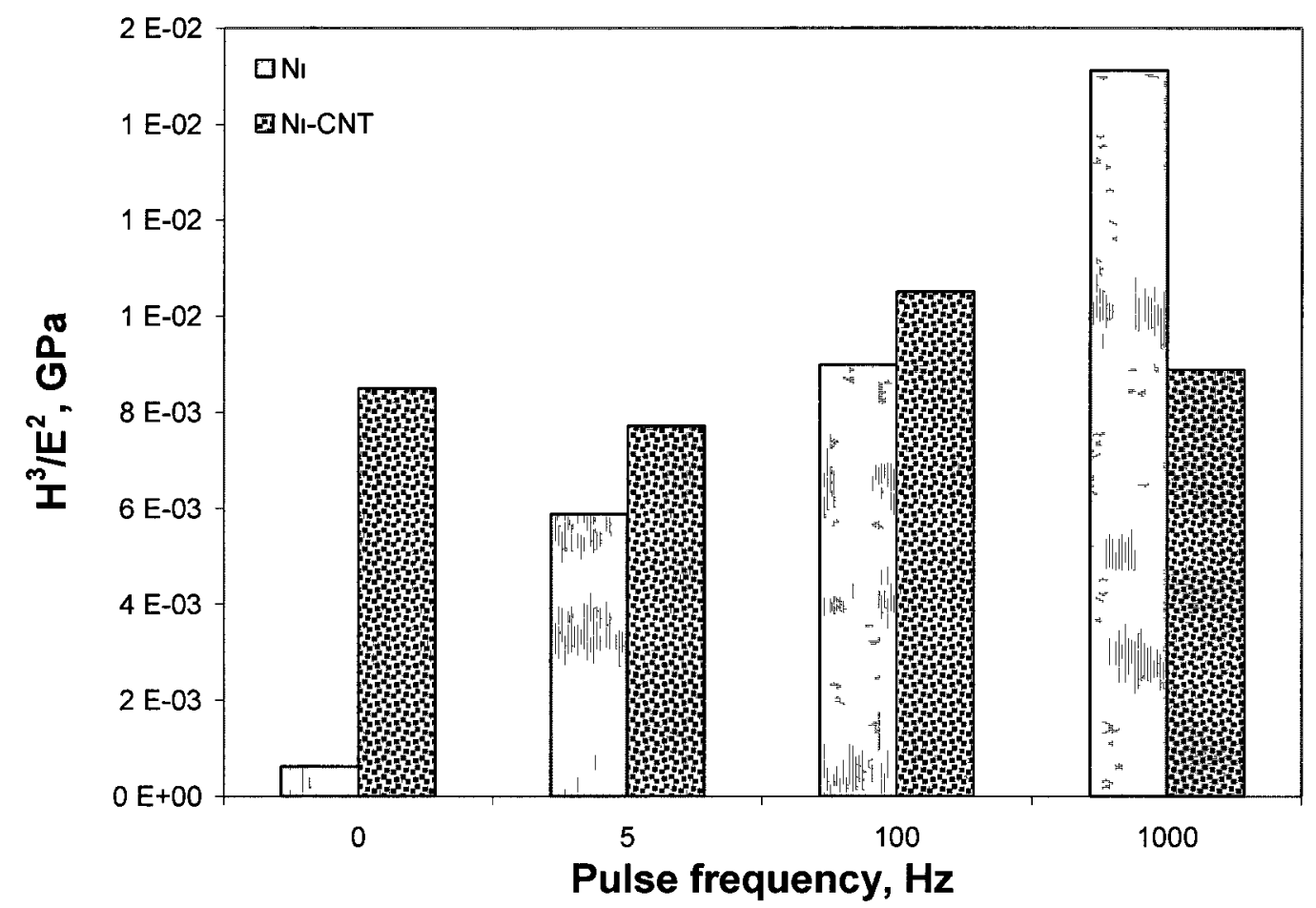

Figure 4-18 $\mathrm{H}^{3} / \mathrm{E}^{2}$ ratios for Ni plated and Ni-CNT plated samples.

The experimentally obtained hardness, modulus of elasticity, which were averaged from 12 measruements (3.5.4), and the caculated $\mathrm{H}^{3} / \mathrm{E}^{2}$ ratios for the Ni plated and Ni-CNT plated samples are summarized in Table 4-7.

Table 4-7 Summary of Vickers hardness and elastic modulus of Ni plated samples

\begin{tabular}{|l|c|c|c|}
\hline Coating system & Hardness $\mathrm{H}(\mathrm{GPa})$ & Elastic modules E (GPa) & $\mathrm{H}^{3} / \mathrm{E}^{2}(\mathrm{GPa})$ \\
\hline AISI 1040 (MS) & 2.6 & 200.59 & $4.2 \times 10^{-4}$ \\
\hline Ni-0 & 3.7 & 286.34 & $6.3 \times 10^{-4}$ \\
\hline Ni-5 & 6.6 & 221.34 & $5.9 \times 10^{-3}$ \\
\hline Ni-100 & 8.1 & 244.15 & $9.0 \times 10^{-3}$ \\
\hline Ni-1000 & 9.2 & 225.07 & $1.5 \times 10^{-2}$ \\
\hline Ni-CNT-0 & 8.2 & 254.44 & $8.5 \times 10^{-3}$ \\
\hline Ni-CNT-5 & 7.7 & 245.26 & $7.7 \times 10^{-3}$ \\
\hline Ni-CNT-100 & 8.4 & 238.43 & $1.1 \times 10^{-2}$ \\
\hline Ni-CNT-1000 & 7.3 & 210.45 & $8.9 \times 10^{-3}$ \\
\hline
\end{tabular}


The measured harnesses $(\mathrm{H})$ and Young's modulus (E) for the PVD coating samples, which were averaged from 12 measurements (3.5.4), are summarized in Table 4-8. The hardness values of the TiN and $\mathrm{CrN}$ based coating systems were obviously higher than the 17-4PH steel substrate $(4.42 \mathrm{GPa})$. The TiN coatings demonstrated higher hardnesses and Young's moduli compared with the $\mathrm{CrN}$ based coating systems. Of the two TiN coating systems, due to the effect of ion bombardment in the deposition process [323, 324], the CA sample showed a higher hardness and Young's modulus than the EB sample. The addition of $\mathrm{Si}$ and $\mathrm{C}$ influenced the Young's modulus (E) of $\mathrm{CrN}$ based coating systems.

The TiN coating systems showed higher $\mathrm{H}^{3} / \mathrm{E}^{2}$ ratios than the $\mathrm{CrN}$ based coating systems, indicating their higher resistances to plastic deformation [395, 396], and are therefore expected to offer better resistances to wear and erosion.

Table 4-8 Summary of Vickers hardness and elastic modulus of PVD coating systems

\begin{tabular}{|l|c|c|c|}
\hline Coating system & Hardness $\mathrm{H}(\mathrm{GPa})$ & Elastic modules E $(\mathrm{GPa})$ & $\mathrm{H}^{3} / \mathrm{E}^{2}(\mathrm{GPa})$ \\
\hline $17-4 \mathrm{PH}$ & 4.42 & 220.88 & 0.002 \\
\hline EB TiN & 31.94 & 368.10 & 0.24 \\
\hline CA TiN/SS & 33.98 & 456.73 & 0.20 \\
\hline $\mathrm{CrN}$ & 19.97 & 309.87 & 0.08 \\
\hline $\mathrm{CrSiCN}(1)$ & 21.59 & 313.12 & 0.10 \\
\hline CrSiCN(2) & 14.04 & 233.41 & 0.05 \\
\hline CrSiCN(3) & 13.86 & 218.33 & 0.06 \\
\hline
\end{tabular}

In the $\mathrm{CrN}$ based coatings, as shown in Figure 4-19, hardness and Young's modulus (E) slightly increased with a small addition of $\mathrm{Si}(1.3$ at.\%), i.e., $\mathrm{CrSiCN}(1)$. With further 
increases in $\mathrm{Si}$ content (from 1.3 to 3.6 at.\%), i.e., $\mathrm{CrSiCN}(2)$ and $\mathrm{CrSiCN}(3)$, the hardness and Young's modulus significantly decreased.

The increases in hardness and Young's modulus from coating $\mathrm{CrN}$ to $\mathrm{CrSiCN}(1)$, with a slight addition of $\mathrm{Si}(1.3$ at.\%), were associated with the alloying effect of $\mathrm{Si}$ and grain refinement (from 7.6 to $5 \mathrm{~nm}$ )(Table 4-6)(Figure 4-15) [397, 398]. With further increase in $\mathrm{Si}$, the hardness and Young's modulus reductions can be explained by the phase transformation to a more ductile fcc structure, an increase in grain size, and the formation of $\mathrm{Si}_{3} \mathrm{~N}_{4}$, which resulted in $\mathrm{Si}$ depletion from solid solution [249, 399].

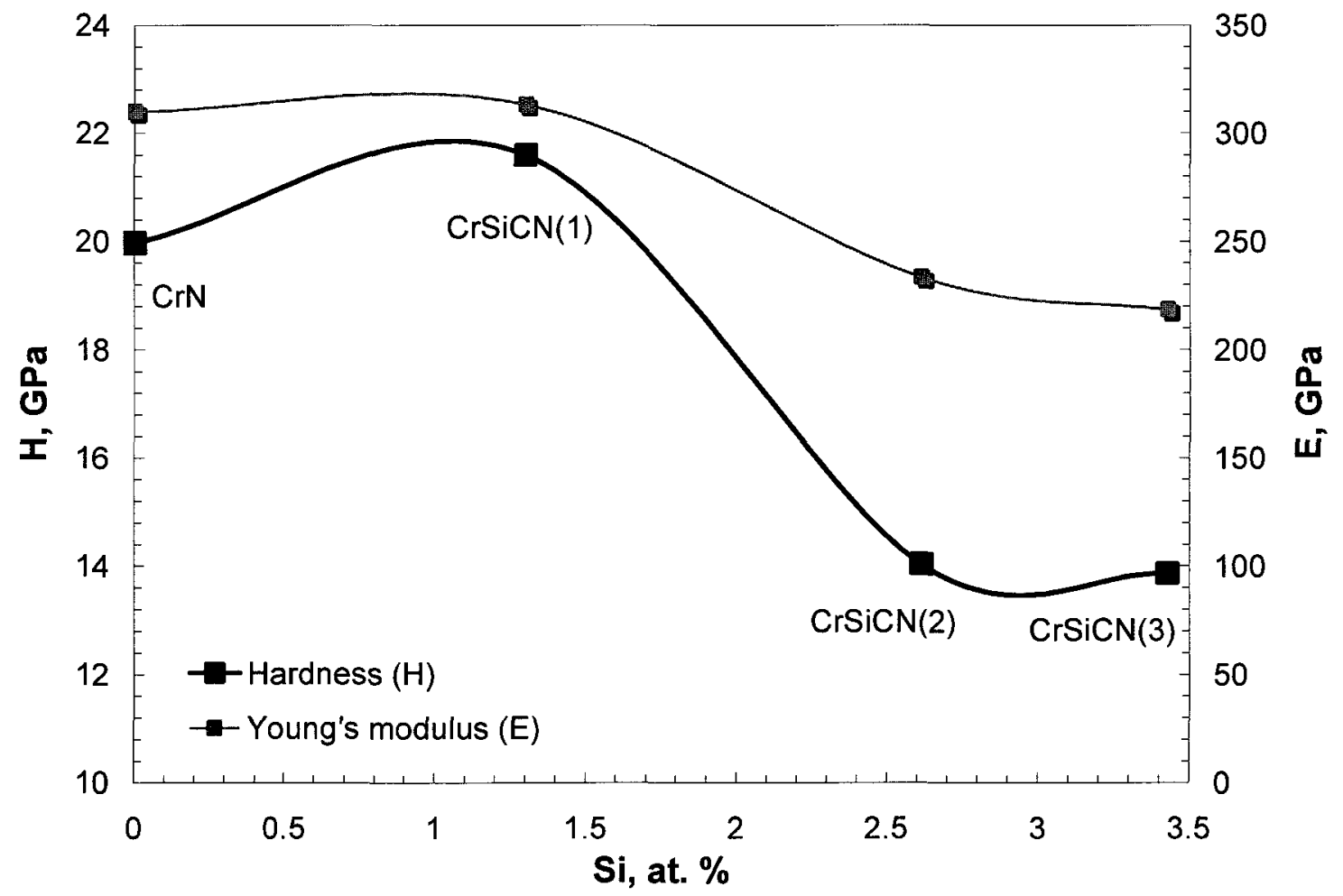

Figure 4-19 Hardness and Young's modulus vs. Si content in CrN based coating systems. 


\subsection{Coating System Tribological Behaviours}

\subsubsection{Wear}

In this study, four $\mathrm{CrN}$ based coating systems were tested using the pin-on-disc method to evaluate their dry sliding wear resistance (Section 3.6.1). For comparison, the CA TiN coating was also evaluated in this test. The tested coatings and the test conditions are summarized in Table 4-9. The loads applied to all the samples were $2 \mathrm{~N}$; and the sliding distances for the four $\mathrm{CrN}$ based coatings were 2,000 meters. To avoid wearing through the relatively thin CA TiN coating, its sliding distance was set to 200 meters.

Table 4-9 Wear test coatings and test conditions

\begin{tabular}{|l|c|c|}
\hline \multirow{2}{*}{ Coating system } & \multicolumn{2}{|c|}{ Testing conditions } \\
\cline { 2 - 3 } & Load (N) & Sliding distance (m) \\
\hline CA TiN & 2 & 200 \\
\hline $\mathrm{CrN}$ & 2 & 2,000 \\
\hline $\mathrm{CrSiCN}(1)$ & 2 & 2,000 \\
\hline $\mathrm{CrSiCN}(2)$ & 2 & 2,000 \\
\hline $\mathrm{CrSiCN}(3)$ & 2 & 2,000 \\
\hline
\end{tabular}

Coefficients of friction vs. sliding distance for the tested samples are plotted in Figure 420. It can be seen that all test coating samples exhibited three sliding regimes. At the initial running-in stage, sliding went smoothly with low coefficients of friction. During the second stage, friction suddenly increased accompanied by fluctuations. In the last stage, which covered about the latter half of the sliding distance, the fluctuations 
moderated, indicating the sliding process entered a steady state. In order to evaluate the sliding behaviours in steady state, average coefficients of friction were calculated over the latter half of the sliding distance and are summarized in Table 4-10 and Figure 4-21. The TiN and $\mathrm{CrN}$ coatings have similar coefficient of friction values (around 0.81 ).

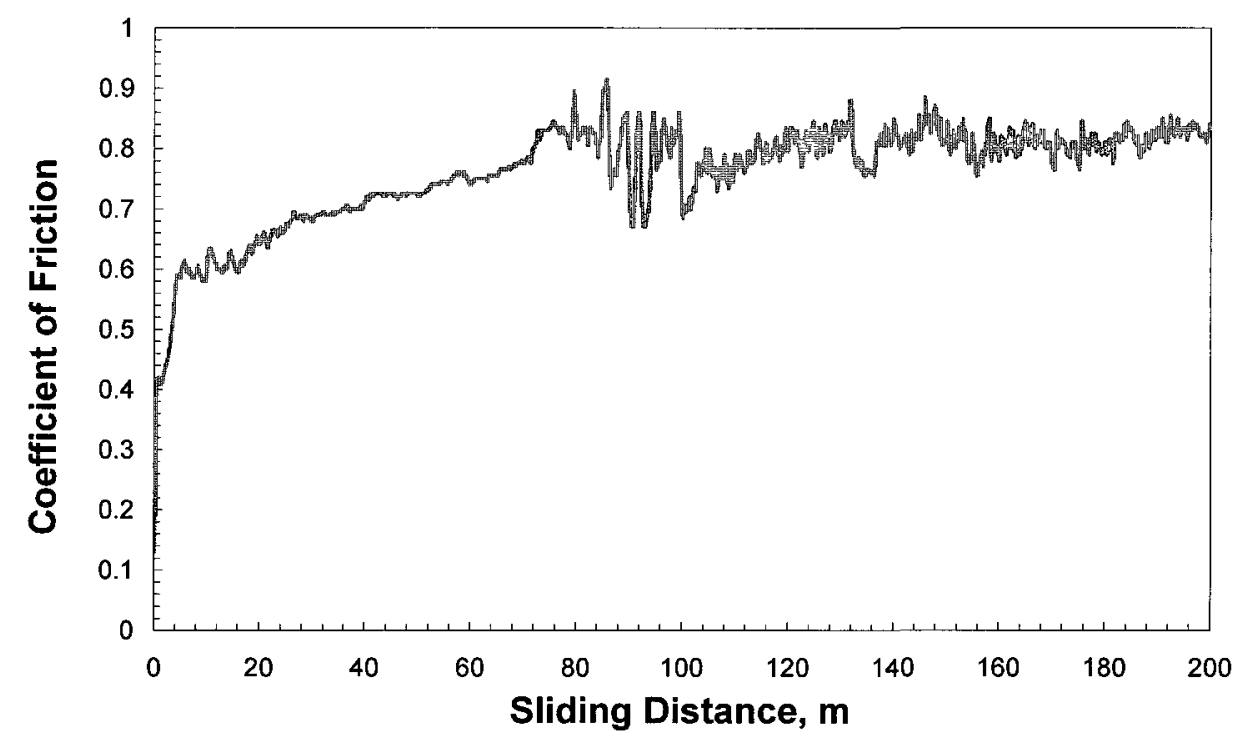

(a) CA TiN coating

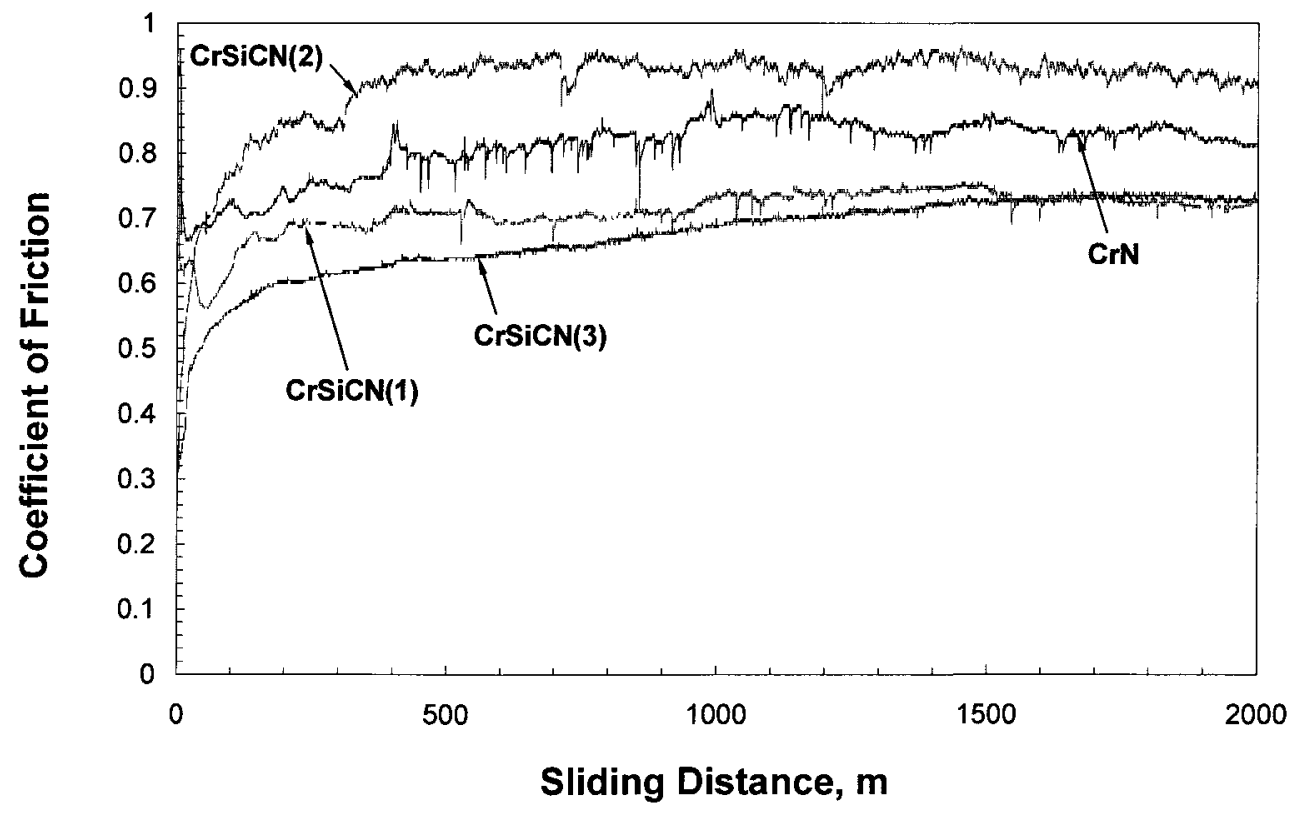

(b) $\mathrm{CrN}$ based coating systems

Figure 4-20 Coating oefficient of friction vs. sliding distance. 
In the $\mathrm{CrN}$ based coating systems, the addition of $\mathrm{Si}$ and $\mathrm{C}$ caused the coefficient of friction to decrease initially for the coating $\mathrm{CrSiCN}(1)$. However, at a Si concentration of 2.6 at. $\%$, the $\mathrm{COF}$ for the coating $\mathrm{CrSiCN}(2)$ rose significantly to 0.91 . With a further increase in the Si concentration to 3.7 at. $\%(\mathrm{CrSiCN}(3))$, the $\mathrm{COF}$ dropped to 0.67 . The decrease in the coefficient of friction with the increase in Si content could be associated with the presence of a silicon nitride $\left(\mathrm{Si}_{3} \mathrm{~N}_{4}\right)$ phase $[206,400]$. Further studies are needed for the role of crystalline $\mathrm{Si}_{3} \mathrm{~N}_{4}$ in the reduction of $\mathrm{COF}$ in the $\mathrm{CrSiCN}$ coating system. The fluctuation in COF with Si content also indicates that the composition alone can not be used to predict the coating system properties; microstructure changes associated with different compositions must be taken into consideration.

As can be seen from Figure 4-21, both the minimum $\mathrm{H}^{3} / \mathrm{E}^{2}$ ratio and the maximum $\mathrm{COF}$ occurred at a $\mathrm{Si}$ concentration of 2.6 at.\% (i.e., $\mathrm{CrSiCN}(2)$ ). This suggests that the high COF could be a result of plastic deformation on the interacting surface, which offsets the effect of the silicon nitride phase $\left(\mathrm{Si}_{3} \mathrm{~N}_{4}\right)$ in reducing $\mathrm{COF}$. The $\mathrm{CrN}$ and TiN coating systems had higher $\mathrm{H}^{3} / \mathrm{E}^{2}$ ratios than the $\mathrm{CrSiCN}(2)$ and $\mathrm{CrSiCN}(3)$ coating systems, but the lack of the effect of silicon nitride led to their higher $\mathrm{COF}$ (than $\mathrm{CrSiCN}(3)$ coating). 


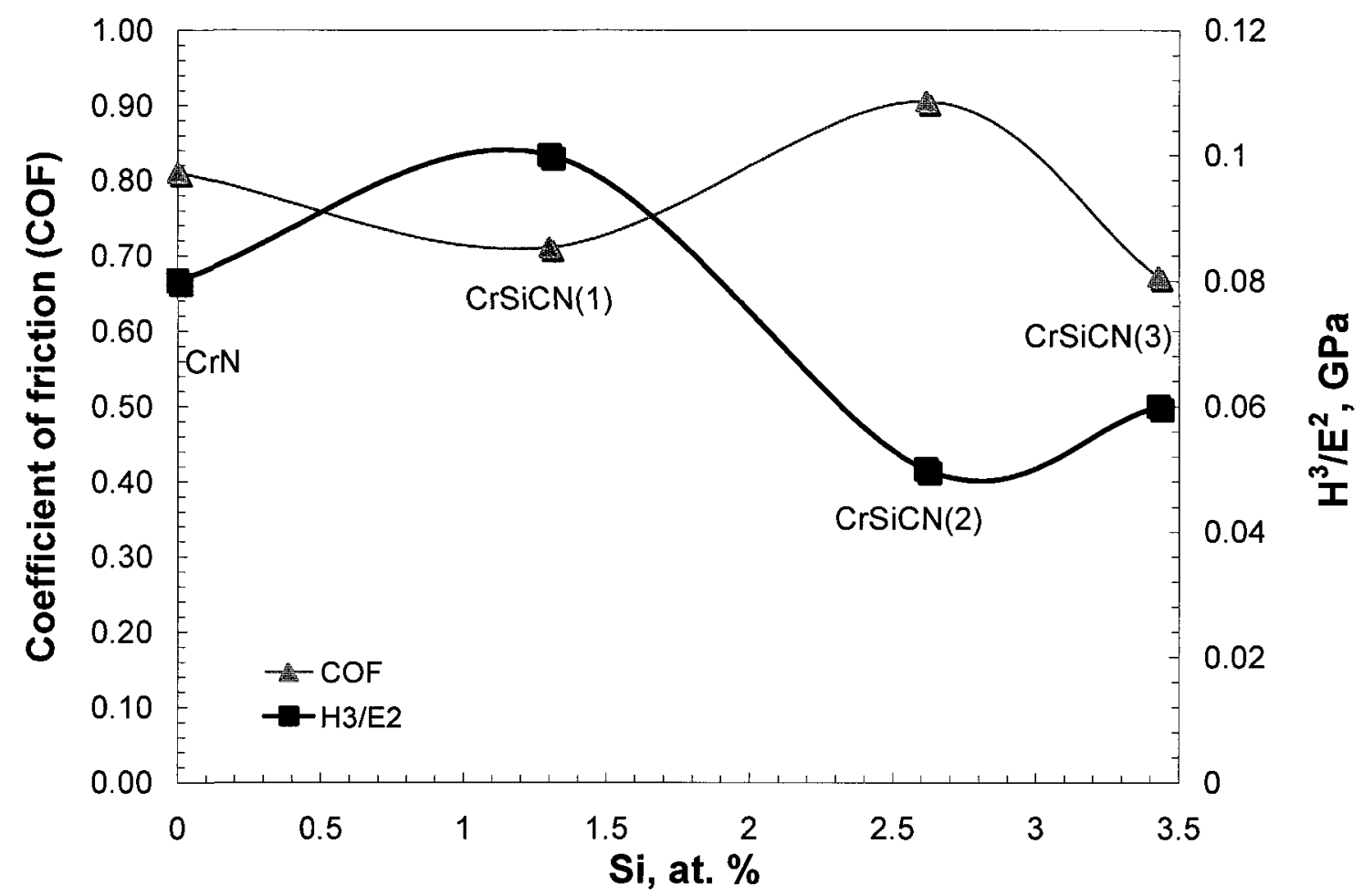

Figure 4-21 Coefficient of friction and $\mathrm{H}^{3} / \mathrm{E}^{2}$ ratio vs. Si content in $\mathrm{CrN}$ based coating systems.

Typical cross-sectional profiles of wear tracks on the test coating samples are shown in Figure 4-22. All wear track cross-sectional profiles exhibit typical Hertzian contact [332]. Hertzian contact occurs between an elastic sphere (wear pin) and a plane (hard coating surface), the normal pressure semi-elipsoidally distributes in a circular contact area, and therefore the maximum wear depth takes place at the center of the contact zone [84]. Among the four $\mathrm{CrN}$ based coating samples, $\mathrm{CrN}$, which had the second highest coefficient of friction, exhibited the largest depth of wear track; whereas $\operatorname{CrSiCN}(2)$, which had the highest coefficient of friction, demonstrated the greatest width and significant depth in its wear track. 


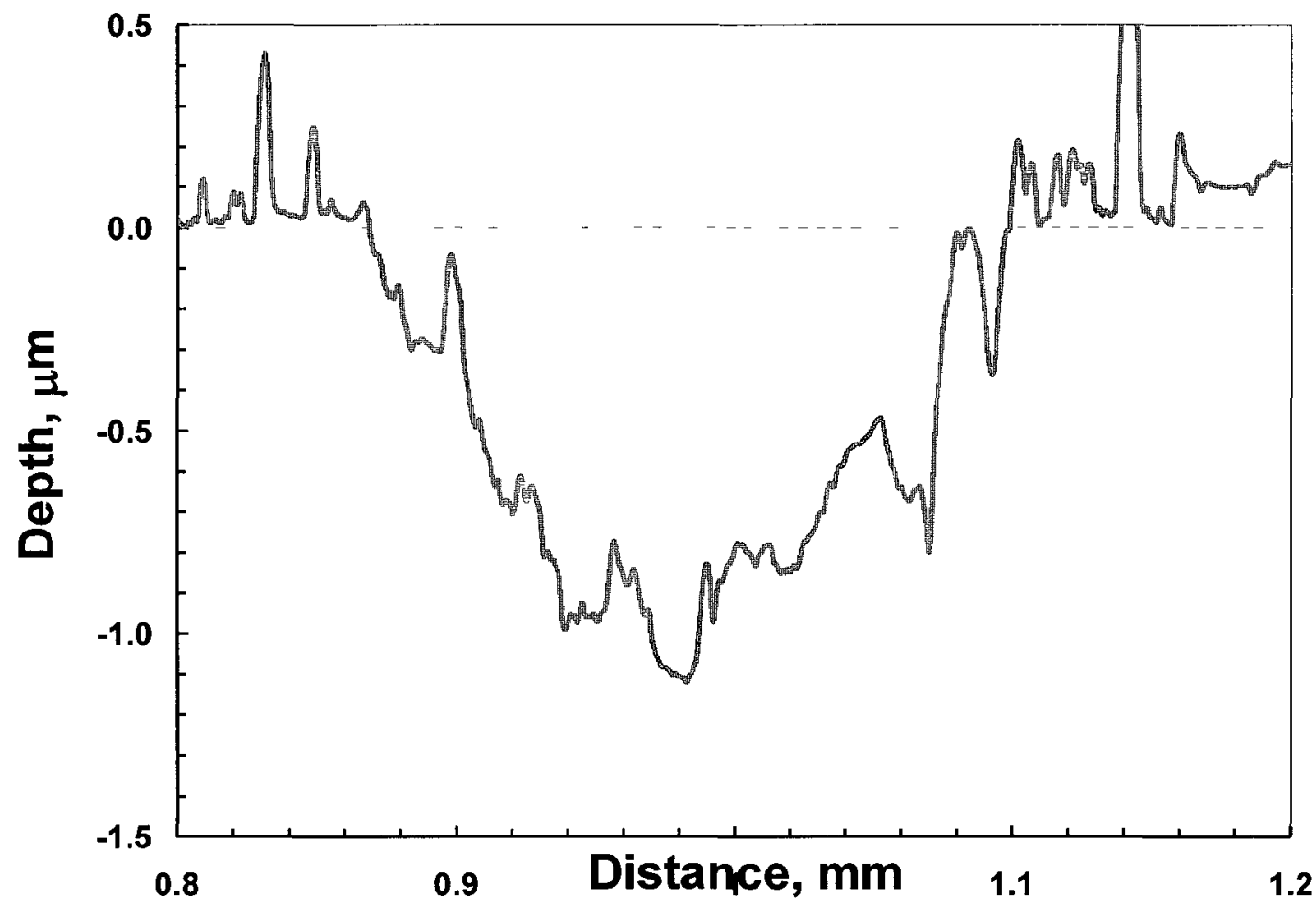

(a) CA TiN coating

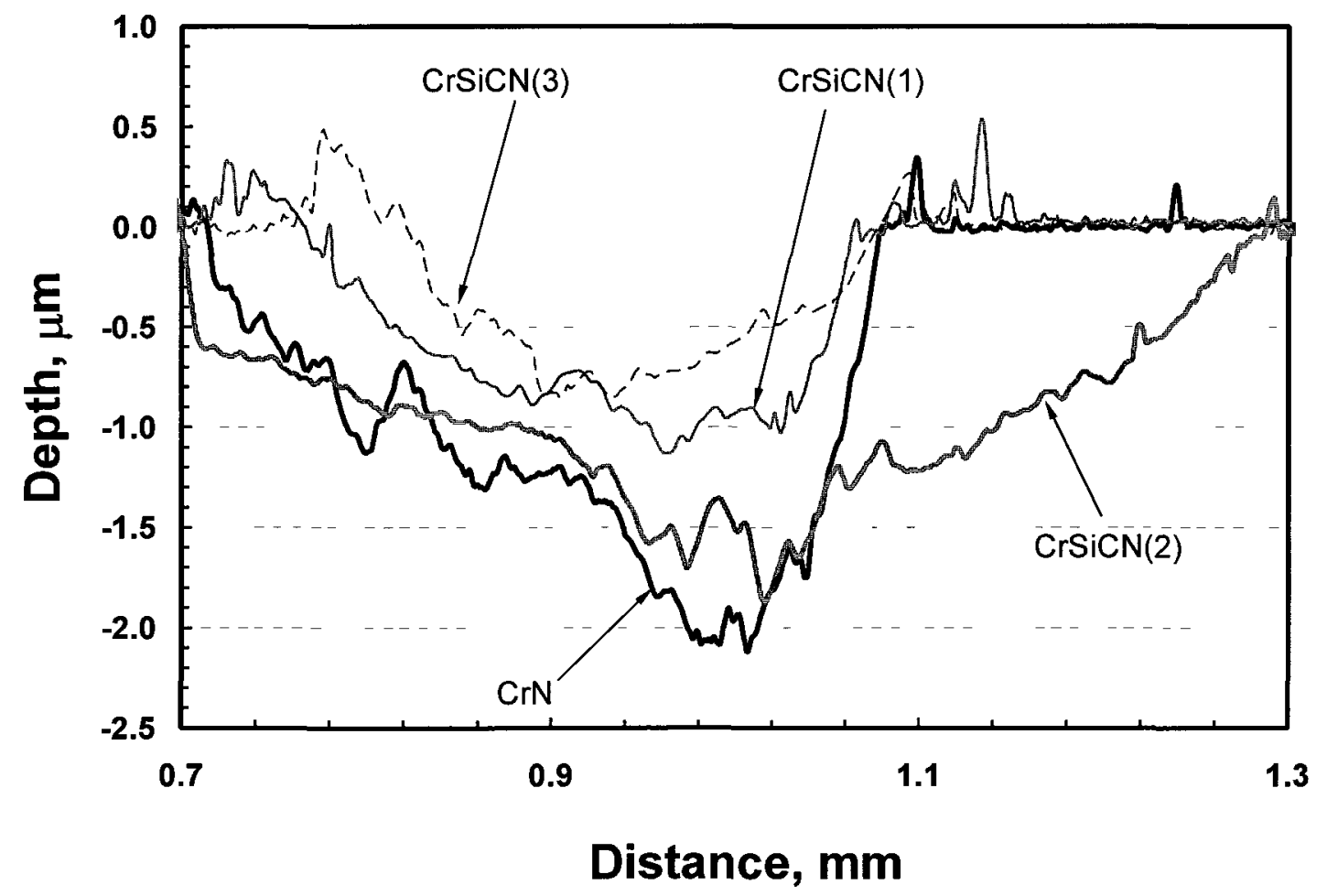

(b) $\mathrm{CrN}$ based coating systems

Figure 4-22 Wear track cross-sectional profiles for test coating samples. 
For each test coating sample, 3 wear tracks obtained at different locations, and for each wear track 3 measurements were taken to obtain the average values of wear cross sectional area, and with which the average specific wear rates were calculated using Eq. (3-7). The results are summarized in Table 4-10 and plotted in Figure 4-23. From Eq. (39), the wear coefficient $\left(k_{A}\right)$ was obtained, and the results are provided in Table 4-10. For the four $\mathrm{CrN}$ based coating systems, the specific wear rate, wear coefficient $k_{A}$, and $\mathrm{COF}$ demonstrated a consistent trend, indicating a correlation between $\mathrm{COF}$ and wear rates.

Table 4-10 Summaries of wear testing data for the tested coating systems

\begin{tabular}{|l|c|c|c|c|}
\hline \multicolumn{1}{|c|}{ Coating } & COF & $\begin{array}{c}\text { Specific wear rate } \\
\left(\mathrm{mm}^{3} / \mathrm{Nm}\right)\end{array}$ & $\begin{array}{c}\text { Wear coefficients } \\
k_{A}\end{array}$ & $\begin{array}{c}\text { W in wear debris } \\
\text { (at.\%) }\end{array}$ \\
\hline $\mathrm{CA} \mathrm{TiN}$ & 0.81 & $8.76 \times 10^{-7}$ & $2.98 \times 10^{-5}$ & - \\
\hline $\mathrm{CrN}$ & 0.81 & $1.29 \times 10^{-6}$ & $2.57 \times 10^{-5}$ & 8.79 \\
\hline $\mathrm{CrSiCN}(1)$ & 0.71 & $1.12 \times 10^{-6}$ & $2.42 \times 10^{-5}$ & 5.61 \\
\hline $\mathrm{CrSiCN}(2)$ & 0.91 & $2.12 \times 10^{-6}$ & $2.98 \times 10^{-5}$ & 13.66 \\
\hline $\mathrm{CrSiCN}(3)$ & 0.67 & $1.10 \times 10^{-6}$ & $1.53 \times 10^{-5}$ & 10.69 \\
\hline
\end{tabular}

A similarly inverse relationship between specific wear rate and the $\mathrm{H}^{3} / \mathrm{E}^{2}$ ratio for the TiN anc CrN based coating systems can be observed in Table 4-10. The CA TiN coating exhibited several times higher $\mathrm{H}^{3} / \mathrm{E}^{2}$ ratio and a significant lower specific wear rate as compared with $\mathrm{CrN}$ based coating systems. In the $\mathrm{CrN}$ based coating systems, specific wear rate showed an inverse relationship with $\mathrm{H}^{3} / \mathrm{E}^{2}$ ratio and a similar trend to the $\mathrm{COF}$ with respect to the content of $\mathrm{Si}$ (Figure 4-23). The lowest $\mathrm{H}^{3} / \mathrm{E}^{2}$ ratio corresponds to the highest specific wear rate (i.e., $\mathrm{CrSiCN}(2)$ ), indicating an increased wear damage due to plastic deformation $[395,396]$. 


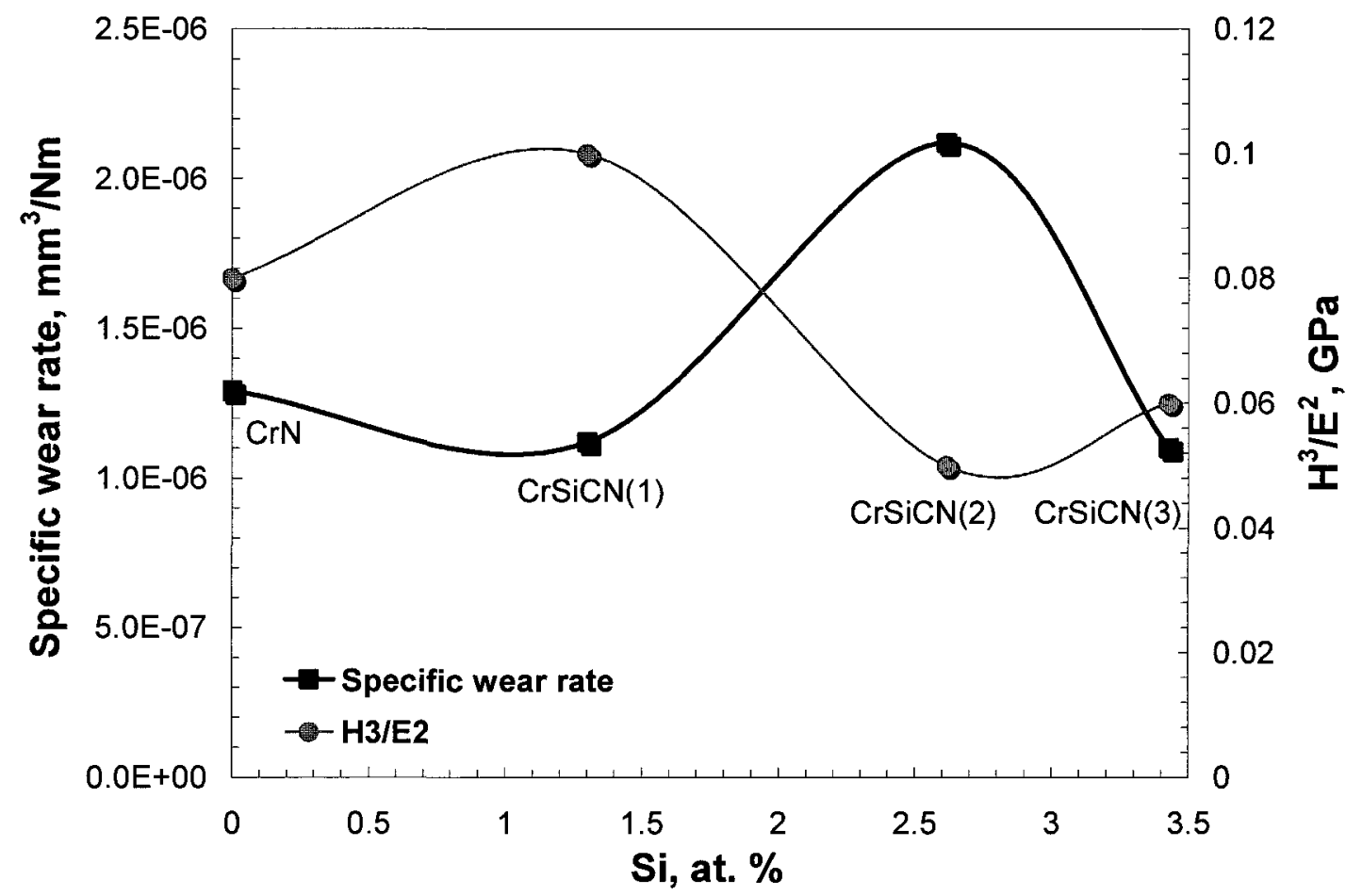

(a) Specific wear rate and $\mathrm{H}^{3} / \mathrm{E}^{2}$ vs. Si content

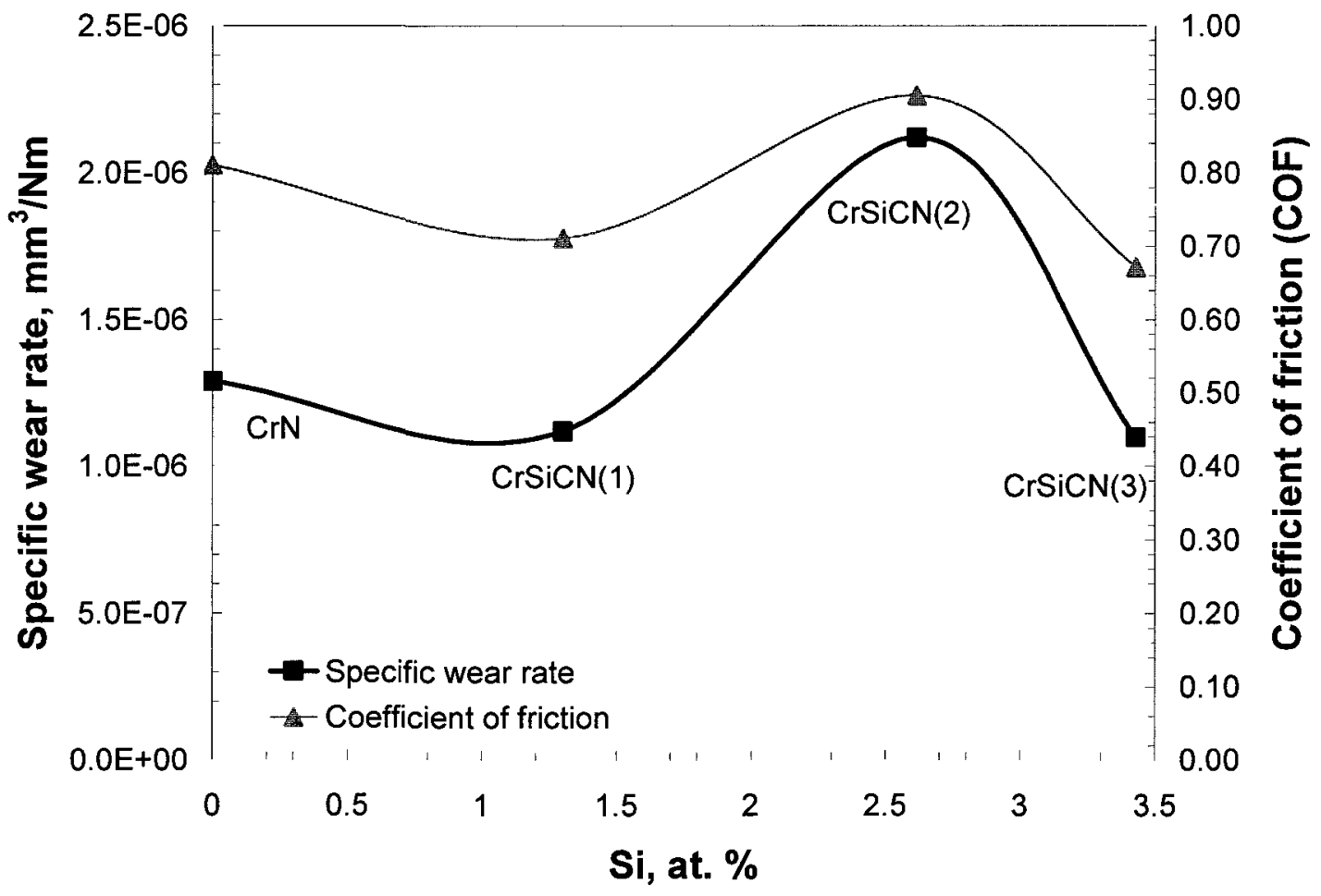

(b) Specific wear rate and coefficient of friction vs. Si content

Figure 4-23 Wear behaviours vs. Si content in $\mathrm{CrN}$ based coating systems. 
However the CA TiN coating exhibited different trends in specific wear rate and Archard wear coefficients $\left(k_{A}\right)$ compared with the $\mathrm{CrN}$ based coating systems (Table 4-10). The specific wear rate of the CA TiN coating was significantly lower than the CrN based coating systems, while its Archard wear coefficient $\left(k_{A}\right)$, was higher than those for the CrN based coatings. Archard wear coefficient $\left(k_{A}\right)$ (Eq. 3-9) involves certain wear test conditions (i.e., load and sliding distance), properties of interacting materials (i.e., hardness), and final wear result (i.e., worn volume). Thus the wear rate evaluated using these two criteria could differ.

The physical interpretation of the Archard wear coefficient $\left(k_{A}\right)$ is the probability of generating debris in a rubbing pair with asperity contacts. Among the factors governing the Archard wear coefficient $\left(k_{A}\right)$, hardness is more important for ductile materials, whereas fracture toughness is dominant for brittle materials [401]. However, in a sliding process, due to plastic deformation near the surface, the dominant role of hardness is diminished because of fatigue; and this is a limit to the application of Archard wear coefficient $\left(k_{A}\right)$. By contrast, specific wear rate is more commonly valid in all wear conditions.

After dry sliding tests, the four $\mathrm{CrN}$ based coating samples were examined using SEM, and compared in secondary electron (SE) images and back-scattered electron (BSE) modes (Figure 4-24). Morphologies of the wear tracks and adjacent wear debris were observed, and the compositions for the adjacent wear debris were analyzed using EDS, an 
example of the EDS spectrum is shown in Figure 4-24 (a-6). The wear debris on the four samples were found to be rich in oxygen $(\mathrm{O})$, indicating oxidation processes occurred during the dry sliding wear tests. In addition to the elements $\mathrm{Cr}$ and $\mathrm{N}$, a considerable amount of $\mathrm{W}$ was found in the wear debris, indicating that the wear debris generated durıng slidıng is a mixture of heavily oxidized $\mathrm{W}\left(\mathrm{i}\right.$ e., $\mathrm{WO}_{3}$ ) from the wear pin (WC-Co ball) in addition to the elements from the coatings The presence of $\mathrm{Cr}$ and $\mathrm{W}$ were consequences of wear in the rubbing pair of coatings and WC-Co balls due to material transfer during the sliding process $[85,402,403]$
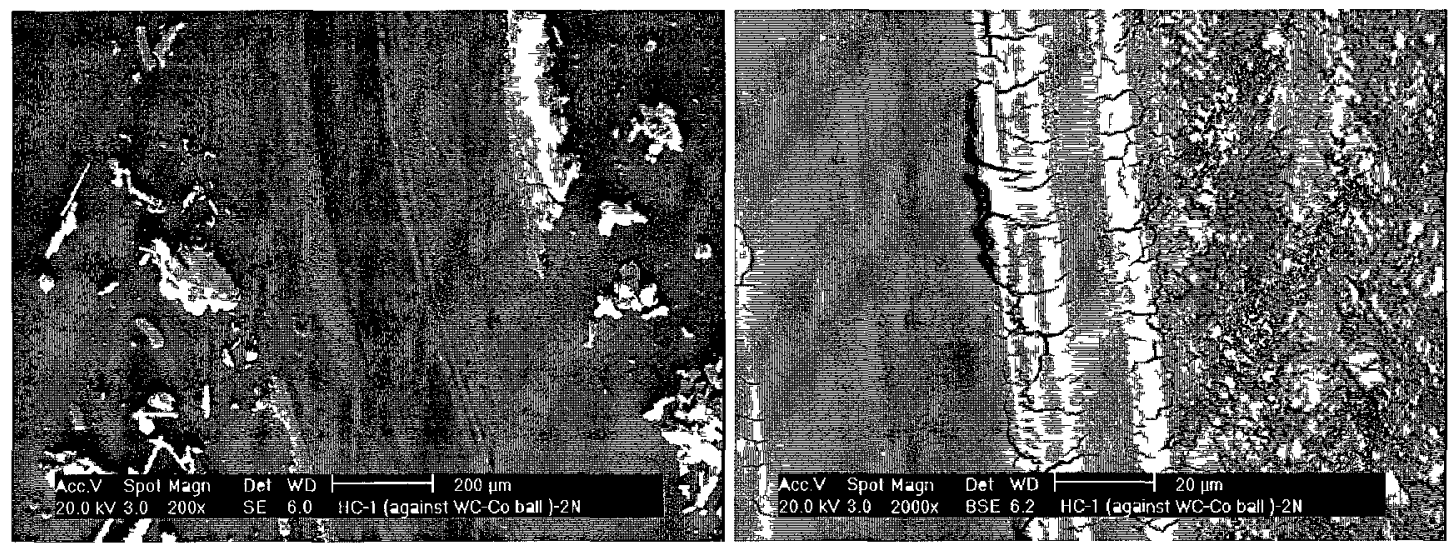

(a)-1 (low magnification, SE mode)

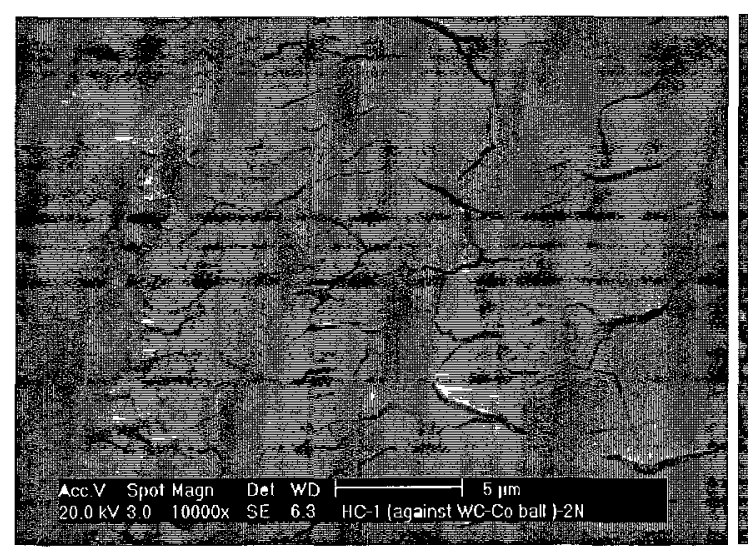

(a)-2 (mid magnification BSE mode)

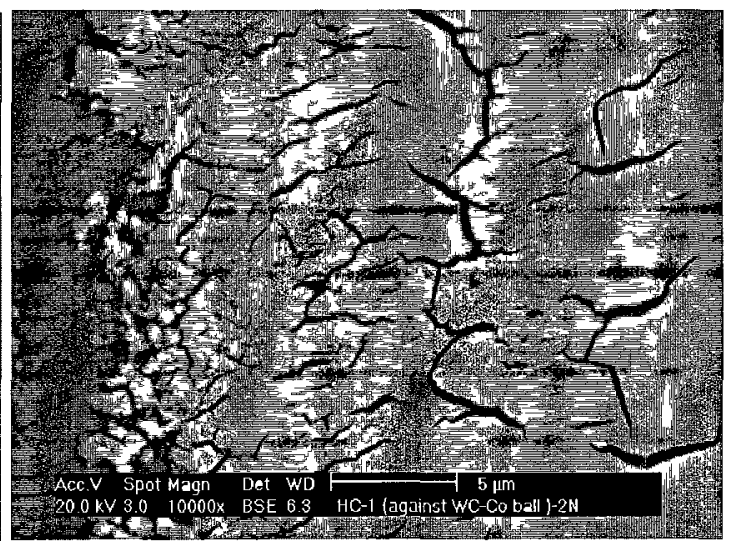

(a)-3 (high magnification, SE mode)

(a)-4 (high magnification, BSE mode)

Figure 4-24 to be continued 

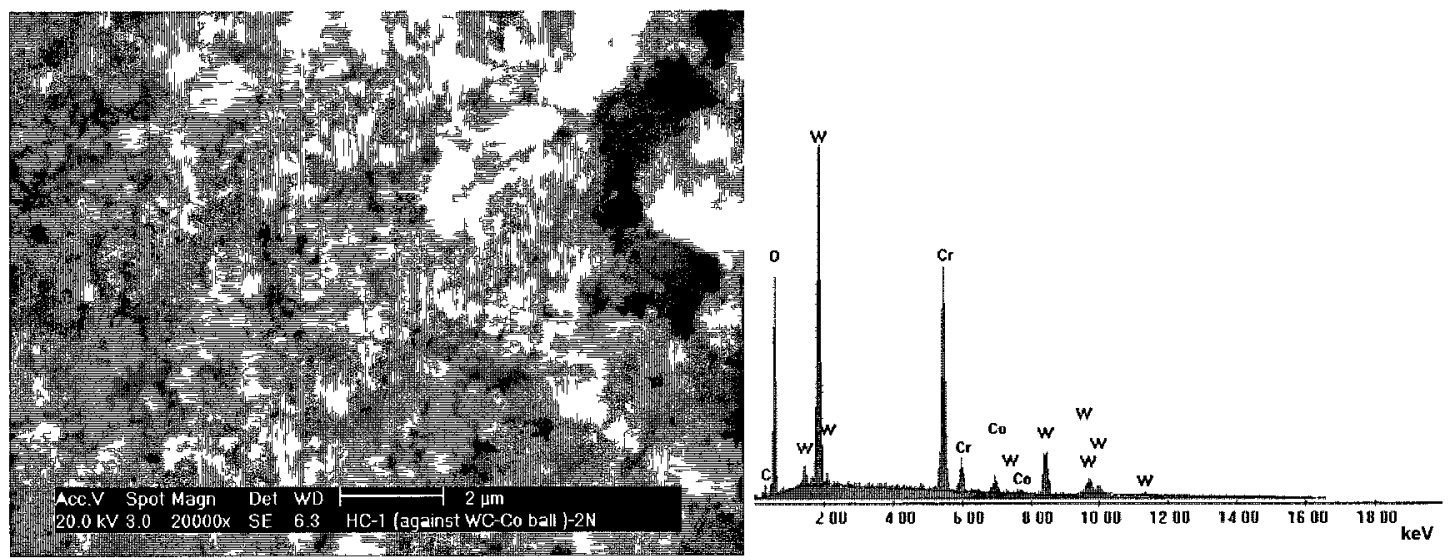

(a)-5 (worn debris)

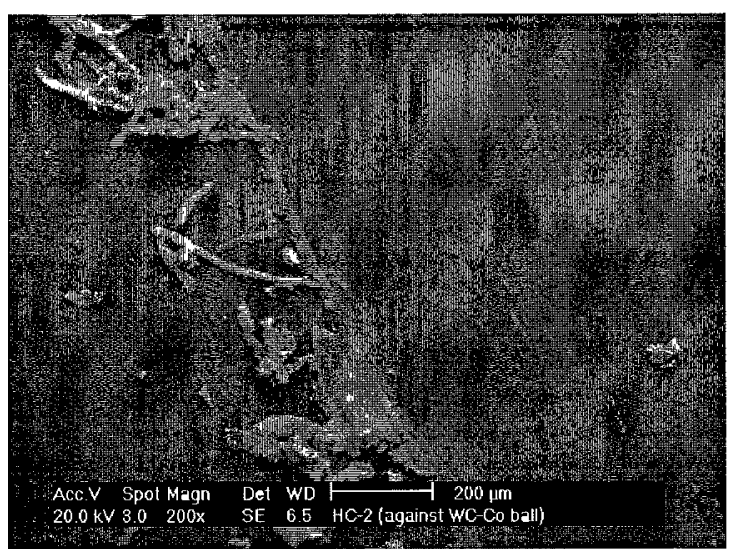

(b)-1(low magnification, SE mode)

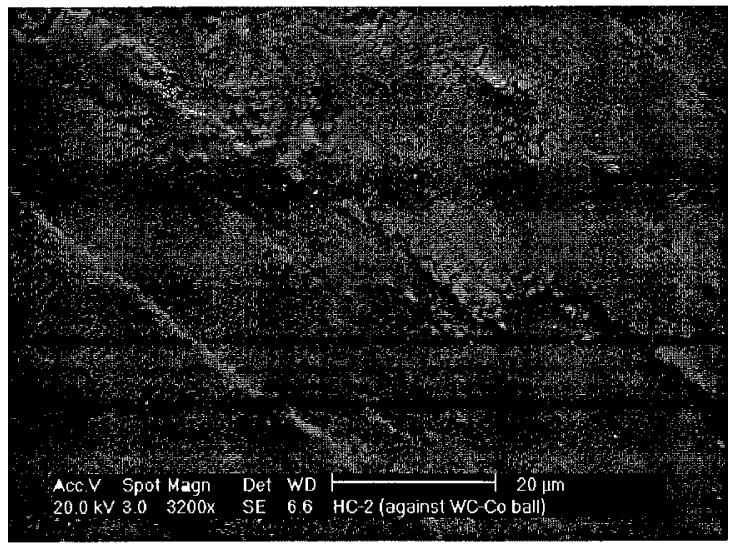

(b)-3 (high magnification, SE mode) (a)-6 (EDS spectrum on wear debris)

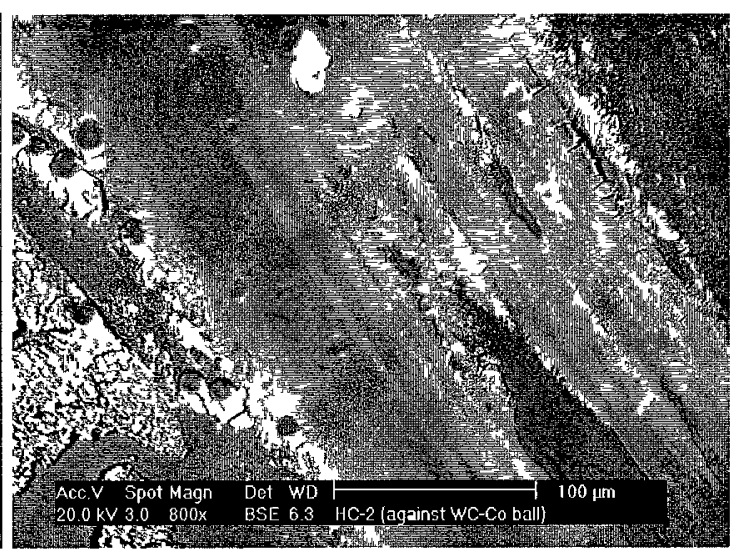

(b)-2 (mid magnification, BSE mode)

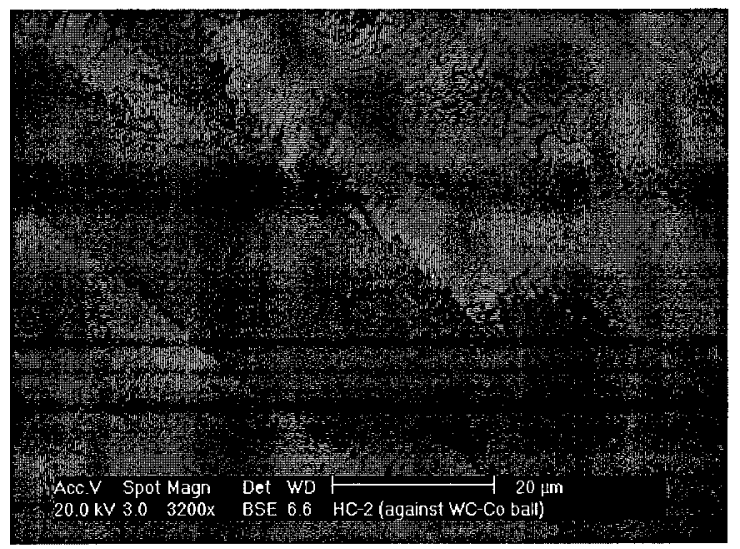

(b)-4 (high magnification, BSE mode)

Figure 4-24 to be continued 

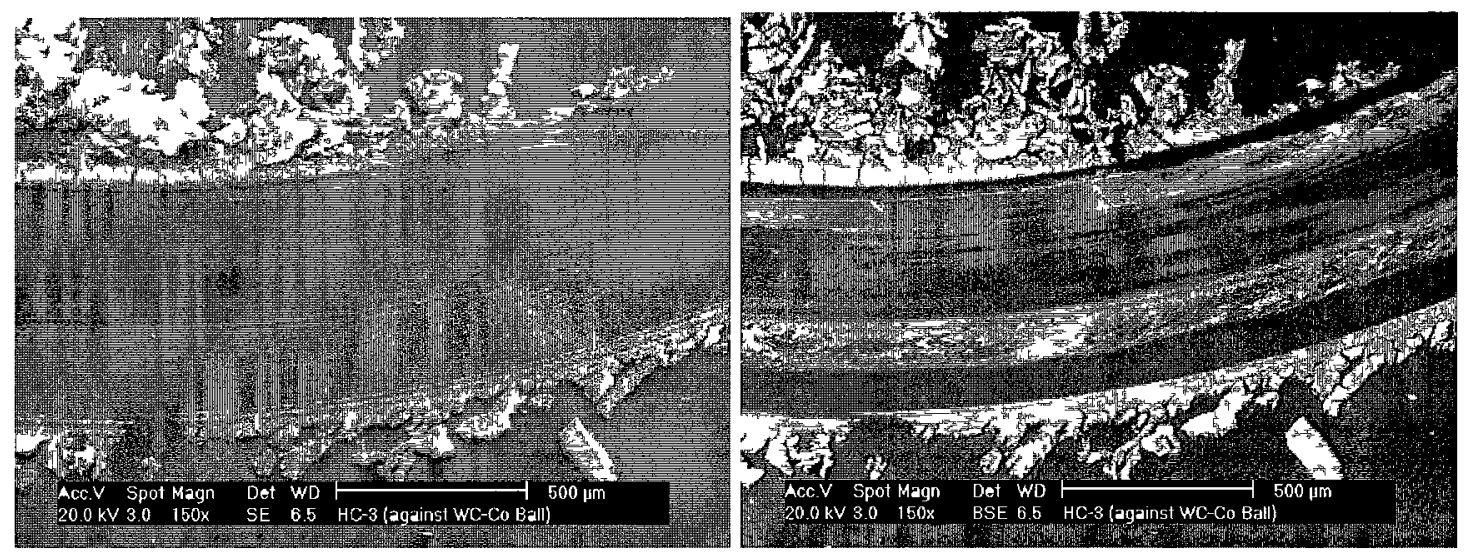

(c)-1 (low magnification, SE mode)

(c)-2 (low magnification, BSE mode)
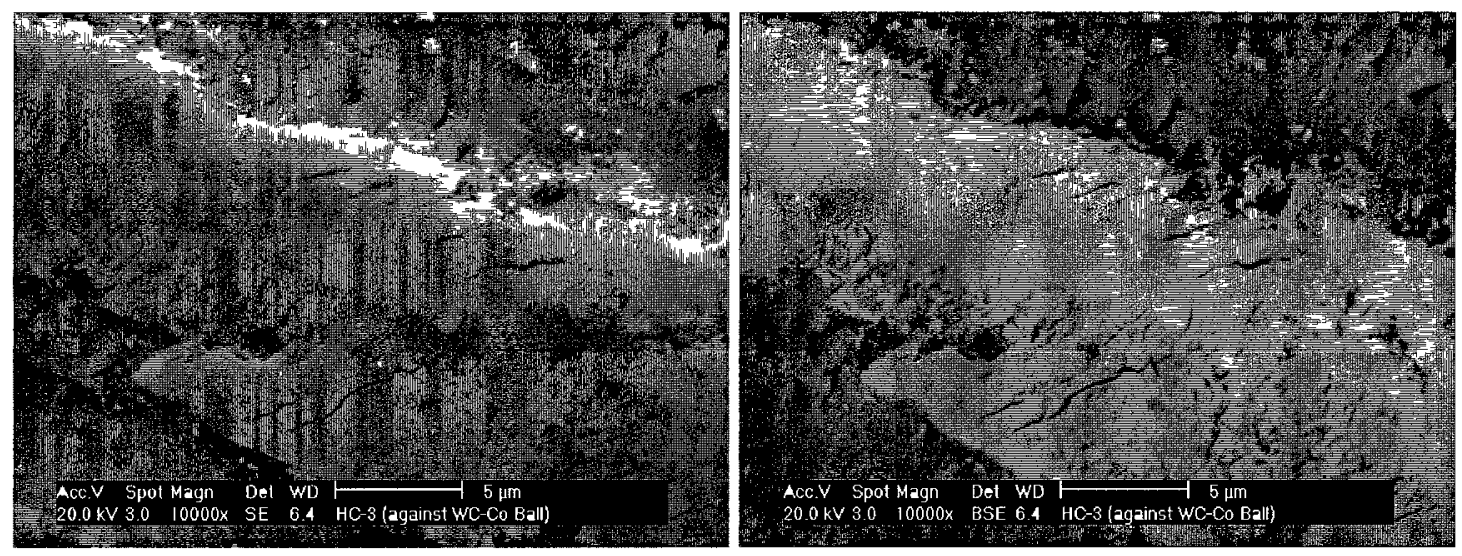

(c)-3 (high magnification, SE mode)

(c)-4 (high magnification, BSE mode)
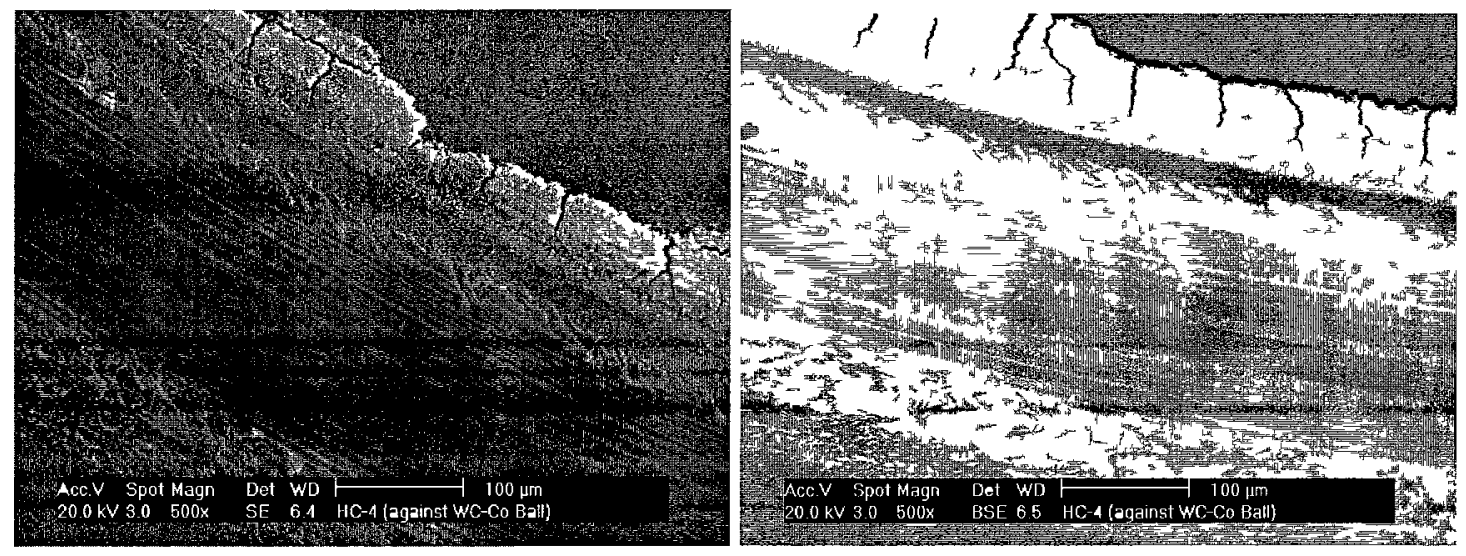

(d)-1 (low magnification, SE mode)

(d)-2 (low magnification, BSE mode)

Figure 4-24 to be continued 


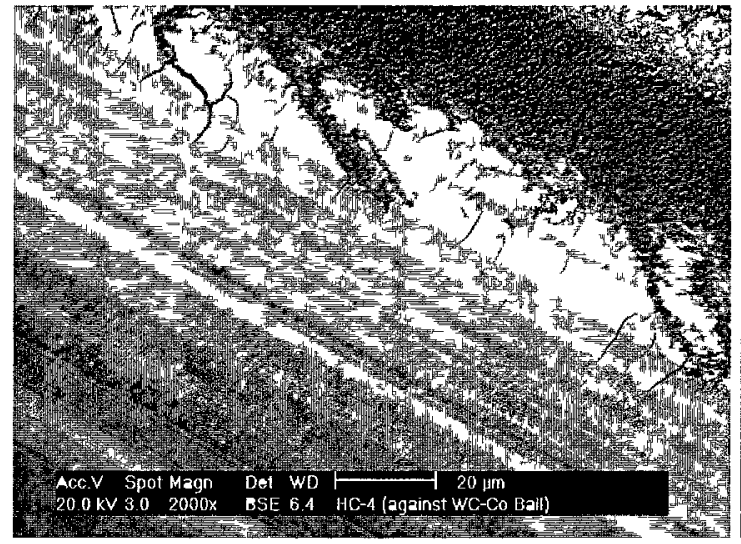

(d)-3 (high magnification, BSE mode)

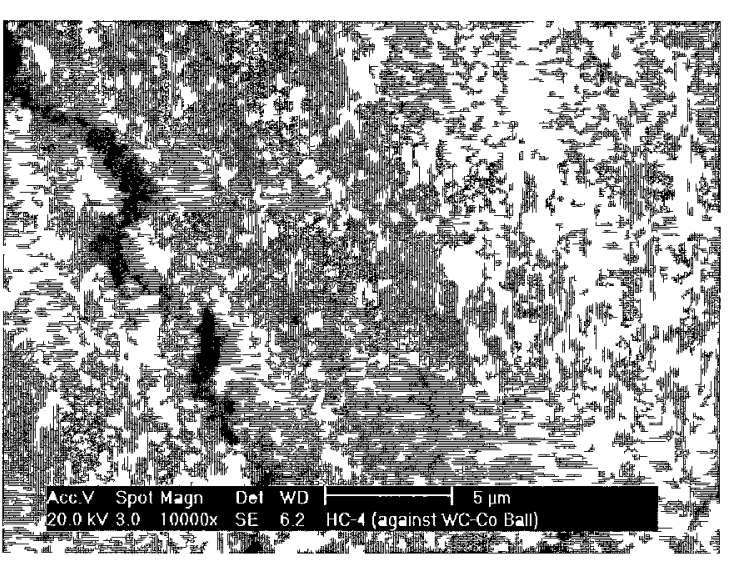

(d)-4 (worn debris)

Figure 4-24 SEM images of wear tracks and EDS spectrum on worn debris for the test coatıng systems (a) $\mathrm{CrN}$, (b) $\mathrm{CrSiCN}(1)$ and (c) $\mathrm{CrSiCN}(2)$, and (d) $\mathrm{CrSiCN}(3)$.

The SEM images at higher magnifications revealed patch-like transferred layers on the worn track surface. In the BSE mode of SEM analysis, a heavy metal (such as tungsten (W) in this study) demonstrates bright contrast. Therefore, the bright layers in BSE mode shown in Figure 4-24 ((a)-1, (a)-4, (b)-2, (b)-4, (c)-2, (c)-4, (d)-2, (d)-3)) indicate the presence of $\mathrm{W}$ transferred from the WC-Co sliding ball. During the wear process, the transferred materials from the sliding ball can be compacted and mechanically mixed with the coating material, and this mixing action may contribute to a reduction in wear [374]. The friction generated during the dry sliding wear test also caused localized high temperature and subsequent surface oxidation (oxide formation). Similar surface oxidation phenomenona were observed [209]. This tribo-oxidation process also plays a dominant role in changing the friction in the rubbing pair, therefore affecting wear behaviour. 
The amounts of $\mathrm{W}$ in the wear debris for the test coating samples are summarized in Table 4-10. The highest $\mathrm{W}$ content is found in the wear debris of $\mathrm{CrSiCN}(2)$, which had the highest specific wear rate. This observation indicates that material transfer was an important phenomenon in the high wear rate dry sliding tests.

SEM images of the CA TiN coating show relatively smooth wear tracks, with no patchlike layers (Figure 4-25 a, b) like those found on the $\mathrm{CrN}$ based coatings during the dry sliding wear test. The SEM image in SEB mode does not show any bright contrasts, indicating the lack of $\mathrm{W}$ on the worn surfaces (Figure 4-25 b). EDS analyses reveal a rich oxygen $(\mathrm{O})$ content in the debris (Figure 4-25 d), suggesting oxidation during the dry sliding. A small amount of W was observed on the EDS spectrum but the amount was insufficient for quantitative results, which is in agreement with the BSE image analysis where no significant $\mathrm{W}$ was found to have been transferred from the WC-Co ball. Therefore no significant material transfer occurred between the rubbing pair of TiN coating and WC-Co ball.

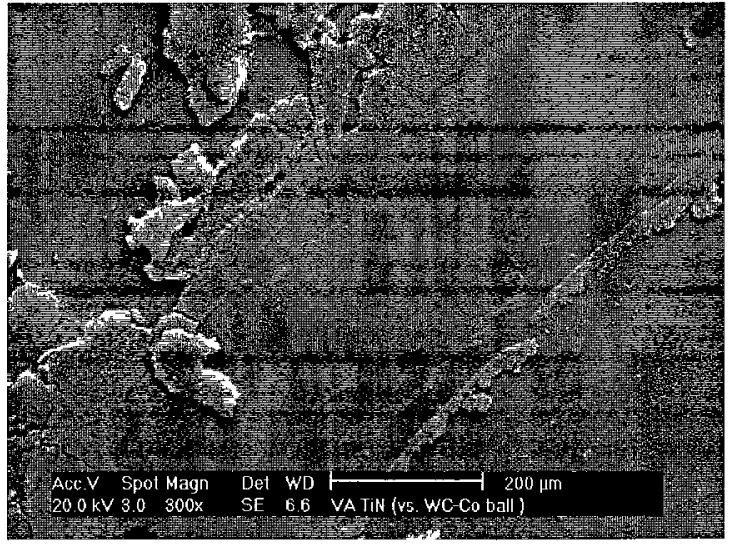

(a) SE Image

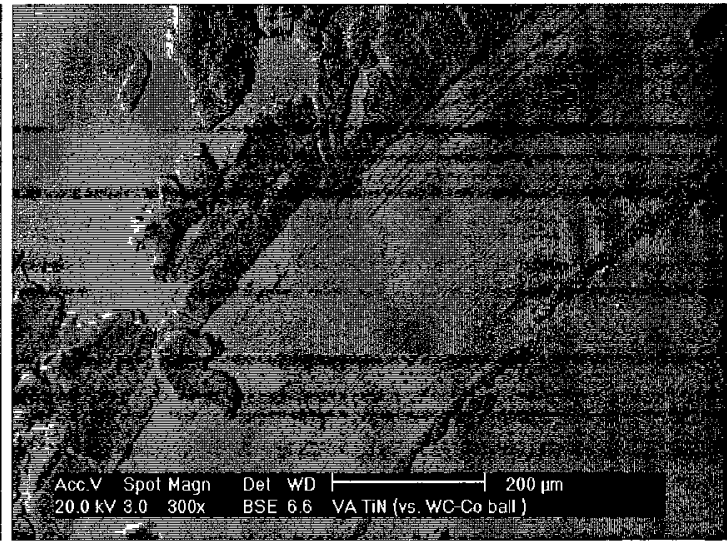

(b) BSE image

Figure 4-25 to be continued 


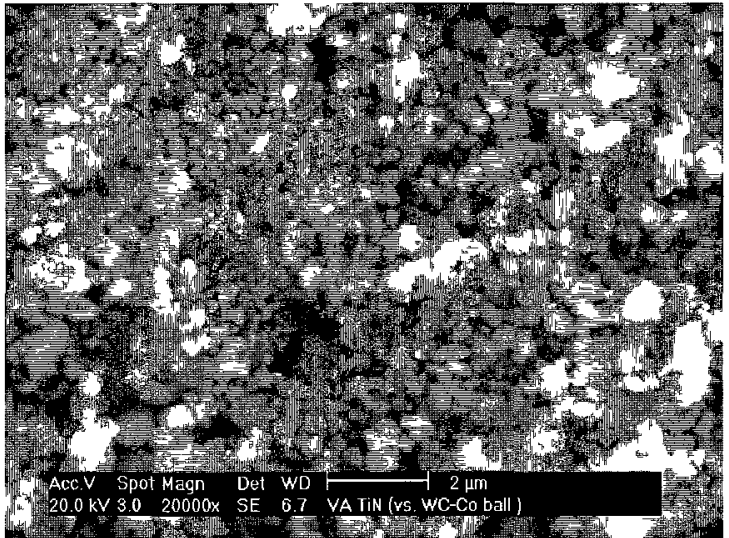

(c) SE image of worn debris

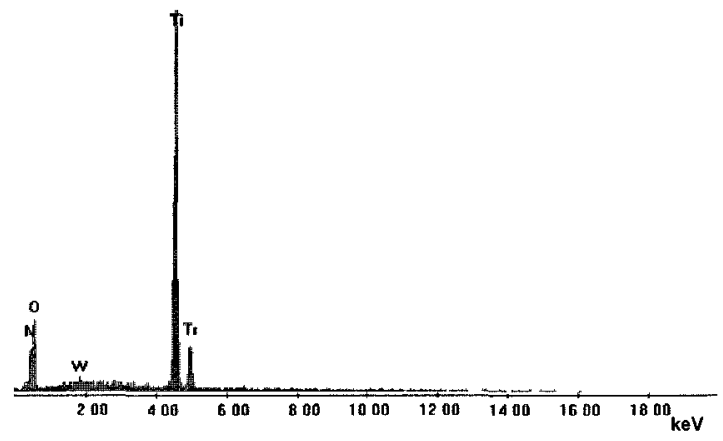

(d) EDS spectrum

Figure 4-25 Wear tracks and EDS spectrum on wear debris of the CA TiN coating.

SEM images of the worn surfaces of WC-Co balls are shown in Figure 4-26. Circular wear scars can be observed on the contact surfaces. Scar diameters were measured using high magnification SEM images, and are summarized in Table 4-11. It can be seen that among the four $\mathrm{CrN}$ based coatings, scar diameters correspond well with the wear rate of the balls measured by the $\mathrm{W}$ concentration in the wear debris. With the measured scar diameters, the corresponding specific wear rates of WC-Co balls were calculated using Eq. (3-6) and Eq. (3-7), and the results are the summarized in Table 4-11. For the CrN based coatings, specific wear rates of the WC-Co balls follow the same trend as the amounts of $\mathrm{W}$ detected on the coating surface (Table 4-10) and the scar diameters on the balls (Table 4-11).

Table 4-11 Wear test data for WC-Co ball sliding against test coatings

\begin{tabular}{|l|c|c|}
\hline Counterpart coating & $\begin{array}{c}\text { Scar diameter on ball } \\
(\mu \mathrm{m})\end{array}$ & $\begin{array}{c}\text { Specific wear rate of WC-Co Ball } \\
\left(\mathrm{mm}^{3} / \mathrm{Nm}\right)\end{array}$ \\
\hline $\mathrm{CA} \mathrm{TiN}$ & 277 & $2.89 \times 10^{-8}$ \\
\hline $\mathrm{CrN}$ & 510 & $3.33 \times 10^{-7}$ \\
\hline $\mathrm{CrSiCN}(1)$ & 500 & $3.08 \times 10^{-7}$ \\
\hline $\mathrm{CrSiCN}(2)$ & 560 & $4.85 \times 10^{-7}$ \\
\hline $\mathrm{CrSiCN}(3)$ & 520 & $3.60 \times 10^{-7}$ \\
\hline
\end{tabular}




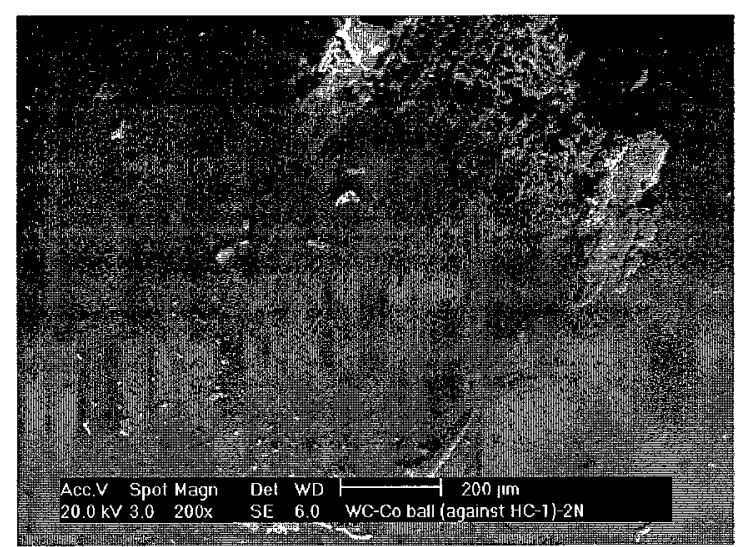

(a) Against $\mathrm{CrN}$ coating

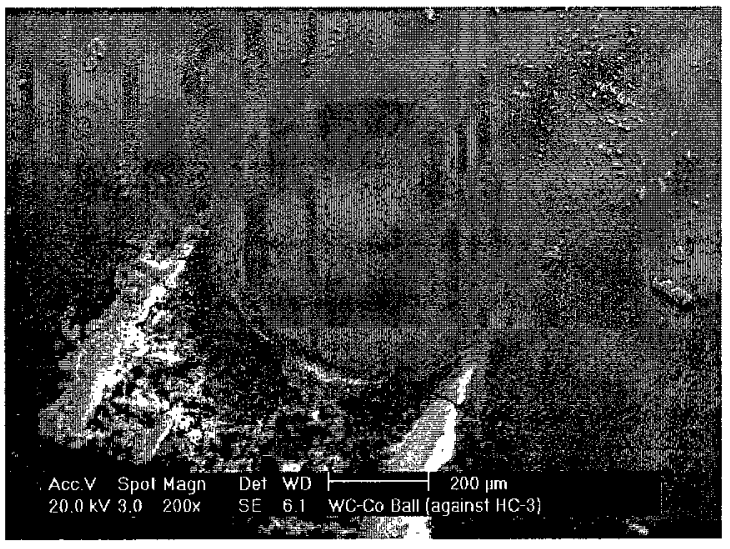

(c) Against $\mathrm{CrSiCN}(2)$ coating

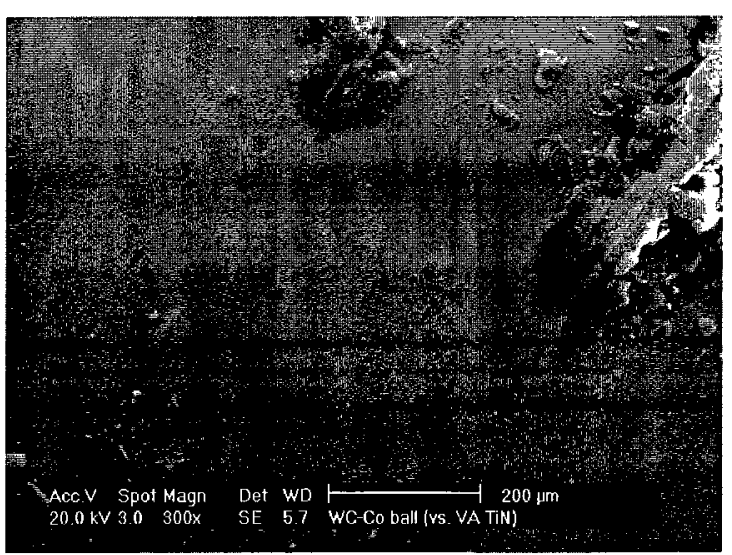

(e) Against CA TiN coating

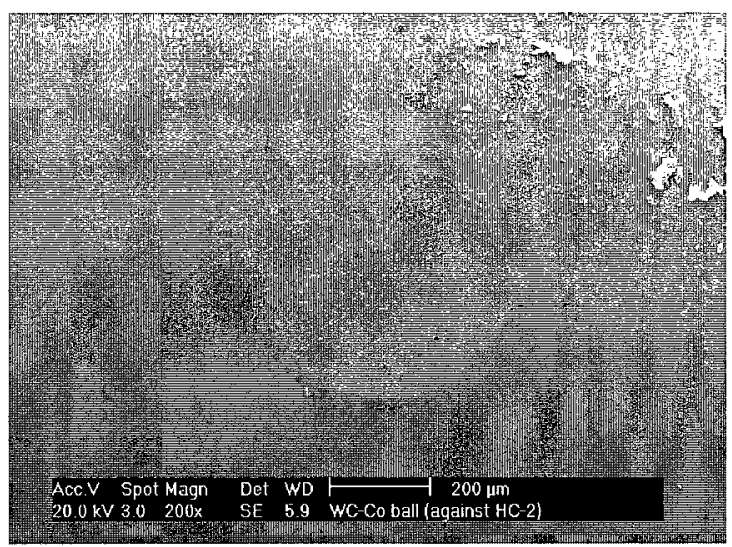

(b) Against $\mathrm{CrSiCN}$ (1) coating

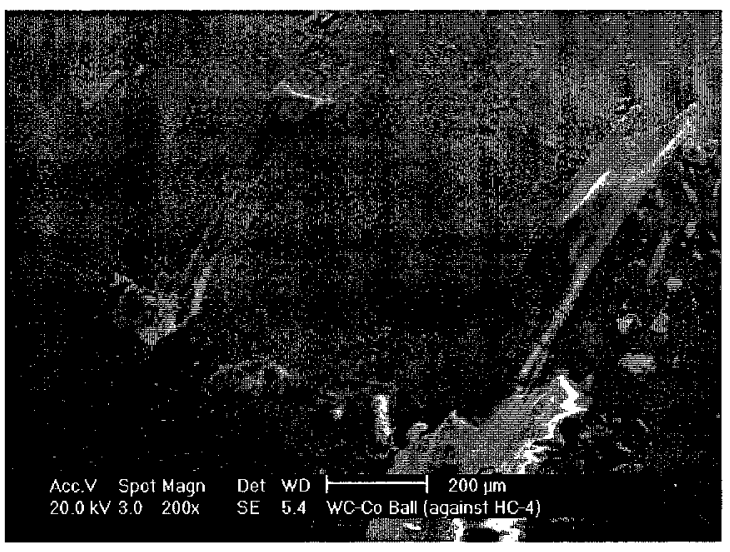

(d) Against $\mathrm{CrSiCN}$ (3) coating

Figure 4-26 SEM images of wear scars on WC-Co balls. 


\subsubsection{Erosion}

In this study, solid partical erosion tests were conducted on Ni plated, Ni-CNT plated samples and physical vapor deposition (PVD) coatings. Erodent particle velocity of 30 $\mathrm{m} / \mathrm{s}$ at impingement angle of $30^{\circ}$ were applied to all the Ni and Ni-CNT plated samples; and erodent particle velocity of $60 \mathrm{~m} / \mathrm{s}$ at impingement angles of $30^{\circ}, 45^{\circ}, 60^{\circ}, 75^{\circ}$, and $90^{\circ}$ were applied to the $\mathrm{CA}$ TiN and $\mathrm{CrN}$ based PVD coating systems.

\subsubsection{Nickel Plated Samples}

The test results for both Ni plated (solid lines) and Ni-CNT plated samples (dished lines) are shown in Figure 4-27. The sample Ni-5 exhibited the lowest erosion rate in terms of weight loss. The other three samples, $\mathrm{Ni}-0, \mathrm{Ni}-100$, and $\mathrm{Ni}-1000$, demonstrate similar but slightly greater erosion rates. As detailed Section 4.2, the sample Ni-5 has substantially higher hardness as compared with $\mathrm{Ni}-0$. The reduced erosion rate for the sample Ni-5 is most likely due to this high hardness value. Since both samples Ni-100 and Ni-1000 have higher hardness values than $\mathrm{Ni}-5$, it is speculated that the increased erosion rates for $\mathrm{Ni}$ 100 and Ni-1000 were associated with their small thicknesses. Examination by SEM (Figure 4-28) showed that the depth of penetration of the solid particles is a few micrometers. As the thicknesses for both $\mathrm{Ni}-100$ and Ni-1000 samples were $3.3 \mu \mathrm{m}$ (Table 4-1), which were much thinner than those for Ni-0 $(20 \mu \mathrm{m})$ and Ni-5 $(13 \mu \mathrm{m})$, it is likely that the solid particles also caused deformation to the mild steel substrates. Since the hardness for the mild steel $(2.56 \mathrm{GPa})$ is lower than the lowest hardness for the $\mathrm{Ni}$ plating samples ( $3.73 \mathrm{GPa}$ ), the erosion resistance of the thin plating can be influenced by the underlying substrate which has much lower resistance to plastic deformation. 
The erosion behaviours of the Ni plating and Ni-CNTs plating, with the same pulse conditions are shown in Figure 4-27. The differences in erosion rates of the samples Ni-0 and Ni-CNT-0, and the samples Ni-5 and Ni-CNT-5 are not significant. Despite the higher values of hardness for both Ni-CNT-0 and Ni-CNT-5, the erosion rates did not change. It is not clear at present what role CNTs played in erosion process, particularly when the dispersion of CNTs is limited and the bond strength between Ni and CNTs are not known.

With a pulse frequency of $100 \mathrm{~Hz}$, an obvious reduction in erosion rate for the sample NiCNT-100, compared to Ni-100, occurred (Figure 4-27). Sample Ni-CNT-100 demonstrated the lowest erosion rate of all samples. The increase in pulse frequency from 5 to $100 \mathrm{~Hz}$ resulted in increased hardness for Ni-CNT-100 and somewhat reduced thickness (from $5.2 \mu \mathrm{m}$ (Ni-CNT-5) to $4.8 \mu \mathrm{m}$ (Ni-CNT-100). The increased hardness definitely contributed to the lower erosion rate for sample Ni-CNT-100 and the coating thickness may have been sufficient to not have its erosion rate affected by the mild steel substrate. Further increasing the pulse frequency to $1000 \mathrm{~Hz}$ resulted in reduced thickness for Ni-CNT-1000 to $1.6 \mu \mathrm{m}$. With the thinnest plating thickness among all the tested samples, Ni-CNT-1000 exhibited the highest erosion rate due to the affect of the softer mild steel substrate. 


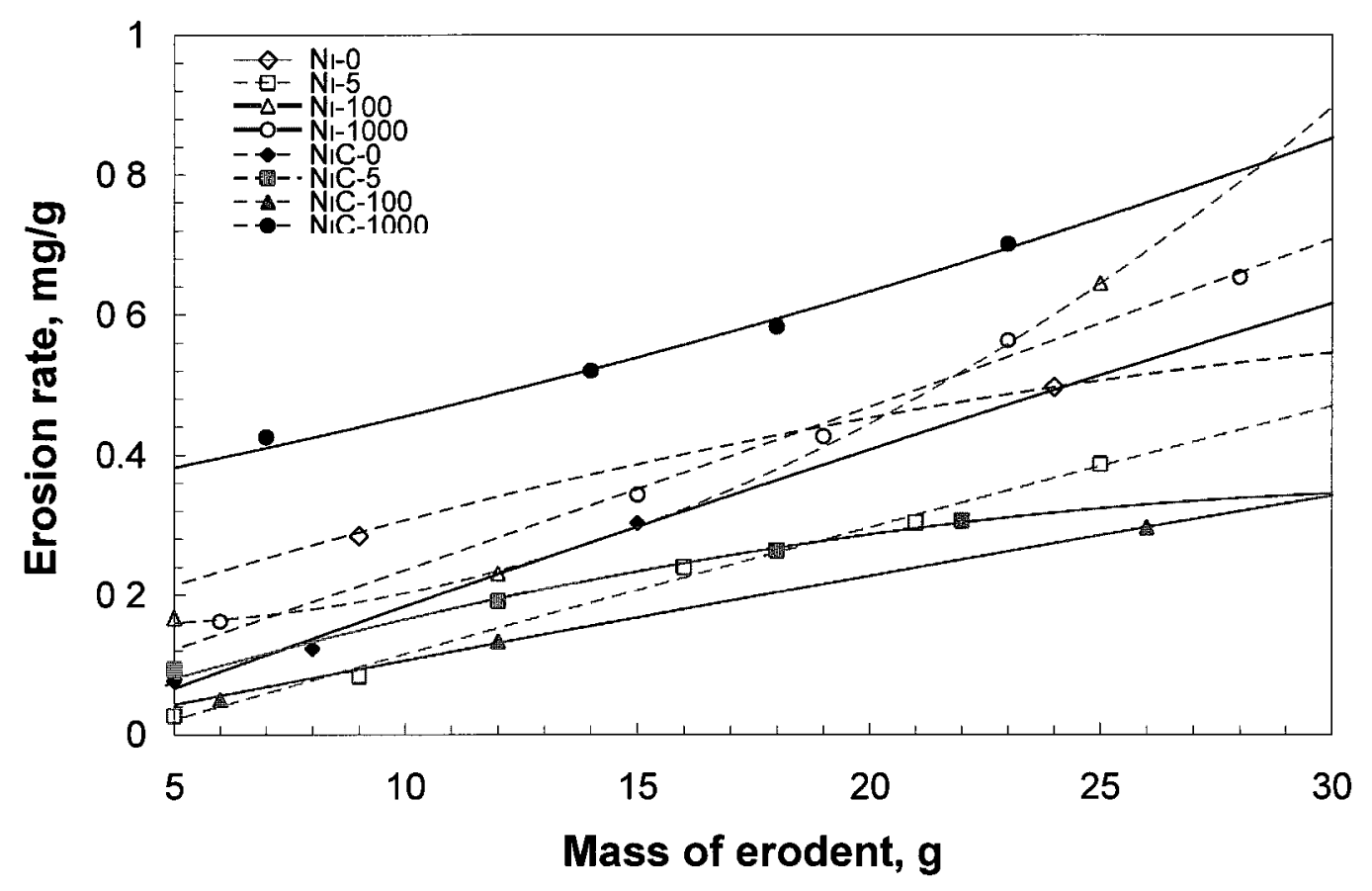

Figure 4-27 Erosion rates (at impingement angle $30^{\circ}$ ) for Ni plated and Ni-CNT plated samples.

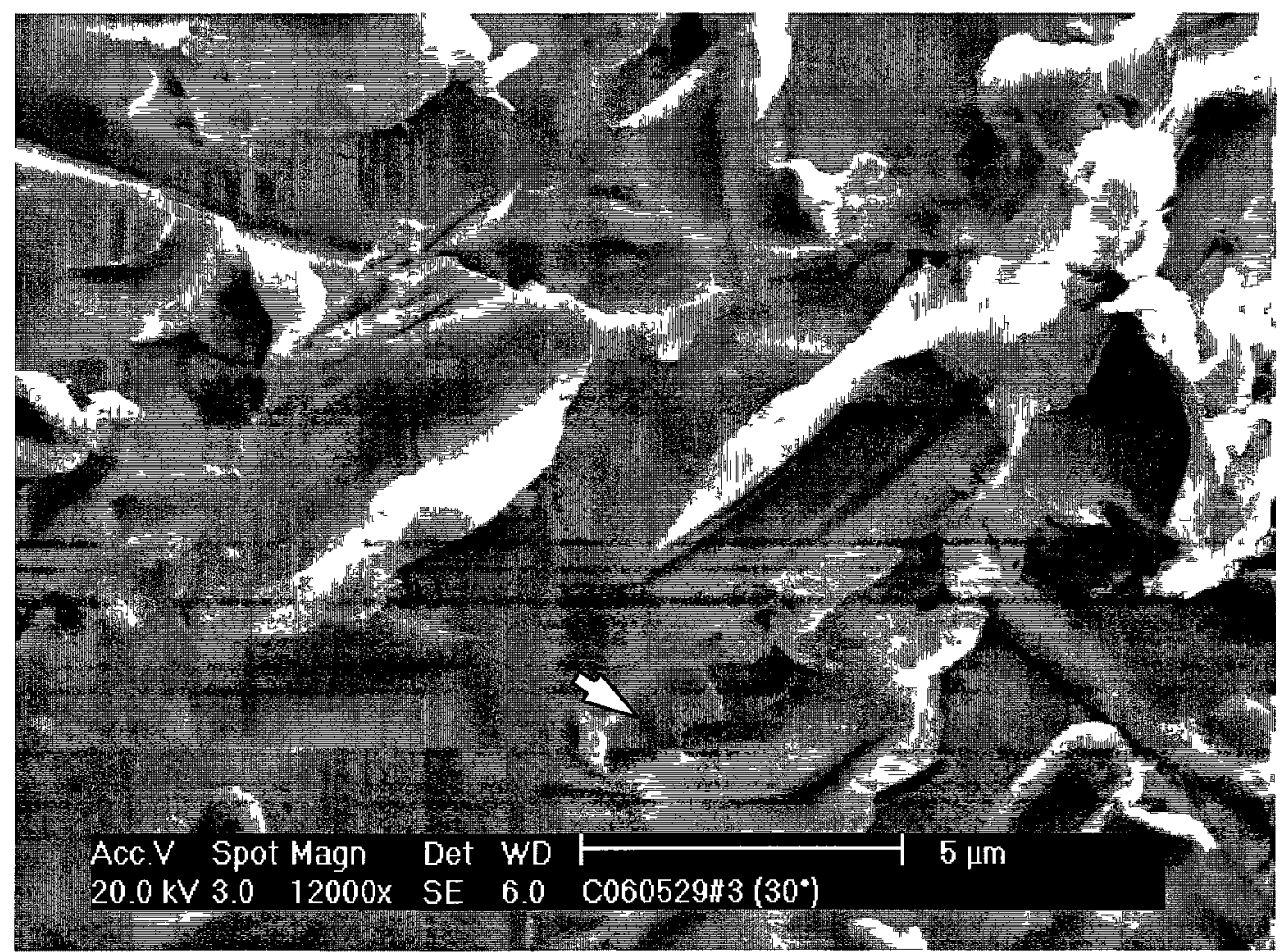

Figure 4-28 SEM image of erosion tested (at impingement angle $30^{\circ}$ ) Ni plated sample (Ni-100) 


\subsubsection{Physical Vapor Deposition (PVD) Coatings}

The erosion rates for hard coatings are commonly expressed in the term of weight loss per unit of erodent as shown in Figure 4-29. It can be seen that the overall erosion rates for the CA TiN coating are significantly lower, at various impingement angles, than those for the CrN based coatings. This is mainly due to its mechanical properties, specifically higher hardness and Young's modulus (Section 4.2).

The erosion rate for the $\mathrm{CA}$ TiN coating also increased with impingement angle and approached the highest value at $90^{\circ}$, as shown in Figure 4-29. This erosion behaviour is indicative of a brittle material (Section 4.2). The formation and intersection of cracks under impact sites determines the erosion process and the final rates [106, 404].

In the $\mathrm{CrN}$ based coating systems, erosion rates versus impingement angle covered a wide range of values. At low impingement angles, the erosion rates for $\mathrm{CrN}$ and $\mathrm{CrSiCN}(1)$ are lower then those for $\mathrm{CrSiCN}(2)$ and $\mathrm{CrSiCN}(3)$, which is attributed to their higher hardness, especially for $\mathrm{CrSiCN}(1)$. Near the impingement angle of $60^{\circ}$, their erosion rates begin exceeding those for $\mathrm{CrSiCN}(2)$ and $\mathrm{CrSiCN}(3)$ and reach peak values at impingement angles of $75^{\circ}$ for $\mathrm{CrN}$ (semi-brittle) and $90^{\circ}$ for $\mathrm{CrSiCN}(1)$ (brittle). These observed erosion behaviours of $\mathrm{CrN}$ and $\mathrm{CrSiCN}(1)$ resemble a brittle erosion mode, where more material is removed in the form of cracking under erodent impacts at higher impingement angles. 


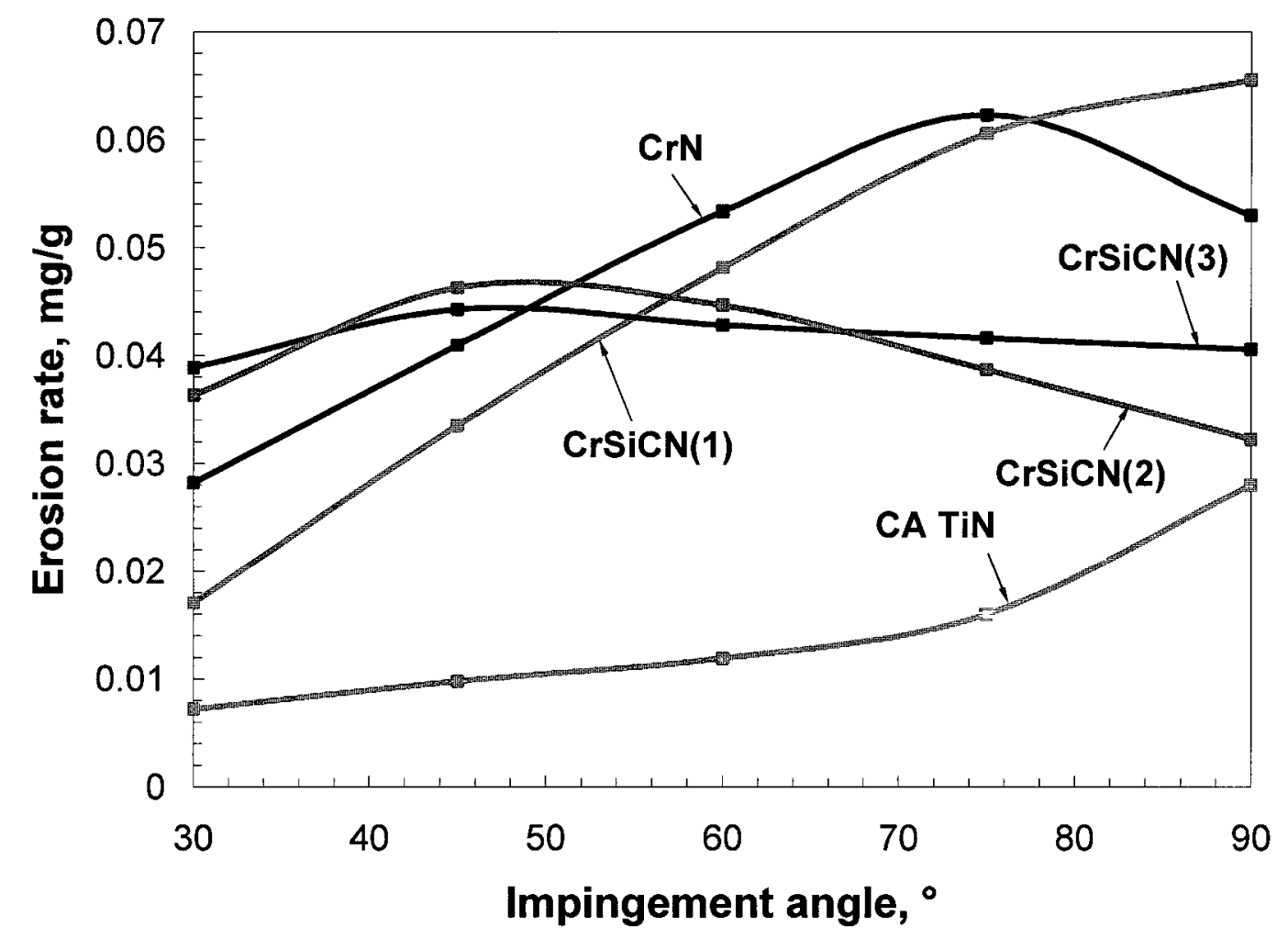

Figure 4-29 Erosion rates for test PVD coating systems.

Coating samples $\mathrm{CrSiCN}(2)$ and $\mathrm{CrSiCN}(3)$, on the other hand, demonstrated a different trend in erosion rate with impingement angle from $\mathrm{CrN}$ and $\mathrm{CrSiCN}(1)$. At low impingement angles, erosion rates for $\mathrm{CrSiCN}(2)$ and $\mathrm{CrSiCN}(3)$ were higher than those of $\mathrm{CrN}$ and $\mathrm{CrSiCN}(1)$, then gently increased with impingement angle, and reached peaks near $45^{\circ}$. At impingement angles over 50 and $55^{\circ}$, the erosion rates for $\operatorname{CrSiCN}(2)$ and $\mathrm{CrSiCN}(3)$ began to reduce to well below those for $\mathrm{CrN}$ and $\mathrm{CrSiCN}(1)$. This type of impingement angle dependent erosion is characteristic of a more ductile behaviour.

The transition of the erosion mode from ductile to brittle reflects changes in the mechanical properties, especially elasticity. The Young's modulus values for the PVD 
coatings are in an increasing order of $\mathrm{CrSiCN}(3)<\mathrm{CrSiCN}(2)<\mathrm{CrN}<\mathrm{CrSiCN}(1)<\mathrm{CA}$ TiN (Table 4-8). With the increase in hardness, the brittleness increases as well. Following the order of the hardness values, the erosion mode changes from ductile $(\mathrm{CrSiCN}(3)$ and $\mathrm{CrSiCN}(2))$ to semi-brittle $(\mathrm{CrN})$ and completely brittle $(\mathrm{CrSiCN}(1)$ and $\mathrm{CA} \mathrm{TiN})$.

Under low impingement angles, materials are subject to erosion by cutting or material displacement through plastic deformation. In designing coatings against plastic deformation induced erosion failure, a combination of a high hardness and a low elasticity is preferred $[395,396]$. Coating materials with high ratios of $\mathrm{H}^{3} / \mathrm{E}^{2}$, such as $\mathrm{CA}$ TiN and $\mathrm{CrSiCN}(1)$, possess higher resistance to plastic deformation at low impingement angle and consequently lower erosion rates (Figure 4-29). With increased impingement angles, normal impact force increases. As it reaches a sufficient value, the erosion mode begins switching from cutting to crack initiation and coalescence. Under this mode, it is the fracture toughness that plays a dominant role in erosion resistance. A material with lower fracture toughness, or increased brittleness, would be subject to excessive erosion [207]. It is speculated that the coatings $\mathrm{CA} \mathrm{TiN}, \mathrm{CrN}$ and $\mathrm{CrSiCN}(1)$ may have insufficient fracture toughness due to their relatively high values of hardness and modulus of elasticity. The erosion test results demonstrated such coatings exhibited high erosion rates at high impingement angles (Figure 4-29). In contrast, with lower values in both hardness and modulus of elasticity, the coatings with reasonable ductilities, such as $\mathrm{CrSiCN}(2)$ and $\mathrm{CrSiCN}(3)$, exhibited much lower erosion rates at high impingement angles compared with $\mathrm{CrN}, \mathrm{CrSiCN}(1)$ and $\mathrm{CA}$ TiN coating systems. 
The surfaces of eroded $\mathrm{CrN}$ based coatings were examined using SEM, and the images are shown in Figure 4-30. At a low impingement angle $\left(30^{\circ}\right)$, most of the surface materials are removed by the erodent in a typical cutting action on all four samples (Figure 4-30 a-1, b-1, c-1, d-1). At the impingement angle of $90^{\circ}$, cracks are observed on the coating surfaces ( $\mathrm{CrN}$ and $\mathrm{CrSiCN}(1))$ (Figure 4-30 a-2, b-2), indicating aggravated localized material removal in the forms of cracking and crack intersection. On the softer coatings $\mathrm{CrSiCN}(2)$ and $\mathrm{CrSiCN}(3)$, no cracks or localized coating removal was found, instead only indentations were present (Figure 4-30 c-2, d-2), indicating a lower rate of material removal and a higher resistance to cracking.
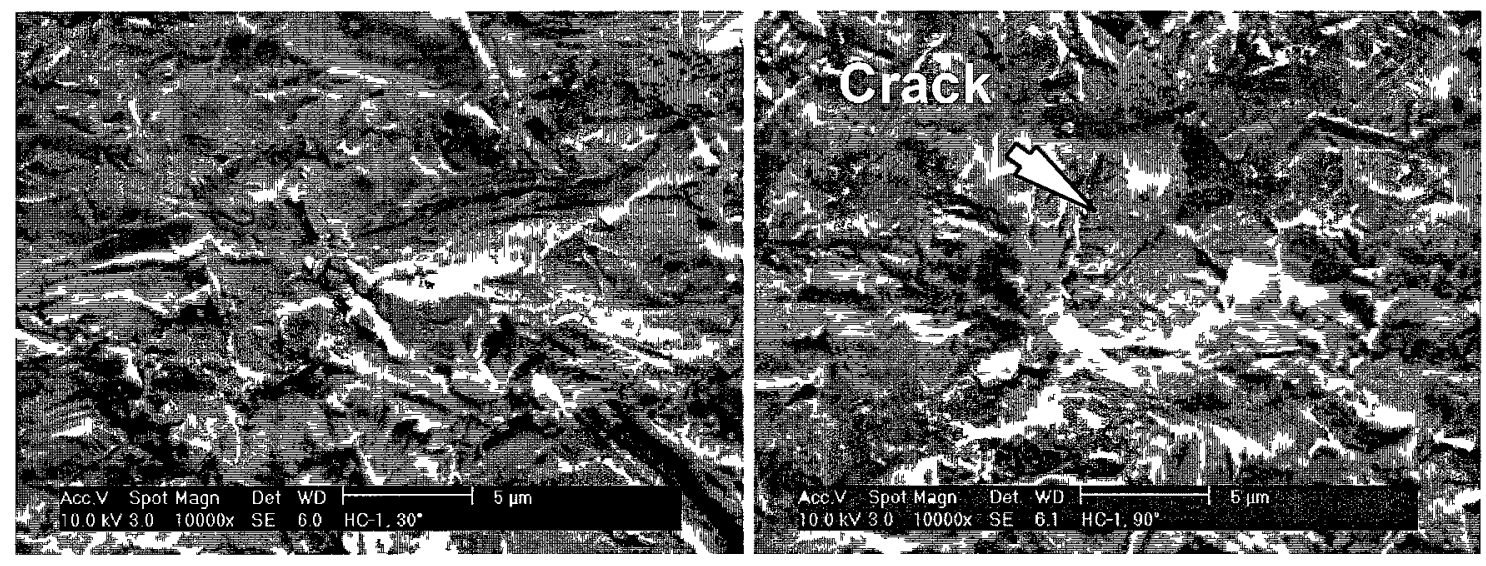

(a) $-1 \mathrm{CrN}\left(30^{\circ}\right)$

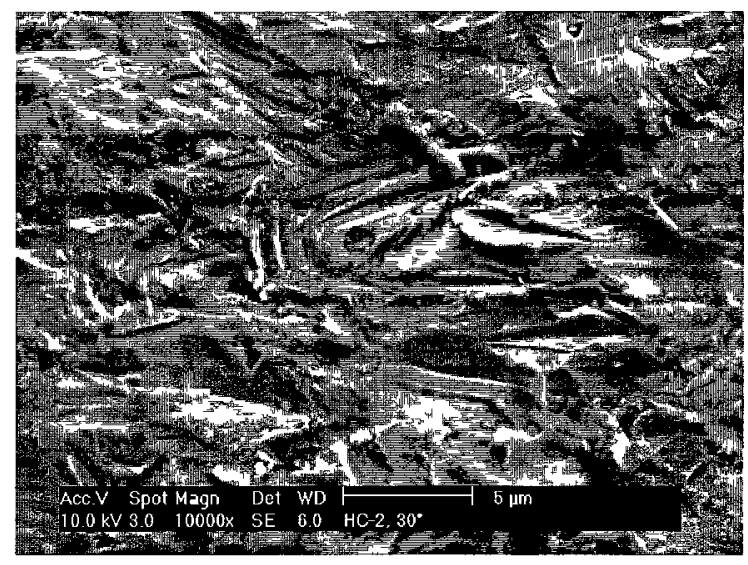

(b)-1 $\mathrm{CrSiCN}(1)\left(30^{\circ}\right)$ (a) $-2 \operatorname{CrN}\left(90^{\circ}\right)$

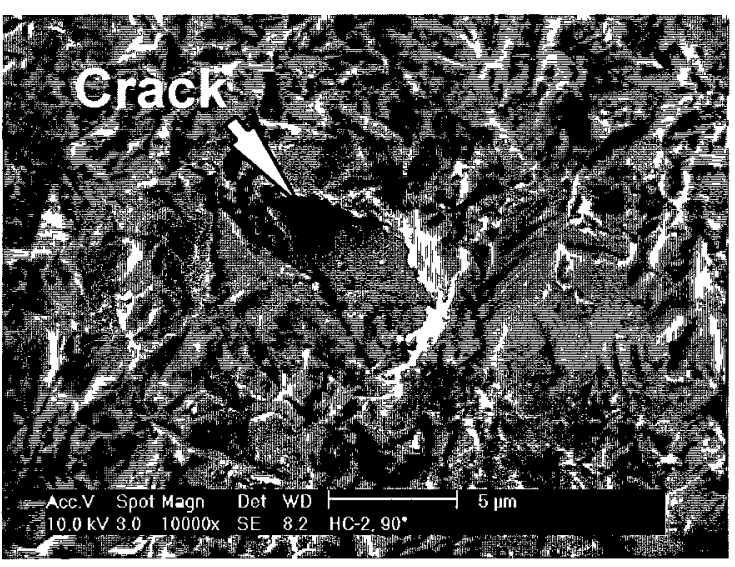

(b) $-2 \mathrm{CrSiCN}(1)\left(90^{\circ}\right)$

Figure 4-30 to be continued 


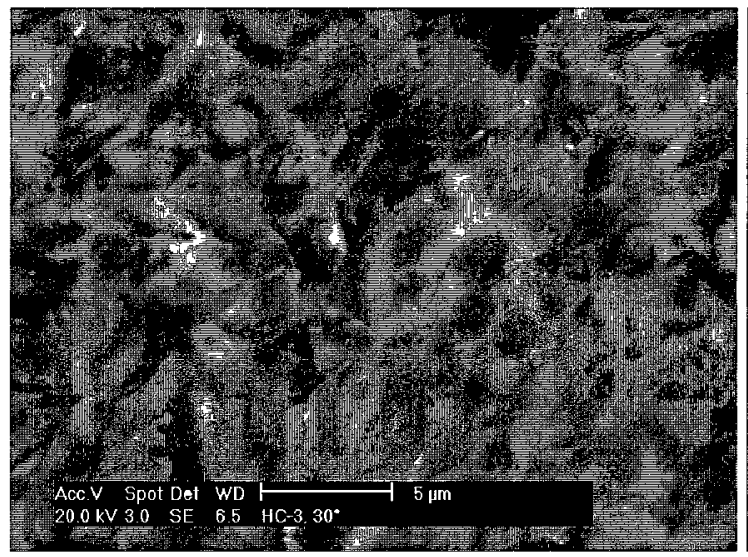

(c) $-1 \mathrm{CrSiCN}(2)\left(30^{\circ}\right)$

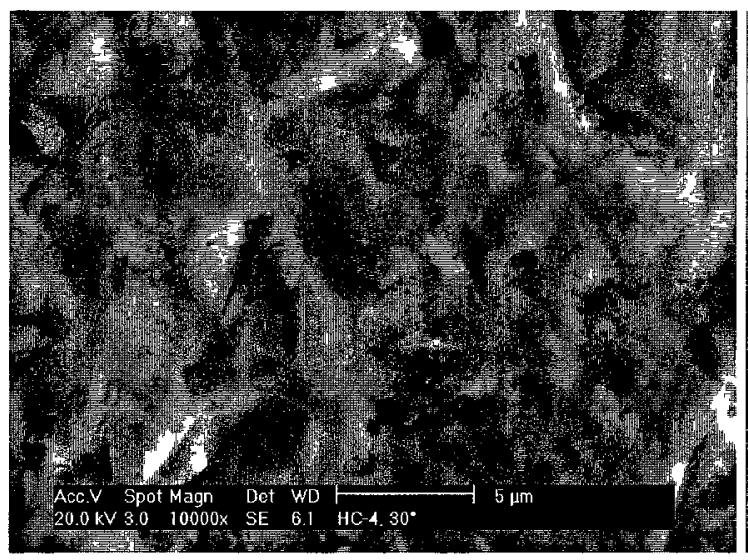

(d) $-1 \mathrm{CrSiCN}(3)\left(30^{\circ}\right)$

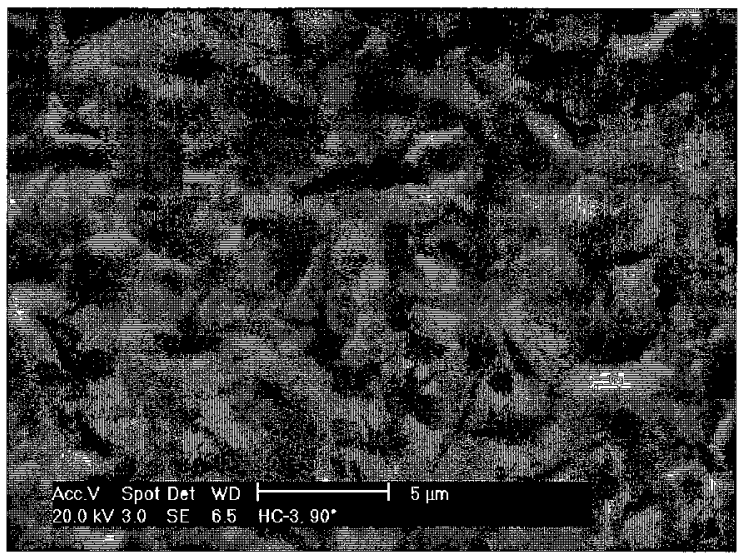

(c) $-2 \mathrm{CrSiCN}(2)\left(90^{\circ}\right)$

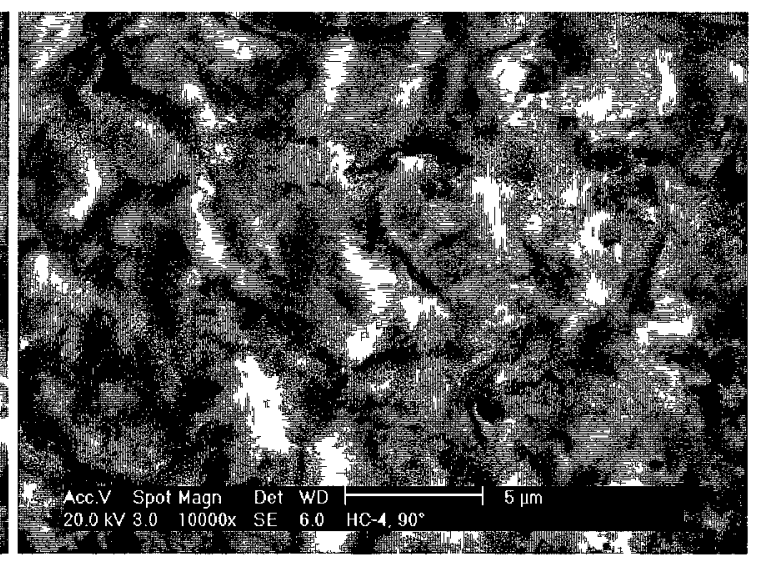

(d) $-2 \mathrm{CrSiCN}(3)\left(90^{\circ}\right)$

Figure 4-30 SEM images of surface characteristics of $\mathrm{CrN}$ based coating systems after erosion tests at different impingement angles.

\subsubsection{Correlations between $\mathrm{H}^{3} / \mathbf{E}^{2}$ Ratio and Tribological Properties of PVD Coating Systems}

In PVD coatings, the $\mathrm{H}^{3} / \mathrm{E}^{2}$ ratio is an important indicator in evaluating tribological behaviour. As shown in this chapter, the specific wear rate increased with a decrease in the $\mathrm{H}^{3} / \mathrm{E}^{2}$ ratio. As for erosion rate in terms of weight loss/erodent used, a general trend of inverse relationship with the magnitude of the $\mathrm{H}^{3} / \mathrm{E}^{2}$ ratio was also observed. The CA TiN coating, being one order of magnitude higher in the $\mathrm{H}^{3} / \mathrm{E}^{2}$ ratio than the $\mathrm{CrN}$ based coatings, demonstrated significantly lower erosion rates at all impingement angles. 
However, when the $\mathrm{H}^{3} / \mathrm{E}^{2}$ ratios of different coatings were similar, their erosion rates demonstrated complex behaviour with impingement angle. The erosion rate at low impingement angles, showed an inverse relationship with this ratio, however this relationship switched to proportional as the impingement angle exceeded a critical value.

These observations suggest that material properties other than just hardness and elasticity combined with test conditions could be used to derive a more accurate indicator for overall tribological behaviour. Examples of these additional factors are the magnitude and direction of the forces (or erodent) and the surface condition of the material since that determines the frictional force. A high $\mathrm{H}^{3} / \mathrm{E}^{2}$ ratio is only beneficial in wear or erosion resistance when plastic deformation is the main contributing factor in wear or erosion.

\subsection{Coating System Corrosion Behaviours}

Potentiodynamic polarization measurements and EIS tests were conducted on TiN and $\mathrm{CrN}$ based coating systems for short- and long-term corrosion behaviours, respectively. TiN coatings, deposited using EB and $\mathrm{CA}$ techniques, with different microstructures, were used to investigate the effect of microstructures on corrosion behaviour. CA TiN coatings deposited on mild steel and 17-4PH stainless steel substrates were investigated in order to reveal the substrate influence on corrosion protection due to the presence of coating defects. For both the EB and CA TiN coatings, and with both mild steel and 174PH steel substrates, PMMA-treated counterparts were also produced to investigate the outcome of defect clogging on corrosion performance. In $\mathrm{CrN}$ based coating systems, the 
microstructural changes with the addition of $\mathrm{Si}$ and $\mathrm{C}$, and the effects of these changes on their corrosion behaviour were explored.

To study their physical and chemical responses to corrosion, the tested samples were examined using SEM and EDS to detect corrosion products if there were any, and to evaluate post-corrosion surface morphology. For quantitative study, EIS test data were fitted using equivalent electric circuit (EEC) technique. The fitted results were utilized to identify and interpret the corrosion mechanisms.

\subsubsection{Potentiodynamic Polarization Tests}

\section{Substrates}

Potentiodynamic polarization curves for mild steel and 17-4PH stainless steel substrates are shown in Figure 4-31. The 17-4PH steel substrate sample demonstrated a lower corrosion current density and a wider passive region with a lower current density than did the mild steel substrate sample. This indicates the higher and more stable corrosion resistance of the $17-4 \mathrm{PH}$ steel. The mild steel did not establish an obvious passive region. The higher corrosion resistance and passivation characteristics of the 17-4PH stainless steel are mainly the results of the formation of a dense passive film with a high dielectric constant on the surface, which would protect the steel from further corrosion. Due to the electrochemically active characteristic of the mild steel, the corrosion takes place in the form of anodic dissolution [37]. Therefore passivity is generally not observed in a corrosion process on mild steel. 
The corresponding electrochemical parameters determined from the potentiodynamic polarization curves in Figure 4-31 using Eq. (3-10) and Eq. (3-11) (Section 3.7.3) are displayed in Table 4-12. It can be seen that the mild steel demonstrated an obvious lower corrosion potential, a higher corrosion current density, and consequently a lower polarization resistance when compared with the 17-4PH steel.

Table 4-12 Poltetiodynamic polarization parameters for substrate steels

\begin{tabular}{|c|c|c|c|}
\hline Substrate & $E_{\text {corr }}(\mathrm{mV}$ vs.SCE $)$ & $I_{\text {corr }}\left(\mu \mathrm{A} / \mathrm{cm}^{2}\right)$ & $R_{p}\left(\mathrm{k} \Omega \cdot \mathrm{cm}^{2}\right)$ \\
\hline MS & -416 & 2.50 & 1.5 \\
\hline $17-4 \mathrm{PH}$ & -374 & 1.70 & 18.9 \\
\hline
\end{tabular}

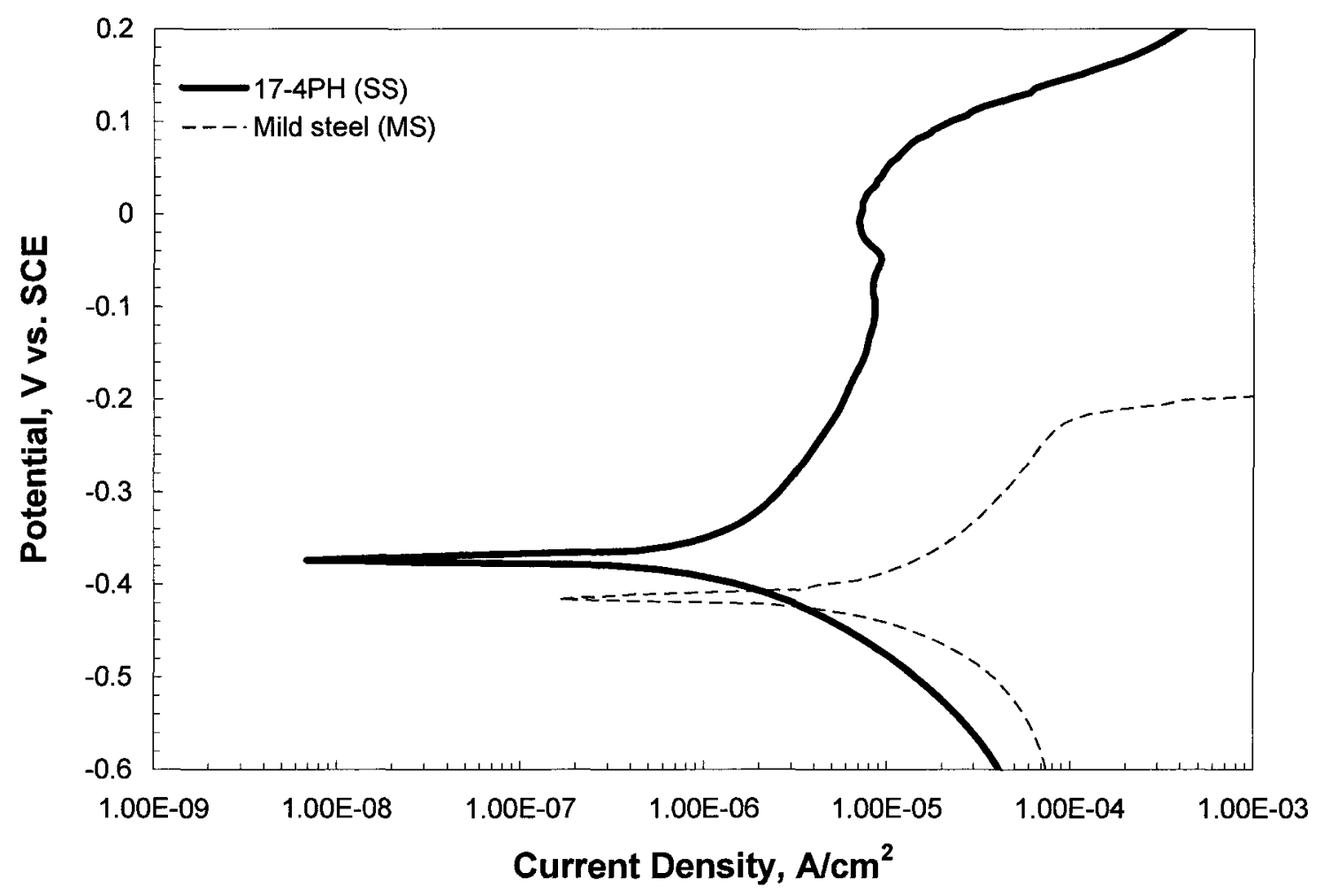

Figure 4-31 Potentiodynamic polarization curves of uncoated mild steel and 17-4PH stainless steel. 


\section{TiN Coating Systems}

Potentiodynamic polarization curves of the as-deposited and PMMA-treated EB and CA TiN coating systems are shown in Figure 4-32, which reveals that the three as-deposited TiN coatings improved the corrosion resistance when deposited on the substrates, mild steel and 17-4PH stainless steel, as indicated by their lower passive current densities and wider passive regions. Note that, the mild steel substrate did not show a passive region in its potentiodynamic polarization curve (Figure 4-31); however, a passive region can be observed in its coated counterpart (i.e., CA TiN/MS system) (Figure 4-32 c). This indicates that applying the TiN coating did not only improve the corrosion resistance, but changed the corrosion mechanism as well. It can also be observed that the three PMMAtreated TiN coatings showed considerably lower passive current densities and significantly wider passive regions when compared with their as-deposited counterparts (Figure 4-32 a, b, c), demonstrating that the PMMA treatment further enhanced corrosion resistances of the TiN coating systems.

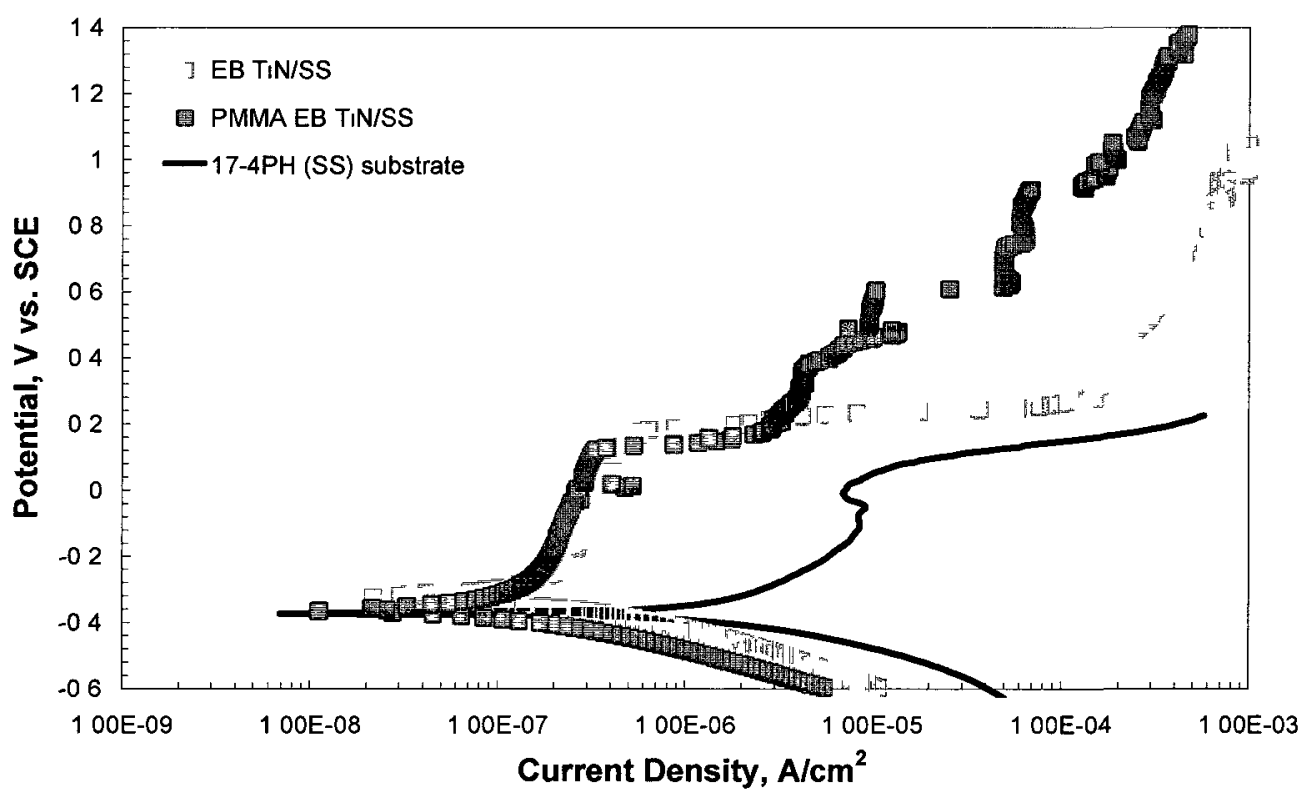

(a) EB TiN/SS

Figure 4-32 to be continued 


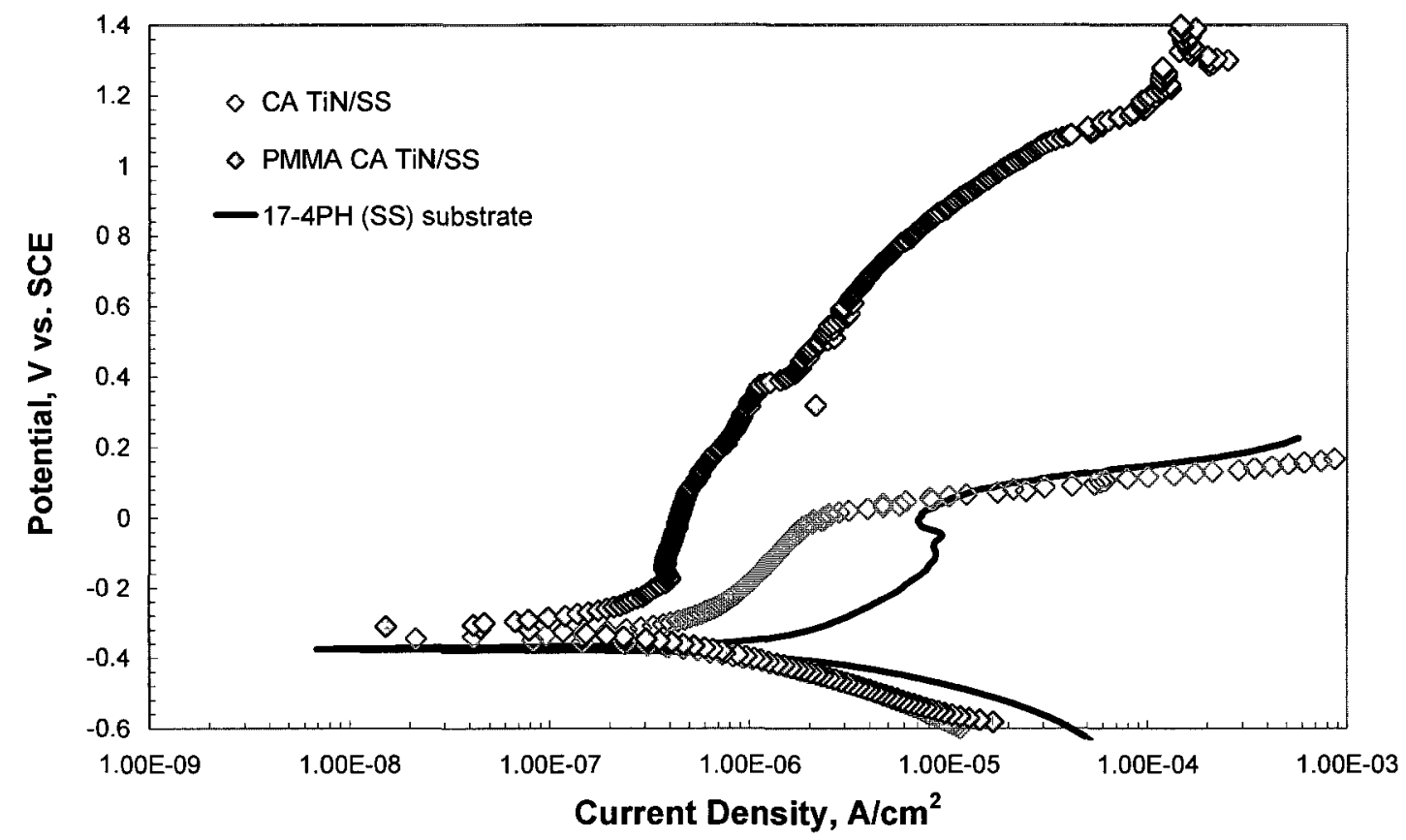

(b) $\mathrm{CA} \mathrm{TiN} / \mathrm{SS}$

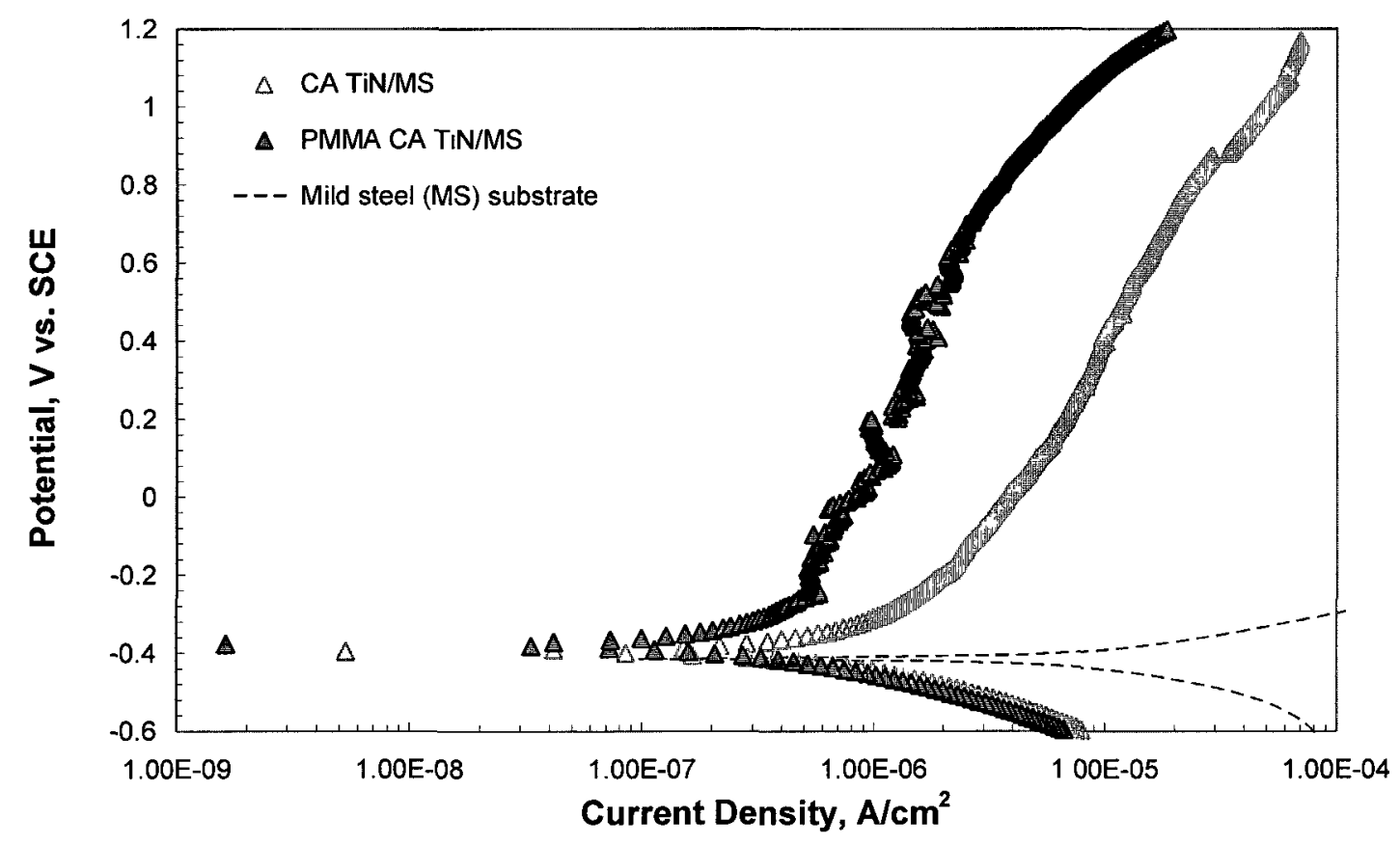

(c) CA TiN/MS

Figure 4-32 Potentiodynamic polarisation curves of TiN coating systems. 
The comparison of the potentiodynamic polarization behaviour of TiN coatings is presented in Figure 4-33. The CA TiN/SS coating system showed a lower passive current density than that of the CA TiN/MS coating system (Figure 4-33 a). This difference is associated with the corrosion characteristics of their substrates because of the presence of coating defects. The PMMA-treated samples exhibited lower current densities relative to their as-deposited counterparts, indicating an improvement in corrosion resistances due to PMMA filling the open pores in the coating and preventing the corrosive media penetrating to the substrate surface.

The passive current density for the EB TiN/SS coating system was lower than that of the CA TiN/SS coating system (Figure 4-33 b), indicating a better anti-corrosion performance as a result of its lower defect density (Table 4-4), and further demonstrating the correlation between corrosion resistance and defect density in TiN coating systems.

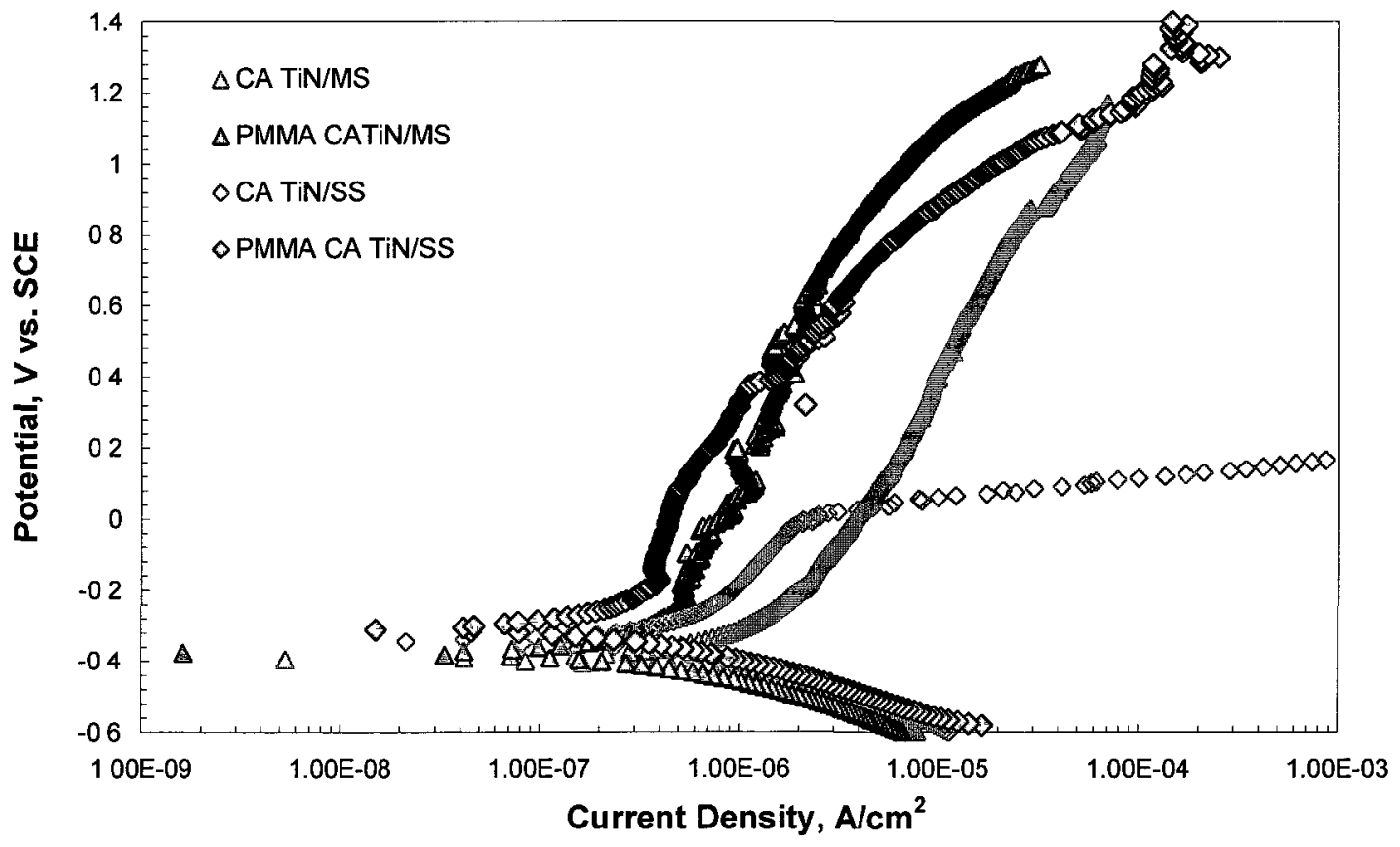

(a) CA TiN coatings: MS vs. SS substrates

Figure 4-33 to be continued 


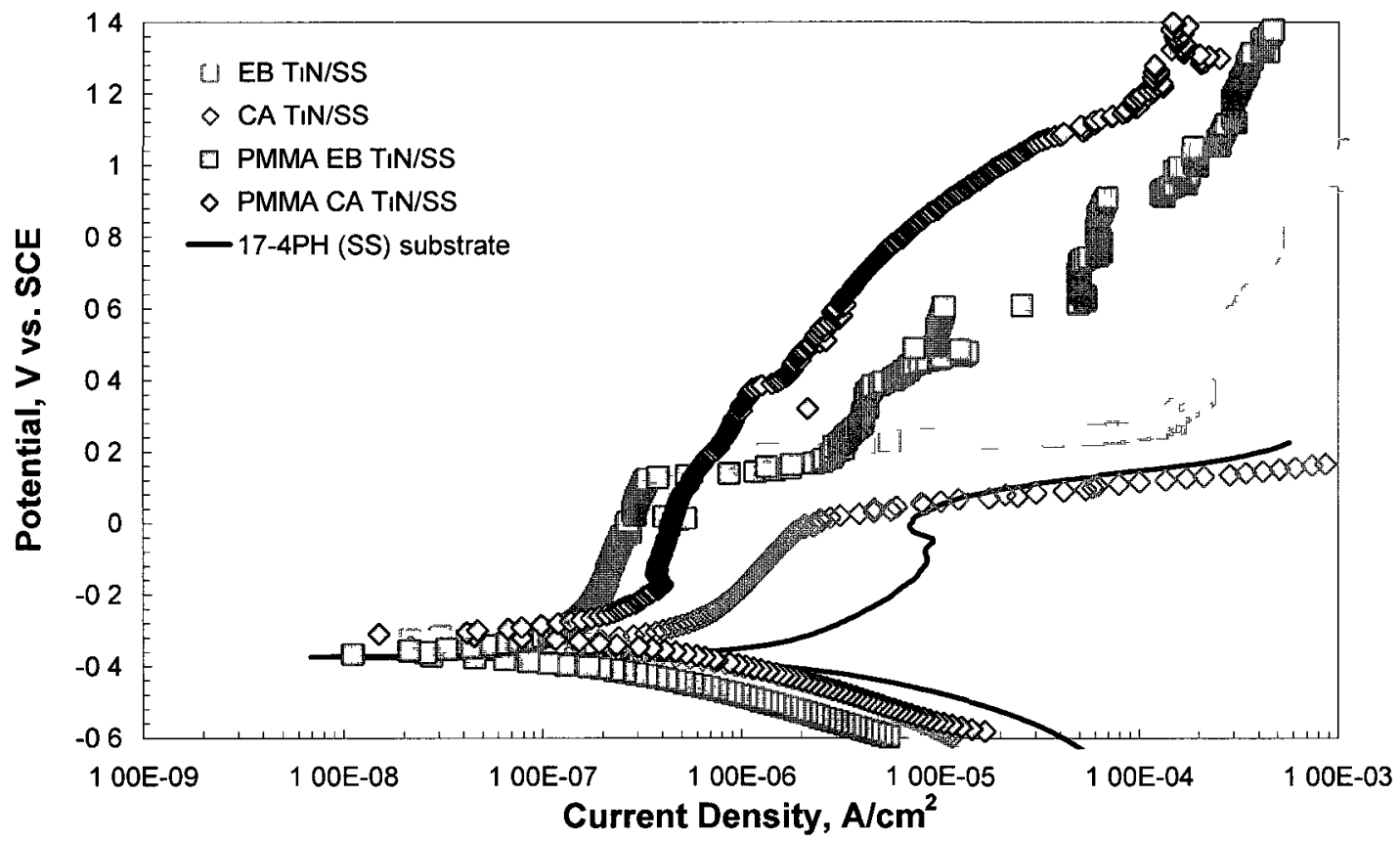

(b) TiN coatings: $\mathrm{EB}$ vs. $\mathrm{CA}$ deposition techniques

Figure 4-33 Potentiodynamic polarization curves of TiN coating systems with various deposition conditions.

From the electrochemical parameters in Table 4-13, it can be found that both EB and CA TiN coatings led to shifts of corrosion potential towards the noble direction when compared with their substrates (mild steel: $E_{\text {corr }}=-416 \mathrm{mV}$, and $17-4 \mathrm{PH}$ steel: $E_{\text {corr }}=-$ $374 \mathrm{mV})$. The CA TiN/SS $(-345 \mathrm{mV})$ and CA TiN/MS (-396mV) coating systems demonstrated lower corrosion potential than their PMMA-treated counterparts $(-311 \mathrm{mV}$ and $-377 \mathrm{mV}$, respectively). The PMMA-treated EB TiN/SS sample $(-367 \mathrm{mV})$ had a lower corrosion potential than the as-deposited one $(-327 \mathrm{mV})$. A general trend can be observed in Table 4-13 that all TiN coatings demonstrated lower corrosion current densities in comparison with the bare substrates; and PMMA-treated samples showed lower corrosion current densities than their as-deposited counterparts. 
Corrosion current density, in a coating/substrate system, is a measurement of the ion exchange rate in reactions at the electrolyte-substrate interface in a unit area of tested surface; the lower corrosion current densities revealed lower corrosion reactions in the coating/substrate systems. The PMMA-treated samples had considerably lower porosity than their as-deposited counterparts, because the PMMA treatment filled the open pores, thereby reducing the porosity and corrosion current density, and increasing the polarization resistance. This further revealed a clear correlation between the porosity (defect) in a coating system and its corrosion behaviour. A coating isolates the substrate from the bulk electrolyte, thus reducing the reaction rate and the ion current density. However, through the coating defects, some amount of electrolyte can still reach the substrate surface through the open pore channels in the coating. The PMMA treatment was shown to effectively seal the defects, reducing access of the electrolyte to the substrate.

Table 4-13 Potentiodynamic polarization test data for TiN coating systems

\begin{tabular}{|l|c|c|c|c|}
\hline \multicolumn{1}{|c|}{ Coating system } & $\mathrm{E}_{\text {cопr }}(\mathrm{mV}$ vs.SCE $)$ & $\mathrm{I}_{\text {corr }}\left(\mu \mathrm{A} / \mathrm{cm}^{2}\right)$ & $\mathrm{R}_{\mathrm{p}}\left(\mathrm{k} \Omega \cdot \mathrm{cm}^{2}\right)$ & $\mathrm{P}(\%)$ \\
\hline EB TiN/SS & -327 & 0.058 & 358 & 0.00036 \\
\hline PMMA EB TiN/SS & -367 & 0.037 & 570 & 0.00031 \\
\hline CA TiN/SS & -345 & 0.069 & 169 & 0.00088 \\
\hline PMMA CA TiN/SS & -311 & 0.035 & 640 & 0.00018 \\
\hline CA TiN/MS & -396 & 0.085 & 117 & 0.00001 \\
\hline PMMA CA TiN/MS & -331 & 0.055 & 170 & $1 \times 10^{-8}$ \\
\hline
\end{tabular}




\section{CrN Based Coating Systems}

Potentiodynamic polarization curves for the $\mathrm{CrN}$ based coating systems are displayed in Figure 4-34. It can be observed that all four coating samples showed wider passive regions than the substrate (17-4PH steel). $\mathrm{CrN}$ and $\mathrm{CrSiCN}(1)$ demonstrated low passive current densities, indicating considerable improvements in polarization resistance. Furthermore, $\mathrm{CrSiCN}(1)$ exhibited a notably higher corrosion potential and lower passive current density, revealing a better anti-corrosion property than the $\mathrm{CrN}$ coating. However, the passive current densities of the $\mathrm{CrSiCN}(2)$ and $\mathrm{CrSiCN}(3)$ coatings were higher than the $17-4 \mathrm{PH}$ steel substrate, indicating that the presence of coatings resulted in degraded corrosion resistances.

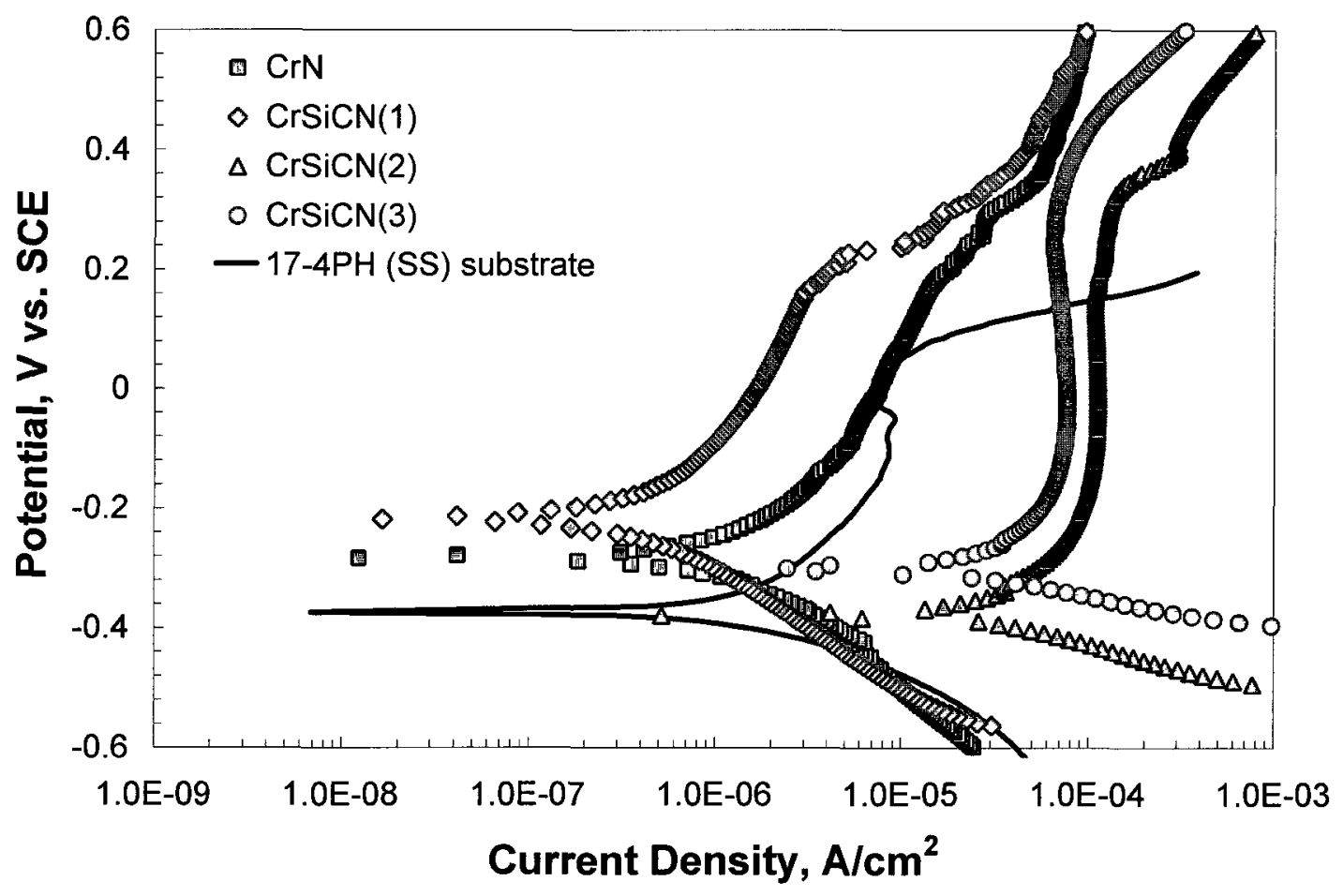

Figure 4-34 Potentiodynamic polarization curves of $\mathrm{CrN}$ based coating systems. 
When the potentiodynamic polarization test data (Table 4-14) were compared, the $\mathrm{CrN}$ coating showed a higher corrosion potential, a lower corrosion current density, and an increased polarization resistance than the $17-4 \mathrm{PH}$ steel substrate. The coating $\mathrm{CrSiCN}(1)$, as compared with the coating $\mathrm{CrN}$, demonstrated a higher corrosion potential, a slightly lower corrosion current density, and consequently a significant increase in polarization resistance over that of the substrate. The coatings $\mathrm{CrSiCN}(2)$ and $\mathrm{CrSiCN}(3)$ showed much higher current densities than $\mathrm{CrN}, \mathrm{CrSiCN}(1)$ and also the $17-4 \mathrm{PH}$ steel substrate (Table 4-12), demonstrating a degradation in anti-corrosion performance associated with their high porous microstructures as revealed by SEM (Figure 4-9 and Figure 4-10).

Open pores in a nitride coating can facilitate the formation of micro galvanic cells between the exposed substrate (anode) and the coating (cathode). In the pores of the coatings, the oxygen diffusion rates are lower than those in the bulk electrolyte outside the coating, thus resulting in deaeration in the pores, where the acidity of the electrolyte increases due to hydrolysis. As a result, the concentration of chloride cation $\left(\mathrm{Cl}^{-}\right)$in the pores increases, leading to an increase in the conductivity of the electrolyte [139]. With the increased conductivity of the electrolyte and sufficient mass transfer through the porous coatings, the corrosion processes in the pores at the electrolyte-substrate interface accelerate, and as a result, the substrate may not be able to re-passivate. The corrosion process in the pores is further accelerated by the large area ratio of cathode to anode. 
The high porosities in the $\mathrm{CrSiCN}(2)$ and $\mathrm{CrSiCN}(3)$ coatings caused high pitting densities, resulting in high corrosion current densities. This further confirms there is a close correlation between the coating porosity and its corrosion behaviour.

Table 4-14 Poltetiodynamic polarization test data for $\mathrm{CrN}$ based coating systems

\begin{tabular}{|l|c|c|c|}
\hline Coating system & $E_{\text {corr }}(\mathrm{mV}$ vs. SCE $)$ & $\mathrm{I}_{\text {corr }}\left(\mu \mathrm{A} / \mathrm{cm}^{2}\right)$ & $\mathrm{R}_{\mathrm{p}}\left(\mathrm{k} \Omega \cdot \mathrm{cm}^{2}\right)$ \\
\hline $\mathrm{CrN}$ & -284 & 0.550 & 64.06 \\
\hline $\mathrm{CrSiCN}(1)$ & -218 & 0.370 & 175.63 \\
\hline $\mathrm{CrSiCN}(2)$ & -301 & 13.00 & 1.15 \\
\hline $\mathrm{CrSiCN}(3)$ & -379 & 12.00 & 1.16 \\
\hline
\end{tabular}

\subsubsection{Electrochemical Impedance Spectroscopy (EIS)}

\section{Substrates}

EIS spectra for the mild steel and 17-4PH steel substrates are displayed in Figure 4-35. For the mild steel substrate, the semicircles (arcs) in the Nyquist plots (Figure 4-35 a) indicate a typical charge-transfer controlled general corrosion process. The rapidly collapsing semicircles indicate a fast degradation in corrosion resistance with immersion time. After 7 hours, the sample surface became so rusty that the test had to be terminated. The arcs broadening to straight lines in the Nyquist plots for the 17-4PH steel substrate (Figure 4-35 b) indicate a diffusion controlled reaction process (referred as to Warburg diffusion behaviour [405]) at low frequencies. The presence of a diffusion controlled reaction process was attributed to the formation of a dense passive oxide film on the surface of $17-4 \mathrm{PH}$ steel substrate. 
From the Bode modulus plots (Figure 4-35 c, d), it can be observed that in the low frequency region the impedance values of $17-4 \mathrm{PH}$ steel substrate increased from $\sim 50$ to $100 \mathrm{k} \Omega \mathrm{cm}^{2}$ in five days of immersion, which were up to three orders of magnitude higher than those of the mild steel substrate (between $\sim 0.035$ and $1.9 \mathrm{k} \Omega \mathrm{cm}^{2}$ in 7 hours of immersion), indicating a significantly higher corrosion resistance of the $17-4 \mathrm{PH}$ steel relative to that of the mild steel substrate. From the Bode phase plots (phase vs. $\log f$ ), it can be found that the minimum phase angles of the mild steel substrate (between $-69^{\circ}$ and $-56^{\circ}$ ) (Figure 4-35 e) were higher than those of the 17-4PH steel (between $-74^{\circ}$ and $-79^{\circ}$ ) (Figure 4-35 f), revealing that the surface of mild steel was more inhomogeneous than 17-4PH steel. The increase of the minimum phase angles from $-69^{\circ}$ to $-56^{\circ}$ (Figure 4-35 e) with immersion time show that the surface of the mild steel became inhomogeneous during the exposure. This could be associated with the dissolution of the mild steel [37] and formation of corrosion products (rust), which resulted in an increase in the quantity and size of pores on the surface. The electrolyte in the pores resulted in a localized changes in capacitance and charge transfer resistance at the pore sites, thus making the surface inhomogeneous $[38,406]$. For the $17-4 \mathrm{PH}$ steel substrate, the minimum phase angles were stable and remained in a narrow range of $-74^{\circ}$ to $-79^{\circ}$ (Figure 4-35 f), indicating a stable and homogeneous surface throughout the whole immersion period owing to the presence of a dense passive oxide surface film. 


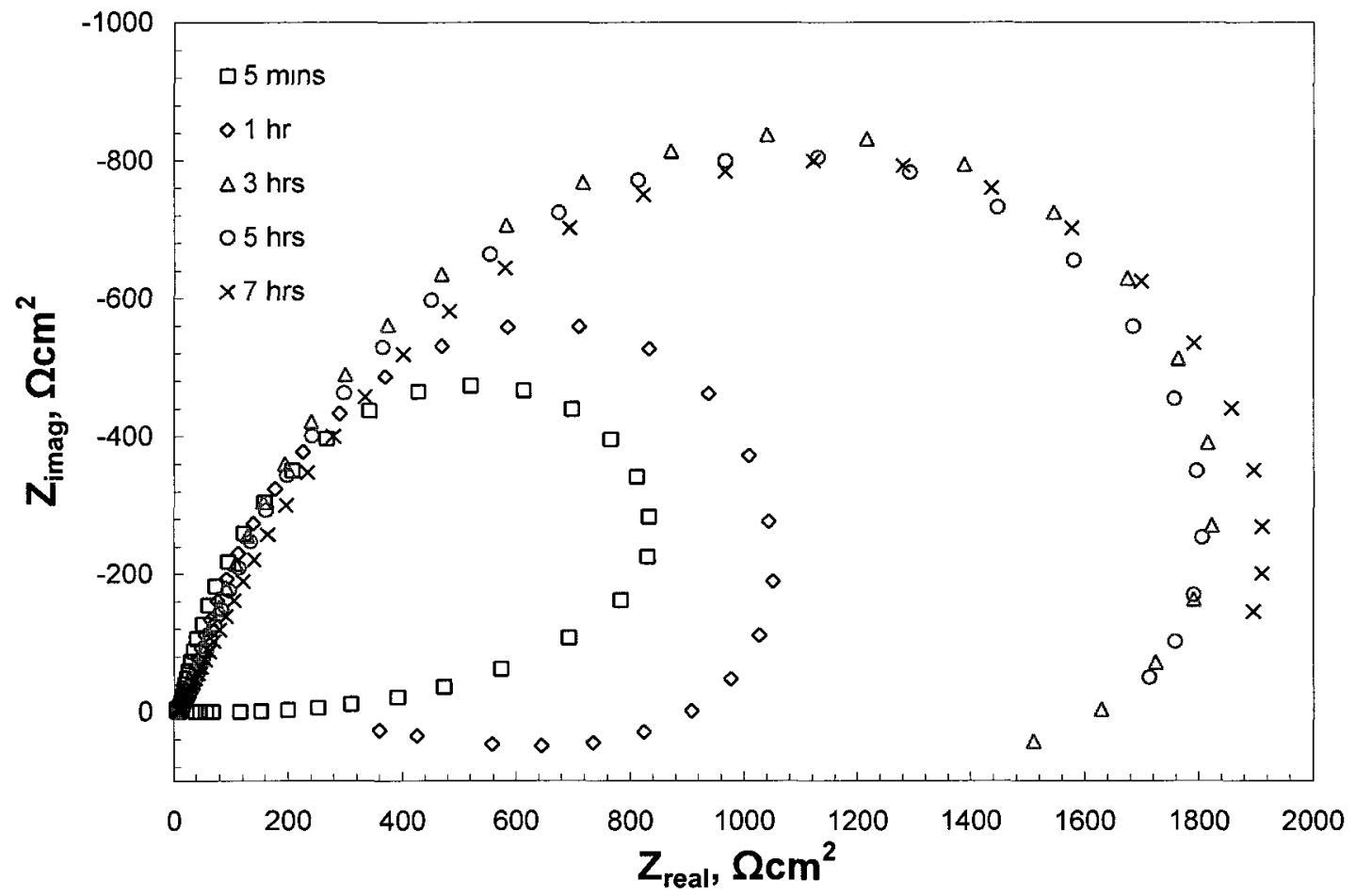

(a) Nyquist plots of MS

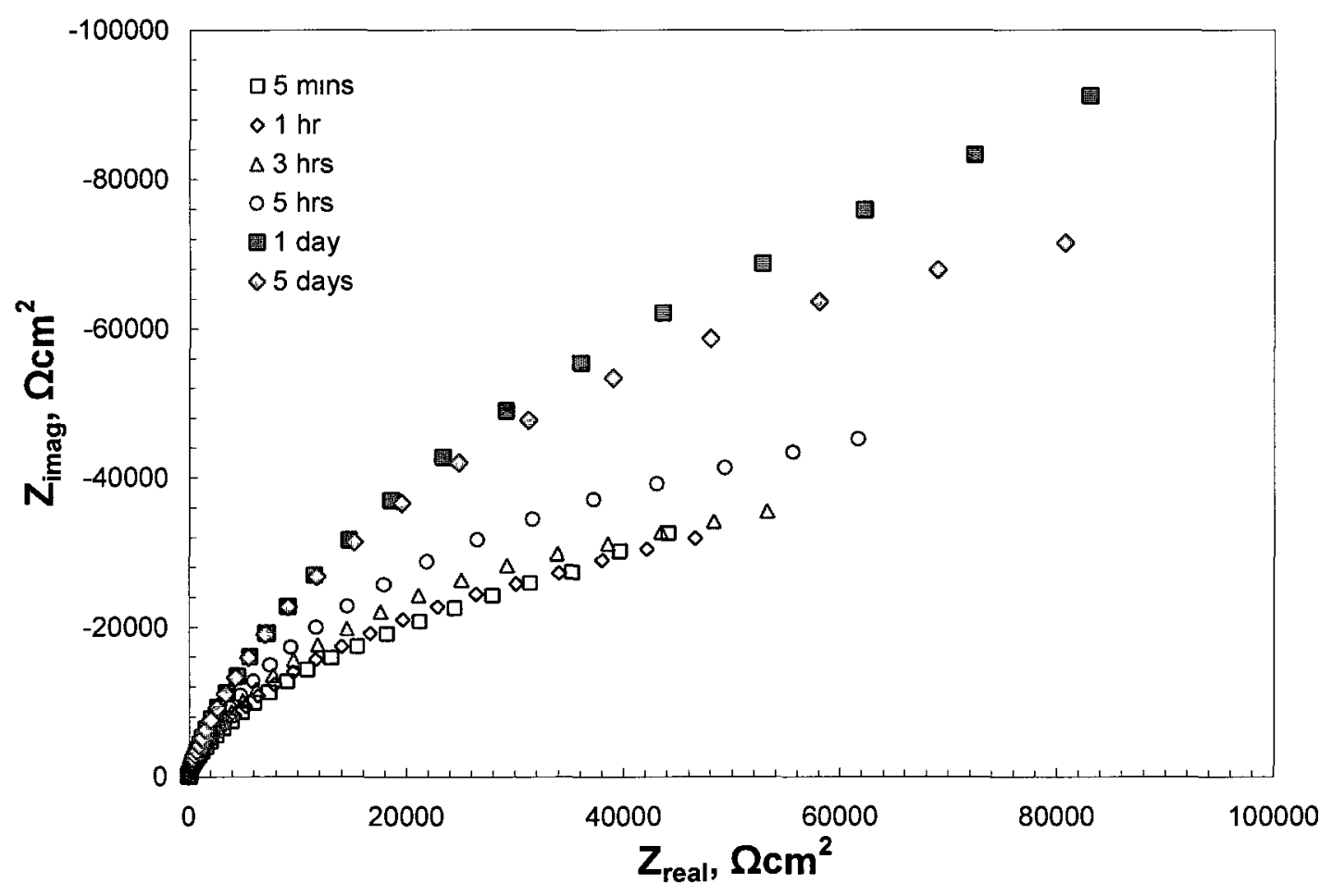

(b) Nyquist plots of 17-4PH steel

Figure 4-35 to be continued 


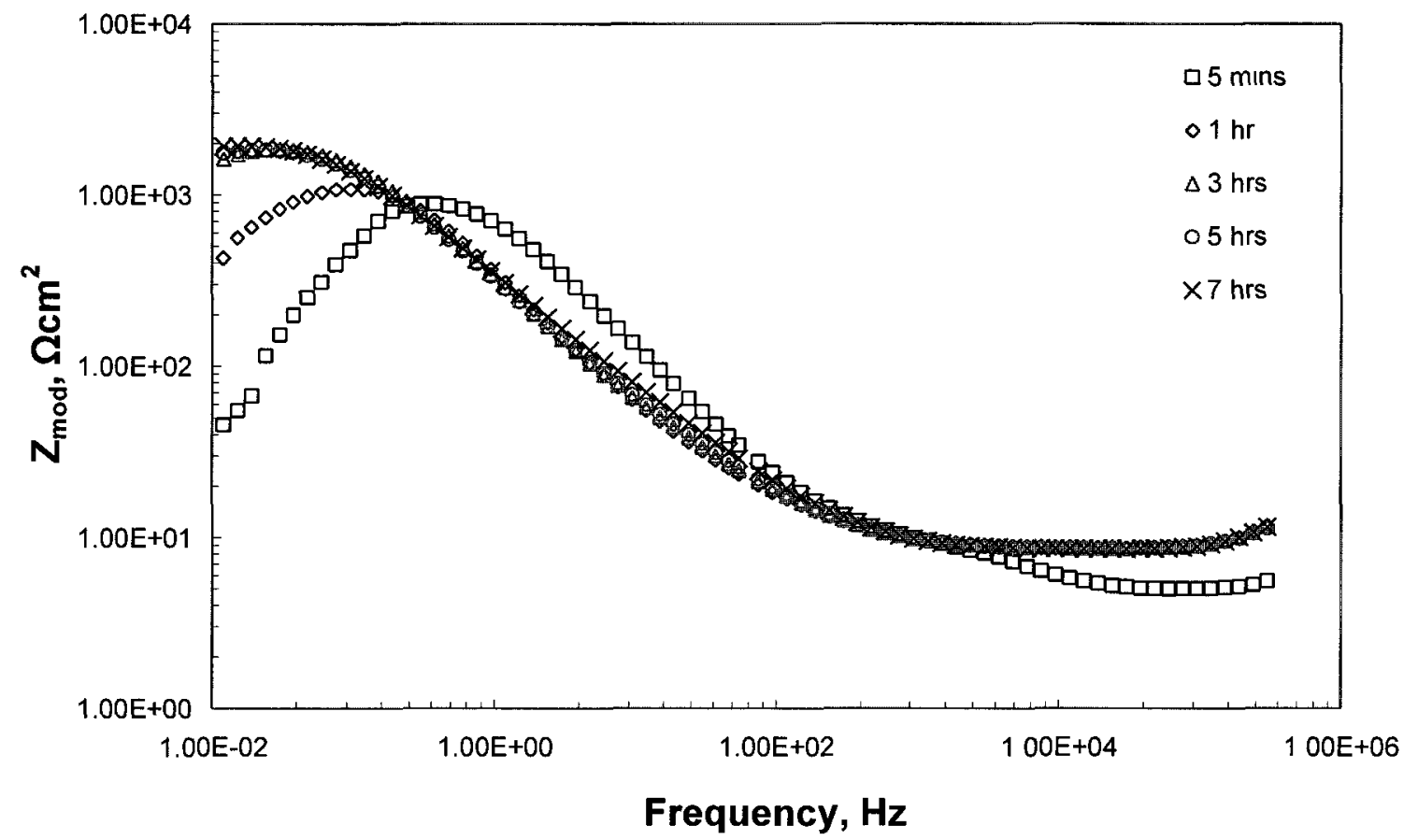

(c) Bode modulus plots of MS

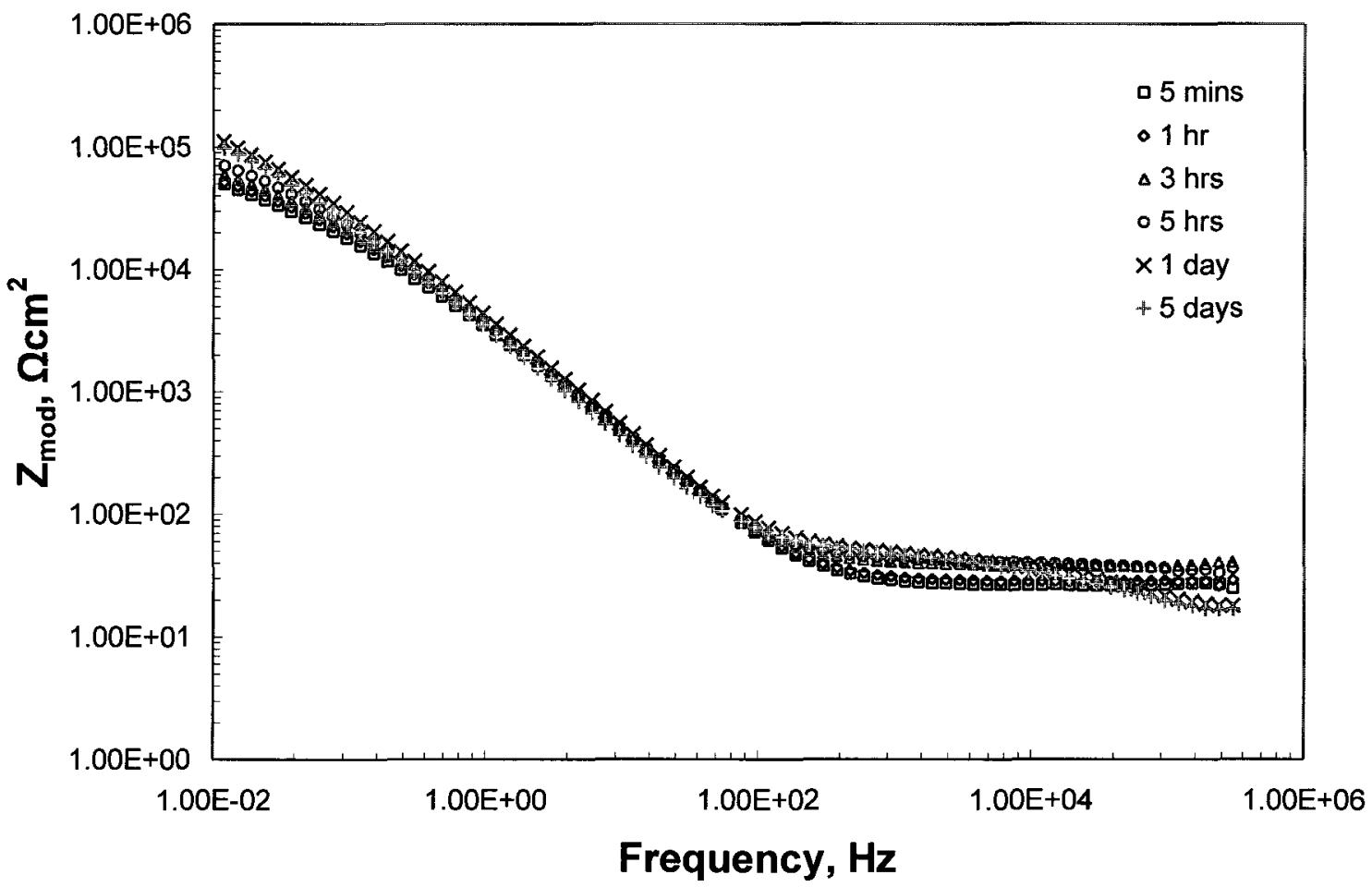

(d) Bode modulus plots of $17-4 \mathrm{PH}$ steel

Figure 4-35 to be continued 


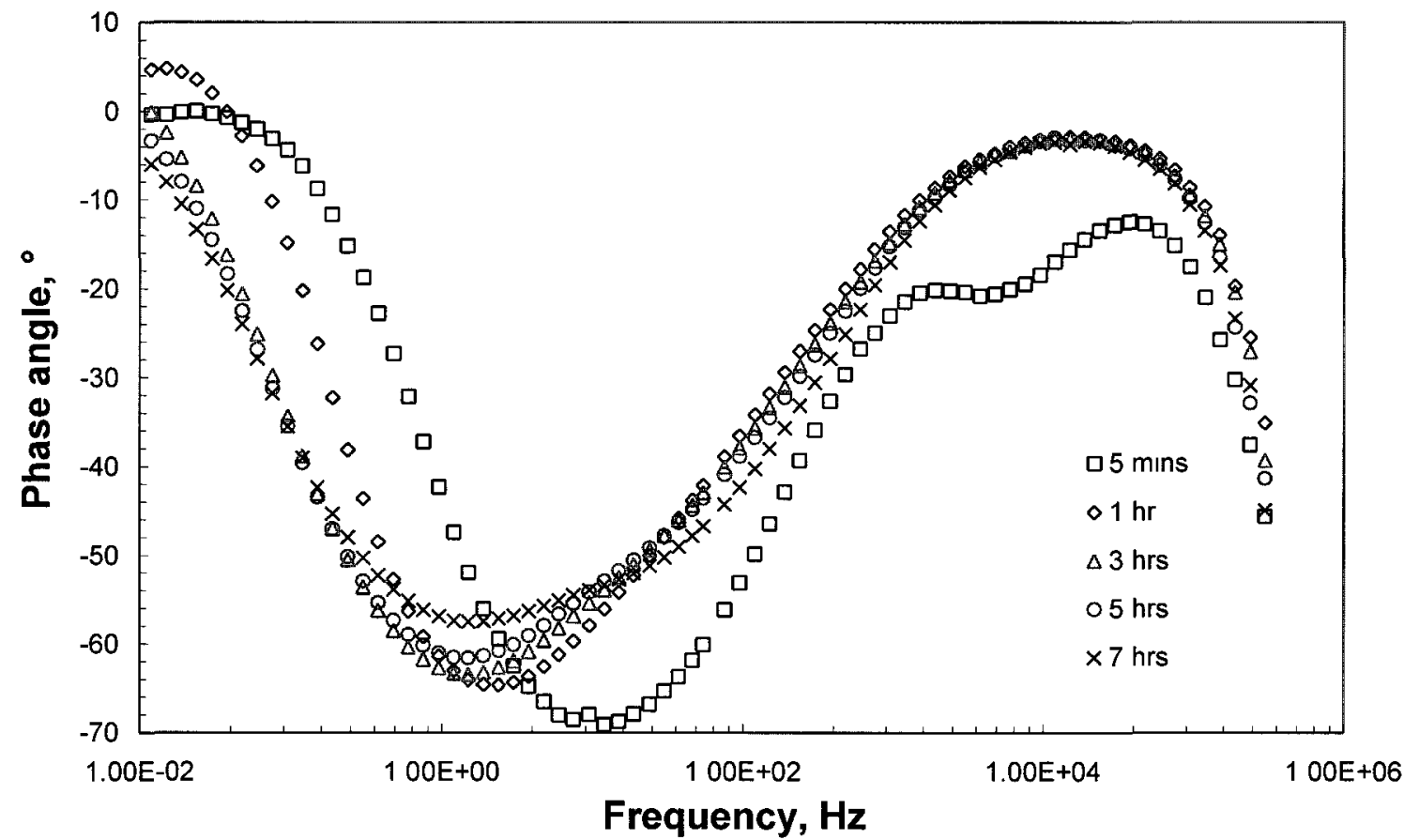

(e) Bode phase plots of MS

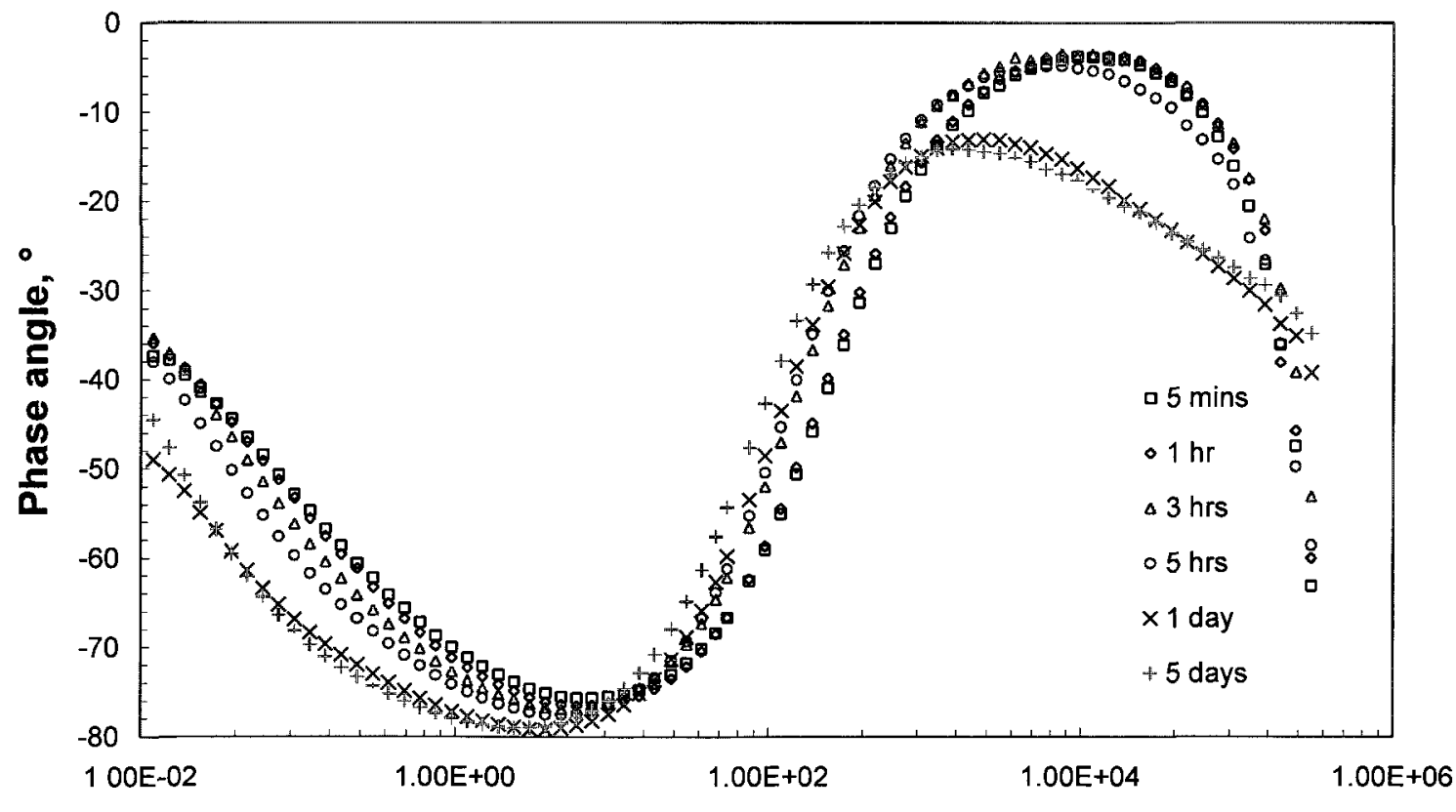

Frequency, $\mathrm{Hz}$

(f) Bode phase plots of 17-4PH steel

Figure 4-35 EIS spectra of mild steel and 17-4PH steel substrates. 


\section{TiN Coating Systems}

EIS spectra of the as-deposited and PMMA-treated EB TiN/SS coating systems are displayed in Figure 4-36. The approximate straight lines in the Nyquist plots in the initial stage of the immersion (Figure 4-36 a) indicated a diffusion controlled corrosion characteristic of the as-deposited TiN coating. In the course of immersion, the Nyquist plots changed from approximate straight lines to semicircles (arcs), revealing the corrosion process changed from diffusion controlled to charge-transfer controlled. The straight lines (Figure 4-36 b) revealed a diffusion dominated corrosion process for the PMMA-treated counterpart. The quickly diminishing semicircles in the course of immersion (Figure 4-36 a) indicated a notable drop in corrosion resistance of the asdeposited EB TiN/SS system. The impedance values of the as-deposited sample at 10 $\mathrm{mHz}$ decreased from $60 \mathrm{k} \Omega \mathrm{cm}^{2}$ (after five minutes) to the lowest value of $\sim 4 \mathrm{k} \Omega \mathrm{cm}^{2}$ (1 day), indicating a degradation in corrosion resistance (Figure 4-36c); whereas, the Nyquist plots for the PMMA-treated sample kept the straight-line features even after twenty days of immersion, demonstrating a high and stable corrosion resistance (222 to $250 \mathrm{k} \Omega \mathrm{cm}^{2}$ at $10 \mathrm{mHz}$ ) (Figure 4-36 b, d). The Bode modulus plots show that the impedance of the as-deposited TiN/SS coating system (Figure 4-36 c) was less stable than the impedance of the 17-4PH steel substrate (Figure 4-35 d).

The potentiodynamic polarization measurement (Figure 4-32 a) (Table 4-13) demonstrated an improved corrosion resistance for the 17-4PH steel substrate with the EB TiN coating, these EIS test results, however, exhibited a continued degradation in corrosion resistance with immersion time. This indicated the different short and long term 
corrosion behaviour of the EB TiN/SS coating system, which can be attributed to defects in the EB TiN coating. In the short term potentiodynamic polarization measurement, there was insufficient time for the $\mathrm{NaCl}$ electrolyte to penetrate the through-coating pores to reach the substrate steel; therefore the coating defects did not cause an obvious degradation in corrosion resistance during the short-term corrosion test. However, in the long term EIS test, sufficient $\mathrm{NaCl}$ electrolyte was able to penetrate; therefore galvanic cells were able to establish at the electrolyte-substrate interface, resulting in increased corrosion of the substrate materials.

The PMMA treatment increased impedance values by over one order of magnitude for the EB TiN coating, and exhibited a better and stable resistance to corrosion (Figure 4-36 c, d). No obvious degradation in corrosion resistance was observed even after an immersion of twenty days, indicating PMMA-treated EB TiN coating can provide a long term and durable protection against corrosion.

The increase of the minimum phase angle with immersion time $\left(-78^{\circ}\right.$ to $\left.-66^{\circ}\right)$ (Figure 436 e) revealed that the surface of the coating EB TiN/SS became more inhomogeneous with the immersion period. This was attributed to the coating pores, through which electrolyte could reach the substrate steel, resulting in localized decreases in capacitance and increases in charge transfer resistance at the pore sites; and furthermore, with immersion time, due to the formation of galvanic cells, the pore size could be increased. 


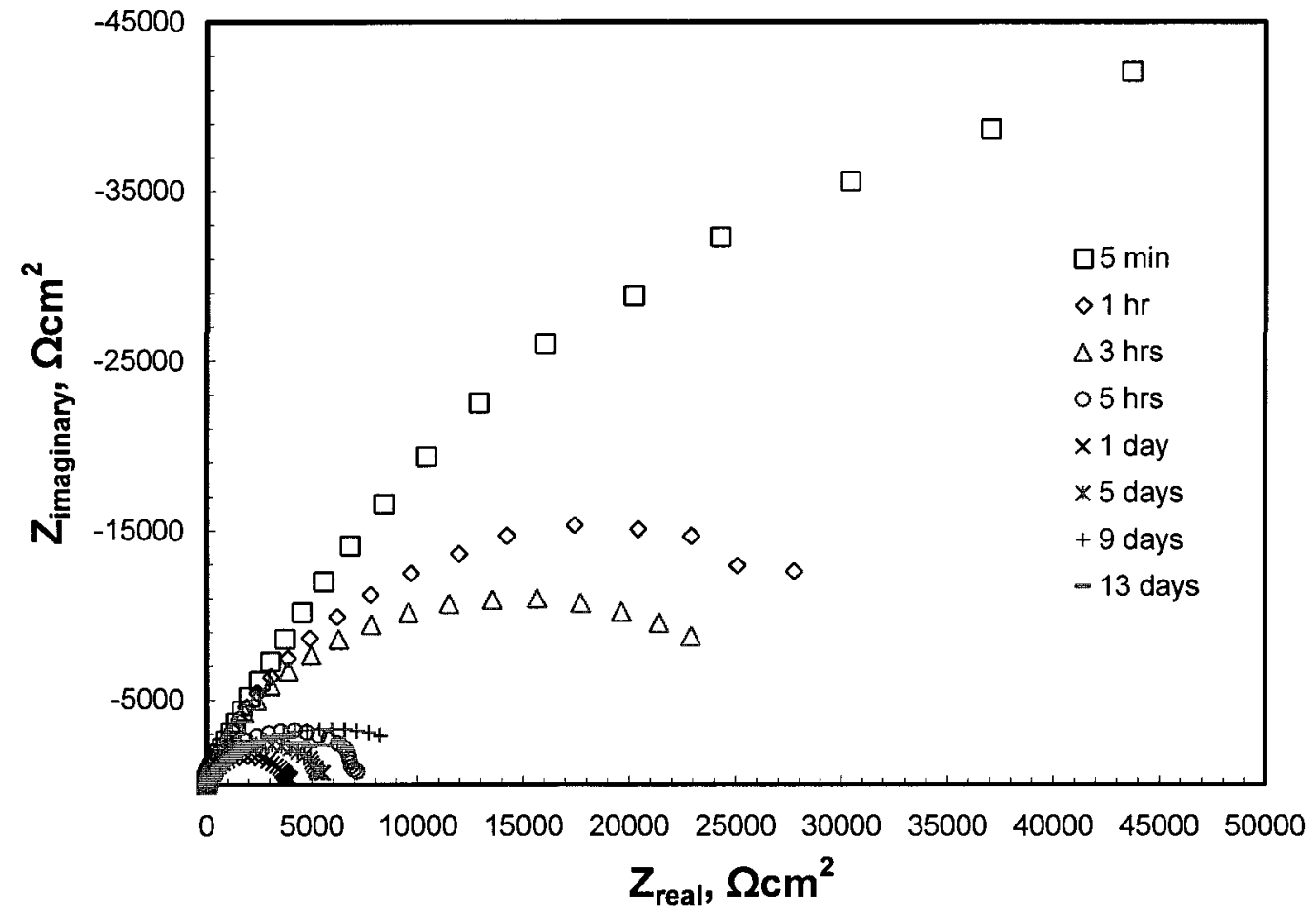

(a) Nyquist plots of EB TiN/SS

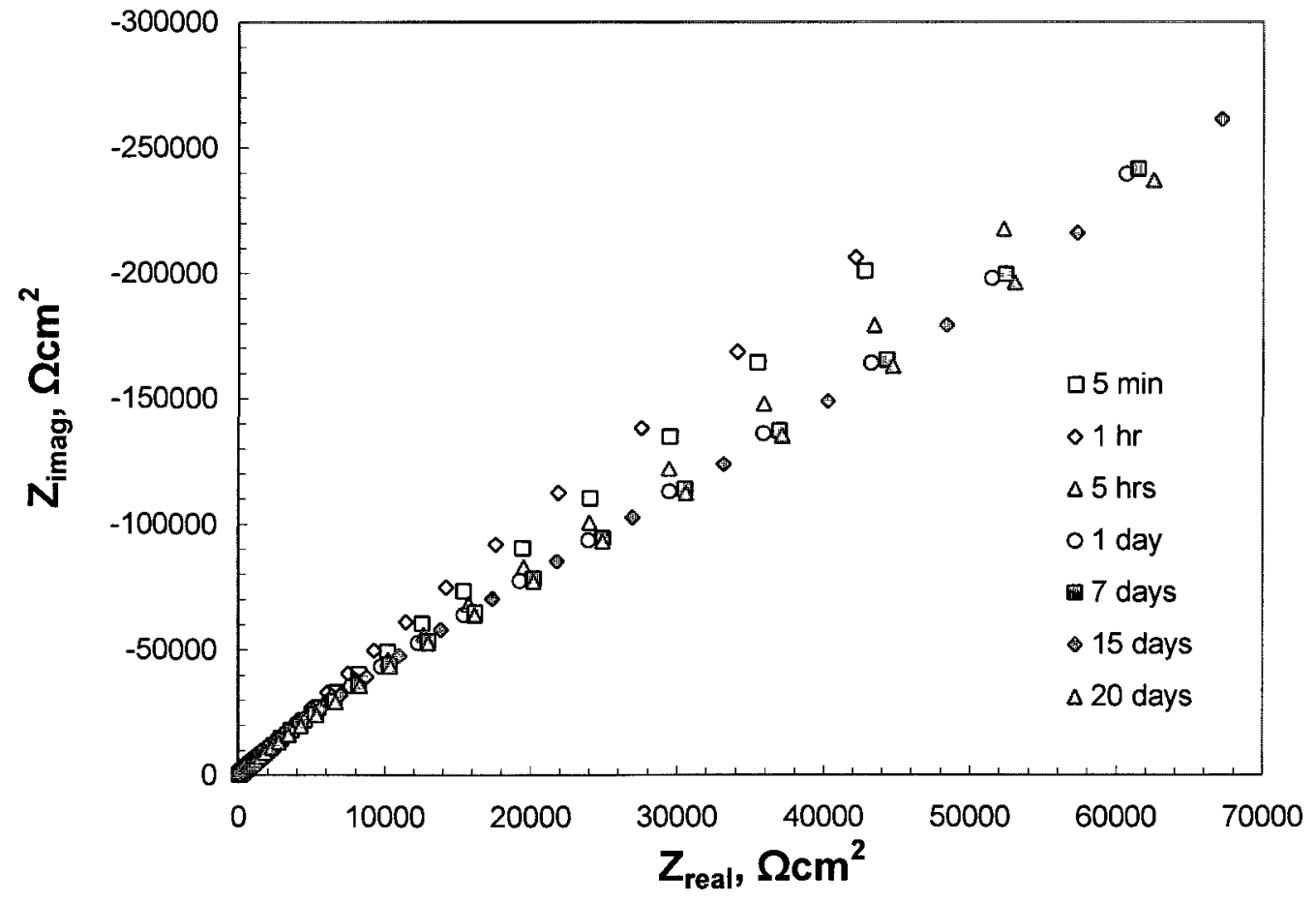

(b) Nyquist plots of PMMA EB TiN/SS

Figure 4-36 to be continued 


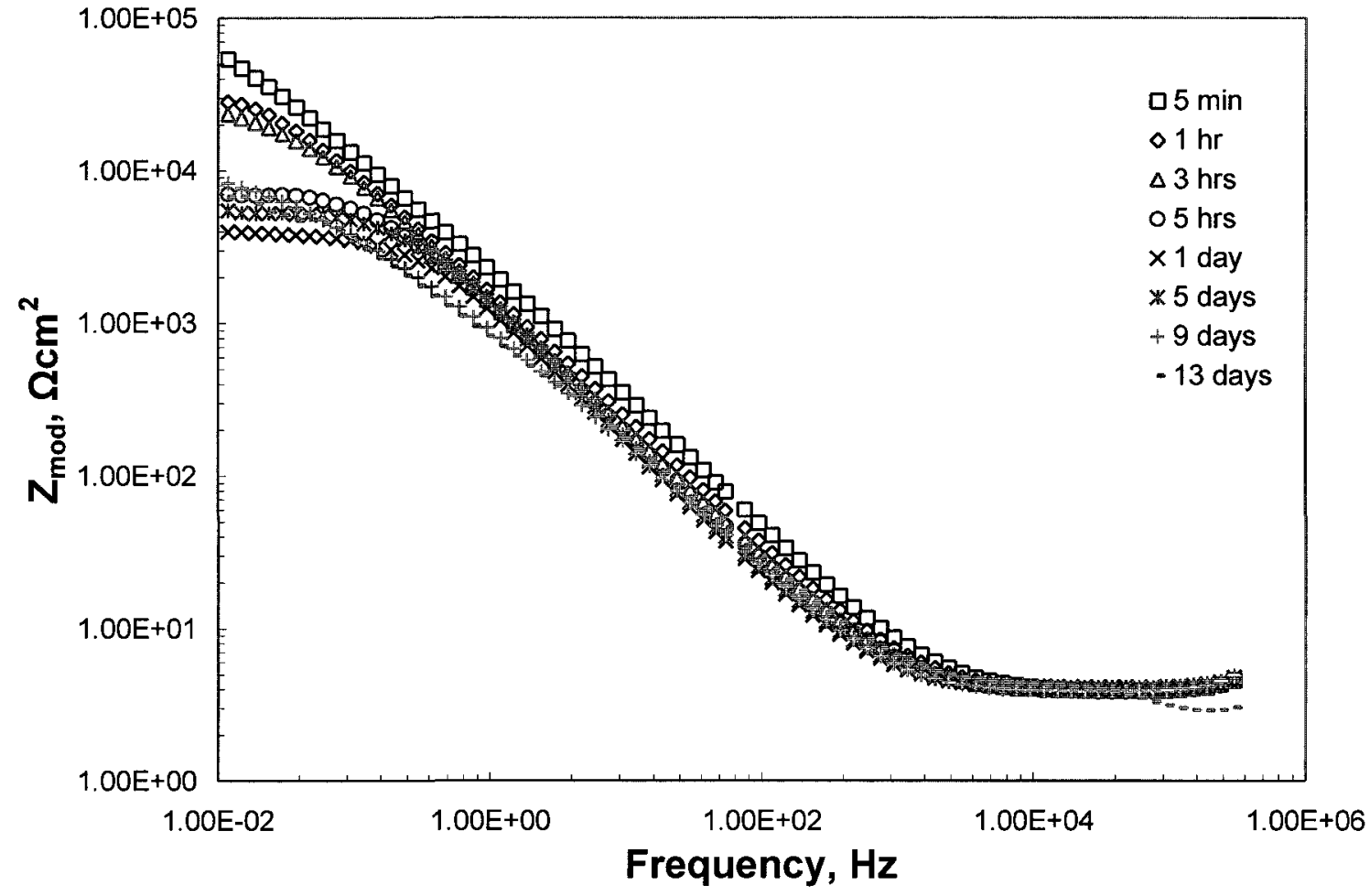

(c) Bode modulus plots of EB TiN/SS

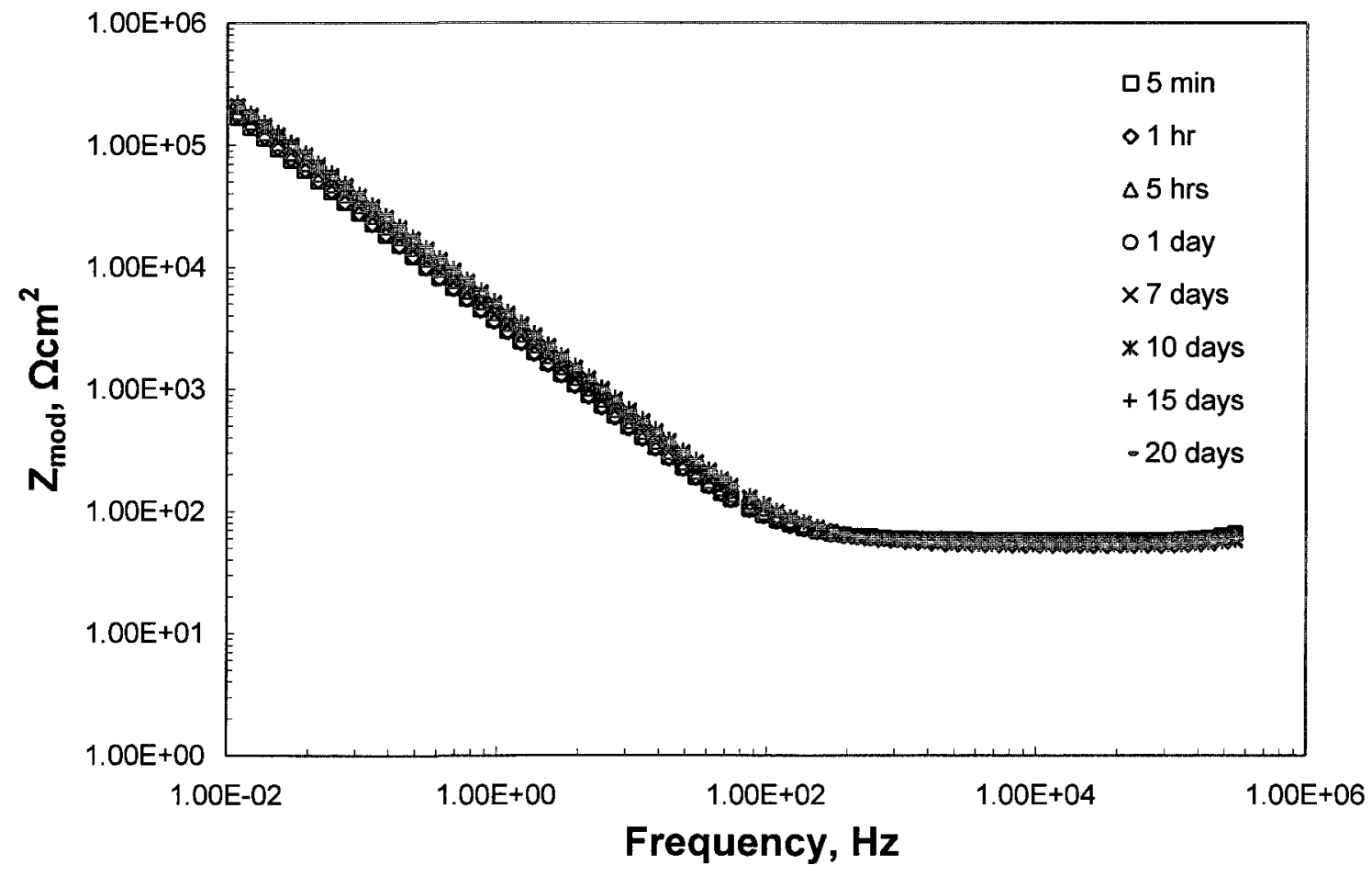

(d) Bode modulus plots of PMMA EB TiN/SS

Figure 4-36 to be continued 


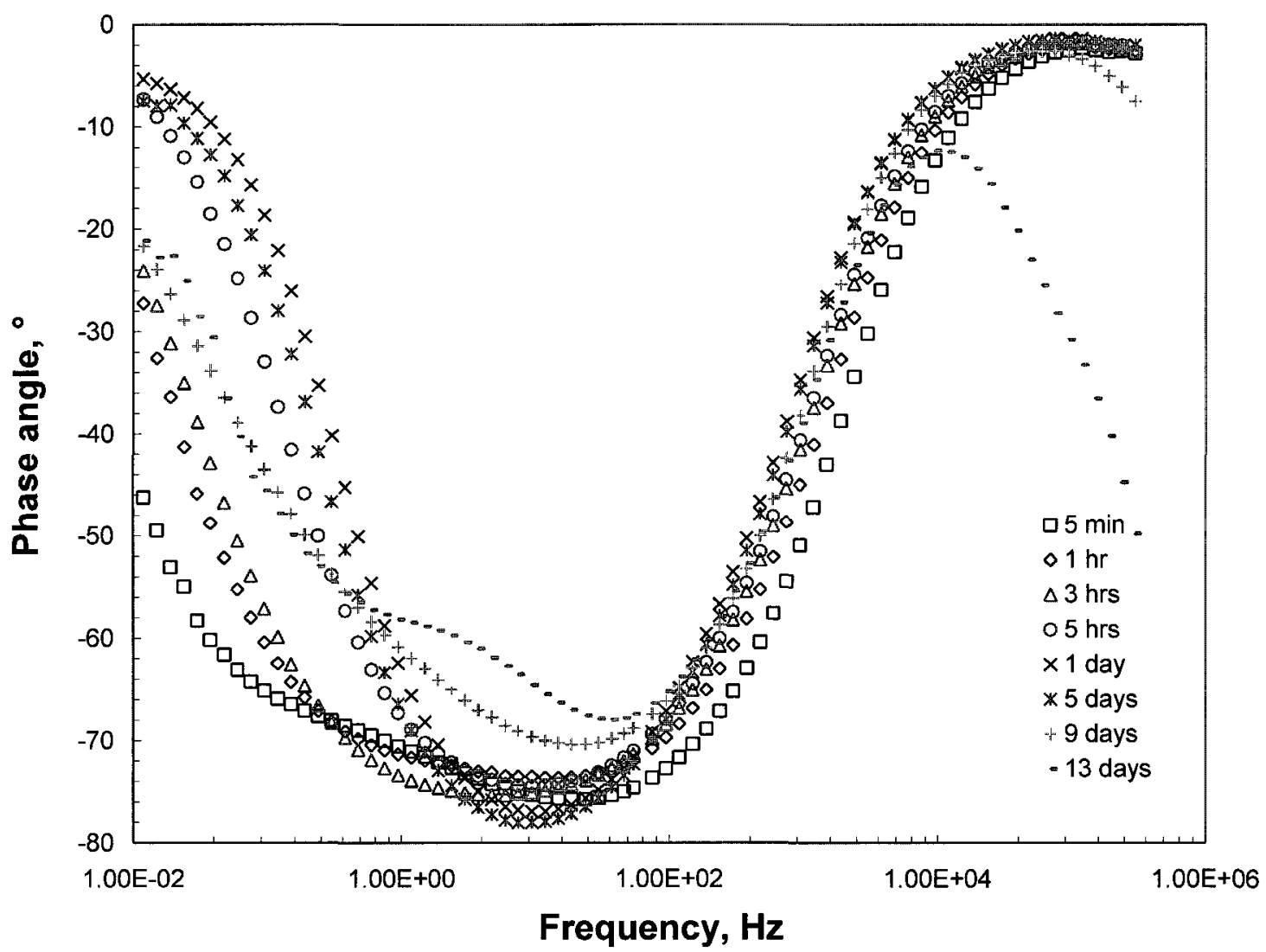

(e) Bode phase plots of EB TiN/SS

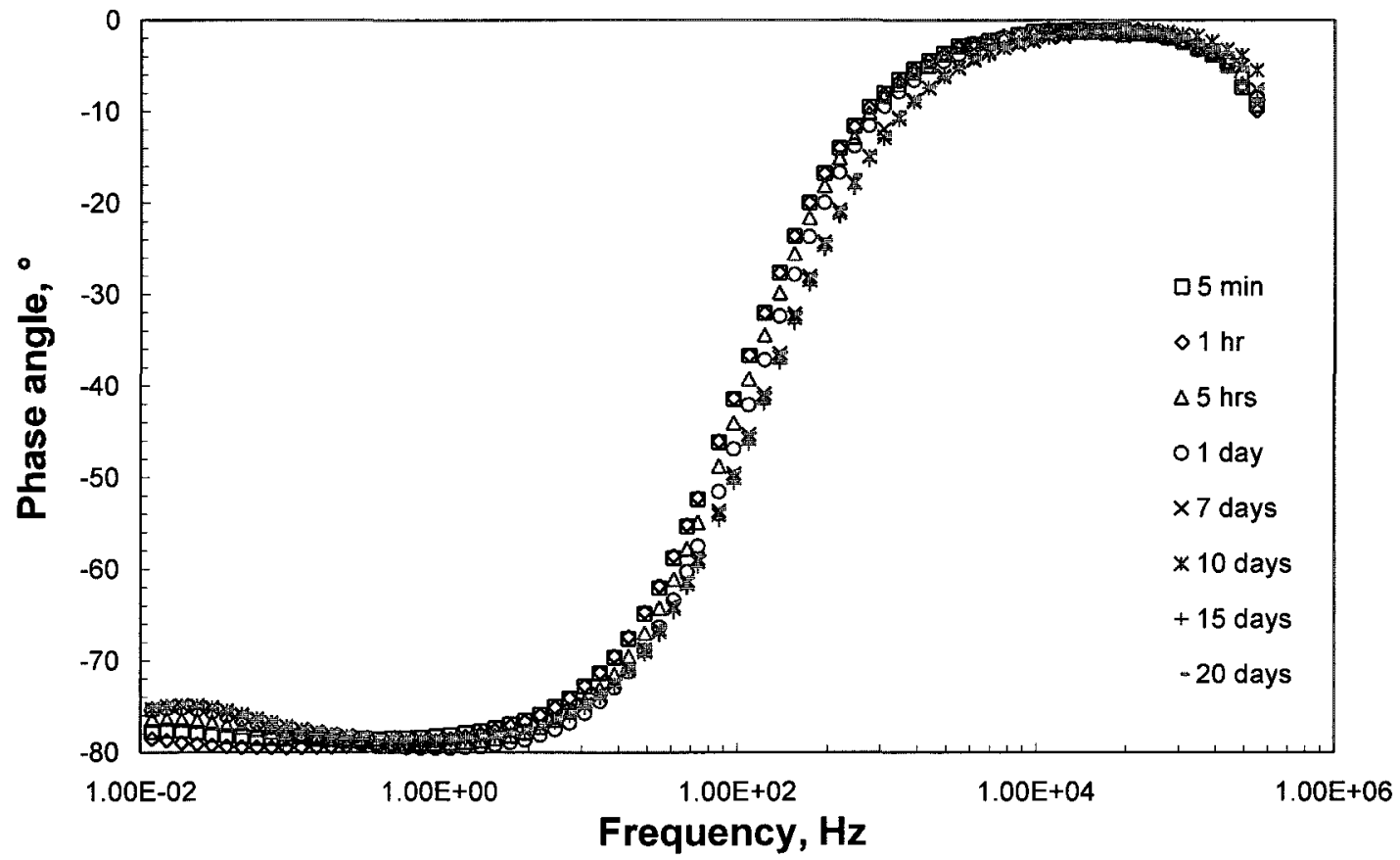

(f) Bode phase plots of PMMA-treated EB TiN/SS

Figure 4-36 EIS spectra of EB TiN/SS coating systems. 
The minimum phase angles of the PMMA-treated EB TiN/SS sample remained unchanged at around $-78^{\circ}$ during the whole immersion period (Figure 4-35 f), indicating a stable and homogeneous surface characteristic as the result of PMMA effectively clogging the coating pores, and therefore protecting the coating surface from localized corrosion damage. The Bode plots showed that the PMMA-treated TiN/SS sample demonstrated a relatively stable surface homogenity (Figure 4-36 f) and impedance modulus (Figure 4-36 d) as compared with those for the as-deposited TiN/SS coatings (Figure 4-36 $\mathrm{c}$ and e). This indicated there is a good correlation between the coating surface condition (e.g., porosity, defect, roughness, etc.) and coating corrosion behaviour, and modifying the coating surface condition (such as a PMMA treatment) would improve the corrosion behaviour.

For the CA TiN/SS coating system, both the as-deposited and PMMA-treated samples demonstrated a straight-line feature in the low frequency region in Nyquist plots (Figure 4-37 a, b), indicating a diffusion dominated corrosion processes. The impendence values for the as-deposited sample decreased from 184 to $72 \mathrm{k} \Omega \mathrm{cm}^{2}$ (Figure 4-37 c), indicating a degradation in corrosion resistance during the two days of immersion, whereas the impedance for the 17-4PH steel increased in five days of immersion (Figure 4-35 d). Like the EB TiN/SS coating, this long term degradation in corrosion resistance was also due to the presence of defects in the coating. The values in impedance for the PMMAtreated sample increased from $82 \mathrm{k} \Omega \mathrm{cm}^{2}$, and stayed around $120 \mathrm{k} \Omega \mathrm{cm}^{2}$ during seven days of immersion (Figure 4-37 d), showing a similar trend to that for the 17-4PH steel, and indicating an improvement in corrosion resistance unlike the as-deposited coating. 


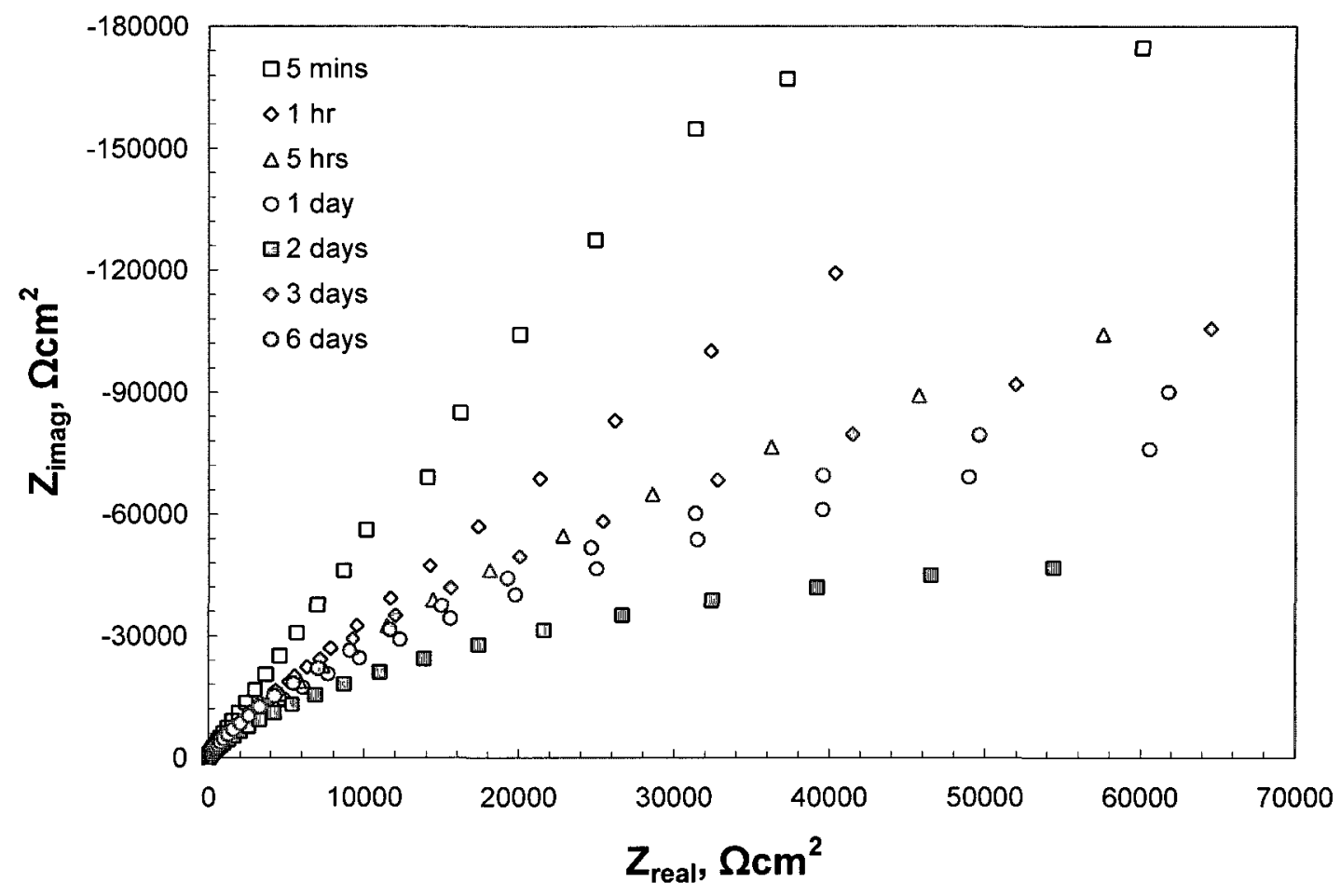

(a) Nyquist plots of CA TiN/SS

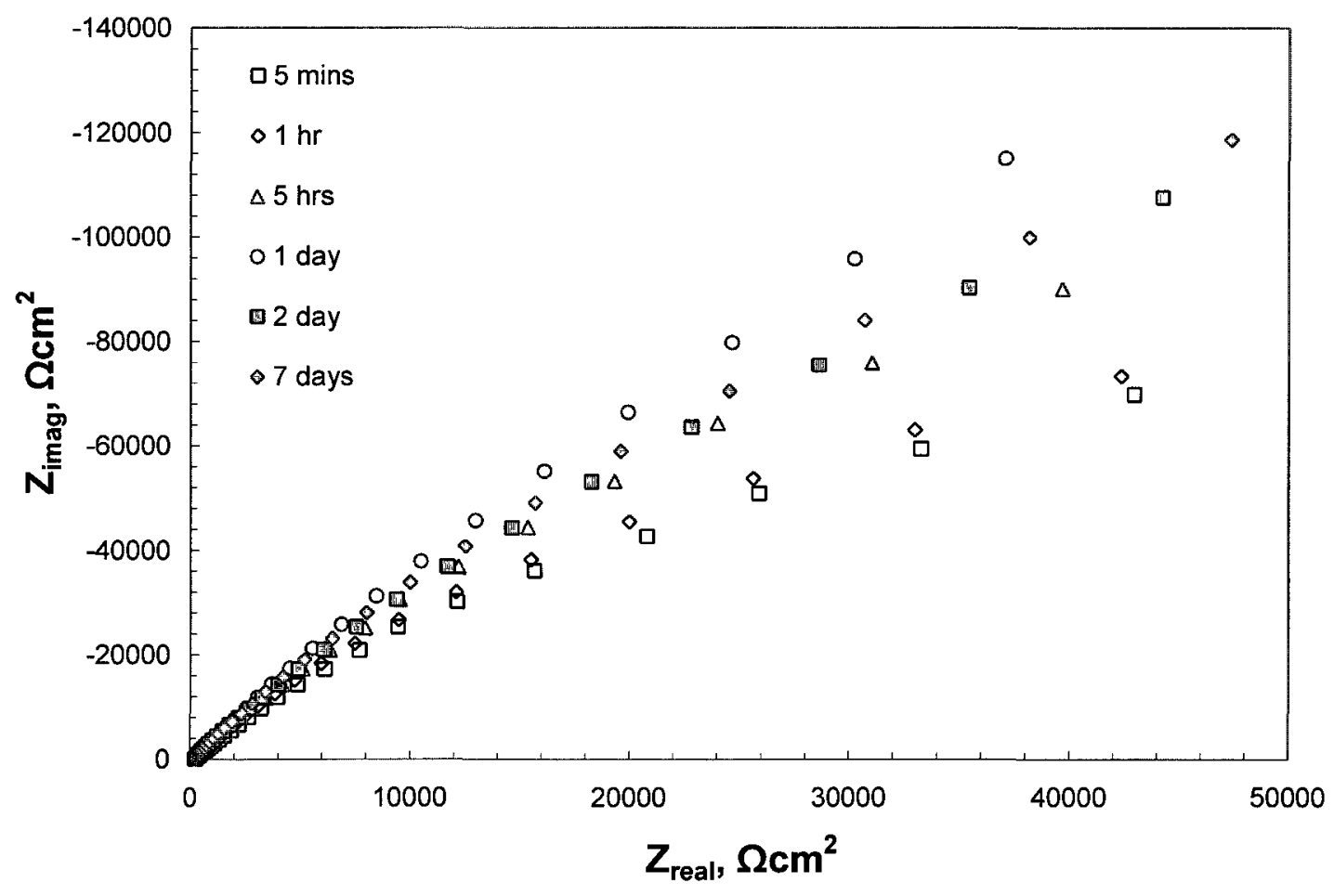

(b) Nyquist plots of PMMA CA TiN/SS

Figure 4-37 to be continued 


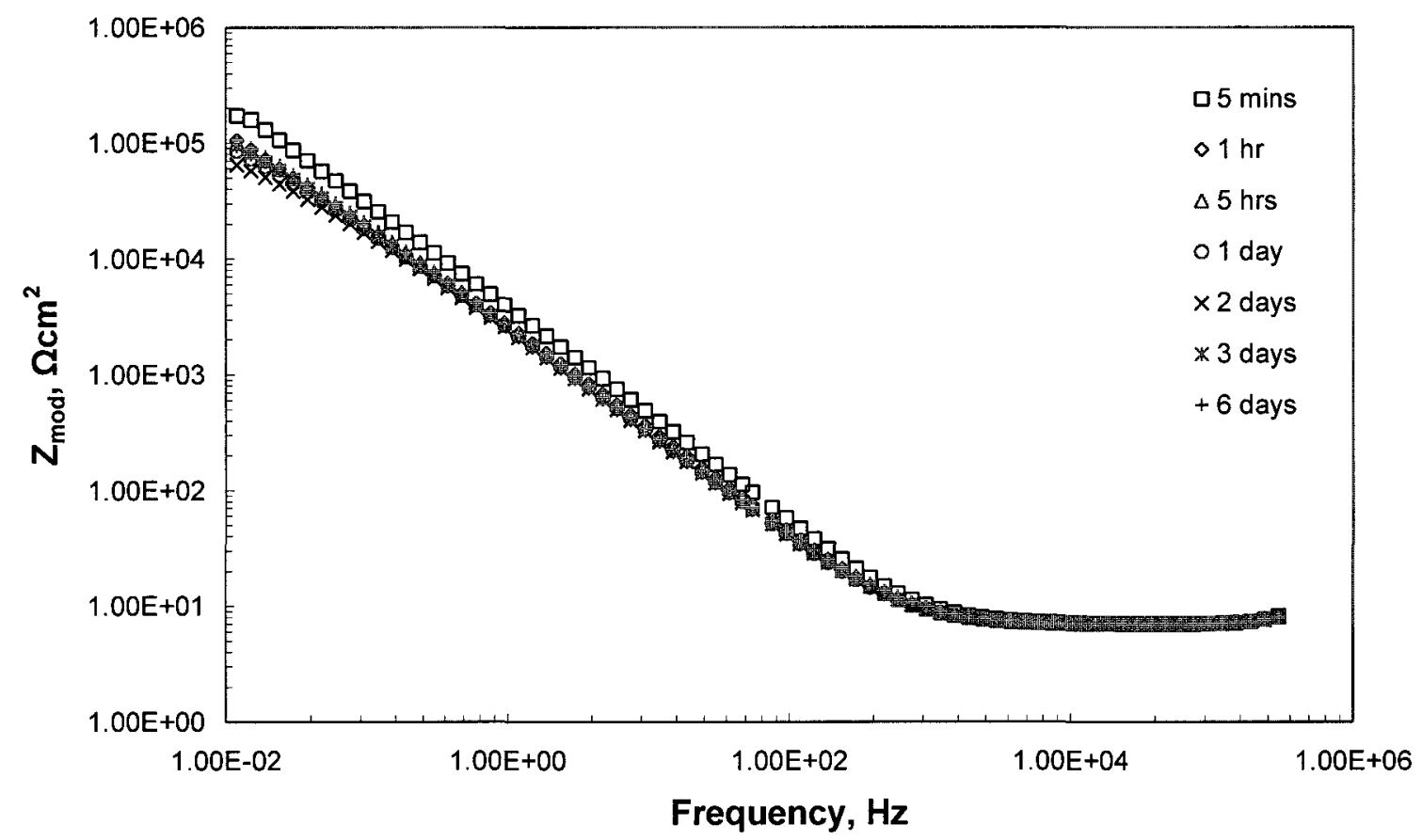

(c) Bode modulus plots of CA TiN/SS

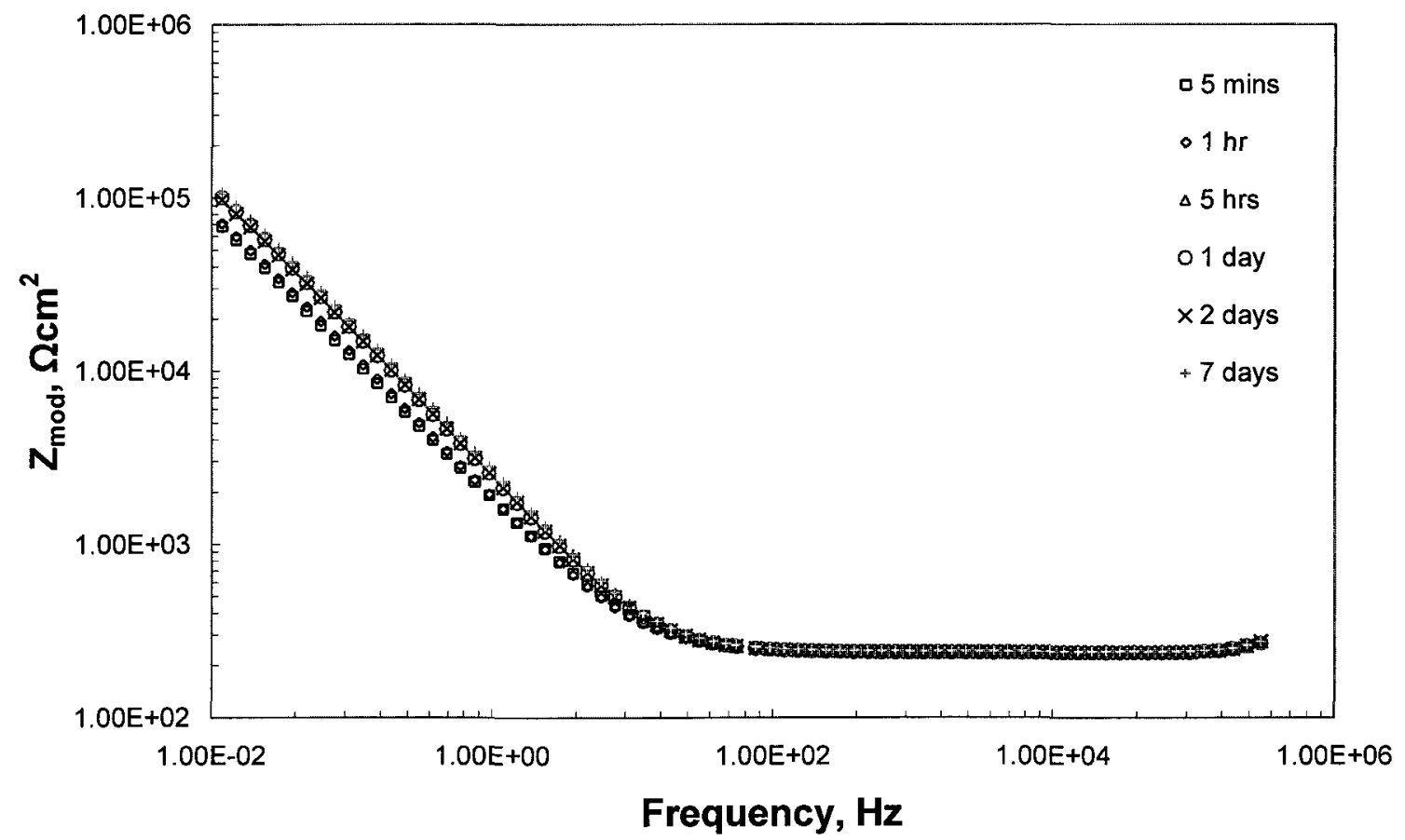

(d) Bode modulus plots of PMMA CA TiN/SS

Figure 4-37 to be continued 


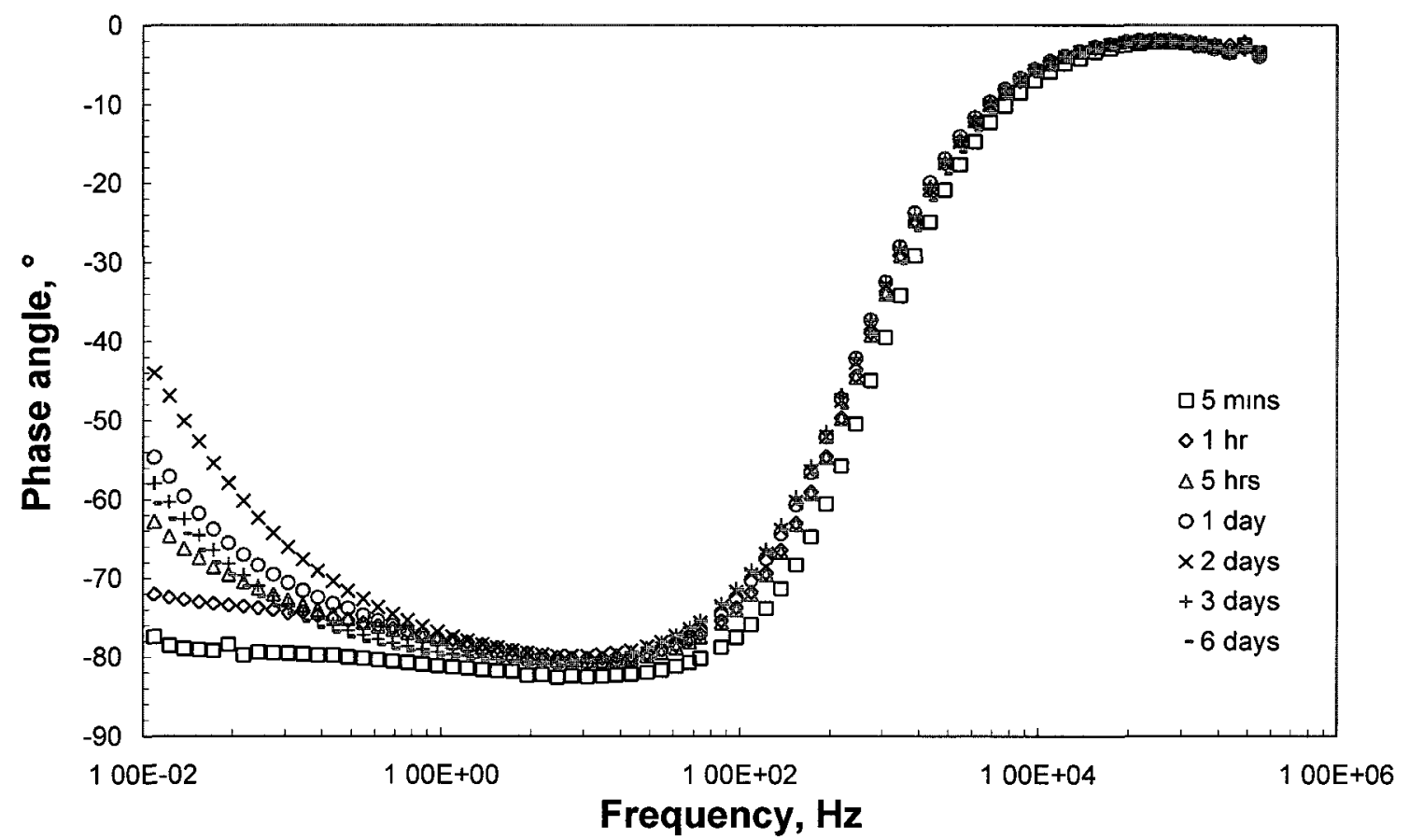

(e) Bode phase plots of CA TiN/SS

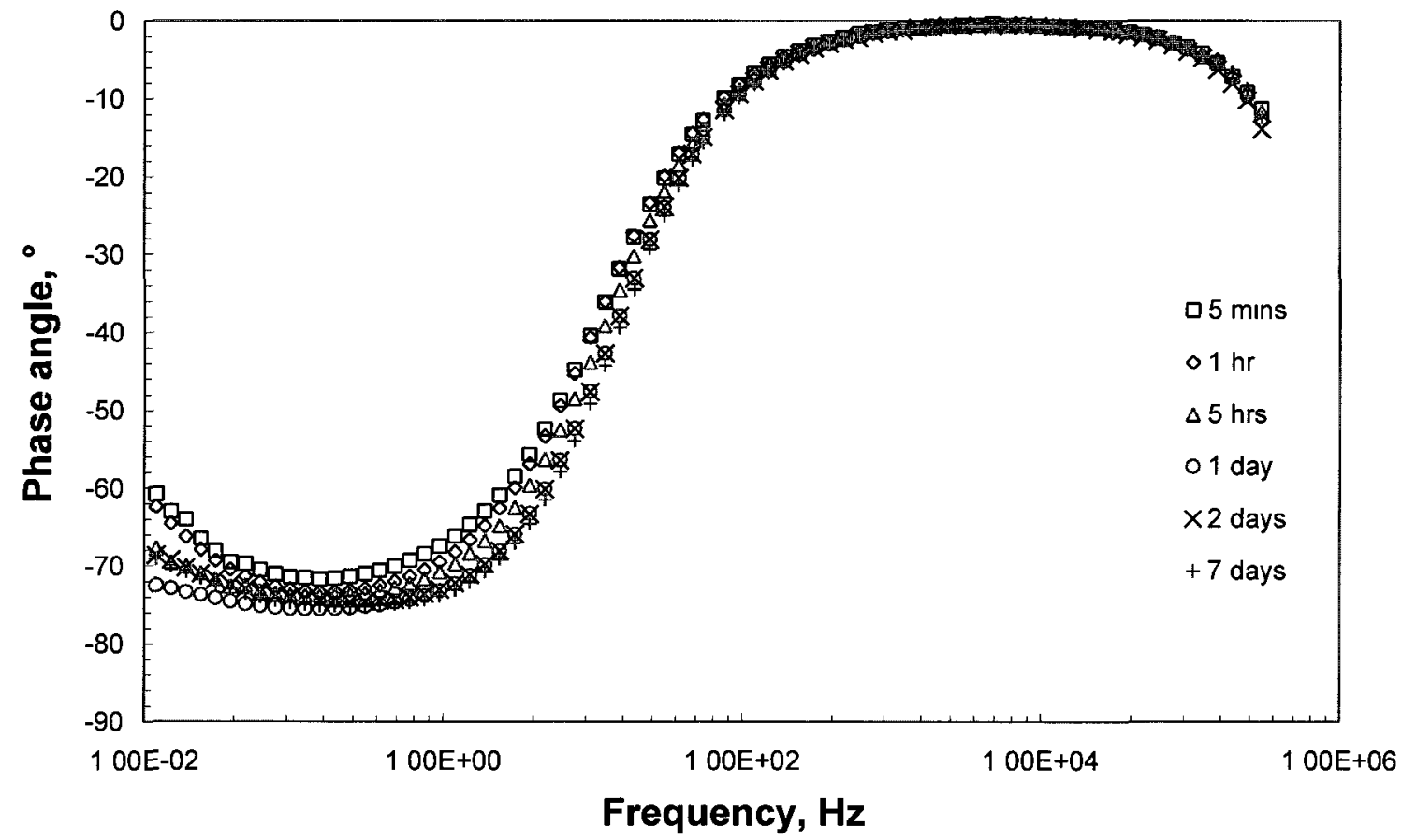

(f) Bode phase plots of PMMA CA TiN/SS

Figure 4-37 EIS spectra of CA TiN/SS coating systems. 
This again provided evidence to prove PMMA-treated coating systems had long term anti-corrosion stability. In the Bode phase plots, the minimum phase angles of the asdeposited $\mathrm{CA}$ TiN/SS sample $\left(-82^{\circ}\right.$ to $\left.-78^{\circ}\right)$ (Figure 4-37 e) remained relatively stable as compared with those of EB TiN/SS $\left(-78^{\circ}\right.$ to $\left.-66^{\circ}\right)($ Figure 4-36 e). This revealed its more stable surface homogeneity during the immersion period. The minimum phase angles of the PMMA-treated CA TiN/SS sample $\left(-76^{\circ}\right.$ to $\left.-72^{\circ}\right)($ Figure $4-37 \mathrm{f})$ indicated its similar inhomogeneous surface characteristics relative to those of the PMMA-treated EB TiN/SS sample (around $-78^{\circ}$ )(Figure 4-36 f).

In the Nyquist plot, the semicircles for the as-deposited CA TiN/MS sample indicated typical charge-transfer corrosion (Figure 4-38 a). The impedance arcs diminished rapidly with immersion time (Figure 4-38 a), which can be confirmed in the Bode modulus plots (Figure 4-38 c), where the impedance at $10 \mathrm{mHz}$ decreased from $\sim 6.5$ to $2.5 \mathrm{k} \Omega \mathrm{cm}^{2}$ (two orders of magnitude higher than those for the mild steel substrate (Figure 4-35 c)), in one day of immersion, indicating a rapid degradation in corrosion resistance.

The PMMA-treated sample showed circular arcs which grew to straight lines and radii increased with longer immersion time (Figure 4-38 b). Impedance values at $10 \mathrm{mHz}$ gradually increased from $\sim 35$ to $70 \mathrm{k} \Omega \mathrm{cm}^{2}$ in two days of immersion (Figure 4-38 d), indicating that PMMA treatment significantly enhanced the corrosion resistance of the coating/substrate system. 


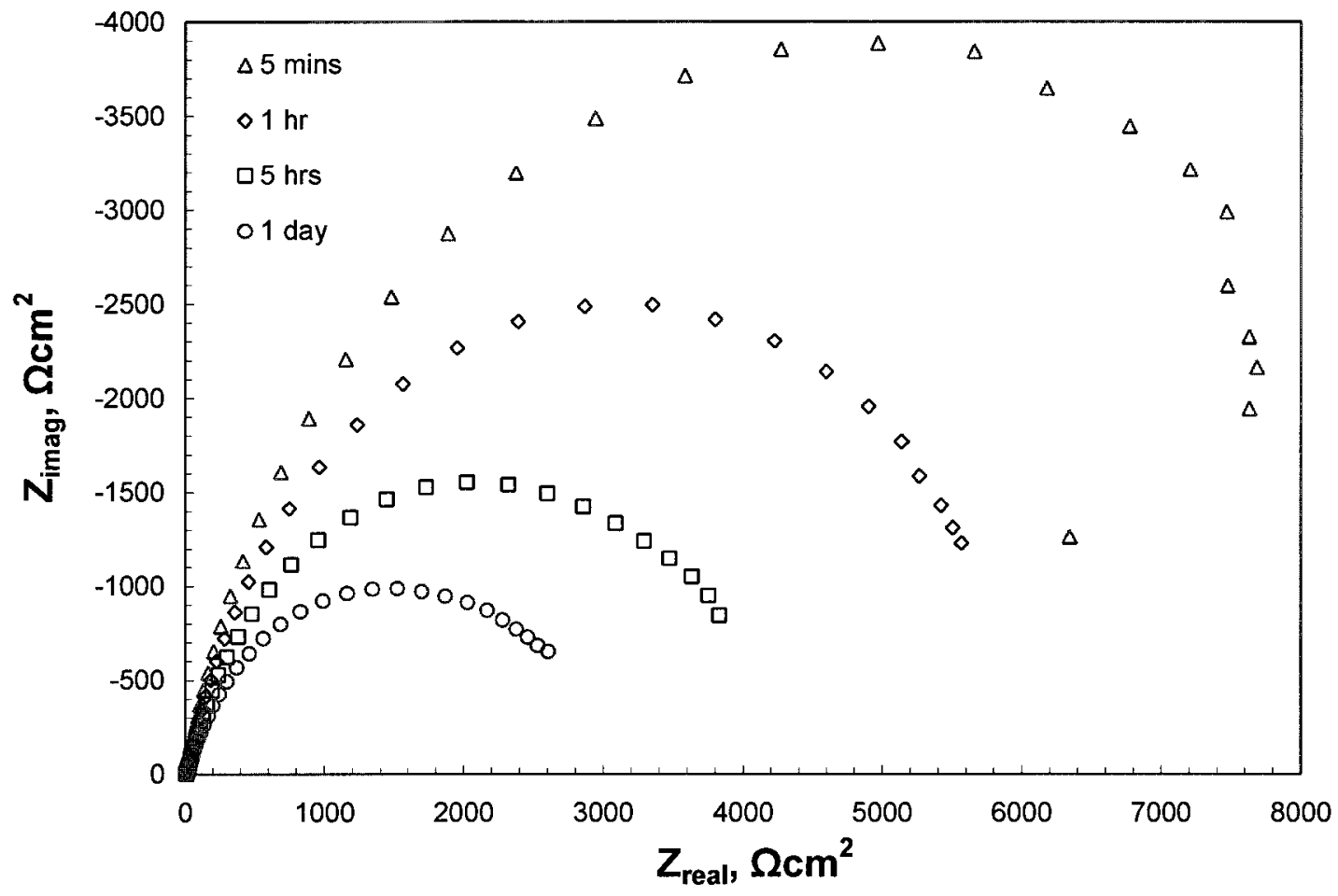

(a) Nyquist plots of CA TiN/MS

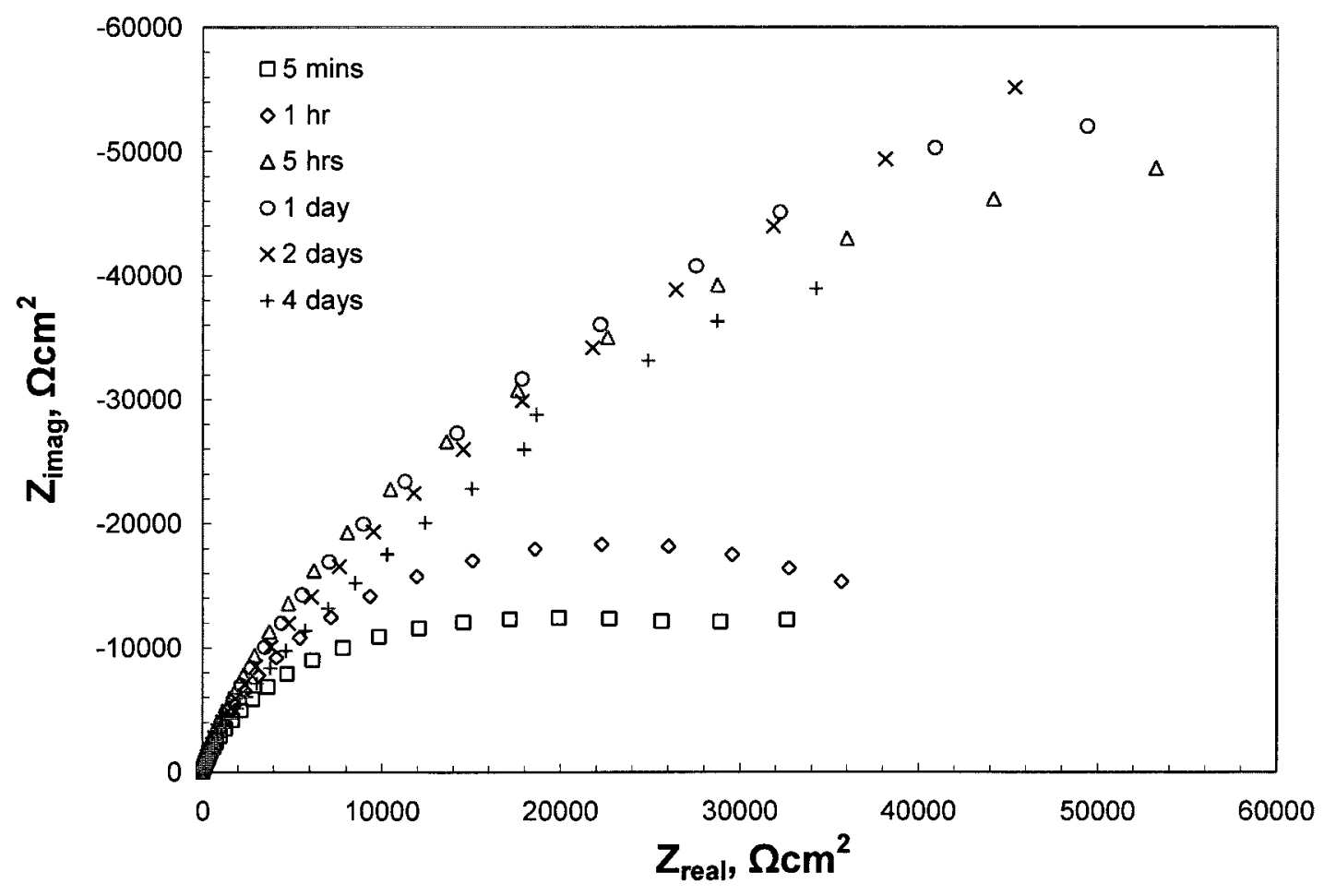

(b) Nyquist plots of PMMA CA TiN/MS

Figure 4-38 to be continued 


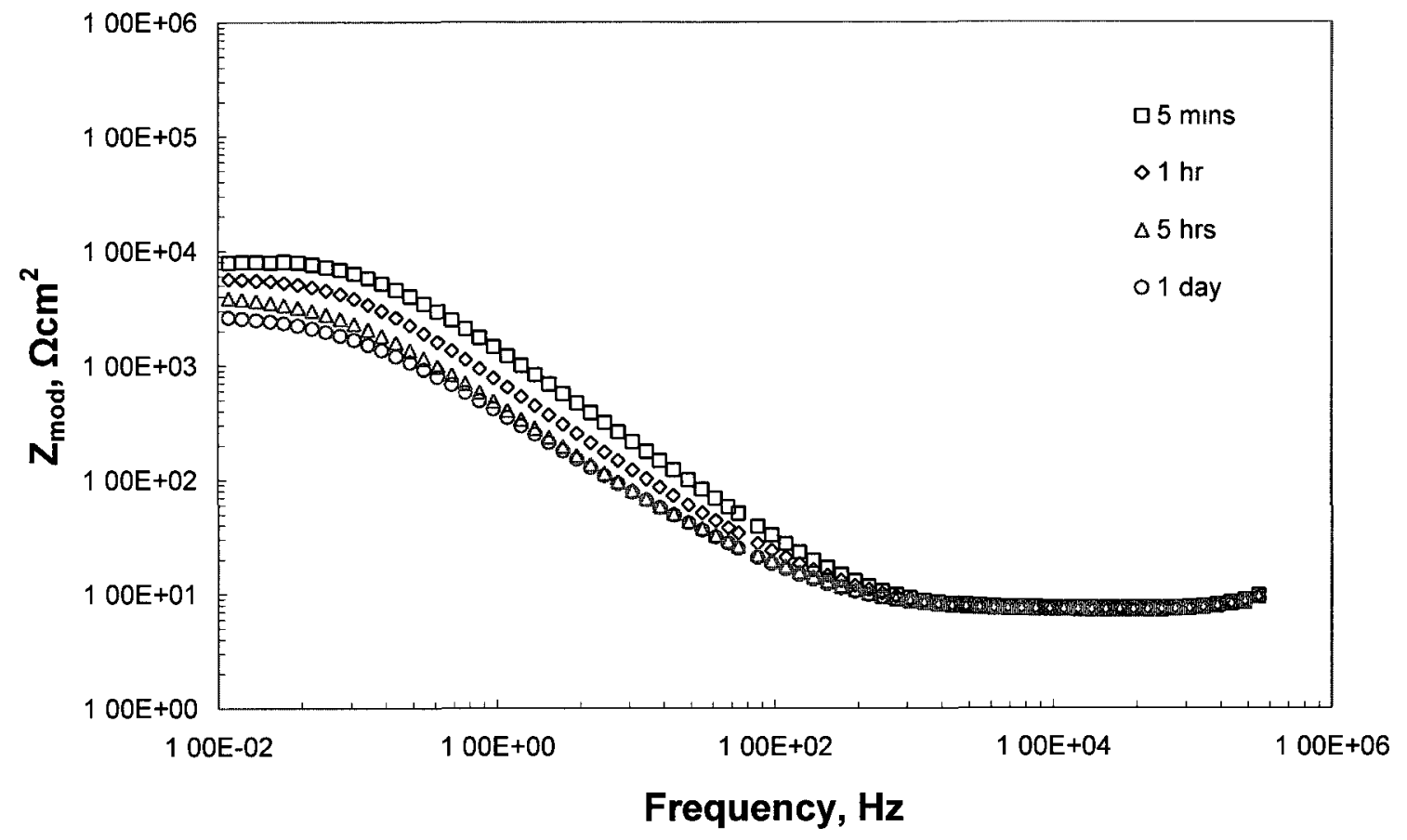

(c) Bode modulus plots of CA TiN/MS

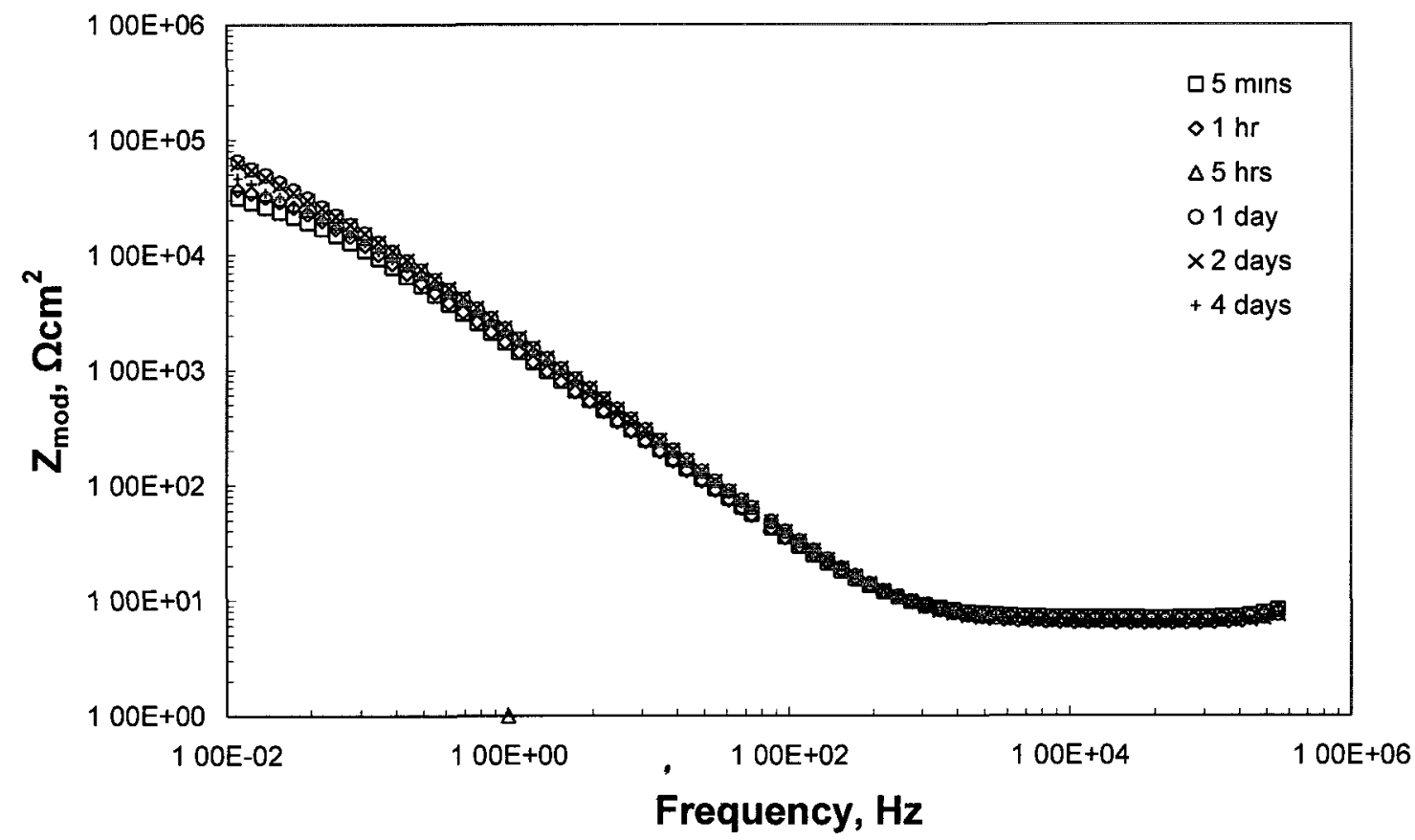

(d) Bode modulus plots of PMMA CA TiN/MS

Figure 4-38 to be continued 


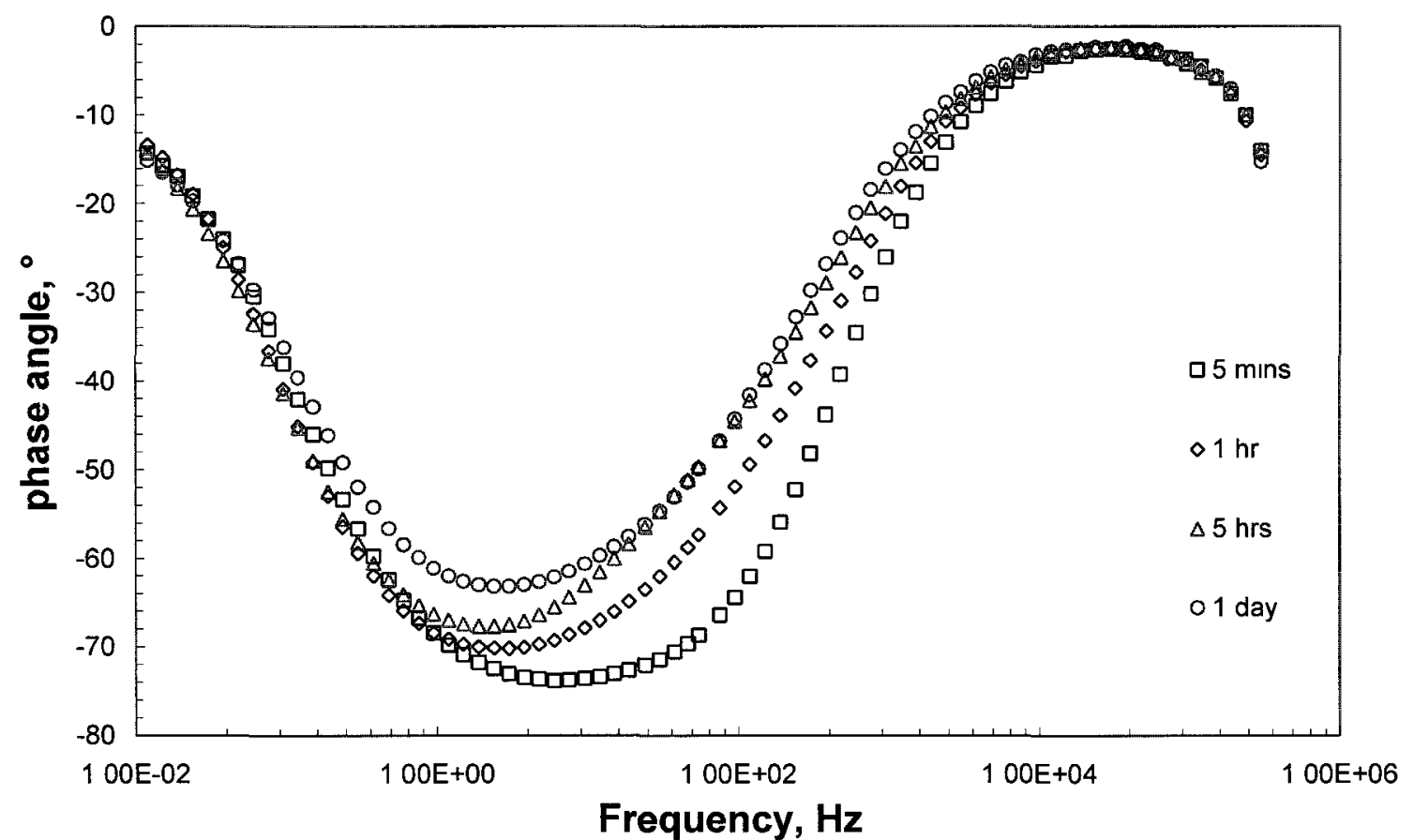

(e) Bode phase plots of CA TiN/MS

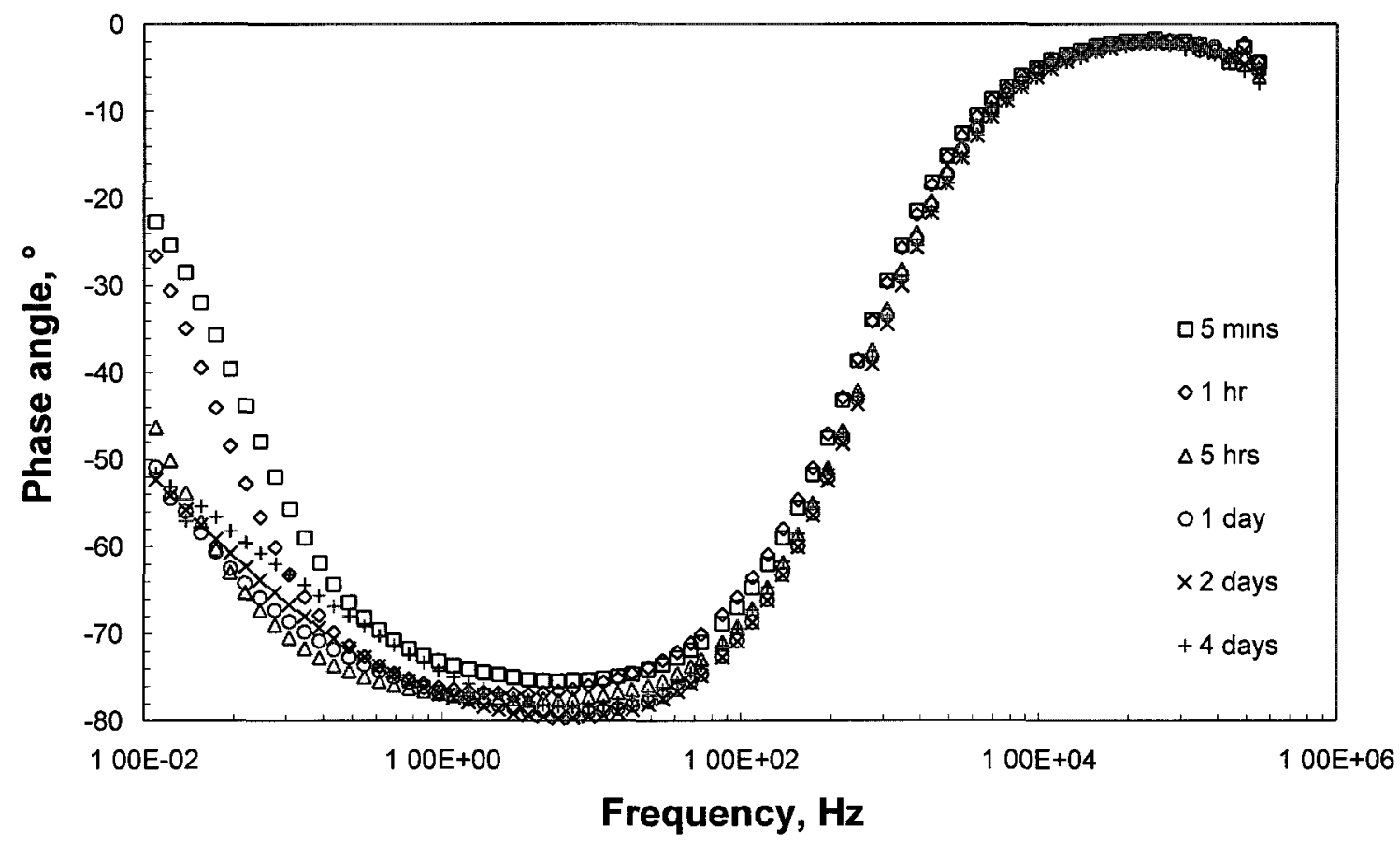

(f) Bode phase plots of PMMA CA TiN/MS

Figure 4-38 EIS spectra of CA TiN/MS coating systems. 
In the Bode phase plots, the lower minimum phase angles of the as-deposited CA TiN/MS sample $\left(-76^{\circ}\right.$ to $\left.-62^{\circ}\right)$ (Figure 4-38 e) revealed its more homogeneous surface when compared with the mild steel substrate $\left(-69^{\circ}\right.$ to $\left.-56^{\circ}\right)$ (Figure 4-35 e). The increasing minimum phase angle (from $-76^{\circ}$ to $-62^{\circ}$ ) with immersion time for the asdeposited CA TiN/MS sample indicated that its surface became more inhomogeneous during the immersion; this was due to the accelerated corrosion process through the coating pores to the mild steel substrate, which therefore resulted in localized changes in capacitance and charge transfer resistance at the pores.

The minimum phase angles of the PMMA-treated CA TiN/MS sample stayed around $76^{\circ}$ to $-80^{\circ}$ (Figure 4-38 f), revealing a relatively stable homogeneous surface feature as compared with its as-deposited counterpart. This surface homogeneity is believed to be associated with the PMMA clogging the coating pores, and therefore experiencing less localized corrosion at the pore sites.

It can be observed that, while the impedance values of the as-deposited samples decreased with immersion time, all the PMMA-treated samples demonstrated initial increases in impedance, and then reached a steady state with the elapse of immersion time, because the PMMA seals the through-coating defects that act as the direct access paths for corrosive media, therefore limited mass transfer and ion current throughout the coating.

Of the deposition techniques (EB and $\mathrm{CA}$ ) and the substrates (MS and SS), the CA TiN/SS coatings demonstrated the best anti-corrosion behaviour in term of diffusion 
impedance, while the CA TiN/MS performed the worst. Clearly the coating microstructure and substrate properties affected the corrosion performance of the coated systems. On one hand, although the CA coatings possessed much higher defect densities than the EB coatings (Table 4-4), the high bias voltages applied in the cathodic arc process increased the surface diffusivity of the deposited species and hence promoted the formation of a coating structure with fewer through-coating voids $[32,98,349]$. On the other hand, the two types of substrate steels exhibited different corrosion mechanisms. Corrosion on a mild steel substrate usually takes a form of anodic dissolution that would generate porous corrosion products at the electrolyte-substrate interface. On a stainless steel substrate, a dense and high dielectric constant passive film isolates the bulk from the corrosive media. Therefore, the corrosion process on the $17-4 \mathrm{PH}$ steel based coating systems were diffusion controlled and the coating system demonstrated much higher resistance to corrosion as compared with coating systems deposited on the mild steel substrate.

The PMMA-treated EB and CA TiN/SS coating systems demonstrated similar corrosion responses; whereas, the PMMA-treated CA TiN/MS coating system performed worse than the PMMA-treated CA TiN/SS coating system. This illustrated a combined effect of coating microstructure, substrate properties, and post-deposition treatments on corrosion behaviours. 


\section{CrN Based Coating Systems}

The straight-line Nyquist plots (Figure 4-39 a to d) indicate diffusion dominated corrosion processes for the four $\mathrm{CrN}$ based coatings, particularly at the electrolytesubstrate interfaces (low frequencies response in the upper-right corner). This was attributed to the factors of both coating and substrate. For the $\mathrm{CrN}$ based coatings, the dense microstructure restricted the penetration of reactive species through coatings. In addition, in the $\mathrm{CrN}$ based coatings, the formation of dense chromium oxides would also increase the electrical impedance. As a result, the electrolyte concentration gradient is high crossing the coating structure [346]; therefore the mass transfers in electrolyte through coatings to substrates were diffusion dominated due to the presence of the Nernst diffusion layer that resulted from a higher electrolyte concentration gradient (which will be discussed in a later section).

For the 17-4PH steel substrates, the electrochemical mass transfers take the form of diffusion through the electrolyte-substrate interfaces. The impedance modulus values for the $\mathrm{CrN}$ and $\mathrm{CrSiCN}(1)$ coatings were found to remain on the order of $100 \mathrm{k} \Omega \mathrm{cm}^{2}$, with the $\mathrm{CrSiCN}(1)$ coating exhibiting a better stability in impedance modulus than the $\mathrm{CrN}$ sample during the seven days of immersion (Figure 4-39 e, f); and were slightly higher than that for the bare $17-4 \mathrm{PH}$ steel substrate (increasing from $\sim 50$ to $100 \mathrm{k} \Omega \mathrm{cm}^{2}$ in the five days of immersion) (Figure 4-35 d), demonstrating their stable corrosion resistances, a result of their $(\mathrm{CrN}$ and $\mathrm{CrSiCN}(1))$ fine dense microstructures (with less throughcoating defects, e.g., voids at grain boundaries). With coarse microstructures, the coatings $\mathrm{CrSiCN}(2)$ and $\mathrm{CrSiCN}(3)$ demonstrated very low impedance modulus values (at the 
orders of 0.7 and $0.5 \mathrm{k} \Omega \mathrm{cm}^{2}$, respectively) (Figure 4-39 $\mathrm{g}, \mathrm{h}$ ), which were more than two orders of magnitude lower than those of the $\mathrm{CrN}$ and $\mathrm{CrSiCN}(1)$ coatings; these values were even lower than that of the $17-4 \mathrm{PH}$ steel substrate $\left(\sim 50\right.$ to $\left.100 \mathrm{k} \Omega \mathrm{cm}^{2}\right)$, indicating their poor corrosion resistance.

The observations from the $\mathrm{AC}$ impedance results were consistent with the potentiodynamic polarization measurements (Figure 4-34)(Table 4-14), showing that the porous coating microstructures were responsible for both the short- and long-term inferior corrosion resistance of the $\mathrm{CrSiCN}(2)$ and $\mathrm{CrSiCN}(3)$ coatings. The Bode phase plots showed that the minimum phase angles of the $\mathrm{CrN}$ and $\mathrm{CrSiCN}(1)$ coating systems stayed at $\sim-76^{\circ}$ (Figure 4-39 $\mathrm{i}, \mathrm{j}$ ), indicating their surface homogenity was similar to the 17-4PH steel substrate $\left(-74^{\circ}\right.$ to $\left.-79^{\circ}\right)$ (Figure 4-35 f); whereas minimum phase angles slightly fluctuated between $-76^{\circ}$ and $-73^{\circ}$ (Figure 4-39 $\mathrm{k}$ ), and $-72^{\circ}$ and $-68^{\circ}$, (Figure 439 1), respectively for the $\mathrm{CrSiCN}(2)$ and $\mathrm{CrSiCN}(3)$ coating systems, revealing their relatively inhomogeneous and less stable surface features. 


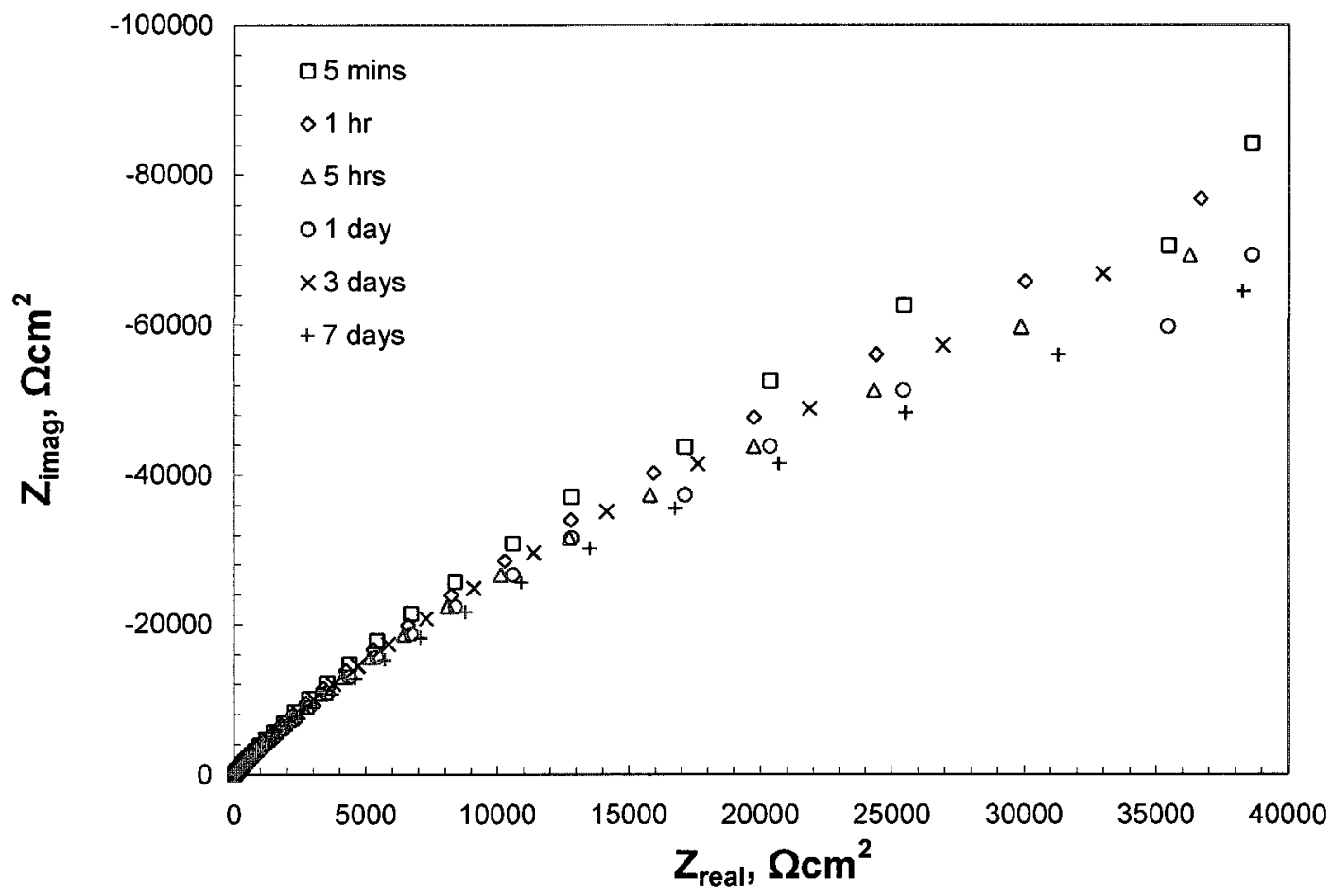

(a) Nyquist plots of $\mathrm{CrN}$

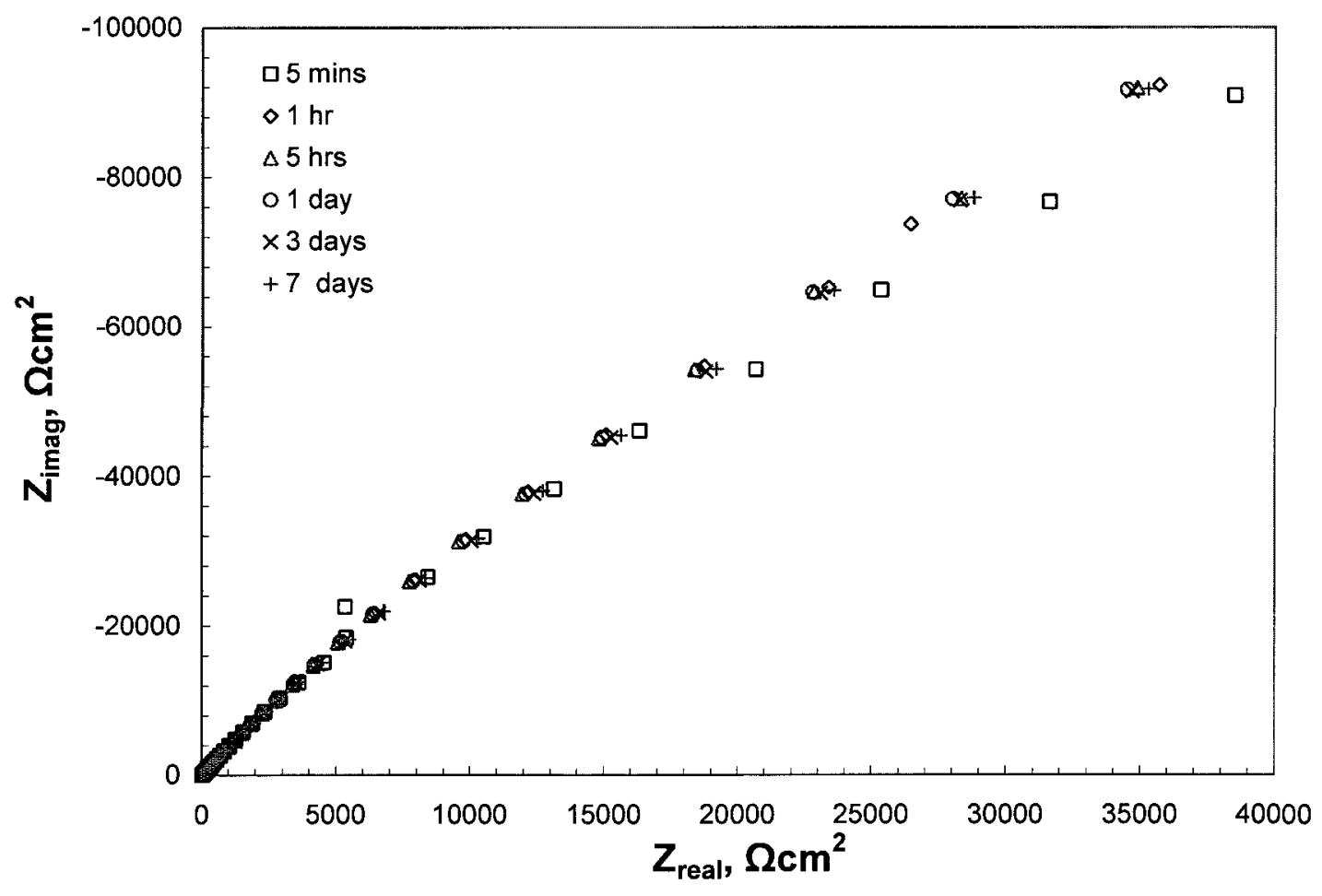

(b) Nyquist plots of CrSiCN(1)

Figure 4-39 to be continued 


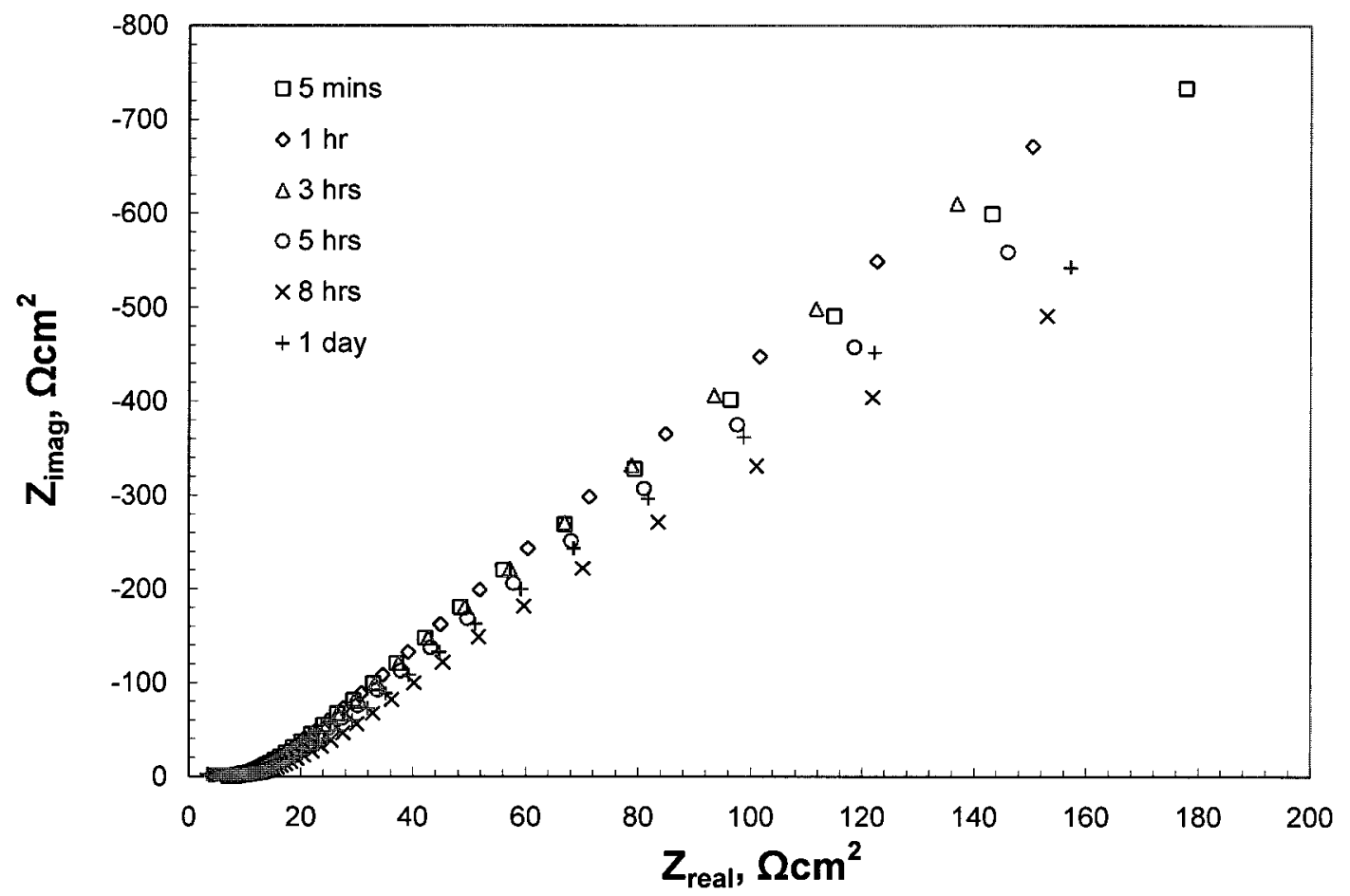

(c) Nyquist plots of $\mathrm{CrSiCN}(2)$

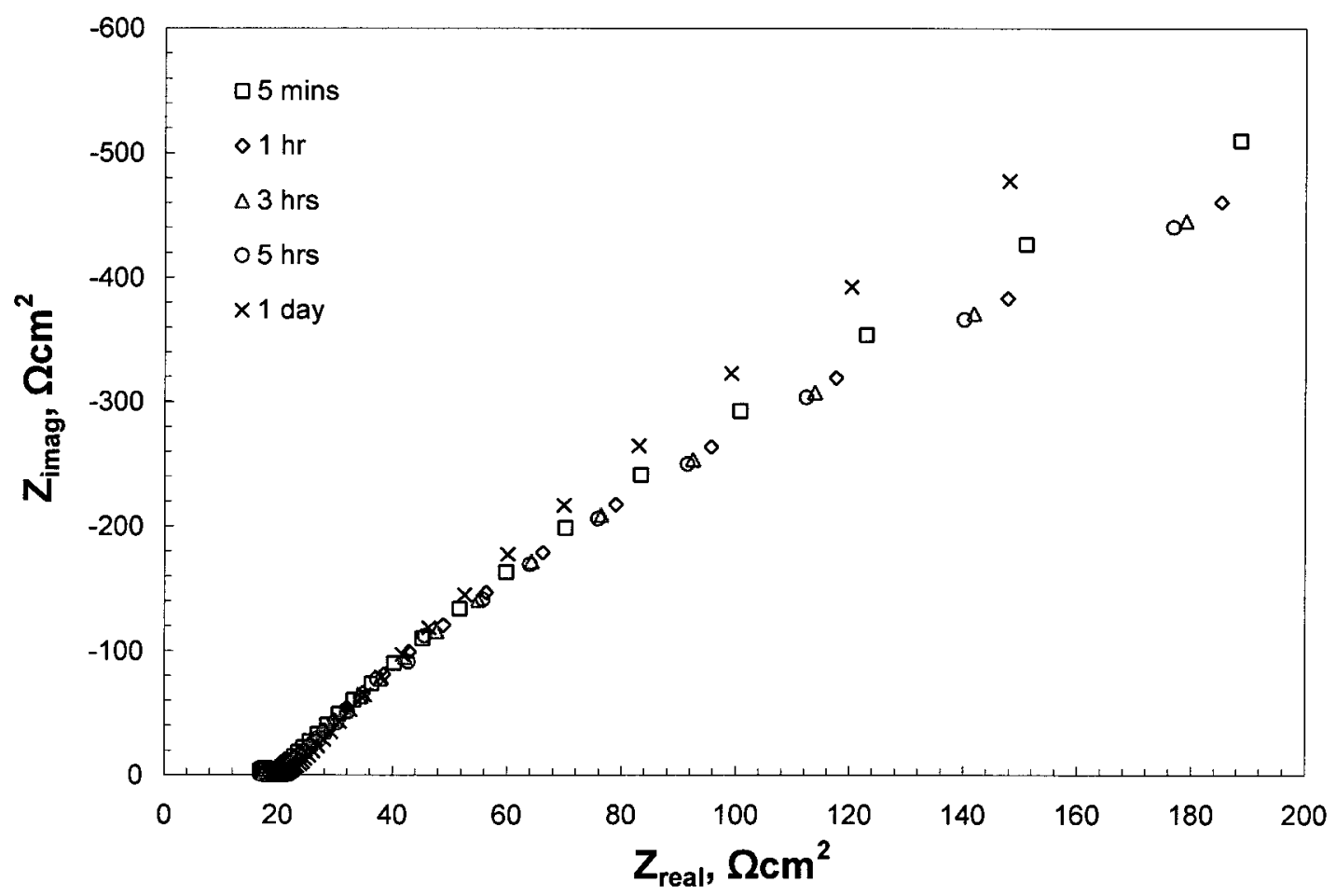

(d) Nyquist plots of $\mathrm{CrSiCN}(3)$

Figure 4-39 to be continued 


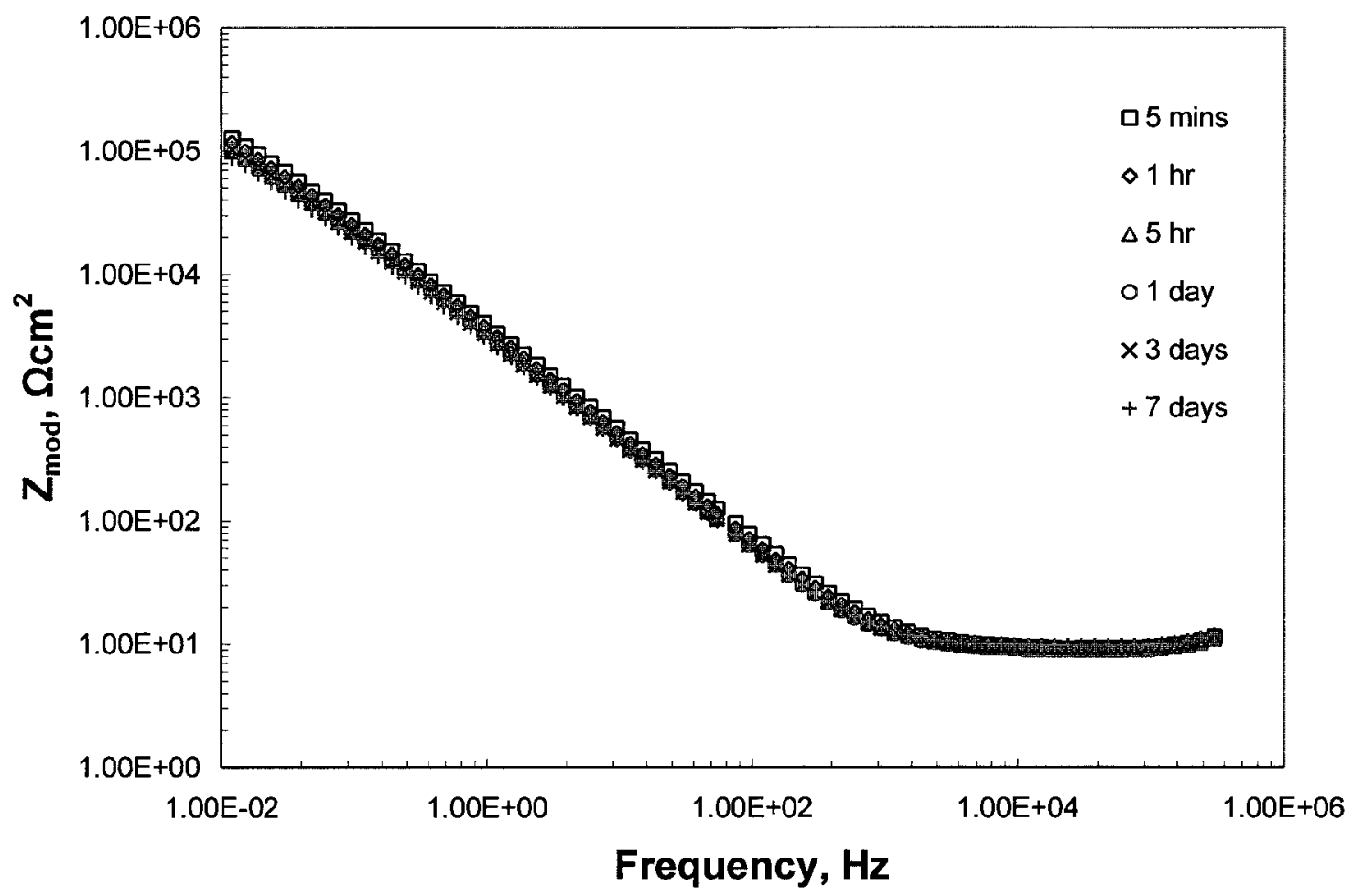

(e) Bode modulus plots of $\mathrm{CrN}$

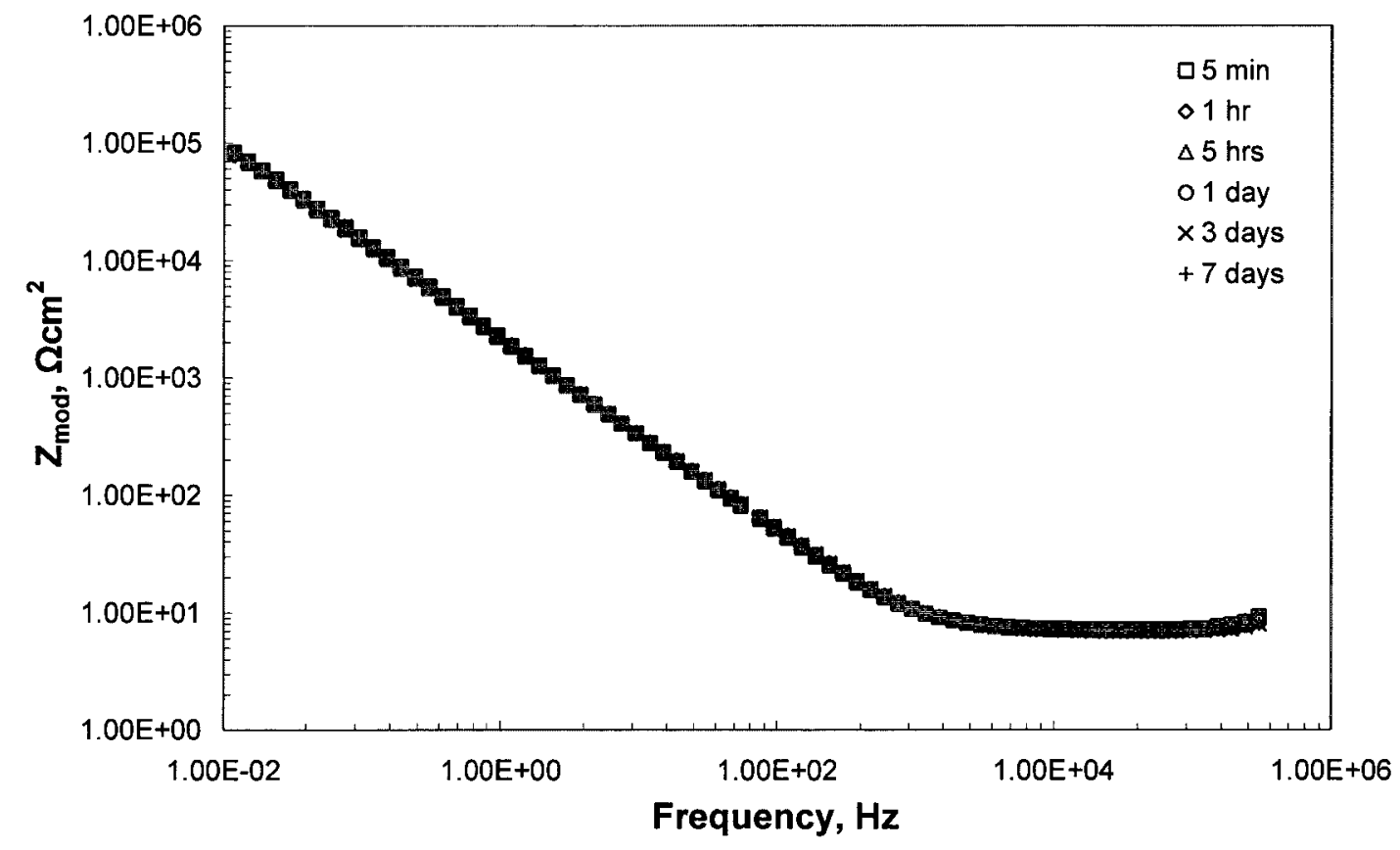

(f) Bode modulus plots of $\mathrm{CrSiCN}(1)$

Figure 4-39 to be continued 


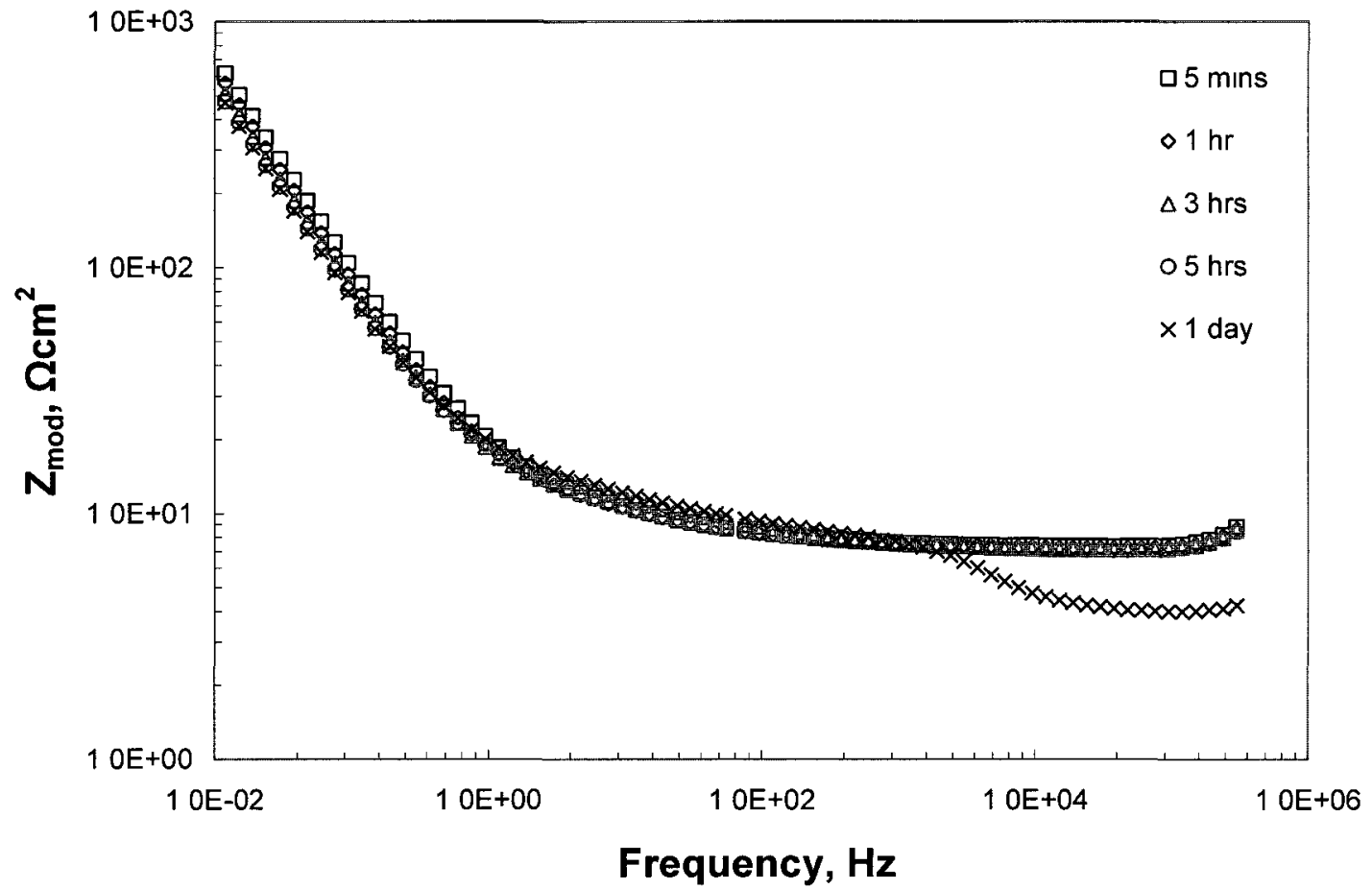

(g) Bode modulus plots of $\mathrm{CrSiCN}(2)$

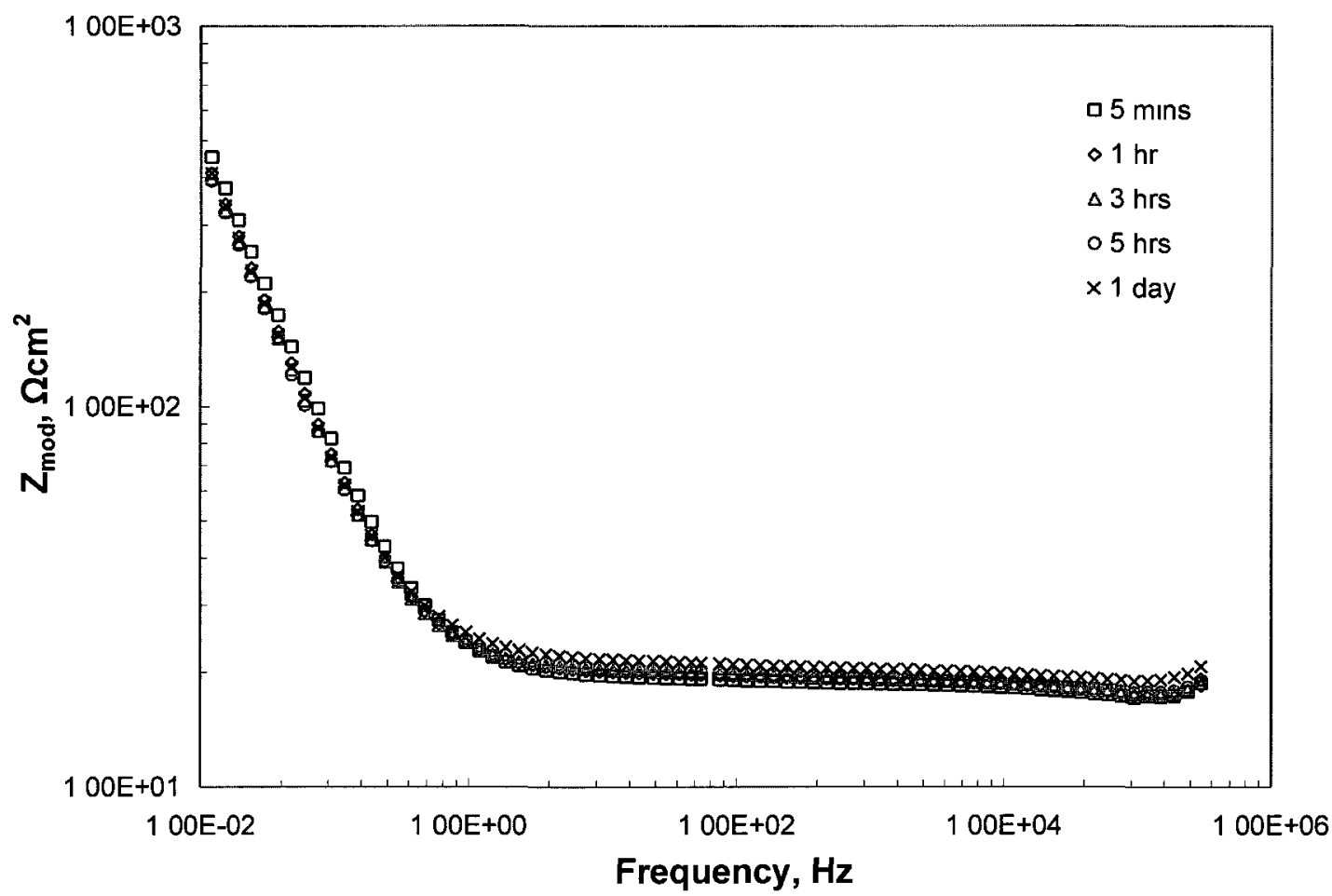

(h) Bode modulus plots of $\mathrm{CrSiCN}$ (3)

Figure 4-39 to be continued 


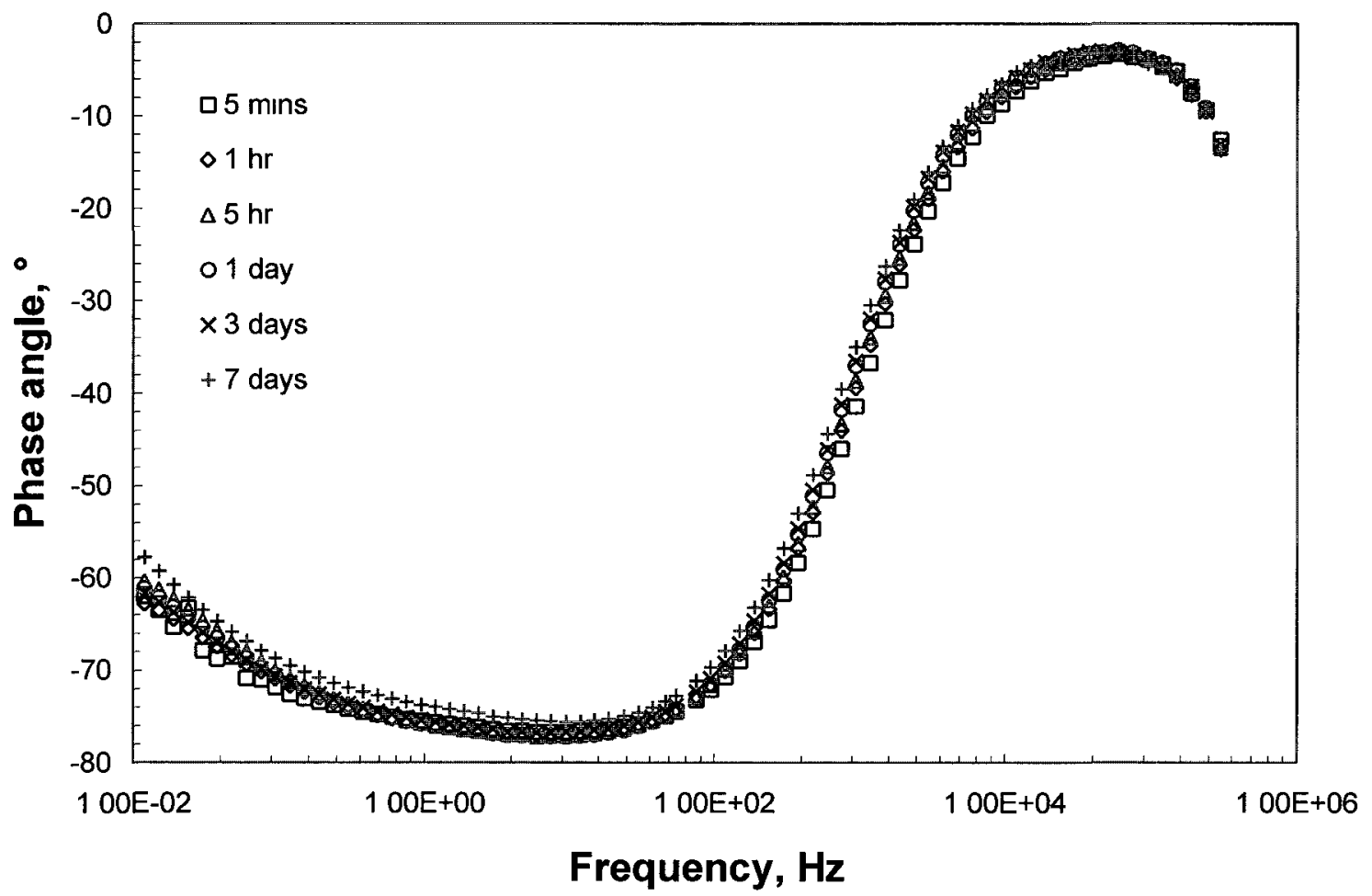

(i) Bode phase plots of $\mathrm{CrN}$

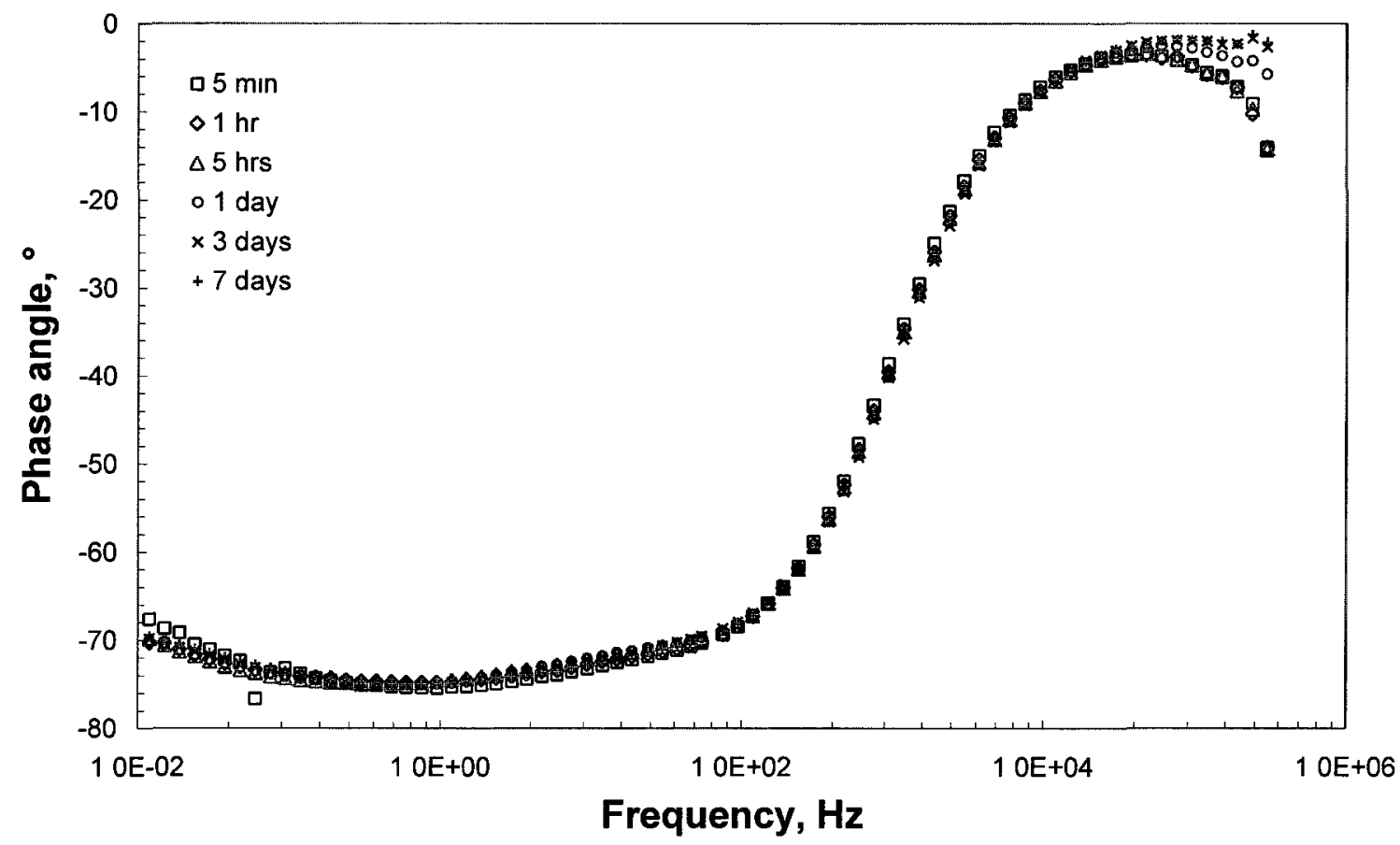

(j) Bode phase plots of $\mathrm{CrSiCN}(1)$

Figure 4-39 to be continued 


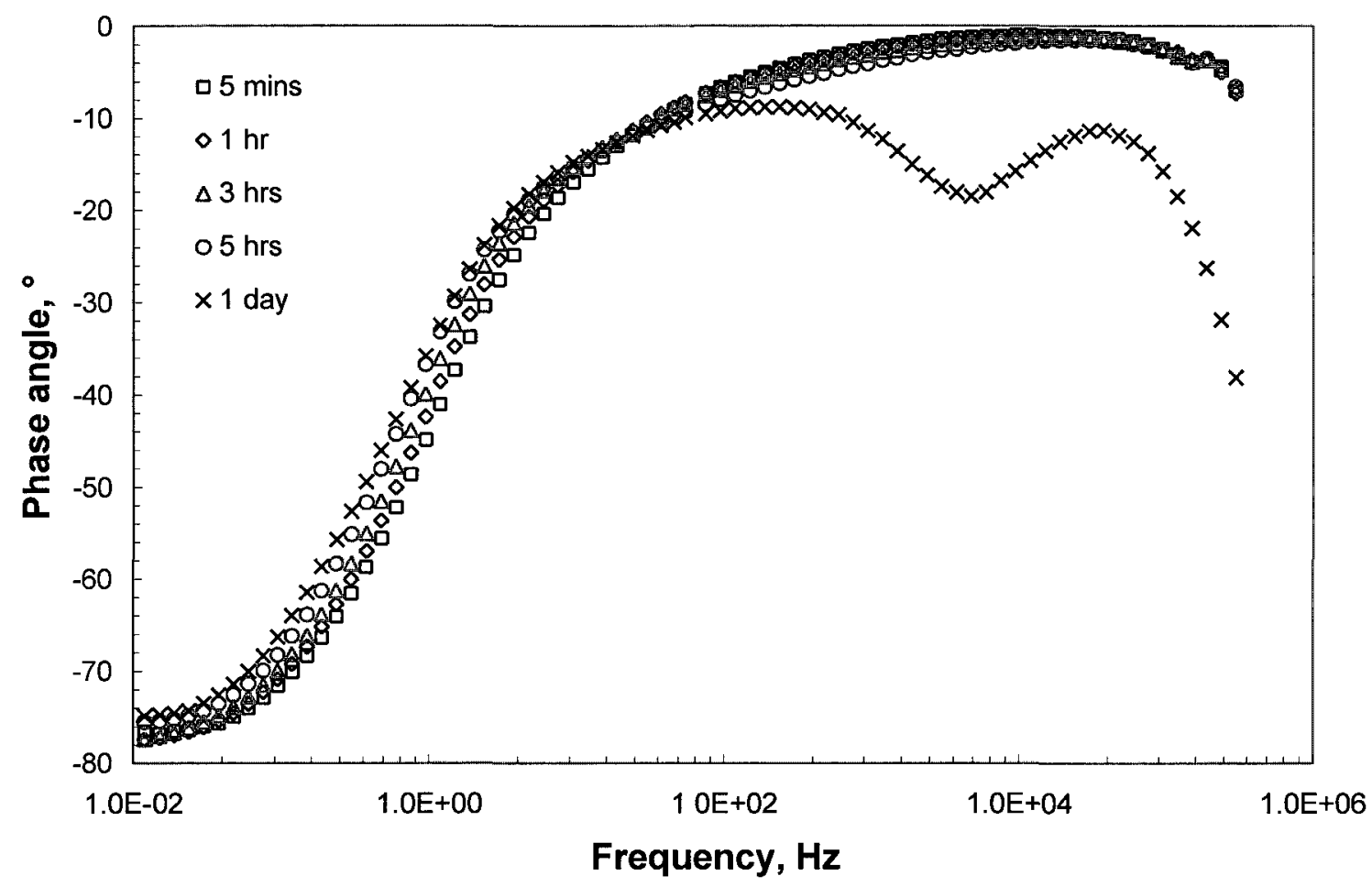

(k) Bode phase plots of $\mathrm{CrSiCN}(2)$

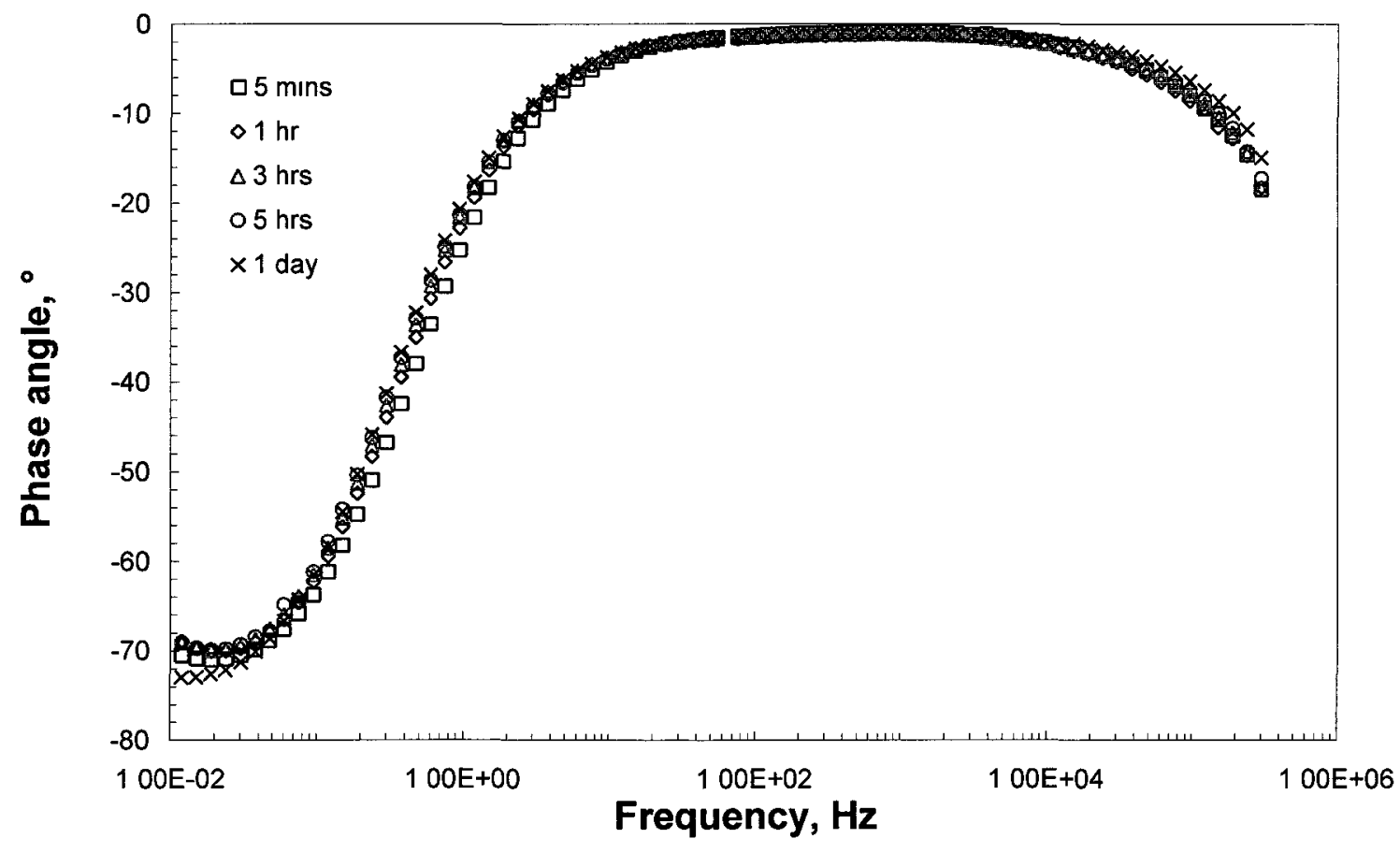

(l) Bode phase plots of $\mathrm{CrSiCN}(3)$

Figure 4-39 EIS spectra of $\mathrm{CrN}$ based coating systems. 


\subsubsection{Scanning Electron Microscope (SEM) Analyses after Corrosion Tests}

\section{TiN Coating Systems}

On the surface of the EB TiN/SS coating, corrosion products were observed around nodules (Figure 4-40 a, b), which were the result of the corrosion attack during thirteen days of immersion, and were confirmed to be mainly ferrous oxide by the presence of $\mathrm{Fe}$ and $\mathrm{O}$ peaks in the EDS spectrum (Figure 4-40 c). As the penetration depth of the electron beam is only on the order of $1 \mu \mathrm{m}$ at an acceleration voltage of $20 \mathrm{kV}$ [407], the observed Ti, W and N EDS peaks originated from the coating under the thin corrosion product at the location "A" (Figure 4-40 a, b); the EDS analysis conducted at the adjacent corrosion product free area only showed $\mathrm{Ti}, \mathrm{W}$ and $\mathrm{N}$ peaks. The presence of corrosion products around the defect vicinity clearly demonstrated that the corrosive media ( $\mathrm{NaCl}$ solution) did penetrate through the coating defects, and resulted in corrosion of the substrate steel.

After seventeen days of immersion for the PMMA-treated EB TiN/SS coating system, no corrosion product was found on the surface (Figure 4-40 d, f), and the PMMA layer was intact as confirmed by EDS analyses (Figure 4-40 e, g). The crater and the adjacent areas surrounding nodules were still covered with the PMMA layer. This observation provided a physical explanation for the large and stable arcs in the Nyquist plots (Figure 4-36 b), indicating that PMMA effectively sealed the pores in the coating, and hence clogged the access paths of corrosive media to the substrate steel. 


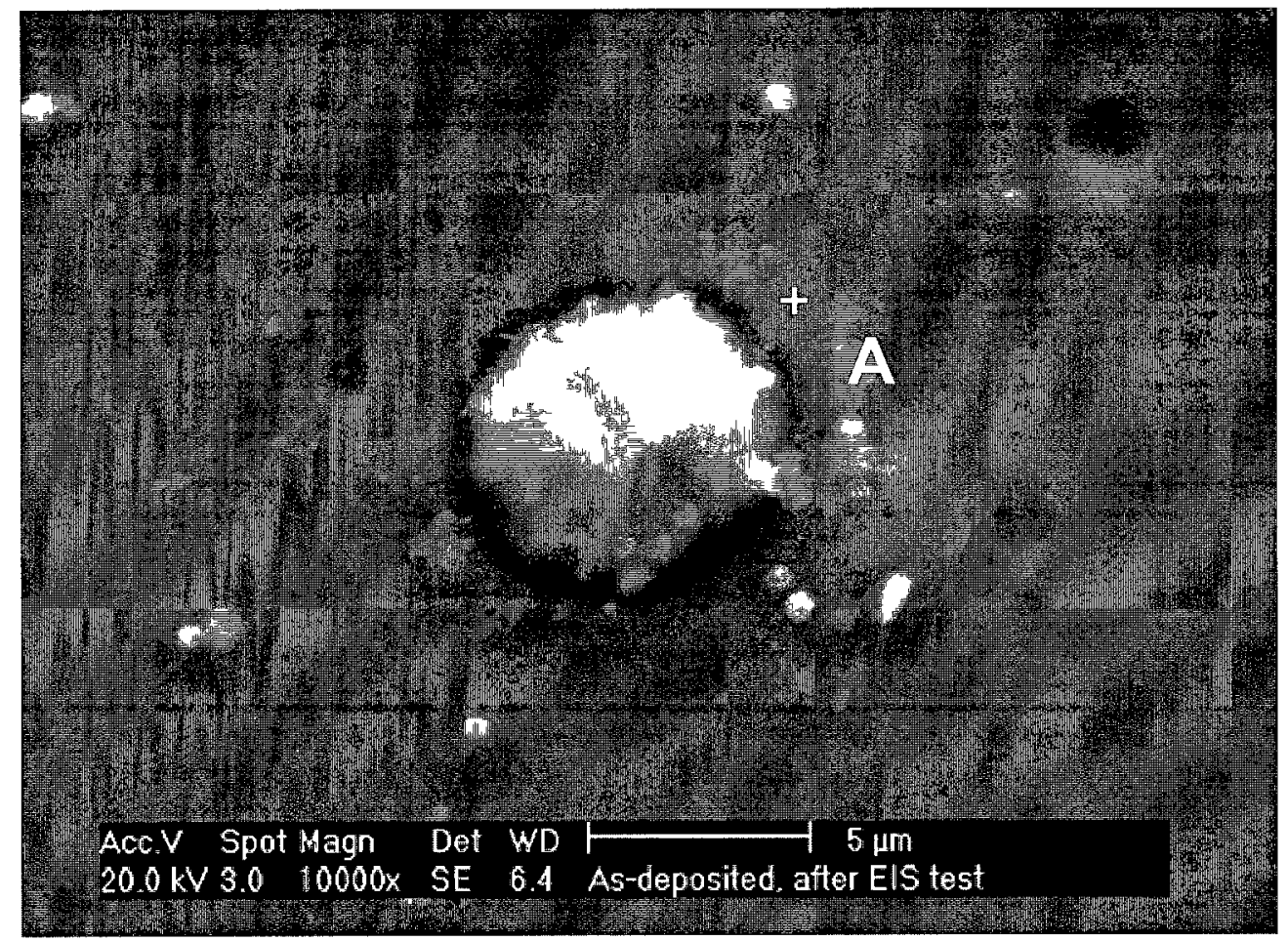

(a) As-deposited sample after thirteen days of immersion ( $\mathrm{Cl}$ containing corrosion product was observed around nodule as indicated in (c))

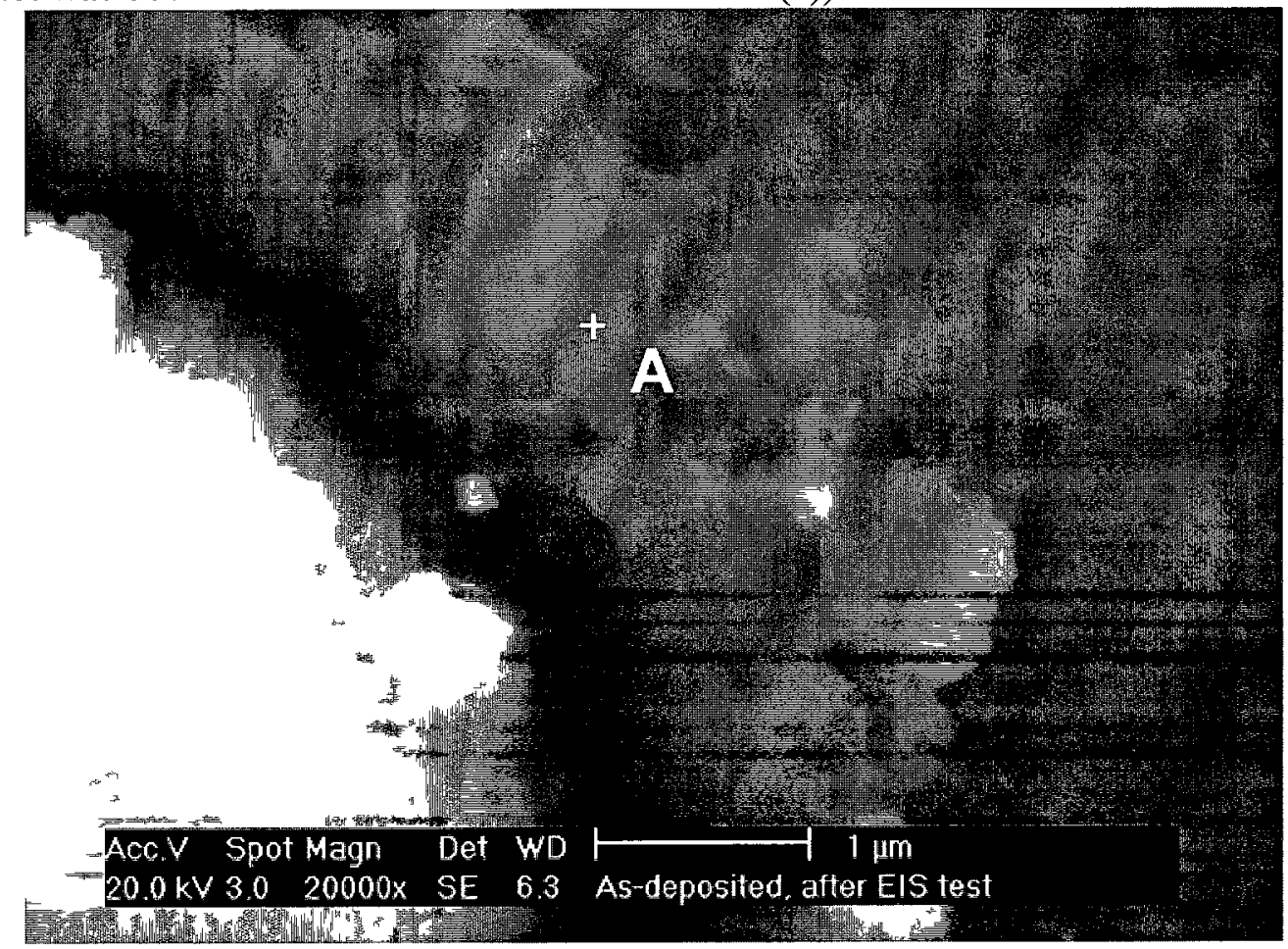

(b) As-deposited sample after thirteen days of immersion ( $\mathrm{Cl}$ containing corrosion product was observed around nodule as indicated in (c))

Figure 4-40 to be continued 


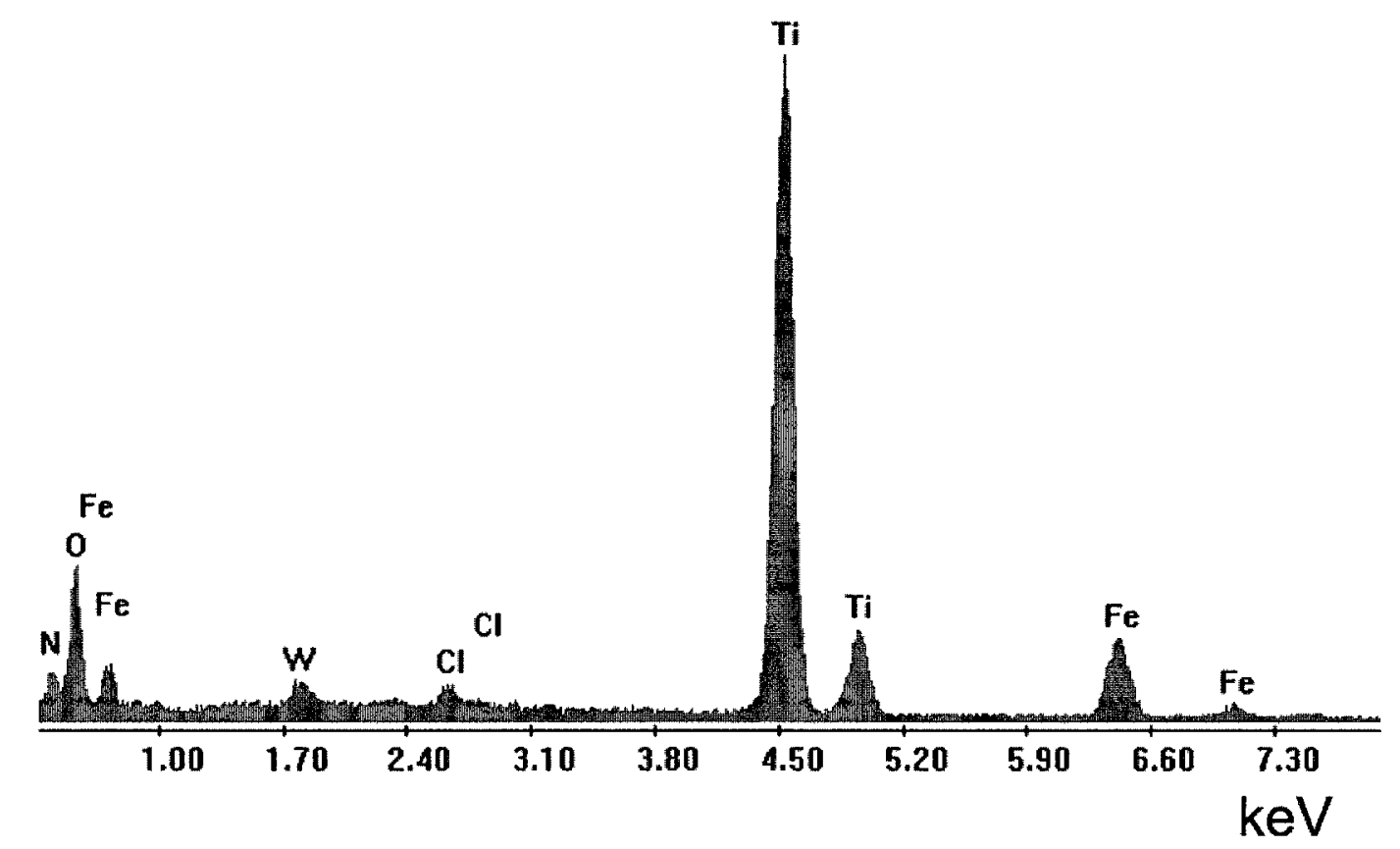

(c) EDS spectrum of corrosion product measured at location A in (a) and (b)

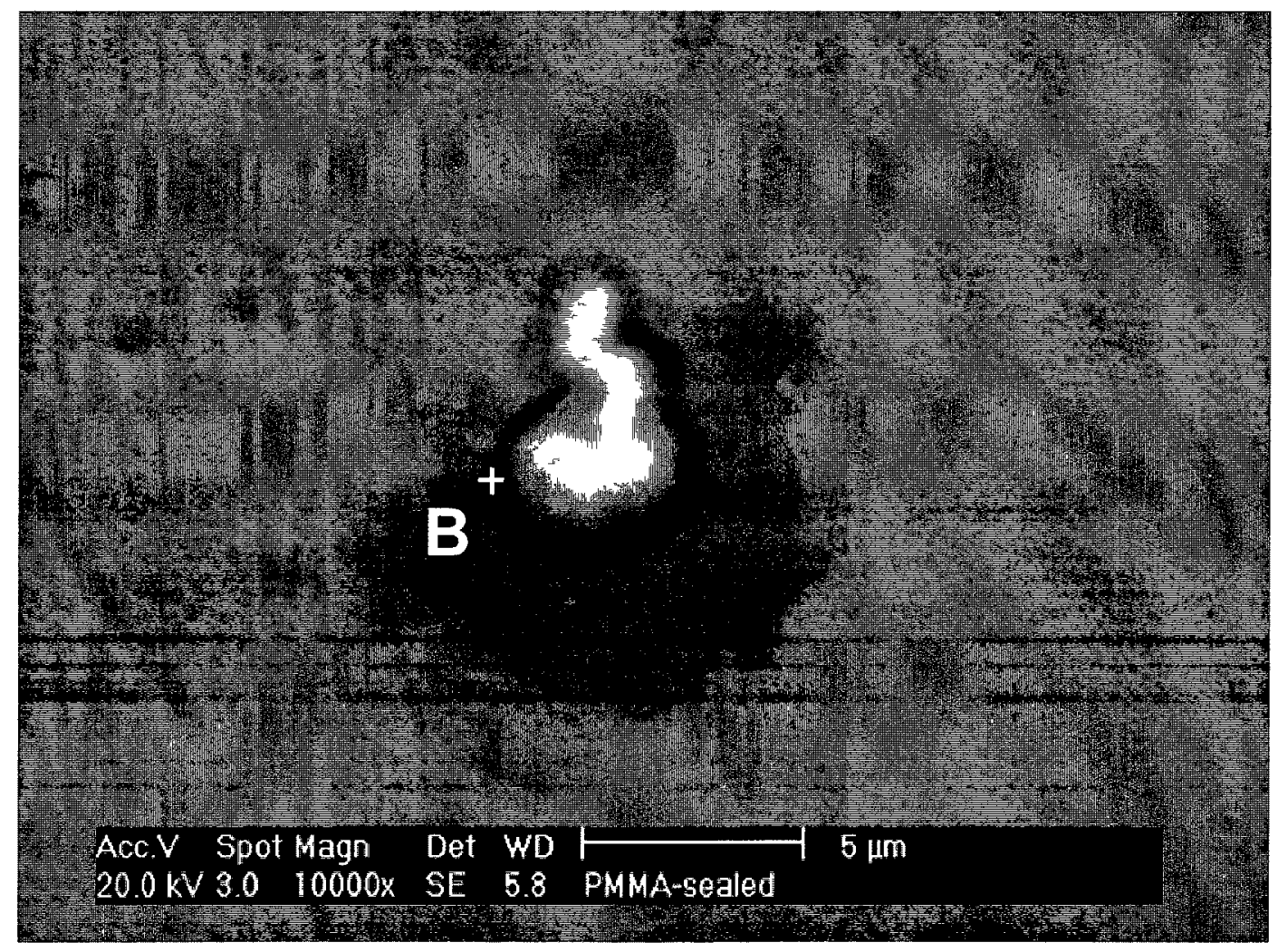

(d) PMMA-treated sample after seventeen days of immersion (no corrosion product was detected around nodule as indicated in (e))

Figure 4-40 to be continued 


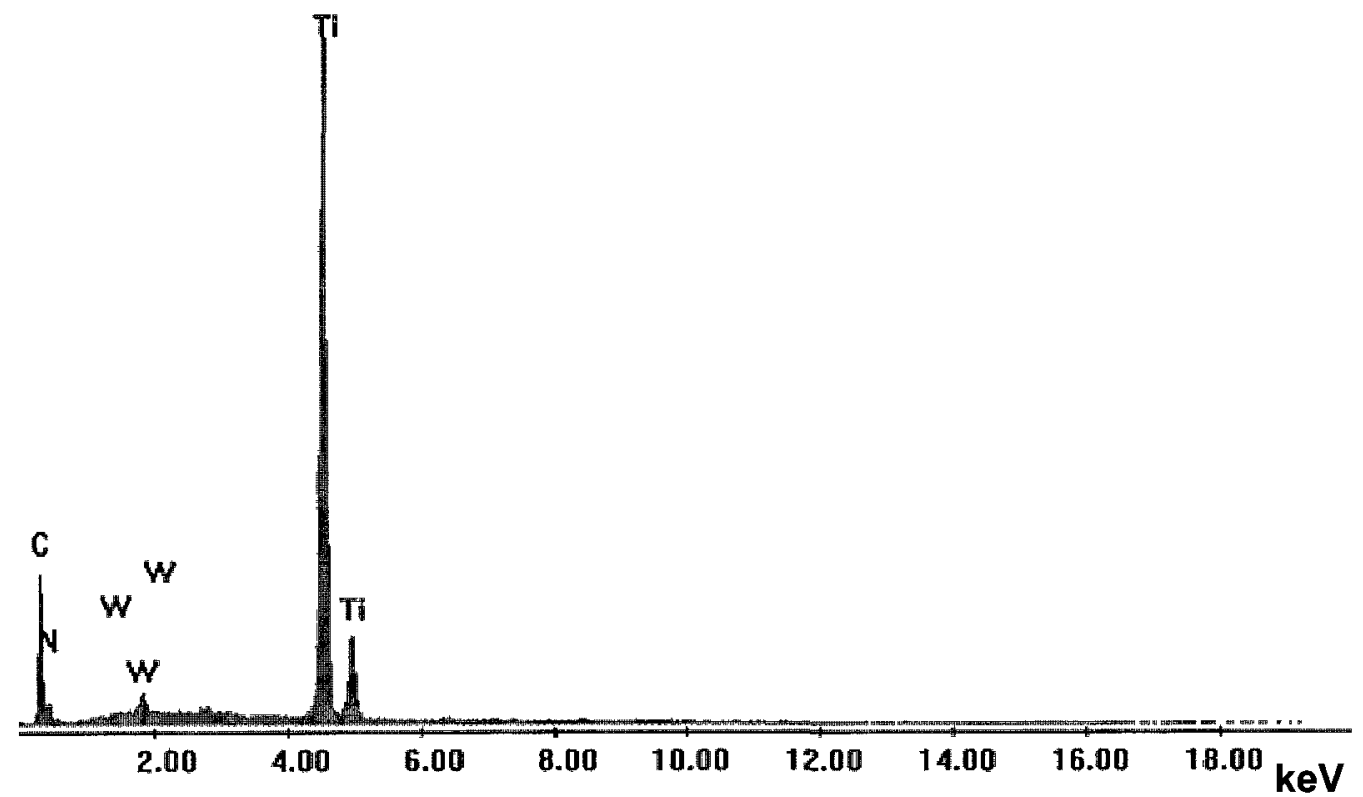

(e) EDS spectrum measured at location B in (d)

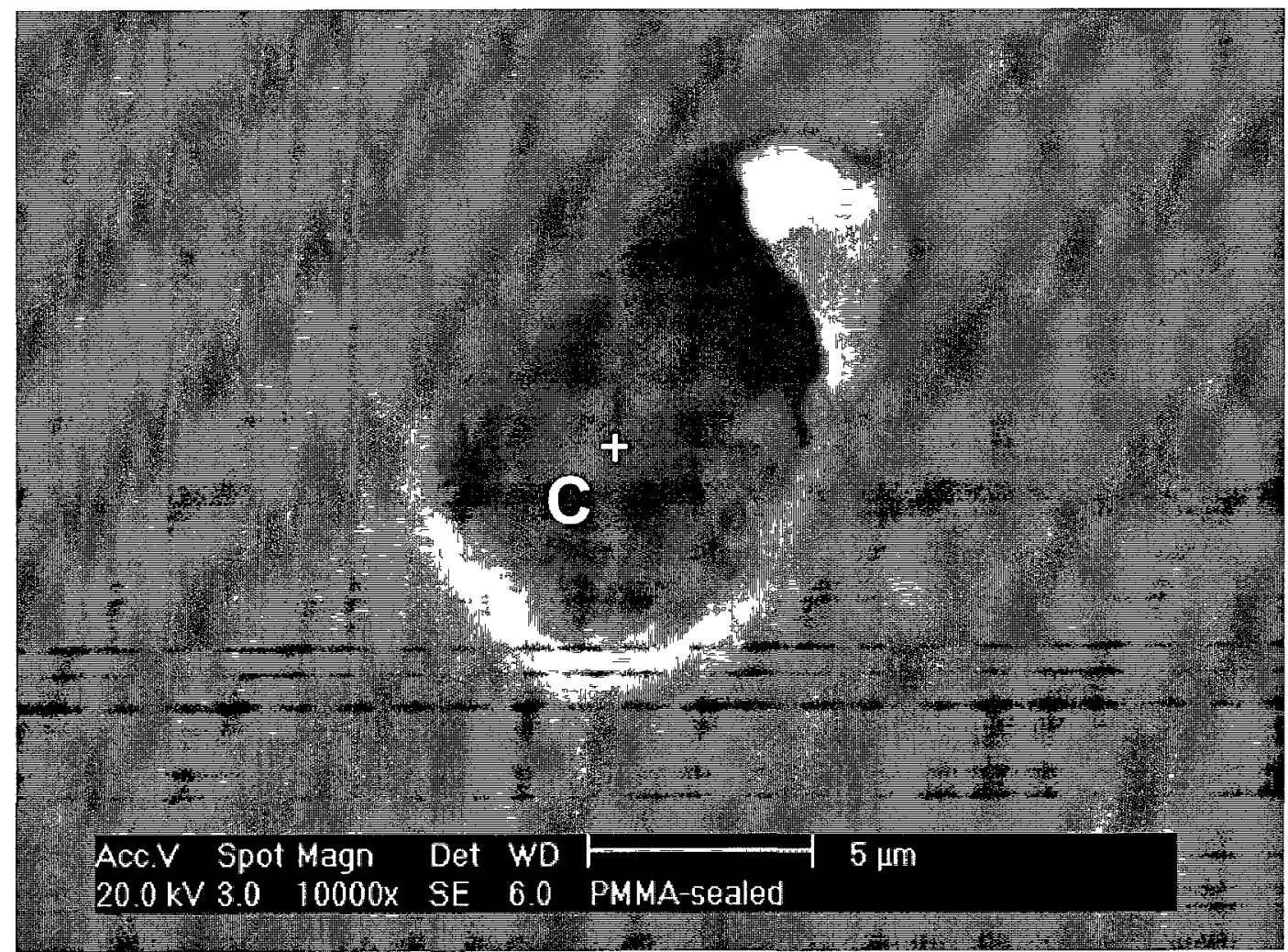

(f) PMMA-treated sample after seventeen days of immersion (no corrosion product was detected in the cancave area as indicated in (e))

Figure 4-40 to be continued 


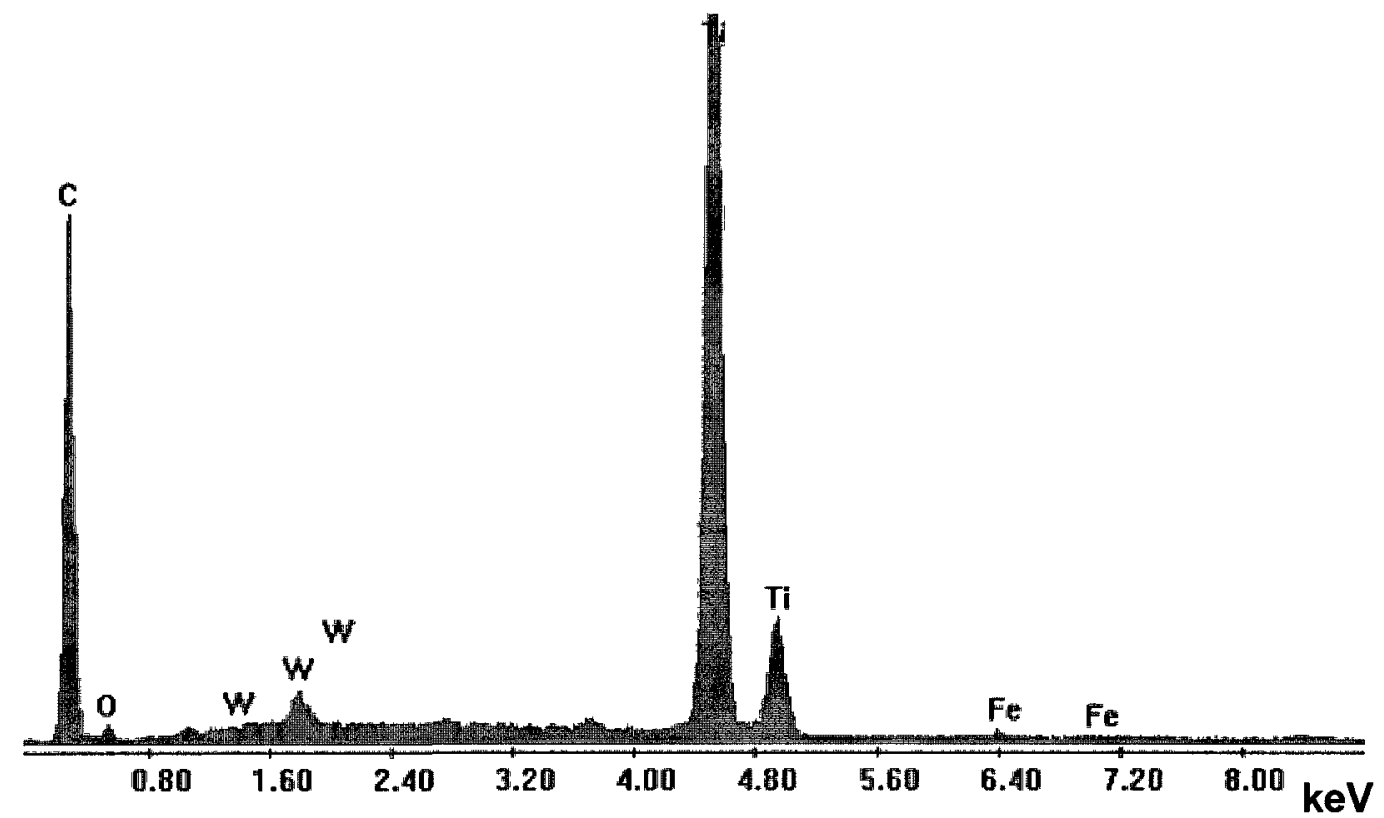

(g) EDS spectrum measured at location $\mathrm{C}$ in (f)

Figure 4-40 SEM analysis images for EB TiN/SS coating systems after immersions.

Throughout the surface of the CA TiN/SS coating (six days of immersion), the defects (nodules and concaves) appeared the same as before the test, and no corrosion product was detected around the nodule and the nodule-detached concavities (Figure 4-41 a). Tiny amounts of the corrosion products $\mathrm{Cl}, \mathrm{Fe}$ and $\mathrm{O}$ can be observed near a nodule (Figure 4-41 b), as is illustrated in EDS spectrum (Figure 4-41 c).

No corrosion product was found on the PMMA-treated sample (seven days of immersion) around nodules and nodule-detached concavities (Figure 4-41 d, f), which was in agreement with the large and stable arcs in the Nyquist plots (Figure 4-37 b) and further demonstrates the effectiveness of the PMMA treatment in sealing the through-coating defects. 


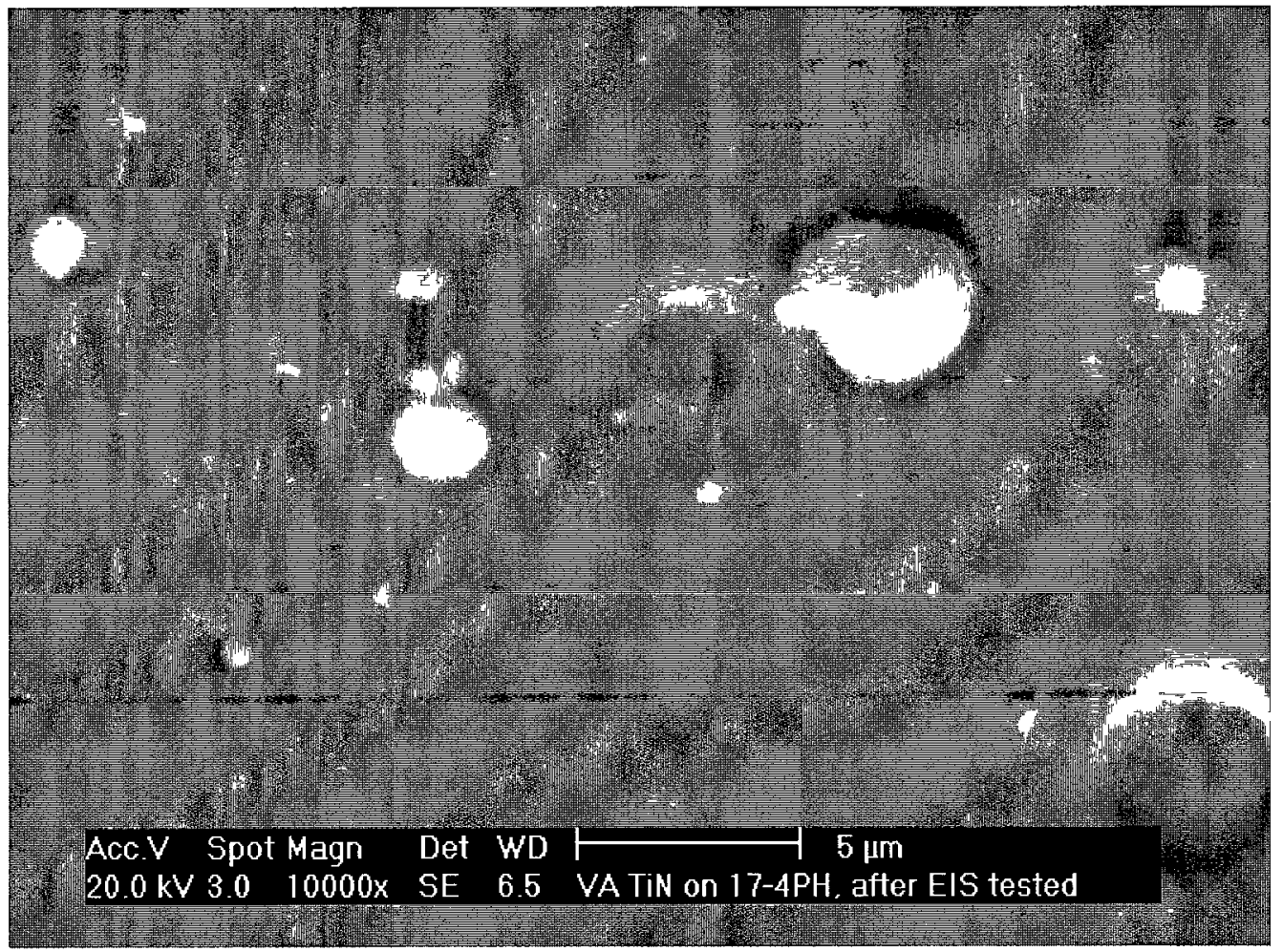

(a) as-deposited sample after six days of immersion (no corrosion product was observed)

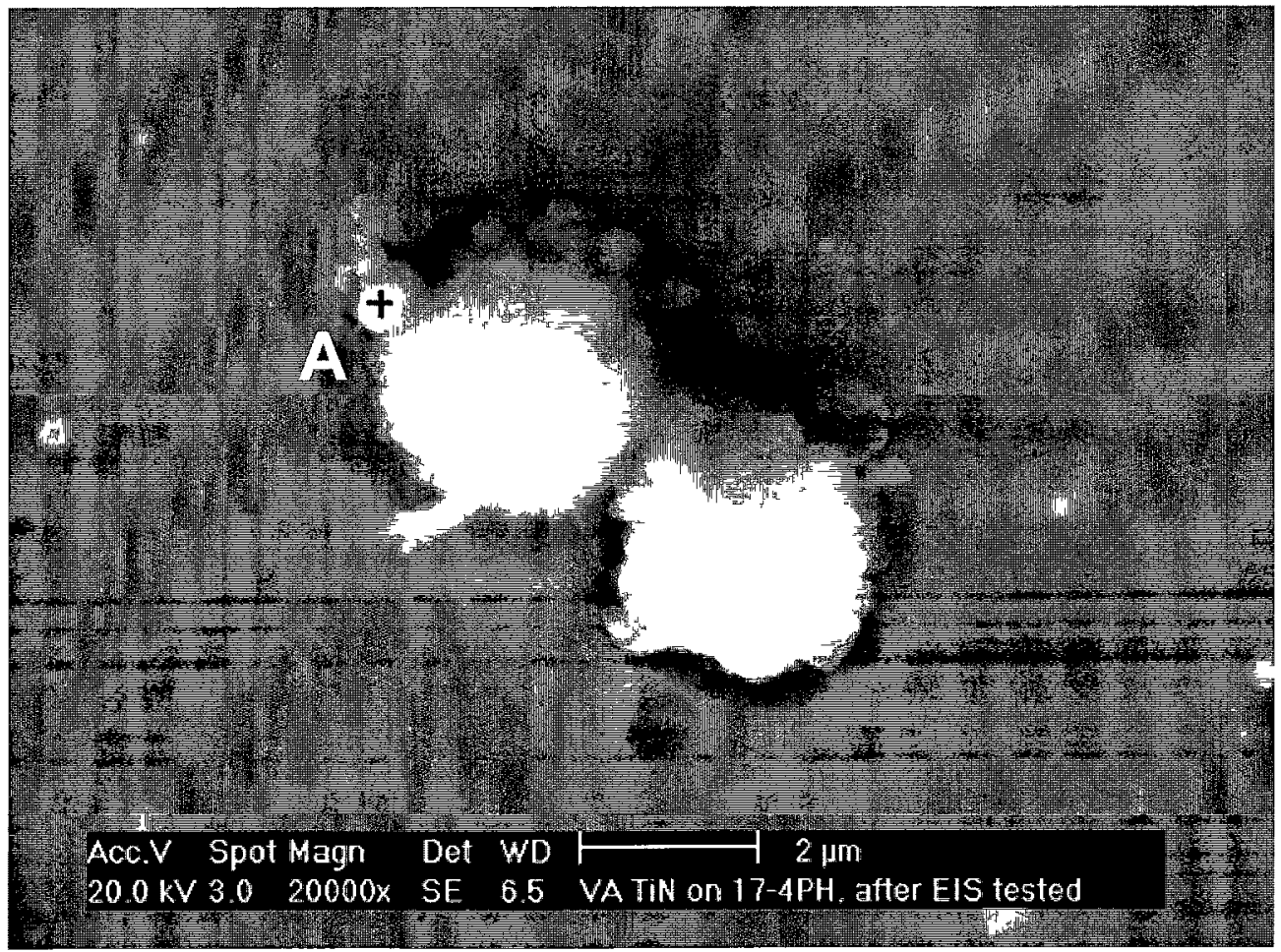

(b) as-deposited sample after six days of immersion, suspected corrosion product containing tiny amount of $\mathrm{Cl}, \mathrm{Fe}$ and $\mathrm{O}$ was observed near a nodule, as indicated in EDS spectrum (c) measured in the location A

Figure 4-41 to be continued 


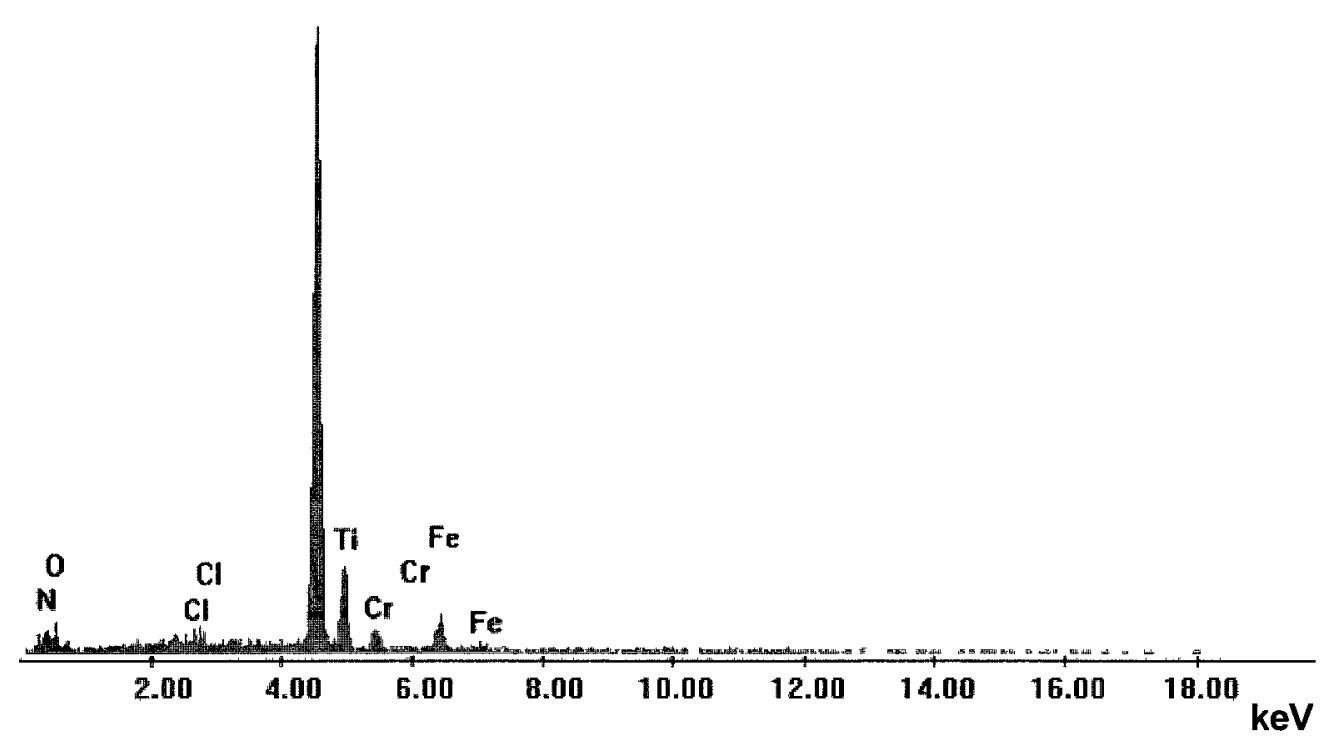

(c) EDS spectrum measured in the location A in (b)

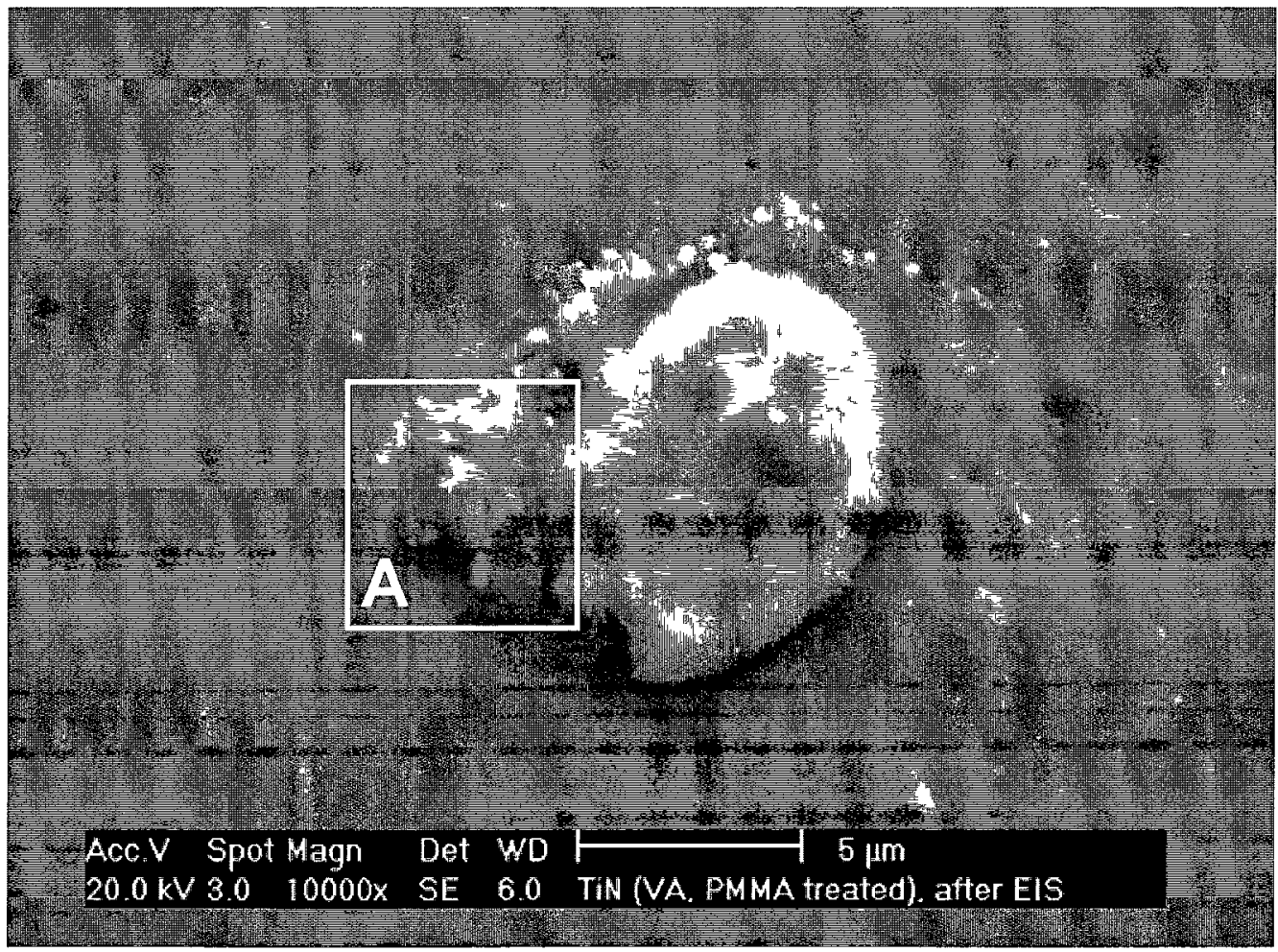

(d) PMMA-treated specimen after seven days of immersion, no detectable corrosion product around nodules and nodule-detached concave areas respectively, with assistance of EDS spectrum (e) measured in the scan area A

Figure 4-41 to be continued 


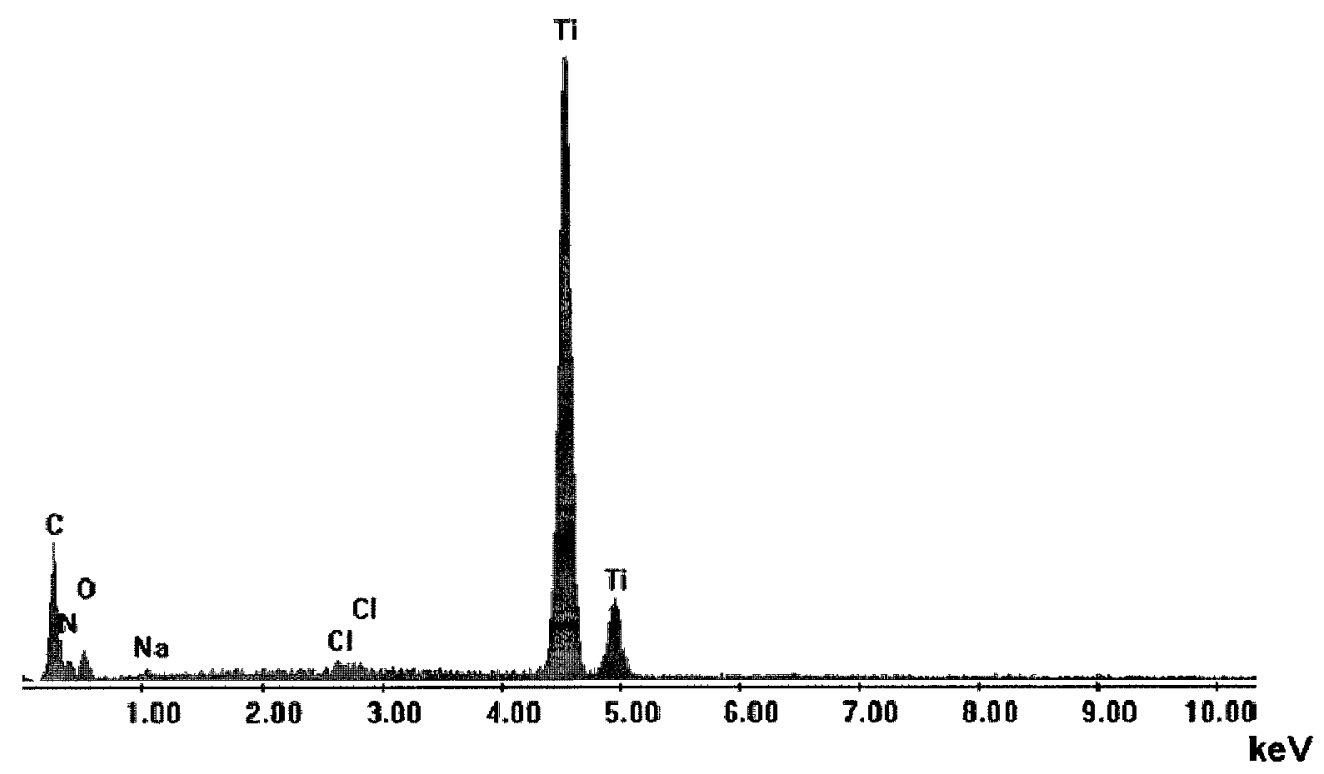

(e) EDS spectrum measured in the scan area A in (d)

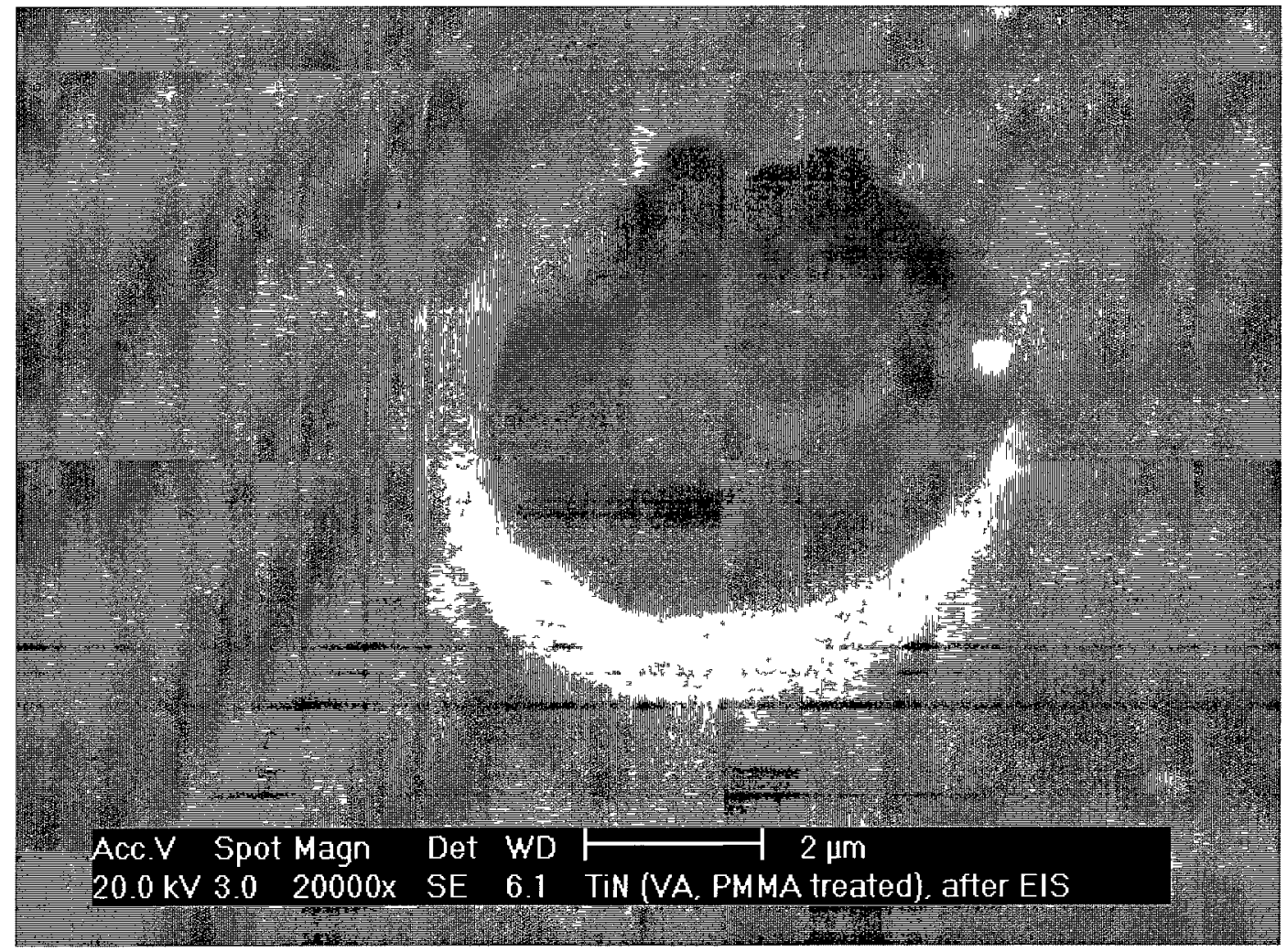

(f) PMMA-treated specimen after seven days of immersion, no detectable corrosion product in nodule-detached concave area

Figure 4-41 SEM analysis images for CA TiN/SS coating system after immersions. 
On the surface of the CA TiN/MS coating (after one day of immersion), serious corrosion pits were observed (Figure 4-42 a, b, c), and corrosion product was detected in the surrounding region (Figure 4-42 d) with oxygen $(\mathrm{O})$ and iron (Fe) by EDS analysis (Figure 4-42 e). This revealed that a corroding electrolyte-substrate interface was established due to the corrosive media penetrating through pores in the coating. Furthermore, the corrosion process was accelerated due to a large area ratio of cathode to anode, which was responsible for the serious degradation in corrosion resistance of the TiN/MS coating system.

SEM examinations of all three PMMA-treated TiN coated specimens (EB TiN/SS, CA TiN/SS and CA TiN/MS) did not reveal the presence of any corrosion product (Figure 4$40 \mathrm{~d}$, f, Figure 4-41 d, f and Figure 4-42 $\mathrm{f}, \mathrm{h}$ ) on the surface. This was confirmed with EDS analyses (Figure 4-40 e, g, Figure 4-41 c, e and Figure 4-42 g, i). The coating defects had the same appearance as before the immersion tests, and PMMA still covered the adjacent areas surrounding nodules and nodule-detached concavities. These results strongly indicate that the PMMA-treated TiN coatings maintain a high resistance to corrosion, and show little degradation in performance over long exposure times in $\mathrm{NaCl}$ solution. All these observations were consistent with the EIS results (Figure 4-38 a, b, Figure 4-37 a, b and Figure 4-38 a, b), demonstrating that PMMA effectively clogged or limited the permeable paths for the corrosive media to reach the substrate, and consequently improved the anti-corrosion performance in the coating/substrate systems. 


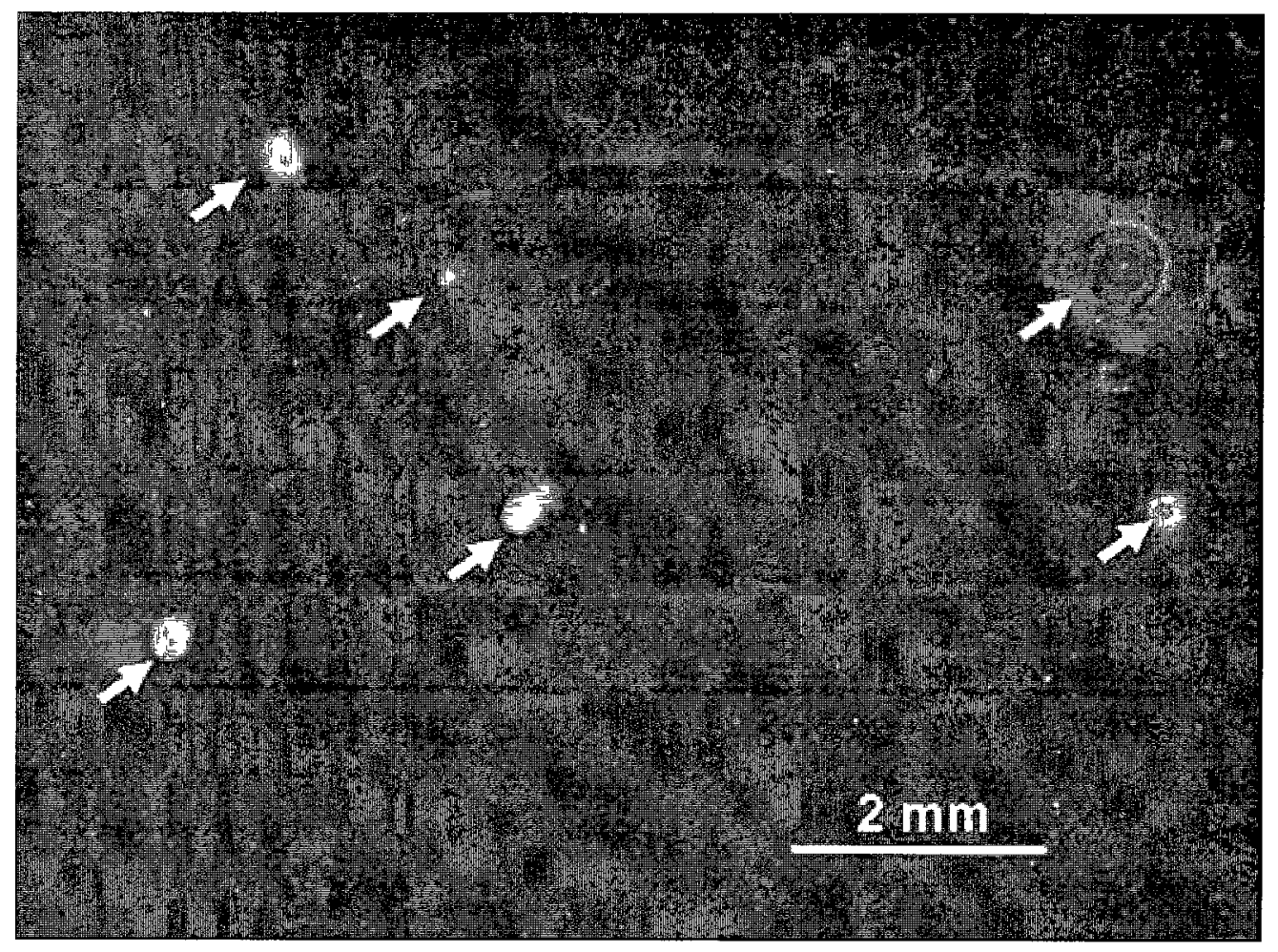

(a) Optical image of as-deposited CA TiN/MS coating system showing pits after one day of immersion

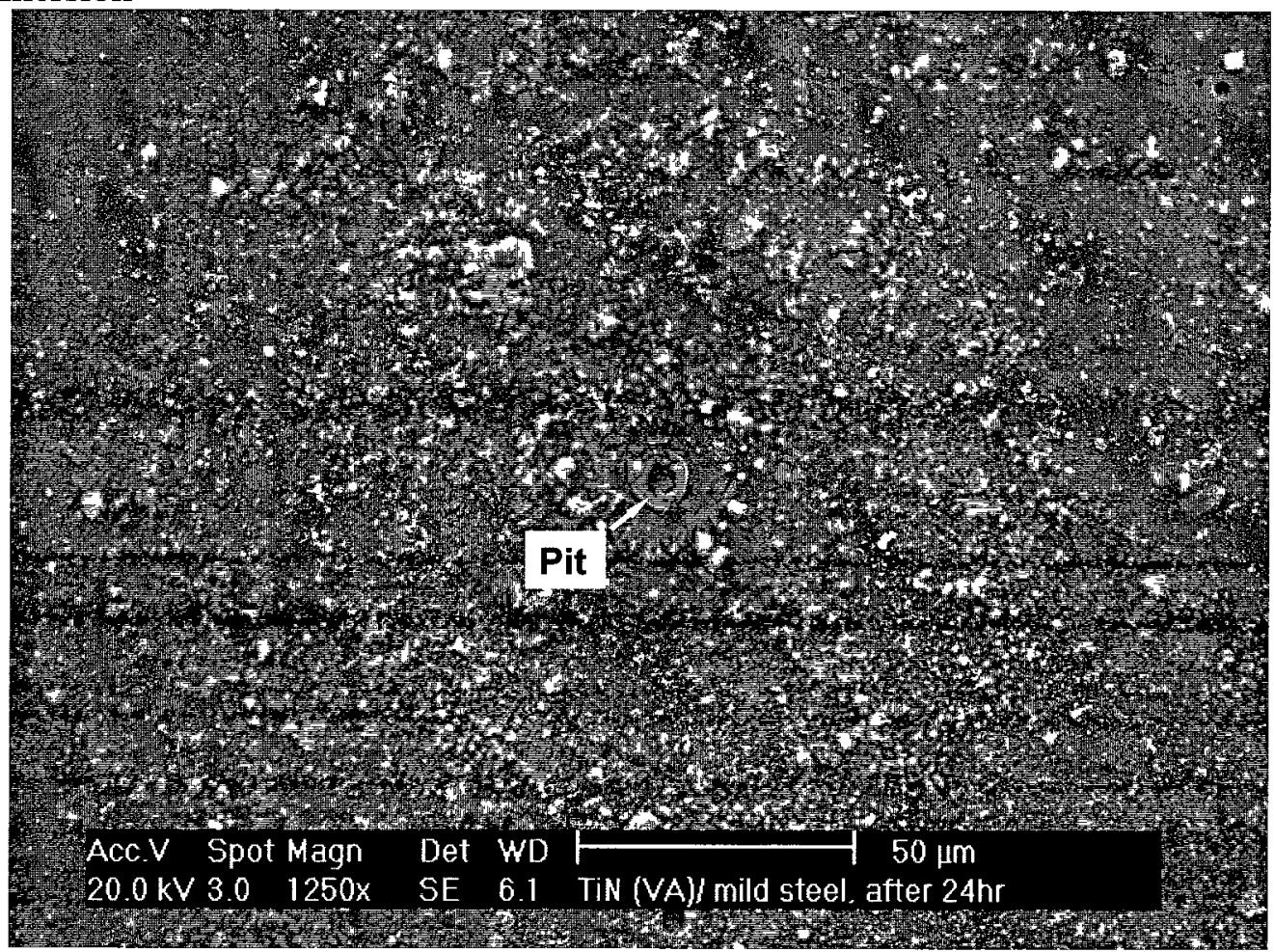

(b) SEM image of as-deposited CA TiN/MS coating system showing a pit after one day of immersion

Figure 4-42 to be continued 


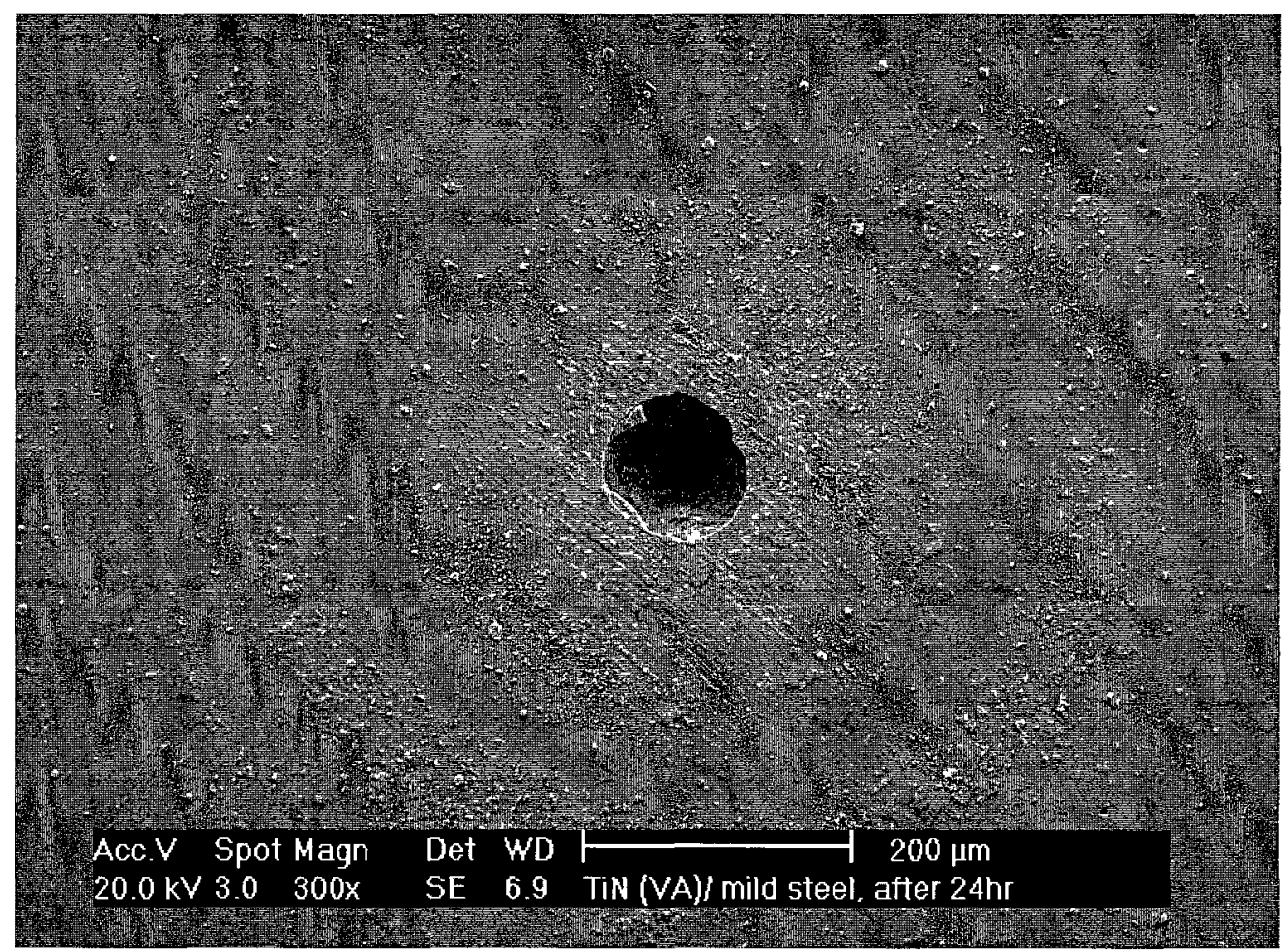

(c) SEM image of as-deposited CA TiN/MS coating system showing a pit hole due to the detachment of a nodule after one day of immersion

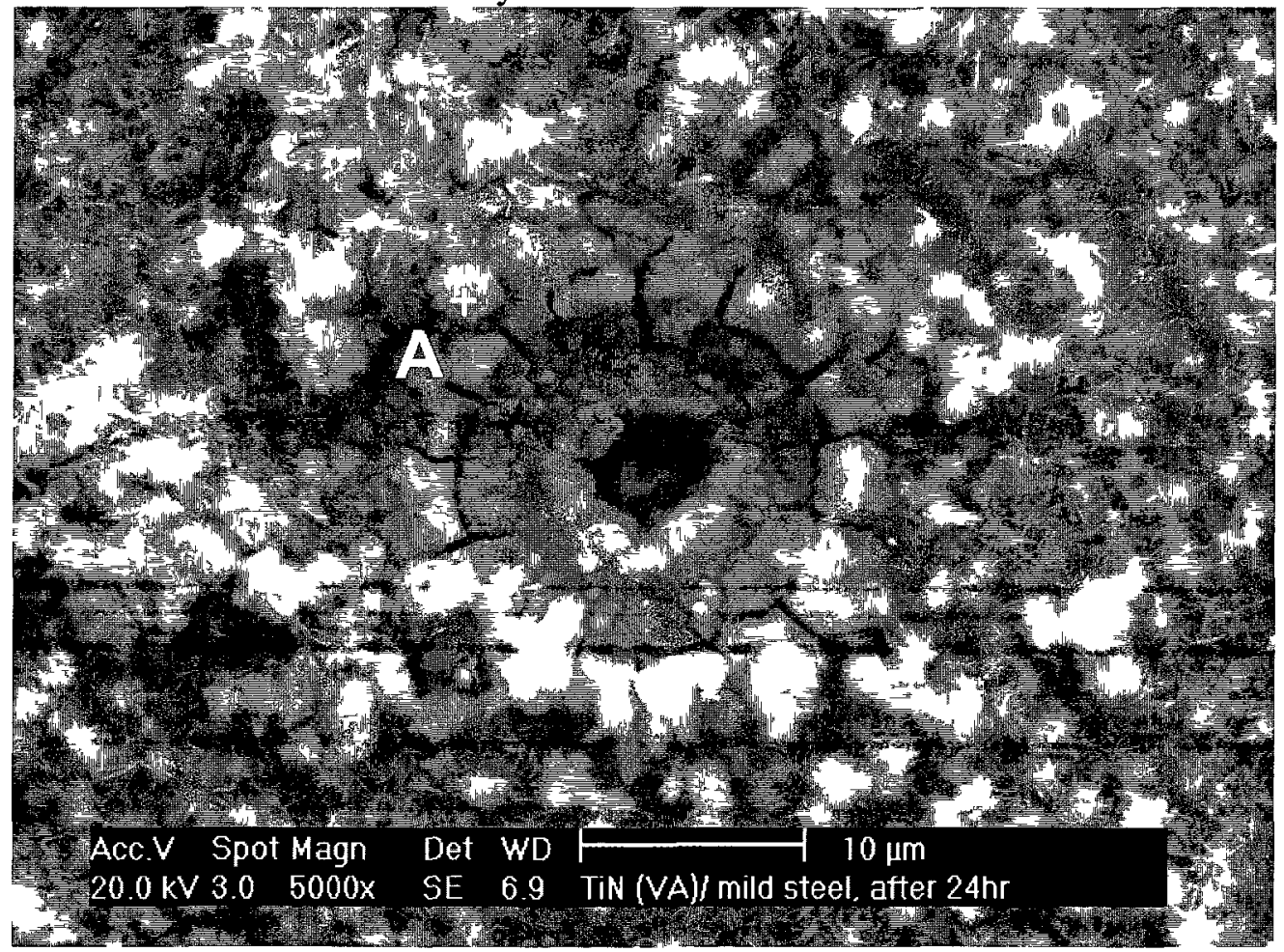

(d) SEM image of as-deposited CA TiN/MS coating system after one day of immersion, $\mathrm{Cl}$ contained corrosion product was observed around the pit hole, as indicated in EDS spectrum (e)

Figure 4-42 to be continued 


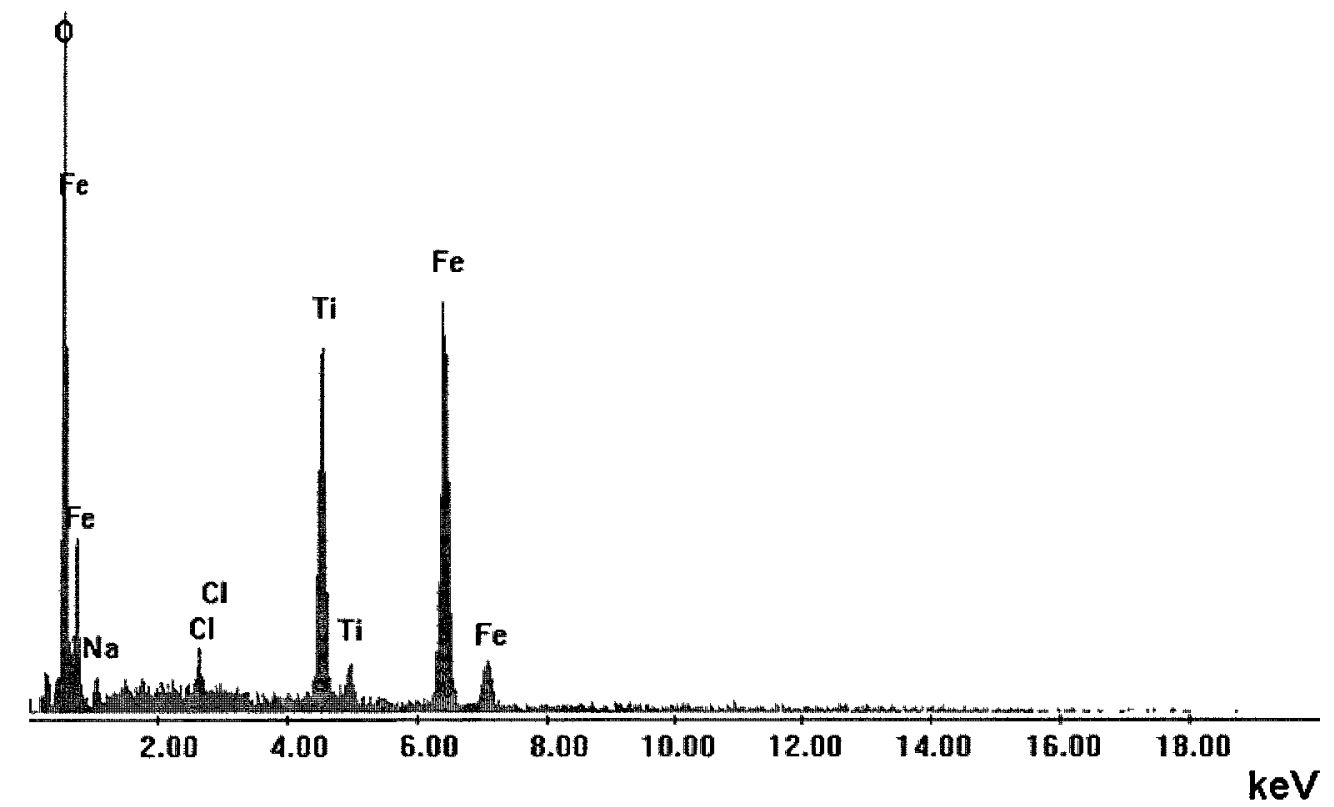

(e) EDS spectrum measured near the pit area at A in (d)

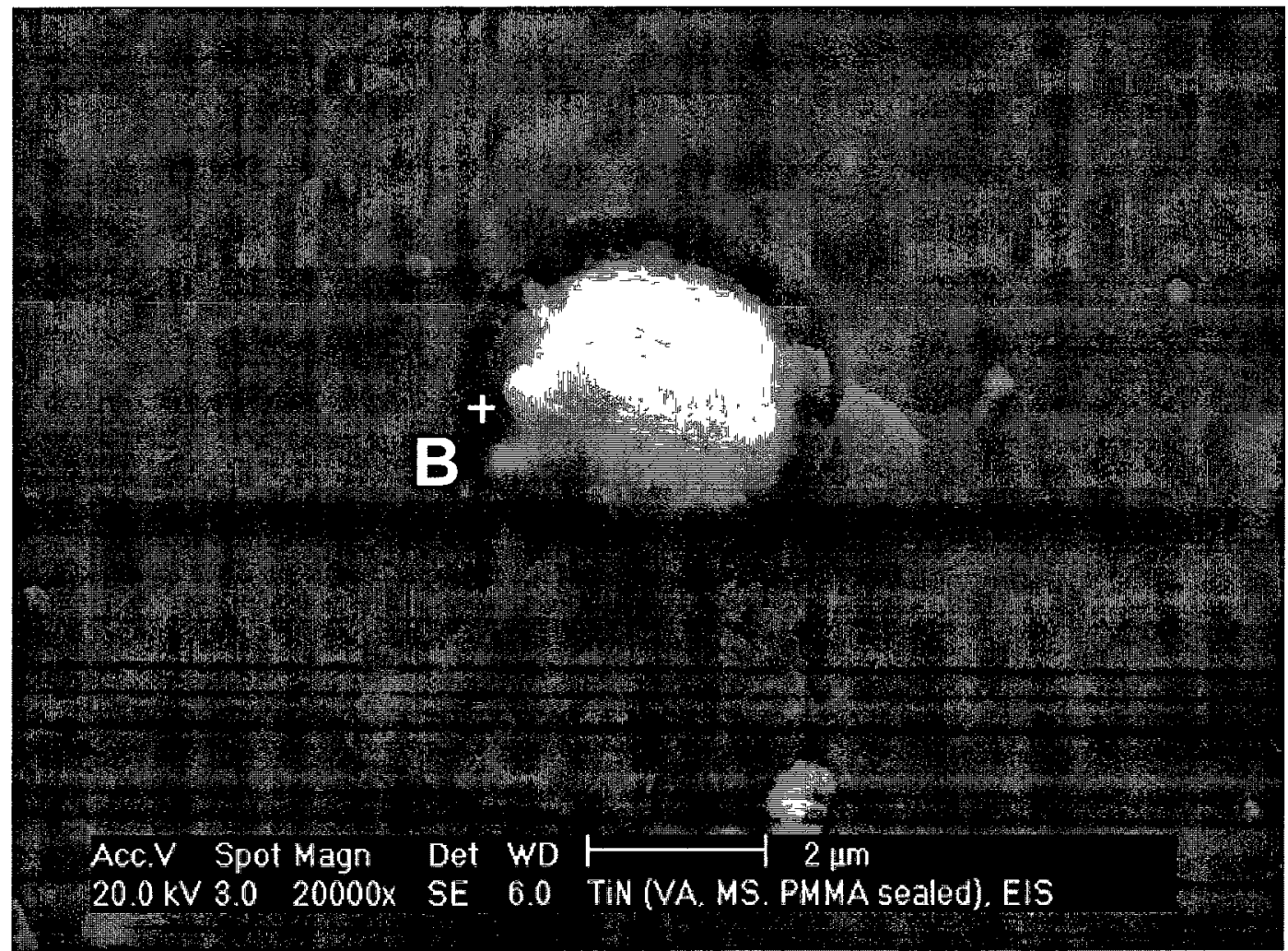

(f) PMMA-treated CA TiN/MS coating system after four days of immersion, no corrosion product around the nodule was observed, as confirmed with EDS spectrum (g)

Figure 4-42 to be continued 


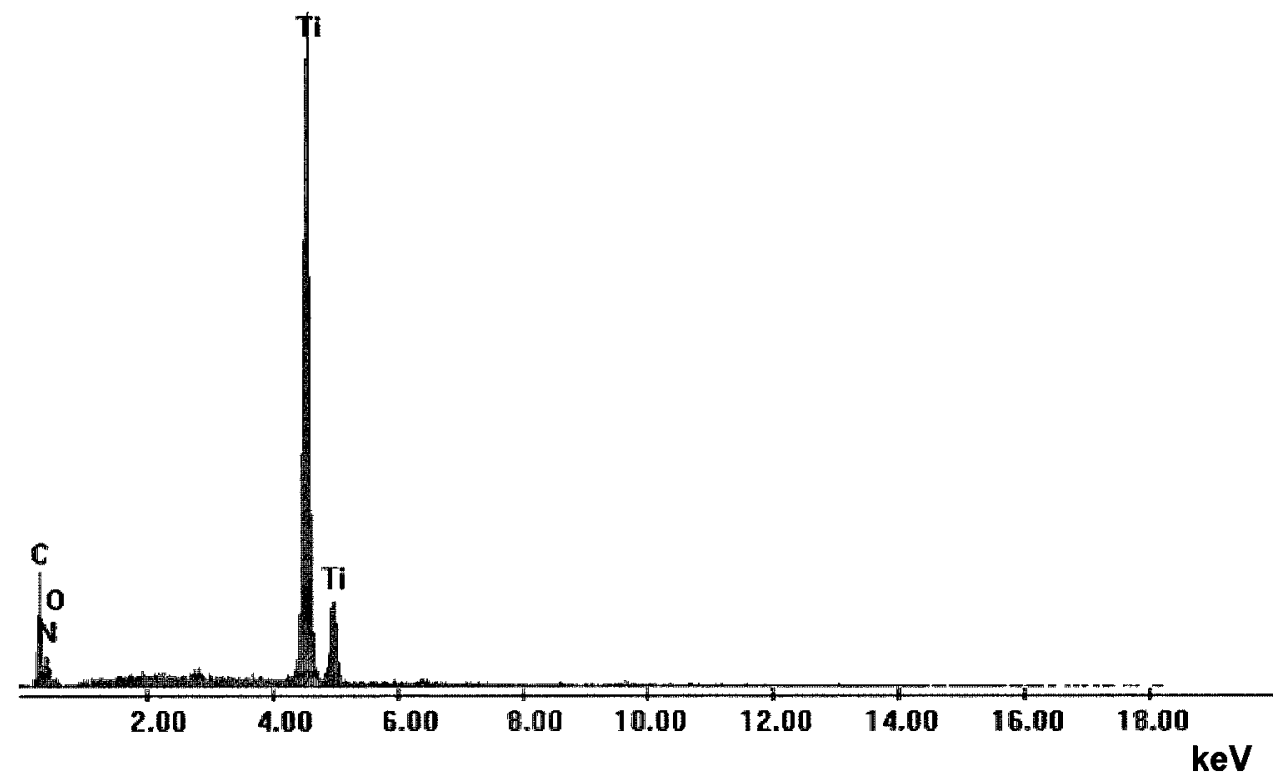

(g) EDS spectrum measured at the gap near the particle at B in (f)

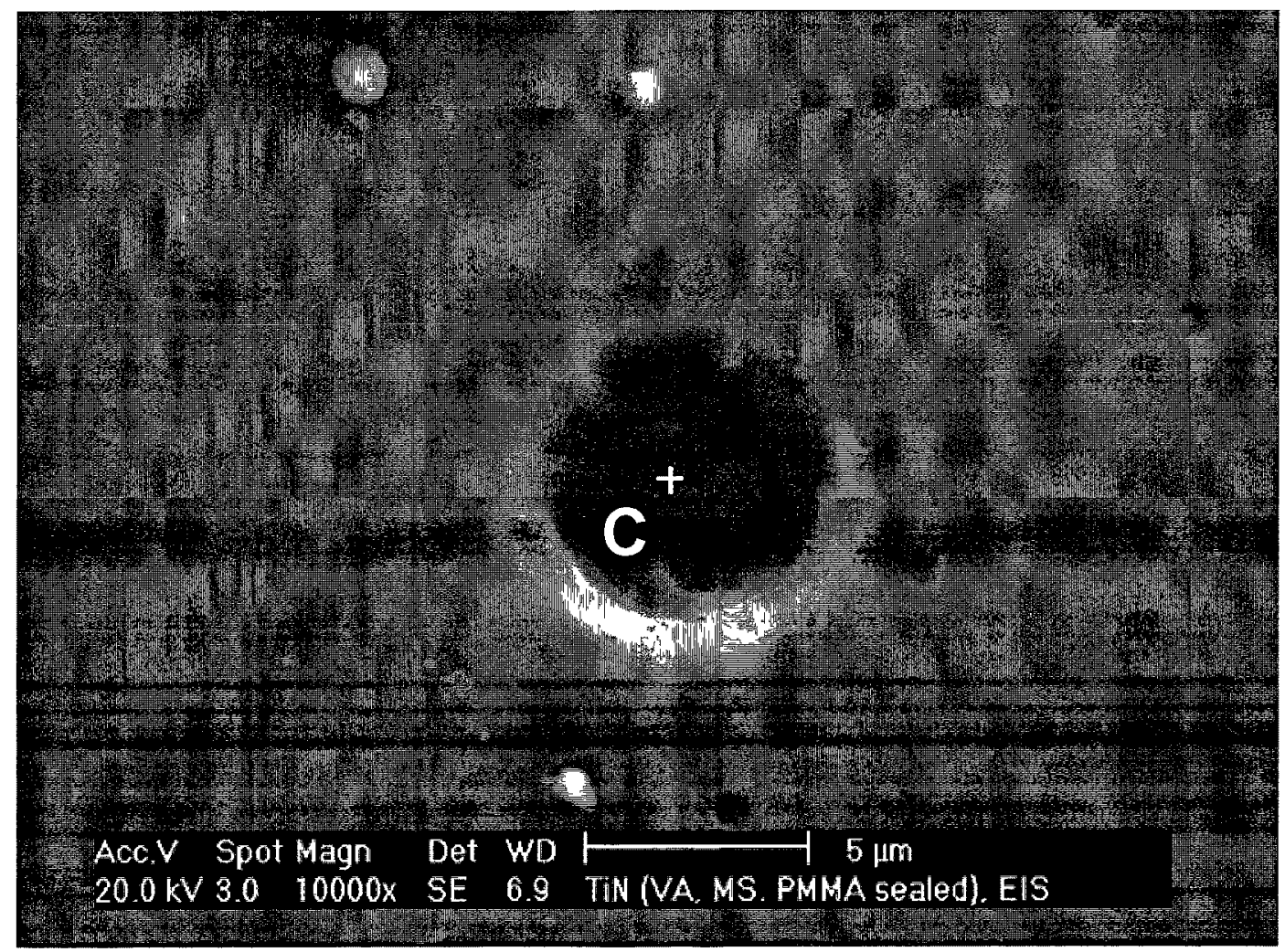

(h) PMMA-treated CA TiN/MS coating system after four days of immersion, no corrosion product in nodule-detached crater was observed, as confirmed with EDS spectrum (i)

Figure 4-42 to be continued 


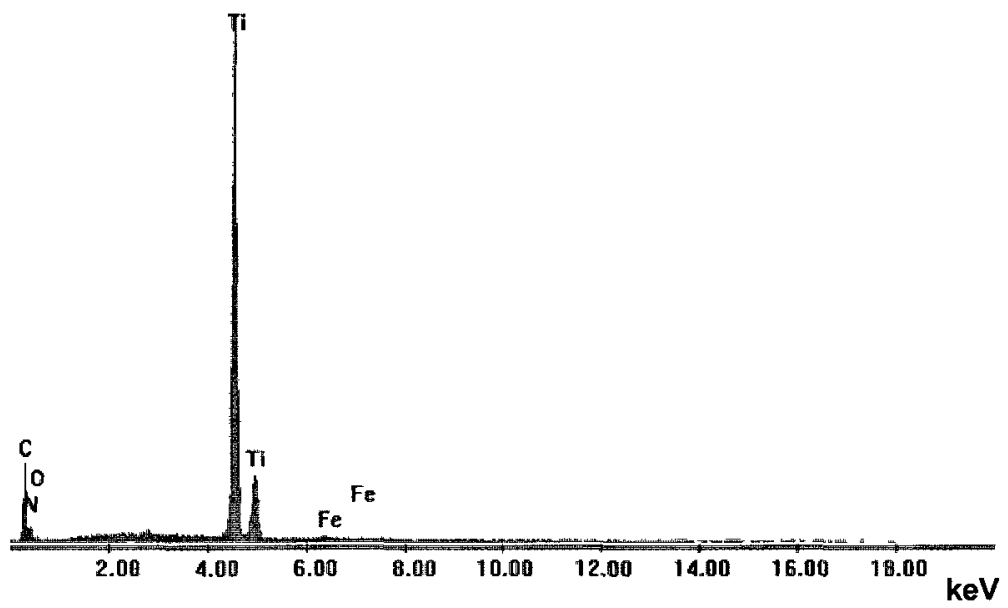

(i) EDS spectrum measured at the crater bottom area at $\mathrm{C}$ in (h)

Figure 4-42 Optical and SEM images for CA TiN/MS coating system after immersion.

\section{CrN Based Coating Systems}

SEM images (Figure 4-43 a to d) reveal that the surfaces of the $\mathrm{CrN}$ and $\mathrm{CrSiCN}(1)$ coatings remained unchanged after ten days and three days of immersions, respectively, in $\mathrm{NaCl}$ solution. EDS analyses did not detect any corrosion products, indicating their superior and stable resistance to corrosion. Pits were observed by visual inspection on $\mathrm{CrSiCN}(2)$ and $\mathrm{CrSiCN}(3)$ coatings after only one hour of immersion, then significant rust, which was confirmed by EDS analyses that showed it to be rich in oxygen $(O)$ and iron (Fe) (Figure 4-43 g, h), was observed surrounding the pits after a few hours of immersion (Figure 4-43 e, f). Cracks can be observed near the pits, which were due to the volume expansion of the corrosion products. The detrimental pitting corrosion was due to the large number of pore defects in the coatings, where highly localized corrosion occurred on the 17-4PH steel substrates, eventually resulting in the failure of its passive oxide thin film. These observations (Figure 4-43 e to h) are consistent with the potentiodynamic polarization and EIS tests, which revealed the serious degradations in corrosion resistance for the coatings $\mathrm{CrSiCN}(2)$ and $\mathrm{CrSiCN}(3)$. 


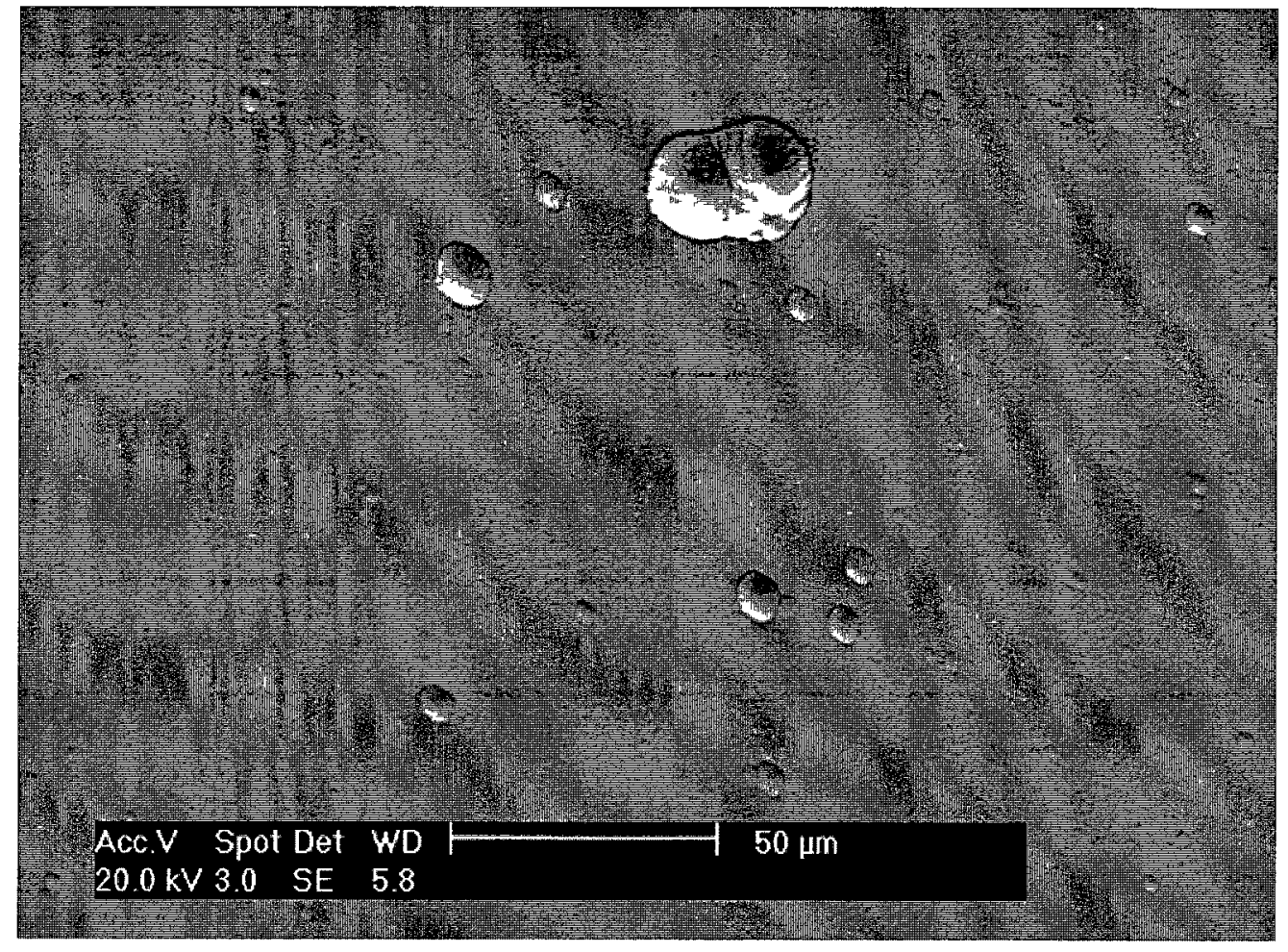

(a) Surface of $\mathrm{CrN}$ coating where no corrosion product was found after ten days of immersion

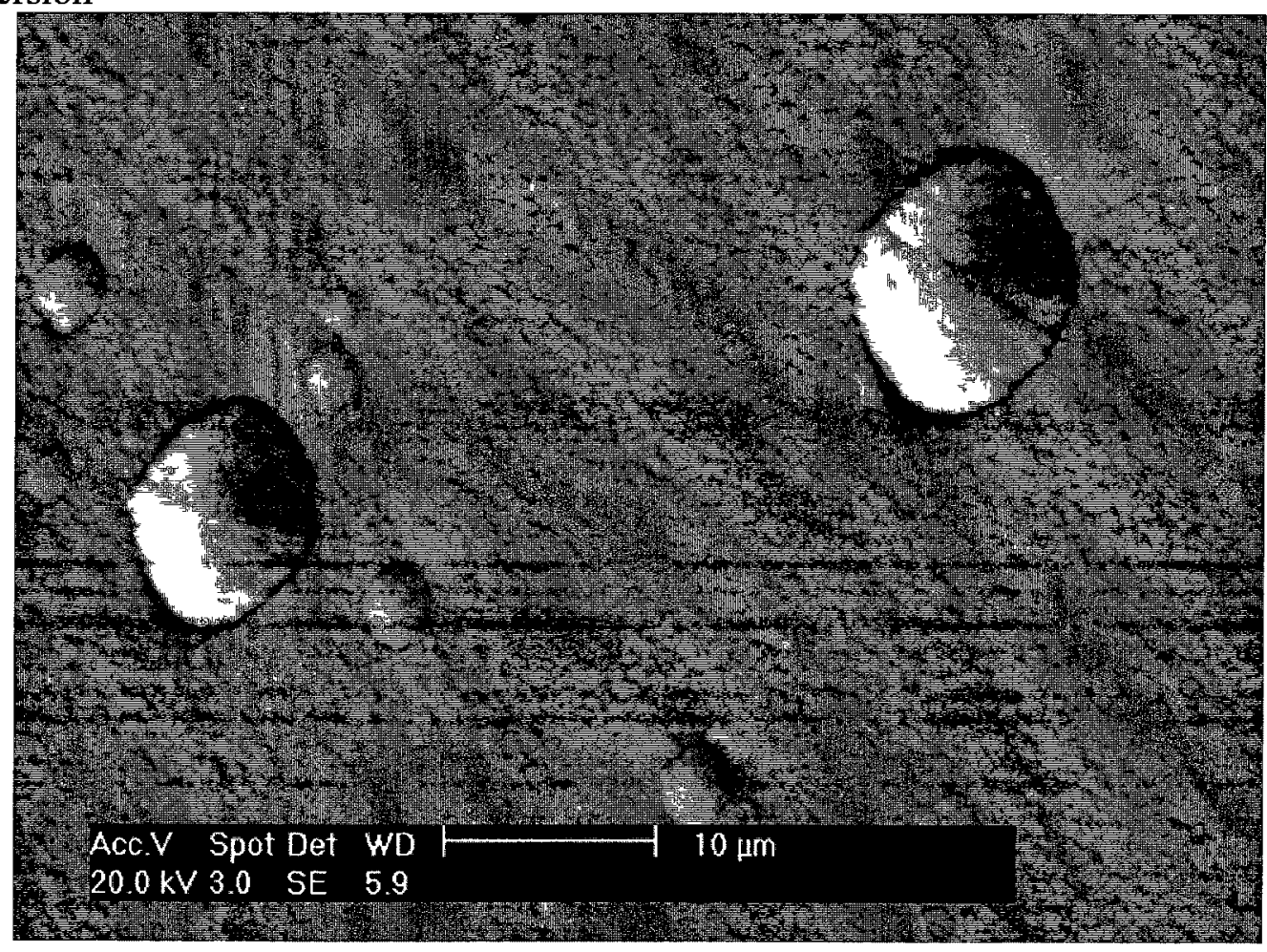

(b) Surface of $\mathrm{CrN}$ coating where no corrosion product was found after ten days of immersion

Figure 4-43 to be continued 


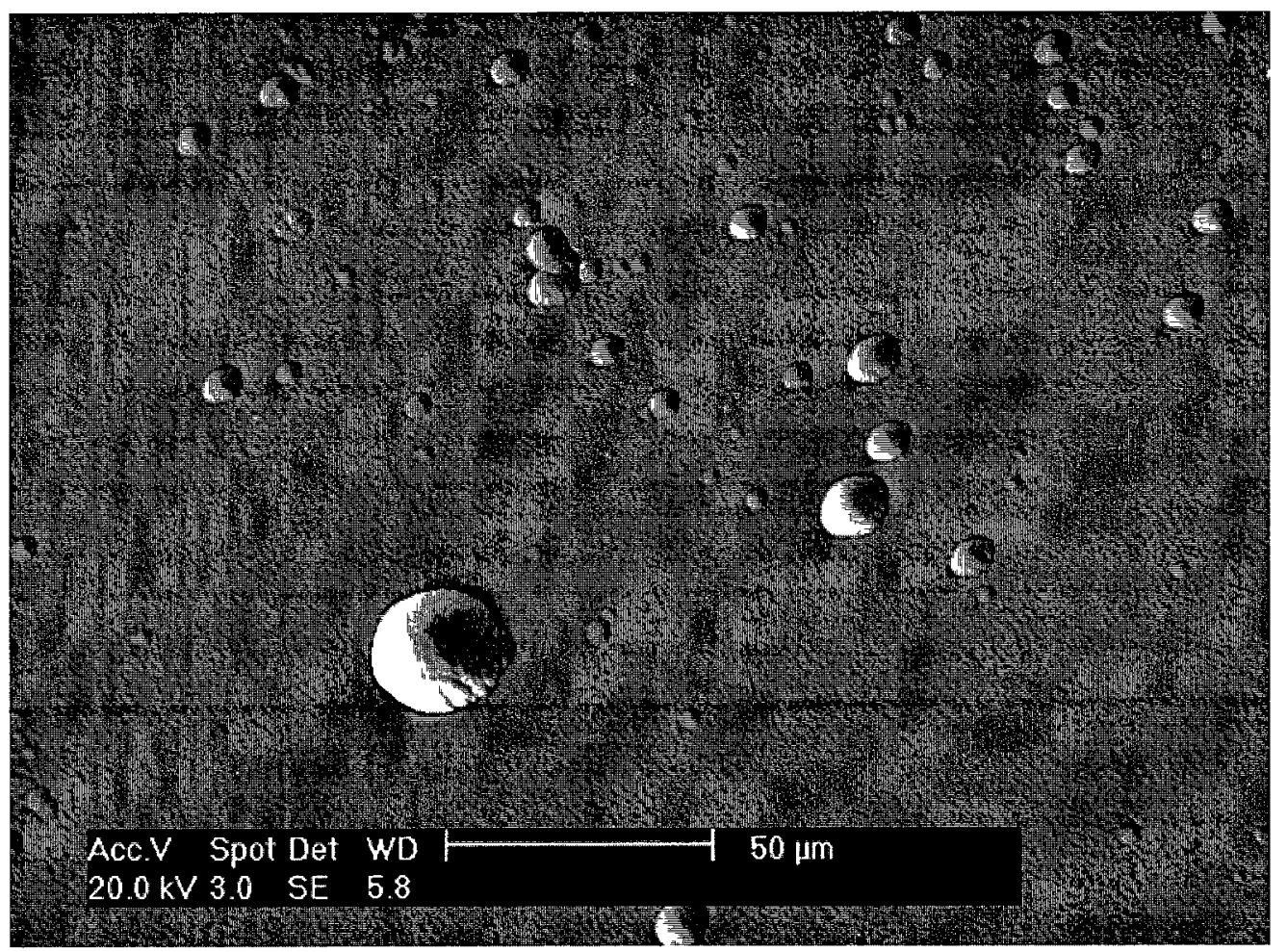

(c) Surface of $\mathrm{CrSiCN}(1)$ coating where no corrosion product was found after three days of immersion

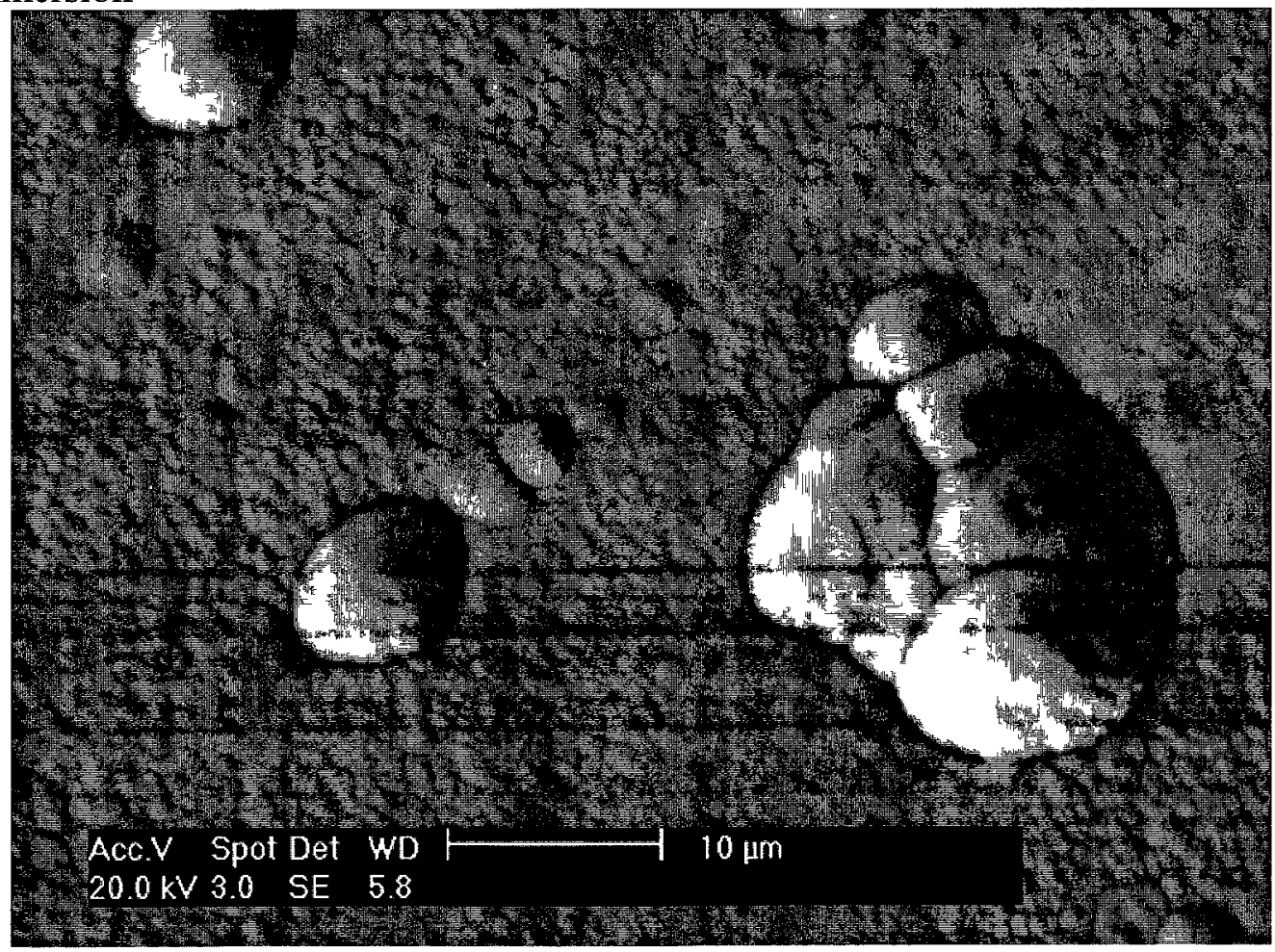

(d) Surface of $\mathrm{CrSiCN}(1)$ coating where no corrosion product was found after three days of immersion

Figure 4-43 to be continued 


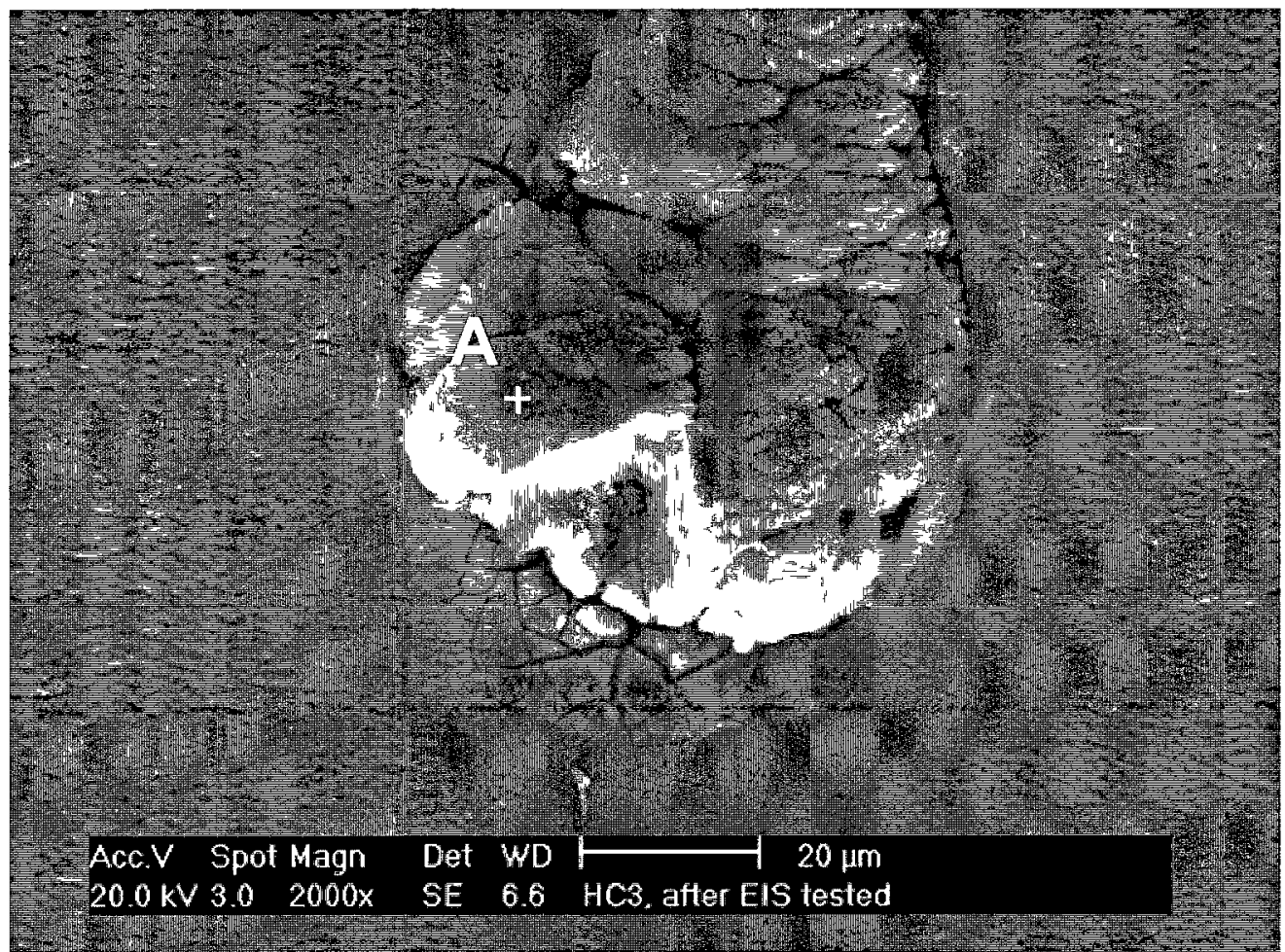

(e) Surface of CrSiCN(2) coating where corrosion product was found near a nodule after one day of immersion

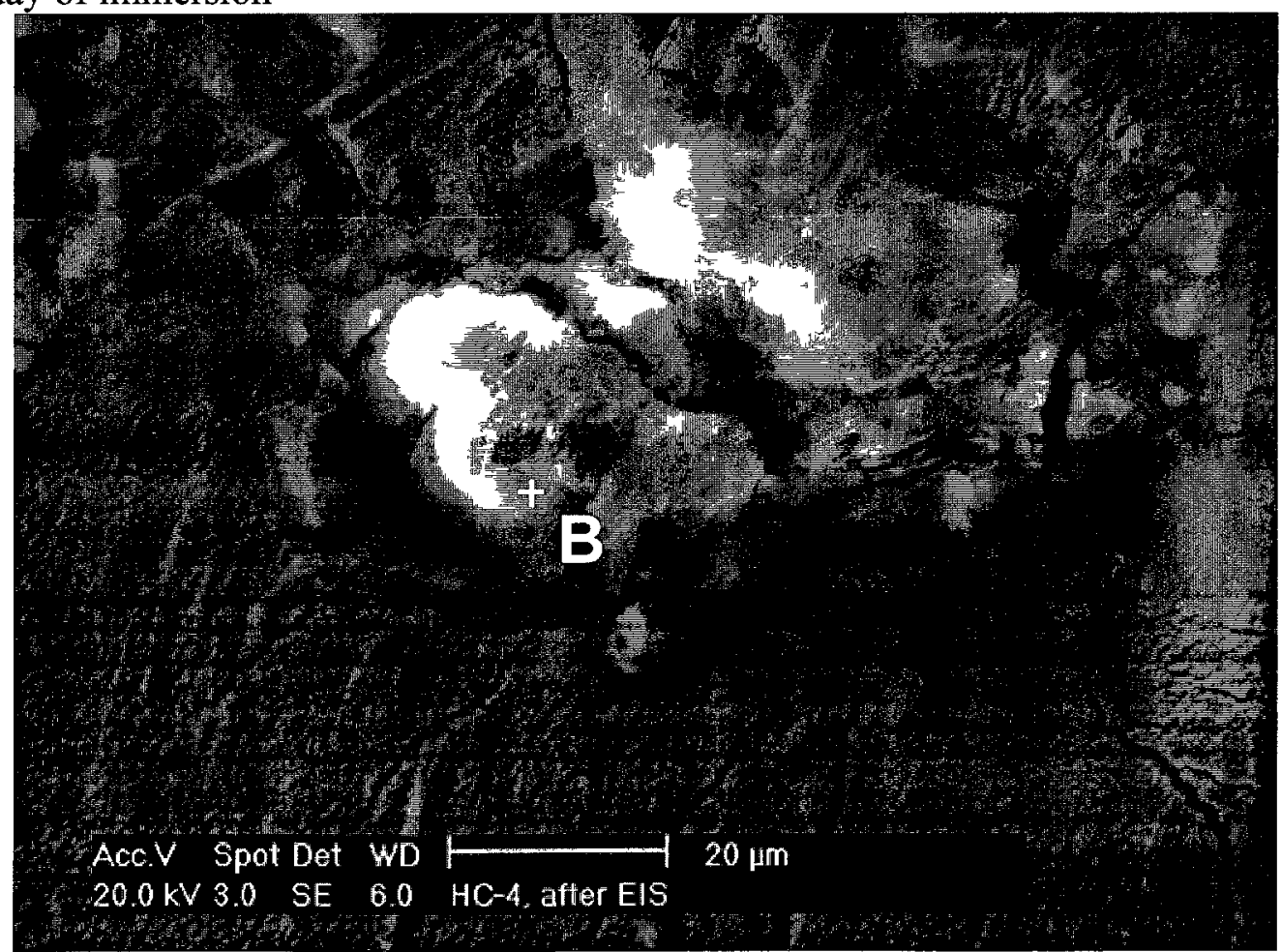

(f) Surface of $\mathrm{CrSiCN}(3)$ showing cracking in the coating due to the volume expansion associated with the formation of corrosion product in the defect after one day immersion

Figure 4-43 to be continued 


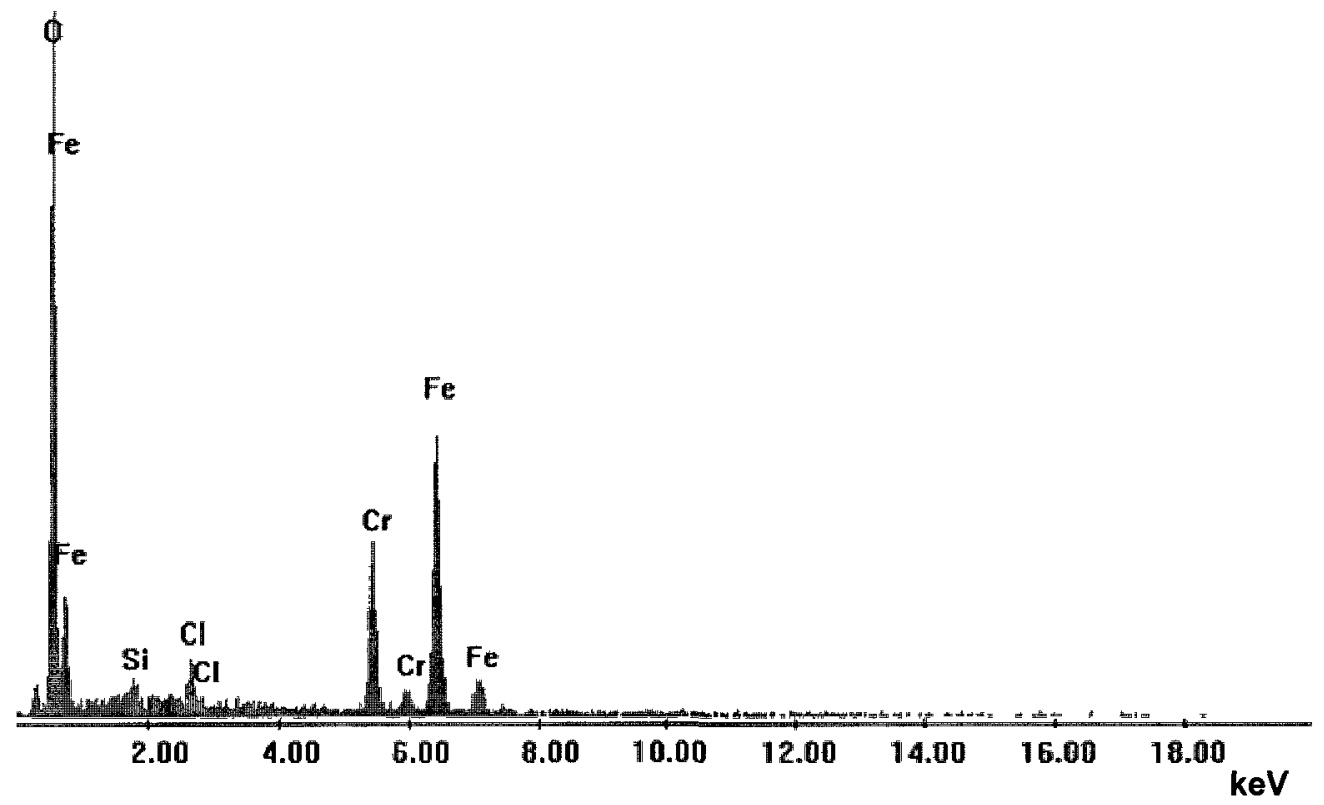

(g) EDS spectrum measured at "A" in (e)

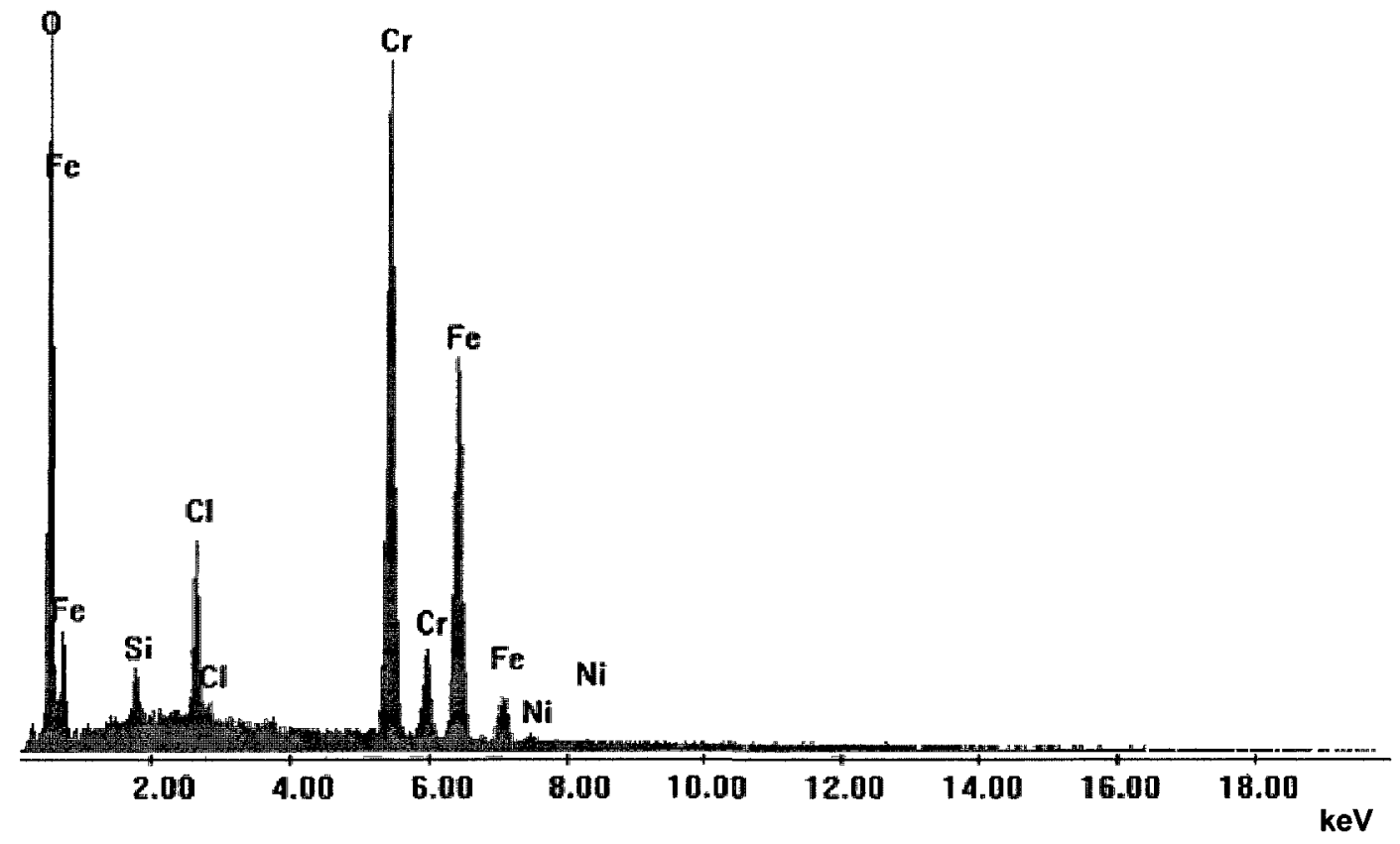

(h) EDS spectrum measured at " $B$ " in (f)

Figure 4-43 SEM/EDS analysis results for $\mathrm{CrN}$ based coatings after immersions. 


\subsubsection{Electrical Equivalent Circuit (EEC) Analysis}

In order to quantitatively investigate the electrochemical corrosion process at interface levels, electrical equivalent circuit (EEC) analyses were conducted using EIS test data on TiN and $\mathrm{CrN}$ based coating systems as well as substrate steels. These analyses further understand and explain corrosion mechanisms in the coating systems, verify, and better interpret the results from the short and long term immersion tests.

\subsubsection{Substrates}

Mild steel, because of its inability to passivate, is an electrochemically active material. Corrosion on mild steel takes the form of anodic dissolution, and results in a uniform macro-scale removal of materials, therefore its corrosion process is usually chargetransfer controlled. The constant phase element (CPE) circuit model (Figure 4-44) was employed in this study to analyze the corrosion mechanism on mild steel. The CPE model was developed from a Randle circuit [408], which consists of an electrolyte resistance $\left(R_{e}\right)$ in series with a parallel sub-circuit of an electrical double layer capacitance $\left(C_{d l}\right)$ and an impedance of a faradic reaction charge (normally called charge transfer, or generally referred as to polarization resistance, $R_{p}$ ).

The electrical double layer capacitance describes the surface charge at the electrolyteelectrode (substrate) interface. In an AC electrochemical system, the net contribution of polarization to the dielectric constant is frequency dependent. Therefore, to account for the non-ideal electrical capacitive behaviour, the double layer capacitance $\left(C_{d l}\right)$ is 
replaced with a CPE. The circuit description code (CDC) for the CPE model is $R_{e}(C P E$ $\left.c, R_{p}\right)$, and the impedance $Z$ in this model is described as:

$$
Z(\omega)=R_{e}+\frac{1}{\frac{1}{R_{p}}+\left(Y_{0}-Q_{c}\right)(j \omega)^{n-Q_{c}}}
$$

where

$R_{e}: \quad$ resistance of electrolyte,

$R_{p}: \quad$ polarization resistance,

$Y_{0}-Q_{c}: \quad$ (modulus of) electrochemical admittance of CPE,

$n-Q_{c}: \quad$ empirical exponent of CPE,

$\omega: \quad$ radial (angular) frequency of potential perturbation,

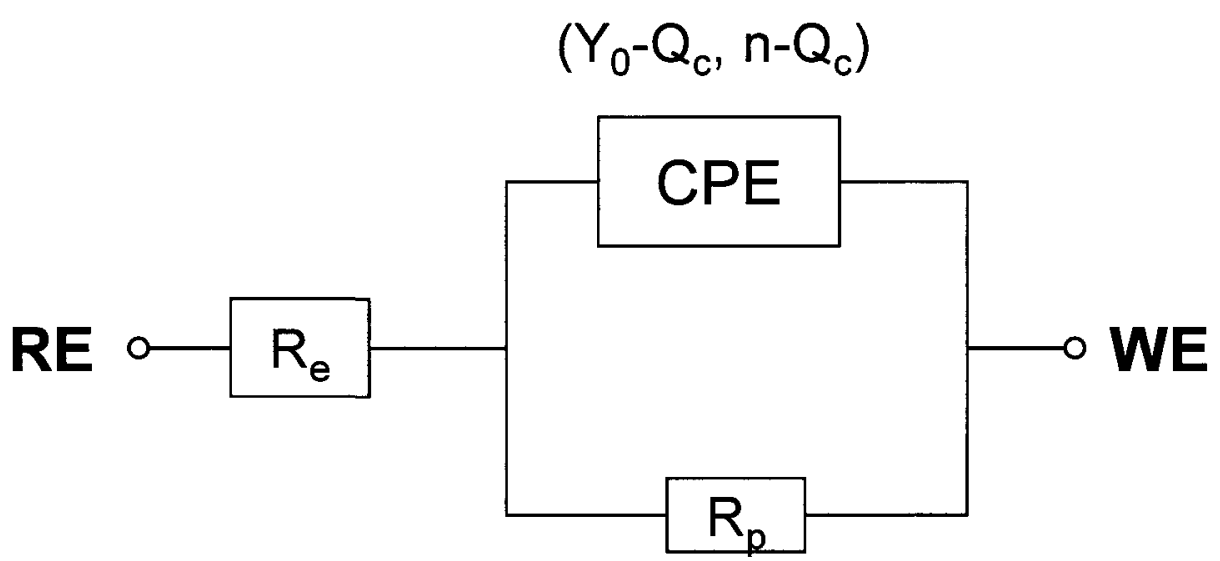

Figure 4-44 Schematic of CPE Model.

Applying the CPE model to a mild steel substrate, results in fitted curves as displayed in

Figure 4-45, and fitted data with fitting errors as summarized in Table 4-15. The fitting errors decreased with immersion time, from $36.5 \%$ (at five minute) to $12.85 \%$ (at one hour), and hence went to lower than the acceptable level of $10 \%$ (Table 4-15). Typical sources of errors in an EEC fitting include the use of incorrect EEC model, poor estimation of initial values and noise introduced [409]. In this study each EEC fitting was tried with various initial values to minimize poor estimations of initial values and noise levels. The attainment of acceptable errors (less than $10 \%$ after one hour) (Table 4-15) 
indicated that the applied EEC model is valid. High error (36.5\%) only occurred at short immersion time (five minute) when the test was still under a non-steady-state condition; and this can be evidenced by the relative low value of the empirical exponent of CPE ( $\mathrm{n}-$ Qs)(0.6780)(at five minute) (Table 4-15), which revealed different surface conditions in the initial period of immersion. These fitting results obtained by applying the CPE model to the mild steel confirmed a charge-transfer controlled corrosion process on the mild steel substrate.

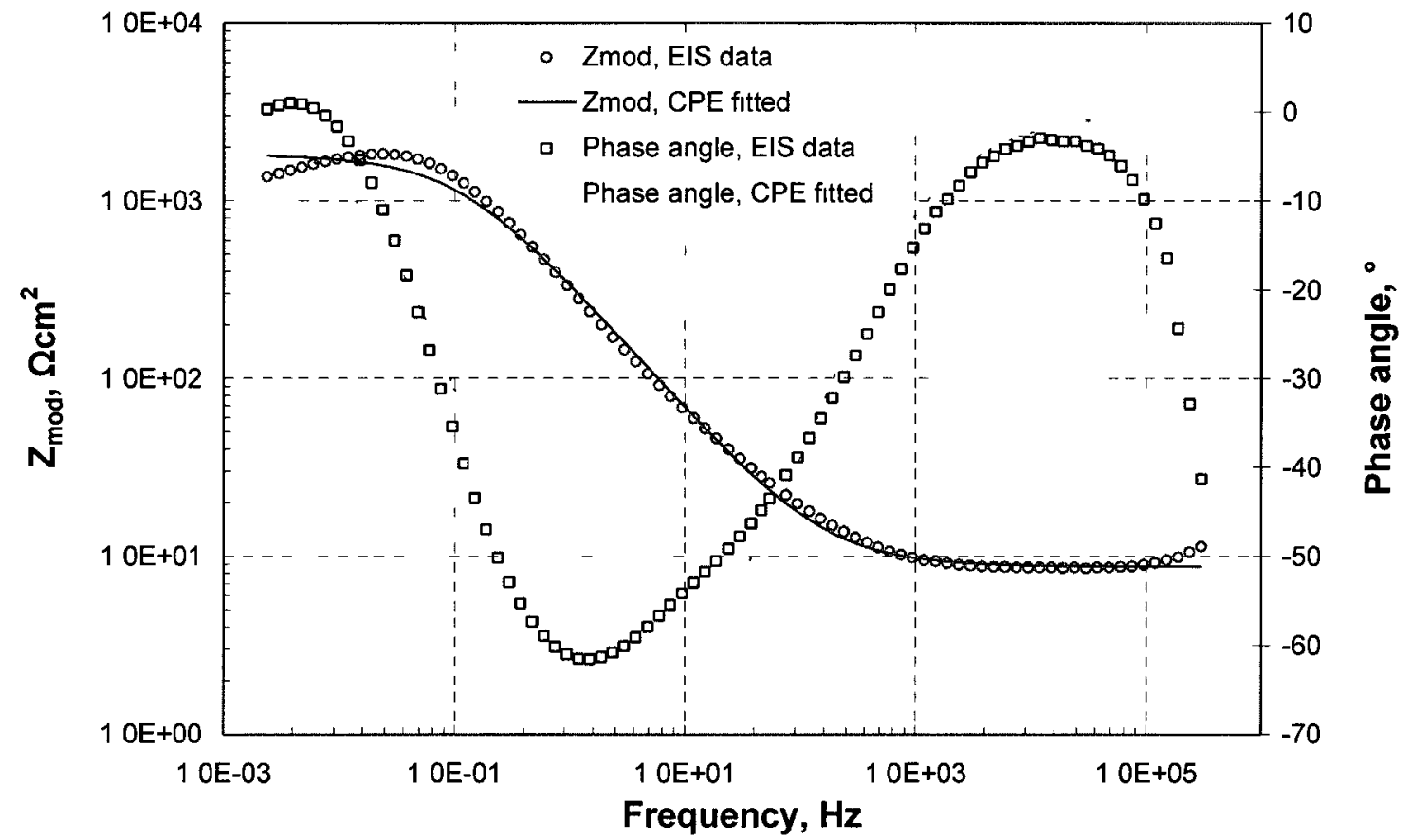

Figure 4-45 EEC fitting curves on MS substrate (CPE model) (at $5 \mathrm{hr}$ ).

Table 4-15 EEC fitted data for mild steel (MS) substrate (CPE)

\begin{tabular}{|l|c|c|c|c|c|}
\hline Time & $\mathrm{R}_{\mathrm{e}}\left(\Omega \mathrm{cm}^{2}\right)$ & $\mathrm{Y}_{0}-\mathrm{Q}_{\mathrm{s}}\left(\mu \mathrm{F} / \mathrm{cm}^{2}\right)$ & $\mathrm{n}-\mathrm{Q}_{\mathrm{s}}$ & $\mathrm{R}_{\mathrm{p}}\left(\mathrm{k} \Omega \mathrm{cm}^{2}\right)$ & Fitting error (\%) \\
\hline $5 \mathrm{~min}$ & 4.89 & 538.60 & 0.6780 & 0.059 & 36.51 \\
\hline $1 \mathrm{hr}$ & 9.13 & 555.41 & 0.8101 & 0.377 & 12.85 \\
\hline $3 \mathrm{hr}$ & 8.96 & 649.30 & 0.7695 & 1.470 & 3.98 \\
\hline $5 \mathrm{hr}$ & 8.74 & 704.33 & 0.7420 & 1.816 & 2.54 \\
\hline $24 \mathrm{hr}$ & 3.51 & 520.76 & 0.7397 & 0.236 & 6.81 \\
\hline
\end{tabular}


On stainless steel, unlike on mild steel, (due to the presence of a dense, passive and high dielectric constant oxide film on the surface of stainless), the mass transfers in the electrochemical corrosion process take the form of diffusion through the diffusion layer (Nernst diffusion layer - NDL)[410] at the electrolyte-steel interface to reach the steel surface. This diffusion process would create an impedance (referred as to the Warburg impedance, $W$ ) [405]. Therefore, to investigate the corrosion of coating systems on a stainless steel substrate, this diffusion impedance must be taken into account.

According to the boundary conditions of diffusion theory, the Warburg impedance corresponds to an infinite or semi-infinite length diffusion process [37, 405], which is similar to an unrestricted diffusion to an infinite planar electrode. The Warburg impedance is described by the Warburg equation [411]:

$$
Z_{W}=\sigma / \sqrt{\omega}-j \sigma / \sqrt{\omega}
$$

from which the modulus of Warburg impedance is given as:

$$
\left|Z_{W}\right|=\sqrt{2} \cdot \sigma / \sqrt{\omega}
$$

where,

$\omega$ : radial frequency of the potential perturbation,

$\sigma$ : Warburg coefficient, which is determined by diffusion coefficients of oxidant and reductant, surface area of the electrode, and concentration of the diffusing species, and is expressed as [411]:

$$
\sigma=\frac{R T}{z^{2} F^{2} A \sqrt{2}}\left(\frac{1}{C_{O} \sqrt{D_{O}}}+\frac{1}{C_{R} \sqrt{D_{R}}}\right)
$$

where,

$D_{O}$ : diffusion coefficient of oxidant;

$D_{R}$ : diffusion coefficient of reductant;

$A$ : surface area of the electrode;

$F$ : Faraday's constant, $F=96,485$ Coulombs;

$z$ : number of electrons transferred;

$C_{O}$ : bulk concentration of the diffusing oxidant;

$C_{R}$ : bulk concentration of the diffusing reductant. 
Eq. (4-4) indicates that theWarburg impedance is inversely related to the diffusion coefficients of oxidant and reductant, and the concentrations of species in the electrolyte.

In a Warburg plot (impedance vs. $1 / \omega^{1 / 2}$ ), the Warburg coefficient $(\sigma)$ represents the slopes of real and imaginary impedances [387, 411], and is written as:

$$
\sigma=1 /\left(\sqrt{2} \cdot Y_{0}\right)
$$

where, $Y_{0}$ : admittance

therefore, Eq. (4-3) can be written as:

$$
Z_{W}=1 /\left(Y_{0} \sqrt{j \omega}\right)
$$

Eq. (4-6) describes the correlation between Warburg impedance $\left(Z_{W}\right)$ and admittance $\left(Y_{0}\right)$; and indicates that Warburg impedance is dependant on the frequency of potential perturbation. In high frequencies $(\omega \rightarrow \infty)$, the modulus of the Warburg impedance is low, thus the electrolyte resistance $\left(R_{e}\right)$ dominates; at low frequencies $(\omega \rightarrow 0)$, the modulus of the Warburg impedance is high, and dominates. To take this impedance into account, a diffusion impedance element (Warburg impedance, $W$ ) is introduced into the CPE circuit, being in series with the charge transfer resistance (or in general polarization resistance, $R_{p}$ ), to account for the infinite or semi-infinite length diffusion of the charging species [412] (Figure 4-46 a). 
This EEC is termed the OtcWar model, describing an electrochemical system, where polarization combines with the kinetic and diffusion processes. The CDC for the OtcWar model is $R_{e}\left(C P E-c\left(R_{p}, W\right)\right)$, and the impedance is described as:

$$
Z=R_{e}+\frac{1}{\left(Y_{0}-Q_{c}\right)(j \omega)^{n-Q_{c}}+\frac{1}{R_{p}+\frac{1}{Y_{0} \sqrt{j \omega}}}}
$$

where,

$R_{e}:$ resistance of electrolyte,

$R_{p}$ : polarization resistance,

$Y_{0}-Q_{c}$ : admittance of CPE for coating,

$n-Q_{c}$ : empirical exponent of CPE for coating,

However, the Warburg impedance $(W)$ (Eq. 4-2) is only valid for an infinite or semiinfinite length diffusion process (corresponding to an infinite or semi-infinite thickness diffusion layer). As for a finite length diffusion process (corresponding to a bounded diffusion layer), Eq. (4-2) is no longer valid, especially at low frequencies $(\omega \rightarrow 0)$; thus the finite length diffusion impedance is then described as [413]:

$$
Z_{O}(\omega)=\left[1 /\left(Y_{0} \sqrt{j \omega}\right)\right] \cdot \tanh [B \sqrt{j \omega}]
$$

or $\quad Z_{o}(\omega)=Z_{W} \cdot \tanh [B \sqrt{j \omega}]$

where

$\omega:$ angular frequency of potential perturbation,

$B$ : characteristic diffusion parameter [413], which characterizes the diffusion time for a reactant to diffuse through the diffusion layer [38], and is defined as:

$$
B=\delta / \sqrt{D}
$$

where,

$D$ : diffusion coefficient (area/time);

$\delta$ : diffusion length in general [413]; with respect to a diffusion layer, it represents the thickness of the diffusion layer [414] (referred as to Nernst diffusion layer NDL)[387]; in the case as a presence of a coating, it approximates the thickness of the coating [38]. 
From Eq. (4-3) and Eq. (4-9), the modulus of OFLD (Open Finite-Length Diffusion) impedance is:

$$
\left|Z_{o}\right|=\left|Z_{W}\right| \cdot\left|\tanh \left[\frac{\delta}{\sqrt{D}} \sqrt{j \omega}\right]\right|
$$

The equations (Eq. 4-8, Eq. 4-8 a, and Eq. 4-8 b) describe the finite length impedance (referred as to Open Finite-Length Diffusion (OFLD) element, abbreviated as " $O$ ") (also referred as to Nernst circuit element) and indicates that OFLD impedance is a function of frequency, diffusion coefficient and diffusion length. At a given frequency, $\left|Z_{O}\right|$ would decrease due to either a high diffusion coefficient $(D)$ or a thin diffusion layer $(\delta)$, conversely, $\left|Z_{O}\right|$ would increase due to either a low diffusion coefficient $(D)$ or a thick diffusion layer $(\delta)$.

In an extreme case as either $D \rightarrow 0$ (no diffusion) or $\delta \rightarrow \infty$ (infinite length diffusion) the item $|\tanh [(\delta / \sqrt{ } D)(\sqrt{ } j \omega)]| \rightarrow 1$, thus $\left|Z_{O}\right| \rightarrow\left|Z_{W}\right|$ (which is the infinite length diffusion impedance $W$; note that as $D \rightarrow 0, \sigma \rightarrow \infty$, and $\left.\left|Z_{W}\right| \rightarrow \infty\right)$. Another extreme case is that as either diffusion coefficient $D \rightarrow \infty$ (no resistance to diffusion across a diffusion layer, which means the mass transfer is not in the form of diffusion) or diffusion layer thickness $\delta \rightarrow 0$ (no diffusion layer exists), the item $|\tanh [(\delta / \sqrt{ } D)(\sqrt{ } j \omega)]| \rightarrow 0$, thus $\left|Z_{O}\right| \rightarrow 0$, indicating that the corrosion process is not diffusion-controlled.

The EEC model which describes this OFLD diffusion is called Otc-Cot-Hyper model. A typical schematic for Otc-Cot-Hyper model is illustrated in Figure 4-46 (b), its CDC is $R_{e}\left(C P E-c\left(R_{p}, O\right)\right)$, and the impedance is described as: 


$$
Z=R_{e}+\frac{1}{\left(Y_{0}-Q_{c}\right)(j \omega)^{n-Q_{c}}+\frac{1}{R_{p}+\frac{1}{Y_{0} \sqrt{j \omega} \cdot \operatorname{coth}[B \sqrt{j \omega}]}}}
$$

where,

$R_{e}$ : resistance of electrolyte,

$R_{p}$ : polarization resistance,

$Y_{0}-Q_{c}$ : admittance of $\mathrm{CPE}$ for coating,

$n-Q_{c}$ : empirical exponent of CPE for coating.

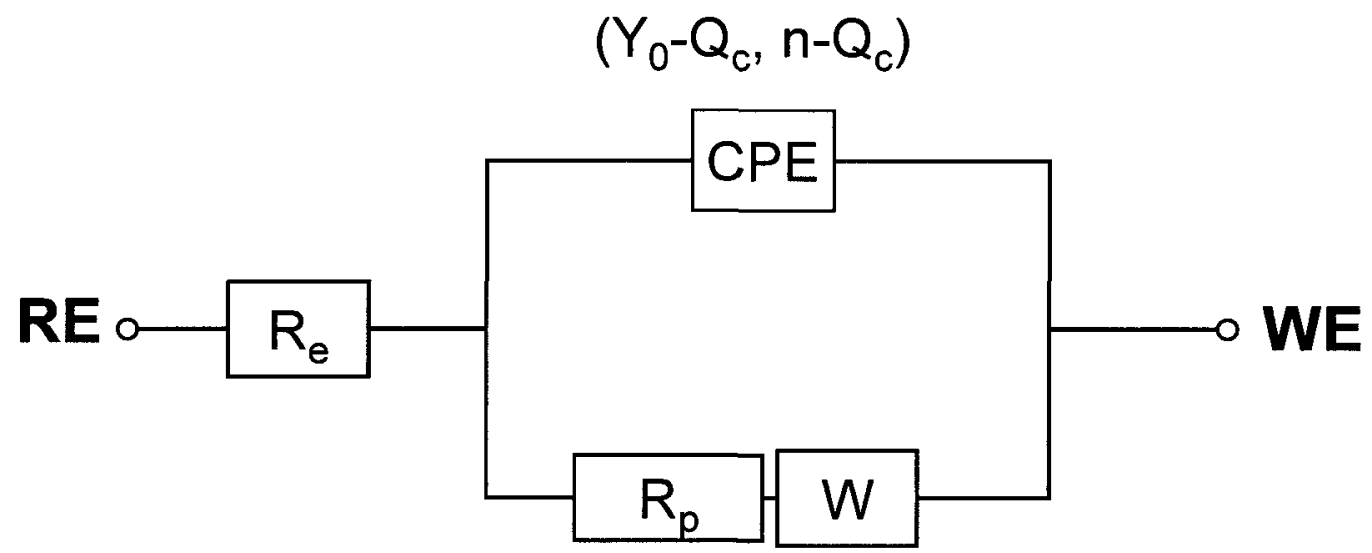

(a) $\mathrm{OtcWar}$

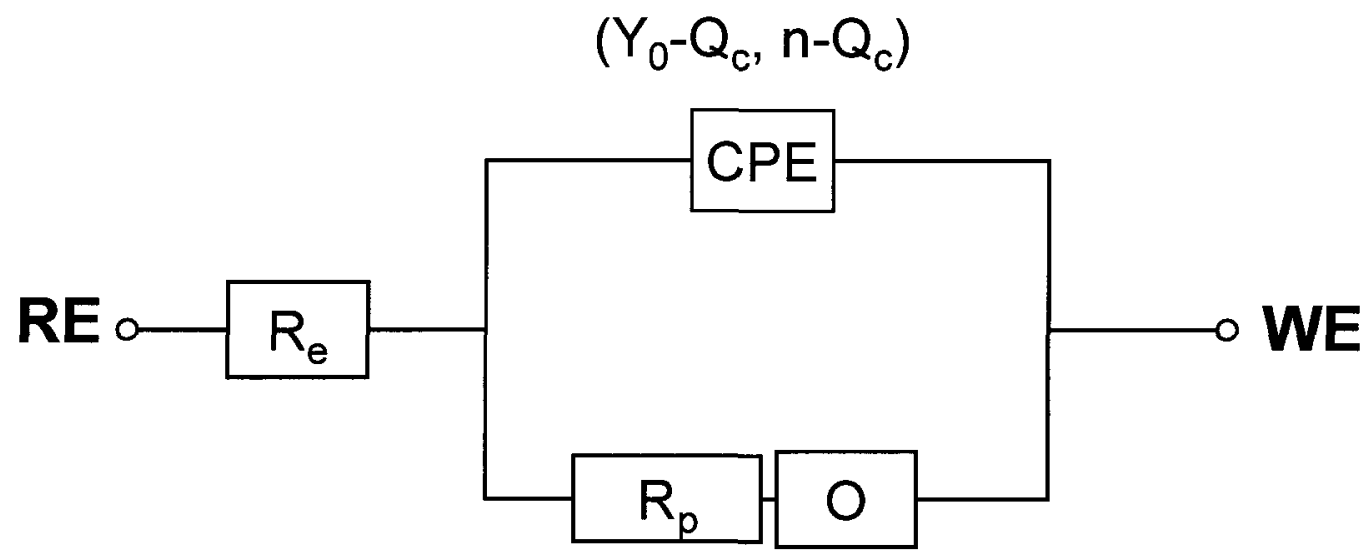

(b) Otc-Cot-Hyper

Figure 4-46 Schematic of EEC models for diffusion controlled corrosions. 
Whereas, the OFLD element $(O)$, in addition to the angular frequency of the potential perturbation $(\omega)$, is also sensitive to the diffusion factors such as the Nernst diffusion layer (NDL) thickness $(\delta)$, and the diffusion coefficient $(D)$, which are dependent on the coating microstruture. Therefore, for application to corrosion in coating/substrate systems, the OtcWar model (with Warburg diffusion element $(W)$ ) (Eq. 4-7) (Figure 4-46 a) was proposed to describe an infinite or semi-infinite length diffusion process that is an unrestricted or less restricted diffusion through the pore defects in the coating to a passivated substrate steel (e.g., stainless steel). This coating usually has a columnar grain microstructure that contains more open (through coating) pores and straight grain boundaries, such as TiN based coating systems [346]. The Otc-Cot-Hyper model (with the OFLD element $(O)$ ) (Eq. 4-10) (Figure 4-46 b) was suggested for a bounded diffusion that is microscopically confined within pores or grain boundaries, for example in $\mathrm{CrN}$ based coatings that have an equiaxed grain microstructure [346, 387].

As for the bare 17-4PH steel substrate, the corrosion process on the surface can be treated as unrestricted diffusion governed. Thus in this study the OtcWar model was applied to the 17-4PH steel substrate to investigate its corrosion mechanism, which will be used as a baseline for the further studies of the TiN and $\mathrm{CrN}$ based coating systems. The fitted curves for 17-4PH steel using the OtcWar model are shown in Figure 4-47, and the fitted data with fitting errors are displayed in Table 4-16. 


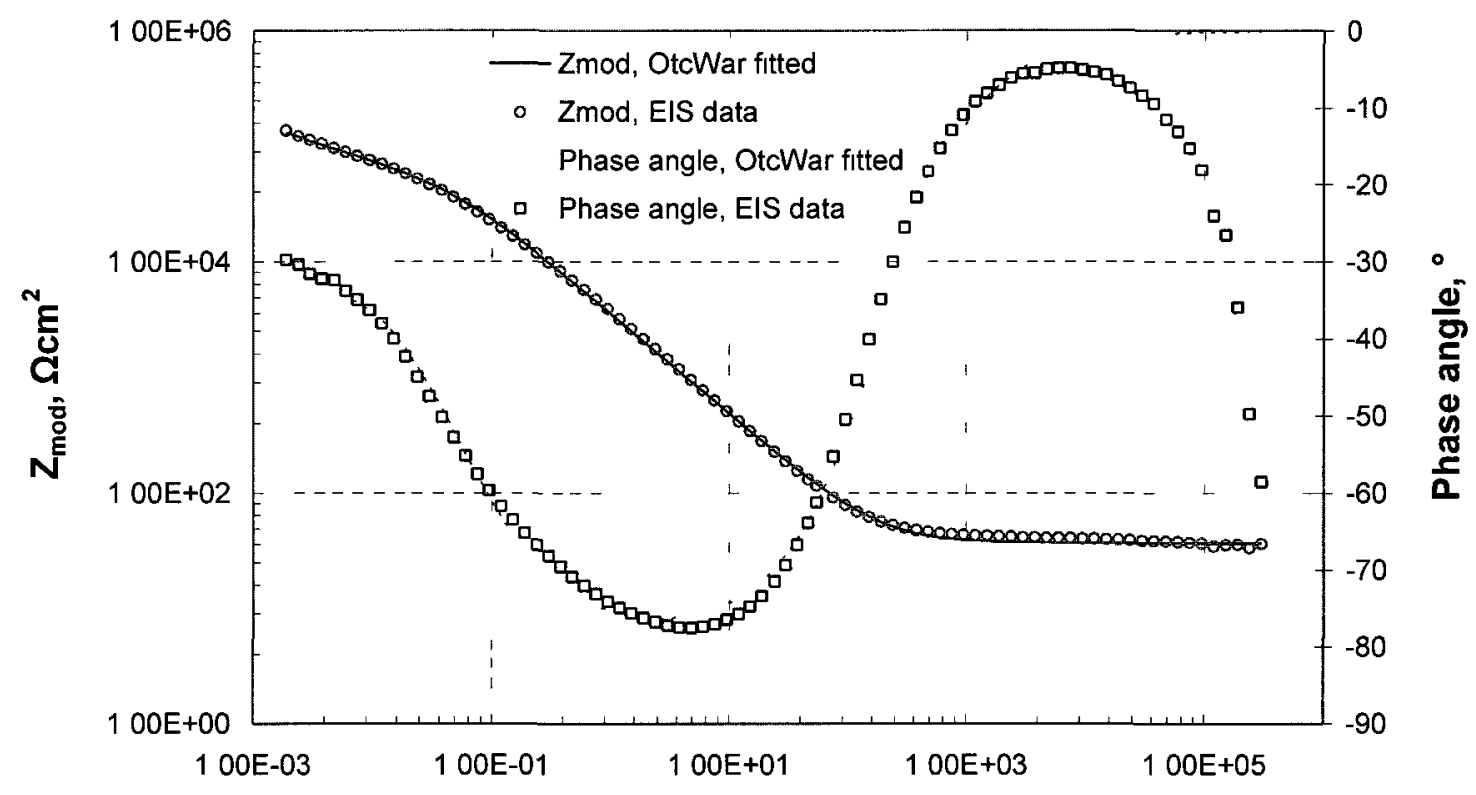

Frequency, $\mathrm{Hz}$

Figure 4-47 EEC fitting curves on 17-4PH steel substrate (OctWar model) (at $5 \mathrm{hr}$ ).

Table 4-16 EEC fitted data for 17-4PH steel substrate (OctWar)

\begin{tabular}{|l|c|c|c|c|c|c|}
\hline Time & $\begin{array}{c}\mathrm{R}_{\mathrm{e}} \\
\left(\Omega \mathrm{cm}^{2}\right)\end{array}$ & $\begin{array}{c}\mathrm{Y}_{0}-\mathrm{Q}_{\mathrm{s}} \\
\left(\mu \mathrm{F} / \mathrm{cm}^{2}\right)\end{array}$ & $\mathrm{n}-\mathrm{Q}_{\mathrm{s}}$ & $\begin{array}{c}\mathrm{R}_{\mathrm{p}} \\
\left(\mathrm{k} \Omega \mathrm{cm}^{2}\right)\end{array}$ & $\begin{array}{c}\mathrm{Y}_{0}-\mathrm{W} \\
\left(\mu / \mathrm{cm}^{2} \Omega \sqrt{s}\right)\end{array}$ & $\begin{array}{c}\text { Fitting error } \\
(\%)\end{array}$ \\
\hline $5 \mathrm{~min}$ & 24.95 & 58.50 & 0.7818 & 27.554 & 96.34 & 3.39 \\
\hline $1 \mathrm{hr}$ & 26.87 & 56.03 & 0.8738 & 35.254 & 110.03 & 3.02 \\
\hline $3 \mathrm{hr}$ & 37.10 & 55.68 & 0.8789 & 46.441 & 112.69 & 2.32 \\
\hline $5 \mathrm{hr}$ & 36.75 & 55.21 & 0.8779 & 61.301 & 94.28 & 3.80 \\
\hline $24 \mathrm{hr}$ & 25.27 & 51.73 & 0.8589 & 127.249 & 35.55 & 8.86 \\
\hline
\end{tabular}

From the fitted data (Table 4-15 and Table 4-16), it can be seen that both mild steel and 17-4 PH steel showed stable values of electrolyte resistance $\left(R_{e}\right)$ throughout the immersion. Comparing polarization resistance $\left(R_{p}\right)$ (Table 4-15 and Table 4-16), it can be observed that the values for 17-4PH steel $\left(\sim 40 \mathrm{k} \Omega \mathrm{cm}^{2}\right)($ Table 4-16) were up to three orders of magnitude higher than those of the mild steel $\left(0.06\right.$ to $\left.1.8 \mathrm{k} \Omega \mathrm{cm}^{2}\right)$; this result was in agreement with the potentiodynamic polarization tests (Table 4-12). 
The admittance constant $\left(Y_{0}-Q_{s}\right)$ reflects the capacitive characteristics of an electrolyteelectrode (steel) interface; and the empirical exponent of CPE $\left(n-Q_{s} \leq 1\right)$ is associated with surface morphology of the electrode (steel) [346]. In this study, the values in $\left(Y_{O^{-}} Q_{s}\right)$ for

the 17-4PH steel $\left(\sim 55 \mu \mathrm{F} / \mathrm{cm}^{2}\right)$ (Table 4-16) were lower (by an order of magnitude) than those for the mild steel $\left(\sim 550 \mu \mathrm{F} / \mathrm{cm}^{2}\right)$ (Table 4-15), indicating that $17-4 \mathrm{PH}$ has a less porous surface feature [346]. The lower values of the exponent $\left(n-Q_{\mathrm{s}}\right)(\sim 0.75)($ Table 415) for the mild steel indicated its less capacitive electrolyte-surface interfacial characteristics [346]. The higher (closer to 1) and stable values of $\left(n-Q_{s}\right)(\sim 0.85)$ (Table 4-16) for the $17-4 \mathrm{PH}$ steel revealed the stable capacitance of its electrolyte-surface interface and can be attributed to it passivation.

\subsubsection{Titanium Nitride (TiN) Coating Systems}

\section{TiN/SS Coating Systems}

As discussed in the preceding section, due to the dense passive and high dielectric constant oxide film on the surface, diffusion is the controlling factor for the electrochemical mass transfer. TiN/SS systems such as EB and CA TiN/SS in this study, due to their combination of both a columnar coating structure and passive substrate, were expected to undergo (simi-) infinite length diffusion (Warburg diffusion) controlled corrosion processes. Therefore the OtcWar model (Figure 4-46 a) was applied to the EB and CA TiN/SS coating systems and their PMMA-treated counterparts. The fitted curves are shown in Figure 4-48 and Figure 4-49, and the fitted data with fitting errors are displayed in Table 4-17 to Table 4-18, respectively. 


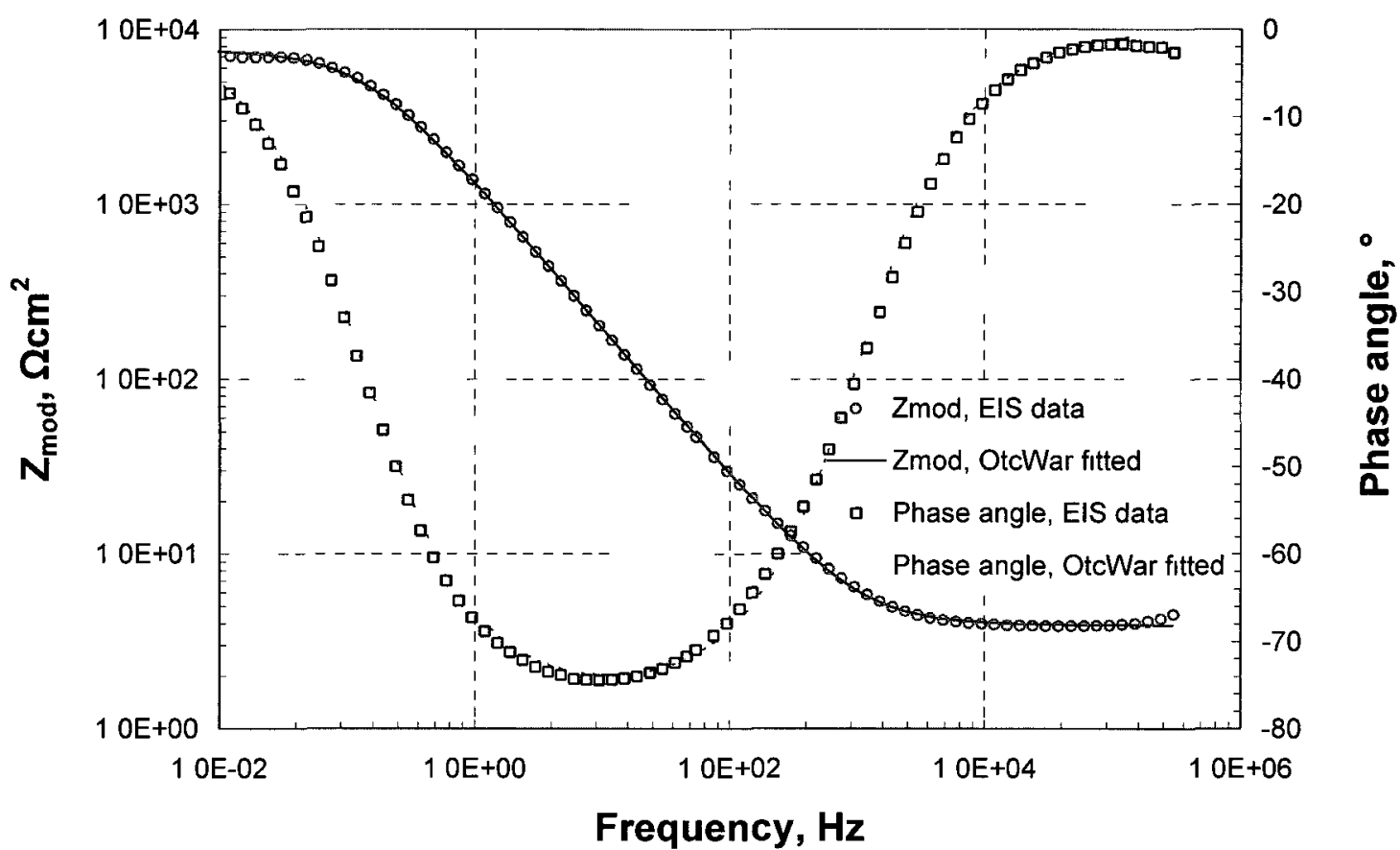

(a) EB TiN/SS

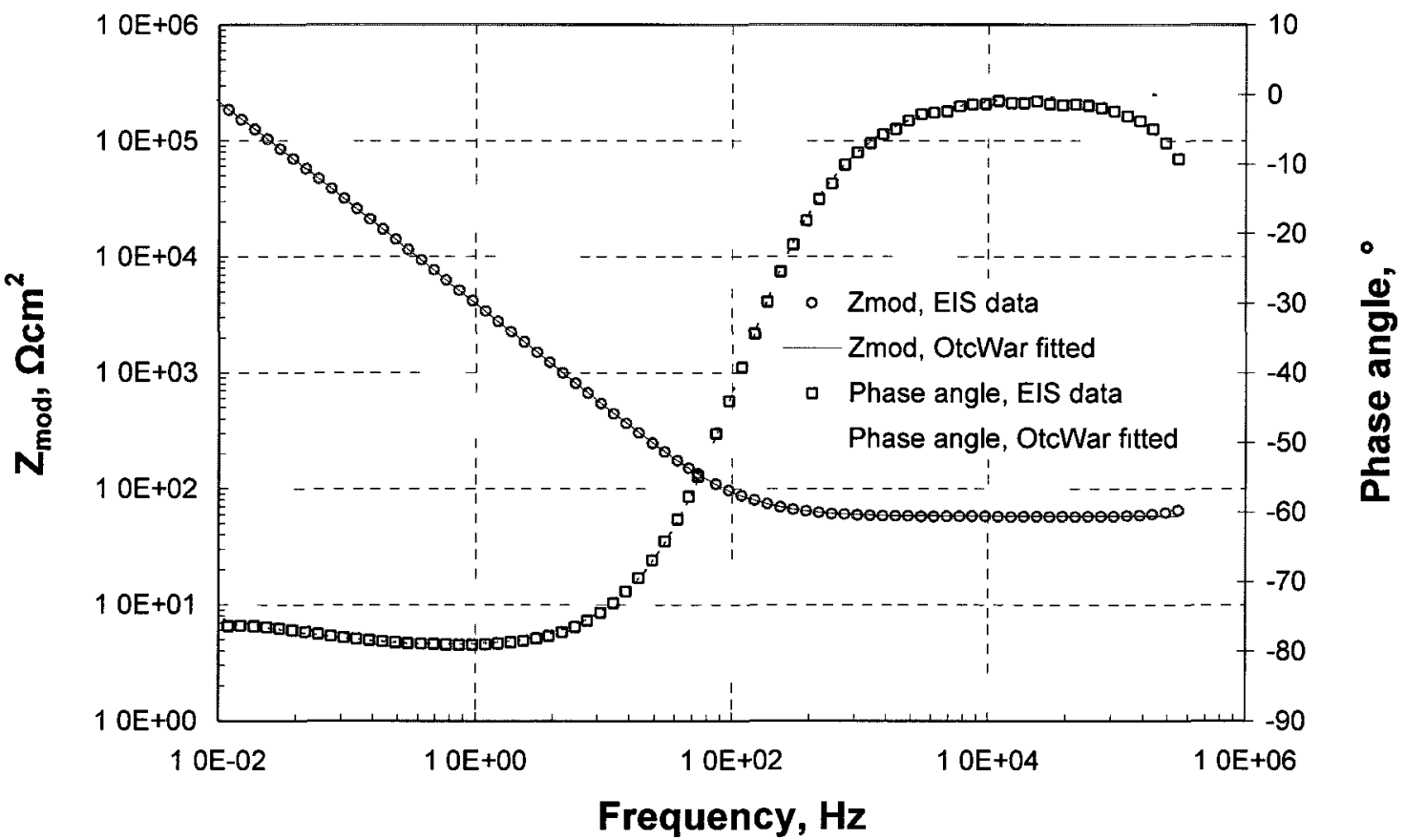

(b) PMMA EB TiN/SS

Figure 4-48 EEC fitting curves on EB TiN/SS coating systems (OtcWar model)(at $5 \mathrm{hr}$ ). 


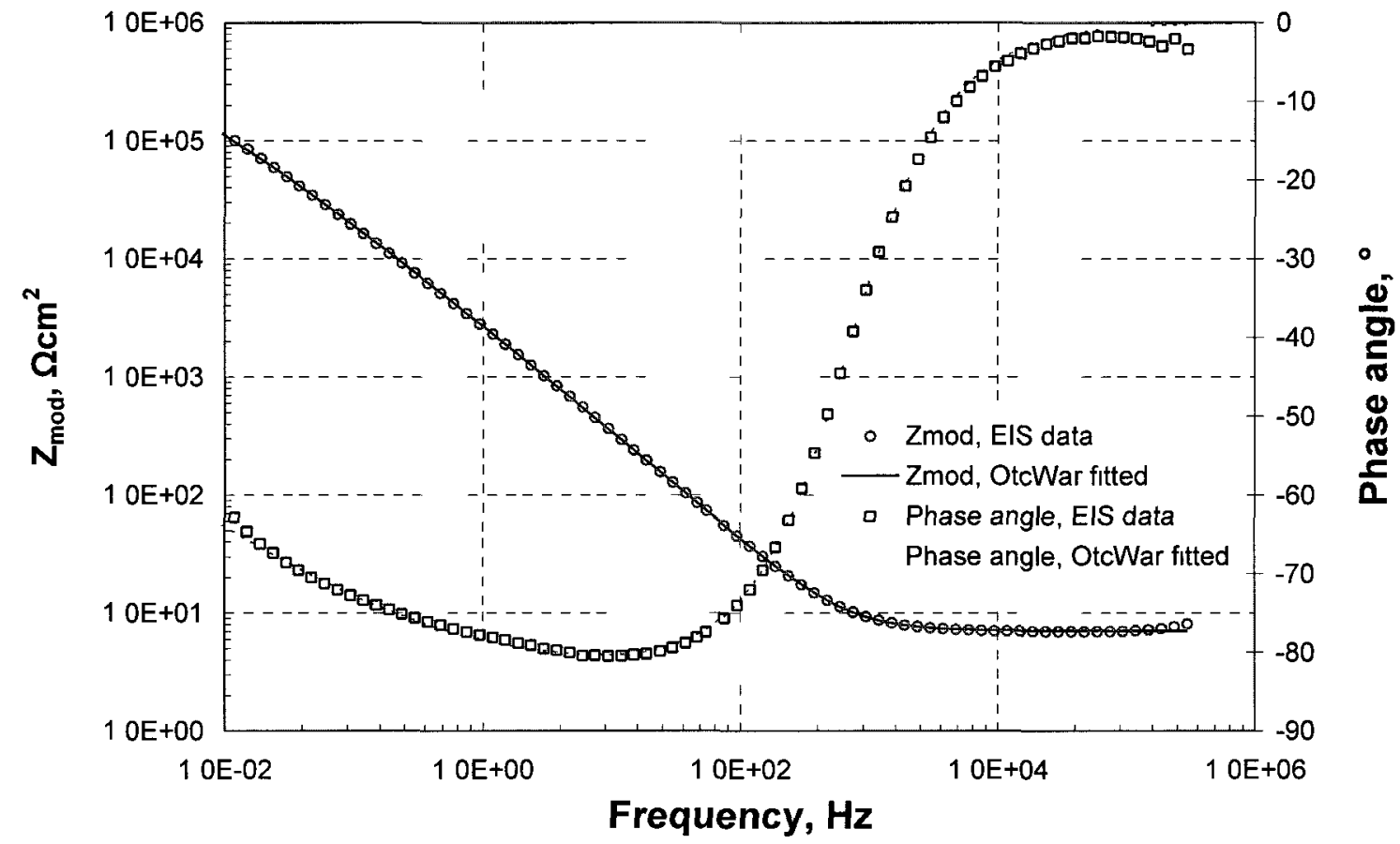

(a) $\mathrm{CA}$ TiN/SS

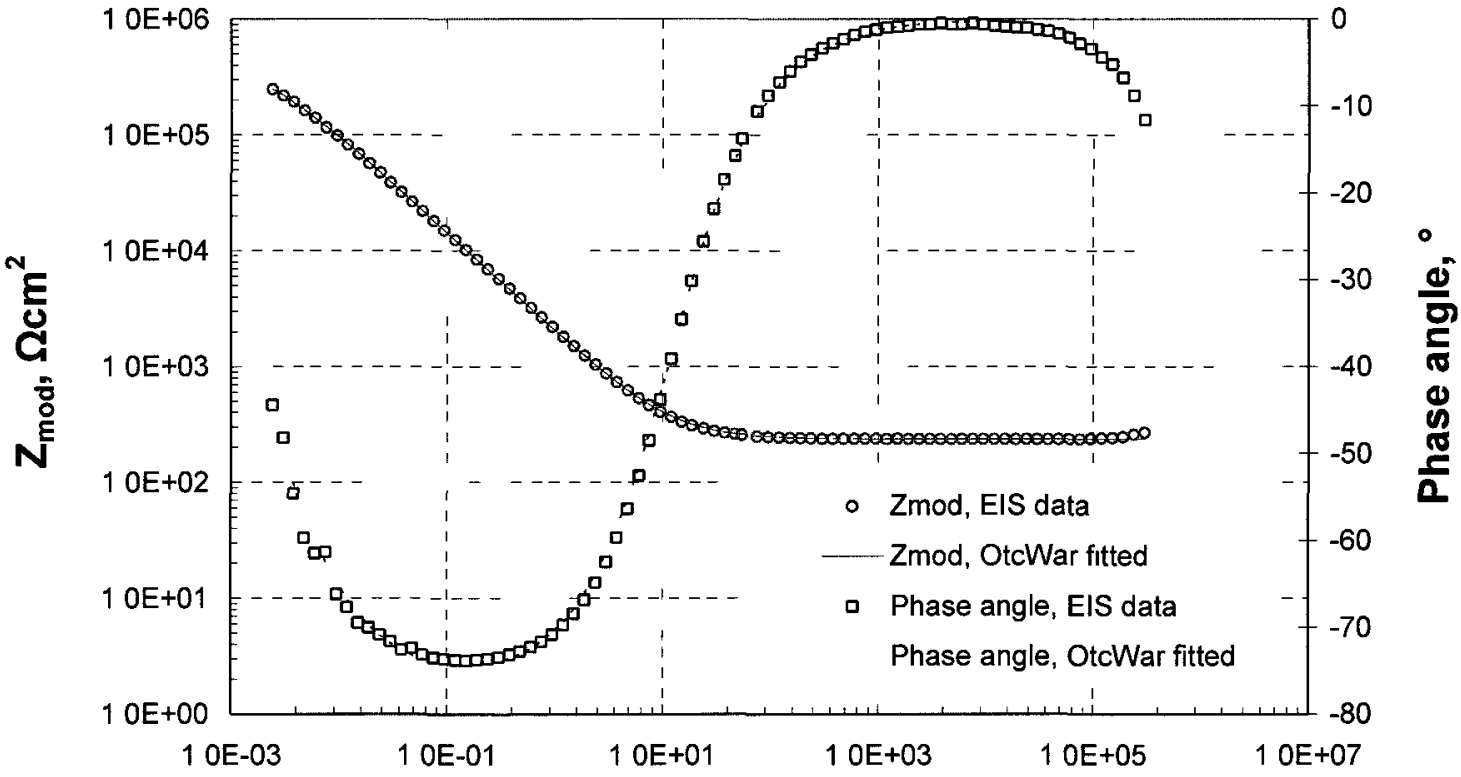

Frequency, $\mathrm{Hz}$

(b) PMMA CA TiN/SS

Figure 4-49 EEC fitting curves on CA TiN/SS coating systems (OtcWar model)(at $5 \mathrm{hr}$ ). 
Table 4-17 EEC fitted data for EB TiN/SS coating systems (OctWar)

\begin{tabular}{|c|c|c|c|c|c|c|}
\hline Time & $\begin{array}{c}\mathrm{R}_{\mathrm{e}} \\
\left(\Omega \mathrm{cm}^{2}\right)\end{array}$ & $\begin{array}{c}Y_{0}-Q_{c} \\
\left(\mu F / \mathrm{cm}^{2}\right)\end{array}$ & $n-Q_{c}$ & $\begin{array}{c}\mathrm{R}_{\mathrm{p}} \\
\left(\mathrm{k} \Omega \mathrm{cm}^{2}\right)\end{array}$ & $\begin{array}{c}Y_{0}-W \\
\left(\mu / \mathrm{cm}^{2} \Omega V_{s}\right)\end{array}$ & $\begin{array}{c}\text { Fitting error } \\
(\%)\end{array}$ \\
\hline \multicolumn{7}{|c|}{ EB TiN/SS } \\
\hline $1 \mathrm{hr}$ & 3.93 & 135.92 & 0.8297 & 36.770 & 1377.07 & 0.09 \\
\hline $3 \mathrm{hr}$ & 3.92 & 146.75 & 0.8449 & 27.130 & 2085.35 & 0.07 \\
\hline $24 \mathrm{hr}$ & 4.09 & 152.87 & 0.8815 & 3.981 & 54101.9 & 0.15 \\
\hline $48 \mathrm{hr}$ & 3.82 & 144.71 & 0.8875 & 3.866 & 30509.6 & 0.15 \\
\hline $120 \mathrm{hr}$ & 4.21 & 138.47 & 0.8867 & 5.506 & 16101.9 & 0.15 \\
\hline $168 \mathrm{hr}$ & 5.19 & 323.06 & 0.7606 & 7.655 & 1290.45 & 0.38 \\
\hline $192 \mathrm{hr}$ & 3.71 & 259.75 & 0.7929 & 5.860 & 949.936 & 0.49 \\
\hline $216 \mathrm{hr}$ & 3.79 & 234.01 & 0.8049 & 5.968 & 947.516 & 0.45 \\
\hline $315 \mathrm{hr}$ & 4.04 & 283.95 & 0.7620 & 6.003 & 1394.9 & 0.65 \\
\hline \multicolumn{7}{|c|}{ PMMA EB TiN/SS } \\
\hline $5 \mathrm{~min}$ & 57.82 & 56.36 & 0.8845 & 4.178 & 1.176 & 0.10 \\
\hline $3 \mathrm{hr}$ & 56.50 & 51.82 & 0.8887 & 469.509 & 1.722 & 0.10 \\
\hline $5 \mathrm{hr}$ & 57.87 & 48.60 & 0.8935 & 181.178 & 2.804 & 0.11 \\
\hline $24 \mathrm{hr}$ & 57.45 & 42.06 & 0.8995 & 157.079 & 3.519 & 0.10 \\
\hline $48 \mathrm{hr}$ & 55.98 & 42.15 & 0.8939 & 186.281 & 2.208 & 0.21 \\
\hline $72 \mathrm{hr}$ & 55.52 & 47.92 & 0.8689 & 1370.61 & 0.588 & 0.16 \\
\hline $144 \mathrm{hr}$ & 52.29 & 46.40 & 0.8688 & 736.723 & 0.913 & 0.18 \\
\hline $168 \mathrm{hr}$ & 52.51 & 43.85 & 0.8699 & 1044.05 & 0.570 & 0.17 \\
\hline $192 \mathrm{hr}$ & 54.56 & 44.64 & 0.8705 & 880.77 & 0.800 & 0.20 \\
\hline $221 \mathrm{hr}$ & 51.43 & 42.18 & 0.8792 & 506.718 & 2.800 & 0.10 \\
\hline $240 \mathrm{hr}$ & 52.86 & 42.50 & 0.8705 & 1226.17 & 0.565 & 0.14 \\
\hline $336 \mathrm{hr}$ & 54.42 & 40.80 & 0.8719 & 1192.42 & 0.584 & 0.15 \\
\hline $360 \mathrm{hr}$ & 53.42 & 40.62 & 0.8721 & 1192.42 & 0.595 & 0.17 \\
\hline $384 \mathrm{hr}$ & 56.69 & 41.53 & 0.8712 & 676.042 & 0.986 & 0.16 \\
\hline
\end{tabular}

Table 4-18 EEC fitted data for CA TiN/SS coating systems (OtcWar)

\begin{tabular}{|c|c|c|c|c|c|c|}
\hline Time & $\begin{array}{c}R_{e} \\
\left(\Omega \mathrm{cm}^{2}\right)\end{array}$ & $\begin{array}{c}Y_{0}-Q_{c} \\
\left(\mu F / \mathrm{cm}^{2}\right)\end{array}$ & $\mathrm{n}-\mathrm{Q}_{\mathrm{c}}$ & $\begin{array}{c}R_{p} \\
\left(k \Omega \mathrm{cm}^{2}\right)\end{array}$ & $\begin{array}{c}Y_{0}-\mathrm{W} \\
\left(\mu / \mathrm{cm}^{2} \Omega \sqrt{ }\right)\end{array}$ & $\begin{array}{c}\text { Fitting error } \\
(\%)\end{array}$ \\
\hline \multicolumn{7}{|c|}{ CA TiN/SS } \\
\hline $5 \mathrm{~min}$ & 7.10 & 46.48 & 0.9291 & 20.206 & 5.77 & 0.17 \\
\hline $1 \mathrm{hr}$ & 7.08 & 68.69 & 0.9073 & 9.671 & 13.37 & 0.11 \\
\hline $3 \mathrm{hr}$ & 7.05 & 65.71 & 0.9153 & 5.2132 & 18.12 & 0.07 \\
\hline $5 \mathrm{hr}$ & 7.07 & 62.47 & 0.9228 & 4.4761 & 19.00 & 0.06 \\
\hline $24 \mathrm{hr}$ & 7.07 & 81.81 & 0.8889 & 154.567 & 100.00 & 0.30 \\
\hline $48 \mathrm{hr}$ & 7.00 & 84.47 & 0.8847 & 84.702 & 147.64 & 0.25 \\
\hline $72 \mathrm{hr}$ & 7.12 & 81.61 & 0.8923 & 214.462 & 76.41 & 0.19 \\
\hline $142 \mathrm{hr}$ & 7.10 & 73.00 & 0.8999 & 152.447 & 28.03 & 0.08 \\
\hline \multicolumn{7}{|c|}{ PMMA CA TiN/SS } \\
\hline $3 \mathrm{hr}$ & 233.93 & 110.05 & 0.8558 & 283.542 & 92.34 & 0.27 \\
\hline $5 \mathrm{hr}$ & 235.97 & 104.13 & 0.8552 & 446.901 & 264.59 & 0.17 \\
\hline $24 \mathrm{hr}$ & 237.31 & 81.69 & 0.8771 & 37.272 & 7.26 & 0.18 \\
\hline $48 \mathrm{hr}$ & 237.23 & 87.16 & 0.8661 & 494.629 & 32.76 & 0.41 \\
\hline $72 \mathrm{hr}$ & 236.60 & 85.78 & 0.8663 & 446.351 & 39.25 & 0.25 \\
\hline $95 \mathrm{hr}$ & 237.86 & 83.35 & 0.8702 & 288.880 & 23.75 & 0.30 \\
\hline $166 \mathrm{hr}$ & 242.49 & 66.92 & 0.9080 & 8.973 & 12.18 & 0.17 \\
\hline
\end{tabular}


In Table 4-17 and Table 4-18, all four TiN coating samples showed stable values in electrolyte resistance $\left(R_{e}\right)$. Comparing the two groups of TiN/SS coating systems, EB TiN/SS (Table 4-17) quickly decreased in polarization resistance $\left(R_{p}\right)$ from 37 to 8 $\mathrm{k} \Omega \mathrm{cm}^{2}$ in the initial hours, and then dropped to $4 \mathrm{k} \Omega \mathrm{cm}^{2}$ in the following two days of immersion; it gently rose to $5 \mathrm{k} \Omega \mathrm{cm}^{2}$ by the $5^{\text {th }}$ day; and maintained an increasing trend until the $13^{\text {th }}$ day. The Warburg admittance $(W)$ slightly increased and reached the peak value after one day immersion, then started decreasing. Diffusion impedance showed a similar trend to that of polarization resistance, indicating a simultaneous change between polarization resistance and diffusion impedance. For the CA TiN/SS system (Table 418), polarization resistance $\left(R_{p}\right)$ decreased from 20 to $4 \mathrm{k} \Omega \mathrm{cm}^{2}$ in the initial hours, and then fluctuated between 155 and $215 \mathrm{k} \Omega \mathrm{cm}^{2}$ in the following three days. This indicated a synergetic effect of the stainless steel substrate and better surface diffusivity in the cathodic arc deposition process (Section 3.3) that prevented the formation of a columnar coating structure, which reduced the conductance for mass transfer and ion current through the coating pinholes. The Warburg impedance $W$ also showed a similar trend to that for polarization resistance $\left(R_{p}\right)$.

For the EB and CA TiN/SS coating systems, the PMMA-treated samples showed higher and stable polarization resistances $\left(R_{p}\right)$ (two to three orders of magnitude higher for EB TiN/SS and several times higher for CA TiN/SS) (Table 4-17 and Table 4-18) as the result of PMMA clogging the pores in the coating, therefore limiting the mass transfer conductance and ion current through the coating. The high $\left(R_{p}\right)$ values for PMMA-treated 
coating systems are in agreement with those of the potentiodynamic polarization measurements. The more significant effect of PMMA treatment on $\left(R_{p}\right)$ for EB TiN/SS than for CA TiN/SS implied that there were more through coating defects (pinholes or voids) in the as-deposited EB TiN/SS coating system which was supported by the fact that the as-deposited EB TiN/SS coating system in general had lower $\left(R_{p}\right)$ values than the as-deposited CA TiN/SS coating system.

For the EB TiN/SS coating system, the PMMA-treated sample showed two to three orders of magnitude lower in Warburg diffusion admittance $\left(Y_{0^{-}} W\right)$ or higher in Warburg diffusion impedance than its as-deposited counterpart (Table 4-17), again indicating that PMMA clogged the coating pores, lowered the diffusion coefficients, and therefore increased the Warburg coefficient $(\sigma)$ (Eq. 4-4) and consequently the Warburg impedance (Eq. 4-3). The Warburg admittance of the PMMA-treated CA TiN/SS coating system was similar to that of its as-deposited counterpart. The small effect of PMMA on polarization resistance $\left(R_{p}\right)$ and Warburg admittance $(W)$ on the CA TiN/SS coating system could be because of fewer through-coating pores.

In a coating system, the admittance constant $\left(Y_{0}-Q_{c}\right)$ indicates the capacitive characteristics of the electrolyte-coating interface. In this study, the $\left(Y_{0^{-}} Q_{c}\right)$ values for the EB TiN/SS sample increased from 136 to $323 \mu \mathrm{F} / \mathrm{cm}^{2}$ with immersion time (Table 4-17), revealing the increases in the porosity of the exposed substrate steel due to the penetration of corrosive media and the increase in exposed coating area due to the increased number of pore walls [346]. The $\left(Y_{0}-Q_{c}\right)$ values for the system CA TiN/SS, 
however, were lower and stabilized in a relatively narrow range (70 to $80 \mu \mathrm{F} / \mathrm{cm}^{2}$ ) (Table 4-18), which indicated less change in the porosity of the exposed substrate and is consistent with the evolution trends of polarization resistance $\left(R_{p}\right)$ and Warburg diffusion impedance $\left(Y_{0^{-}} W\right)$.

The exponent of CPE $\left(n-Q_{c}\right)$ is associated with coating surface morphology. In this study, the values of $\left(n-Q_{c}\right)$ remained relatively constant with immersion time for both EB and CA as-deposited TiN/SS samples (Table 4-17 and Table 4-18), indicating constant capacitive characteristics of the electrolyte-coating interface. Similarly, comparing with the as-deposited counterparts, the smaller $\left(Y_{0}-Q_{c}\right)$ for the PMMA-treated EB TiN/SS system correspond to less exposed coating area due to the reduced number of pores. This verified that PMMA did enter and fill the coating pores. As for the CA TiN/SS coating system, PMMA did not cause notable changes in the values of $\left(n-Q_{c}\right)$.

\section{TiN/MS Coating Systems}

Due to the anodic dissolution corrosion mechanism of mild steel, corrosion of a coating on a mild steel substrate usually occurs locally at defect locations of the coating where the electrolyte penetrates and reaches the substrate; the coating therefore demonstrates a leaky capacitance characteristic. Thus the effect of charge transfer must be taken into account.

As is demonstrated in Figure 4-50, in a TiN/MS coating system, the parallel elements $C P E_{-S}$ (substrate capacitance) and $R_{s}$ (charge-transfer resistance of the substrate) are 
involved to describe the charge transfer effect through the coating pores. Warburg impedance element ( $W$ ) (in series with $R_{s}$ ) describes the semi-infinite length diffusion behaviour for the TiN coating at the electrolyte-substrate interface [37]. In series with the parallel sub circuit $\left(C P E-s, R_{s}\right), R_{\text {pore }}$ is the resistance to the ionic current through the coating pores, and $C P E-c$ represents the coating capacitance. These two parallel elements, $\left(C P E-c, R_{\text {pore }}\right)$ and $\left(C P E-s, R_{s}\right)$, correspond to two time constants representing the two localized corrosion processes at the electrolyte-coating interface (at high frequencies) and electrolyte-substrate interface (at low frequencies), respectively [37]. This EEC model $R_{e}\left(C P E-c\left(R_{\text {pore }}\left(C P E-s, R_{s} W\right)\right)\right)$ is termed the localized corrosion model, and the impedance $(Z)$ is presented as:

$$
Z=R_{e}+\frac{1}{\left(Y_{0}-Q_{c}\right)(j \omega)^{n-Q c}+\frac{1}{R_{\text {pore }}+\frac{1}{\left(Y_{0}-Q_{s}\right)(j \omega)^{n-Q s}+\frac{1}{R_{s}+\frac{1}{Y_{0} \sqrt{j \omega}}}}}}
$$

where,

$Y_{0} Q_{C}:$ admittance for coating, $n$-Qc: empirical exponent of CPE at coating, $Y_{0} Q_{S}$ : admittance for substrate, $n$-Qs: empirical exponent of CPE at substrate. 


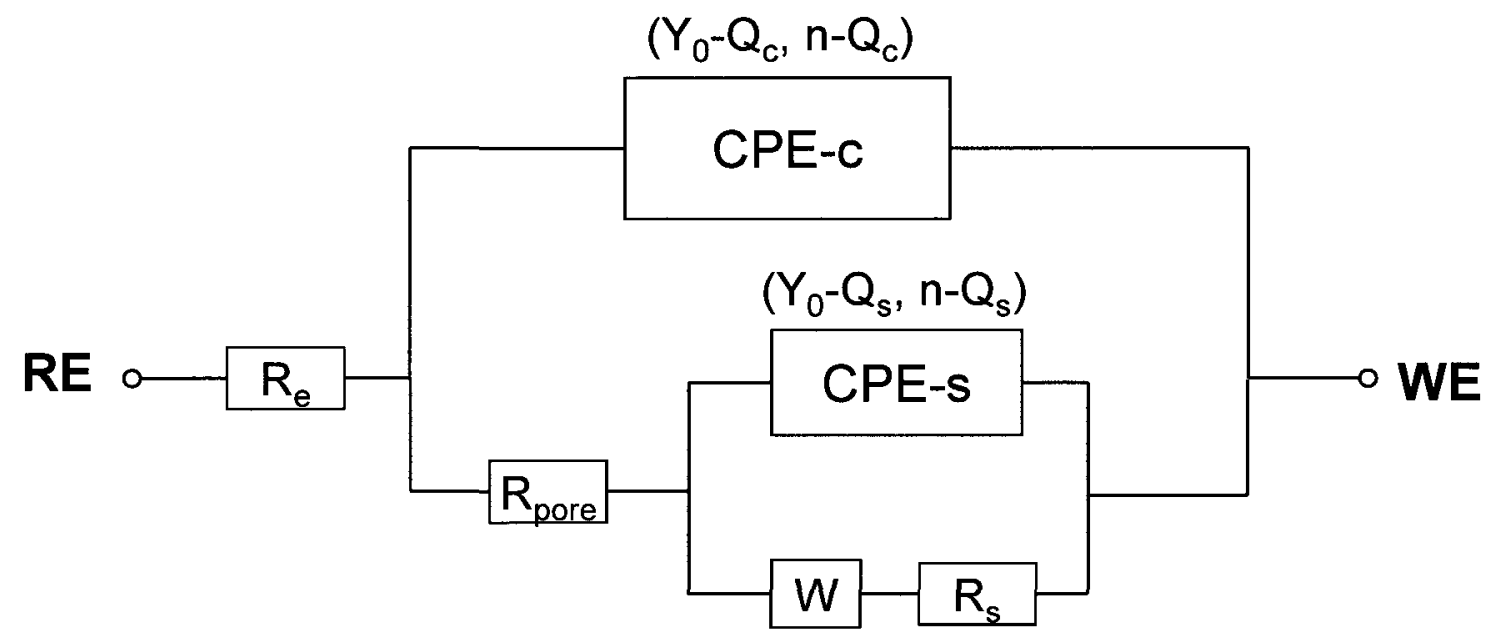

Figure 4-50 Schematic of EEC model for localized corrosion model (for PVD coating/MS system).

In this study, the typical charge-transfer corrosion feature as shown in EIS Nyquist plots (Figure 4-38 a) and the serious pitting corrosion observed in SEM analyses (Figure 4-42 a to d) indicated typical localized corrosions on the CA TiN/MS coating system. Therefore the localized corrosion model (Figure 4-50) was applied to the CA TiN/MS coating. The EIS Nyquist plot for the PMMA-treated CA TiN/MS coating system (Figure 4-38 b) with depressed semicircles followed by straight lines, combined with the fact that no pitting corrosion was found in SEM analyses (Figure 4-42 e to i), implied that diffusion controlled corrosion rather than localized corrosion occurred on this sample. Therefore the OtcWar model (Figure 4-46 a) was employed to the PMMAtreated CA TiN/MS sample.

The fitted curves are shown in Figure 4-51, and fitted data with fitting errors are displayed in Table 4-19 and Table 4-20. The very low fitting errors confirm good matches of these two EEC models on the two coating samples. 


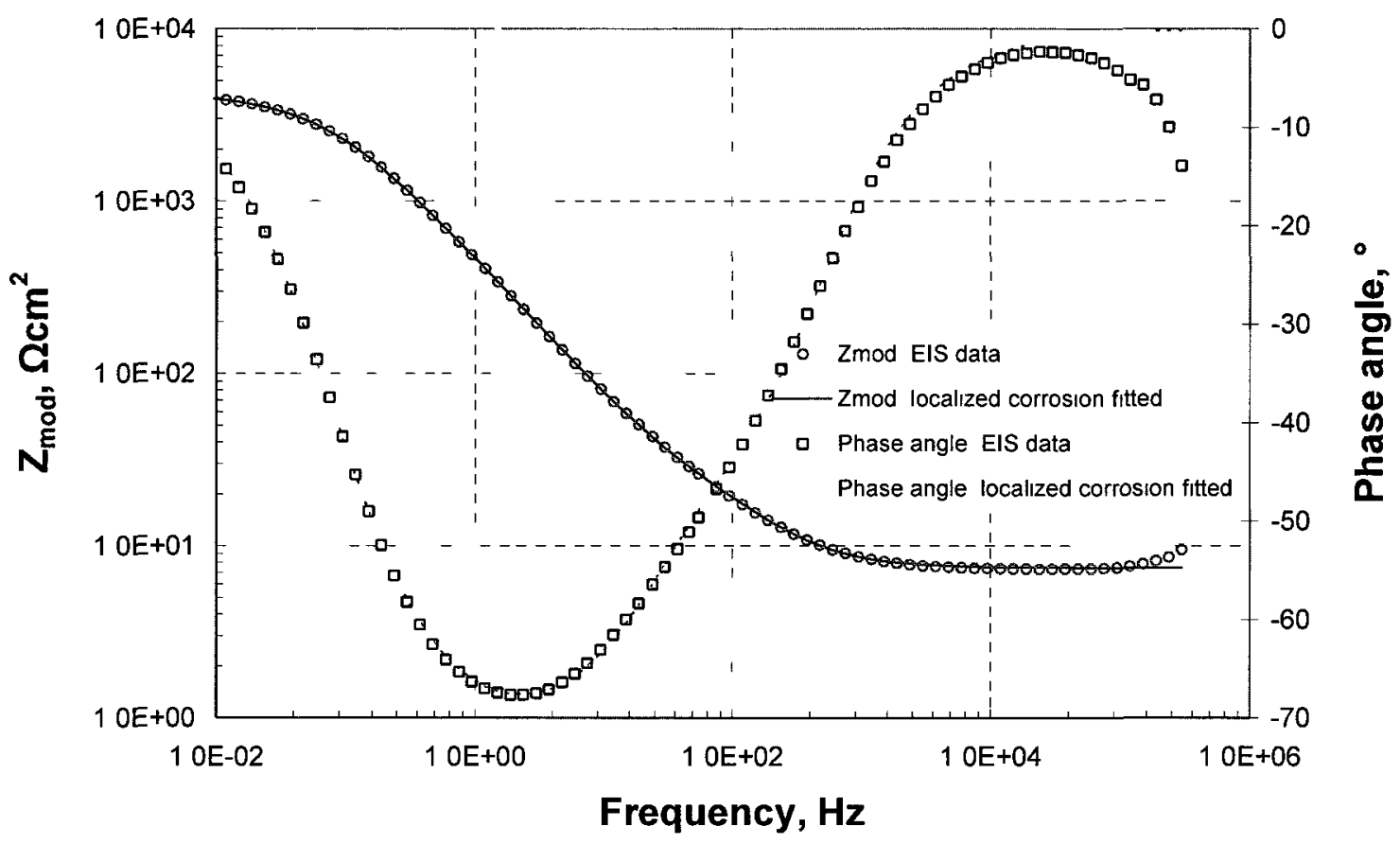

(a) CA TiN/MS (localized corrosion)(at $5 \mathrm{hr}$ )

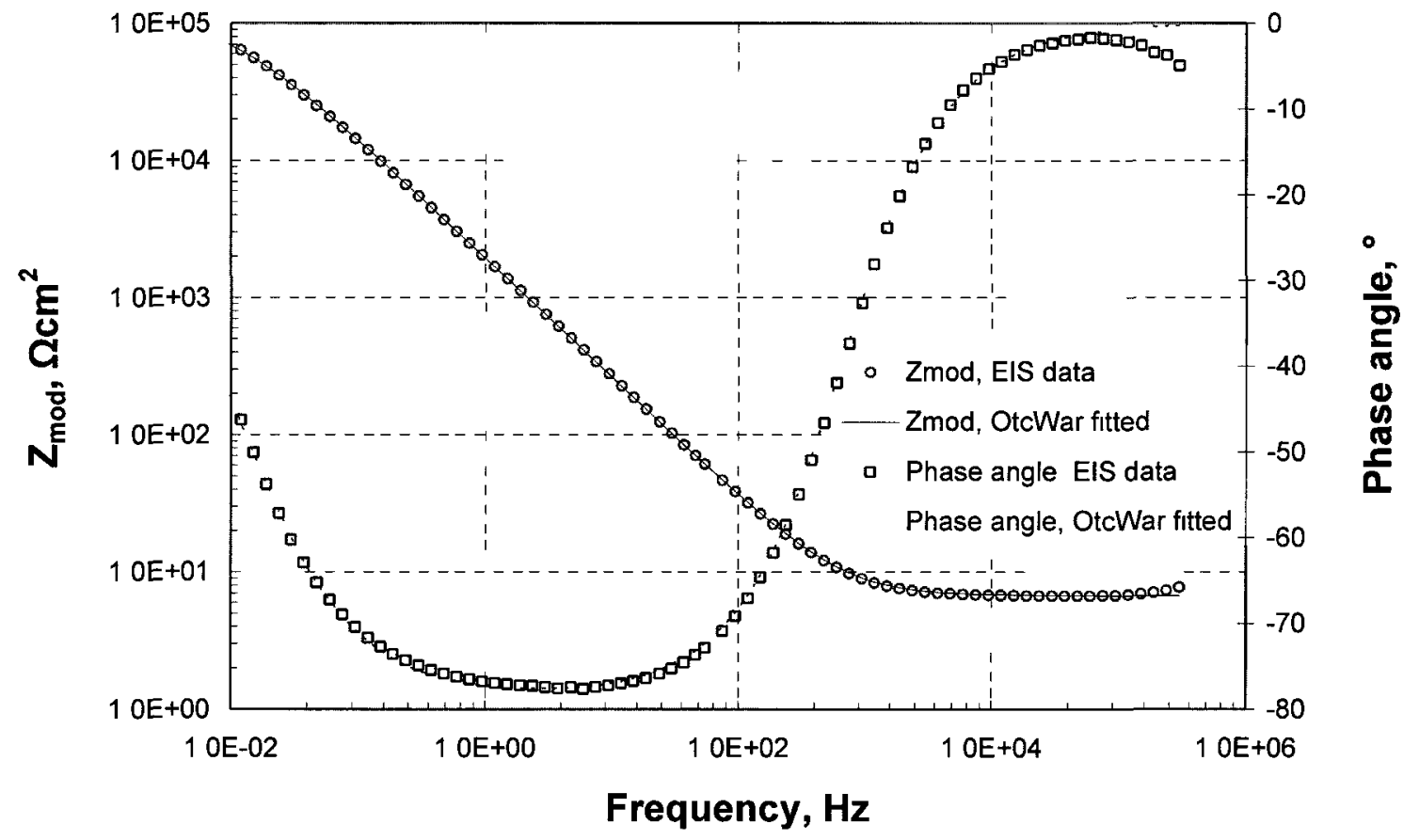

(b) PMMA CA TiN/MS (OtcWar)(at $5 \mathrm{hr}$ )

Figure 4-51 EEC fitting curves on CA TiN/MS coating systems. 
Table 4-19 EEC fitted data for CA TiN/MS coating systems (localized corrosion)

\begin{tabular}{|l|c|c|c|c|c|c|c|c|c|}
\hline Time & $\begin{array}{c}\mathrm{R}_{\mathrm{e}} \\
\left(\Omega \mathrm{cm}^{2}\right)\end{array}$ & $\begin{array}{c}\mathrm{Y}_{0}-\mathrm{Qc} \\
\left(\mu \mathrm{F} / \mathrm{m}^{2}\right)\end{array}$ & $\mathrm{n}-\mathrm{Qc}$ & $\begin{array}{c}\mathrm{R}_{\text {pore }} \\
\left(\mathrm{k} \Omega \mathrm{cm}^{2}\right)\end{array}$ & $\begin{array}{c}\mathrm{Y}_{0}-\mathrm{Qs} \\
\left(\mu \mathrm{F} / \mathrm{cm}^{2}\right)\end{array}$ & $\mathrm{n}-\mathrm{Qs}$ & $\begin{array}{c}\mathrm{R}_{\mathrm{s}} \\
\left(\mathrm{k} \Omega \mathrm{cm}^{2}\right)\end{array}$ & $\begin{array}{c}\mathrm{Y}_{0}-\mathrm{W} \\
\left(\mu / \mathrm{cm}^{2} \Omega{ }_{\mathrm{s}}\right)\end{array}$ & $\begin{array}{c}\text { Fitting } \\
\text { error } \\
(\%)\end{array}$ \\
\hline $5 \mathrm{~min}$ & 7.65 & 247.01 & 0.8789 & 1.103 & 281.63 & 0.8300 & 6.955 & 3480.3 & 0.54 \\
\hline $1 \mathrm{hr}$ & 7.68 & 1446.9 & 0.8599 & 0.075 & 357.24 & 0.8193 & 5.946 & 9003.8 & 0.29 \\
\hline $3 \mathrm{hr}$ & 7.39 & 1554.0 & 0.8196 & 0.087 & 2727.26 & 0.8817 & 4.517 & 25388.5 & 0.26 \\
\hline $5 \mathrm{hr}$ & 7.43 & 1536.3 & 0.8331 & 0.048 & 2746.12 & 0.8146 & 3.969 & 13210.2 & 0.26 \\
\hline $24 \mathrm{hr}$ & 7.58 & 1654.1 & 0.8026 & 0.081 & 2736.94 & 0.7534 & 2.592 & 10710.8 & 0.26 \\
\hline
\end{tabular}

Table 4-20 EEC fitted data for CA PMMA TiN/MS coating systems (OtcWar)

\begin{tabular}{|l|c|c|c|c|c|c|}
\hline Time & $\begin{array}{c}\mathrm{R}_{\mathrm{e}} \\
\left(\Omega \mathrm{cm}^{2}\right)\end{array}$ & $\begin{array}{c}\mathrm{Y}_{0}-\mathrm{Q}_{\mathrm{c}} \\
\left(\mu \mathrm{F} / \mathrm{cm}^{2}\right)\end{array}$ & $\mathrm{n}-\mathrm{Q}_{\mathrm{c}}$ & $\begin{array}{c}\mathrm{R}_{\mathrm{p}} \\
\left(\mathrm{k} \Omega \mathrm{cm}^{2}\right)\end{array}$ & $\begin{array}{c}\mathrm{Y}_{0}-\mathrm{W} \\
\left(\mu / \mathrm{cm}^{2} \Omega \sqrt{ } \mathrm{s}^{2}\right.\end{array}$ & $\begin{array}{c}\text { Fitting error } \\
(\%)\end{array}$ \\
\hline $5 \mathrm{~min}$ & 7.04 & 122.03 & 0.8602 & 32.52 & 2816.56 & 0.17 \\
\hline $3 \mathrm{hr}$ & 6.85 & 109.75 & 0.8688 & 81.48 & 1137.45 & 0.07 \\
\hline $5 \mathrm{hr}$ & 6.69 & 103.07 & 0.8728 & 100.17 & 206.24 & 0.07 \\
\hline $24 \mathrm{hr}$ & 6.59 & 94.01 & 0.8798 & 87.84 & 93.43 & 0.19 \\
\hline $48 \mathrm{hr}$ & 6.43 & 90.00 & 0.8855 & 61.38 & 62.47 & 0.24 \\
\hline $72 \mathrm{hr}$ & 6.84 & 95.35 & 0.8784 & 17.20 & 94.34 & 0.72 \\
\hline $95 \mathrm{hr}$ & 6.55 & 99.68 & 0.8723 & 35.29 & 81.92 & 0.42 \\
\hline
\end{tabular}

For the as-deposited CA TiN/MS coating, the continuous decreases in $R_{\text {pore }}$ and $R_{S}$ (Table 4-19) with immersion time as well as the continuous increases in Warburg admittance $\left(Y_{0^{-}} W\right)$, diffusion admittance constants $\left(Y_{0^{-}} Q_{c}\right)$ and $\left(Y_{0^{-}} Q_{s}\right)$ indicated a continual degradation in corrosion resistance in the CA TiN/MS coating system.

The OtcWar model was applied to the PMMA-treated CA TiN/MS coating, and confirmed that the corrosion process was diffusion controlled as shown in Figure 4-38 (b). This can be confirmed by the fitted data obtained using OtcWar model (Table 4-20), where the values of $R_{p}$ were higher (by two orders of magnitude) than those of $R_{s}$ (Table 4-19) for the as-deposited samples. Clearly the PMMA treatment caused a significant increase in polarization resistance. Furthermore, these improvements were maintained for a long immersion time (95 hours). The values in $\left(Y_{0^{-}}-W\right)$ (Table 4-20) for the PMMA- 
treated CA TiN/MS sample were substantially lower than those for the as-deposited counterpart (Table 4-19), indicating a significant improvement in diffusion impedance due to the PMMA treatment. More importantly, the values of $\left(Y_{0^{-}} W\right)$ (Table 4-20) are of the same order of magnitude as those for the PMMA-treated CA TiN/SS sample (Table 4-18). This further indicated that the corrosion mechanism changed from a chargetransfer controlled process (for the as-deposited sample) to a diffusion controlled process. The results above clearly demonstrated that the PMMA post-deposition treatment to the CA TiN/MS coating system not only increased corrosion resistance, but more importantly changed the corrosion mechanism as well.

The CA TiN/MS coating system exhibited much lower polarization resistance $\left(R_{s}\right)$ (Table 4-19) than the CA TiN/SS system (Table 4-18). This was due to anodic dissolutive corrosion of the mild steel substrate. The passive oxide film on the 17-4PH steel substrate acted as a barrier to the electrolyte-substrate corrosion through the coating pores, so it therefore demonstrated higher values of polarization resistance. This is in agreement with the potentiodynamic polarization test results (Table 4-13).

Of the three TiN coating systems, the CA TiN/SS sample (Table 4-18) has a polarization resistance that is notably higher than the EB TiN/SS sample (Table 4-17), and is one order of magnitude higher than the CA TiN/MS sample (Table 4-19). The PMMA treatment resulted in significant increases in polarization resistance for all the three TiN coating systems, and changed the corrosion mechanism for the CA TiN/MS coating system to diffusion dominated. 


\subsubsection{Chromium Nitride Based Coating Systems}

The Otc-Cot-Hyper model (Figure 4-46 b) was applied to the four CrN based coating systems with equiaxed microstructures and with $17-4 \mathrm{PH}$ steel substrates, which have diffusion controlled corrosion features. Fitted curves are shown in Figure 4-52, and the fitted data with fitting errors are displayed in Table 4-21 to Table 4-24.

Applying the Otc-Oct-Hyper model to the four CrN based coating systems (Figure 4-52) showed good agreement with measured data. In the fitted data (Table 4-21 to Table 424), all four coatings showed stable electrolyte resistance $\left(R_{e}\right)$. In three days of immersion, the polarization resistance of the $\mathrm{CrN}$ coating increased from 380 to 430 $\mathrm{k} \Omega \mathrm{cm}^{2}$ (Table 4-21), and $\mathrm{CrSiCN}(1)$ rose from 530 to $1,400 \mathrm{k} \Omega \mathrm{cm}^{2}$ (Table 4-22). The increases in polarization resistance $\left(R_{p}\right)$ for these two coatings were attributed to passivation during the immersion period. The formation of chromium oxide on the coating surfaces limited mass transfer and ion current. The coating $\mathrm{CrSiCN}(1)$ demonstrated a considerable improvement in corrosion resistance, as evidenced by its polarization resistance $\left(R_{p}\right)$, when compared with the $\mathrm{CrN}$ coating. This was due to the denser microstructure of the $\mathrm{CrN}$ coating. These results are in agreement with the results in potentiodynamic polarization tests (Table 4-13). 


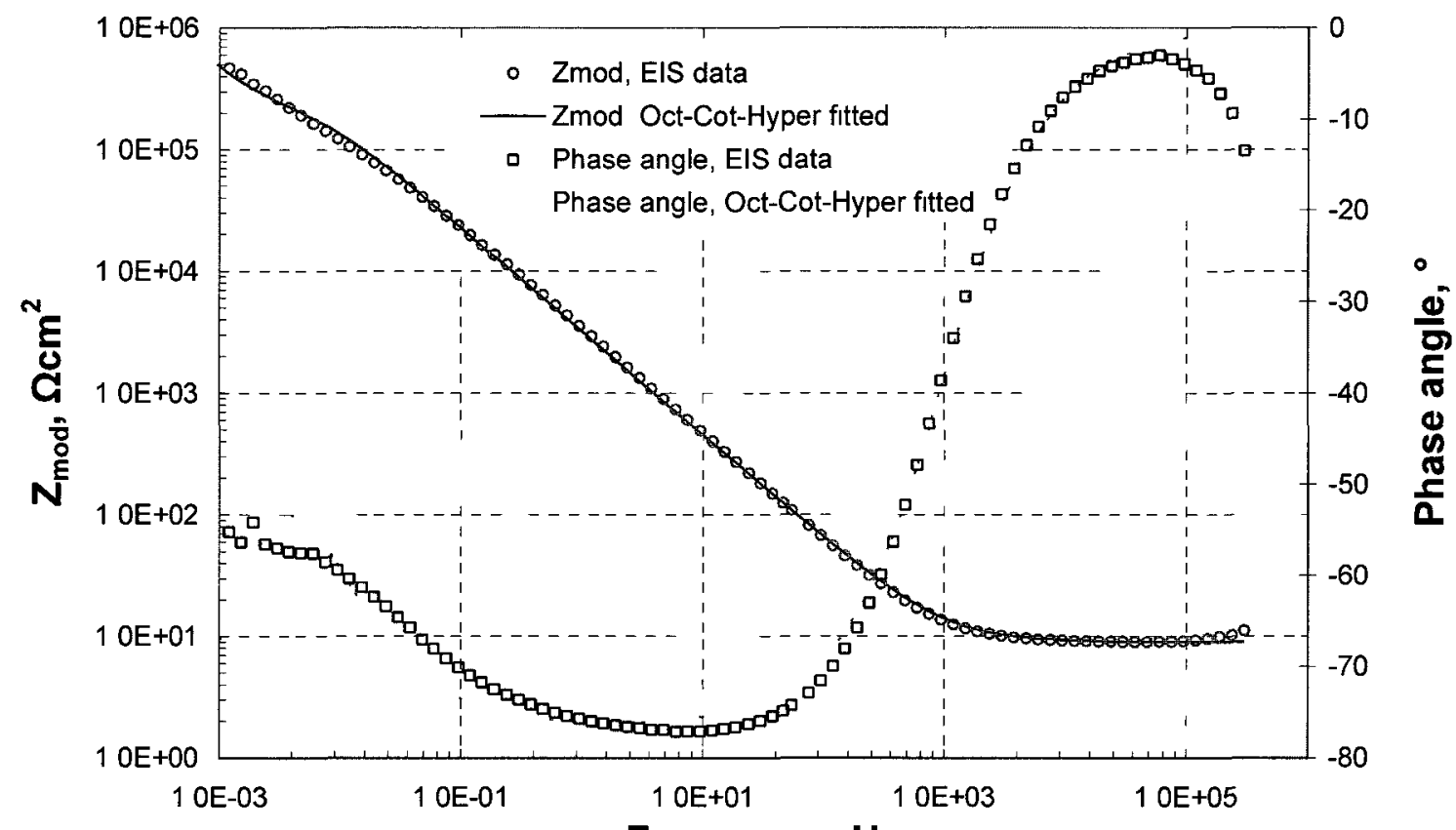

(a) $\mathrm{CrN}$

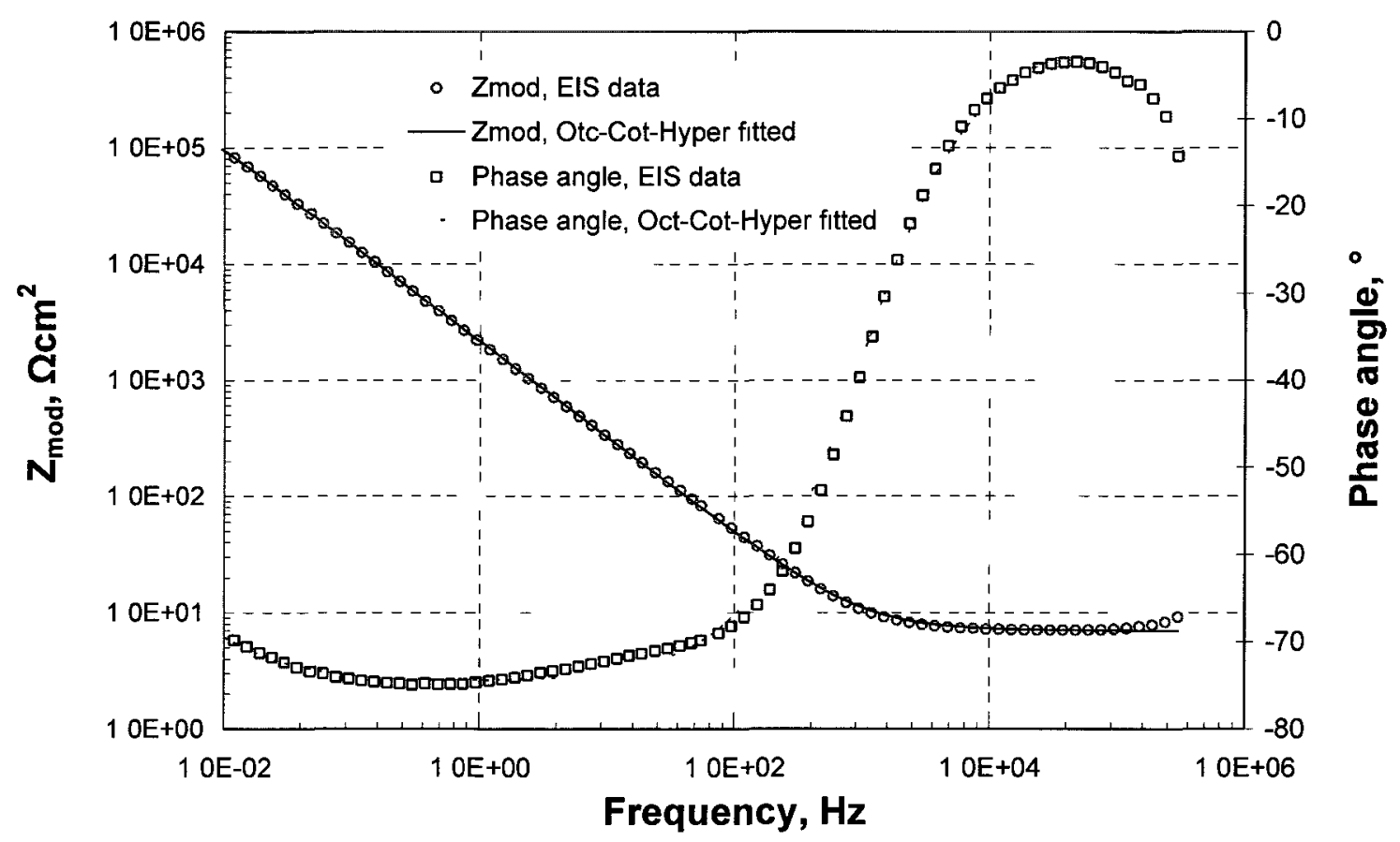

(b) $\mathrm{CrSiCN}(1)$

Figure 4-52 to be continued 


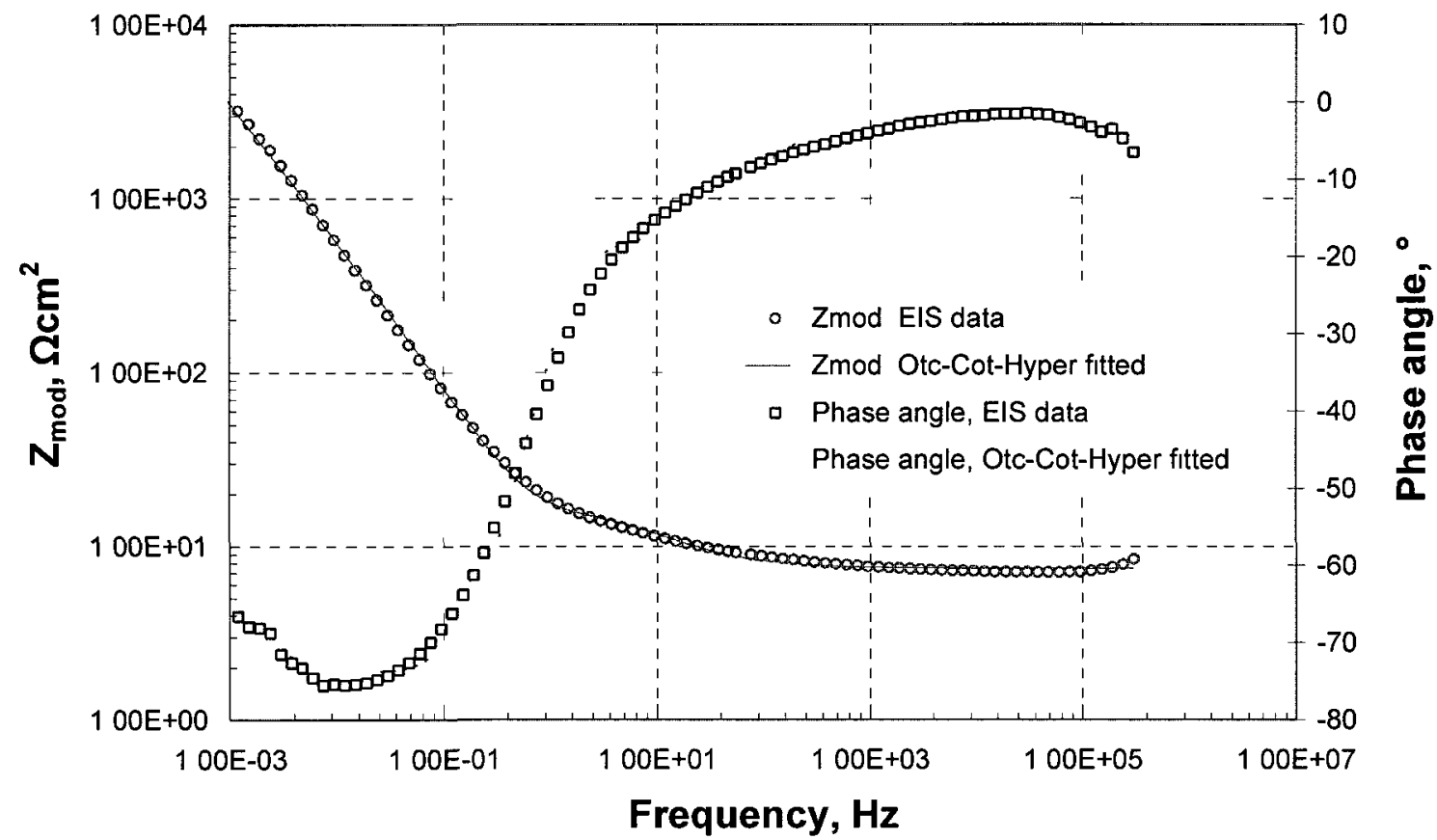

(c) $\mathrm{CrSiCN}(2)$

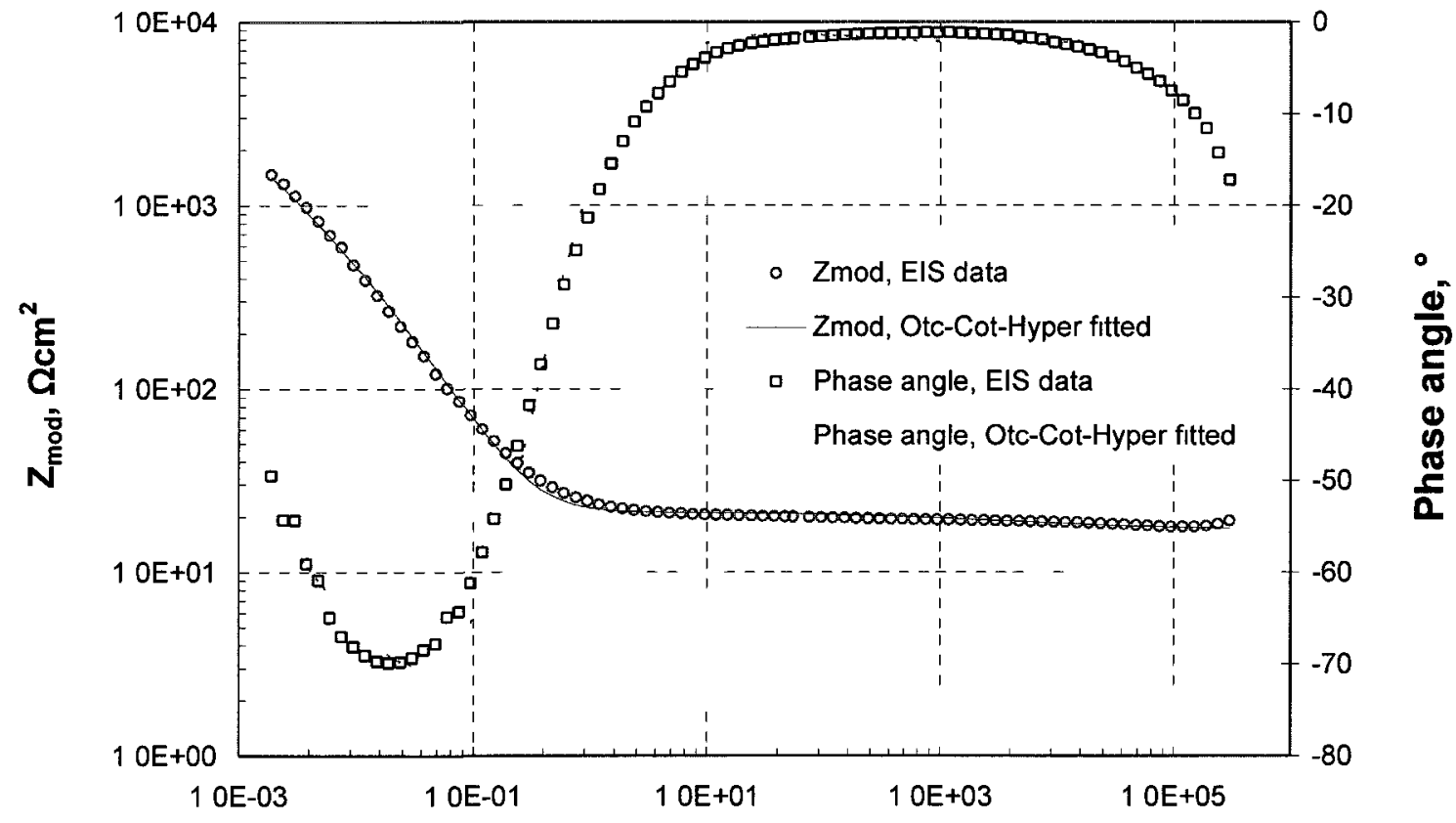

Frequency, $\mathrm{Hz}$

\section{(d) $\mathrm{CrSiCN}(3)$}

Figure 4-52 EEC fitting curves for $\mathrm{CrN}$ based coating systems (Otc-Cot-Hyper model)(at $5 \mathrm{hr}$ ). 
Table 4-21 EEC fitted data for CrN coating system (Otc-Cot-Hyper)

\begin{tabular}{|l|c|c|c|c|c|c|c|}
\hline Time & $\begin{array}{c}\mathrm{R}_{\mathrm{e}} \\
\left(\Omega \mathrm{cm}^{2}\right)\end{array}$ & $\begin{array}{c}\mathrm{Y}_{0}-\mathrm{Q}_{\mathrm{c}} \\
\left(\mu \mathrm{F} / \mathrm{cm}^{2}\right)\end{array}$ & $\mathrm{n}-\mathrm{Q}_{\mathrm{c}}$ & $\begin{array}{c}\mathrm{R}_{\mathrm{p}} \\
\left(\mathrm{k} \Omega \mathrm{cm}^{2}\right)\end{array}$ & $\begin{array}{c}\mathrm{Y}_{0}-\mathrm{O} \\
\left(\mu / \mathrm{cm}^{2} \Omega \sqrt{ } \mathrm{s}\right)\end{array}$ & $\begin{array}{c}\mathrm{B} \\
(\sqrt{\mathrm{s}})\end{array}$ & $\begin{array}{c}\text { Fitting } \\
\text { error }(\%)\end{array}$ \\
\hline $5 \mathrm{~min}$ & 9.22 & 55.36 & 0.8568 & 382.845 & 192.73 & 0.6147 & 0.29 \\
\hline $1 \mathrm{hr}$ & 9.28 & 58.46 & 0.8615 & 181.963 & 354.14 & 0.4760 & 1.85 \\
\hline $3 \mathrm{hr}$ & 9.07 & 63.33 & 0.8528 & 386.613 & 198.73 & 0.9767 & 0.50 \\
\hline $5 \mathrm{hr}$ & 8.93 & 65.30 & 0.8517 & 400.743 & 200.13 & 1.125 & 0.68 \\
\hline $24 \mathrm{hr}$ & 9.00 & 67.33 & 0.8562 & 369.814 & 204.33 & 0.9620 & 0.58 \\
\hline $48 \mathrm{hr}$ & 9.11 & 69.67 & 0.8513 & 431.593 & 221.53 & 1.016 & 0.62 \\
\hline $72 \mathrm{hr}$ & 9.22 & 70.13 & 0.8500 & 429.395 & 222.17 & 0.9699 & 0.59 \\
\hline $144 \mathrm{hr}$ & 6.51 & 73.67 & 0.8332 & 217.602 & 542.93 & 1.289 & 5.13 \\
\hline $192 \mathrm{hr}$ & 9.64 & 82.36 & 0.8296 & 326.403 & 662.42 & 1.139 & 0.65 \\
\hline $240 \mathrm{hr}$ & 9.66 & 85.73 & 0.8256 & 328.837 & 601.66 & 1.163 & 0.62 \\
\hline
\end{tabular}

Table 4-22 EEC fitted data for $\mathrm{CrSiCN}(1)$ coating system (Otc-Cot-Hyper)

\begin{tabular}{|l|c|c|c|c|c|c|c|}
\hline Time & $\begin{array}{c}\mathrm{Re} \\
\left(\Omega \mathrm{cm}^{2}\right)\end{array}$ & $\begin{array}{c}\mathrm{Y}_{0}-\mathrm{Q}_{\mathrm{c}} \\
\left(\mu \mathrm{F} / \mathrm{cm}^{2}\right)\end{array}$ & $\mathrm{n}-\mathrm{Q}_{\mathrm{c}}$ & $\begin{array}{c}\mathrm{Rp} \\
\left(\mathrm{k} \Omega \mathrm{cm}^{2}\right)\end{array}$ & $\begin{array}{c}\mathrm{Y}_{0^{-}} \mathrm{O} \\
\left(\mu / \mathrm{cm}^{2} \Omega{ }_{\mathrm{s}}\right)\end{array}$ & $\begin{array}{c}\mathrm{B} \\
\left(ل_{\mathrm{s}}\right)\end{array}$ & $\begin{array}{c}\text { Fitting } \\
\text { error }(\%)\end{array}$ \\
\hline $5 \mathrm{~min}$ & 7.07 & 97.21 & 0.8388 & 531.210 & 955.41 & 0.1101 & 0.31 \\
\hline $1 \mathrm{hr}$ & 7.06 & 98.27 & 0.8334 & 667.015 & 353.63 & 0.1351 & 0.32 \\
\hline $3 \mathrm{hr}$ & 7.17 & 99.77 & 0.8311 & 932.580 & 1092.10 & 0.0947 & 0.33 \\
\hline $5 \mathrm{hr}$ & 6.99 & 100.33 & 0.8303 & 929.440 & 1369.43 & 0.0836 & 0.34 \\
\hline $24 \mathrm{hr}$ & 6.87 & 101.19 & 0.8273 & 1431.055 & 1968.15 & 0.1343 & 0.16 \\
\hline $48 \mathrm{hr}$ & 7.99 & 101.38 & 0.8262 & 1423.990 & 1912.10 & 0.1318 & 0.10 \\
\hline $72 \mathrm{hr}$ & 6.81 & 100.48 & 0.8271 & 1428.700 & 1750.32 & 0.1248 & 0.12 \\
\hline
\end{tabular}

Table 4-23 EEC fitted data for $\mathrm{CrSiCN}(2)$ coating system (Otc-Cot-Hyper)

\begin{tabular}{|l|c|c|c|c|c|c|c|}
\hline Time & $\begin{array}{c}\mathrm{R}_{\mathrm{e}} \\
\left(\Omega \mathrm{cm}^{2}\right)\end{array}$ & $\begin{array}{c}\mathrm{Y}_{0}-\mathrm{Q}_{\mathrm{c}} \\
\left(\mu \mathrm{F} / \mathrm{cm}^{2}\right)\end{array}$ & $\mathrm{n}-\mathrm{Q}_{\mathrm{c}}$ & $\begin{array}{c}\mathrm{R}_{\mathrm{p}} \\
\left(\mathrm{k} \Omega \mathrm{cm}^{2}\right)\end{array}$ & $\begin{array}{c}\mathrm{Y}_{0}-\mathrm{O} \\
\left(\mu / \mathrm{cm}^{2} \Omega \sqrt{ }\right)\end{array}$ & $\begin{array}{c}\mathrm{B} \\
(\sqrt{ } \mathrm{s})\end{array}$ & $\begin{array}{c}\text { Fitting } \\
\text { error } \\
(\%)\end{array}$ \\
\hline $5 \mathrm{~min}$ & 7.62 & 8191.08 & 0.7691 & 0.01499 & 325477 & 0.0211 & 0.23 \\
\hline $1 \mathrm{hr}$ & 7.52 & 8253.50 & 0.7615 & 0.01270 & 301019 & 0.0284 & 0.25 \\
\hline $3 \mathrm{hr}$ & 7.45 & 8143.95 & 0.7436 & 0.01177 & 245477 & 0.0420 & 0.29 \\
\hline $5 \mathrm{hr}$ & 7.41 & 7512.10 & 0.7002 & 0.01227 & 220891 & 0.0544 & 0.35 \\
\hline $8 \mathrm{hr}$ & 7.28 & 8589.81 & 0.6379 & 0.01511 & 196433 & 0.0640 & 0.43 \\
\hline $24 \mathrm{hr}$ & 3.00 & 2472.61 & 0.4613 & 0.01079 & 618089 & 0.0306 & 2.54 \\
\hline
\end{tabular}

Table 4-24 EEC fitted data for $\mathrm{CrSiCN}(3)$ coating system (Otc-Cot-Hyper)

\begin{tabular}{|l|c|c|c|c|c|c|c|}
\hline Time & $\begin{array}{c}\mathrm{Re} \\
\left(\Omega \mathrm{cm}^{2}\right)\end{array}$ & $\begin{array}{c}\mathrm{Y}_{0}-\mathrm{Q}_{\mathrm{c}} \\
\left(\mu \mathrm{F} / \mathrm{cm}^{2}\right)\end{array}$ & $\mathrm{n}-\mathrm{Q}_{\mathrm{c}}$ & $\begin{array}{c}\mathrm{R}_{\mathrm{p}} \\
\left(\mathrm{k} \Omega \mathrm{cm}^{2}\right)\end{array}$ & $\begin{array}{c}\mathrm{Y}_{0}-\mathrm{O} \\
\left(\mu / \mathrm{cm}^{2} \Omega{ }_{\mathrm{s}}\right)\end{array}$ & $\begin{array}{c}\mathrm{B} \\
(\sqrt{\mathrm{s}})\end{array}$ & $\begin{array}{c}\text { Fitting } \\
\text { error } \\
(\%)\end{array}$ \\
\hline $5 \mathrm{~min}$ & 17.98 & 7937.58 & 0.6667 & 0.00285 & 222165 & 0.0568 & 0.52 \\
\hline $1 \mathrm{hr}$ & 16.54 & 3141.40 & 0.4240 & 0.00433 & 880127 & 0.0238 & 0.58 \\
\hline $3 \mathrm{hr}$ & 17.11 & 3597.45 & 0.4500 & 0.00403 & 973630 & 0.0219 & 0.55 \\
\hline $24 \mathrm{hr}$ & 19.52 & 5633.12 & 0.6096 & 0.00358 & 870700 & 0.0211 & 0.42 \\
\hline
\end{tabular}


For the $\mathrm{CrSiCN}(2)$ and $\mathrm{CrSiCN}(3)$ coatings, the polarization resistance $\left(R_{p}\right)$ remained as low as 0.010 and $0.004 \mathrm{k} \Omega \mathrm{cm}^{2}$ (Table 4-23 and Table 4-24) respectively, irrespective of immersion time. These low polarization resistance values indicated failure in corrosion protection for these two coating systems, which experienced the serious pitting corrosion that were observed by SEM (Figure 4-43 e, f). The corrosion performance of these two coating systems strongly reflects their coating microstructures: coarser grains (Table 4-6) and dual-phase structure (Figure 4-14) together with presence of pores and nodules (Figure 4-9 and Figure 4-10), which were responsible for the rapid degradation in corrosion resistance.

The CrN coating had low values of $\left(Y_{0^{-}} O\right)\left(\sim 200 \mu / \mathrm{cm}^{2} \Omega \sqrt{ } \mathrm{s}\right)$ and high values of $B$ (about $1 \sqrt{ }$ ) (Table 4-21) (Otc-Oct-Hyper model fitted data) indicating a high diffusion impedance level. Where the low values in diffusion admittance indicate high diffusion impedance; and high values in $B(B=\delta / \sqrt{D})$ (Eq. 4-9) corresponded to relatively thicker diffusion layers $(\delta)$ or relatively low diffusion coefficients $(D)$, both of which lower the diffusion rate. The values of $\left(Y_{0^{-}} O\right)$ for the coating $\mathrm{CrSiCN}(1)$ increased from 950 to $1,750 \mu / \mathrm{cm}^{2} \Omega V_{\mathrm{S}}$ in three days of immersion, and those of $B$ remained as low as $0.1 \mathrm{Vs}$ (Table 4-22)(Otc-Oct-Hyper model fitted data) indicating a trend in decreasing diffusion impedance with immersion time. The increases in diffusion admittances $\left(Y_{0^{-}} O\right)$ for the sample of $\mathrm{CrSiCN}(1)$ compared with the $\mathrm{CrN}$ coating were due primarily to its denser microstructure and therefore higher polarization resistance $\left(R_{p}\right)$, which resulted in a lower mass flow rate through the diffusion layer. Diffusion layer and diffusion coefficient are two important factors governing diffusion admittance in an $\mathrm{AC}$ electrochemical process, and will be discussed in the following section. 
As for the $\mathrm{CrSiCN}(2)$ and $\mathrm{CrSiCN}(3)$ coatings, the relatively high values of $\left(Y_{0^{-}} O\right)(>$ $\left.200,000 \mu \Omega \mathrm{cm}^{2} \vee_{\mathrm{s}}\right)$ and lower values of $B\left(<0.07 \sqrt{s}_{\mathrm{s}}\right)$ (Table 4-23 and Table 4-24) revealed relatively low diffusion impedances compared with the $\mathrm{CrN}$ and $\mathrm{CrSiCN}(1)$ coatings $\left(\mathrm{Y}_{0}-\mathrm{O}<700\right.$ and $<2000 \mu \Omega \mathrm{cm}^{2} \sqrt{s}_{\mathrm{s}}$, respectively) $(\mathrm{B}>0.4$ and $>0.08 \mathrm{~V} \mathrm{~s}$, respectively) (Table 4-21 and Table 4-22). The high values in admittance (for $\mathrm{CrSiCN}(2)$ and $\mathrm{CrSiCN}(3)$ systems) were associated with the porous coating structure.

The admittance constant $\left(Y_{0^{-}} Q_{c}\right)$ for the coatings $\mathrm{CrN}\left(\sim 65 \mu \mathrm{F} / \mathrm{cm}^{2}\right)$ (Table 4-21) and $\mathrm{CrSiCN}(1)\left(\sim 100 \mu \mathrm{F} / \mathrm{cm}^{2}\right)$ (Table 4-22) remained quite low after three days of immersion; whereas the the values for the $\mathrm{CrSiCN}(2)$ and $\mathrm{CrSiCN}(3)$ coatings were much higher (both over 2,500 $\mu \mathrm{F} / \mathrm{cm}^{2}$ ) (Table 4-23 and Table 4-24) after one day of immersion. The low values of $\left(Y_{0^{-}} Q_{c}\right)$ for the $\mathrm{CrN}$ and $\mathrm{CrSiCN}(1)$ coatings were attributed to their relatively small effective surface areas because of their dense microstructures. In contrast, the high values in $\left(Y_{0}-Q_{c}\right)$ of the $\mathrm{CrSiCN}(2)$ and $\mathrm{CrSiCN}(3)$ coatings were due to their relatively large exposed surface area as a result of their high porosity.

The stable and similar values of empirical exponent $\left(n-Q_{c}\right)$ for the $\mathrm{CrN}$ and $\mathrm{CrSiCN}(1)$ coatings (Table 4-21 and Table 4-22) demonstrated their stable and similar electrolytecoating capacitive interfaces. The lower values of $\left(n-Q_{c}\right)$ for the $\operatorname{CrSiCN}(2)$ and CrSiCN(3) coating systems (Table 4-23 and Table 4-24) indicated their leaky capacitance electrolyte-coating interfacial characteristics [346], a result associated with their relatively porous microstructure. 


\subsubsection{Diffusion and Coating System Corrosion Resistance}

In an electrochemical cell, the process of mass transfer to an electrode surface can take the forms of diffusion, migration, and convection. Since an electrical force is the driving force for charge migration, the migration can be negligible in a situation where an electrical field is absent or weak. Also, at a location near the electrode surface, convection is not applicable to the boundary layer at the electrolyte-electrode interface (where a stagnant layer exists). Therefore, diffusion is the dominant factor governing the mass transport reaching the electrode surface, and determines the rate of an electrochemical corrosion process. In a PVD coating/substrate system, especially one with an inert ceramic coating deposited on a stainless steel substrate, electrochemical diffusion impedance is a critical factor that determines the corrosion resistance.

According to Fick's First Law, a diffusion process is described by equation:

$$
J=-D \frac{\partial C(x, t)}{\partial x}
$$

where,

$J$ : flux of diffusing specie, i.e., moles passing a unit plane at a point in a unit time $t$, $D$ : diffusion coefficient,

$C$ : ion concentration as a function of $x$ (the distance from the electrode surface), $t$ : time since power is applied, $x$ : distance from electrode surface.

Fick's First Law (Eq. 4-12) states that the flux of the diffusing species $J$ is proportional to the concentration gradient $\partial C / \partial x$ with a certain diffusion coefficient $(D)$. The diffusion 
coefficient $(D)$ is proportional to the velocities of the diffusing species, which, according to the Stokes-Einstein principle, depends on the temperature, viscosity of the fluid, and the size of the species. In a coating system, the diffusion of the reactant(s) occurs in the pores in the coating. Therefore, the coating microstructure is another important factor governing the diffusion process. To describe a diffusion process through a porous structure, the effective diffusion coefficient (transporting through pores) was introduced, which is written as [415]:

$$
D_{e}=\frac{D \varepsilon_{t} c}{\tau}
$$

where

$D$ : diffusion coefficient (area/time) in the fluid that fills the pores;

$\varepsilon_{t}:$ the porosity available for the transport;

$c$ : the constrictivity;

$\tau$ : the tortuosity.

In the effective diffusion coefficient $\left(D_{e}\right)$ (Eq. 4-13), the porosity available $\left(\varepsilon_{t}\right)$ is the measure of the available pore spaces for the diffusing species. A high available porosity indicates a large capacity in the pore spaces of the porous media, which provide paths for the diffusing species. The constrictivity $\mathrm{c}$ describes transport processes in porous media. Its value depends on the fluid viscosity, the pore diameter and the size of the diffusing particles. The tortuosity $\tau$ is treated as the ratio of the curve length through the pore to the straight distance between the ends of the through-pore channel. A high tortuosity indicates that the diffusing particles need longer and more complex paths to transit a pore with a given straight length; thus the resistance is high to the diffusing particle. 
Eq. (4-13) reveals a proportional relationship between porosity in a given coating system and the corresponding effective diffusion coefficient $\left(D_{e}\right)$ through the coating to the substrate under a given electrochemical cell condition. This effective diffusion coefficient $\left(D_{e}\right)$ describes the actual rate of diffusion to the substrate through the coating, where the diffusion condition is different from that in the bulk electrolyte outside the coating; hence the diffusion coefficients in Eq. (4-4), Eq. (4-8 b), Eq. (4-9), and Eq. (4-12) should be treated as the effective diffusion coefficient (Eq. 4-13). Thus, a coating with a high porosity corresponds to a high effective diffusion coefficient $\left(D_{e}\right)$ (Eq. 4-13), and is responsible for a low Warburg diffusion coefficient $(\sigma)$ (Eq. 4-4), and consequently low Warburg impedance $W$ (Eq. 4-2 and Eq. 4-3), also a low OFLD impedance $(O)$ (Eq. 4-8 b); and vice versa.

With regard to the factor of diffusion length or diffusion layer thickness, for a coating system with a given porosity (or density), (due to that the mass transfer via throughcoating pores to the substrate is only in the form of diffusion), actual diffusion layer thickness to the substrate is related to the coating thickness [38]. A thick coating is responsible for a thick diffusion layer to the substrate, and therefore a long diffusion length; this consequently results in an increase in the finite length diffusion impedance $(O)$ (Eq. 4-8 b). In addition to the issue of diffusion layer thickness, a thick coating has a relative lower probability of forming through-coating defects compared with a thinner coating, and this would also lower the effective diffusion coefficient $\left(D_{e}\right)$ (Eq. 4-13). This is another major factor (being associated with coating thickness) that further lowers the diffusion impedance through the coating. It was reported that the critical passivation 
current densities of TiN/SS systems were significantly affected by coating thickness, and the corrosion resistance were remarkably improved when the coating was made thicker $[35,134]$

The above discussions suggest that for a coating system, either a thick coating or a dense (less-porous) coating is preferred for anti-corrosion applications. In this study, the four $\mathrm{CrN}$ based coatings were significantly thicker $(\sim 20 \mu \mathrm{m})$ than the EB $(1.5 \mu \mathrm{m})$ and $\mathrm{CA}$ $(2.8 \mu \mathrm{m})$ TiN coatings (Table 4-2). The thicker CrN (Table 4-21) and $\mathrm{CrSiCN}(1)$ (Table 4-22) coatings have better corrosion resistances than the (as-deposited) TiN coatings (Table 4-17, Table 4-18, and Table 4-19). However, with similar coating thicknesses (Table 4-2), the coatings $\mathrm{CrSiCN}(2)(21.1 \mu \mathrm{m})$ and $\mathrm{CrSiCN}(3)(19.6 \mu \mathrm{m})$ demonstrated four to five orders of magnitude lower polarization resistance $\left(R_{p}\right)$, and three orders of magnitude higher diffusion admittance $\left(Y_{0^{-}} O\right)$ (indicating lower diffusion impedance, $\left.Z_{O}\right)$, than the coatings $\mathrm{CrN}(20.2 \mu \mathrm{m})$ and $\mathrm{CrSiCN}(1)(18.9 \mu \mathrm{m})($ Table 4-21 to Table 4-24). The lower impedances of $\mathrm{CrSiCN}(2)$ and $\mathrm{CrSiCN}(3)$ coating systems were attributed to their higher porosities $\left(\varepsilon_{t}\right)$ which resulted in high effective diffusion coefficients $\left(D_{e}\right)$ (Eq. 4-13). The high porosities of these two coating systems were due to their microstructures became less dense as the contents of $\mathrm{Si}$ exceed the optimal amount (around 1.3 at.\%) where the densest microstructure was observed, i.e., $\mathrm{CrSiCN}(1))$.

For the TiN coating systems, CA TiN coating system demonstrated the same order of magnitude in polarization resistance $\left(R_{p}\right)$ as EB TiN coating system, and two to three orders of magnitude lower diffusion impedance, $Z_{O}$ than EB TiN coating system (Table 
4-17 and Table 4-18). This is associated with the thicker thickness of CA TiN coating $(2.8 \mu \mathrm{m})$ than EB TiN coating $(1.5 \mu \mathrm{m})$ (Table 4-2) and its coating structure with fewer through-coating voids due to the cathodic arc process with high bias voltages that improved the surface diffusivity of the deposited species $[32,98,349]$.

The PMMA-treated TiN coating samples demonstrated increased polarization resistance ( $\left.R_{p}\right)$ (Table 4-13) and decreased diffusion admittance $\left(Y_{0^{-}}-W\right)$ (Table 4-17 to Table 4-20) (indicating increased diffusion impedance, $Z_{W}$ ) as the result of the PMMA clogging the pores in coatings, consequently lowering porosity available $\left(\varepsilon_{t}\right)$ for the transport through the coating and the effective diffusion coefficients $\left(D_{e}\right)($ Eq. 4-13). This lower effective diffusion coefficients would cause lower diffusion coefficient of oxidant $\left(D_{O}\right)$ and diffusion coefficient of reductant $\left(D_{R}\right)$, thus resulted in higher Warburg coefficients $(\sigma)$ (Eq. 4-4), therefore increased Warburg diffusion impedance $\left|Z_{\text {W }}\right|$ (Eq. 4-3). 


\subsection{Summary}

In this research work, two categories of transition metal nitride PVD coating systems, namely $\mathrm{TiN}$ and $\mathrm{CrN}$ based coating systems $(\mathrm{CrSiCN})$, have been studied with respect to microstructure, mechanical properties, tribological and corrosion behaviours. Two deposition techniques, i.e., EB and CA, were used to apply TiN coating systems in order to study the effects of deposition process on microstructure and mechanical properties as well as tribological and corrosion behaviours. Both mild steel and 17-4 PH stainless steel substrates were used for the CA TiN coating systems. Post-deposition treatment with PMMA was applied to the TiN coating systems in order to further study the effect of defects on the corrosion behaviour of the coating systems and to find an effective approach to the improvement of corrosion resistance of the coating systems. In the $\mathrm{CrN}$ based coating systems, various contents of $\mathrm{Si}$ and $\mathrm{C}$ were incorporated into the coatings in order to study the effect of composition on microstructure, mechanical properties, tribological and corrosion behaviours.

The results revealed that the $\mathrm{CA}$ TiN coating has a higher defect density than EB TiN coating. This was due to the cathodic arc deposition technique which usually generates large amounts of droplets during the deposition process. In the $\mathrm{CrN}$ based coating systems, the coating was dense with the initial addition of $\mathrm{Si}(1.3$ at.\%), and then became coarser and more porous with further increase in $\mathrm{Si}$, indicating that the changes in composition resulted in microstructure changes, which eventually led to changes in mechanical properties of the coating systems. 
Mechanical and tribological properties of the PVD coating systems tested in this research are summarized in Table 4-25, and illustrated in Figure 4-54 to Figure 4-55.

Table 4-25 Summary of mechanical and wear properties of PVD coating systems

\begin{tabular}{|l|c|c|c|c|c|}
\hline $\begin{array}{c}\text { Coating } \\
\text { system }\end{array}$ & $\begin{array}{c}\text { Hardness } \mathrm{H} \\
(\mathrm{GPa})\end{array}$ & $\begin{array}{c}\text { Elastic modules E } \\
(\mathrm{GPa})\end{array}$ & $\begin{array}{c}\mathrm{H}^{3} / \mathrm{E}^{2} \\
(\mathrm{GPa})\end{array}$ & $\mathrm{COF}$ & $\begin{array}{c}\text { Specific wear rate } \\
\left(\mathrm{mm}^{3} / \mathrm{Nm}\right)\end{array}$ \\
\hline $\mathrm{CA} \mathrm{TiN}$ & 33.98 & 456.73 & 0.20 & 0.81 & $8.76 \times 10^{-7}$ \\
\hline $\mathrm{CrN}$ & 19.97 & 309.87 & 0.08 & 0.81 & $1.29 \times 10^{-6}$ \\
\hline $\mathrm{CrSiCN}(1)$ & 21.59 & 313.12 & 0.10 & 0.71 & $1.12 \times 10^{-6}$ \\
\hline $\mathrm{CrSiCN}(2)$ & 14.04 & 233.41 & 0.05 & 0.91 & $2.12 \times 10^{-6}$ \\
\hline $\mathrm{CrSiCN}(3)$ & 13.86 & 218.33 & 0.06 & 0.67 & $1.10 \times 10^{-6}$ \\
\hline
\end{tabular}

In the $\mathrm{CrN}$ based coating systems, relationships between the composition, grain size, hardness and elastic modulus have been observed. With the initial addition of Si (1.3 at.\%) and C, grain size decreased while hardness and elastic modulus increased. However, with further increases in $\mathrm{Si}$ and $\mathrm{C}$ contents, grain size increased but the hardness and elastic modulus decreased (Figure 4-53 a, b). These results demonstrated that compositions determine the microstructures which in turn influence the mechanical properties of the coating systems. It was also found that, with the change in Si content, specific wear rates showed an inverse proportional relationship with the $\mathrm{H}^{3} / \mathrm{E}^{2}$ ratios, and a similar trend to coefficient of friction (Figure 4-54 a, b). These results indicated that wear behaviours of the coating systems depend on their hardness, modulus of elasticity (i.e., resistance to plastic deformation) and coefficient of friction. 


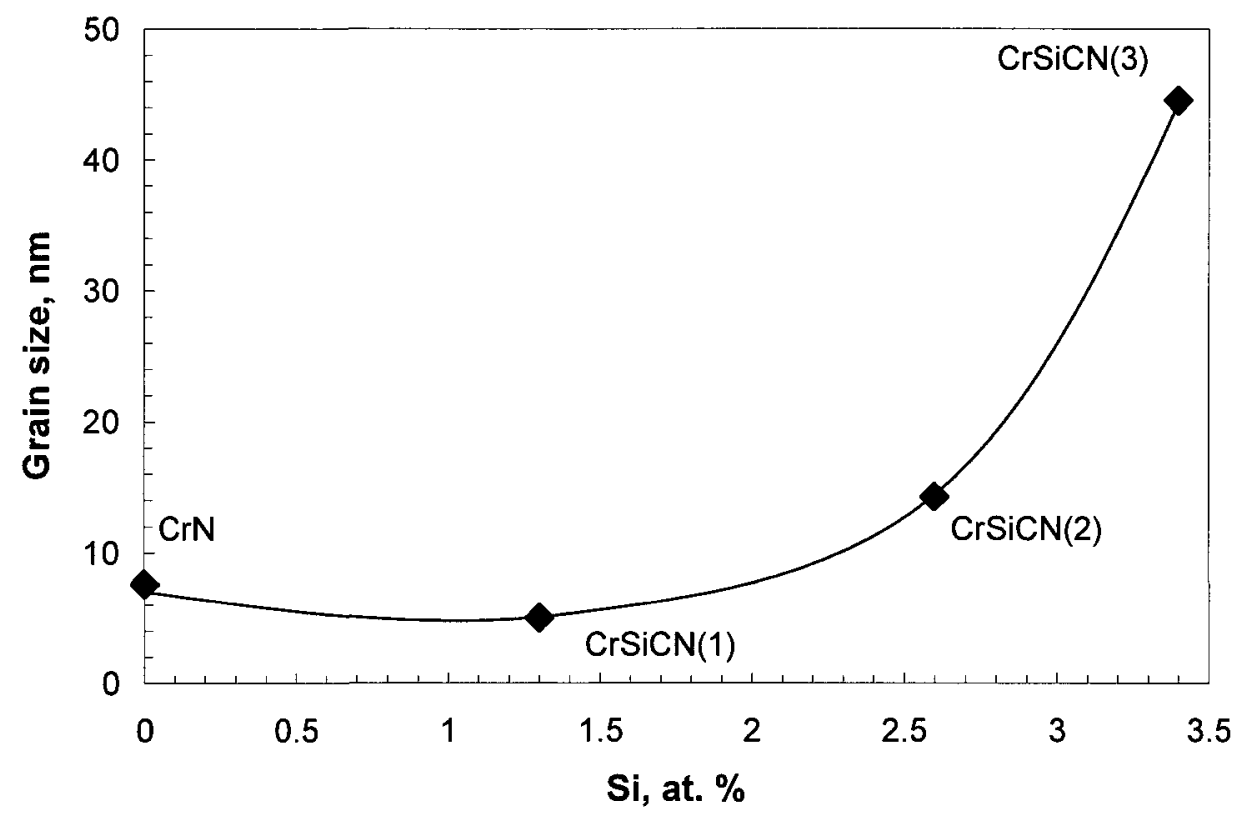

(a) Grain size

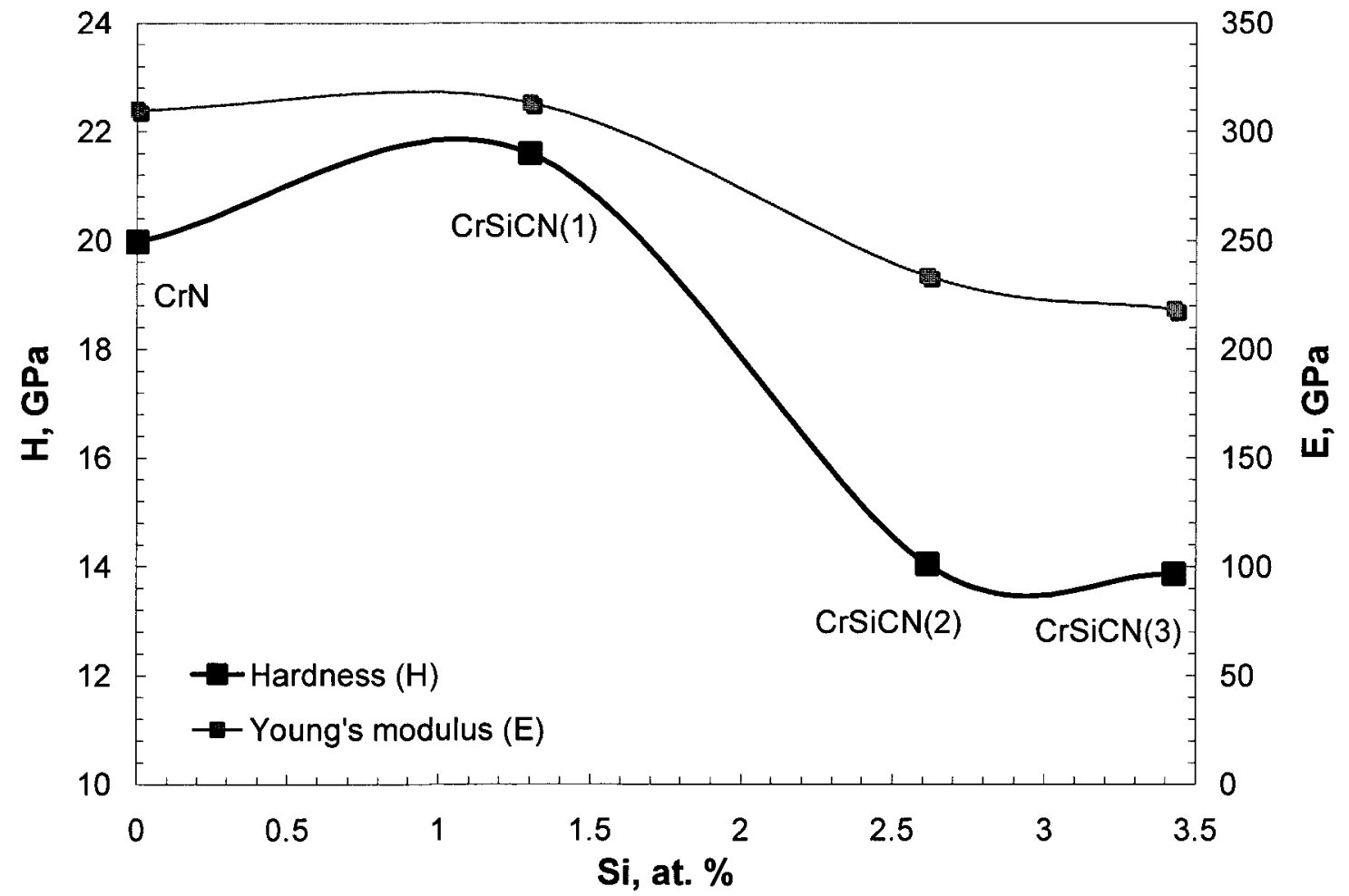

(b) Hardness and modulus of elasticity

Figure 4-53 Mechanical properties vs. Si content in $\mathrm{CrN}$ based coating systems. 


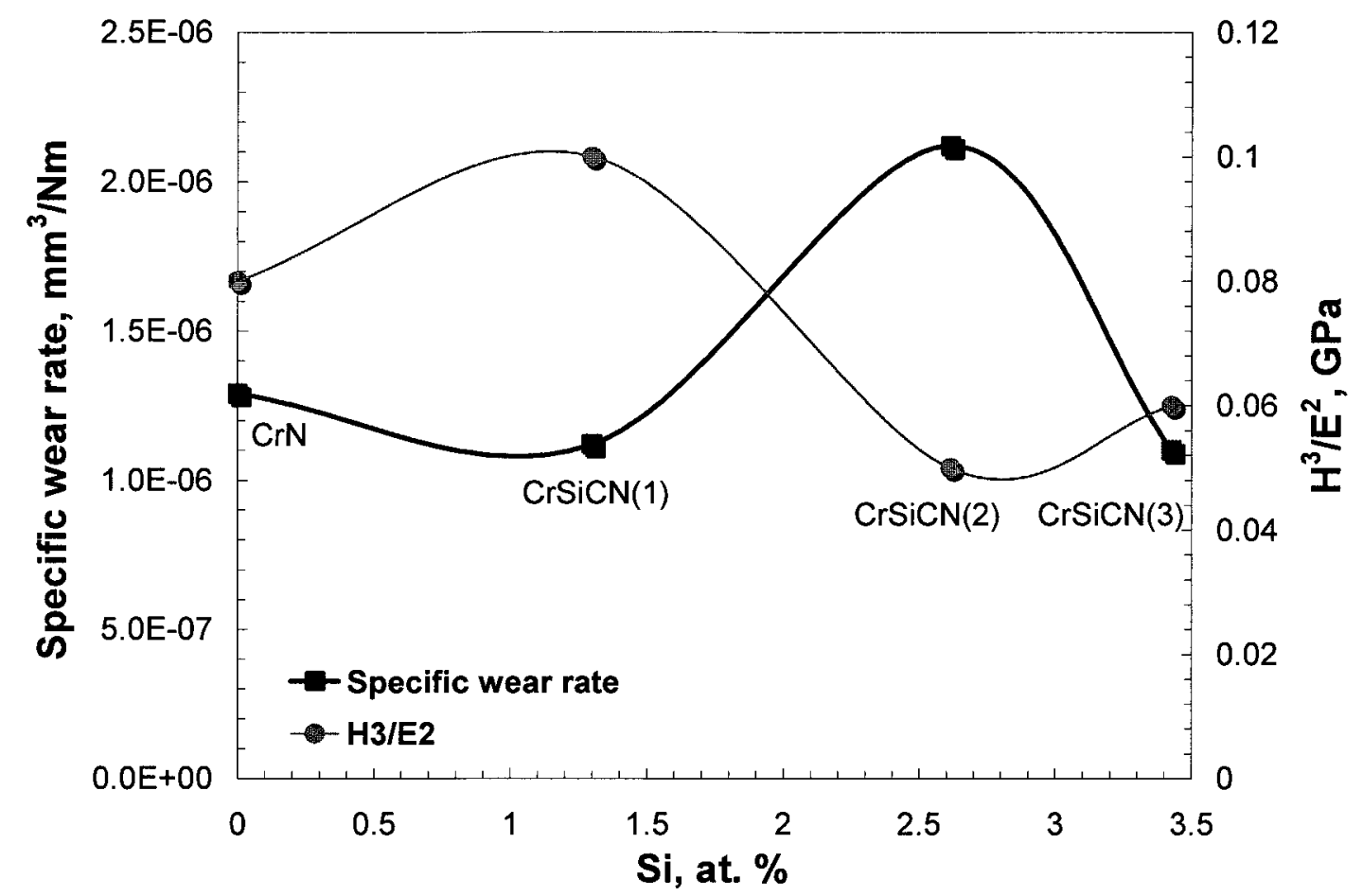

(a) Specific wear rate and $\mathrm{H}^{3} / \mathrm{E}^{2}$ ratio

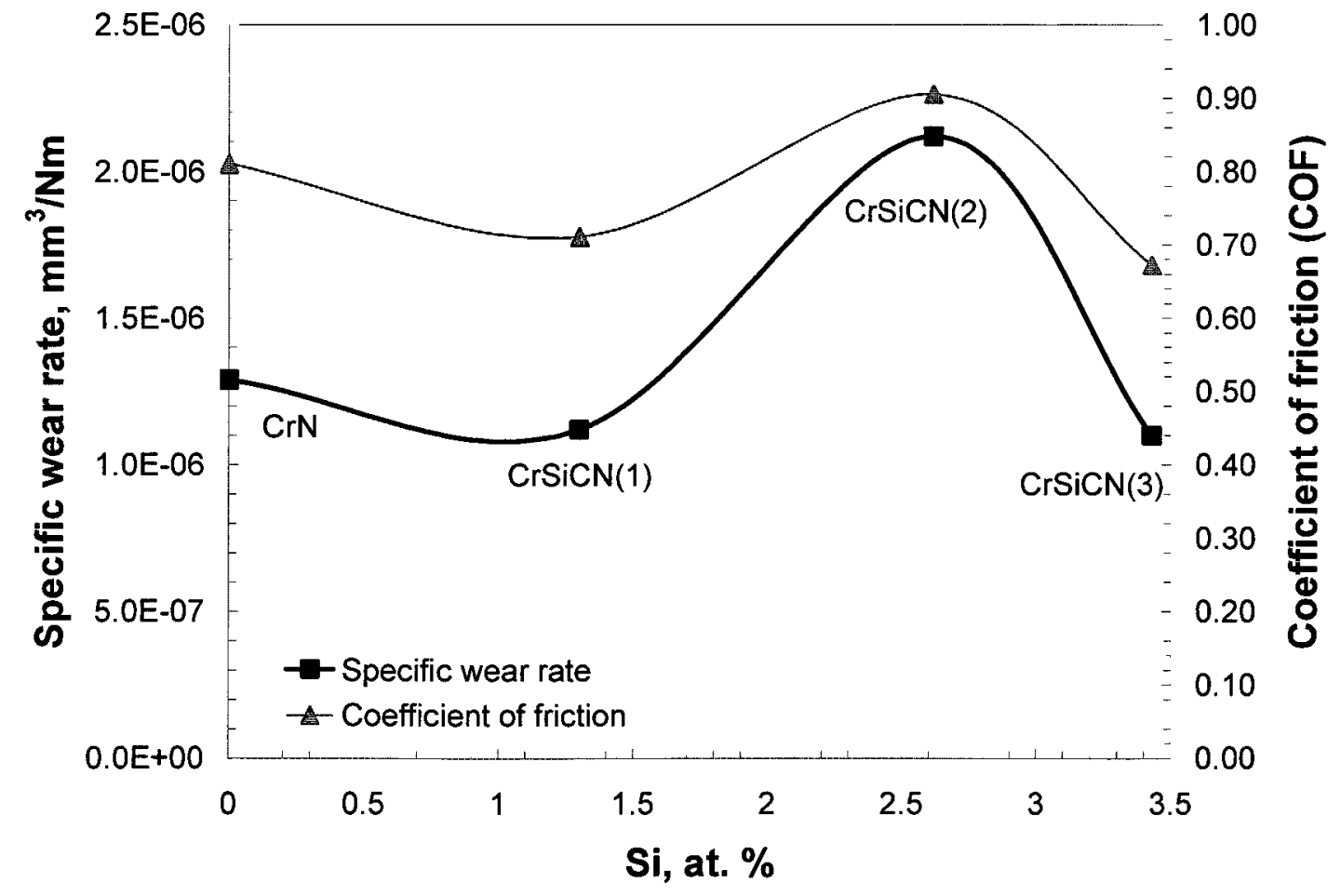

(b) Specific wear rate and COF

Figure 4-54 Wear behaviour vs. Si content in $\mathrm{CrN}$ based coating systems. 
Due to higher hardness and $\mathrm{H}^{3} / \mathrm{E}^{2}$ ratio, the $\mathrm{CA}$ TiN coating system showed a lower specific wear rate (Table 4-25) and erosion rates (Figure 4-55 a) comparing with the $\mathrm{CrN}$ based coating systems. Also due to its high hardness, the CA TiN coating system demonstrated a brittle erosion characteristic, with increasing erosion rates at high impingement angles (Figure 4-55 a).

The erosion behaviours of the $\mathrm{CrN}$ based coating systems also showed strong correlations with the composition and mechanical properties. The $\mathrm{CrN}$ and $\mathrm{CrSiCN}(1)$ (with higher hardness) coatings showed the maximum erosion rates at high impingement angles; whereas $\mathrm{CrSiCN}(2)$ and $\mathrm{CrSiCN}(3)$ (with lower hardness) showed peak erosion rates at low impingement angles (Figure 4-55 b). At low impingement angles, $\mathrm{CrN}$ and $\mathrm{CrSiCN}(1)$ had lower erosion rates than $\mathrm{CrSiCN}(2)$ and $\mathrm{CrSiCN}(3)$; whereas, at high impingement angles, they exhibited higher erosion rates than $\mathrm{CrSiCN}(2)$ and $\mathrm{CrSiCN}(3)$ (Figure 4-55 b). The differences in erosion behaviour are attributed to both coating properties and erosion mechanisms with respect to impingement angle. At low impingement angles, cutting dominates erosion damages (Figure 4-55 b), where the harder coating systems such as $\mathrm{CrN}$ and $\mathrm{CrSiCN}(1)$ possess higher resistance to cutting, resulting in lower erosion rates. At high impingement angles, cracking and crack propagation dominate erosion damages for brittle materials. The coating samples tested at higher impingement angles were examined, and cracks were consistently found on harder coatings such as $\mathrm{CrN}$ and $\mathrm{CrSiCN}(1)$; whereas no cracks were found on the coatings $\mathrm{CrSiCN}(2)$ and $\mathrm{CrSiCN}(3)$ ) (Figure 4-55 b) These results indicated a combination of high hardness and good toughness is required in order to design a coating system with 
enhanced erosion resistance at all impingement angles; and such a system can be obtained by modifying both the coating process and the composition of the coating system.

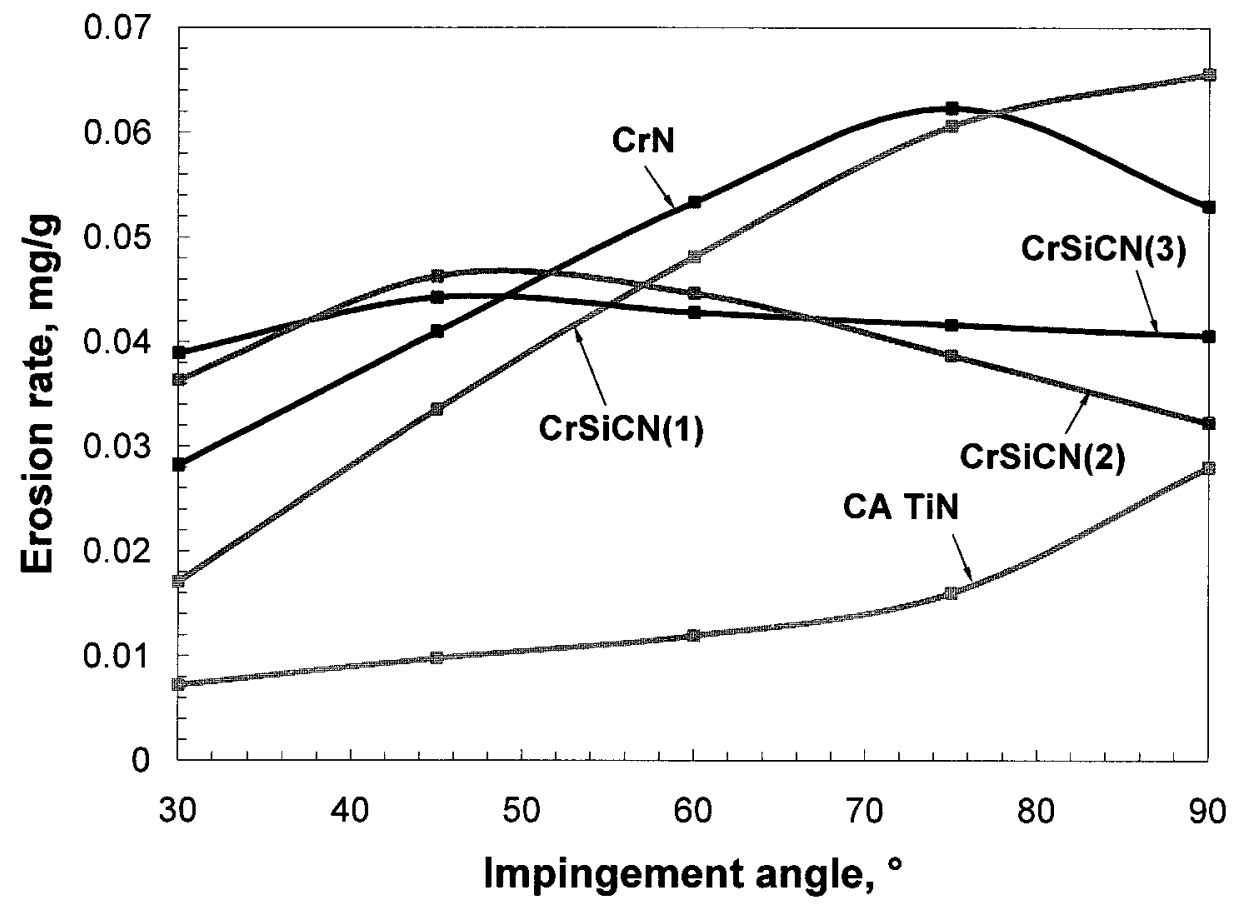

(a) Erosion behaviours of test coating systems

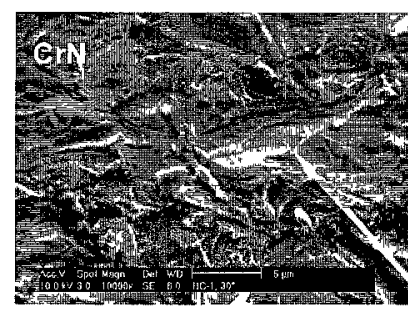

At low impingement angles Cuttıng dominates
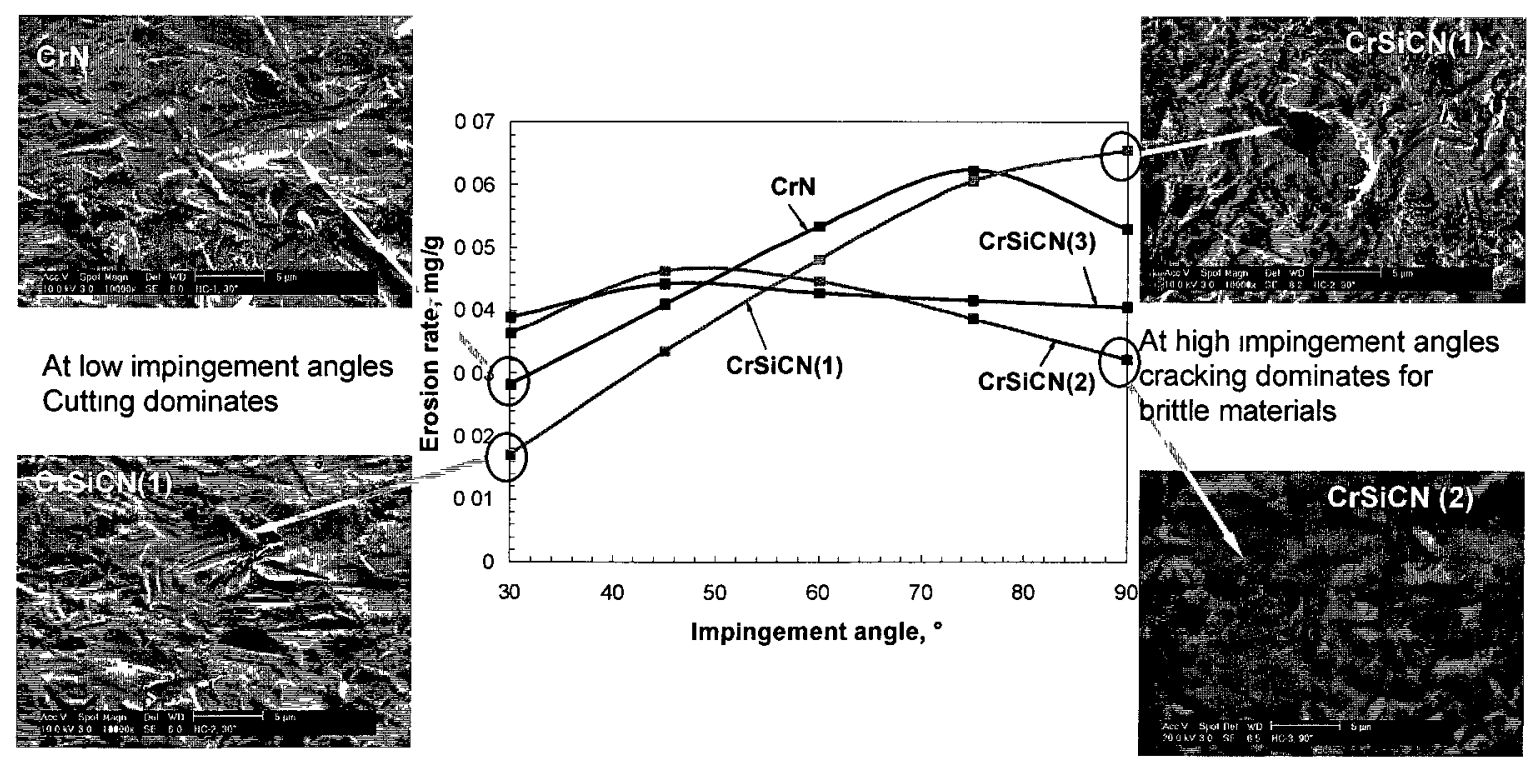

(b) Erosion behaviours vs. Si content in $\mathrm{CrN}$ based coating systems

Figure 4-55 Erosion behaviours of test coating systems. 
To study the corrosion behaviours, the two coating systems were subjected to short- and long- term corrosion tests using potentiodynamic polarization and EIS measurements, respectively.

Short term corrosion studies with potentiodynamic polarization method (Figure 4-56) demonstrated that in the TiN coating systems, the EB TiN/SS coating shows a lower corrosion current density than $\mathrm{CA}$ TiN/SS, indicating that EB TiN/SS coating has a higher corrosion resistance than CA TiN/SS coating when same substrate material is used; and this was due to its lower defect density as compared with CA TiN/SS coating. In the CA TiN coating systems with different substrates, CA TiN/SS coating system showed a lower current density than CA TiN/MS, indicating a higher corrosion resistance of the CA TiN/SS coating system than CA TiN/MS, as mild steel lacks of the ability to form passive oxide film. All the PMMA-treated coating samples showed significantly lower current densities than the as-deposited counterparts as PMMA effectively clogged the through-coating defects and improved the corrosion resistance of the treated coating systems. These results also suggest that the presence of defects is a leading cause in compromising corrosion resistance of any given coating system.

In $\mathrm{CrN}$ based coating systems, $\mathrm{CrSiCN}(1)$ showed the lowest corrosion current density, as it had the densest microstructure. Whereas $\mathrm{CrSiCN}(2)$ and $\mathrm{CrSiCN}(3)$ showed higher corrosion current densities due to their porous microstructures. 


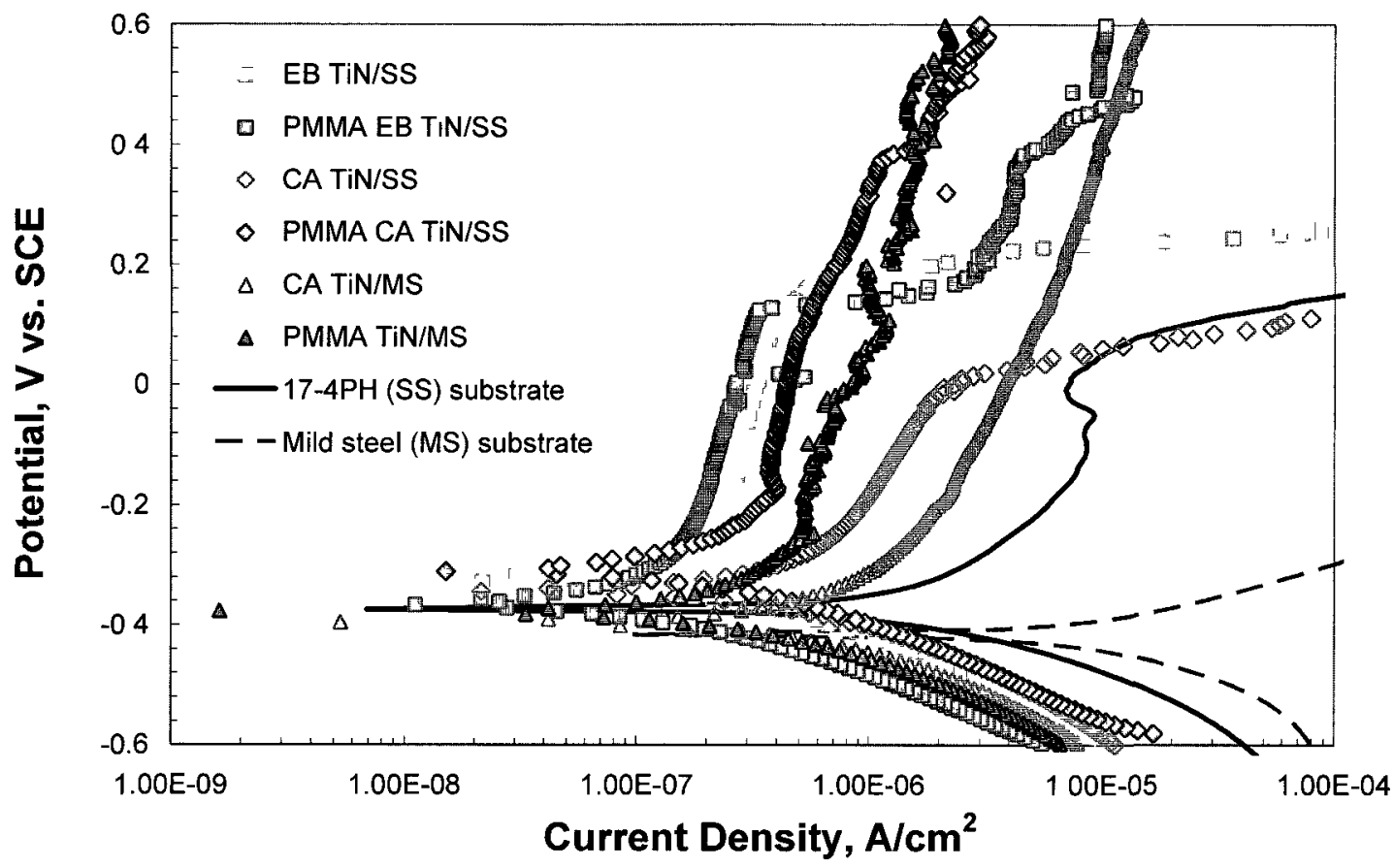

(a) TiN coating systems and PMMA-treated counterparts

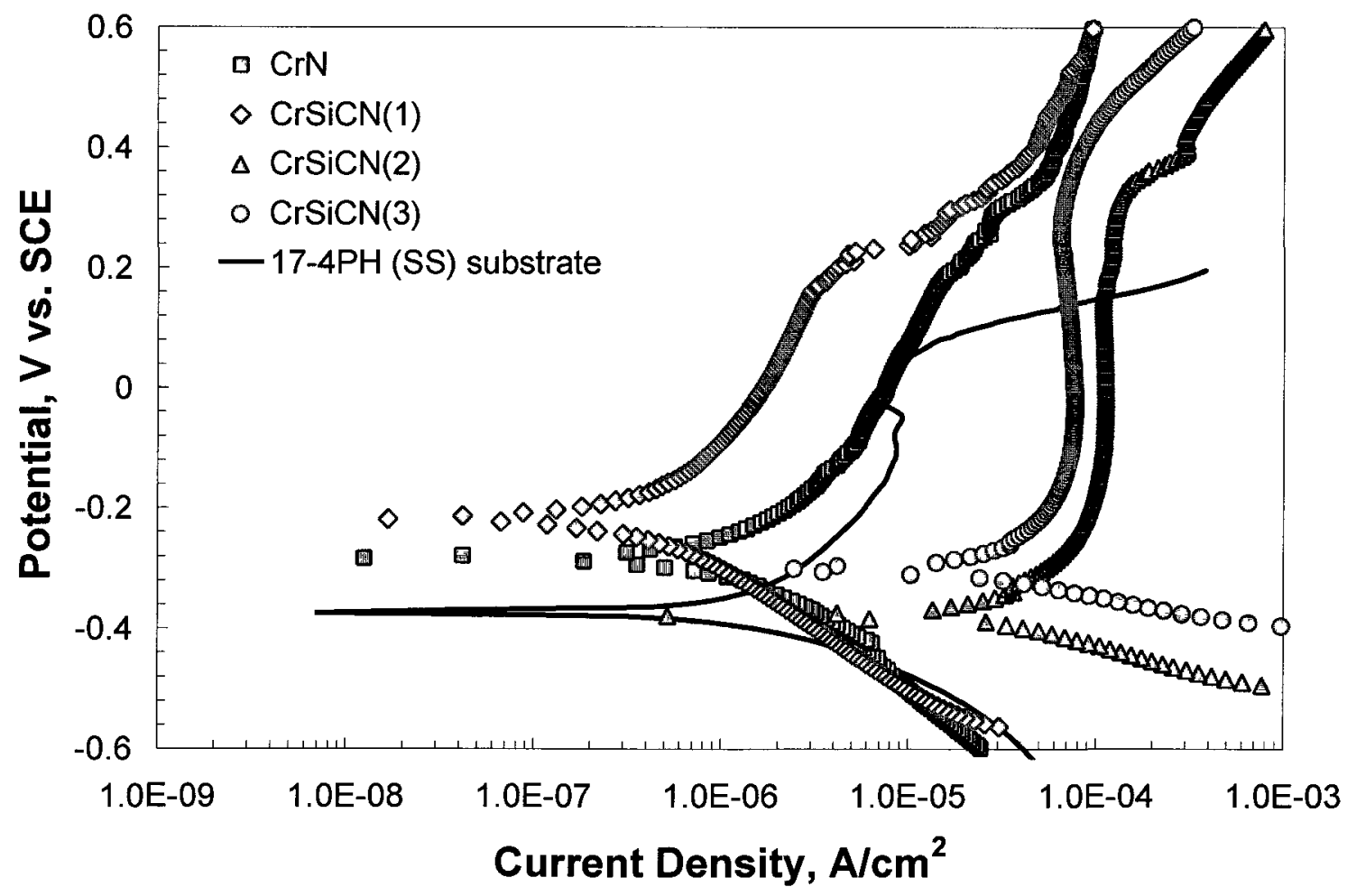

(b) $\mathrm{CrN}$ based coating systems

Figure 4-56 Polarization behaviours of test PVD coating systems. 
Mathematical analyses were conducted, and EIS tests were applied with respect to electrochemical diffusion impedance in coating systems. Post testing data processing and modeling studies revealed that the two factors diffusion coefficient and diffusion length played controlling effects on corrosion behaviour of a coating system. It was found that in a coating system effective diffusion coefficient was proportional to coating porosity, and diffusion layer thickness increased with coating thickness (when the coating is thicker than the Nernst Diffusion Layer (NDL) on a given substrate); thus a lower coating porosity or a greater coating thickness would result in an increase in diffusion impedance.

The summaries of typical EIS plots (twenty four hours of immersion) are given in Figure 4-57. In the Nyquist plots as shown in Figure 4-57 (a), the small semicircle (arc) for the CA TiN/MS indicates a typical charge transfer controlled corrosion behaviour and low diffusion impedance; whereas the straight line shown for CA TiN/SS system indicates a diffusion dominated corrosion behaviour and high diffusion impedance. The distinguishable corrosion characteristics stemmed from the different corrosion mechanisms of the substrates. The corrosion on stainless steel is diffusion controlled due to the formation of passive oxide film; while the corrosion on mild steel is a charge transfer controlled mode because of anode dissolution. For the EB TiN/SS coating system, semicircle (arc) in the Nyquist plots revealed a charge transfer featured corrosion behaviour after twenty four hours of immersion; its diffusion impedance was found between those of $\mathrm{CA}$ TiN/MS and CA TiN/SS coating systems. Its higher diffusion impedance comparing to $\mathrm{CA}$ TiN/MS was attributed to the stainless steel substrate that 
provided a protection with a passive oxide film; whereas its lower diffusion impedance comparing to $\mathrm{CA}$ TiN/SS was due to its thinner coating thickness $(1.5 \mu \mathrm{m})$. High bias voltages applied in the CA process resulted in a higher surface diffusivity of the deposited coating, thus led to fewer through-coating voids, and eventually a lower effective diffusion coefficient of the CA TiN coating systems.

All the PMMA-treated TiN coating samples show increased diffusion impedances (Figure 4-57 a), revealed that PMMA indeed clogged through-coating defects, thus resulted in lower effective diffusion coefficients. Also note that for the coating systems EB TiN/SS (after twenty four hours of immersion) and CA TiN/MS, after PMMA treatment, the EIS Nyquist plots changed from semicircles (arc) to the combination of a semicircle and straight line. This suggests a shift in corrosion process from typical charge transfer controlled mode to a combination of charge transfer and diffusion controlled modes; and indicates that post-deposition treatment with PMMA not only increased the diffusion impedance but also modified the corrosion mechanism in the coating systems.

In $\mathrm{CrN}$ based coating systems, $\mathrm{CrN}$ and $\mathrm{CrSiCN}(1)$ showed significant higher impedances than $\mathrm{CrSiCN}(2)$ and $\mathrm{CrSiCN}(3)$ (Figure 4-57 b). The high diffusion impedances of the $\mathrm{CrN}$ and $\mathrm{CrSiCN}(1)$ coating were attributed to their denser microstructures that therefore lower effective diffusion coefficients. In contrast, the very low diffusion impedances of the $\mathrm{CrSiCN}(2)$ and $\mathrm{CrSiCN}(3)$ coating systems were due to their larger grain sizes and porous microstructures that resulted in the higher effective diffusion coefficients. 


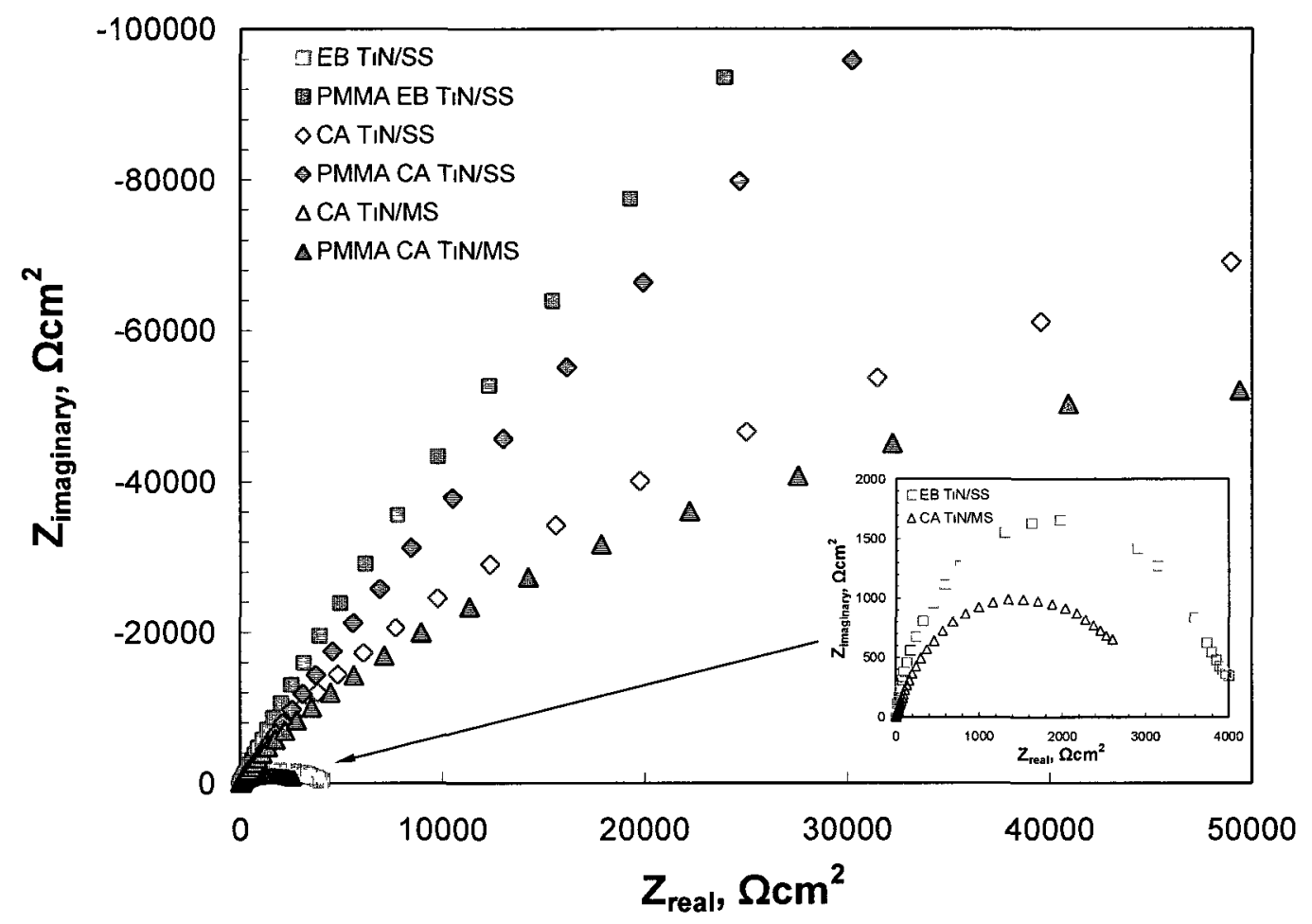

(a) TiN coating systems and PMMA-treated counterparts

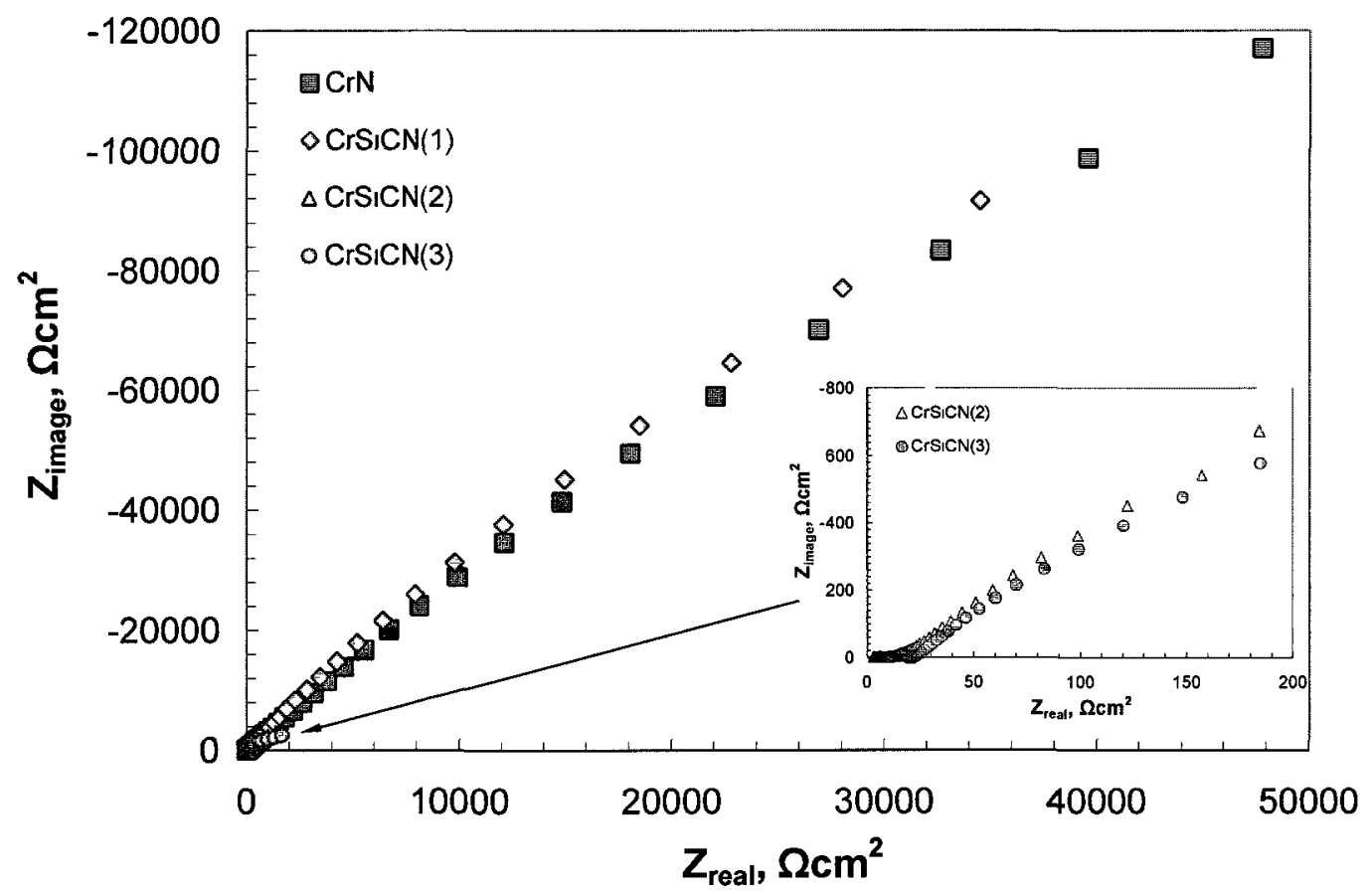

(b) $\mathrm{CrN}$ based coating systems

Figure 4-57 EIS (Nyquist) plots of test PVD coating systems (at $24 \mathrm{hr}$ ). 
Based on the EIS analyses, Warburg diffusion and Open Finite-Length Diffusion (OFLD) EEC models were applied to TiN and $\mathrm{CrN}$ based coating systems for infinite length diffusion and finite length diffusion, respectively. The results, in form of simplified magnitude level, are summarized in Table 4-26. CA TiN/SS was observed to have almost twice the thickness of the EB TiN/SS coating system and about two orders of magnitude higher diffusion impedance than the EB TiN/SS coating system (Table 4-26). This was mainly attributed to the increased electrochemical diffusion length (i.e., thicker coating thickness of CA TiN/SS coating system). This result proved the modeling studies which revealed that the coating thickness has a direct influence on diffusion impedance; and a sufficient coating thickness is therefore needed to increase the electrochemical diffusion impedance for the coating system.

Table 4-26 Summaries of coating thickness, porosities, polarization resistance $\left(R_{p}\right)$ and diffusion impedance $(Z)$ for the test PVD coating systems

\begin{tabular}{|c|c|c|c|}
\hline Coating system & $\begin{array}{c}\text { Thickness } \\
(\mu \mathrm{m})\end{array}$ & $\begin{array}{c}R_{p} \\
\left(\mathrm{k} \Omega \mathrm{cm}^{2}\right)\end{array}$ & $\begin{array}{c}Z \\
\left(\mathrm{~cm}^{2} \Omega \sqrt{ } \mathrm{s}\right)\end{array}$ \\
\hline EB TiN/SS & 1.5 & $3.87-3.68 \times 10$ & $1.85 \times 10-1.06 \times 10^{+3}$ \\
\hline PMMA EB TiN/SS & 1.5 & $4.18-1.21 \times 0^{+3}$ & $2.84 \times 10^{+5}-1.77 \times 10^{+6}$ \\
\hline \multicolumn{4}{|c|}{} \\
\hline CA TiN/SS & 2.8 & $4.48-2.15 \times 10^{+2}$ & $6.77 \times 10^{+3}-1.73 \times 10^{+5}$ \\
\hline PMMA CA TiN/SS & 2.8 & $8.97-4.95 \times 10^{+2}$ & $3.78 \times 10^{+3}-1.38 \times 10^{+5}$ \\
\hline \multicolumn{4}{|c|}{} \\
\hline CA TiN/MS & 2.8 & $2.59-6.96$ & $7.57 \times 10-2.87 \times 10^{+2}$ \\
\hline PMMA CA TiN/MS & 2.8 & $1.72 \times 10-1.0 \times 10^{+2}$ & $3.56 \times 10^{+2}-1.6 \times 10^{+4}$ \\
\hline \multicolumn{4}{|c|}{} \\
\hline CrN & 20.2 & $1.82 \times 10^{+2}-4.32 \times 10^{+2}$ & $1.51 \times 10^{+3}-5.19 \times 10^{+3}$ \\
\hline CrSiCN(1) & 18.9 & $5.31 \times 10^{+2}-1.43 \times 10^{+3}$ & $5.08 \times 10^{+2}-2.83 \times 10^{+3}$ \\
\hline CrSiCN(2) & 21.1 & $1.1 \times 10^{-2}-1.5 \times 10^{+2}$ & $1.62-5.09$ \\
\hline CrSiCN(3) & 19.6 & $2.85 \times 10^{-3}-4.33 \times 10^{+2}$ & $1.03-4.50$ \\
\hline
\end{tabular}

* The data for diffusion impedance is reciprocal of that for obtained diffusion admittance 
PMMA-treated TiN coating samples demonstrate significantly increased electrochemical diffusion impedances and polarization resistances (Table 4-26) as PMMA clogged through-coating defects and lowered the effective diffusion coefficients. The results demonstrated the role of coating defect in governing electrochemical diffusion impedance, and suggested that coatings must be designed with lower porosity or defect population in order to increase their corrosion resistance.

In the $\mathrm{CrN}$ based coating systems, $\mathrm{CrSiCN}(2)$ and $\mathrm{CrSiCN}(3)$ show one to two orders of magnitude lower electrochemical diffusion impedance and polarization resistance than $\mathrm{CrN}$ and $\mathrm{CrSiCN}(1)$ (Table 4-26). These results were due to their larger grain sizes and porous microstructures that led to higher effective diffusion coefficients through the coatings. It also concluded that coating composition could result in changes in coating microstructure, and consequently corrosion behaviours.

The outcomes from this research provided major findings in terms of effects of coating porosity and thickness in governing the corrosion resistance. They also indicated that lowering coating defect density is an essential approach to corrosion prevention in coating systems. 


\section{Conclusions and Future Work}

A series of coatings were deposited using techniques including Ni electroplating, EB and CA TiN coatings, and PEMS CrN based coatings. The coating samples were examined for the effects of coating compositions and deposition process on coating microstructure and mechanical properties. Further, they were evaluated for wear, erosion and corrosion behaviours using a series of systematical approaches. The correlation between the composition, deposition method and condition, microstructure and the behaviours of coating systems were analyzed.

\subsection{Summary of the Findings}

\section{Effects of Composition and Deposition Condition on Coating Microstructure Properties}

The grain size of the Ni electroplated coatings was found to decrease when the deposition current was pulsed, and decrease with pulse frequency; the grain size was also observed to decrease when nano-scaled particulates (i.e, CNTs in this study) were added into the electrolyte. Surface morphology was observed to be finer with an increase in pulse frequency, which is associated with the reduction in grain size with pulse frequency. The plating thickness was also found to decrease due to pulsed deposition current and addition of CNTs (for a given deposition time). However with CNTs present, the thickness reduction effect of pulse frequency was found to lessen. The attenuated effect 
of the increase in pulse frequency with the presence of CNTs indicated that a high rate of nucleation plays a dominant role in the reduction of grain size for $\mathrm{Ni}$ electroplated coatings.

The TiN coatings deposited using EB and $\mathrm{CA}$ techniques demonstrated different morphologies. The CA deposited TiN coating systems had a coarser surface morphology, higher defect density, and larger defects than the EB TiN coating systems, phenomena which are known to be associated with the effect of the cathodic arc on the growth of the deposition.

The study of the $\mathrm{CrN}$ based coating systems with varying concentrations of $\mathrm{Cr}, \mathrm{Si}, \mathrm{C}$, and $\mathrm{N}$ deposited using plasma enhanced magnetron sputtering (PEMS) showed that coating composition also affects coating microstructure in terms of surface morphology, grain size and porosity. Particularly, an optimal Si content (1.3 at.\% in this study) was found to exist where the grain size was finest.

These results indicate that deposition process and composition are the two major factors governing the microstructure of a coating system.

In addition to deposition process, post-deposition treatment is an approach to modifying the microstructure of a coating system. In this study, post-deposition treatment with PMMA clogged the exposure pores and gaps surrounding nodules in the TiN coating systems, thus reducing the permeability of the TiN coating system for corrosive media. 


\section{Effects of Composition and Deposition Condition on Coating Mechanical Properties}

For Ni plated samples, the hardness $(H)$, Young's modulus $(E)$ and the $\mathrm{H}^{3} / \mathrm{E}^{2}$ ratio were found to increase with the application of pulsed deposition current or the addition of CNTs. These changes are associated with grain size reduction due to pulse frequency or the addition of CNTs.

The TiN coating systems deposited using $\mathrm{EB}$ and $\mathrm{CA}$ techniques also demonstrated different mechanical properties. The effect of ion bombardment in the CA process resulted in higher hardness and Young's modulus in the CA TiN coating than was obtained with EB deposition.

The examinations of the $\mathrm{CrN}$ based coating systems with varying concentrations of $\mathrm{Cr}, \mathrm{Si}$, $\mathrm{C}$, and $\mathrm{N}$ deposited using plasma enhanced magnetron sputtering (PEMS) showed that coating composition also affects mechanical properties. And an optimal Si content (1.3 at.\% in this study) was found to exist where the hardness (H), Young's modulus (E) and the $\mathrm{H}^{3} / \mathrm{E}^{2}$ ratio were the highest.

These results indicate that as a consequence of the influence on microstructure, composition and deposition technique affect the mechanical properties of coating systems, which will determine the performances of the coating systems in particular applications, for example wear, erosion and corrosion behaviour. 


\section{Tribological Properties and Their Relationship with Mechanical Properties of Coating Systems}

For both TiN and $\mathrm{CrN}$ based coating systems, the specific wear rate obtained from drysliding wear tests showed an inverse proportional relationship with the $\mathrm{H}^{3} / \mathrm{E}^{2}$ ratio. This trend confirms that the resistance to plastic deformation (that is indicated by a combined effect of hardness $(H)$ and Young's modulus $(E)$, the $H^{3} / E^{2}$ ratio) is an essential factor governing wear behaviour.

The erosion tests show that high hardness $(\mathrm{H})$, Young's modulus $(\mathrm{E})$ and $\mathrm{H}^{3} / \mathrm{E}^{2}$ ratio (e.g. as in the $\mathrm{CA}$ TiN coating) result in a high resistance to erosion at all impingement angles. This suggests that a high resistance to plastic deformation ensures a high resistance to erosion. The erosion characteristics shifted from ductile to brittle with an increase in hardness $(\mathrm{H})$ and $\mathrm{H}^{3} / \mathrm{E}^{2}$ ratio (e.g., $\mathrm{CrN}$ and $\mathrm{CrSiCN}$ coatings); and the erosion rates for different coating systems with varying hardness $(H)$, Young's modulus(E) and $\mathrm{H}^{3} / \mathrm{E}^{2}$ ratio are a function of the impingement angles due to the erosion mechanism change.

Because the values of hardness $(\mathrm{H})$ and Young's modulus $(\mathrm{E})$ of a coating system can be modified through composition and process changes, it follows that the tribological properties behaviours can be governed by composition and deposition process. 


\section{Electrochemical Corrosion Properties of Coating Systems}

\section{Corrosion Mechanism and Coating Microstructure}

Electrochemical corrosion studies show that the corrosion behaviour of a coating system is determined by both the coating microstructure and the substrate properties. The substrate composition, microstructure and surface morphology are the essential properties determining the corrosion mechanism. The coating microstructure affects the mode of mass transfer of corrosive media through the coating to the substrate, and is the key factor governing the extent of the corrosion process and the corrosion mechanism.

In this study, some of the coating systems with a mild steel substrate (for example CA TiN/MS coating system) show charge-transfer featured corrosion. This is due to the mild steel substrate on which the corrosion is in the form of metal dissolution, and is chargetransfer controlled. The coating systems with stainless steel substrate show typical diffusion dominated corrosion processes. This is due to the passive and dielectric oxide film on the stainless surface, thus corrosion is diffusion controlled. Furthermore, through EEC analysis applying the Warburg diffusion and Open Finite-Length Diffusion (OFLD) EEC models, it was found that the corrosion processes in the TiN and $\mathrm{CrN}$ based coating systems were controlled by infinite length diffusion and finite length diffusion, respectively. This difference in corrosion mechanism is due to their distinctive coating microstructures (i.e., columnar structure for $\mathrm{TiN}$ and equiaxed structure for $\mathrm{CrN}$; where the equiaxed microstructure is more beneficial in providing high diffusion impedance). 


\section{Polarization Resistance and Its Relationship with Coating Microstructure}

Potentiodynamic polarization tests show that EB TiN coating systems have a lower corrosion current density and higher polarization resistance than CA TiN coating systems; these results are due to the higher defect density of the CA TiN coating system. In $\mathrm{CrN}$ based coating systems, it was observed that corrosion current density decreased (consequently polarization resistance increased) with a decrease in average grain size of the coatings.

These observations indicate that coating microstructure, particularly the presence of coating defects affects the polarization resistance in a coating system; and suggest that a small grain size and low defect density offers better corrosion resistance.

\section{EIS Measurement and Properties of Coating Systems}

EIS measurements also showed that CA TiN coating systems demonstrated higher diffusion impedance than the EB TiN coating systems. This is because the CA TiN coating is thicker than the EB TiN coating, and the high bias voltages applied in the CA process resulted in greater surface diffusivity of the deposited species and hence allowed fewer through-coating voids in the CA TiN coating systems.

In CrN based coating systems, EIS measurements also found that the coating systems with small average grain sizes and dense microstructures (e.g., $\mathrm{CrSiCN}(1)$ and $\mathrm{CrN}$ ) demonstrated high diffusion impedances. In general, any coating system with a small 
average grain size, dense microstructure, and few through-coating defects has high electrochemical diffusion impedance.

\section{Electrochemical Diffusion Impedance and Properties of Coating Systems}

In this study, theoretical analyses and experimental studies were conducted to examine the relationship between coating microstructure, diffusion factors and the diffusion impedance of the coating system. The effects of diffusion coefficient and diffusion layer thickness on the diffusion impedance of an electrochemical diffusion process were studied. The correlations between the coating microstructure in a coating system and the two electrochemical diffusion factors (diffusion coefficient and diffusion layer thickness) were analyzed. It was found that in a coating/substrate system the effective diffusion coefficient is proportional to the coating porosity, and the diffusion layer thickness on a given substrate would be increased with a sufficient coating thickness (when the coating thickness is thicker than the Nernst Diffusion Layer (NDL) thickness on the substrate). In an electrochemical corrosion process, for high electrochemical impedance, a low effective diffusion coefficient or a thick diffusion layer is required. Accordingly, for a coating/substrate system, a thick and dense coating system would be able to provide a high resistance to corrosion because of its high electrochemical diffusion impedance. This observation was made clear in this study where the relatively thick $(18.9 \mu \mathrm{m})$ and dense coating system $(\mathrm{CrSiCN}(1))$ exhibited the highest electrochemical impedance among all the as-deposited coating systems. 


\section{Effect of PMMA Treatment on Corrosion Behaviour of Coating Systems}

A major finding is that post-deposition treatment with PMMA on the CA and EB TiN coating systems resulted in significant increases in polarization resistance and electrochemical diffusion impedance by clogging the through-coating defects. Furthermore the PMMA treatment on the CA TiN/MS coating system also changed the corrosion mechanism from charge-transfer controlled to diffusion governed, which was confirmed with the EEC model fitted results. It also demonstrates that the coating defect density is the essential factor determining corrosion behaviour of coating systems. Thus modifying the defect density in a coating system would change not only the resistance to corrosion, but also the corrosion mechanism to some extent.

The results of the PMMA treatment suggest that this treatment is a worthwhile approach to improving the anti-corrosion performance of a coating system, especially in mild temperature and non-wear/erosion applications.

\subsection{Major Contributions}

\section{Tribological and Corrosion Studies on TiN and CrN Based Coating Systems}

Through this thesis work, correlations between microstructure and tribological and corrosion behaviours in CA and EB TiN coating systems as well as PEMS CrN based coating systems were revealed. It was confirmed that it is essential to increase resistance to plastic deformation (indicated by a high $\mathrm{H}^{3} / \mathrm{E}^{2}$ ratio) for superior anti-wear and antierosion behaviour, and to lower porosity (defect density) for superior anti-corrosion 
behaviour. Defect density and the $\mathrm{H}^{3} / \mathrm{E}^{2}$ ratio are determined by the coating composition and the coating process (deposition method and deposition parameters).

The properties of $\mathrm{CrN}$ based coating systems can be modified by adjusting the composition (i.e., $\mathrm{Cr}, \mathrm{Si}, \mathrm{C}$, and $\mathrm{N}$ ); as a critical element in composition, an optimal $\mathrm{Si}$ content (around 1.3 at.\%) is suggested for the optimized properties for anti-wear/erosion and anti-corrosion applications. For the same coating composition this research suggests that $\mathrm{CA}$ deposition provides a better TiN coating than $\mathrm{EB}$ deposition in anti-wear/erosion and anti-corrosion applications.

\section{Correlation between Diffusion Factors and Diffusion Impedance in Coating Systems}

The analyses of the correlation between the diffusion factors (effective diffusion coefficient and diffusion layer thickness) and the overall diffusion impedance for a coating system reveals the relationship between coating microstructure, thickness and its resistance to corrosion; it provides a fundamental interpretation, at a diffusion layer level, of the roles of thickness and porosity in governing the electrochemical impedance of the coating system. The result suggests that the characteristic diffusion parameter in the diffusion impedance (Eq. 4-8 a) should be taken into account in the coating system design; and also that the coating thickness and porosity should be included in determining the characteristic diffusion parameter in the diffusion impedance. 


\section{Application of PMMA Treatment}

The post-deposition treatment with PMMA showed a significant effect in providing anticorrosion properties to a coating system by clogging pores in the coating. Combining the method of PMMA treatment with electrochemical tests and EEC analyses, an effective methodology for the study of a coating system with respect to the influence of defects on the coating corrosion behaviours has been developed.

\section{Systematic Thinking in Coating Systems}

This study illustrates that a coating is a system, where the composition and the deposition method determine the microstructure and therefore determine the mechanical properties. Tribological and corrosion behaviours of a coating system are influenced by both the microstructure and the properties of the coating and substrate. To study or design a coating system for a specific application, all of these factors and relationships must be considered. For wear resistance applications, the coating system is basically required to have high resistance to plastic deformation, which is indicated by the $\mathrm{H}^{3} / \mathrm{E}^{2}$ ratio. In addition, a high hardness and low coefficient of friction is preferred. Also an oxidation resistant composition is beneficial to reduce chemical reactions due to the elevated temperature of the wear process. For erosion resistance applications, the coating systems are also required to have high resistances to plastic deformation. Additionally, in some particular erosion conditions, brittleness and ductility should be taken into account with consideration of the effect of impingement angle. For corrosion resistance applications of a coating system, in addition to anti-corrosion compositions, dense microstructure and sufficient thickness are required. 


\subsection{Future Work}

This study successfully accomplished the goal in exploring the factors governing the tribological and corrosion behaviours and their correlations to the microstructure and properties of coating systems. However, some issues need further study.

In this study, because the $\mathrm{Ni}$ electroplating processing time was kept constant (120 minutes), the plating thickness was not constant for different deposition current frequencies. Some of the thinner plates were not able to effectively protect the substrate steel from erosion. Further experiments would be needed to generate plated samples with uniform and sufficient thickness in order to avoid the influence from the substrate.

Further work could include a measurement of the coating fracture toughness. A correlation between fracture toughness and erosion resistance of a hard coating system should be established, particularly for erosion at high impingement angles.

Multiple layered coating systems should also be explored. Future work on multiple layered coating systems, including superlattice structures, are expected to show enhanced overall resistance to wear and corrosion. The expected high resistance to wear is attributed to a high overall hardness and resistance to plastic deformation. The resistance to corrosion is expected to be higher than that of a single layered coating system for the same coating thickness and porosity, based on the expected lower overall effective diffusion coefficients through the multiple layered coating systems. 


\section{Reference}

1 M.B. Peterson, Classification of Wear Processes, M.B. Peterson and W.O. Winer (Eds.), Wear Control Handbook, ASME, New York, 1980.

2 G.H. Koch, M.P.H. Brongers, N.G. Thompson, Y. Paul Virmani, J.H. Payer, Corrosion Costs and Preventive Strategies in the United States, Supplement to Materials Performance, July 2002, Report No. FHWA-RD-01-156, Federal Highway Administration, McLean, VA, 2002.

3 Battelle Columbus Laboratories and the National Institute of Standards and Technology, Economic Effects of Metallic Corrosion in the United States, 1978, and Battelle updates in 1995.

4 J.R. Davis (Ed.), Surface Engineering for Corrosion and Wear Resistance, Maney Publishing, London, 2001.

5 S.J. Findlay and N. D. Harrison, Why Aircraft Fail, Mater. Today 5 (2002) 18.

6 Bundesministerium fur Forschung und Technologie (Federal Ministry for Research and Technology), Research Report (T76-38) Tribologie (Code BMFT-FBT76-38), West Germany, 1976.

7 V.R. Parameswaran, J.-P. Immarigeon, D. Chow, D. Morphy, Erosion Coatings for Compressor Applications, in A.K. Koul, V.R. Parameswaran, J.-P. Immarigeon, W. Wallace (Eds.), Advances in High Temperature Structure Materials and Protective Coatings, National Research Council of Canada, Ottawa, Canada, 1994.

8 A.W. Batchelor, Loh Nee Lam, Margam Chandrasekaran, Materials Degradation and its Control by Surface Engineering ( ${ }^{\text {nd }}$ Ed.), Imperial College Press, London, 2002.

9 J. Kruger, Cost of metallic corrosion, in Uhlig's Corrosion Handbook (2nd Ed.), R.W. Revie (Ed.), Wiley, New York, 2000.

10 ASM International Handbook Committee, ASM Handbook Vol.18: Friction, Lubrication and Wear Technology, Materials Park, Ohio, 1992.

11 Jeff Th. M. De Hosson and Albano Cavaleiro, Galileo Comes to the Surface, Nanostructured Coatings, Albano Cavaleiro and Jeff Th. M. De Hosson (Eds.), Springer Science Business Media, LLC, New York, 2006.

$12 \mathrm{~K}$. Holmberg and A. Matthews, Coatings Tribology, in Tribology Series 28, D. Dowson (Ed.), Elsevier, Amsterdam, 1994.

13 K.N. Strafford, P.K. Datta, J.S. Gray, Surface Engineering Practice, Ellis Horwood, New York, 1990.

14 C.M. Cotell and J.A. Sprague, Preface, Surface Engineering, Vol. 5, ASM Handbook, ASM International, 1994.

15 K. Funatani, Heat Treatment of Automotive Components: Current and Future Trends, Trans. Indian Inst. Met. 57 (2004) 381. 
16 M. Hetmańczyk, L. Swadźba, B. Mendala, Advanced Materials and Protective Coatings in Aero-engines Application, Journal of Achievements in Materials and Manufacturing Engineering 24 (2007) 372

17 D. Zhu and R.A. Miller, Thermal Barrier Coatings for Advanced Gas Turbine and Diesel Engines, NASA/TM (1999) 209453.

18 R.G. Wellman and J.R. Nicholls, On the Effect of Ageing on the Erosion of EB-PVD TBCs, Surf. Coat. Technol. $177-178$ (2004) 80.

19 R.G. Wellman and J.R. Nicholls, Some Observations on Erosion Mechanisms of EBPVD TBCs, Wear 242 (2000) 89.

20 A.G. Davis, D.H. Boone, A.V. Levy, Erosion of Ceramic Thermal Barrier Coatings, Wear 110 (1986) 101.

21 J.R. Nicholls, Y. Jaslier, D.S. Rickerby, Erosion of EB-PVD Thermal Barrier Coatings, Mater. High Temp. 15 (1998) 15.

22 T. Rhys-Jones, F.C. Toriz, Thermal Barrier Coatings for Turbine Use in Aero Engines, High Temp. Technol. 7 (1989)73.

23 A. Thiruvengadam, The Concept of Erosion Strength, Erosion by Cavitation or Impingement, ASTM STP 408, Am. Soc. Testing Mats. (1967) 22.

24 V.R. Parameswaran, J.-P. Immarigeon, D. Nagy, Titanium Nitride Coating for Aero Engine Compressor Gas Path Components, Surf. Coat. Technol. 52 (1992) 251.

25 J.T. DeMasi-Marcin and D.K. Gupta, Protective Coatings in the Gas Turbine Engine, Surf. Coat. Technol. 68-69 (1994) 1.

26 H.I.H. Saravanamuttoo, G.F.C. Rogers, H. Cohen, Gas Turbine Theory (5th Ed.), Prentice Hall, Harlow, England, 2001.

27 R. Buhl, H.K. Pulker, E. Moll, TiN Coatings on Steel, Thin Solid Films 80 (1981) 265.

28 J.E. Restall, Surface Degradation and Protective Treatments, G.W. Meetham (Ed.), the Development of Gas Turbine Materials, Applied Science Publishers Ltd., London, 1981.

29 C. Rebholz, H. Ziegele, A. Leyland, A. Matthew, Structure, Mechanical and Tribological Properties of Nitrogen-containing Chromium Coatings Prepared by Reactive Magnetron Sputtering, Surf. Coat. Technol. 115 (1999) 222.

30 R.L. Hatschek, Am. Mach. Special Report 752 (1983) 129.

31 H. Boone, T.E. Strangman, L.W. Wilson, Some Effects of Structure and Composition on the Properties of Electron Beam Vapor Deposited Coatings for Gas Turbine Superalloys, J. Vac. Sci. Technol. A 11 (1974) 641.

32 R. Aharonov, M. Chhowalla, S. Dhar, R.P. Fontana, Factors Affecting Growth Defect Formation in Cathodic Arc Evaporated Coatings, Surf. Coat. Technol. 82 (1996) 334. 
33 C. Liu, Q. Bi, A. Matthews, EIS Comparison on Corrosion Performance of PVD TiN CrN Coated Mild Steel in 0.5N NaCl Aqueous Solution, Corros. Sci. 43 (2001) 1953.

34 H. Ahn, Y. S. Choi, J. G. Kim, J. G. Han, A Study on Corrosion Resistance Characteristics of PVD Cr-N Coated Steels by Electrochemical Method, Surf. Coat. Technol. 150 (2002) 319.

35 H. Uchida, S. Inoue, K. Koterazawa, Electrochemical Evaluation of Pinhole Defects in TiN Films Prepared by r.f. Reactive Sputtering, Mater. Sci. Eng. A 234-236 (1997) 649.

36 H.A. Jehn, Improvement of the Corrosion Resistance of PVD Hard Coating-Substrate Systems, Surf. Coat. Technol. 125 (2000) 212.

37 C. Liu, Q. Bi, A. Leyland, A. Matthews, An Electrochemical Impedance Spectroscopy Study of the Corrosion Behaviour of PVD Coated Steels in $0.5 \mathrm{~N} \mathrm{NaCl}$ Aqueous Solution: Part I. Establishment of Equivalent Circuits for EIS Data Modeling, Corros. Sci. 45 (2003) 1243.

38 V.K.W. Grips, H.C. Barshilia, V. Ezhil Selvi, Kalavati, K.S. Rajam, Electrochemical Behaviour of Single Layer CrN, TiN, TiAlN Coatings and Nanolayered TiAlN/CrN Multilayer Coatings Prepared by Reactive Direct Current Magnetron Sputtering, Thin Solid Films 514 (2006) 204.

39 Z.-J. Jin, C.-Q. Li, L. Yu, W.-T. Wu, Corrosion Performance of Ion-Plated Titanium and Yttrium-Modified TiN Coatings, Surf Coat. Technol. 46 (1991) 307.

40 H.A. Jehn and M.E. Baumgärtner, Corrosion Studies with Hard Coating-Substrate Systems, Surf. Coat. Technol. 54-55 (1992) 108.

41 C. Liu, A. Leyland, Q. Bi, A. Matthews, Corrosion Resistance of Multi-Layered PAPVD TiN and CrN Coatings, Surf. Coat. Technol. 141 (2001) 164.

42 J. Warren, C. Gorton, S. Hoff, F. Alby, Annex B - Air, Land, Sea and Space FOD ISSUES, NATO RTO Technical Report TR-AVT-094 (2004).

43 R. Chattopadhyay, Surface Wear - Analysis, Treatment, and Prevention. OH, ASMInternational, 2001.

44 R. Chattopadhyay, Advanced Thermally Assisted Surface Engineering Processes. MA, Kluwer Academic Publishers, Norwell, MA, 2004.

45 ASTM G40 - 10 Standard Terminology Relating to Wear and Erosion.

46 D.M. Mattox, Chapter 1 Introduction, Handbook of Physical Vapor Deposition (PVD) Processing: Film Formation, Adhesion, Surface Preparation and Contamination Control, Noyes Publications, Westwood, New Jersey, 1998.

47 J.-Y. Lin and H.S. Cheng, An Analytical Model for Dynamic Wear, ASME J. Tribol. 111 (1989) 468.

48 J. Jamari, Running-in of Rolling Contacts, $\mathrm{PhD}$ Thesis, University of Twente, Enschede, The Netherlands, 2006. 
49 D.J. Whitehouse, The Effect of Surface Topography on Wear, Fundamentals of Tribology, Suh and Saka (Eds.), MIT (1980) 17.

50 V. Kraghelsky, M.N. Dobychun, V.S. Kombalov, Friction and Wear Calculation Methods, Pergamon Press, Oxford, 1982.

51 J.A. Williams, Wear Modeling, Analytical, Computational and Mapping, a Continuum Mechanics Approach, Wear 225-229 (1999) 1.

52 J. Xu, K. Kato, T. Hirayama, The Transition of Wear Mode During Running-in Process of Silicon Nitride Sliding in Water, Wear 205 (1997) 55.

53 R. Kumar, B. Prakash, A. Sethuramiah, A Systematic Methodology to Characterize the Running-In and Steady-State Wear Processes, Wear 252 (2002) 445.

54 W. Wang, P.L. Wong, Z. Zhang, Experimental Study of the Real Time in Surface Roughness During Running-in for PEHL Contacts, Wear 244 (2000) 140.

55 P.J. Blau, Friction and Wear Transition of Materials, Noyes, Park Ridge, NJ, 1989.

56 D. Zhu, A. Martini, W. Wang, Y. Hu, B. Lisosky, J.Q. Wang, Simulation of Sliding Wear in Mixed Lubrication, ASME J. Tribol. 129 (2007) 544.

57 K. Adachi, K. Kato, N. Chen, Wear Map of Ceramics, Wear 203-204 (1997) 291.

58 K.P. Purushotham, L.P. Ward, N. Brack, P.J. Pigram, P. Evans, H. Noorman, R.R. Manory, Wear Behaviour of CrN Coatings MEVVA Ion Implanted with Zr, Wear 257 (2004) 901.

59 S.H. Li and V. Palekar, Quantum Mechanochemistry Understanding of Tribochemical Reactions, Tribol. Mater. Surf. Interfaces 3 (2009) 132.

60 S.M. Hsu, J. Zhang, Z. Yin, The Nature and Origin of Tribochemistry, Tribol. Lett. 13 (2002) 131.

61 S.M. Hsu, M.C. Shen, A.W. Ruff, Wear Prediction for Metal, Tribol. Int. 30 (1997) 377.

62 G. Remond, C. Nockolds, M. Phillips, C. Roques-Carmes, Implications of Polishing Techniques in Quantitative X-Ray Microanalysis, J. Res. Natl. Inst. Stand. Technol. 107 (2002) 639.

63 E. Rabinowicz, Friction and Wear of Materials, John Willey \& Sons, Inc., Canada, 1995.

64 I. Garcia, A. Ramil, J.P. Celis, A Mild Oxidation Model Valid for Discontinuous Contacts in Sliding Wear Test: Role of Contact Frequency, Wear 254 (2003) 429.

65 J.F. Archard, Contact and Rubbing of Flat Surfaces, J. Appl. Phys. 24 (1953) 981.

66 A. Ruina and P. Rudra, Introduction to Statics and Dynamics, Oxford University Press, 2002.

67 R.C. Hibbeler, Engineering Mechanics (11th Ed.), Pearson, Prentice Hall, 2007.

68 R.W. Soutas-Little and B. Inman, Engineering Mechanics, Thomson, 2008. 
69 F.P. Beer and E.R. Johnston, Jr., Vector Mechanics for Engineers (6th Ed.), McGrawHill, 1996.

70 J.L. Meriam and L.G. Kraige, Engineering Mechanics (5th Ed.), John Wiley \& Sons, 2002.

71 R.T. Spurr and T.P. Newcomb, The Adhesion Theory of Friction, Proc. Phys. Soc. B 70 (1957) 98.

72 B. Bhushan, Principles and Applications of Tribology, John Wiley \& Sons, New York, 1999.

73 B.N.J. Persson, Sliding Friction: Physical Principles and Applications, SpringerVerlag, Berlin, Germany, 1998.

74 Z.S. Andras Fluid Film Lubrication: Theory and Design, Cambridge University Press, Cambridge, UK, 1998.

75 BJ. Hamrock, S.R. Schmid, B.O. Jacobson, Fundamentals of Fluid Film Lubrication ( $2^{\text {nd }}$ Ed.), Marcel Dekker, Inc., New York, 2004.

76 M.F.J. Bohan, T.C. Claypole, D.T. Gethin, The Application of Roughness Model to A Soft EHL Contact, Int. J. Numer. Methods Heat Fluid Flow 12 (2002) 356.

77 W.A. Glaeser (Ed), Characterization of Tribological Materials. Materials Characterization Series. Boston, Butterworth-Heinemann, 1993.

78 G.W. Stachowiak and A.W. Batchelor, Engineering Tribology, Burlington, Elsevier Butterworth-Heinemann, 2005.

79 J. F. Archard and W. Hirst, The Wear of Materials under Unlubricated Conditions, Proc, Royal Soc. A-236 (1958) 71.

80 M.M. Kruschov, Resistance of Metal to Wear by Abrasion, as Related to Hardness, Proc. Int. Conf. on Lubrication and Wear, I. Mech. E., London, (1957) 655.

81 K. Kato and K. Hokkirigawa, Abrasive Wear Diagram, Proceedings of the Eurotrib (1985) 1 .

82 D.-Y. Wang, K.-W. Weng, C.-L.g Chang, W.-Y. Ho, Synthesis of $\mathrm{Cr}_{3} \mathrm{C}_{2}$ Coatings for Tribological Applications, Surf. Coat. Technol. 120-121 (1999) 622.

83 R. Holm, The Friction Force over the Real Area of Contact (in German), Wiss. Veroff. Siemens-Werk 17 (1938) 38.

84 A. Zmitrowicz, Wear Patterns and Laws of Wear - A Review, J. of Theor. Appl. Mech. 44 (2006) 219.

85 D.A. Rigney, The Roles of Hardness in the Sliding Behaviour of Materials, invited for Festschrift honoring D. Dowson, Wear 175 (1994) 63.

86 J.F. Archard and W. Hirst, Wear of Metals under Unlubricated Conditions, Proceedings of the Royal Society of London. Series A, Mathematical and Physical Sciences 236 (1956) 397. 
87 M. Öqvizt, Numerical Simulation of Mild Wear Using Update Geometry with Different Step Size Approaches, Wear 249 (2001) 6.

88 A.B.J Hugnel, S. Bjorklund, S. Andersson, Simulation of the Mild Wear in a Camfollower Contact with Follower Rotation, Wear 199 (1996) 202.

89 A. Flodin and S. Andersson, Simulation of Mild Wear in Spur Gears, Wear 207 (1997) 16.

90 U. Olofsson, S. Andersson, S. Bjorklund, Simulation of Mild Wear in Boundary Lubricated Spherical Roller Thrust Bearings, Wear 241 (2000) 180.

91 A. Flodin and S. Andersson, Simulation of Mild Wear in Helical Gears, Wear 241 (2000) 123.

92 R.G. Bayer, Comment on Engineering Needs and Wear Models, In Tribological Modeling for Mechanical Designers, in K.C. Ludema and R.G. Bayer (Eds.), ASTM, Philadelphia, ASTM STP 1105 (1991) 3.

93 H.C. Meng and K.C. Ludema, Wear Models and Predictive Equation, Their Form and Content, Wear 443 (1995) 181.

94 I.M. Hutchings, Abrasive and Erosive Wear Tests for Thin Coatings: A Unified Approach, Tribol. Int. 31 (1998) 5.

95 J.W. Dini, Electrodeposition: The Materials Science of Coatings and Substrates, Noyes Publications, New Jersey, 1993.

96 ASTM G99-05, Standard Test Method for Wear Testing with a Pin-on-disk Apparatus.

97 S. Lakel, K. Almi, Y. Berriche, Micro-scale Abrasive Wear Testing of Cr-Nx Coatings, Rom. Rep. Phys. 59 (2007) 113.

98 D.M. Mattox, Chapter 10 Film Characterization and Some Basic Film Properties, Handbook of Physical Vapor Deposition (PVD) Processing: Film Formation, Adhesion, Surface Preparation and Contamination Control, Noyes Publications, Westwood, New Jersey, 1998.

99 E.N. Simons, Metal Wear: A Brief Outline, Frederick Muller Limited, London, 1972.

100 A.R. Lansdown and A.L. Price, Materials to Resist Wear, Pergamon Press, Oxford, 1986.

101 M. Ohring, The Materials Science of Thin Films, Academic Press, Inc., San Diego, 1992.

102 B.A. Lindsley and A.R. Marder, Solid Particle Erosion of an $\mathrm{Fe}^{-} \mathrm{Fe}_{3} \mathrm{C}$ Metal Matrix Composite, Metall. Mater. Trans. A 29 (1998) 1071.

103 A.V. Levy, Solid Particle Erosion and Erosion-Corrosion of Materials, ASM International, 1995.

104 R.J.K. Wood, B.G. Mellor, M.L. Binfield, Sand Erosion Performance of Detonation Gun Applied Tungsten Carbide/Cobalt-Cbxomium Coatings, Wear 211 (1997) 70. 
105 G.P. Tilly, Erosion Caused by Airborne Particles, Wear 14 (1969) 63.

106 I. Finnie, Some Reflections on the Past and Future of Erosion, Wear 186-187 (1995) 1.

107 I. Finnie, Erosion of Surface by Solid Particles, Wear 3 (1960) 87.

108 D. Aquaro and E. Fontani, Erosion of Ductile and Brittle Materials, Meccanica 36 (2001) 651.

109 R.A. Andrievski and G.V. Kalinnikov, Synthesis and Properties of $\mathrm{TiB}_{2} / \mathrm{TiN}$ and $\mathrm{TiB}_{2} / \mathrm{B}_{4} \mathrm{C}$ Films, A.A. Voevodin, D.V. Shtansky, E.A. Levashov, J.J. Moore (Eds.), Nanostructured Thin Films and Nanodispersion Strengthened Coatings, Kluwer Academic Publishers, Dordrecht, 2004.

110 A.V. Reddy and G. Sundararajan, The Influence of Grain Size on the Erosion Rate of Metals, Metall. Mater. Trans. A 22 (1991) 1043.

111 H.W. Wang, M.M. Stack, S.B. Lyon, P. Hovsepian, W.-D. Münz, Wear Associated with Growth Defects in Combined Cathodic Arc/Unbalanced Magnetron Sputtered $\mathrm{CrN} / \mathrm{NbN}$ Superlattice Coatings During Erosion in Alkaline Slurry, Surf. Coat. Technol. 135 (2000) 82.

112 V. Shanov and W. Tabakoff, Erosion Resistance of Coatings for Metal Protection at Elevated Temperatures, Surf. Coat. Technol. 86-87 (1996) 88.

113 R. Bellman Jr. and A. Levy, Erosion Mechanism in Ductile Metals, Wear 70 (1981) 1.

114 B.R. Lawn and S.M. Wiederhorn, Contact Fracture in Brittle Materials, Conference: Contact Mechanics and Wear of Rail/Wheel Systems, Proceedings of the International Symposium, Vancouver, BC. 1983.

115 Y. Bailout, J.A. Mathis, J.E, Talia, Solid Particle Erosion Mechanism in Glass, Wear 196 (1996) 263.

116 H. Wensik and M.C. Elwenspoek, A Closer Look at the Ductile-Brittle Transition in Solid Particle Erosion, Wear 253 (2002) 1035.

117 K. Bose, R.J.K. Wood, D.W. Wheeler, High Energy Solid Particle Erosion Mechanisms of Superhard CVD coatings, Wear 259 (2005) 135.

118 D.J. Stephenson and J.R. Nicholls, Modelling the Influence of Surface Oxidation on High Temperature Erosion, Wear 186-187 (1995) 284.

119 L.G. Wright, V.K. Sethi, A.J. Markworth, A Generalized Description of the Simultaneous Processes of Scale Growth by High-Temperature Oxidation and Removal by Erosive Impact, Wear 186-187 (1995) 230.

120 I.M. Hutchings, Tribology: Friction and Wear of Engineering Materials. Edward Arnold, London, 1992.

121 P.A. Engel, Impact Wear of Materials, Elsevier Scientific Publishing Company, Amsterdam, 1976. 
122 ASTM Standard G76, Standard Test Method for Conducting Erosion Tests by Solid Particle Impingement Using Gas Jets, ASTM International, West Conshohocken, PA, DOI: 10.1520/G0076-07 (2007).

123 T. Deng, M.S. Bingley, M.S.A. Bradley and S.R. De Silva, A Comparison of the Gas-blast and Centrifugal-accelerator Erosion Testers: The Influence of Particle Dynamics, Wear 265 (2008) 945.

124 P. Hedenqvist and A. Roos, Wear of Pyrolytic Tin Oxide and Anodized Aluminium Oxide Coatings on Aluminium, Surf. Coat. Technol. 48 (1991) 41.

125 E. Bardal, Corrosion and Protection, Springer, London, 2004.

126 R.W. Revie and H.H. Uhlig, Corrosion and Corrosion Control, John Wiley \& Sons, Inc., 2008.

127 N. Perez, Electrochemistry and Corrosion Science, Kluwer Academic Publishers, 2004.

128 D.L. Klarstrom. In Metals Handbook, ASM International, Metals Park, OH, 1987.

129 S.L. Pohlman, General Corrosion, in Corrosion, Vol. 13 ( $9^{\text {th }}$ Ed.), ASM International, (1987) 80.

130 S.C. Dexter, Localized Corrosion, in Corrosion, Vol. 13, ASM International, (1987) 104.

131 W.D. Callister, Jr., Materials Science and Engineering: An Introduction, John Wiley \& Sons, Inc., 2002.

132 K. Jüttner, Electrochemical Impedance Spectroscopy (EIS) of Corrosion Processes on Inhomogeneous Surfaces, Electrochim. Acta 35 (1990) 1501.

133 S.H. Ahn, J. H. Lee, J. G. Kim, J. G. Han, Localized Corrosion Mechanisms of the Multilayered Coatings Related to Growth Defects, Surf. Coat. Technol. 177-178 (2004) 638.

134 H. Uchida and M. Yamashita, Pinhole Defect Evaluation of TiN Films Prepared by Dry Coating Process, Vacuum 59 (2000) 321.

135 C.L. Li, H.X. Zhao, T. Takahashi, M. Matsumura, Improvement of Corrosion Resistance of Materials Coated with $\mathrm{A} \mathrm{Cr}_{2} \mathrm{O}_{3} / \mathrm{NiCr}$ Dilayer Using A Sealing Treatment, Mater. Sci. Eng. A308 (2001) 268.

136 H.S. Khatak and B. Raj (Eds.), Corrosion of Austenitic Stainless Steel: Mechanism, Mitigation and Monitoring, Woodhead Publishing, 2002.

137 D. Jones, Principles and Prevention of Corrosion ( ${ }^{\text {nd }}$ Ed.), Prentice-Hall, Upper Saddle River, NJ, 1996.

138 R.E. Melchers, Mathematical Modelling of the Diffusion Controlled Phase in Marine Immersion Corrosion of Mild Steel, Corros. Sci. 45 (2003) 923. 
139 C.C. Lin, K.-L. Chang, H.C. Shih, Corrosive Behaviour of Chromium Carbide-Based Films Formed on Steel Using A Filtered Cathodic Vacuum Arc System, Appl. Surf. Sci. 253 (2007) 5011.

140 G.T. Burstein and K. Sasaki, Effect of Impact Angle on the Slurry Erosion-Corrosion of 304L Stainless Steel, Wear 240 (2000) 80.

141 G.T. Burstein and K. Sasaki, The Birth of Corrosion Pits as Stimulated by Slurry Erosion, Corros. Sci. 42 (2000) 841.

142 M.M. Stack, Y. Purandare, P. Hovsepian, Impact Angle Effects on the ErosionCorrosion of Superlattice CrN/NbN PVD Coatings, Surf. Coat. Technol. 188-189 (2004) 556.

143 J.C. Oliveira, A. Cavaleiro, C.M.A. Brett, Influence of Sputtering Conditions on Corrosion of Sputtered W-Ti-N Thin Film Hard Coatings: Salt Spray Tests and Image Analysis, Corros. Sci. 42 (2000) 1881.

144 C.M.A. Brett, A. Cavaleiro, A Comparison of the Electrochemical Behaviour of WM-N (M=Ni, Ti, Al) Thin Film Coatings on High Speed Steel, Thin Solid Films 322 (1998) 263.

145 M.G. Fontana, Corrosion Engineering, McGraw-Hill Book Company, 1986.

146 D.A. Jones, Principles and Prevention of Corrosion, Macmillan Publishing Company, New York, 1992.

147 J.O'M. Bockris, A.K.N. Reddy, M.E. Gamboa-Aldeco, Modern Electrochemistry 2A: Fundamentals of Electrodics ( $2^{\text {nd }} E d$.), Springer, 2001.

148 M. Stern and A.L. Geary, Electrochemical Polarization, A Theoretical Analysis of the Shape of Polarization Curves, J. electrochem. Soc. 104 (1957) 56.

149 C. Wagner and W. Traud, On the Interpretation of Corrosion Processes through Superposition of Electrochemical Partial Processes and on the Potential of Mixed Electrodes, Z. Elektrochem. Ang. Physik. Chemie, 44 (1938) 391.

150 M. Stern, A Method For Determining Corrosion Rates From Linear Polarization Data, Corrosion 14 (1958) 440t.

151 M. Fenker, M. Balzer, H. Kappl, Corrosion Behaviour of Decorative and Wear Resistant Coatings on Steel Deposited by Reactive Magnetron Sputtering-Tests and Improvements, Thin Solid Films 515 (2006) 27.

152 W.-J. Chou, G.-P.Yu, J.-H. Huang, Corrosion Resistance of ZrN Films on AISI 304 Stainless Steel Substrate, Surf. Coat. Technol. 167 (2003) 59.

153 M. Kouril, P. Novak, M. Bojko, Limitations of the Linear Polarization Method to Determine Stainless Steel Corrosion Rate in Concrete Environment, Cem. Concr. Compos. 28 (2006) 220.

154 R.H. Hausler, Practical Experiences with Linear Polarization Measurements, Corrosion 33 (1977) 117. 
155 J.-Y. Chen, G.-P. Yu, J.-H. Huang, Corrosion Behaviour and Adhesion of Ion-Plated TiN Film on AISI 304 Stainless Steel, Mater. Chem. Phys. 65 (2000) 310.

156 L.A.S. Ries, D.S. Azambuja, I.J.R. Baumvol, Corrosion Resistance of Steel Coated with Ti/TiN Multilayers, Surf. Coat. Technol. 89 (1997) 114.

157 Y.M. Chen, G.P. Yu, J.H. Huang, Comparison of Electrochemical Porosity Test Methods for TiN-Coated Stainless Steel, Surf. Coat. Technol. 150 (2002) 309.

158 B. Elsener, A. Rota, H. Böhni, Impedance Study of PVD and CVD Titanium Nitride coatings, J. Mater. Sci. Forum. 44-45 (1989) 29.

159 B. Matthes, E. Broszeit, J. Aromaa, H. Ronkainen, S.P. Hannula, A. Leyland, A. Matthews, Corrosion Performance of Some Titanium-based Hard Coatings, Surf. Coat. Technol. 49 (1991) 489.

$160 \mathrm{~W}$. Tato, D. Landolt, Electrochemical Determination of the Porosity of Single and Duplex PVD Coatings of Titanium and Titanium Nitride on Brass, J. Electrochem. Soc. 145 (1998) 4173.

161 D.D. Macdonald, A Brief History of Electrochemical Impedance Spectroscopy, International Symposium on Corrosion Science: A Retrospective and Current Status in Honor of Robert P. Frankenthal May 13-16, Philadelphia, PA, 2002.

162 D.D. Macdonald, Chapter 11, Techniques for Characterization of Electrodes and Electrochemical Processes, John Wiley \& Sons, Inc., New York, 1991.

163 F. Mansfeld, S.L. Jeanjaquet, M.W. Kendig, An Electrochemical Impedance Spectroscopy Study of Reactions at the Metal/Coating Interface, Corros. Sci. 26 (1986) 735.

164 F. Mansfeld, Electrochemical Impedance Spectroscopy (EIS) as A New Tool for Investigating Methods of Corrosion Protection, Electrochim. Acta 35 (1990) 1533.

165 A.T. Kuhn (Ed.), Techniques in Electrochemistry, Corrosion and Metal Finishing - A Handbook, Wiley, Chichester, 1987.

166 M.K. Gabel and D.M. Donovan, Wear Resistant Coating and Treatments, in M.B. Peterson and W.O. Winer (Eds.), Wear Control Handbook, ASME, New York, 1980.

167 C.A. Krier, Protective Coatings, in C.F. Powell, J.H. Oxley, J.M. Blocher, Jr. (Eds.), Vapor Deposition, John Wiley \& Sons, Inc. New York, 1966.

168 D.G. Bhat, Application of CVD and PVD Technologies to Cutting Tools and Evaluation of Tool Failure Modes, Revista de la facultad de ingeniería, 13 (1998) 15.

169 D.L. Snyder, Electroplating Nickel/Chromium for the Automotive Industry, Met. Finish. 95 (1997) 29.

170 J. Musil, Hard and Superhard Nanocomposite Coatings, Surf. Coat. Technol. 125 (2000) 322.

171 G. Perugini, Ceramic Self Sealing Coatings for High Temperature Surfaces, Ceramurgia International 4 (1978) 3. 
172 P. Hancock, Current High Temperature Corrosion-resistant Materials and A Personal View of Their Future Prospects, Materials Science and Engineering 88 (1987) 303.

173 S. Seal, Transition Metal Nitride Functional Coatings, JOM 53 (2001) 51.

174 P.W. Schilke, Advanced Gas Turbine Materials and Coating, 39th GE Turbine Stateof-the-Art Technology Semina, GE Power Systems, EGR-3569F (1996) 22.

175 J.F. Nejedlik and J.D. Gadd, Coatings for Long Term Intermediate Temperature Protection of Columbium Alloys, NTIS, Virginia, 1968.

176 H.M. Green, Manufacturing Techniques for Application of Erosion Resistant Coatings to Turbine Engine Compressor Components. NTIS, Virginia, 1969.

177 R.C. Cook, R.H. MacDonald, L.F. Ncrris, Development of Precipitation Hardened Dispersion Strengthened Nickel-Chromium Alloys, NTIS, Virginia, 1969.

178 S. Priceman and R. Kubick, Development of Protective Coatings for Columbium Alloy Gas Turbine Blades, NTIS, Virginia, 1971.

179 W.J. McAnally, Erosion-Resistant Coating for Titanium, NTIS Virginia, 1972

180 N.S. Bornstein, M.A. DeCrescente, H.A. Roth, Effect of Vanadium and Sodium Compounds on Accelerated Oxidation of Nickel-base Alloys, NTIS, Virginia, 1973.

181 V.S. Moore and A.R. Stetson, Hot Corrosion Resistant Aluminide Coatings of Controlled Composition for Nickel-Base Superalloys, NTIS, Virginia, 1973.

182 C.C. McComas and I.S. Sckol, Research for Engine Demonstration of ErosionResistant Coating Effectiveness, NTIS, Virginia, 1974.

183 F.S. Galasso, Chemical Vapor Deposited Materials, CRC Press, Inc. Boca Raton, 1991.

184 J. Aromaa, H. Ronkainen, A. Mahiout and S.-P. Hannula, Identification of Factors Affecting the Aqueous Corrosion Properties of (Ti, Al) N-coated Steel, Surf. Coat. Technol. 49 (1991) 353.

185 P. Bhardwaj, O.J. Gregory, K. Bragga, M.H. Richman, Sputtered TiN Thin Films for Improved Corrosion Resistance, Appl. Surf. Sci. 48-49 (1991) 555.

186 P.W. Schilke, A.D. Foster, J. J. Pepe, A.M. Beltran, Advanced Materials Propel Progress in Land-based Gas Turbines, Adv. Mater. Processes 141 (1992) 22.

187 US Pat. 3948697, C23F 7/14, April 6, 1976

188 US Pat. 4141760, C23F 7/10, February 27, 1979.

189 US Pat. 4350719, C23C 11/4, September 21, 1982.

190 L. Swadzba, B. Formanek, H.M. Gabriel, P. Liberski and P. Podoiski, Erosion and Corrosion Resistant Coatings for Aircraft Compressor Blades, Surf. Coat. Technol. 62 (1993) 486.

191 J.S. Colligon, Applications of Ion-assisted Deposition to Surface Modification on the Micro- and nano-scale, in A.A. Voevodin, D.V. Shtansky, E.A. Levashov and J.J. 
Moore (Eds.), Nanostructured Thin Films and Nanodispersion Strengthened Coatings, Kluwer Academic Publishers, Dordrecht, 2004.

192 S.H. Ahn J.H. Yoo, Y.S. Choi, J. G. Kirn, J. G. Han, in A. Kumar, Y-W. Chung, J.J. Moore, G.L. Doll, K. Yasui and D.S. Misra (Eds.), Surface Engineering: Science \& Technology of Interfaces II, TMS (The Minerals, Metals \& Materials Society), 2002.

193 S.-C. Lee, W.-Y. Hob, F.D. Lai, Effect of Substrate Surface Roughness on the Characteristics of CrN Hard Film, Mater. Chem. Phys. 43 (1996) 266.

194 Y.L Su and S.H. Yao, On the Performance and Aapplication of CrN Coating, Wear 205 (1997) 112.

195 S. Veprek, P. Nesladek, A. Niederhofer, F. Glatz, M. Jilek, M. Sima, Hardness of TiN/BN Composites, Surf. Coat. Technol. 108-109 (1998) 138.

196 C. Louro and A. Cavaliero, Hardness versus Structure in W-Si-N Sputtered Coatings, Surf. Coat. Technol. 119 (1999) 74.

197 M. Diserens, J. Patscheider, F. Lévy, Improving the Properties of Titanium Nitride by Incorporation of Silicon, Surf. Coat. Technol. 108-109 (1998) 241.

198 J. Musil, P. Zeman, H. Hruby, P. Mayrhofer, ZrN/Cu Nanocomposite Film - Novel Superhard Material, Proc. ICMCTF'99, April 12-18, San Diego, CA (1999) B1-2-9.

199 J. Musil, H. Hruby, Superhard Nanocomposite Ti1-xAlxN Films Prepared by Magnetron Sputtering, Proc. 14th Int. Symp. on Plasma Chemistry, August 2-6, Praha, Czech Republic, Vol. III (1999) 1605.

200 J. Musil, I. Leipner, M. Kolega, Nanocrystalline and Nanocomposite $\mathrm{CrCu}$ and $\mathrm{CrCu}-\mathrm{N}$ Films Prepared by Magnetron Sputtering, Surf. Coat. Technol. 115 (1999) 32.

201 C.L. Li, H.X. Zhao, M. Matsumura, T. Takahashi, M. Asahara, H. Yamaguchi, The Effect of NiCr Intermediate Layer on Corrosion Behaviour of $\mathrm{Cr}_{2} \mathrm{O}_{3}$ Ceramic Coated Materials, Surf. Coat. Technol. 124 (2000) 53.

202 P. Ajayan, Bulk Metal and Ceramics Nanocomposites, in P.M. Ajayan, L.S. Schadler, P.V. Braun (Eds.), Nanocomposite Science and Technology, Wiley-VCH $\mathrm{GmbH} \& \mathrm{Co} . \mathrm{KGaA}$, Weinheim, 2003.

203 S. PalDey and S.C. Deevi, Single Layer and Multilayer Wear Resistant Coatings of (Ti, Al)N: A Review, Mater. Sci. Eng. A 342 (2003) 58.

204 L. Hultman, Materials Science of Wear-Protective Nanostructured Thin Films, in A.A. Voevodin, D.V. Shtansky, E.A. Levashov, J.J. Moore (Eds.), Nanostructured Thin Films and Nanodispersion Strengthened Coatings, Kluwer Academic Publishers, Dordrecht, 2004.

205 Z. Werner, W. Szymczyk, J. Piekoszewski, Ion Implanted Nanolayers in Alloys and Ceramic Coatings for Improved Resistance to High-temperature Corrosion, in A.A. Voevodin, D.V. Shtansky, E.A. Levashov, J.J. Moore (Eds.), Nanostructured Thin 
Films and Nanodispersion Strengthened Coatings, Kluwer Academic Publishers, Dordrecht, 2004.

206 J.-H. Jeon, C.S. Jang, S.-Y. Yoon, B.-C. Shin, K.H. Kim, Effects of Si Addition on the Characteristic Evolution and Syntheses of nanocomposite $\mathrm{Cr}-\mathrm{Si}-\mathrm{C}-\mathrm{N}$ coatings prepared by a hybrid coating system, Surf. Coat. Technol. 200 (2005) 1635.

207 A.A. Voevodin, J. S. Zabinski, C. Muratore, Recent Advances in Hard, Tough, and Low Friction Nanocomposite Coatings, Tsinghua Sci. \& Technol. 10 (2005) 665.

208 D. Rafaja, M. Dopita, M. Rủžička, V. Klemm, D. Heger, G. Schreiber, M. Šíma, Microstructure Development in $\mathrm{Cr}-\mathrm{Al}-\mathrm{Si}-\mathrm{N}$ Nanocomposites Deposited by Cathodic Arc Evaporation, Surf. Coat. Technol. 201 (2006) 2835.

209 I.-W. Park, D.S. Kang, J.J. Moore, S.C. Kwon, J.J. Rha, K.H Kim, Microstructures, Mechanical Properties, and Tribological Behaviours of $\mathrm{Cr}-\mathrm{Al}-\mathrm{N}, \mathrm{Cr}-\mathrm{Si}-\mathrm{N}$, and $\mathrm{Cr}-$ Al-Si-N Coatings by A Hybrid Coating System, Surf. Coat. Technol. 201 (2007) 5223.

210 R. Wei, Plasma Enhanced Magnetron Sputter Deposition of Ti-Si-C-N Based Nanocomposite Coatings, Surf. Coat. Technol. 203 (2008) 538.

211 S. Carvalho, L. Rebouta, E. Ribeiro, F. Vaz, C.J. Tavares, E. Alves, N.P. Barradas, J.P. Riviere, Structural Evolution of Ti-Al-Si-N Nanocomposite Coatings, Vacuum 83 (2009) 1206.

212 G.K. Dosbaeva, S.C. Veldhuis, K. Yamamoto, D.S. Wilkinson, B.D. Beake, N. Jenkins, A. Elfizy, G.S. Fox-Rabinovich, Oxide Scales Formation in Nano-crystalline TiAlCrSiYN PVD Coatings at Elevated Temperature, Int. J. Refract. Met. Hard Mater $28(2010) 133$.

213 L.E. Toth, Transition Metal Carbides and Nitrides, Academic Press, New York, 1971.

214 E.K. Storms, The Refractory Carbides, Academic Press, New York, 1967.

215 L. Cunha, M. Andritschky, K. Pischow, Z. Wang, Microstructure of CrN Coatings Produced by PVD Techniques, Thin Solid Films 355-356 (1999) 465.

216 F. Lévy, P. Hones, P.E. Schmid, R. Sanjinés, M. Diserens, C. Wiemer, Electronic States and Mechanical Properties in Transition Metal Nitrides, Surf. Coat. Technol. 120-121 (1999) 284.

217 A. Zaoui, S. Kacimi, B. Bouhafs, A. Roula, First-principles Study of Bonding Mechanisms in the Series of Ti, V, Cr, Mo, and Ttheir Carbides and Nitrides, Physica B: Condensed Matter B 358 (2005) 63.

218 C. Kral, W. Lengauer, D. Rafaja, P. Ettmayer, Critical Review on the Elastic Properties of Transition Metal Carbides, Nitrides and Carbonitrides, J. Alloys Compd. 265 (1998) 215.

219 H.A. Jehn, J.-H. Kim, S. Hofmann, Composition and Properties of Transition Metal Nitride Thin Films (ZrNx, NbNx, MoNx), Surf. Coat. Technol. 36 (1988) 715. 
220 J.C. Grossman, A. Mizel, M. Côté, M.L. Cohen, S.G. Louie, Transition Metals and Their Carbides and Nitrides: Trends in Electronic and Structural Properties, Phys. Rev. B: Condens. Matter 60 (1999) 6343.

221 S.-H. Jhi, S.G. Louie, M.L. Cohen, J. Ihm, Vacancy Hardening and Softening in Transition Metal Carbides and Nitrides, Phys. Rev. Lett. 86 (2001) 3348.

222 J.C. Slater and G.F. Koster, Simplified LCAO Method for the Periodic Potential Problem, Phys. Rev. 94 (1954) 1498.

223 J.-L. Colot, APW Bands and Bonding in Transition Metal Oxides, Nitrides and Carbides, J. Phys. C: Solid State Phys. 10 (1977) 1675.

224 A.P. Sutton, Chapter 6, Electronic Structure of Materials, Oxford University Press, Oxford, UK, 1993.

225 Daniel Jädernäs, Investigation of Interfacial Microstructure of CrN Coatings on HSS Substrates Pretreated by HIPIMS for Adhesion Enhancement, Master's Thesis, Department of Physics, Chemistry and Biology, Linköping University, Linköping, Sweden, 2006.

226 H. Holleck, Material Selection for Hard Coatings. J. Vac. Sci. Technol. A 4 (1986) 2661.

227 J. Haglund, A. Fernandez Guillermet, G. Grimvall, M. Korling, Theory of Bonding in Transition-metal Carbides and Nitrides, Phyeical Review B48 (1993)11685.

228 E. Vogelzang, J. Sjollema, H. J. Boer, J. Th. M. De Hosson, Optical Absorption in TiNxOy Compounds, J. Appl. Phys. 61 (1987) 4606.

229 S. Zhang, H.L. Wang, S.-E. Ong, D. Sun, X.L. Bui, Hard yet Tough Nanocomposite Coatings - Present Status and Future Trends, Plasma Process. Polym. 4 (2007) 219.

230 V.K.W. Grips, V.E. Selvi, H.C. Barshilia, K.S. Rajam, Effect of electroless nickel interlayer on the electrochemical behaviour of single layer $\mathrm{CrN}$, TiN, TiAIN coatings and nanolayered $\mathrm{TiAlN} / \mathrm{CrN}$ multilayer coatings prepared by reactive dc magnetron sputtering, Electrochimica Acta 51 (17) (2006) 3461.

231 B. Navinsek, P. Panjan, I. Milosev, Industrial Applications of CrN (PVD) Coatings, Deposited at High and Low Temperatures, Surf. Coat. Technol. 97 (1997) 182.

232 B. Navinsek and P. Panjan, Novel applications of CrN (PVD) Coatings Deposited at $200^{\circ} \mathrm{C}$, Surf. Coat. Technol. 74-75 (1995) 919.

233 S. Baragetti, G.M. La Vecchia, A. Terranova, Fatigue Behaviour and FEM Modeling of Thin-coated Components, Int. J. Fatigue 25 (2003) 1229.

234 L. Hultman and J.E. Sundgren, Chapter 4 Structure/Property Relationships for Hard Coatings, in R.F. Bunshah (Ed.), Handbook of Hard Coatings: Deposition Technologies, Properties and Applications, Noyes Publications, New Jersey, 2001.

235 H.O. Pierson, Handbook of Refractory Carbides and Nitrides: Properties, Characteristics, Processing, and Applications, Noyes Publications, Park Ridge, New Jersey, 1996. 
236 K.I. Portnoi, A.A. Mukaseev, V.N. Gribkov, Yu.V. Levinskii and S.A. Prokof'ev, Modulus of Elasticity of Some Refractory Compounds, Powder Metall. Met. Ceram. 7 (1968) 185.

237 B. Wielage, J. Wilden, T. Schnick, Manufacture of SiC Composite Coatings by HVOF, in C.C. Berndt, K.A. Khor, E.F. Lugscheider (Eds.), Thermal Spray 2001: New Surfaces for a New Millennium, ASM International, Materials Park, Ohio, USA, 2001.

238 A.I. Gusev, A.A. Rempel and A.J. Magerl, Disorder and Order in Strongly Nonstoichiometric Compounds: Transition Metal Carbides, Nitrides and Oxides (Springer Series in Materials Science), Springer, New York, 2010.

239 H.O. Pierson, Handbook of Chemical Vapor Deposition (CVD) Principles, Technology, and Applications, Noyes Publications, Park Ridge, NJ, 1992.

240 S.H. Yao, Y.L. Su, The Tribological Potential of $\mathrm{CrN}$ and $\mathrm{Cr}(\mathrm{C}, \mathrm{N})$ Deposited by Multi-arc PVD Process, Wear 212 (1997) 85.

241 S. Veprek, The Search for Novel, Superhard Materials, J. Vac. Sci. Technol. A 17 (1999) 2401.

242 J.H. Park, W.S. Chung, Y.R. Cho, K.H. Kim, Synthesis and Mechanical Properties of $\mathrm{Cr}-\mathrm{Si}-\mathrm{N}$ Coatings Deposited by A Hybrid System of Arc Ion Plating and Sputtering Techniques, Surf. Coat. Technol. 188-189 (2004) 425.

243 J. Patscheider, T. Zehnder, M. Diserens, Structure-Performance Relations in Nanocomposite Coatings, Surf. Coat. Technol. 146-147 (2001) 201.

244 E. Martìnez, R. Sanjinés, A. Karimi, J. Esteve, F. Lévy, Mechanical Properties of Nanocomposite and Multilayered $\mathrm{Cr}-\mathrm{Si}-\mathrm{N}$ Sputtered Thin Films, Surf. Coat. Technol. 180-181 (2004) 570.

245 R. Sanjinés, M. Benkahoul, C.S. Sandu, F. Lévy, Relationship between the Physical and Structural Properties of NbzSiyNx Thin Films Deposited by DC Reactive Magnetron Sputtering, J. Appl. Phys. 98 (2005) 123511.

246 C.S. Sandu, R. Sanjinés, M. Benkahoul, F. Medjani, F. Lévy, Formation of Composite Ternary Nitride Thin Films by Magnetron Sputtering Co-deposition, Surf Coat. Technol. 201 (2006) 4083.

247 D. Mercs, P. Briois, V. Demange, S. Lamy, C. Coddet, Influence of the Addition of Silicon on the Structure and Properties of Chromium Nitride Coatings Deposited by Reactive Magnetron Sputtering Assisted by RF Plasmas, Surf. Coat. Technol. 201 (2007) 6970.

248 D. Mercs, N. Bonasso, S. Naamane, J.-M. Bordes, C. Coddet, Mechanical and Tribological Properties of $\mathrm{Cr}-\mathrm{N}$ and $\mathrm{Cr}-\mathrm{Si}-\mathrm{N}$ Coatings Reactively Sputter Deposited, Surf. Coat. Technol. 200 (2005) 403.

249 S. Veprek and S. Reiprich, A Concept for the Design of Novel Superhard Coatings, Thin Solid Films 268 (1995) 64. 
250 J. Musil, R. Daniel, J. Soldan, P. Zeman, Properties of Reactively Sputtered W-Si-N Films, Surf. Coat. Technol. 200 (2006) 3886.

251 P. Steyer, D. Pilloud, J.F. Pierson, J.-P. Millet, M. Charnay, B. Stauder, P. Jacquot, Oxidation Resistance Improvement of Arc-evaporated TiN Hard Coatings by Silicon Addition, Surf. Coat. Technol. 201 (2006) 4158.

252 S. Veprek and M. Jilek, Superhard Nanocomposite Coatings. From Basic Science toward Industrialization, Pure Appl. Chem. 74 (2002) 475.

253 Kyung H. Nam, Min J. Jung, Jeon G. Han, A Study on the High Rate Deposition of CrNx Films with Controlled Microstructure by Magnetron Sputtering, Surf. Coat. Technol. 131 (2000) 222.

254 S.M. Aouadi, D.M. Schultze, S.L. Rohde, K.-C. Wong, K.A.R. Mitchell, Growth and Characterization of Cr N/CrN Multilayer Coatings, Surf. Coat. Technol. 140 (2001) 269.

255 E. Martìnez, J. Romero, A. Lousa, J. Esteve, Wear Behaviour of Nanometric CrN/Cr Multilayers, Surf. Coat. Technol. 163-164 (2003) 571.

256 F.R. Lamastra, F. Leonardi, R. Montanari, F. Casadei, T. Valente, G. Gusmano, Xray Residual Stress Analysis on $\mathrm{CrN} / \mathrm{Cr} / \mathrm{CrN}$ Multilayer PVD Coatings Deposited on Different Steel Substrates, Surf. Coat. Technol. 200 (2006) 6172.

257 L.S. Palatnik and A.I. Il'inskii, Stabilization of High-strength Vacuum-deposited Films. Soviet Physics Doklady 9 (1964) 93.

258 L.S. Palatnik, A.I. Il'inskii, N.P. Sapelkin, Strength of Multilayered Vacuum Condensates, Sov. Pys. Solid State 8 (1967) 2016.

259 K.S. Chan, M.Y. He, J.W. Hutchinsson, Cracking and Stress Redistribution in Ceramic Layered Composites, Mater. Sci. Eng. A 167 (1993) 576.

260 Y. Huang and H.W. Zhang, A Correlation between Stability of Compounds and Structure of Hydrogen-induced Amorphous Alloys in GdM2 (M = Fe, Co, Ni), Acta Metall. Mater. 41 (1993) 1523.

261 H. Holleck, Designing Advanced Coatings for Wear Protection, Surf. Eng. 7 (1991) 137.

262 H. Holleck and H. Schulz, Advanced Layer Material Constitution. Thin Solid Films 153 (1987) 11.

$263 \mathrm{H}$. Holleck, H. Schulz, Preparation and Behaviour or Wear-resistant TiC/TiB 2 , $\mathrm{TiN} / \mathrm{TiB}_{2}$ and TiC/TiN Coatings with High Amounts of Phase Boundaries, Surf. Coat. Technol. 36 (1988) 707.

264 X. Chu and S.A. Barnett, A Model of Superlattice Yield Stress and Hardness Enhancements. Mater. Res. Soc. Symp. Proc. 382 (1995) 291.

265 J.S. Koehler, Attempt to Design A Strong Solid. Phys. Rev. B 2 (1970) 547.

266 R.W. Springer, D.S. Catlett, Structure and Mechanical Properties of AlxOy Vacuumdeposited Laminates. Thin Solid Films 54 (1978) 197. 
267 B.A. Movchan, A.V. Demchishin, G.F. Badilenko, R.F. Bunshah, C. Sans, C. Deshpandey, H.J. Doerr, Structure-property Relationships in Microlaminate $\mathrm{TiC} / \mathrm{TiB}_{2}$ Condensates. Thin Solid Films 97 (1982) 215.

268 W.D. Sproul, Multilayer, Multicomponent, and Multiphase Physical Vapor Deposition Coatings for Enhanced Performance. J. Vac. Sci. Technol. A 12 (1994) 1595.

269 I. Efeoglu, Deposition and Characteriztion of A Multilayered-composite Solid Lubricant Coating, Rev. Adv. Mater. Sci. 15(2007) 87.

270 Q. Luo, G. Robinson, M. Pittman, M. Howarth, W.-M. Sim, M.R. Stalley, H. Leitner, R. Ebner, D. Caliskanoglu, P.Eh. Hovsepian, Performance of Nano-structured Multilayer PVD Coating TiAlN/VN in Dry High Speed Milling of Aerospace Aluminium 7010-T7651, Surf. Coat. Technol. 200 (2005) 123.

271 J. Birch, T. Joelsson, F. Eriksson, N. Ghafoor, L. Hultman, Single Crystal CrN/ScN Superlattice Soft X-ray Mirrors: Epitaxial Growth, Structure, and Properties, Thin Solid Films 514 (2006) 10.

272 R.A. Andrievski, Nanostructured Superhard Films as Typical Nanomaterials, Surf. Coat. Technol. 201 (2007) 6112.

273 J. Xu, K. Hattori, Y. Seino, I. Kojima, Microstructure and Properties of $\mathrm{CrN} / \mathrm{Si}_{3} \mathrm{~N}_{4}$ Nano-structured Multilayer films, Thin Solid Films 414 (2002) 239.

274 M.D. McCluskey, L.T. Romano, B.S. Krusor, N.M. Johnson, Interdiffusion of In and Ga in InGaN Quantum Wells, Appl. Phys. Lett. 73 (1998) 1281.

275 U. Egger, M. Schultz, P. Werner, O. Breitenstein, T.Y. Tan, U. Gosele, R. Franzheld, M. Uematsu, H. Ito, Interdiffusion Studies in $\mathrm{GaAsP} / \mathrm{GaAs}$ and $\mathrm{GaAsSb} / \mathrm{GaAs}$ Superlattices under Various Arsenic Vapor Pressures, J. Appl. Phys. 81 (1997) 6056.

276 J. Musil and L. Vicek, Magnetron Sputtering of Films with Controlled Texture and Grain Size, Mater. Chem. Phys. 54 (1998) 116.

277 A. Gupper, A. Fernandez, C. Fernandez-Ramos, F. Hofer, C. Mitterer, P. Warbichler, Characterization of Nanocoposite Coatings in the System Ti-B-N by Analytical Electron Microscopy and X-Ray Photoelectron Spectroscopy, H. Hofmann, Z. Rahman and U. Schubert (Eds.) Nanostructured Materials, Springer-Verlag Wein, New York, 2002.

278 P.H. Mayrhofer, C. Mitterer, J. Musil, Structure-property Relationships in Singleand Dual-phase Nanocrystalliine Hard Coatings, Surf. Coat. Technol. 174-175 (2003) 725.

279 J. Patscheider, T. Zehnder, J. Matthey, M. Diserns, Nanostructured Hard Coatings Form Nanocomposites to Nanomultilayer, in A.A. Voevodin, D.V. Shtansky, E.A. Levashov, J.J. Moore (Eds.), Nanostructured Thin Films and Nanodispersion Strengthened Coatings, Kluwer Academic Publishers, Dordrecht, 2004.

280 J. Musil, Hard Nanocomposite Films Prepared by Reactive Magnetron Sputtering, in A.A. Voevodin, D.V. Shtansky, E.A. Levashov, J.J. Moore (Eds.), Nanostructured 
Thin Films and Nanodispersion Strengthened Coatings, Kluwer Academic Publishers, Dordrecht, 2004.

281 Y. Ryabkov, P. Istomin, N. Chezhina, Structural Design and Properties of Layered Nanocomposite Titanium Carbide-silicide Materials, Mater. Phys. (2001) 101.

282 D.V. Shtanskii, S.A. Kulinich, E.A. Levashov, J.J. Moore, Structure and PhysicalMechanical Properties of Nanostructured Thin Films, Phys. Solid State 45 (2003) 1177.

283 A.A. Voevdin and J.S. Zabinski, Smart Nanocomposite Coatings with Chameleon Surface Adaptation in Tribological Applications, in A.A. Voevodin, D.V. Shtansky, E.A. Levashov, J.J. Moore (Eds.). Nanostructured Thin Films and Nanodispersion Strengthened Coatings, Kluwer Academic Publishers, Dordrecht, 2004.

284 G. Cao, Nanostructures \& Nanomaterials: Synthesis, Properties \& Applications, Imperial College Press, London, 2004.

285 A. Agarwal and N.B. Dahotre, Pulse Electrode Deposition of Superhard Boride Coatings on Ferrous Alloys, Surf. Coat. Technol. 106 (1998) 242.

286 M. Schwartz, Deposition from Aqueous Solutions: An Overview, in R.F. Bunshah (Ed.), Handbook of Deposition Technologies for Films and Coatings, Science, Technology and Applications ( $\left.2^{\text {nd }} E d.\right)$, Noyes Publications, New Jersey, 1994.

287 J.C. Puippe, Qualitative Approach to Pulse Plating, in J.C. Puippe and F. Leaman (Eds.), Theory and Practice of Pulse Plating, American Electroplaters \& Surface Finishing Society, 1986.

288 F. Barlow, A. Elshabini-Riad, R. Brown, Film Deposition Techniques and Processes, in A. Elshabini-Riad and F. D. Barlow (III Ed.), Thin Film Technology Handbook, McGraw-Hill, New York, 1998.

289 E. Toth-Kadar, I. Bakonyi, L. Pogany, A. Cziraki, Microstructure and Electrical Transport Properties of Pulse-plated Nanocrystalline Nickel Electrodeposits, Surf. Coat. Technol. 88 (1996) 57.

290 K.P. Wong, K.C. Chan, and T.M. Yue, Modeling the Effect of Complex Waveform on Surface Finishing in Pulse Current Electroforming of Nickel, Surf. Coat. Technol. 135 (2000) 91.

291 Y.M. Yeh, G.C. Tu, T.H. Fang, Nanomechanical Properties of Nanocrystalline NiFe Mold Insert, J. Alloys Compd. 372 (2004) 224.

292 M. Paunovic and M. Schlesinger, Fundamentals of Electrochemical Depositiono (2nd Ed.), John Wiley \& Sons, Inc., Hoboken, New Jersey, 2006.

293 A. Panda and E. J. Podlaha, Nanoparticles to Improve Mass Transport inside Deep Recesses, Electrochem. Solid-State Lett. 6 (2003) C149.

294 L. Shi, C. Sun, P. Gao, F. Zhou, W. Liu, Mechanical Properties and Wear and Corrosion Resistance of Electrodeposited $\mathrm{Ni}-\mathrm{Co} / \mathrm{SiC}$ Nanocomposite Coating, Appl. Surf. Sci. 252 (2006) 3591. 
295 S. Arai, M. Endo, N. Kaneko, Ni-deposited Multi-walled Carbon Nanotubes by Electrodeposition, Carbon 42 (2004) 641.

296 L.-N. Tsai, Y.-T. Cheng, W. Hsu, and W. Fang, Ni-Carbon Nanotubes Nanocomposite for Robust Microelectromechanical Systems Fabrication, J. Vac. Sci. Technol. B 24 (2006) 205.

297 M. Verelst, J.P. Bonino, M. Brieu, A. Rousset, Thermomechanical Properties of Ni$\mathrm{Al}_{3} \mathrm{O}_{3}$ Metal Matrix Composites Produced by Electroforming, Mater. Sci. Eng. A 191 (1995) 165.

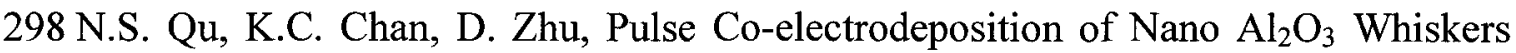
Nickel Composite Coating, Scr. Mater. 50 (2004) 1131.

299 Y. Gan, D. Lee, X. Chen, J.W. Kysar, Structure and Properties of Electrocodeposited $\mathrm{Cu}-\mathrm{Al}_{2} \mathrm{O}_{3}$ Nanocomposite Thin Films, J. Eng. Mater. Technol. 127 (2005) 451.

300 A. Moller, H. Hahn, Synthesis and Characterization of Nanocrystalline $\mathrm{Ni} / \mathrm{ZrO}_{2}$ Composite Coatings, Nanostruct. Mater. 12 (1999) 259.

301 L. Benea, P.L. Bonora, A. Borello, S. Martelli, Wear Corrosion Properties of Nanostructured SiC-Nickel Composite Coatings Obtained by Electroplating, Wear 249 (2002) 995.

302 P. Gyftou, M. Stroumbouli, E.A. Pavlatou, P. Asimidis, N. Spyrellis, Tribological Study of Ni Matrix Composite Coatings Containing Nano and Micro SiC Particles, Electrochim. Acta 50 (2005) 4544.

303 S. Arai and M. Endo, Carbon Nanofiber-Copper Composite Powder Prepared by Electrodeposition, Electrochem. Commun. 5 (2003) 797.

304 R.F. Bunshah, Chapter 14 Metallurgical Applications, in R.F. Bunshah (Ed.), Handbook on Deposition Technologies for Films and Coatings, Science, Technology and Applications ( $2^{\text {nd }}$ Ed.), Noyes Publications, Park Ridge, NJ, 1994.

305 J.E. Mahan, Physical Vapor Deposition of Thin Films, John Wilet \& Sons, Inc., New York, 2000.

306 R.F. Bunshah, Evaporation, in R.F. Bunshah et al (Eds.), Deposition Technologies for Films and Coatings: Developments and Applications, Noyes Publications, Park Ridge, NJ, 1982.

307 R.F. Bunshah, Vapor Deposition Technologies, in R.F. Bunshah (Ed.), Handbook of Hard Coatings: Deposition Technologies, Properties and Applications, Noyes Publications / William Andrew Publishing, LLC, Norwich, New York, 2001.

308 K. Wasa, M. Kitabatake, H. Adachi, Thin Film Materials Technology: Sputtering of Compound Materials, William Andrew, Inc., Norwich, NY, 2004.

309 A. Flink, T. Larsson, J. Sjölén, L. Karlsson, L. Hultman, Influence of Si on the Microstructure of Arc Evaporated (Ti, Si)N Thin Films; Evidence for Cubic Solid Solutions and Their Thermal Stability, Surf. Coat. Technol. 200 (2005) 1535. 
310 D.M. Mattox, Chapter 7 Arc Vapor Deposition, Handbook of Physical Vapor Deposition (PVD) Processing: Film Formation, Adhesion, Surface Preparation and Contamination Control, Noyes Publications, Westwood, New Jersey, 1998.

311 M. Balaceanu, M. Braic, D. Macovei, M.J. Genet, A. Manea, D. Pantelica, V. Braic, F. Negoita, Properties of Titanium Based Hard Coatings Deposited by the Cathodic Arc Method, J. Optoelectron. Adv. Mater. 4 (2002)107.

312 M. MacKenzie, A.J. Craven, P. Hatto, Erosion and Deposition During the Sputter Cleaning of Substrates Prior to the Cathodic Arc Evaporation of Transition Metal Nitride Coatings, Thin Solid Films 349 (1999) 176.

313 E.P. Degarmo, J.T. Black, R.A. Kosher, Materials and Processes in Manufacturing ( $9^{\text {th }}$ Ed.), Wiley and Sons, 2003.

314 L.T. Minassian-Saraga, Thin Films Including layers: Terminolgy in Relation to Their Preparation and Characterization, Pure Appl. Chem. 66 (1994) 1667.

315 S. Craig and G.L. Harding, Effects of Argon Pressure and Substrate Temperature on the Structure and Properties of Sputtered Copper Films, J. Vac. Sci. Technol. 19 (1981) 205.

316 J.A. Thorton, High Rate Thick Film Growth, Ann. Rev. Mater. Sci. 7 (1977) 239.

317 B. Window and N. Savvides, Charged Partical Fluxes from Planar Magnetron Sputtering Sources, J. Vac. Sci. Technol. A 4 (1986)196.

318 S.E. Rodil and J.J. Olaya, Unbalanced Magnetic Field Configuration: Plasma and Film Properties, J. Phys.: Condens. Matter 18 (2006) S1703.

319 W.D. Münz, F.J.M. Hauzer, D. Schuize, B. Buil, A New Concept for Physical Vapor Deposition Coating Combining the Methods of Arc Evaporation and UnbalancedMagnetron Sputtering, Surf. Coat. Technol. 49 (1991) 161.

320 J. Gudmundsson, J. Alami, U. Helmersson, Spatial and Temporal Behaviour of the Plasma Parameters in A Pulsed Magnetron Discharge, Surf. Coat. Technol. 161 (2002) 249.

321 K.L. Choy, Chemical Vapour Deposition of Coatings, Progress in Materials Science 48 (2003) 57.

322 L.A. Dobrzanski and M. Adamiak, Structure and Properties of the TiN and Ti(C,N) Coatings Deposited in the PVD Process on High-speed Steels, J. Mater. Process. Technol. 133 (2003) 50.

323 S.J. Bull and D.S. Rickerby, Chapter 5 Characterization of Hard Coatings, in R.F. Bunshah (Ed.), Handbook of Hard Coatings: Deposition Technologies, Properties and Applications, Noyes Publications, New Jersey, 2001.

324 B. Bhushan and B.K. Gupta, Chapter 6 Macro- and Micromechanical and Tribological Properties, in R.F. Bunshah (Ed.), Handbook of Hard Coatings: Deposition Technologies, Properties and Applications, Noyes Publications, New Jersey, 2001. 
325 B. Trindade, A. Cavaleiro, M.T. Vieira, The Influence of the Addition of a Third Element on the Structure and Mechanical Properties of Transition-Metal-Based Nanostructured Hard Films: Part II-Carbides, in A. Cavaleiro and J.Th. M. De Hosson, Nanostructured Coatings, Springer Science+Business Media, LLC, New York, 2006.

326 Q. Yang, D.Y. Seo, L.R. Zhao, X.T. Zeng, Erosion Resistance Performance of Magnetron Sputtering Deposited TiAlN coatings, Surf. Coat. Technol. 188-189 (2004) 168.

327 A.A. Adjaottor, E.I. Meletis, S. Logothetidis, I. Alexandrou, S. Kokkou, Effect of Substrate Bias on Sputter-deposited TiCx, TiNy and TiCxNy Thin Films, Surf. Coat. Technol. 76-77 (1995) 142.

328 H.C. Barshilia, A. Jain, K.S. Rajam, Structure, Hardness and Thermal Stability of Nanolayered TiN/CrN Multilayer Coatings, Vacuum 72 (2004) 241.

329 S.H Kim, H. Park, K.H. Lee, S.H. Jee, D.-J. Kim, Y.S. Yoo, H.B. Chae, Structure and Mechanical Properties of Ttitanium Nitride Thin Films Grown by Reactive Pulsed Laser Deposition, Journal of Ceramic Processing Research 10 (2009) 49.

330 J. Patscheider, Nanocomposite Hard Coating for Wear Protection, MRS Bull. 28 (2003) 180.

331 A. Leyland, A. Matthews, On the Significance of the H/E Ratio in Wear Control: A Nanocomposite Approach to Optimised Tribological Behaviour, Wear 246 (2000) 1.

332 K.L. Johnson, Contact Mechanics, Cambridge University Press, London, 1985.

333 D.S. Rickerby and P.J. Burnett, The Wear and Erosion Resistance of Hard PVD Coatings, Surf. Coat. Technol. 33 (1987) 191.

334 J.W. Cox, Chapter 8 Wear and Corrosion Resistant Hard Coatings For Non-cutting Tool Applications, in R.F. Bunshah (Ed.), Handbook of Hard Coatings: Deposition Technologies, Properties and Applications, Noyes Publications, Park Ridge, New Jersey, 2001.

335 Y.L. Su and T.H. Liu, Effects of Tungsten on the Microstructures and Tribological Properties of CrN-Wx\% Coatings, Mater. Sci. Eng. A 395 (2005) 241.

336 Q. Yang, L.R. Zhao, P.C. Patnaik, X.T. Zeng, Wear Resistant TiMoN Coatings Deposited by Magnetron Sputtering, Wear 261 (2006) 119.

337 J.-P. Immarigeon, D. Chow, V.R. Parameswaran, P. Au, H. Saari, A.K. Koul, Erosion Testing of Coatings for Aero Engine Compressor Components, Adv. Perform. Mater. 4 (1997) 371.

338 D. Garg and P.N. Dyer, Erosive Wear Behaviour of Chemical Vapor Deposited Multilayer Tungsten Carbide Coating, Wear 162-164 (1993) 552.

339 W. Tabakoff, Erosion Resistance of Superalloys and Different Coatings Exposed to Particulate Flows at High Temperature, Surf. Coat. Technol. 120-121 (1999) 542. 
340 R. Wei, E. Langa, C. Rincon, James H. Arps, Deposition of Thick Nitrides and Carbonitrides for Sand Erosion Protection, Surf. Coat. Technol. 201 (2006) 4453.

341 J.P. Tu, The effect of TiN Coating on Erosion-Corrosion Resistance of $\alpha$-Ti Alloy in Saline Slurry, Corros. Sci. 42 (2000) 147.

342 M. Urgen, A.F. Cakir, The Effect of Heating on Corrosion Behaviour of TiN- and CrN-coated Steels, Surf. Coat. Technol. 96 (1997) 236.

343 H.C. Barshilia, N. Selvakumar, B. Deepthi, K.S. Rajam, A Comparative Study of Reactive Direct Current Magnetron Sputtered CrAIN and CrN coatings, Surf. Coat. Technol. 201 (2006) 2193.

344 X.-Z. Ding, A.L.K. Tan, X.T. Zeng, C. Wang, T. Yue, C.Q. Sun, Corrosion Resistance of CrAlN and TiAlN Coatings Deposited by Lateral Rotating Cathode Arc, Thin Solid Films 516 (2008) 5716.

345 R.M. Souto, H. Alanyali, Electrochemical Characteristics of Steel Coated with TiN and TiAlN Coatings, Corros. Sci. 42 (2000) 2201.

346 C. Liu, Q. Bi, A. Leyland, A. Matthews, An Electrochemical Impedance Spectroscopy Study of the Corrosion Behaviour of PVD Coated Steels in $0.5 \mathrm{~N} \mathrm{NaCl}$ Aqueous Solution: Part II. EIS Interpretation of Corrosion Behaviour, Corros. Sci. 45 (2003) 1257.

347 F. Lang and Z. Yu, The Corrosion Resistance and Wear Resistance of Thick TiN Coatings Deposited by Arc Ion Plating, Surf. Coat. Technol. 145 (2001) 80.

348 R. Morita, K. Azuma, S. Inoue, R. Miyano, H. Takikawa, A. Kobayashi, E. Fujiwara, H. Uchida, M. Yatsuzuka, Corrosion Resistance of TiN Coatings Produced by Various Dry Processes, Surf. Coat. Technol. 136 (2001) 207.

349 D.M. Mattox, Chapter 9 Atomistic Film Growth and Some Growth-Related Film Properties, Handbook of Physical Vapor Deposition (PVD) Processing: Film Formation, Adhesion, Surface Preparation and Contamination Control, Noyes Publications, Westwood, New Jersey, 1998.

350 A. Ehiasarian, W.-D. Munz, L. Hultman, U. Helmersson, I. Petrov, High Power Pulsed Magnetron Sputtered CrN films, Surf. Coat. Technol. 163-164 (2000) 267.

351 L. Hultman, Review Paper, Thermal Stability of Nitride Thin Films, Vacuum 57 (2000) 1 .

352 A. Sekiguchi, J. Koike, S. Kamiya, M. Saka, K. Maruyama, Void Formation by Thermal Stress Concentration at Twin Interfaces in $\mathrm{Cu}$ Thin Films, Appl. Phys. Lett. 79 (2001) 27.

353 N.J.M. Carvalho, E. Zoestbergen, B.J. Kooi, J.Th.M. De Hosson, Stress Analysis and Microstructure of PVD Monolayer TiN and Multilayer TiN/(Ti, Al)N Coatings, Thin Solid Films 429 (2003) 179.

354 R.L. Boxman and S. Goldsmith, Macroparticle Contamination in Cathodic Arc Coatings: Generation, Transport and Control, Surf. Coat. Technol. 52 (1992) 39. 
355 G.W. McClure, Plasma Expansion as a Cause of Metal Displacement in Vacuum-arc Cathode Spots, J. Appl. Phys. 45 (1974) 2078.

356 M. Egawa, K. Miura, M. Yokoi, I. Ishigami, Effects of Substrate Bias Voltage on Projection Growth in Chromium Nitride Films Deposited by Arc Ion Plating, Surf. Coat. Technol. 201 (2007) 4873.

357 A. Kawana, H. Ichimura, Y. Iwata, S. Ono, Development of PVD Ceramic Coatings for Valve Seats, Surf. Coat. Technol. 86-87 (1996) 212.

358 E. Galvanetto, F. Borgioli, F.P. Galliano, T. Baccie, Improvement of Wear and Corrosion Resistance of RPS Ti-TiN Coatings by Means of Thermal Oxidation, Surf. Coat. Technol. 200 (2006) 3650.

359 L. Bai, X. Zhu, J. Xiao, J. He, Study on Thermal Stability of CrTiAlN Coating for Dry Drilling, Surf. Coat. Technol. 201 (2007) 5257.

360 Y. Pei, Morphology and Mechanical Properties of Polycarbonate / Carbon Nanotube Composites, Master's Thesis, Carleton Univeristy, Ottawa, Canada, 2003.

361 F. Cai, X. Huang, Q. Yang, and D. Nagy, Effect of Microstructure on the Solid Particle Erosion Properties of Ni Plating, J. Mater. Eng. Perform. 18 (2009) 305.

362 G.R. Shen, Y.-T. Cheng, and L.-N. Tsai, Synthesis and Characterization of Ni-PCNT's Nanocomposite Film for MEMS Applications, IEEE Transactions On Nanotechnology 4 (2005) 539.

363 L. Wang, Y. Gao, Q. Xue, H. Liu, and T. Xua, Effects of Nano-Diamond Particles on the Structure and Tribological Property of Ni-matrix Nanocomposite Coatings, Mater. Sci. Eng. A 390 (2005) 313.

364 D. Anderson, H. Knoedler, S. Tiku, Cycle Time Reduction During Electroplating of Through Wafer Vias For Backside Metallization of III-V Semiconductor Circuits, 2003 International Conference on Compound Semiconductor Manufacturing Technology.

365 T. Fritz, H. S. Cho, K. J. Hemker, W. Mokwa, U. Schnakenberg, Characterization of Electroplated Nickel, Microsyst. Technol. 9 (2002) 87.

366 P. Xiong-Skib, D. Engelhaupt, R. Hulguin, B. Ramsey, Effect of Pulse Plating Parameters on the Composition of Alumina/Nickel Composite, J. Electrochem. Soc. 152 (2005) C571.

367 A.M. El-Sherik, U. Erb, J. Page, Microstructural Evolution in Pulse Plated Nickel Electrodeposits, Surf. Coat. Technol. 88 (1996) 70.

368 F. Cai, Q. Yang, X. Huang, R. Wei, Microstructure and Corrosion Behaviour of CrN and CrSiCN Coatings, J. Mater. Eng. Perform. 19 (2010) 721.

369 F. Cai, X. Huang, Q. Yang, R. Wei and D. Nagy, Microstructure and Tribological Properties of CrN and CrSiCN Coatings, Surf. Coat. Technol. 205 (2010) 182.

370 F. Mark, N.M. Bikales, C.G. Overberger and G. Menges, Encyclopaedia of Polymer Science and Technology, John Wiley and Sons, New York, 1985. 
371 J. Jang, B. Lim, J. Lee, T. Hyeon, Fabrication of a Novel Polypyrrole/Poly (methyl methacrylate) Coaxial Nanocable Using Mesoporous Silica as A Nanoreactor, Chem. Commun. (2001) 83.

372 P. Hoyer and H. Masuda, Electrodeposited Nanoporous $\mathrm{TiO}_{2}$ Film by a Two-step Replication Process from Anodic Porous Alumina, J. Mater. Sci. Lett. 15 (1996) 1228.

373 S. Kang, P. C. Su, Y. I. Park, Y. Saito, F. B. Prinz, Thin-film Solid Oxide Fuel Cells on Porous Nickel Substrates with Multistage Nanohole Array, J. Electrochem. Soc. 153 (2006) A554.

374 Q. Yang, F. Cai, L.R. Zhao, X. Huang, Improving Corrosion Resistance of CrTiAlN Coating by Post-deposition Treatments, Surf. Coat. Technol. 203 (2008) 606.

375 P.S. Alexopoulos and T.C. O'Sullivan, Mechanical Properties of Thin Films, Annu. Rev. Mater. Sci. 20 (1990) 391.

376 W.C. Oliver and G.M. Pharr. Measurement of Hardness and Elastic Modulus by Instrumented Indentation: Advances in Understanding and Refinements to Methodology. J. Mater. Res. 19 (2004) 3.

377 H. Jehn, G. Reiners, N. Siegel, Charakterisierungdiinner Schichten, DIN Fachbericht 39, Beuth Verlag, Berlin 1993.

378 Günter Berg, Christoph Friedrich, Erhard Broszeit, Christina Berger, Development of Chromium Nitride Coatings Substituting Titanium Nitride, Surf. Coat. Technol. 8687 (1996) 184.

379 W.C. Oliver and G.M. Pharr, An Improved Technique for Determining Hardness and Elastic Modulus Using Load and Displacement Sensing Indentation Experiments, J. Mater. Res. 7 (1992) 1564.

380 ASTM G99-04 Standard Test Method for Wear Testing with a Pin-on-Disk Apparatus, American Society for Testing and Materials, West Conshohocken, Pennsylvania, 2004.

381 ASTM G76-02 Standard Test Method for Conducting Erosion Tests by Solid Particle Impingement Using Gas Jets, American Society for Testing and Materials, West Conshohocken, Pennsylvania, 2002.

382 N. Birks, F.S. Pettit, D.M. Rishel, Erosion-Corrosion and Wear, Journal de Physique IV, Colloque C9, supplément au Journal de Physique III 3 (1993) 667.

383 A.W. Bott, Practical Problems in Voltammetry 3: Reference Electrodes for Voltammetry, Current Separations 14 (1995) 64.

384 ASTM G59-97 (reapproved 2003) Standard Test Method for Conducting Potentiodynamic Polarization Resistance Measurements, American Society for Testing and Materials, West Conshohocken, Pennsylvania, 2003. 
385 ASTM G102-89 (reapproved 1999), Standard Practice for Calculation of Corrosion Rates and Related Information from Electrochemical Measurements, American Society for Testing and Materials, West Conshohocken, Pennsylvania, 1999.

386 ASTM G5-94, Standard Reference Test Method for Making Potentiostatic and Potentiodynamic Anodic Polarization Measurements, American Society for Testing and Materials, West Conshohocken, Pennsylvania, 2002.

387 Gamry Instruments, Inc., Gamry Instruments Software Tutorials and Primers, Rev. 4.2, 2003.

388 Gamry Instruments, Inc., Equivalent Circuit Modeling Using the Gamry EIS300 Electrochemical Impedance Spectroscopy Software, Gamry Application Notes 2003.

389 S.K. Ghosh, A.K. Grover, G.K. Dey, M.K. Totlani, Nanocrystalline Ni-Cu Alloy Plating by Pulse Electrolysis, Surf. Coat. Technol. 126 (2000) 48.

390 I.I. Aksenov, V.M. Khoroshikh, N.S. Lomino, V.O. Ovcharenko, Yu.A. Zadneprovskij, Transformation of Axial Vacuum-arc Plasma Flows into Radial Streams and Their Use in Coating Deposition Processes, in Discharges and Electrical Insulation in Vacuum, Proceedings ISDEIV. XVIIIth International Symposium 2, Eindhoven, Netherlands (1998) 566.

391 I.I. Aksenov, V.A. Belous, V.M. Khoroshikh, Formation of Filtered Intense Vacuumarc Plasma Flows, presented at XVIIth Int. Symp. Discharges and Electrical Insulation in Vacuum, Berkeley, CA, 1996, 895.

392 C. Suryanarayana and M.G. Norton, X-Ray Diffraction: A Practical Approach. Plenum Press, New York and London, 1998.

393 J.T.M. De Hosson, N.J.M. Carvalho, Y. Pei, and D. Galvan, Electron Microscopy Characterization of Nanostructured Coatings, edited by Albano Cavaleiro and Jeff Th. M. De Hosson, Springer Science+Business Media, LLC, New York, 2006.

394 J. Musil, P. Dohnal, and P. Zeman, Physical properties and high-temperature oxidation resistance of sputtered $\mathrm{Si}_{3} \mathrm{~N}_{4} / \mathrm{MoNx}$ nanocomposite coatings, Vac. Sci. Technol. B 23 (2005) 1568.

395 T. Y. Tsui, G. M. Pharr, W. C. Oliver, C. S. Bhatia, R. L. White, S. Anders, A. Anders, I. G. Brown, Mechanical Behaviour of Diamond and Other Forms of Carbon, Mat. Res. Soc. Symp. Proc. 383 (1995) 447.

396 J. Musil, F. Kunc, H. Zeman, H. Poláková, Relationships between Hardness, Young's Modulus and Elastic Recovery in Hard Nanocomposite Coatings, Surf. Coat. Technol. 154 (2002) 304.

397 A. Lasalmonie, J.L. Strudel, Influence of Grain on the Mechanical Behaviour of Some High Strength Materials, J. Mater. Sci. 21 (1986) 1837.

398 S. Veprek, S. Reiprich, L. Shizhi, Superhard Nanocrystalline Composite Materials: the TiN/ $\mathrm{Si}_{3} \mathrm{~N}_{4}$ System, Appl. Phys. Lett. 66 (1995) 2640. 
399 J.W. Kim, K. H. Kim, D.B. Lee, J.J. Moore, Study of High-temperature Oxidation Behaviour of Cr-Si-N films, Surf. Coat. Technol. 200 (2006) 6702.

$400 \mathrm{~K}$. Miyoshi, Solid Lubricants and Coatings for Extreme Environments: State-of-theArt Survey, NASA/TM-2007-214668, Glenn Research Center, Cleveland, Ohio, 2007.

401 D.A. Rigney, Some Thoughts on Sliding Wear, Wear 152 (1992) 187.

402 M. Kerridge, J.K. Lancaster, The Stages in A Process of Severe Metallic Wear, Proc. R. Soc. London, Ser. A 236 (1956) 250.

403 D.A. Rigney, Transfer, Mixing and Associated Chemical and Mechanical Processes during the Sliding of ductile Materials, in special issue of Wear, D.A. Rigney (Ed.), based on International Symposium in Hyderabad, India, Wear 245 (2000) 1.

404 B. Bhushan, Chapter 7 Introduction to Tribology, Wiley and Sons, New York, 2002.

405 J.R. Macdonald, Impedance Spectroscopy-Emphasizing Solid Materials and System, John Wiley \& Sons, 1987.

406 N. Wagner, Fuel Cells, in E. Barsoukov and J.R. Macdonald (Eds.), Impedance Spectroscopy Theory, Experiment, and Applications ( $2^{\text {nd }}$ Ed.), John Wiley \& Sons, Inc., Hoboken, New Jersey, 2005.

407 P. Duncumb and P. K. Shields, The Present State of Quantitative X-ray Microanalysis Part 1: Physical Basis, Br. J. Appl. Phys. 14 (1963) 617.

408 J.E. Randles, Kinetics of Rapid Electrode Reactions, Discuss. Faraday Soc. 1 (1947) 11.

409 Gamry Instruments, Inc., Basics of Electrochemical Impedance Spectroscopy, Gamry Application Notes Rev. 1.0, 2010.

410 IUPAC Compendium of Chemical Terminology (2nd ed.) 1997.

411 A.J. Bard and L.R. Faulkner, Electrochemical Methods, Fundamentals and Applications, Wiley, 2000.

412 J. Piippo, B. Elsener, H. Bohni, Impedance Study on Thin Inorganic Coatings, Mater. Sci. Forum 111-112 (1992) 219.

413 F. Mansfeld, L.T. Han, C.C. Lee, G. Zhang, Evaluation of Corrosion Protection by Polymer Coatings Using Electrochemical Impedance Spectroscopy and Noise Analysis, Electrochim. Acta 43 (1998) 2933.

414 T.K. Rout, Electrochemical Impedance Spectroscopy Study on Multi-layered Coated Steel Sheets, Corros. Sci. 49 (2007) 794.

415 P. Grathwohl, Diffusion in Natural Porous Media: Contaminant Transport, Sorption / Desorption and Dissolution Kinetics, Kluwer Academic Publishers, 1998. 


\section{Appendix: List of Publications}

\section{Conference}

[1] F. Cai, X. Huang and Q. Yang, Effect of Microstructure on the Tribological Properties of Nickel Plating, 18th Canadian Materials Science Conference, McGill University, Montreal, Canada, 2006.

\section{Journals}

[1] F. Cai, X. Huang, Q. Yang, R. Wei and D. Nagy, Microstructure and Tribological Properties of CrN and CrSiCN Coatings, Surf. Coat. Technol. 205 (1) (2010) 182.

[2] F. Cai, Q. Yang, X. Huang, and R. Wei, Microstructure and Corrosion Behaviour of $\mathrm{CrN}$ and CrSiCN Coatings, J. Mater. Eng. Perform. 19 (5) (2010) 721.

[3] F. Cai, Q. Yang, X. Huang and L.R. Zhao, Corrosion Behaviour of As-deposited and PMMA Treated TiN Coating Systems Deposited by Cathodic Arc Technique, Corros. Eng. Sci. Technol., (2010), DOI: 10.1179/147842209X12489567719626.

[4] F. Cai, X. Huang, Q. Yang, and D. Nagy, Effect of Microstructure on the Solid Particle Erosion Properties of Ni Plating, J. Mater. Eng. Perform. 18 (3) (2009) 305.

[5] X. Huang, G. Franchi, F. Cai, Characterization of Porous Bi-modal Ni Structures, J. Porous Mater. 16 (2) (2009) 165.

[6] Q. Yang, F. Cai, L.R. Zhao, X. Huang, Improving Corrosion Resistance of CrTiAlN Coating by Post-deposition Treatments, Surf. Coat. Technol. 203 (5-7) (2008) 606.

[7] F. Cai, Q. Yang, X. Huang and D. Nagy, Alleviating Detrimental Effects of Defects on Corrosion Performance of TiN Coating by Post-deposition Treatment Using Polymethyl Methacrylate, Surf. Eng. 24 (6) (2008) 436.

[8] Q. Yang, L.R. Zhao, F. Cai, S. Yang, D.G. Teer, Wear, Erosion and Corrosion Resistance of CrTiAlN Coating Deposited by Magnetron Sputtering, Surf. Coat. Technol. 202 (16) (2008) 3886. 